\title{
Getting Closer to Communities: The Warmth of Community Well-being in the Local Government Act 2002
}

\author{
By
}

\author{
Muhammad Mubashir Mukhtar
}

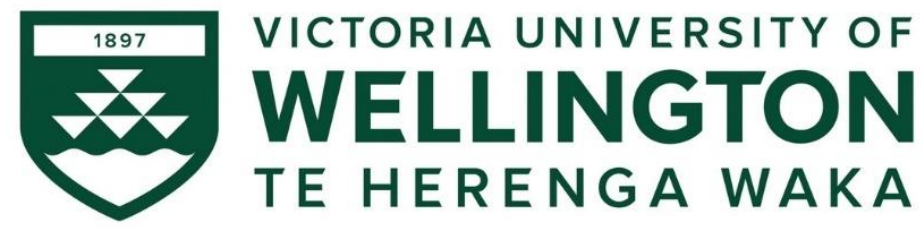

A thesis submitted to the Victoria University of Wellington in fulfilment of the requirements for the degree of Masters by Thesis in Public Policy, School of Government

Victoria University of Wellington

(January 2021) 


\begin{abstract}
The reintroduction of community well-being as a purpose, into the Local Government Act 2002, has created the opportunity for the Councils to engage with their communities in a more holistic way. Although the Councils are consulting within their jurisdictions, this is currently more focused on the core services delivered or assets managed by them. With the community well-being framework in place, the Councils will be able to monitor the developments within their communities from a social, economic, environmental, and cultural perspective. It will enable them to participate on the ground level and to raise the volume with the central government agencies on what matters most for their communities.
\end{abstract}

The enabling factor for this conversation to happen is sitting with the extensiveness of community engagement from the Council side. Through these engagements, the Councils will be able to identify and prioritise the outcomes which their communities' value. These outcomes will be impacting on the Council business as to what and how they will deliver the core services.

In this thesis, we will be looking into the history and role of community well-being in the Local Government Act 2002, and its evolution into the Local Government (Community Well-being) Amendment Act 2019. Along with it, we intend to identify a framework and a set of indicators on community well-being which are common across the local government sector.

This research will primarily help the staff members of the Councils to grasp the dynamics of community well-being and to incorporate it in their various strategic plans and reports. Secondly, it will help the community organisations and contractors to work more closely with the Councils and see how they can have a more positive impact on their communities. Thirdly, this piece will seek to align the well-being work of the local government sector with the work currently undergoing at the central government level; specifically, the Living Standards Framework (LSF) at The Treasury and Indicators Aotearoa New Zealand (IANZ) at Statistics New Zealand. 


\section{Acknowledgements}

Alhamdulillah, all praise to Allah swt who gave the strength and knowledge to undertake this research project. I would like to specifically mention few names in here who encouraged, supported and inspired in this whole journey. Starting from my mother Qaiser Mukhtar who encouraged, pushed and inspired in every way of life. This thesis wouldn't been completed without her prayers. I know that it's not going to stop here as now she wants me to pursue PhD. I miss my father Mukhtar Ahmed but feel his prayers around all the time. Have to definitely give a huge thanks for untiring support from my wife Iqra who sacrificed uncountable weekends and evenings while I kept on working. Also thanks to her in looking after our three young children who sneaked every opportunity to start jumping in my study room and take out printer pages whenever possible.

The unique guidance which I keep on getting from my mentors were also very helpful to keep the balance and focus. Thanks to my Sheikh Hazrat Feroz Abdullah Memon for his ever guidance around life balance and prayers for my success. Am also thankful to Dr Asad Zaman who kept on giving feedbacks and guidance over the time. Also thanks to Dr Shahid Qureshi, Dr Zeeshan Ahmed, Dr Azam Ali, Dr Muhammad Umair and Mufti Sarfraz Nehal who kept on encouraging.

This thesis wouldn't have been started or completed without the support and guidance of various colleagues whom I worked with over the time. A big thankyou to Vincie Billante, Toni Durham (both from Ashburton District Council), Pim Borren (from Masterton District Council and SOLGM), Karen Thomas, Kate Macnaught, Raymond Horan, Chelsey Reid (all four from New Zealand Society of Local Government Managers (SOLGM)).

There are couple of people who inspired me to take this topic and thanks is truly due to them as well. Thankyou Dr Asad Zaman (from Pakistan Institute of Development Economics), Dr Syed Salman Ali (from Islamic Development Bank), Dr Mike Reid (from Local Government New Zealand), Karen Thomas (from SOLGM), Dr Ganesh Nana (from BERL), Hon Minister Nanaia Mahuta (Minister for Local Government, Foreign Affairs and Maori Development). 
The list might not be exhaustive, but it will be absolutely incomplete without a special thanks of gratitude to my thesis supervisor Dr Girol Karacaoglu. I am thankful to the support, wisdom and patience given by you. I am also awed with the level of passion which you have in this area of research and feel lucky to have you as my supervisor. You have inspired me a lot, taught me a lot and keenly looking forward for the next project of PhD. I am really thankful on your support during the two major events (15 March 2019 incident and COVID-19 Local Government Response workload) which diverted me from this project for a while, your checkins and catch ups really helped and motivated me, thank you!

Lastly a huge thanks to my three sisters, family, friends and colleagues who constantly provided me with moral support. I needed every bit of it to keep on going and completing this.

Thank you all! 


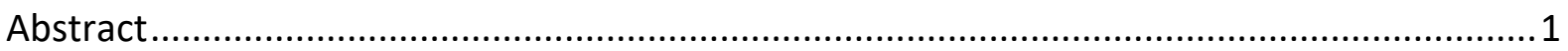

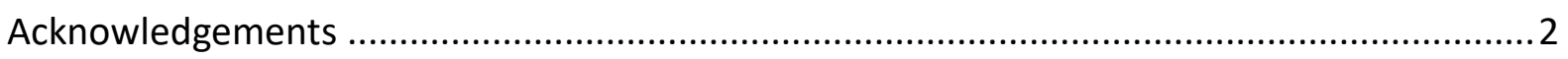

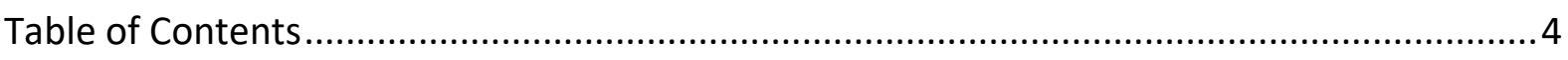

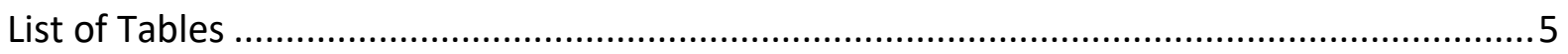

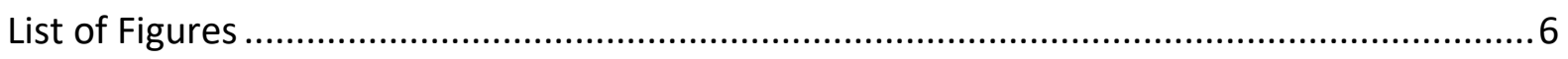

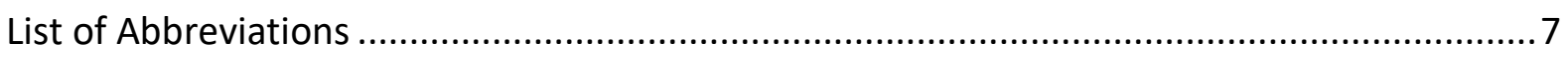

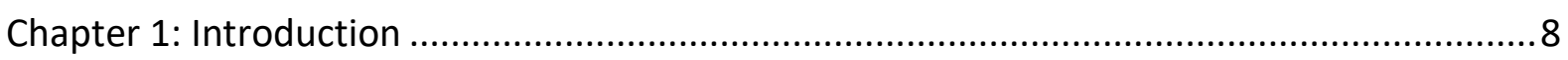

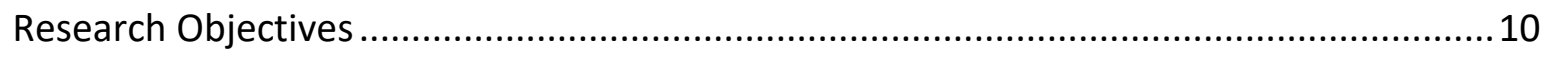

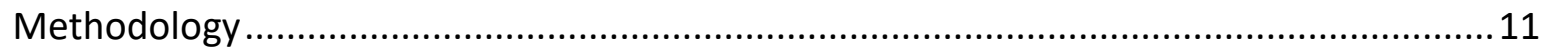

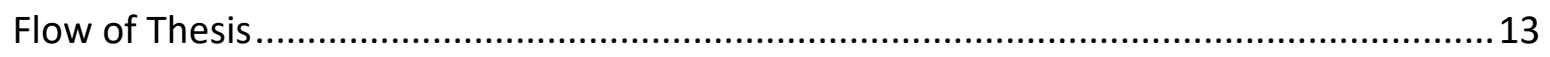

Chapter 2: History of Community Well-being in Local Government Act 2002 ....................... 15

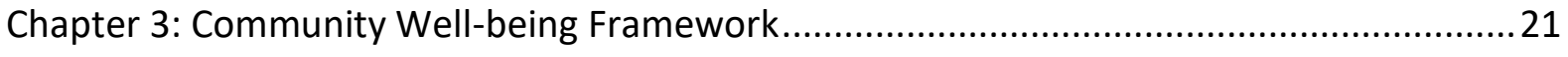

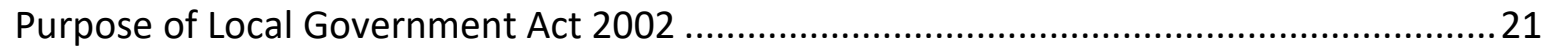

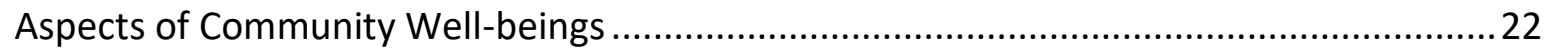

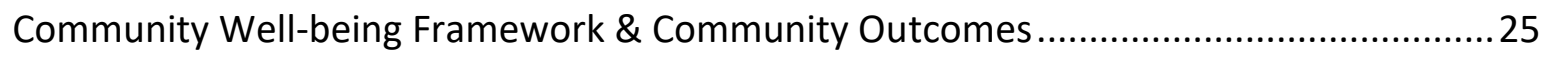

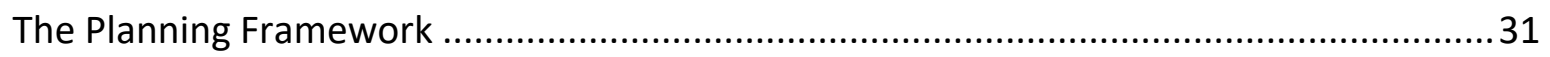

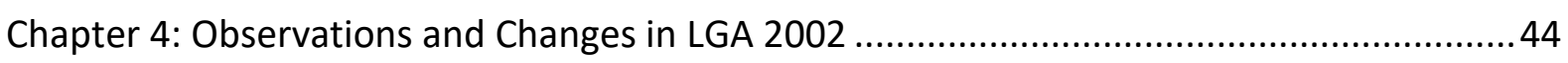

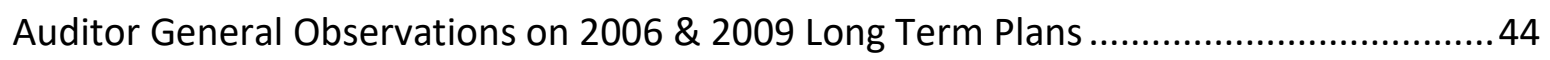

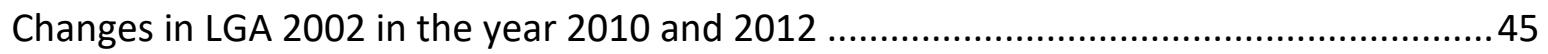

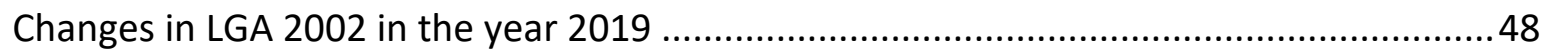

Chapter 5: Set of Measures Common Across the Councils ............................................... 50

Measures selection through long term plan 2009-19 …..............................................50

Chapter 6: Aligning the Community Well-being Measures with LSF and IANZ .....................62

Chapter 7: Case Study: Community Well-beings in Ashburton District Council.....................67

Chapter 8: Conclusion and Further Research Directions................................................ 78

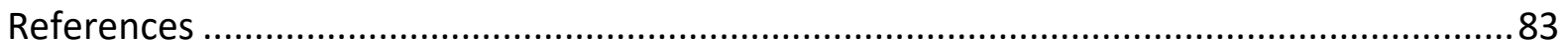

Appendix A: List of Community Well-being Outcomes and Measures by Councils ................ 90

Appendix B: Community Well-being Statements by Councils.............................................28

Appendix C: Coverage of Community Well-being Measures in Living Standard Framework ..343

Appendix D: Coverage of Community Well-being Measures in Indicators Aotearoa New Zealand. 


\section{List of Tables}

Table 1: Existing and Proposed Changes in Local Government Act..........................................20

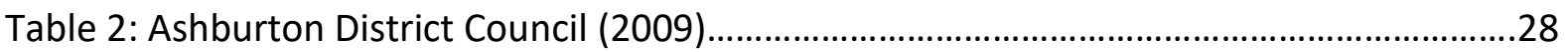

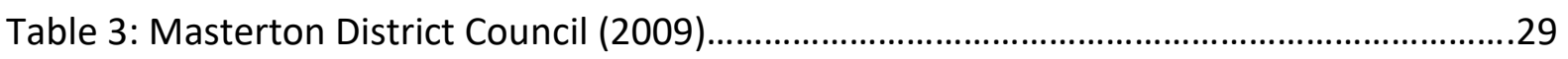

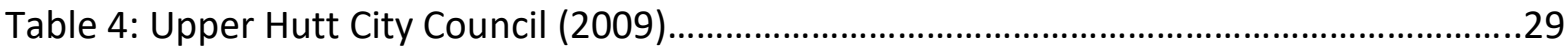

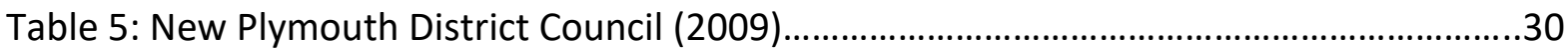

Table 6: Mackenzie District Council (2009).................................................................................

Table 7: Cluster Analysis Model.................................................................................................54

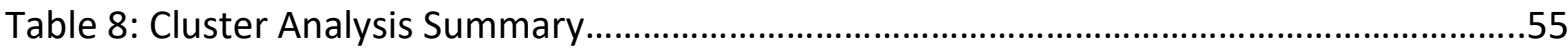

Table 9: Community Outcomes and Measures from Ashburton District Council

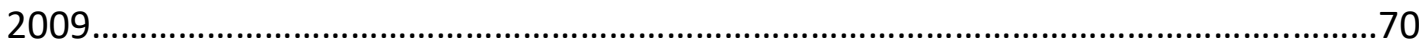

Table 10: Community Outcomes and Strategic Objectives from Ashburton District Council 2009 


\section{List of Figures}

Figure 1: Community Well-being in LGA 2002 over the time............................................

Figure 2: SOLGM Community Well-being Indicator Framework .........................................2 27

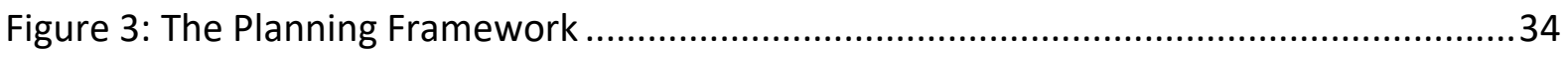

Figure 4: The Performance Framework for Local Government (SOLGM, 2020) ....................36

Figure 5: Components of the City Strategic Framework (Hamilton City Council, 2009) .........37

Figure 6: Council's Contribution to Tauranga Tomorrow (Tauranga City Council, 2009) ........38

Figure 7: Council's Contribution to Outcomes (Grey District Council, 2009) .........................39

Figure 8: Community Outcomes, Details for 1st Outcome (Ashburton District Council, 2009)

Figure 9a: Community Outcomes, Details for 3rd Outcome (Masterton District Council, 2009)

Figure 9b: Community Outcomes, Details for 3rd Outcome (Masterton District Council, 2009)

Figure 10: Community Outcomes, Details for 1st Outcome (Hurunui District Council, 2009) 43

Figure 11: Community Outcomes Data Availability Dashboard ..........................................56

Figure 12: Overview of the Community Well-beings and Comparison with LSF and IANZ .....57

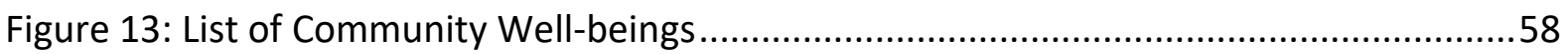

Figure 14: List of Community Well-being Statements ........................................................59

Figure 15: Broad Group of Measures by Number of Measures, Number of Outcomes and

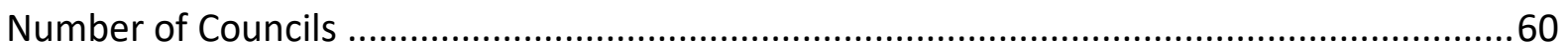

Figure 16: Broad Community Well-being Measures........................................................61

Figure 17: Coverage of Community Well-being Measures in Living Standard Framework.....65

Figure 18: Coverage of Community Well-being Measures in Indicators Aotearoa New Zealand

Figure 19: ADC Community Outcomes and Strategic Planning 72 


\section{List of Abbreviations}

$A D C$

AG

AP

AR

$\mathrm{AU}$

CWB

DIA

GDP

GSS

IANZ

LGA

LGAA

LGAB

LGNZ

LSF

LTCCP

LTFS

LTP

MBIE

NZ

NZTA

OAG

SA2

SMART

SOLGM

Stats NZ
Ashburton District Council

Auditor General

Annual Plan

Annual Report

Area Unit

Community Well-being

Department of Internal Affairs

Gross Domestic Product

General Social Survey

Indicators Aotearoa New Zealand

Local Government Act

Local Government Amendment Act

Local Government Amendment Bill

Local Government New Zealand

Living Standard Framework

Long Term Council Community Plan

Long Term Financial Strategy

Long Term Plan

Ministry of Business, Innovation and Employment

New Zealand

New Zealand Transport Authority

Office of Auditor General

Statistical Area 2

Specific, Measurable, Achievable, Relevant and Timebound

New Zealand Society of Local Government Managers

Statistics New Zealand 


\section{Chapter 1: Introduction}

Community well-being was first introduced in the Local Government Act 2002 (LGA 2002). Then in the year 2004, the local authorities used it for the first time to develop community outcomes and the monitoring reports. These outcomes were then used to develop the Long Term Council Community Plan (LTCCP) of 2006 and 2009. However later in the year 2012 they were repealed from the LGA 2002 and after a gap of around seven years they are now (2019) introduced back into the LGA 2002. These community well-beings will be used again by the local authorities in developing the Long Term Plan (LTP) of 2021. See Figure 1 for the flow of this progress over the time.

Figure 1: Community Well-being in LGA 2002 over the time.

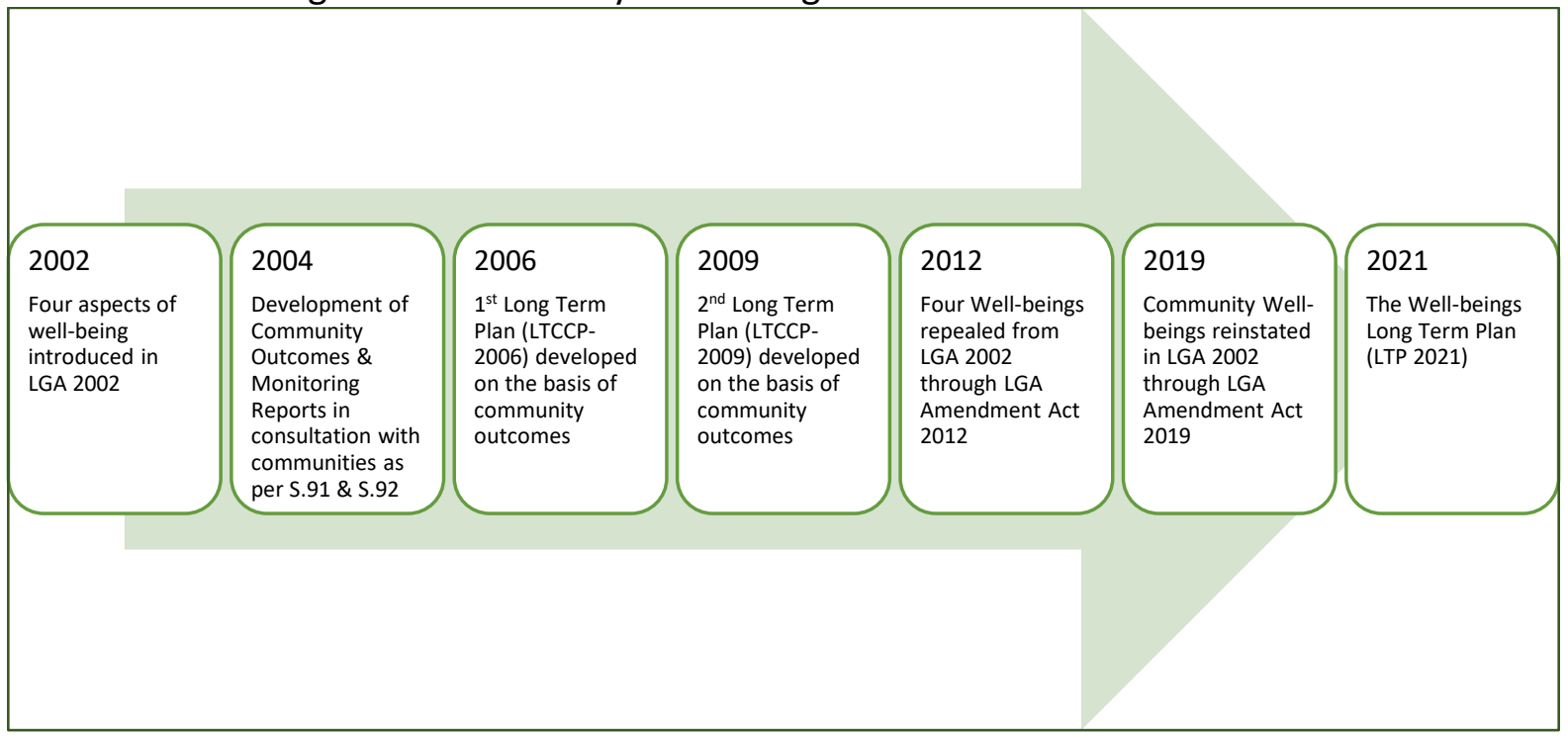

The latest effort of reinstating the community well-being in LGA 2002 started on 11 April 2018 by Hon Nanaia Mahuta, Minister of Local Government, where she introduced the Local Government (Community Well-being) Amendment Bill 2018 (LGAB 2018) in the Parliament. After going through the process, the Bill was passed and assented on 13 May 2019 and is now known as the Local Government (Community Well-being) Amendment Act 2019 (LGAA 2019).

The LGAA 2019 looked into reinstating the purpose of the LGA 2002, which is to "provide for local authorities to play a broad role in promoting the social, economic, environmental, and cultural well-being of their communities (the four aspects of well-being), taking a sustainable 
development approach" (cl. 4) ${ }^{1}$. This Act also reinstates a section under principles that the local authorities have to take into account: "the likely impact of any decision on each aspect of well-being" (cl. 7) ${ }^{2}$. Another significant change made in this Act is on replacing the definition of community outcomes (cl. 5(1)), with: "community outcomes means the outcomes that a local authority aims to achieve in order to promote the social, economic, environmental, and cultural well-being of its district or region in the present and for the future". These amendments have taken the LGA 2002 nearly to its position before to the Local Government Act 2002 Amendment Act 2012 (LGAA 2012).

Broadly, there were three objectives for the Local Government (Community Well-being) Amendment Bill 2018: (i) reinstate the community well-being in the purpose of local government, (ii) restore the power to collect development funds by local authorities to assist in providing facilities to the communities, and (iii) a minor clarification on the financial assistance provided by New Zealand Transport Authority (NZTA) so that it doesn't affect the collection of development funds (previous objective) (LGAB 2018, pg.1). There is a view that local government has a larger role to play as it is the nearest one to the community and can understand the dynamics of it in a much better way than the central government, or more holistically than any other agency (Stewart and Clarke, 1996). Thus, there is much to do by the local government, in addition to service delivery and asset management (Mahuta, 2018).

With the given position of Councils, they can easily engage and consult with their communities. Through this engagement, they can identify the outcomes that the community deems as important and prioritise these as well. The wider purpose being pursued is to identify and then try to meet the immediate and long-term needs of the district or region in terms of community well-being. The revised Act gives a direction to the Council for utilisation of community resources and also aligns it with other organisations. Thus, it creates a relationship between the Council, the communities, Maori, the private sector, local agencies, regional agencies, and central government agencies. These community outcomes are to be reviewed and reported in Long-Term Plans, which are developed every three years, reporting

\footnotetext{
${ }^{1}$ Local Government (Community Well-being) Amendment Act 2019.

${ }^{2}$ Local Government (Community Well-being) Amendment Act 2019.
} 
on progress towards achieving the community outcomes and any significant alignment of them where needed.

The focus of the current government on well-beings increased the priority of a couple of important projects for the central government agencies. The Government wanted to develop the world's first Well-being Budget in 2019. In this regard, the top two projects which were developed by the central agencies were: Living Standards Framework (LSF) at The NZ Treasury and Indicators Aotearoa New Zealand (IANZ) at Statistics New Zealand. The LSF is based on the Organisation for Economic Co-operation and Development's (OECD's) well-being approach, 'Better Life Initiative: Measuring Well-Being and Progress'. It also draws on the consultations with domestic experts on well-being. On the other hand, IANZ is primarily based on a bottom-up approach where Stats NZ have consulted with New Zealanders on what wellbeing means to them, and what aspects of well-being matter most to them. For Stats NZ it was important that the indicators reflected the people's preferences and not only the Government's or the public sector's perspectives on what matters most for their well-being.

There was a very clear message from the Prime Minister in her public address earlier that, "it will no longer be good enough to say a policy is successful because it increases GDP if, at the same time, it also degrades the physical environment, or drives down wages or fractures a community [...] we want New Zealand to be the first place in the world where our budget is not presented simply under the umbrella of pure economic measures, and often inadequate ones at that, but one that demonstrates the overall well-being of our country and its people" (Walters, 2018).

\section{Research Objectives}

There are multiple research objectives for this thesis, which are listed below:

1. To gain insight on community well-being in the Local Government Act 2002;

2. To identify a framework for the local government sector on community well-being;

3. To identify a set of indicators for the local government sector on community wellbeing; 
4. To seek the possible alignment of well-being projects in local and central government; and

5. To carry out a case study on a district council covering community well-beings usage.

\section{Methodology}

For the first objective, we conducted an extensive literature review focusing on the community well-being as in Local Government Act 2002. Looking into the need for reviewing the Local Government Act 1974, the introduction of community well-being in Local Government Act 2002, the developments of community outcomes by the Councils, and using these outcomes for reporting purposes in long-term plans of the Councils. Further, the literature review also looked into the repealing of community well-being from Local Government Act 2002 in the year 2012, and the re-introduction of it through the Local Government (Community Well-being) Amendment Act 2019.

The second objective, of identifying a community well-being theoretical framework, looked a bit deeper into the LGA 2002 and the operationalisation of it from a council perspective. The theoretical framework of community well-being brought together the concept, definitions, and the level of interventions which are needed to operationalise the concept of community well-being.

Further on, the third objective of developing a set of indicators was carried out by compiling community outcomes, indicators and measures within it. They were compiled from the 2009 Long Term Plans (LTP) of the District and Regional Councils. The year 2009 was our best option to compile this information because after the royal assent on Local Government Act 2002 the community outcomes were developed in 2004 by each Council and they were used for the first time in the 2006 LTCCP round. The next time they were used in the 2009 LTP, but afterwards in 2012 the community well-being was repealed from the purpose of LGA 2002. Due to this fact some Councils stopped reporting on it, while some kept on continuing reporting on it. For this reason, the best option for this study was to look into the LTP 2009 and compile the whole set of indicators. Common themes and indicators under each well- 
being across the whole local government sector (i.e., 78 city, district and regional councils) are pulled together and presented.

For the fourth objective a comprehensive view of the top two projects that were developed by the central government agencies was carried out. The aim was to seek an alignment of them with the common set of local government community well-being indicators. We tried to trace the linkages of these projects at the local level or community level. Starting from their framework and down to the list of indicators, we have compiled these projects and then compare them with local government community well-being with a set of criteria based on our learnings from the previous objectives.

Lastly for the fifth objective we carried out a case study of a district council to affirm our findings and see how they have responded to the changes in the LGA 2002 from a community well-being perspective. The long-term plans of 2009 and 2018 along with other published reports and articles of Ashburton District Council were reviewed in detail.

This study has a more qualitative approach that heavily relies on the existing literature. With a tremendous amount of changes in the policy over a relatively short period, this study will help the staff members of the Councils to grasp the dynamics of community well-being and to incorporate it in their various strategic plans and reports. As the community well-being was removed as a purpose in the year 2012, there is a gap in the institutional knowledge within the Councils which can be compensated by this study. On the other hand, from the derived framework and the set of indicators, this study will help the community organisations and contractors to work more closely with the Councils as they will be able to see their role in bringing a positive impact on their communities. Lastly the alignment work between the local government and central government is also beneficial as it uncovers the linkages running across the sectors. 


\section{Flow of Thesis}

This study is divided into five chapters which builds upon each other and takes a deeper view in a certain aspect in each chapter. Starting from the history of inclusion of community wellbeing, the first chapter discusses the major reform in the Local Government Act, where the 1974 version of the Act was updated in the year 2002, thus making it more fit for purpose for the current times. The second chapter discusses the community well-being framework and its operationalisation. In this chapter we also discuss the process of identifying the community outcomes, its usage in the long term plans and the development of strategies for the Council. The third chapter covers the observations from the Auditor General (AG) and the Department of Internal Affairs (DIA) with regard to the community outcomes and the long term plans, and how they can be developed further for the betterment of the sector. In this same chapter we also looked into the rationale of the 2012 government for removing the community wellbeings from the LGA 2002 and subsequent efforts of 2018 government to bring it back in the LGA 2002. The fourth chapter looks into identifying the common set of community outcomes, indicators and measures used by the local authorities in their long term plans of 2009. After identifying these common indicators, we also compared these with the work of other two central government agencies which are on similar lines. In the fifth chapter we carried out a case study on Ashburton District Council in which we looked into their long term plans of 2009 and 2018, their strategic documents and published articles on the utilisation of community well-being in the Council. Lastly, we summarised our work and provided a conclusion along with the further directions of work which could be helpful in providing further insights from an operationalisation perspective for the local authorities. Below is the list of the chapters with major topic headings within each chapter:

1. History of community well-being in Local Government Act 2002

2. Community Well-being Framework
a. Purpose of Local Government Act 2002
b. Aspects of Community Well-beings
c. Community Well-being Framework \& Community Outcomes
d. The Planning Framework

3. Observations and Changes in LGA 2002 
a. Auditor General \& DIA observations for 2006 \& 2009

b. Change of LGA 2002 in the year 2012

c. Change of LGA 2020 in the year 2018

4. Set of indicators common across the councils

a. Indicators selection through LTP 2009

b. Aligning the indicators with LSF and IANZ

5. Case study of Ashburton District Council on their usage of community well-being 


\section{Chapter 2: History of Community Well-being in Local Government Act 2002}

The long winding story of community well-being and the Local Government Act 2002 is full of ups and downs; and twists and turns. In March 2000, it was decided to review the then existing Local Government Act 1974 along with other local government legislations. This generated a huge amount of interest from both the local and central government. The reason behind this interest was that the LGA 1974 was not seen as fit for purpose according to the recent times; it was seen as "cumbersome, contradictory and out of date" (Carter, 2002). It was having a heavy prescriptive touch, which created legal uncertainties thus leading to a constant need for amendments in the Act.

The local government is considered as a key partner of the central government as it plays an important and significant role in delivering national goals and priorities (Department of the Prime Minister and Cabinet, 2019). The LGA 1974 was an important piece of legislation defining the power and responsibility of local authorities but in its current state it was limiting the local government's effectiveness. This raised the requirement of replacing it with a new LGA which would meet the need of the current communities and bring sustainable local development. After deciding to review the LGA the government circulated a consultation document in August 2001 that contained four broad objectives and elements for developing new legislation (Department of Internal Affairs, 2001, p.5-6.):

1. To review the purpose of local government that would reflect a coherent overall strategy of local government;

2. To streamline and enhance the effectiveness of local government so that they would have powers and can meet the needs of their communities;

3. To develop ways which would create partnerships between central and local government;

4. To clarify the local government's relationship between Maori and the Treaty of Waitangi.

The review of the purpose of the local government was a centre stage agenda in this consultation document. The LGA 1974 didn't clearly express the purpose of the local 
government. It was proposed that the purpose of the local government should be "to enable local decision-making by and on behalf of citizens in their local communities to promote their social, economic, cultural and environmental well-being in the present and for the future" (Department of Internal Affairs, 2001, p.6.). Along with the purpose, was a proposal for increasing the powers of the local government. In LGA 1974, due to its prescriptive nature, it was viewed that local government can only involve itself in the things that were mentioned in the LGA, which created bottlenecks for the progressive work. In contrast, it was proposed that the local government have the "scope to choose the activities they undertake and how they should undertake them (subject to public consultation processes)" (Department of Internal Affairs, 2001, p.6.). These powers were restrained through further clauses that local government can only undertake the activities which are consistent with the objective of the new legislation; it has to consult with the community first; and "it complies with the law, including specific provisions in the new Act as well as other statutes" (Department of Internal Affairs, 2001, p.7.).

With the emphasis on the community as the core of the new legislation, it became clearer that "what local government does is directly relevant to the active protection of Maori culture and way of living, and requires ongoing interaction with Maori as an important part of the community" (Department of Internal Affairs, 2001, p.7.). Covering the broad spectrum of agendas, there was also a need to develop some best-practice evolutionary mechanism which would "streamline, enhance and refine the effectiveness of the local government law" (Department of Internal Affairs, 2001, p.7). Overall, the new legislation was seen as a major tool for having a positive impact on communities which would bring back "localness to local government and to enhance its ability to recognise and respond to community aspirations", (Department of Internal Affairs, 2001, p.7).

It was in this context that community aspirations were linked to the community well-beings. Starting from providing a timely, clear, detailed and summarised planning information from the councils to the community and the groups within it. Which would then lead to a better participation of the community in the council decision making process. Councils were asked to "develop their own consultation mechanisms that best suit the needs of their communities" (Department of Internal Affairs, 2001, p.8.). This would give the communities 
an opportunity to let the Council know about their desired outcomes; in return, the Council could inform the communities about the possibilities in achieving their aspirations. It was "proposed that long term council plans will include the identification of desired community outcomes, the role of the council in achieving those outcomes through its services, activities and policies, and through partnerships with other bodies and organisations" (Department of Internal Affairs, 2001, p.8.).

It was intended that the review of LGA 1974 along these lines would create a platform for longer-term changes which would recognise the importance of local government in pursuing sustainable development in New Zealand. It would enable the local government to focus and promote social, economic, environmental, and cultural well-being in the short and long term. The enabling factor comprises of giving empowering and flexible powers so that the Councils could respond to the diverse needs of their communities. The revenue and financing decisions were seen to be integrated with the service delivery and planning processes and had a broad range of objectives to consider. It was in total contrast to the then current process that was too much reliant on economic analysis and leaned towards selling off Council owned assets or increasing the user pay scenario as a default funding options.

Efforts to push for rewriting the LGA 1974 was not a month or year task; discussions around the need for updating the Act can be traced back to at least the Local Government Association Conference held in 1996 (Reid, 2010). The other substantive effort can be traced to a forum organised by the Christchurch City Council in 1999, titled 'Community Governance: the Christchurch Forum' (Reid, 2010). After three years of the former conference, it started to gain some momentum due to the fact that it was adopted as a manifesto by the Labour and Alliance parties for the general elections of 1999. The stance of the Labour party on this issue was, that the "local bodies [should] operate with autonomy and freedom within a collaborative framework that allows levels of government to work for the best outcomes for communities" (Labour Party Election Manifesto, 1999). The need for reform was a matter of such significance that later down the road, it was acknowledged by the Government that: "the challenges facing New Zealand in areas such as sustainable development cannot be met by central government making decisions and acting on its own. They require a partnership 
approach within which central government, local government and the voluntary and business sectors can work together" (Lee 2001, p. 13).

In the forum organised by the Christchurch City Council, the main focus was on the communities' role in the local government. Along with international and local speakers, there were some papers also provided in this forum. A particularly interesting one was, 'Community Governance: Resource Kit' by Mike Richardson (1999). It outlined a new form of local government that was more focused on the community outcomes in contrast to its role of a service provider, which brings into context the role as a facilitator, developing networks, and participating in such a way that it covers a wide range of groups and individuals within the community (Reid, 2010). This theme of community governance can well be observed in the LGA 2002 where the community stands in the centre of all the things and the "explicit reference to promoting well-being in the purpose statement, the recognition of sustainability in the principles and the requirement to identify community outcomes - a form of community strategic planning - suggest an awareness of the instruments commonly associated with community governance" (Reid, 2010).

The consultation on the new Local Government Act was sought by the Department of Internal Affairs (DIA) in August 2001, and a subsequent report on its submission was released in October 2001 (Department of Internal Affairs, 2001b). In total, DIA received 655 submissions from various organisations, groups and individuals. Out of these 655 submissions, 312 covered the purpose of Local Government and there was a clear or qualified support in this regard (i.e., 197 (63\%) submissions out of 312). The idea that the Councils need to work closely with their communities was clearly supported. For example, from one of the submissions on the statement of purpose it was mentioned that "This purpose statement clearly establishes councils as an integral part of the governance system of New Zealand and provides flexibility in application for different parts of New Zealand" (Department of Internal Affairs, 2001b, p.13). There were some minor comments on making the LGA a bit clearer, e.g., removing the word citizen as that would be seen to address only those people who are legal citizens of New Zealand, and therefore exclude the other residents living in the area. As for submissions from Maori groups, it was advocated that the LGA must acknowledge the Treaty of Waitangi; and the Treaty must be acknowledged in the purpose of the LGA and in all of the policy objectives. 
There were some submissions from individuals, businesses and other groups that opposed the purpose statement and advocated that the councils should keep their focus on core services (Department of Internal Affairs, 2001b).

The key aspects of the consultation document were planning, consultation, and reporting, this is why many questions were to be asked in these regards. Table 1 below summaries the LGA 1974 decision-making process and the proposed changes (DIA 2001 p.38). Encouraging participation of communities and individuals in the local government decision-making was seen important and to make it happen it requires conveying information or data from the Council to these groups and keep them updated on the progress on the decisions made by the Council over the time. There were mixed sentiments regarding this aspect of the consultation document, where Councils had a view that they were doing pretty well in consulting with their communities. On the other hand, the communities felt that they were consulted in the later part of the proposed plans, where it failed to incorporate their opinions in the documents; however, it can be resolved by engaging them at the start of the process rather than later. Overall, the concept of long term plans was agreed upon by the councils and further suggested that there should be some directions in the LGA on community participation and engaging them through developing a partnership approach. The long term plan was seen as an integral document which will bring accountability of the Council to the community. There was also some proposal to develop regular monitoring reports that would give insights on the councils' performance and the results of community outcomes over the time, as stated in the long term plans (Department of Internal Affairs, 2001b).

The new Local Government Bill was introduced in the Parliament on 18 December 2001 and received royal assent on 24 December 2002; it came to be known as the Local Government Act 2002. The LGA 2002 replaced most of the LGA 1974 and it was the first comprehensive revision of the legislation relating to local government in 28 years. There were other legislative reforms happening around the same time which included Local Government (Rating) Act 2002 and the Local Electoral Act 2001 (DIA, LGNZ and SOLGM, 2003, p.24). 
Table 1: Existing and Proposed Changes in Local Government Act (DIA 2001, p.38)

Existing (LGA 1974)

Proposed (LGA 2002)

\begin{tabular}{|c|c|}
\hline $\begin{array}{l}\text { “Long Term Financial Strategy } \\
\text { - Along with borrowing Management } \\
\text { Policy, Funding Policy }\end{array}$ & $\begin{array}{l}\text { Long Term Council Community Plan } \\
\text { - Existing LTFS processes included plus } \\
\text { increased recognition of social, } \\
\text { environmental, cultural and economic } \\
\text { council work } \\
\text { - [Explicit addition of community outcomes] }\end{array}$ \\
\hline Annual Plan & Focused Annual Plan 3 \\
\hline Annual Report & Annual Report \\
\hline $\begin{array}{l}\text { Consultation } \\
\text { - Special consultative procedure }\end{array}$ & $\begin{array}{l}\text { Consultation } \\
\text { - Modified special consultative procedure } \\
\text { - Summary information for communities }\end{array}$ \\
\hline $\begin{array}{l}\text { Special delivery choices } \\
\text { - General obligation to consult on } \\
\text { specific significant changes }\end{array}$ & $\begin{array}{l}\text { Special delivery choices } \\
\text { - Specific obligations to consult on changes, } \\
\text { and required information to be provided to } \\
\text { community }\end{array}$ \\
\hline $\begin{array}{l}\text { Council-controlled organisations [Local } \\
\text { Authority Trading Enterprises (LATEs)] } \\
\text { - Establishment units required for Local } \\
\text { Authority Trading Enterprises only } \\
\text { - [These are limited to trading } \\
\text { organisations] }\end{array}$ & $\begin{array}{l}\text { Council-controlled organisations } \\
\text { - No establishment units } \\
\text { - Written performance agreements for all } \\
\text { council-controlled organisations } \\
\text { - Modified special consultative procedure for } \\
\text { for-profit organisations - current } \\
\text { information requirements of statement of } \\
\text { corporate intent will continue to apply" }\end{array}$ \\
\hline
\end{tabular}

\footnotetext{
3 "The existence of a detailed LTCCP would mean the annual plan could focus on a summary of the annual budget and the proposed level of rates and charges. It would also signal proposed changes from the long term council plan. The public would be able to use the annual plan to focus on current issues and refer to the LTCCP for other matters", (DIA 2001, p.40).
} 


\section{Chapter 3: Community Well-being Framework}

\section{Purpose of Local Government Act 2002}

The most significant clause introduced in the Local Government Act 2002 was the explicit purpose of the Act and of Local Government: "The purpose of this Act is to provide for democratic and effective local government that recognises the diversity of New Zealand communities; and, to that end, this Act:

a) states the purpose of local government; and

b) provides a framework and powers for local authorities to decide which activities they undertake and the manner in which they will undertake them; and

c) promotes the accountability of local authorities to their communities; and

d) provides for local authorities to play a broad role in promoting the social, economic, environmental, and cultural well-being of their communities, taking a sustainable development approach." (LGA 2002, 2007 \& 2020, Part 1, s.3)

Further to the purpose of the LGA 2002 as mentioned above, the purpose of local government, as stated in the LGA 2002, is:

a) "to enable democratic local decision-making and action by, and on behalf of, communities; and

b) to promote the social, economic, environmental, and cultural well-being of communities, in the present and for the future." (LGA 2002, 2007 \& 2020, Part 2, s.10)

Previously, in the Local Government Act 1974, the purpose of the local government was introduced through an amendment in the year 1989, which stated that, "the purposes of local government in New Zealand are to provide, at the appropriate levels of local government:

a) Recognition of the existence of different communities in New Zealand.

b) Recognition of the identities and values of those communities.

c) Definition and enforcement of appropriate rights within those communities.

d) Scope for communities to make choices between different kinds of local public facilities and services. 
e) For the operation of trading undertakings of local authorities on a competitively neutral basis.

f) For the delivery of appropriate facilities and services on behalf of central government.

g) Recognition of communities of interest

h) For the efficient and effective exercise of the functions, duties, and powers of the components of local government.

i) For the effective participation of local persons in local government." (LGA 1974, 1989, Part IA, s.37K)

Thus, the LGA 2002 was a breath of fresh air and gave a set of directions to the local government to follow on. It shifted the prescriptive theme of the LGA 1974 to a more principled theme (Sheard and McDonald, 2013). Here among others, the focus was on the introduction of community well-beings or more commonly known as four well-beings (i.e., social, economic, environmental and cultural well-beings). It was added in the legislation so that councils could have a flexibility and work like a corporation or an individual under the general law, and to give them broad discretion over the activities which they want to get involved in and deliver for their communities, (New Zealand Government, 2001, p.2). Community well-being was also seen as having a sustainable development approach, which has been defined internationally as: "development which meets the needs of the present without compromising the ability of future generations to meet their own needs" (DIA, LGNZ and SOLGM, 2003, p. $28^{4}$ ).

\section{Aspects of Community Well-beings}

As for the community well-beings there is no fixed definition of it in LGA 2002. It is because each community is different to others and it has to define its own set of well-beings which it deems to suit its particular circumstances. For each category there can be many aspects of well-beings which follows the criteria of sustainable development. According to the

\footnotetext{
${ }^{4}$ https://www.ukssd.co.uk/sustainable-development
} 
Department of the Prime Minister and Cabinet (2019) these community well-beings can be described as:

a) "Social Well-being: encompasses those factors that enables individuals, their families, whanau, iwi, hapu and a range of communities to set goals and achieve them - factors such as education, health, the strength of community networks, connectedness, financial and personal security equity of opportunity, rights and freedoms could all be expressed in this well-being.

b) Economic Well-being: encompasses factors relating to the capacity of the economy to generate employment and wealth necessary to provide many of the pre-requisites for social well-being, such as health and financial security and equity of opportunity.

c) Environmental Well-being: encompasses factors that relate ultimately to the capacity of the natural environment to support, in a sustainable way, the activities that constitute community life. (i.e., air quality, freshwater, uncontaminated land, control of pollution etc.).

d) Cultural Wellbeing: encompasses the shared beliefs, values, custom behaviors and identities reflected through language, stories, visual and performing arts, ceremonies and heritage (i.e., the diversity of a district, with inclusiveness reflected in the cultural activities undertaken)." (Department of the Prime Minister and Cabinet, 2019, p.19)

From the Council perspective the concept of well-being and defining it depends on the Council's own understanding, and it is ideally developed bottom up, through a process of identifying the community outcomes. There can be cases where change or impact in one wellbeing will impact on the other well-beings, and this is where the balance and prioritisation of the activities or projects are required. Historically the economic well-being had the most of the weightage on decision-making. There are possibilities that with innovation and experimentation, economic well-being can be achieved along with positive outcomes in social, environmental and cultural well-beings (Central Hawke's Bay District Council, 2009, p.23).

As for the four community well-beings and Councils' role in it, some aspects have been dealt with in great detail and some have not been addressed to a great extent. The Council's role in assisting with social well-being has been limited, and primarily left to central government 
and community groups to deal with. In the space of social well-being, Councils have been actively playing their role in the context of providing social housing and grants to community groups. Councils can also provide a networking opportunity between the different groups within the community and with the central government agencies as well. From the economic well-being perspective, councils have been playing their role through regulating land use, developing infrastructure, housing construction, as well as providing different types of social places. They also have been promoting businesses and tourism within their cities or districts. In this context a combination of all of these organisations provides a network and connections among all which then facilitates economic activity. As for the well-beings that pertains to the environmental, Councils have a statutory requirement to look after the environment through the Resource Management Act 1991. Further actions can be taken in the context of climate change, and this is where networking and collaborative working with the community helps in providing a cleaner environment for all people. Councils are also involved in cultural wellbeing by providing services like libraries and museums, and supporting cultural events and arts, and cultural activities in general. While the other activities are also of importance, it becomes a balancing act for the Council to focus on culture related activities, with a broad range of other competing Council initiatives (Central Hawke's Bay District Council, 2009, p.23).

Along with these aspects of well-being, Councils also have the role of decision making in such a way that it fulfils the requirement of sustainable development. Therefore, sustainable development is "both an approach to decision-making and concerned with an ideal outcome to be pursued", (DIA, LGNZ and SOLGM, 2003, p.28). The main points to consider in pursuing a sustainable development approach in the local government context, is to develop a strategic document which adopts a long-term view (e.g. a ten year financial strategy or 50 year asset management plan or a ten year long term plan); integrating these strategic documents with the overall community well-beings (i.e. connecting Council activities with the community wellbeings); developing partnerships with central government, Maori, businesses, community groups and volunteers; and regularly measuring the progress of community well-being, and assessing what has been achieved (DIA, LGNZ and SOLGM, 2003). 


\section{Community Well-being Framework \& Community Outcomes}

After going through the purpose of LGA 2002 and understanding the four aspects of community well-beings, we now turn towards understanding the framework through which the community well-beings are developed in the local authority context. It starts by asking the individuals and community groups what they intend to be the outcomes for their city, district or community. Those outcomes which they deem to be important in the present and in the future; their aspirations which would increase their well-being. The local government has to have an engagement process with the community through which they identify the set of desired community outcomes. These outcomes then become an integral part of the councils' long-term plan. The purpose of going through the exercise of identifying desired community outcomes is firstly, to engage with the communities, provide them with opportunities to discuss their views concerning four well-beings, to know that what things deem essential to them, determine the level of priority of each outcome. After that decide on measures of progress over time, better application of community resources through a coordinated way, and to set the expectations with the Council regarding the services and activities with which it is involved with (LGA 2002, 2007 p.75).

In the previous version ${ }^{5}$ of LGA 2002 under Part 6, there was a full section that outlined the process for identifying community outcomes and the obligation to report the progress accordingly, but it was repealed with the Local Government Act 2002 Amendment Act 2012, and it was not added back in with the Local Government (Community Well-being) Amendment Act 2019. This is more or less kept open and flexible so that each Council could decide what methodology suits them better. Each district or city is different from the other; they have different resources, capacities and capabilities, and therefore they would aspire to different sets of community outcomes. If there is a sort of similar community outcomes across different places, they would may have a different priority or levels of satisfaction associated with those outcomes (Karacaoglu and Reid, 2019). It was also noted by the Office of the Auditor-General of New Zealand that, "there is no 'one-size-fits-all' approach. Therefore, we expect local authorities to customise any process for monitoring regional community

\footnotetext{
${ }^{5}$ Before the amendment of 2012.
} 
outcomes by also addressing their own specific community outcomes and their community's local context" (OAG 2010, cl.8.34, p.77). That is why there is no fixed definition of community well-being across the four well-beings.

Councils are encouraged to develop a strong working relationship with the community organisations that can give them further insights on specific things happening within their communities. The consultation exercise can be different for each local authority. Councils can use different mediums to contact the community - e.g., Councils' websites, facebook pages, newspapers, focus groups, kiosks, online surveys, public meetings, community events, festivals, specific consultation websites etc. The responses received are combined according to the themes and then articulated in terms of community outcome statements. These statements then help in selecting the indicators which would be useful in tracking the progress over time. Finally, the progression of indicators tells that how community well-being is progressing over the time. Here, the interesting part is that a single indicator might be impacting multiple spheres of well-being and, in the same way, an increase or decrease in one well-being might impact on other well-beings as well. This is demonstrated in the SOLGM Community Well-being Indicator Framework below (Figure: 2), (The Community Well-being Service, 2019).

To engage with the community and develop these community outcomes, Councils can establish a committee comprising of the Councillors, Council staff members, representatives from local Iwi, and other important groups that it deems significant and useful for this activity. The community outcomes are for the whole community, individuals, groups and all other organisations within the city or district. They are not Councils' outcomes and should not be confused with Council activities or services, although they give the direction to the Council in this regard. Council can play its role by identifying ways through which it could coordinate effort with various community groups, agencies and other organisations in materialising these community outcomes. After having identified these community outcomes, they can be changed, but through another round of consultation with the community. It is also important for the Councils to list down the indicators and measures through which they will be monitoring the progress over the time (Rangitikei District Council, 2009). 
Figure 2: SOLGM Community Well-being Indicator Framework

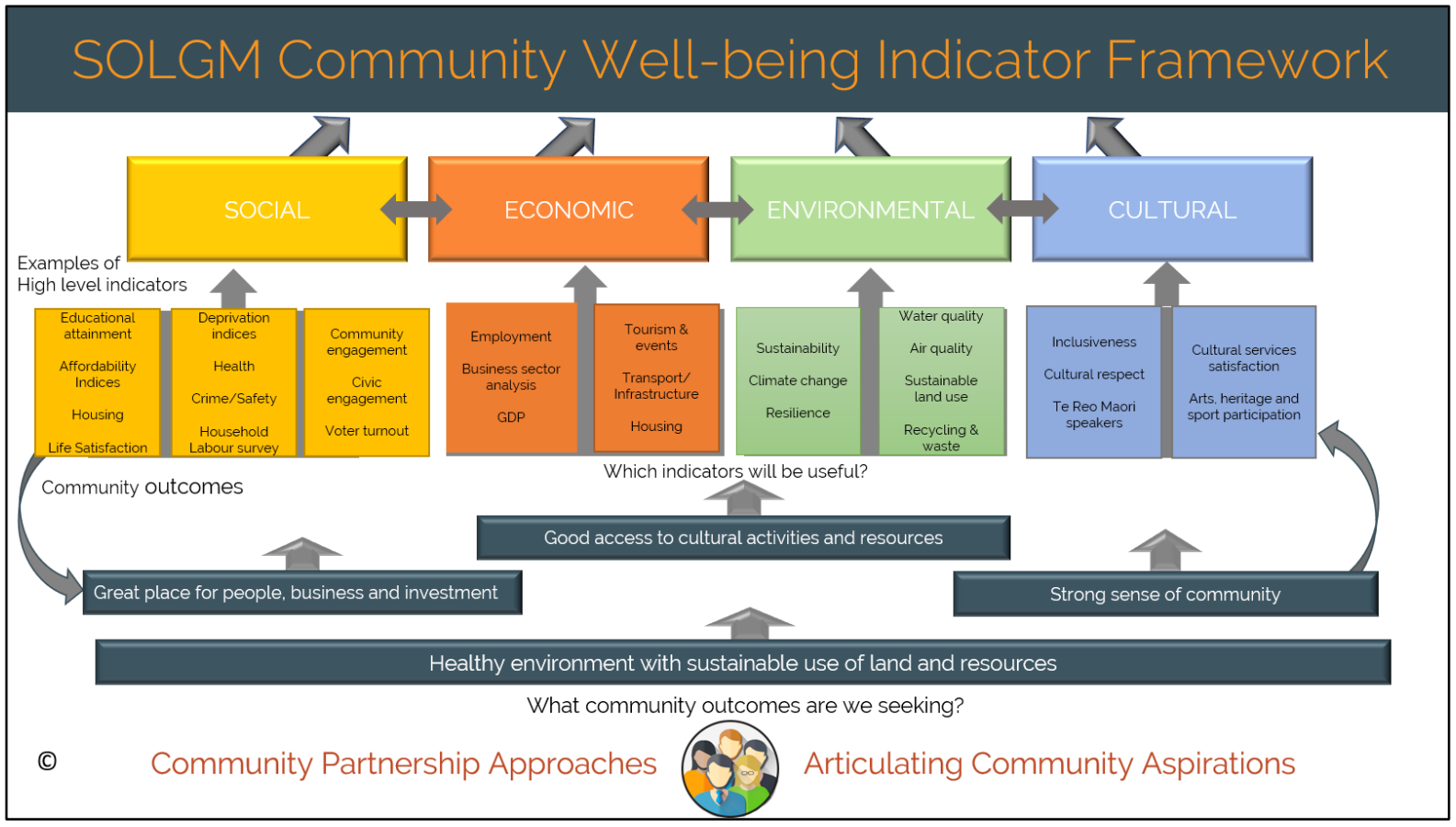

The first set of community outcomes were developed in the years 2004 and 2005 and used for the first time in 2006 for informing Long Term Council Community Plans (LTCCP). Although, the community outcomes had to be reviewed after six years according to the LGA 2002 $(2007)^{6}$, some Councils reviewed them for the 2009 LTCCP. It happened because Councils learned from their experience and also from other Councils' work.

Council must take a leading role in delivering the identified community outcomes; either by itself or in collaboration with others. Networking, allocating resources, managing and monitoring the progress, can make the things happen (Lower Hutt City Council, 2009). Community outcomes are for all and it can be delivered collaboratively - e.g., "by working together on particular projects, the Council and Ministry of Justice jointly fund Neighbourhood Support; Horizons Regional Council and Manawatu District Council work in partnership to looks after Totara Reserve Regional Park; by working together through networks and 'umbrella groups', such as the Regional Inter-Agency Network (central and local government agencies) and Social Issues Network Council of Social Services" (Manawatu District Council (2009, p.15)). As for the specific Council's contribution to community

\footnotetext{
${ }^{6}$ It is not a requirement now as per LGA 2002 (2020).
} 
outcomes, Tararua District Council (2009, p.252), mentions that the Council can be, "a provider or funder of services; regulator and enforcer of rules, legislation and standards; partner or collaborator with other organisations; [facilitator] ${ }^{7}$; advocate and leader for the community; and a monitor to measure the community's progress towards achieving community outcomes. The Council engages in many activities for the continued well-being of the Tararua community, and many of these roles are performed in the normal course of delivering services to the community."

Following are the community outcomes and their explanations from few Councils' LTCCP 20098:

\section{Table 2: Ashburton District Council (2009)}

\section{Community Outcomes}

1. "Economy

\section{Environment}

3. Identity \& Heritage

4. Education

5. Health and Wellbeing

6. Recreation

7. Democracy

\section{Explanation}

A thriving and diverse local economy that provides the foundation for a quality lifestyle

Natural and developed environments are sustained for the enjoyment of current and future generations

A community with a strong sense of identity and heritage, which welcomes new residents and encourages diversity

A community with access to quality education and lifelong learning

Healthy, active people enjoying a good quality of life in a caring and safe environment

A community with access to a variety of cultural, recreational and heritage experiences and facilities that enhance our quality of life A community that contributes to improving our quality of life through democratic participation"

\footnotetext{
${ }^{7}$ Added by author, Masterton District Council (2009, p.6)

${ }^{8}$ Complete list of community outcomes along with full details can be viewed in Appendix A and B. For discussion on the indicators and measures see Chapter 7: Common Indicators and Measures Across the Councils.
} 


\section{Table 3: Masterton District Council (2009)}

\section{Community Outcomes}

1. "Sustainable Use of Environment

2. Educated and Knowledgeable People

3. Equitable Society

4. Vibrant, Strong and Healthy Communities

5. Safe and Efficient Transport

6. Buoyant Local Economy

7. Grow Masterton

\section{Explanation}

Sustainable management and use of natural resources and infrastructure, now and for the future.

Lifelong learning and development, to achieve personal, life, community and work goals.

Diversity of the community appreciated, respected, celebrated and encouraged.

Healthy, caring and creative people who feel connected to others and to the District, creating a positive community where people are involved and contribute.

A transport system that is safe, sustainable, integrated and responsive, enabling safe and efficient passage for people and goods around and through the district.

Sustainable economic activity to create a positive and prosperous local economy that enables improved opportunities and living standards for all.

Sustainable development to create a place that is attractive to new residents and investors who have the potential to make a positive contribution to the District."

\section{Table 4: Upper Hutt City Council (2009)}

\section{Community Outcomes}

1. "Upper Hutt is the city of choice for people from all walks of life

2. Upper Hutt has a vibrant city heart

3. The economy is robust, innovative and growing

4. Upper Hutt offers a 'green' and attractive living environment

5. The community is safe, healthy and strong

6. Leisure opportunities are outstanding

7. Upper Hutt is connected with the world" 


\section{Table 5: New Plymouth District Council (2009)}

\section{Community Outcomes}

1. “Connected (relates to our infrastructure)

2. Prosperous (our economy)

3. Secure and Healthy (our community wellbeing)

4. Skilled (our learning and education opportunities)

5. Sustainable (our environment)

6. Together (our community cohesion)

7. Vibrant (our culture, leisure and recreation opportunities)

\section{Explanation}

A district that delivers accessible and integrated infrastructure, transport and communication systems which meet the needs of residents, businesses and visitors.

A district that boasts a sustainable, resilient and innovative economy that prospers within the natural and social environment.

A district that provides a safe, healthy and friendly place to live, work or visit.

A district that values and supports learning so all people can play a full and active role in social, cultural and economic life.

A district that appreciates its natural environment and its physical and human resources in planning, delivery and protection.

A district that is caring, inclusive and works together and where people have a strong, distinctive sense of identity.

A district that provides high-quality and diverse cultural and recreational experiences and where independence and creativity are encouraged." 


\section{Community Outcomes}

1. "An attractive and highly valued natural environment

2. Thriving economy

3. A democracy which upholds the rights of the individual

4. Fit and healthy community

6. Supportive and contributing community

5. Safe, effective and sustainable

\section{Explanation}

The outcome desired by the community is to have the outstanding natural features of the district preserved, enhanced, accessible, and promoted

The outcome desired by the community is to have a well-balanced economy, providing employment and investment opportunities for all ages, and sustaining a full range of support services

The outcome desired by the community is to have a council which champions the rights of the individual and represents its community

The outcome desired by the community is to have a variety of sporting, recreational, cultural, spiritual, health, welfare and educational resources available to sustain and enrich the lives of its people. The outcome desired by the community is to have safe, effective and sustainable water, waste, communication, energy and transport systems in place when required, through sound long term planning and funding.

The outcomes desired by the community are to foster the unique attributes and strong sense of community that makes the Mackenzie special, and to encourage people to use their skills for the benefit of the community."

\section{The Planning Framework}

Once the community well-being framework and community outcomes are scoped out, then the next step is to integrate these outcomes in the Council's planning and strategic documents. These planning and strategy documents are the ones which give direction to the Council's objectives and day-to-day work programme. Below in figure 3 there is a planning 
framework developed by DIA, LGNZ and SOLGM $(2003)^{9}$. There have been changes in this planning framework in the LGA 2002 (2020) version due to the changes in 2012. These changes were: firstly, that the name of the long-term plan has changed from Long Term Council Community Plan (LTCCP) to Long Term Plan (LTP) ${ }^{10}$; secondly, the monitoring and review stage is not a compulsory stage now as it was back in the earlier version of LGA $2002^{11}$. Apart from these changes the planning framework shown in Figure 3 below remains relevant.

From an LGA 2002 (2020) point of view, this planning framework is covered under 'Part 6: Planning, decision-making, and accountability', and further explained in detail in 'Schedule 10'. The community well-beings provides the basis for initiating the planning framework, which then develops the community outcomes, which are then connected to the Council's activities, then outlined in the long-term plan. Subsequently the actions are communicated through the annual plan and annual report. If there are any significant changes to be made, then the cycle starts again.

The purposes of the long-term plan, annual plan and annual report as in the LGA 2002 (2020) are as follows:

Long-term plan:

a) "describe the activities of the local authority; and

b) describe the community outcomes of the local authority's district or region; and

c) provide integrated decision-making and co-ordination of the resources of the local authority; and

d) provide a long-term focus for the decisions and activities of the local authority; and

e) provide a basis for accountability of the local authority to the community".

Annual plan:

\footnotetext{
${ }^{9}$ There have been slight changes in the LGA 2002 (2020) version for this

${ }^{10}$ Which meant that section $93(f)$ of the Act was repealed and the long term plans were made to focus more on the Councils activities

${ }^{11}$ Which meant that section 91 of the Act was removed which consequently diminished community engagement and nullified the obligation to report on progress
} 
a) "contain the proposed annual budget and funding impact statement for the year to which the annual plan relates; and

b) identify any variation from the financial statements and funding impact statement included in the local authority's long-term plan in respect of the year; and

c) provide integrated decision making and co-ordination of the resources of the local authority; and

d) contribute to the accountability of the local authority to the community".

Annual report:

a) "compare the actual activities and the actual performance of the local authority in the year with the intended activities and the intended level of performance as set out in respect of the year in the long-term plan and the annual plan; and

b) promote the local authority's accountability to the community for the decisions made throughout the year by the local authority".

LGA 2002 (2020) have set a purpose and role for local authorities, which is - along with other things - to take action and perform the duties (section $10 \& 11$ ). In this regard Council must engage in some group of activities ${ }^{12}$, and the community outcomes must be tied to these activities. These groups of activities take a central position in the Council's strategic and planning documents:

1. "A long-term plan must, in relation to each group of activities of the local authority,

a) identify the activities within the group of activities:

b) identify the rationale for delivery of the group of activities (including the community outcomes to which the group of activities primarily contributes):

c) outline any significant negative effects that any activity within the group of activities may have on the social, economic, environmental, or cultural well-being of the local community:

d) include the information specified in clauses 4 and $5^{13}-$

\footnotetext{
12 "group of activities means 1 or more related activities provided by, or on behalf of, a local authority or councilcontrolled organisation" (LGA 2002, (2020), section 5).

${ }^{13}$ Statement of service provision and funding impact statement for group of activities.
} 
i. in detail in relation to each of the first 3 financial years covered by the plan; and

ii. in outline in relation to each of the subsequent financial years covered by the plan.

2. In this schedule, each of the following activities is a group of activities:
a) water supply:
b) sewerage and the treatment and disposal of sewage:
c) stormwater drainage:
d) flood protection and control works:
e) the provision of roads and footpaths.

3. Despite subclause (2), a local authority may treat any other activities as a group of activities" (LGA 2002, (2020, Schedule 10, Part 1, cl.2)).

Figure 3: The Planning Framework

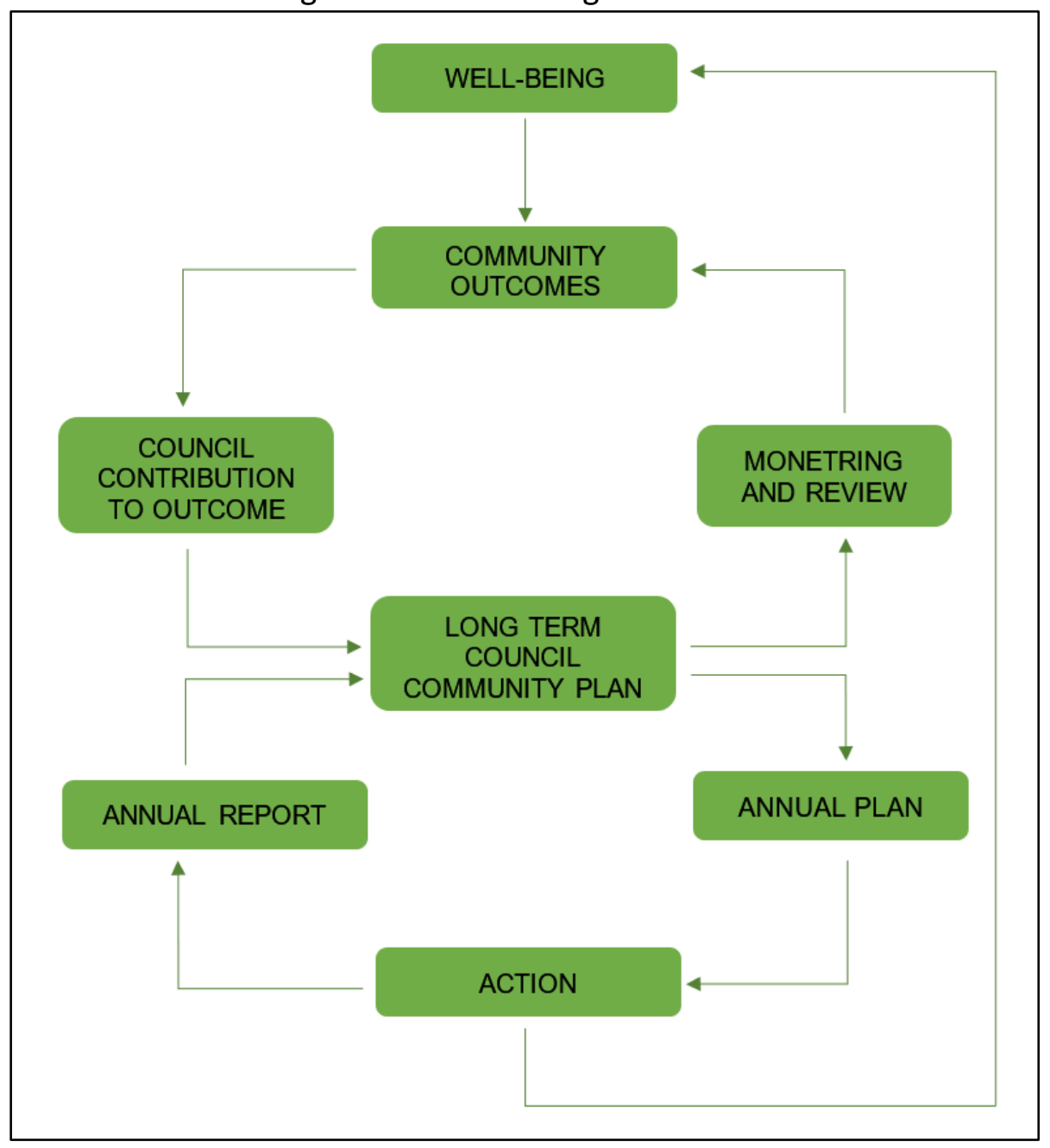


The latest structure on combining the community outcomes with the planning aspects in local authority has been provided by SOLGM (2020) in its Performance Management Framework. It is defined as "a continuous process of determining objectives, measuring progress against those objectives, and using the results to improve your local authority's delivery of services to the community" (SOLGM 2020, p.6). Figure 4 shows the performance management framework which connects the community outcomes with the planning aspects of the Council. It clearly shows that the community well-beings are the overarching principle for the Council's performance. The column on the left side shows the elements of the framework which corresponds to the reporting structure on the right column. The first step is developing the community outcomes, which then connects with the Council's strategic and planning documents, where these documents would show the activities which Council undertakes and how they impact the well-being. The expected levels of services are then set and measured over the time so that it could be seen that either the Council is meeting its target or not. Finally, it is checked that whether the Council was able to achieve what it set out to do and reported back to the community.

According to Audit New Zealand (2020) there are two main parts of this performance framework: identifying community outcomes and setting levels of service, measures and targets for each group of activities. The latter is an important part that forms the basis for public accountability for the Council. Along with reporting the direct and positive impact of activities on the community well-beings, Councils are also required to identify any adverse impact of a groups of activities on the well-beings. It is also recommended that Councils incorporate a future outlook or forecasts on the group of activities which have significant impact on the community. 
Figure 4: The Performance Framework for Local Government (SOLGM, 2020)

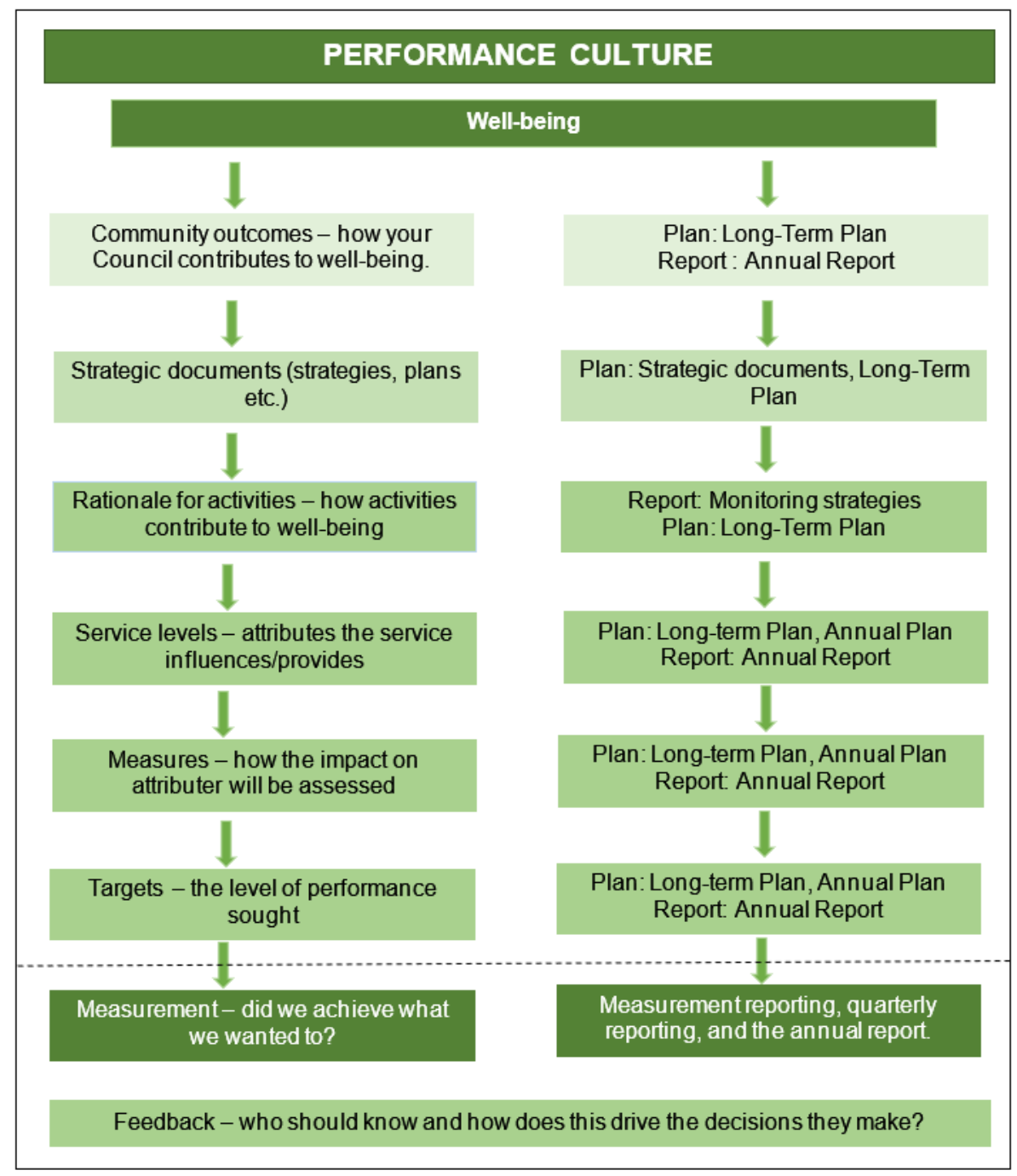

The planning framework can be seen in action in LTP 2009 as developed by the Councils. Below in Figure 5 is the Hamilton City Council strategic framework. It starts from the four wellbeings and connects to the community outcomes (there were seven community outcomes in 2009). The outcomes are integrated with the Council's vision for the city, known as Vibrant Hamilton (which had three components in 2009). This combination then derives strategies for various different plans and service delivery. This strategic framework comprises the input from various stakeholders and legislations, including the community, key stakeholders within the city, strategic partners, central government agencies, and the Council itself. In these types of strategic frameworks, the Council also has to look for the national and regional legislation, strategies, and policies, along with the community feedback. 
Figure 5: Components of the City Strategic Framework (Hamilton City Council, 2009)

\begin{tabular}{|c|c|c|}
\hline KEY DRIVERS AND INPUTS & & RESPONSIBILITY \\
\hline Sustainable Development & CURRENT AND FUTURE COMMUNITY WELL-BEING & \multirow[t]{2}{*}{ The Whole Community } \\
\hline $\begin{array}{l}\text { "Getting more, from less, } \\
\text { for longer" }\end{array}$ & & \\
\hline Community Aspirations & HAMILTON'S COMMUNITY OUTCOMES & $\begin{array}{l}\text { The Whole Community and } \\
\text { Kev Stakeholders }\end{array}$ \\
\hline \multirow{5}{*}{$\begin{array}{l}\text { Collaborative City-wide } \\
\text { Leadership Response }\end{array}$} & & \multirow{5}{*}{$\begin{array}{l}\text { Strategic Partners and } \\
\text { Agencies in Hamilton } \\
\text { (including Hamilton City } \\
\text { Council) }\end{array}$} \\
\hline & NFOR IH & \\
\hline & $\begin{array}{c}\text { Vibrant Hamilton } \\
\text { Investing in Our People Creating Identity \& Prosperity } \\
\text { Protecting Our Future }\end{array}$ & \\
\hline & CITY STRATEGIES & \\
\hline & $\begin{array}{l}\text { Social Well-being Creativity \& Identity Urban Growth } \\
\text { Economic Development CityScope Access Hamilton } \\
\text { Active Communities Environmental Sustainabilitv }\end{array}$ & \\
\hline \multirow{2}{*}{$\begin{array}{l}\text { - National Legislation, } \\
\text { Strategies, } \\
\text { Policies and Standards }\end{array}$} & & \multirow[t]{5}{*}{ Hamilton City Council } \\
\hline & COUNCIL'S POLICY \& PLANNING FRAMEWORK & \\
\hline $\begin{array}{l}\text { Policies and Standards } \\
\text { - Regional Strategies, } \\
\text { Policies and Plans }\end{array}$ & $\begin{array}{c}\text { Council Policies District Plan } \\
\text { Activity Management Plans } \\
\text { LTCCP \& Annual Plan (Including Financial Management } \\
\text { Approach) }\end{array}$ & \\
\hline \multirow{2}{*}{$\begin{array}{l}\text { - Community Consultation } \\
\text { and Feedback }\end{array}$} & COUNCIL'S SERVICE DELIVERY & \\
\hline & 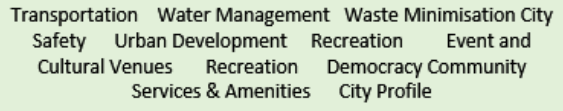 & \\
\hline
\end{tabular}

In the Tauranga City Council (2009) long-term plan, they have listed the community outcomes and the contribution of a group of activities for each of them. The group of activities comprises different services given by the Council. It is visible in the majority of the activities that they are impacting on more than one community outcome and, in the same way each outcome has a role in more than one Council activity. Thus, it shows the close relationship of community outcomes with the Council service delivery - see Figure 6.

A similar theme can be seen for Grey District Council (2009) long-term plan, in which they have shown how the Council contributes to the community outcomes. In their cross-table, they have indicated which Council activity significantly contributes or simply contributes to the community outcomes. In total, the Grey District Council had seven group of activities and 33 individual activities, which corresponded to six community outcomes - see Figure 7. 
Figure 6: Council's Contribution to Tauranga Tomorrow (Tauranga City Council, 2009)

\begin{tabular}{|c|c|c|c|c|c|c|c|c|}
\hline $\begin{array}{l}\text { COMMUNITY OUTCOMES } \\
\text { (by contributing Activity Area) }\end{array}$ & 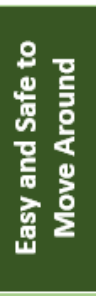 & 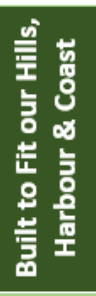 & 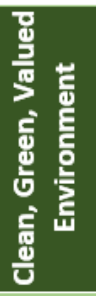 & 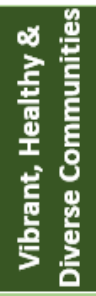 & 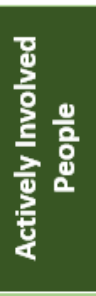 & 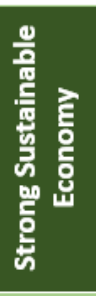 & 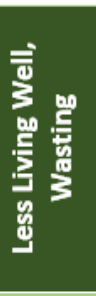 & 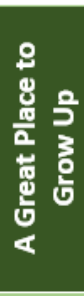 \\
\hline Airport & $\bullet$ & & & & & • & & \\
\hline Animal Service & & & & $\bullet$ & & & & \\
\hline Aquatic & & & & $\bullet$ & & & & $\bullet$ \\
\hline Arts \& Heritage & & & & $\bullet$ & & $\bullet$ & & $\bullet$ \\
\hline Bay park & & & & $\bullet$ & & $\bullet$ & & $\bullet$ \\
\hline Building Service & & $\bullet$ & & & & & & \\
\hline CBD \& Neighborhood Center Revitalization & $\bullet$ & $\bullet$ & & $\bullet$ & & $\bullet$ & & \\
\hline Civil Defense \& Emergency Management & & & & $\bullet$ & $\bullet$ & $\bullet$ & & \\
\hline Community Property Service & & $\bullet$ & $\bullet$ & $\bullet$ & & $\bullet$ & & $\bullet$ \\
\hline Customer Service Center & & & & $\bullet$ & $\bullet$ & & & \\
\hline Democracy \& Civil Service & & & & $\bullet$ & $\bullet$ & $\bullet$ & & $\bullet$ \\
\hline Economic Development & $\bullet$ & & & $\bullet$ & & $\bullet$ & & $\bullet$ \\
\hline Elder Housing & & & & $\bullet$ & & & & \\
\hline Environmental Compliance \& Monitoring & & $\bullet$ & $\bullet$ & $\bullet$ & & & & $\bullet$ \\
\hline Environmental Planning & $\bullet$ & $\bullet$ & $\bullet$ & & & & & \\
\hline Environmental policy & $\bullet$ & $\bullet$ & $\bullet$ & $\bullet$ & & $\bullet$ & & $\bullet$ \\
\hline Indoor Space \& Active Living & & & & $\bullet$ & & & & $\bullet$ \\
\hline Libraries & & & & $\bullet$ & $\bullet$ & & & $\bullet$ \\
\hline Open Space & $\bullet$ & $\bullet$ & $\bullet$ & $\bullet$ & & & & $\bullet$ \\
\hline Solid Waste & & & $\bullet$ & $\bullet$ & & & $\bullet$ & \\
\hline Stormwater & & $\bullet$ & $\bullet$ & $\bullet$ & & & $\bullet$ & \\
\hline Strategic Property & & $\bullet$ & & & & $\bullet$ & & \\
\hline Support Services & $\bullet$ & $\bullet$ & $\bullet$ & $\bullet$ & $\bullet$ & $\bullet$ & $\bullet$ & \\
\hline Transportation & $\bullet$ & $\bullet$ & & $\bullet$ & & $\bullet$ & $\bullet$ & $\bullet$ \\
\hline Venues \& Events & & & & $\bullet$ & & $\bullet$ & $\bullet$ & \\
\hline Wastewater & & $\bullet$ & $\bullet$ & $\bullet$ & & & $\bullet$ & $\bullet$ \\
\hline Water Supply & & • & $\bullet$ & $\bullet$ & & & $\bullet$ & \\
\hline
\end{tabular}


Figure 7: Council's Contribution to Outcomes (Grey District Council, 2009)

\begin{tabular}{|c|c|c|c|c|c|c|c|}
\hline & & $\begin{array}{l}\text { Outrome } \\
\text { One } \\
\text { ENVIROMENT }\end{array}$ & $\begin{array}{l}\text { Outcome } \\
\text { Two } \\
\text { ECONOMY }\end{array}$ & $\begin{array}{l}\text { Outcome } \\
\text { Three } \\
\text { HEALTH }\end{array}$ & $\begin{array}{l}\text { Outcome } \\
\text { Four } \\
\text { EDUCATION }\end{array}$ & $\begin{array}{l}\text { Outrome } \\
\text { Five } \\
\text { SAFETY }\end{array}$ & $\begin{array}{l}\text { Outcome } \\
\text { Six } \\
\text { IDENTITY }\end{array}$ \\
\hline \multirow{3}{*}{\multicolumn{2}{|c|}{$\begin{array}{l}\text { - significant contribution to community outcome } \\
\text { contribution to community outcome }\end{array}$}} & $\begin{array}{l}\text { That the } \\
\text { distinctive } \\
\text { character of } \\
\text { the } \\
\text { environment } \\
\text { is appreciated } \\
\text { and retained }\end{array}$ & $\begin{array}{l}\text { A thriving, } \\
\text { resilient and } \\
\text { innowative } \\
\text { economy } \\
\text { creating } \\
\text { opportunities } \\
\text { for growth } \\
\text { and } \\
\text { employment }\end{array}$ & $\begin{array}{c}\text { Health } \\
\text { communities } \\
\text { with access } \\
\text { to quality } \\
\text { facilities and } \\
\text { services }\end{array}$ & $\begin{array}{l}\text { A district that } \\
\text { values and } \\
\text { supports } \\
\text { learning with } \\
\text { accessible, } \\
\text { relevant } \\
\text { education } \\
\text { and training } \\
\text { opportunities }\end{array}$ & $\begin{array}{l}\text { A District } \\
\text { that is a } \\
\text { safe } \\
\text { place to } \\
\text { live }\end{array}$ & $\begin{array}{c}\text { A } \\
\text { "happening" } \\
\text { district with } \\
\text { a strong } \\
\text { community } \\
\text { spirit and } \\
\text { distinctive } \\
\text { lifestyle. }\end{array}$ \\
\hline & & & \multirow{2}{*}{\multicolumn{3}{|c|}{$\begin{array}{c}\text { How Council contributes } \\
\text { to outcomes }\end{array}$}} & & \\
\hline & & & & & & & \\
\hline $\begin{array}{l}\text { SIGNIFICANTS ACTIVITY GROUP } \\
\text { COUNCLL REPORTS ON }\end{array}$ & \multicolumn{7}{|l|}{ COUNCIL ACTIVITY } \\
\hline Roading & Roading & $\bullet$ & $\bullet$ & $\bullet$ & & $\bullet$ & \\
\hline Stormwater \&Flood Protection & Stormwater \&Flood Protection & $\bullet$ & $\bullet$ & $\bullet$ & & $\bullet$ & \\
\hline Sewerage & Sewerage & $\bullet$ & $\bullet$ & $\bullet$ & & $\bullet$ & \\
\hline Water Supply & Water Supply & $\bullet$ & $\bullet$ & $\bullet$ & & $\bullet$ & \\
\hline Solid Waste Management & Solid Waste Management & $\bullet$ & $\bullet$ & $\bullet$ & & & $\bullet$ \\
\hline Emergency Management & Rural Fire Service & $\bullet$ & $\bullet$ & & & $\bullet$ & \\
\hline Emergency Management & Civil Defense \& Emergency Management & & $\bullet \bullet$ & & & $\bullet$ & \\
\hline Environmental Service & District planning & $\bullet$ & $\bullet$ & & & & $\bullet$ \\
\hline Environmental Service & Building fontrol & $\bullet$ & $\bullet$ & $\bullet$ & & & \\
\hline Environmental Service & Dog and Stock Control & & & & & $\bullet$ & \\
\hline Environmental Service & Amenity Management & $\bullet$ & & $\bullet \bullet$ & & & \\
\hline Environmental Service & Health Regulation & & & $\bullet$ & & & \\
\hline Other Transport & Vermouth Aerodrome & & $\bullet$ & $\bullet$ & & $\bullet$ & \\
\hline Other Transport & Parking & & $\bullet$ & & & $\bullet$ & \\
\hline Other Transport & Port & & $\bullet$ & & & & \\
\hline Property & Retirement Housing & & $\bullet$ & $\bullet$ & & $\bullet$ & $\bullet$ \\
\hline Property & Councils property Portfolio & & $\bullet$ & & & & \\
\hline Community Facilities and Events & Reserves & $\bullet$ & $\bullet$ & $\bullet$ & & & $\bullet$ \\
\hline Community Facilities and Events & Councils In-House Task Force & $\bullet$ & $\bullet$ & & & & $\bullet$ \\
\hline Community Facilities and Events & Rest Rooms \& Public Conveniences & $\bullet$ & $\bullet$ & $\bullet$ & & & $\bullet$ \\
\hline Community Facilities and Events & Heritage \& Culture & & & & $\bullet$ & & $\bullet$ \\
\hline Community Facilities and Events & District Libraries & & & $\bullet$ & $\bullet$ & & $\bullet$ \\
\hline Community Facilities and Events & Swimming Baths & & $\bullet$ & $\bullet$ & & $\bullet$ & $\bullet$ \\
\hline Community Facilities and Events & Cemeteries & $\bullet$ & & $\bullet$ & & & $\bullet$ \\
\hline Community Facilities and Events & Civic Center-Sports Center & & & $\bullet$ & & & $\bullet$ \\
\hline Community Facilities and Events & Events \& Recreation Management & & $\bullet$ & & & & $\bullet$ \\
\hline Democracy \& Administration & Council & $\bullet$ & $\bullet$ & $\bullet$ & $\bullet$ & $\bullet$ & $\bullet$ \\
\hline Democracy \& Administration & Consultation & $\bullet$ & • & $\bullet$ & $\bullet$ & $\bullet$ & $\bullet$ \\
\hline Democracy \& Administration & Access to Official Information & & $\bullet$ & & & & \\
\hline Democracy \& Administration & Council Administration & $\bullet$ & • & $\bullet$ & $\bullet$ & $\bullet$ & $\bullet$ \\
\hline Democracy \& Administration & Economic Development, Marketing \& Youth Dev & & $\bullet$ & & & & $\bullet$ \\
\hline Liaison with Other Agencies & Co-Operation with External Service Providers & & & • & $\bullet$ & $\bullet$ & \\
\hline Liaison with Other Agencies & Community Safety Projects \& Restorative Justice & & $\bullet$ & & & $\bullet$ & \\
\hline
\end{tabular}

A further insight can be gleaned from the long-term plans of Ashburton District Council (2009), Masterton District Council (2009), and Hurunui District Council (2009). Connections of community outcomes with groups of activities and with the strategic planning documents can be seen here. Along with it, all of them have mentioned the key organisations which could contribute towards achieving the respective outcomes. Moreover, Masterton District Council has listed the examples of work being undertaken across the district by all other organisations 
with regard to the outcome, and what can people do further, and what else is planned to do and by whom. Here, Hurunui District Council, after listing the organisations, has also mentioned the expected level of their contribution, in their roles as advocators, funders, service providers, regulators, as well as facilitators or monitors of the progress of the community outcomes. The other noteworthy aspect in this planning framework covered by Masterton District Council is that it has detailed how progress will be measured, what indicators and measures will be used to assess the progress of that particular outcome. Additionally, they have provided the baseline (data when these community outcomes were developed) and the progress made till the writing of LTP 2009. Figures 8,9 and 10 present a table for each of these three Councils.

Figure 8: Community Outcomes, Details for 1st Outcome (Ashburton District Council, 2009)

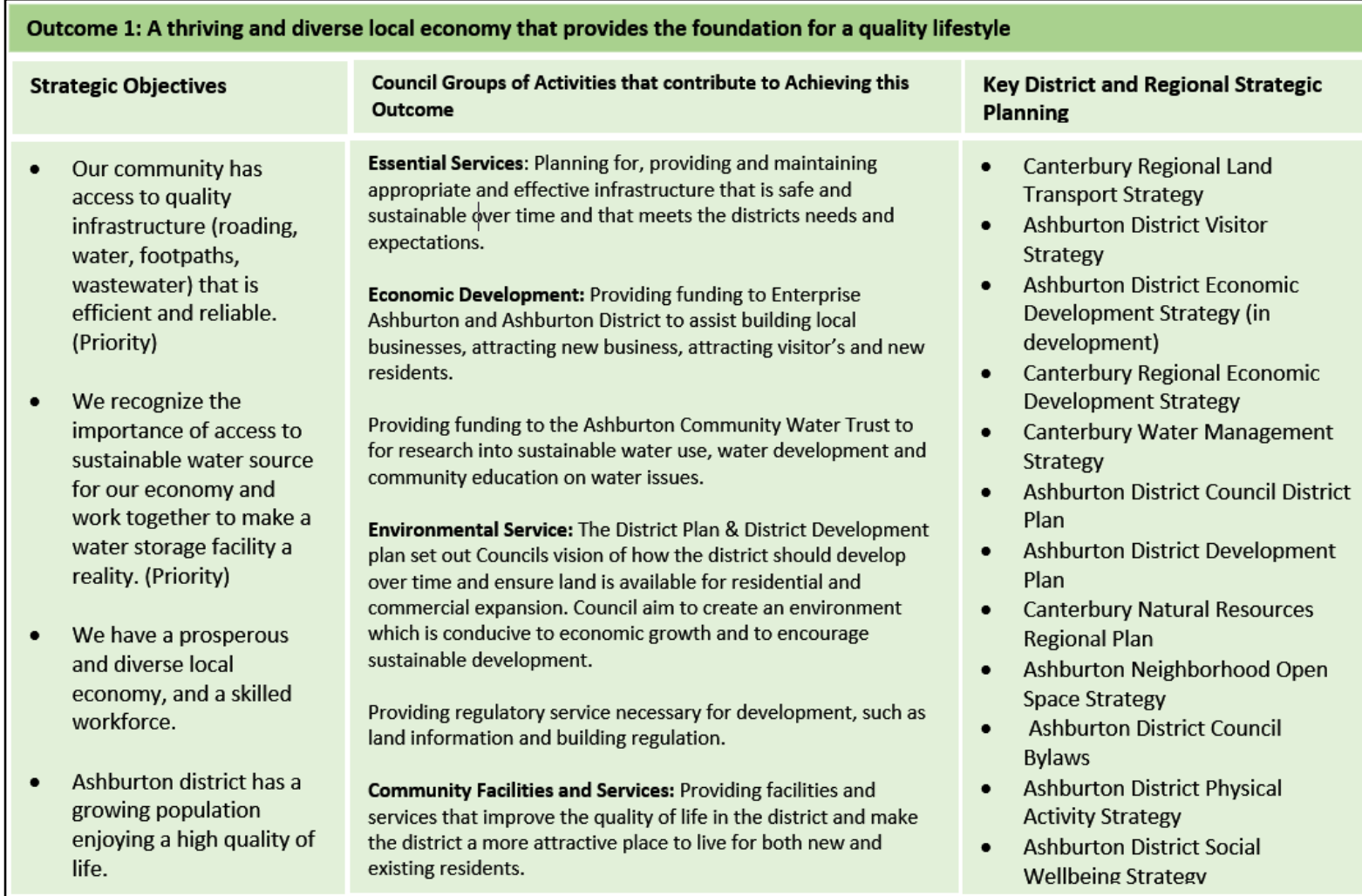

Key organization we work with to help achieve this outcome:

Enterprise Ashburton; Ashburton District Tourism; Ashburton Community Water Trust; RDR Management Ltd; Ashburton Business Association; Federated Farmers; Canterbury Regional Land; Transport Committee; New Zealand Transport Agency; Canterbury Development Corporation; Electricity Ashburton; Ministry of Economic Development; Ministry of Social Development; Environment Canterbury; Canterbury Mayoral Forum; Department of Labor 
Figure 9a: Community Outcomes, Details for 3rd Outcome (Masterton District Council, 2009)

OUTCOME 3: Equitable Society

Diversity of the community appreciated, respected, celebrated and encouraged.

Our goal is to:

- Encourage appreciation of diversity

- Enable people to participate

Improve opportunities and outcomes for al

Some Example of What Is Being Done Now

- Youth council

- Foodbank

- Council Maori Liaison Task Force

- $\quad$ King St Artworks programmed

- Wairarapa Community Primary Health Organization Maori Representative

- Matariki celebrations

- Aratoi exhibitions featuring different culture and community

- Wairarapa Physical Activity Plan

- Health promotion initiatives

- $\quad$ CCS and IDEA Services programmes

- Wairarapa Organization for Older People programmes
- Wairarapa Arts Culture and Heritage Strategy

- Youth Action Project For youth who have committed offences

- Accessibility codes for building

- Council has an Equal Employment Opportunities

- Provide a range of housing options for older people

- Library programmes for children, youth and the elderly, in English and Te Reo

- Programmes offered by Maori providers

- Church programmes and activities

- International Communities Group

- Waitangi Day Origins Festival

What Can You Do?

- Get to know people in our community who have different ethnic, religious, socio-economic backgrounds

- Include and involve older people and youth

- Attend a range of culture events and activities

- Learn Te Reo and/or another language

Visit a marae

Go to a course on the treaty of Waitangi

WHAT ELSE IS PLANNED?

1. Further celebrate, promotion and protection of Maori culture and Te Reo

Further strengthen partnerships with Maori

Community development projects within Masterton

Implementation of Wairarapa of Youth Health Strategy

Implementation of Wairarapa Arts Culture and Heritage Strategy

Implementation of Wairarapa Physical Activity Plan

Implementation of Wairarapa Physical Activity
- Share yours culture with others

- Speaks up if people are disrespectful about others

- Donate food to the Foodbank

- Practice Equal Employment Organization policies when recruiting

- Learn about others' cultural values and beliefs

- Make your home accessible to those with physical disabilities

WHO WILL LEAD THE PROJECT?

Various

Various

Council

District Health Board

Wairarapa Arts Culture and Heritage-Toi Wairarapa Trust

Wairarapa Council, DHB

Council

What Changes Would Show Progress Toward This Outcome?

- Greater appreciation of and support for diversity

- Increased ability to access and express one's culture

- Fewer people living in deprivation

- Increased diversity of people actively involved in community through workforce, sport, other? 
Figure 9b: Community Outcomes, Details for 3rd Outcome (Masterton District Council, 2009)

\begin{tabular}{|c|c|c|}
\hline \multicolumn{3}{|c|}{ How Can We Measure Progress? } \\
\hline Indicator & Baseline & Progress to Date \\
\hline $\begin{array}{l}\text { Number of Languages } \\
\text { spoken }\end{array}$ & $\begin{array}{l}8.1 \% \text { of people speak } 2 \text { language; } 1.3 \% \text { speak three } \\
\text { language and } 88.6 \% \text { speak one language } \\
\text { only(Census,2001) }\end{array}$ & $\begin{array}{l}8.1 \% \text { of people speak } 2 \text { language; } 1.0 \% \text { speak three languages } \\
\text { And } 88.0 \% \text { speak one language only (Ccnsus, } 2006)\end{array}$ \\
\hline $\begin{array}{l}\text { Maori Language } \\
\text { speakers }\end{array}$ & $\begin{array}{l}22.7 \% \text { of Maori in the Masterton District speak Te Reo } \\
\text { Maori (Ccnsus, 2001) }\end{array}$ & $\begin{array}{l}22.3 \% \text { of Maori in the Masterton District speak Te Reo Maori } \\
\text { (Census,2006) }\end{array}$ \\
\hline Social deprivation & $\begin{array}{l}16.6 \% \text { of people in Masterton live in } \\
\text { declie10areas; } 25.2 \% \\
\text { In declies } 7-9 ; \\
44.6 \% \text { in deciles } 4-6 \text { and } \\
13.6 \% \text { in deciles } 1-3 \\
\text { (NZ Dep Measure, 2001) } \\
\text { The average decile rating for Census Area Units in the } \\
\text { Masterton District is :5.8 (NZ Dep Measure, 2001) }\end{array}$ & $\begin{array}{l}16.3 \% \text { of people in Masterton live in decile } 10 \text { areas; } 52 \% \text { in } \\
\text { Deciles } 7-9 \text {; } \\
9.8 \% \text { in deciles } 4-6 \text { and } \\
21.9 \% \text { in deciles } 1-3 \text { (NZ Dep Measure 2006) } \\
\text { The average decile rating for Census Area Units in the } \\
\text { Masterton Districts is : } 6.1 \text { (NZ Dep Measure 2006) } \\
\text { Of the } 12 \text { Census Area Units in the District: } \\
6 \text { received the same decile rating: } 4 \text { declined and } 2 \text { improved. }\end{array}$ \\
\hline $\begin{array}{l}\text { Telephone and internet } \\
\text { access in the home }\end{array}$ & $\begin{array}{l}95.9 \% \text { of people have access to a telephone; and } \\
30.7 \% \text { have access to the internet (Ccnsus,2001) }\end{array}$ & $\begin{array}{l}90 \%{ }^{*} \text { of people have access to a telephone; and } \\
52 \% \text { hve access to the internet (Census 2006) }\end{array}$ \\
\hline $\begin{array}{l}\text { Households with } \\
\text { access to motor vehicle }\end{array}$ & $\begin{array}{l}88.5 \% \text { of households have access to a motor vehicle } \\
\text { (Ccnsus,2001) }\end{array}$ & $\begin{array}{l}90 \% \text { of households have access to a motor vehicle (Census } \\
2006 \text { ) }\end{array}$ \\
\hline
\end{tabular}

${ }^{\star}$ This decrease in the number of people who have access to a landline at home may reflect the increasing use of mobile and cell phones

\section{KEY CONTRIBUTORS}

You! Masterton District Council, Rangitane o Wairarapa Inc, Ngati Kahungun, Work and Income New Zealand, District Health Board, Primary Health organization, Wairarapa Workforce Development Trust, Wairarapa RWAP, Community Groups, Schools, Kohanga Reo, Kura Kaupapa o Wairarapa, Women's Center, CCS, IEAD Services, King Street Artworks, Wairarapa Organization for older people, Tertiary education providers, Masterton Trust Lands Trust, Trust house Charitable Trust 
Figure 10: Community Outcomes, Details for 1st Outcome (Hurunui District Council, 2009)

Community Outcome 1 :

"A Desirable Place to Live, Work and Play"

To make progress in relation to this outcome we need

A strong sense of community that give

people a sense of belonging and encourage

them to take part in local activities, to

support essential volunteer services, and to

be involved in local consultation and

decision making
We will know we ar succeeding when

People are involved

with their local

community
Progress will be measured using these key indicators

1 Percentage of people in paid employment

1.2 Number of volunteers helping outside of the home

1.3 Degree people feel a sense of community with others in the local neighborhood

1.4 Perceptions of people about their quality of life

People actively

participate in public

1.6 Number of people involved in Council meetings, committees and submissions to Council

1.7 Level of confidence in Council's decision making in the past year 1.8 Degree of understanding of Council's decision making

Attractive, well designed villages that

present a positive image encouraging

People to live in and visit the area

People feel the
Hurunui District is an
attractive place to live

1.9 District population and growth rate

1.10 Perception of attractiveness of townships and villages

1.11 Perception of use of subdivisions, coastal environment, Hamner Basin and Hurunui lakes

Opportunities in leisure, art and cultural

activities

Effective planning for future development but ensuring the preservation of local heritage and rural uniqueness

\begin{tabular}{|l|l|}
\hline $\begin{array}{l}\text { What the } \\
\text { Council is } \\
\text { doing } \\
\text { towards } \\
\text { achieving the } \\
\text { outcome }\end{array}$ & Council Activity \\
\cline { 2 - 3 } & $\begin{array}{l}\text { Governance and } \\
\text { Advocacy }\end{array}$ \\
\cline { 2 - 3 } & $\begin{array}{l}\text { Hamner Spring Thermal } \\
\text { Pools and Spa }\end{array}$ \\
\cline { 2 - 3 } & $\begin{array}{l}\text { Library, Local Facilities } \\
\text { and Maintenance, }\end{array}$ \\
& $\begin{array}{l}\text { Property, Public Toilets, } \\
\text { Cemeteries Grants \& } \\
\text { Service Awards }\end{array}$ \\
\hline
\end{tabular}

Main wellbeing areas / dimensions of wellness to which the outcome contributes

\begin{tabular}{|c|c|c|c|c|c|c|c|}
\hline \multirow{5}{*}{$\begin{array}{l}\text { Organisations } \\
\text { and groups } \\
\text { that contribute } \\
\text { towards } \\
\text { achieving this } \\
\text { outcome }\end{array}$} & Organization & Advocate & Funder & $\begin{array}{l}\text { Service } \\
\text { Provider }\end{array}$ & Regulator & Facilities & Monitor \\
\hline & Historic Places Trust & - & & & - & & $\bullet$ \\
\hline & $\begin{array}{l}\text { Ministry of Culture \& } \\
\text { Heritaqe }\end{array}$ & $\bullet$ & & & & & \\
\hline & Federated Farmers of NZ & - & & & - & & - \\
\hline & $\begin{array}{l}\text { Sport \& Recreation NZ } \\
\text { (SPARC) }\end{array}$ & - & $\bullet$ & & & & \\
\hline
\end{tabular}




\section{Chapter 4: Observations and Changes in LGA 2002}

\section{Auditor General Observations on 2006 \& 2009 Long Term Plans}

After the introduction of Local Government Act 2002 with the legislative requirement of developing long term plans with community well-beings and community outcomes, the Office of the Auditor-General New Zealand (2007) compiled a report regarding the job done by the Councils in developing the 2006 LTCCP. As it was the first time that the Councils were writing these plans, OAG (2007) commented that the Councils struggled in developing the performance framework. More than a quarter of the Councils couldn't establish a clear strong connection between the measures and the level of services. It was due to a weak rationale provided on how the services would be impacting on the community outcomes and wellbeing (OAG 2007).

As expected for the 2009 LTCCP, the Councils improved on developing their performance framework and connecting it with their community outcomes (OAG 2010). Almost all of the Councils updated their performance frameworks that resulted in developing a much clearer connection between services delivered and community outcomes. It was recommended that the Councils can further work on effectively disclosing their performance related to service delivery, community outcomes and well-beings. The other main recommendation, while acknowledging the variations between the Councils, was to improve the groupings of the activities. The collaborative working of the Councils with their neighbouring Councils for identifying and monitoring the community outcomes was recognised and supported. It helped in fulfilling the legislative requirement in an efficient way, especially for the small Councils that have limited resources. Here, another notable thing was that the majority of the Councils used their 2006 community outcomes in this round of LTCCP. This was because, according to the LGA 2002, the Councils are able to use the determined community outcomes for six years before they have to review them. Only few Councils went through the whole process of identifying the desired community outcomes, whereas some councils just updated the wordings a bit or changed the measures and targets. 
The other important observation raised in OAG (2007) was regarding the connection of sustainable development with community well-beings and community outcomes. It was observed that although there was an improvement, nevertheless the connections were not clear enough in the 2006 LTCCPs. All of the LTCCPs had referred to the four well-beings but some areas lacked clear connections as compared to others. Larger Councils have done a better job as compared to the smaller Councils because they had developed their community outcomes more recently. The improvements suggested were that sustainable development should be thought in a more localised way at Council and community level; expressed in a way that is more meaningful and specifically show the Council's contribution in it; clearly integrate with Council activities, planning and management; show how, during the decisionmaking, the well-beings for foreseeable future generations were considered.

Looking into the most recent report, along the same lines, from OAG (2019), regarding the development of Council activities and outcomes, we note that the Councils have significantly improved in creating a logical flow of different components of their performance framework. The community outcomes were also connected with what Councils do, but they were so broad that they were added in nearly all of the Council activities. Therefore, it was suggested that the Councils should identify the primary activity to which that community outcome would be impacting the most, and along with it also identify a secondary activity. The other important point raised by OAG was the disclosure of performance measures and targets for each activity which would be clearly linked back to the community outcome. It was also observed that some Councils provided explanations around achieving the community outcomes by setting up some goals, descriptions, objectives, relevant programs and projects. This helped in seeing what Council wants to achieve and how it will get it there.

\section{Changes in LGA 2002 in the year 2010 and 2012}

In the year 2010, the then Minister of Local Government, Hon Rodney Hide, proposed amendments $^{14}$ in the Local Government Act 2002 which were known as Transparency,

\footnotetext{
${ }^{14}$ These amendments were made when National Party was in the government.
} 
Accountability and Financial Management (TAFM)later came to be known as Better Local Government (Hide, 2010). The main reason for these amendments was to improve the transparency, financial management and accountability of the local government. More specifically, the purpose was to go back to the core services module as laid out in the LGA 2002, as the Councils are answerable to the ratepayers. Secondly, to develop a prudent financial strategy which would limit the increase in Councils' rates and debts along with measurable targets for returns on Council investments. Thirdly, to encourage an informed debate during the local government elections, a recommendation to publish a pre-election report. There were a couple of other amendments as well to reduce the procedural requirements (Hide, 2010a).

Among the reasons for the proposed amendments there was one on making a simple planning process (Hide, 2010). It was outlined that the LTCCP developed by the Councils contained a huge amount of information which was of little interest to the ratepayers, and it was difficult to understand and find a proposal of interest. The process of identifying community outcomes as a separate process to LTCCP was seen as duplication of effort, where the former feeds into the latter one. As well, these community outcomes were broad and didn't necessarily involve services which Council was responsible for delivering.

The amendment Act was passed in November 2010 and the main decisions with regard to the planning process were:

1. Changing the name from Long Term Council Community Plan (LTCCP) to Long Term Plan (LTP).

2. Inclusion of financial strategy in LTP so that Councils and ratepayers could focus on and prioritise the projects, services, investments, debts and rates accordingly.

3. Focus on the core services and mandatory disclosure of the information regarding to the five major infrastructure services (i.e., water supply, stormwater drainage, roading, sewage treatment and disposal, and flood protection) $)^{15}$.

\footnotetext{
${ }^{15}$ A new Section 11A was added in LGA 2002 in the Amendment Act of 2010 which listed the core services for the communities as: "in performing its role, a local authority must have particular regard to the contribution that the following core services make to its communities:
} 
4. Merging the community outcomes identification process with the LTP process.

5. Focus on only those community outcomes that the Council will achieve and ratepayers are willing to pay for.

In March 2012 the Minister for local government announced another reform program (DIA, 2012). It was formally known as 'Better Local Government' and was designed to improve the effectiveness and efficiency of local government. The amendment Act was passed in two phases, and the main decisions taken in it were around (DIA, 2013):

1. Change of purpose of local government;

2. Financial prudence requirements;

3. Council governance structure and intervention options;

4. Process of reorganising local government;

5. Development contributions;

6. Auckland Local Boards;

7. Services delivery and its governance;

8. Consultation, decision-making, long term plans and annual plans; and

9. Infrastructure and asset management planning.

The significant change made in the first phase was to modify the second part of the purpose of the local government in the LGA 2002 to, "meet the current and future needs of communities for good-quality local infrastructure, local public services and performance of regulatory functions in a way that is most cost-effective for households and businesses" (DIA, 2012). Thus, with this change, legislatively the focus of local government shifted from community well-beings to the delivery of core services. It was deemed that this change would make the Councils focus only on activities they can perform and, do so in the best possible way.
(a) network infrastructure;
(b) public transport services;
(c) solid waste collection and disposal;
(d) the avoidance or mitigation of natural hazards;
(e) libraries, museums, reserves, recreational facilities, and other community infrastructure." 


\section{Changes in LGA 2002 in the year 2019}

In April 2018 the Minister of Local Government, Hon Nanaia Mahuta proposed amendments ${ }^{16}$ to the LGA 2002. These amendments were mainly to reinstate the purpose of the community well-being back in the LGA 2002; and secondarily to make modifications to the collection of development contributions (Local Government (Community Well-being) Amendment Bill: Explanatory Bill, 2018; DIA, 2018). The proposed amendments were on the basis of a very strong context regarding the role of local government in enhancing the well-being of their communities (New Zealand Parliament, 2018). The purpose statement, to be reinstated, was "to promote the social, economic, environmental, and cultural well-being of communities, in the present and for the future" (DIA, 2018). Here well-being is considered holistically, and the four well-beings are the four aspects of it, which needs to be considered together rather than individually (New Zealand Parliament, 2018). It is envisioned that with these amendments the purpose will become more clearer, coherent and consistent; which will enable the Councils to be more focused towards their communities as it will shift their thinking from 'are we allowed to do this?', to more towards 'should we do this?' (DIA, 2018).

The three main objectives of the Local Government (Community Well-being) Amendment Act 2019 were: to restore the purpose of local government in the context of community wellbeings; to allow for the collection of development contributions from any new development involving the installation of any public amenities needed which results from these new developments; and to remove the focus of Councils from core services to a more open community based services provision (New Zealand Parliament, 2018; Local Government (Community Well-being) Amendment Bill: Explanatory Bill, 2018). The third objective was a technical change in the collection of development contributions (Explanatory Bill, 2018).

The new Section 11A that was added by amendment in 2010 , which made the core services ${ }^{17}$ to be considered in the Council's role in serving its communities was repealed. It gave Councils a more flexible approach to cater to the needs of their communities. As the Councils and

\footnotetext{
${ }^{16}$ These amendments were made when Labour Party was in government.

${ }^{17}$ See footnote 13
} 
communities of each area are in the best position to determine what is important and needed for them, and how it can be approached for addressing or resolving. This change is seen as a more empowering approach for local authorities, with greater flexibility as compared to the instructions given in regard to what can be done with communities (New Zealand Parliament, 2018).

The Local Government (Community Well-being) Amendment Act 2019 was passed in May 2019. This amendment can be seen as unifying the thinking of central government and local government, and how the former views the latter (New Zealand Parliament, 2018a). From a timing perspective, Councils have to prepare LTP 2021-2031 which is due by June 2021. With the community well-being reinstated, councils have to work a bit more for identifying their community outcomes and connecting it with Council activities. It might also change the projects, the priorities and the financial impacts (i.e., the performance framework and performance measures). Decision-making will be the key aspect while incorporating the community well-beings in Councils' plans for the coming years. This situation becomes a bit tricky to handle with the Covid-19 scenario and its economic impact on the communities. There has been a massive support for the local government sector from the Department of Internal Affairs (DIA), Audit New Zealand ${ }^{18}$ and the New Zealand Society of Local Government Managers (SOLGM) ${ }^{19}$, through providing timely guidance on developing the LTP 2021-2031.

As stated by Hon Nanaia Mahuta, "community well-being is at the heart of what local authorities do. So, it only makes sense for the legislation to recognise this important role. To work effectively, all levels of government must listen to the needs of communities. This is the only way to ensure social cohesion, inclusion, and develop thriving and sustainable local communities" (New Zealand Parliament, 2018a).

\footnotetext{
${ }^{18}$ Audit New Zealand (2020)

${ }^{19}$ LG Sector Good Toolkit, Long Term Planning Resources
} 


\section{Chapter 5: Set of Measures Common Across the Councils}

\section{Measures selection through long term plan 2009-19}

In this chapter we intend to collate the community well-being outcomes, their definitions, indicators and measures used across the local government in the long-term Council community plans of 2009. As discussed in the previous chapters, the community well-beings were introduced in the year 2002 and they were developed for the first time in the year 2004, and then they were used for the first in the year 2006 in the long-term Council community plan (LTCCP), and after that, they were used in the year 2009 for LTCCP. After that, with the change in the Local Government Act, they were not used again. Although we see the traces of community well-beings in some Councils' plans and they were kept and used in the subsequent long-term plans ${ }^{20}$, the majority of the Councils stopped reporting on it. Therefore, focusing on the year 2009 is a better option for this study.

Moreover, from the Audit New Zealand reports, it is apparent that the community outcomes were more mature and clearly articulated in the year 2009 as compared to previous years. For the long-term plan (LTP) 2021-31, Audit New Zealand (2020) have given some guidance around the importance of the performance framework and performance measures. They mentioned that there are two parts to the performance framework; the first part connects with the community outcomes and community well-beings, whereas the other describes details about the Council level of service for each group of activities. These two parts must align and show the connection between each other. The group of activities statements is the best place where this link is shown. This guidance document also recommended to have a small set of well-being indicators along with the performance measures, as it will give a strong foundation for the Council to report on the impact of its services on the community wellbeings.

Statistics New Zealand (2006, as cited in Masterton District Council, 2009) defined indicators "as a parameter that can be measured to show the trends or changes in a particular condition...Indicators reduce the number of measurements that are normally required to give

\footnotetext{
${ }^{20}$ For example see long term plans of Christchurch City Council.
} 
a complete picture of a situation." Then there can be some criteria for choosing indicators. By way of example, Masterton District Council (2009) has set this criterion around four things, i.e., available, affordable, applicable and simple and easily understood.

These four criteria are explained as (Masterton District Council, 2009):

1. "Available:

a. Already being measured (existing data and information) wherever possible

2. Affordable:

a. Easy to access and/or measure data.

b. Simple to monitor.

c. Do not require huge expense to obtain and/or measure.

3. Applicable:

a. Able to measure changes on an appropriate scale, e.g. overtime.

b. Relevant to the outcome being measured.

c. Provides information at a level appropriate and useful for decision making.

d. Already recognised (i.e., tested and validated) as a measure of the outcome it is applied to at a national level.

4. Simple and easily understood:

a. Simple to interpret and publicly appealing.

b. Clearly informs about the extent of the issues of interest."

For this study we compiled the list of community outcomes, their definitions, indicators and measures from all the regional, city and district Councils across New Zealand. In some cases, we also came across the data sources from where the measures were collected but this was only available for a couple of Councils. This extensive data collection process was carried out by visiting the council websites one by one through the Internet Archive: Way Back Machine ${ }^{21}$. The Way Back Machine contains a record of websites as it was in the year 2009. By going back in time, we were able to download the long-term plans. It took a bit of time to search for the files but eventually we were able to download all of them. In some cases, there was only one file whereas in others there were a couple of files.

${ }^{21}$ https://archive.org/web/ 
All Councils had a separate chapter on community outcomes, with a discussion around it. There were cases where we found that the community outcomes were properly referred in a separate report which was released a bit earlier than the long-term plan 2009. They were around the progress of community outcomes. In those cases, we had to search the community outcomes report by again using the Way Back Machine within the Council websites.

As shown in the dashboard below (Figure 11$)^{22}$, there were 78 region, city and district Councils. The majority of the data (about 90\%) was collated from the long-term plans of the Councils, while in some cases the community outcomes (8\%) were collected from the reports specifically written on community outcomes or monitoring reports. In a rare case for Auckland Council the community outcomes were gathered from their plan (i.e., The Auckland Plan).

Most of the datasets around community outcomes are based on the year $2009(87 \%)$, then from 2018 (8\%), and the rest from the years 2006, 2007 and 2008. With the community wellbeings back in the Local Government Act 2002, the Councils are at the moment busy in drafting their long-term plan 2021-2031 and will release it by June 2021. In this LTP 2021-31 round we will be able to see some updates in the community outcomes and the measures used, but we do not expect a significant change. All of the Councils have reported community outcomes, whereas $67 \%$ of the Councils have provided a definition or articulated the community aspiration. After this, $62 \%$ or 48 councils have provided a list of indicators to further elaborate the community outcomes. In order to comprehend the progress of wellbeing over time, $90 \%$ (or 70 ) of Councils have provided a specific list of measures which is connected to the community outcomes. Only $24 \%$ of the Councils have given the source of data from where they will be collecting the data. In a small number of cases (14\%) the number of indicators were more than the number of measures.

\footnotetext{
${ }^{22}$ Access the interactive version of these dashboards from: https://public.tableau.com/profile/data.n.dashboards\#!/vizhome/CommunityWell-beings/DataOverview
} 
Since the number of community outcomes and the corresponding measures were more commonly available across the Councils, we took the approach to drop the indicators, and connect the outcomes directly with the measures. In this way, it became easier for us to understand the relationship between what the community wants and how it will be measured. From the 78 councils across New Zealand we compiled, in total, 556 community outcomes and $4,337^{23}$ measures. Whereas there were 987 community outcome definitions or statements, or in other words, we can say community aspirations. To connect all of these outcomes and measures with the four well-beings we created another layer of broad groups of measures. In total there are 74 broad measures - see Figures 12 and 13 . These broad groups comprise measures which are same or very similar to each other. These broad groups were further connected with the four well-beings (i.e., social well-being, economic well-being, environmental well-being and cultural well-being); and one more category of demographics. In this way we were able to summarise this wealth of information in one place (i.e., from measures to broad groups to community outcomes to well-beings). See Figure 12 for the overview dashboard.

Within the broad measures group, the highest number of measures are for 'rivers, lakes, coastal and groundwater', followed by 'environment/environmental attitudes' and then for 'community development'. See Figure 15 for the dashboard and visit the website for the full interactive version of $\mathrm{it}^{24}$. The author of this study developed these broad measures group by making the best judgement on the best fit for each measure. As for the highest number of broad measures group for the Councils are for 'crime/safety' followed by 'rivers, lakes, coastal and groundwater' and then by 'qualification/school'. The former broad measure group was cited by 71 Councils and the latter two by 67 and 66 respectively.

A comprehensive view across all the 78 Councils shows that the average number of community outcomes for a Council is around seven; and there are on the average around 55 measures per Council. These outcomes and measures can be divided into four well-beings and a demographics category. On the basis of our broad measure groups and the number of

\footnotetext{
${ }^{23}$ We have removed 14 measures as they were not completely defined.

${ }^{24}$ Access the interactive version of these dashboards from: https://public.tableau.com/profile/data.n.dashboards\#!/vizhome/CommunityWell-beings/DataOverview
} 
Councils reporting those measures we did a cluster analysis. It was to ascertain which broad measure groups are similar to one another (see Figure 16 for the clusters dashboard).

We used Tableau software to conduct these cluster analyses. Tableau uses k-means algorithm for clustering and Calinski-Harabasz criterion to assess the quality and the numbers of the clusters. "The Calinski-Harabasz criterion is defined as:

$$
\frac{S S_{B}}{S S_{W}} \times \frac{(N-k)}{(k-1)}
$$

Where SSB is the overall between-cluster variance, SSW the overall within-cluster variance, $\mathrm{k}$ the number of clusters and $\mathrm{N}$ the number of observations. The greater the value of this ratio, the more cohesive the clusters (low within-cluster variance) and the more distinct/separate the individual clusters (high between-cluster variance)" (Find Clusters in Data, Tableau). Tableau automatically assigns the number of clusters on the basis of the first local maximum of Calinski-Harabasz.

In Tables 7 and 8 the results of this cluster analysis are presented. The F-statistic and p-value are significant in this analysis.

\begin{tabular}{|l|l|l|l|l|l|l|}
\hline \multicolumn{5}{c}{$\begin{array}{c}\text { Table 7: Cluster Analysis Model } \\
\text { Analysis of Variance }\end{array}$} \\
\hline & & & Model & \\
\hline Variable & F-statistic & p-value & Sum of Squares & DF & Sum of Squares & DF \\
\hline $\begin{array}{l}\text { Number of } \\
\text { Councils }\end{array}$ & 32.08 & $1.186 e-10$ & 5.035 & 2 & 5.572 & 71 \\
\hline
\end{tabular}

Whereas we got three clusters, from which the third cluster has the highest number of Councils (59) but has the least number of broad groups (16), and cluster one has the second highest number of Councils (33) but the highest number of broad groups (31). From these results we can confidently suggest that most of the Councils are measuring their community well-beings in somewhat similar fashion to each other. At the same time the second cluster, 
which represents 10 Councils, have 27 different broad groups. This result verifies the discussion in previous chapters that each district or city is different to the others; they have different challenges, resources, capacities, capabilities and opportunities.

\section{Table 8: Cluster Analysis Summary}

\begin{tabular}{|c|c|c|}
\hline \multicolumn{3}{|l|}{ Inputs for Clustering } \\
\hline Variables: & Number of Councils & \\
\hline Level of Detail: & Broad Measures & \\
\hline Scaling: & Normalised & \\
\hline \multicolumn{3}{|l|}{ Summary Diagnostics } \\
\hline Number of Clusters: & 3 & \\
\hline Number of Points: & 74 & \\
\hline Between-group Sum of Squares: & 5.0355 & \\
\hline Within-group Sum of Squares: & 0.53657 & \\
\hline \multirow[t]{2}{*}{ Total Sum of Squares: } & 5.572 & \\
\hline & & Centres \\
\hline Clusters & Number of Broad Measures & Number of Councils \\
\hline Cluster 1 & 31 & 32.6 \\
\hline Cluster 2 & 27 & 9.8 \\
\hline Cluster 3 & 16 & 59.0 \\
\hline
\end{tabular}


Figure 11: Community Outcomes Data Availability Dashboard

\begin{tabular}{|llll} 
Community Well-beings by Measures, Outcomes \& Councils \\
New Zealand Local Government: Long Term Plans 2009
\end{tabular}


Figure 12: Overview of the Community Well-beings and Comparison with LSF and IANZ

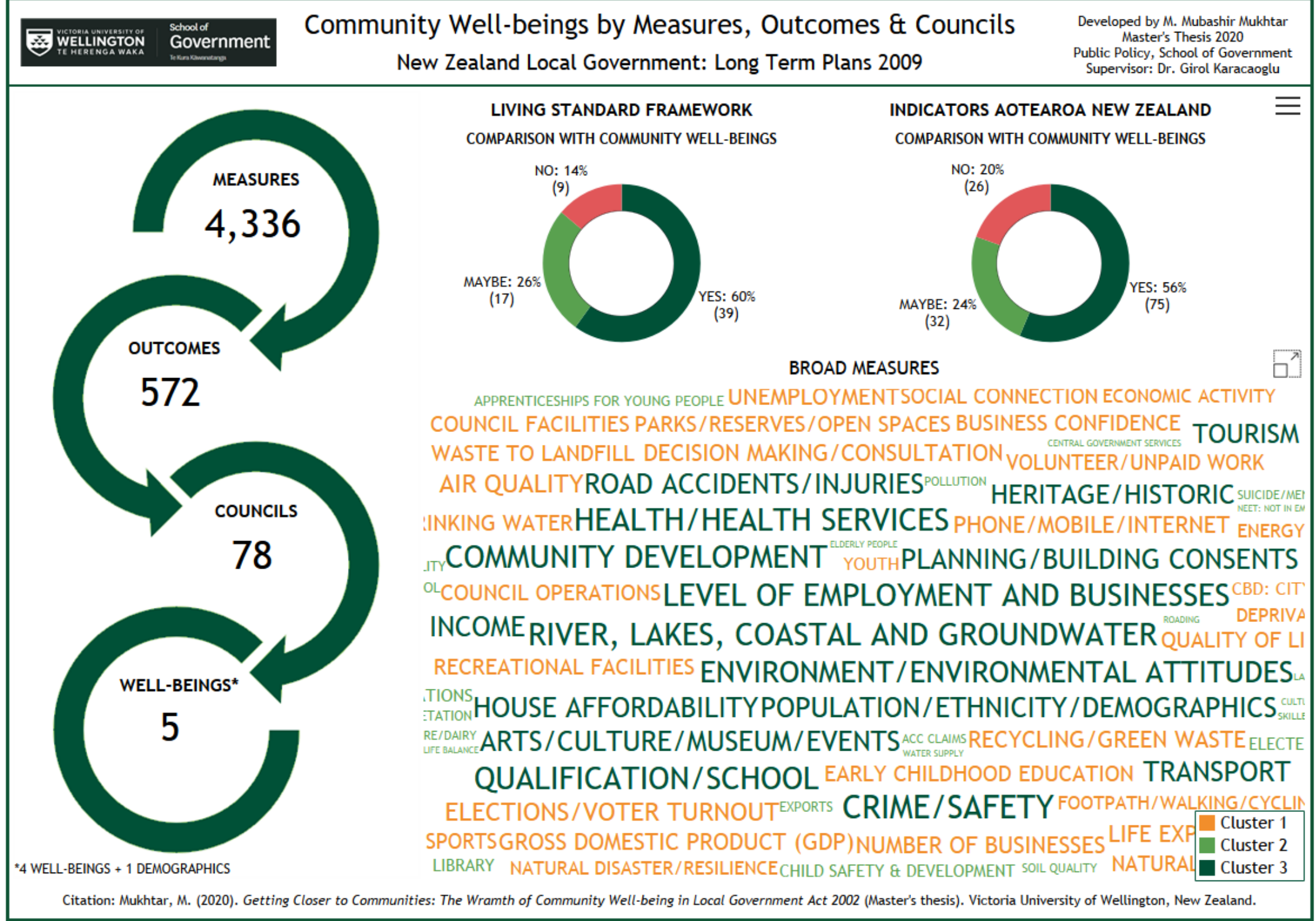


Figure 13: List of Community Well-beings

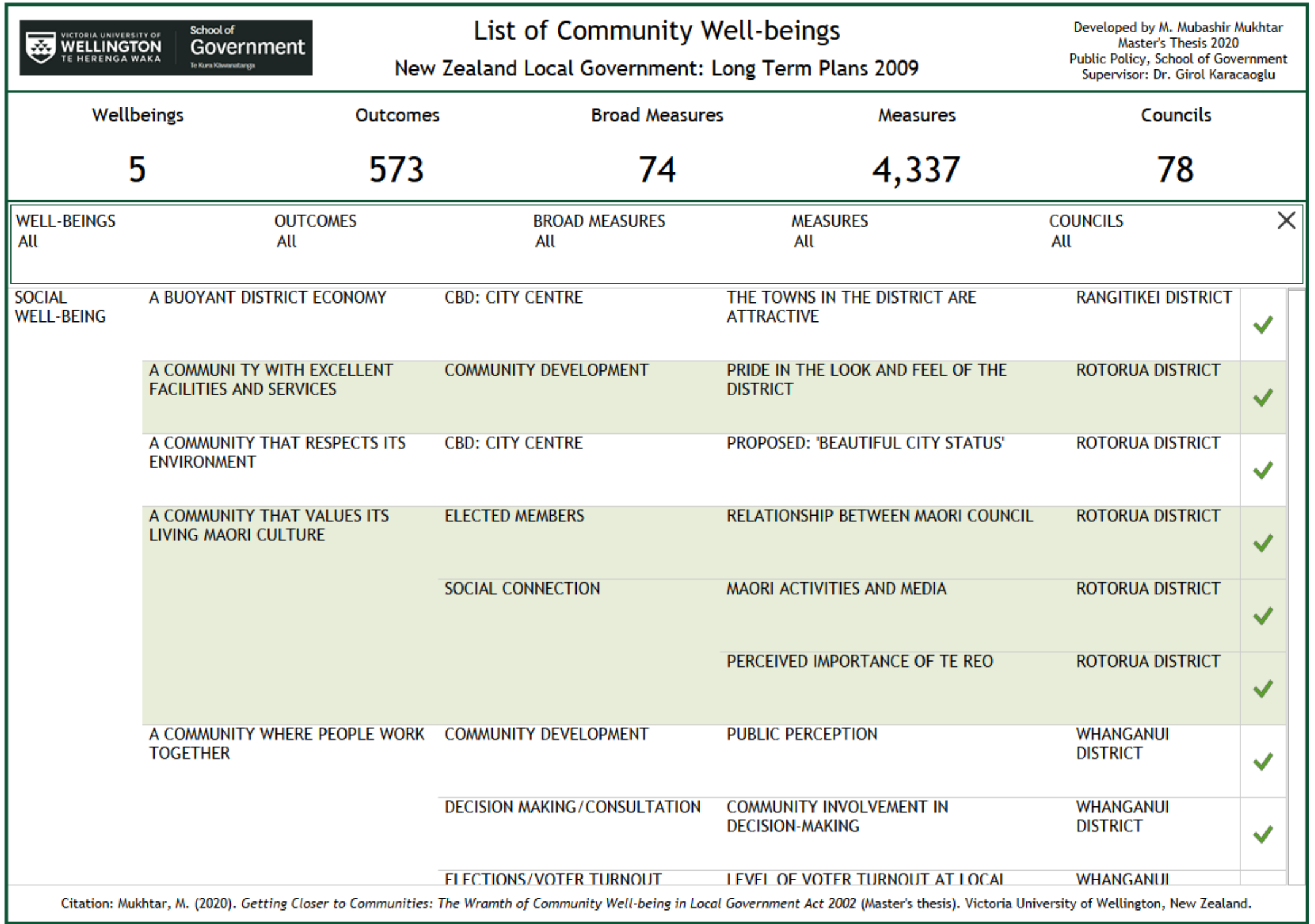


Figure 14: List of Community Well-being Statements

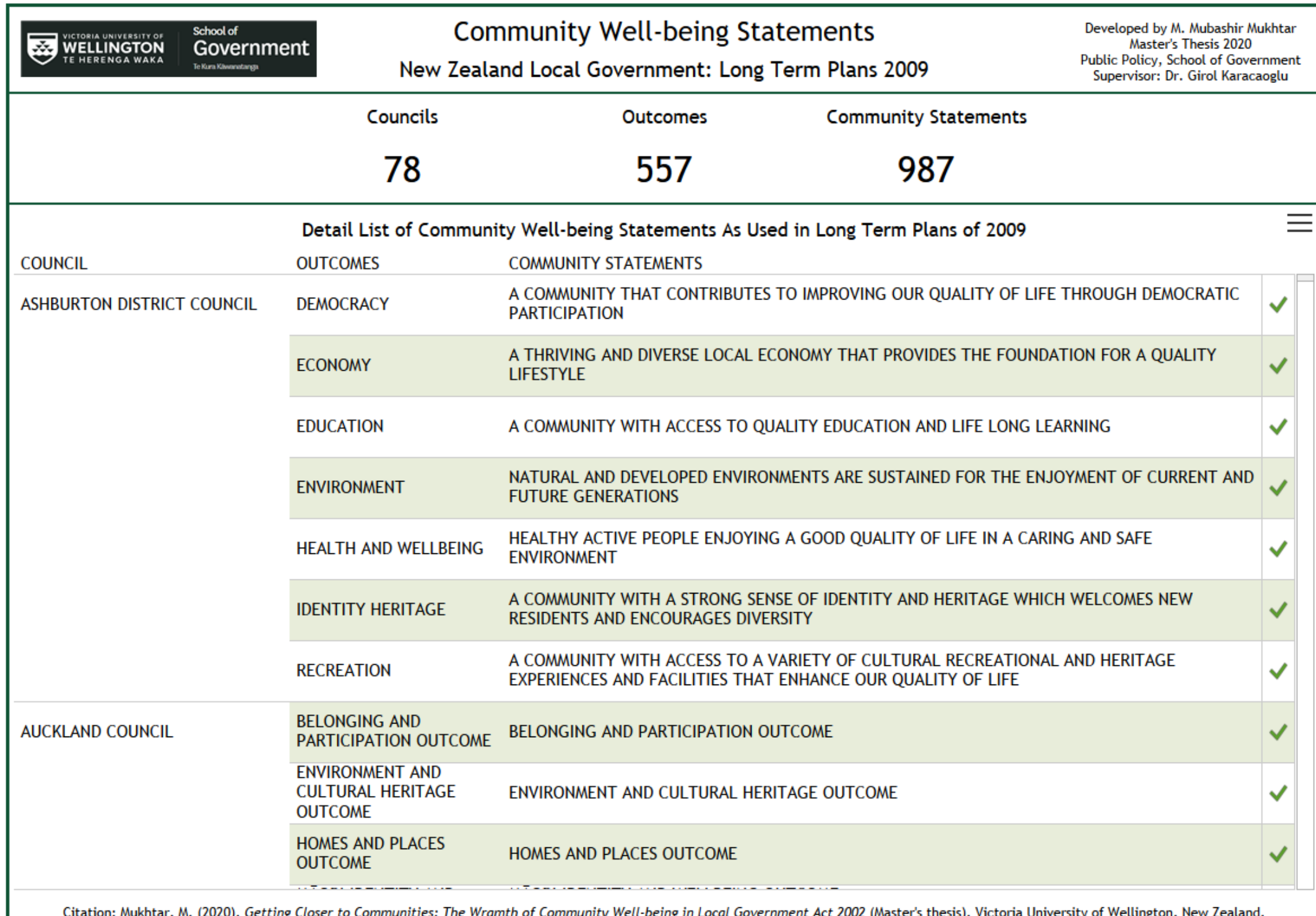


Figure 15: Broad Group of Measures by Number of Measures, Number of Outcomes and Number of Councils

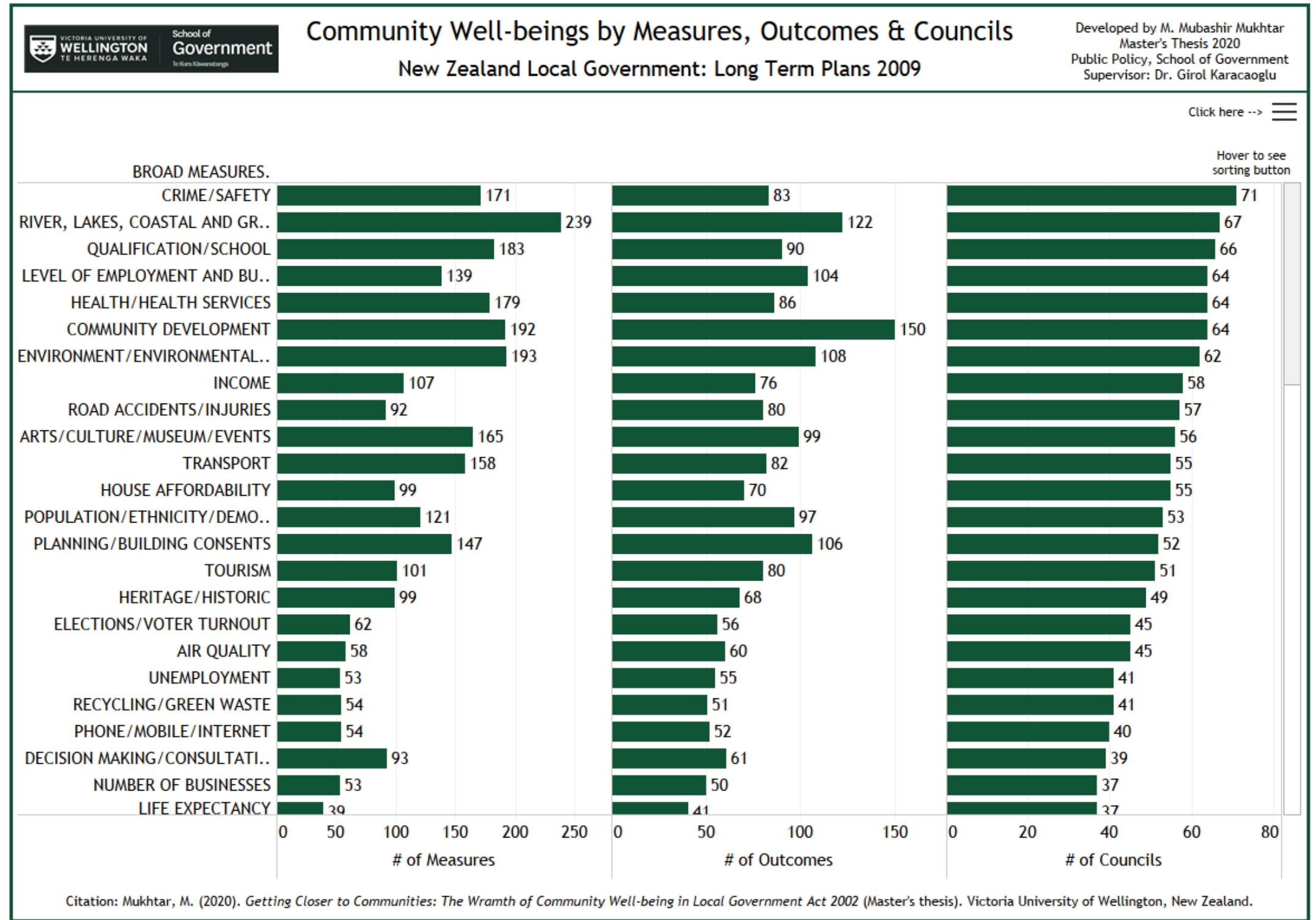


Figure 16: Broad Community Well-being Measures

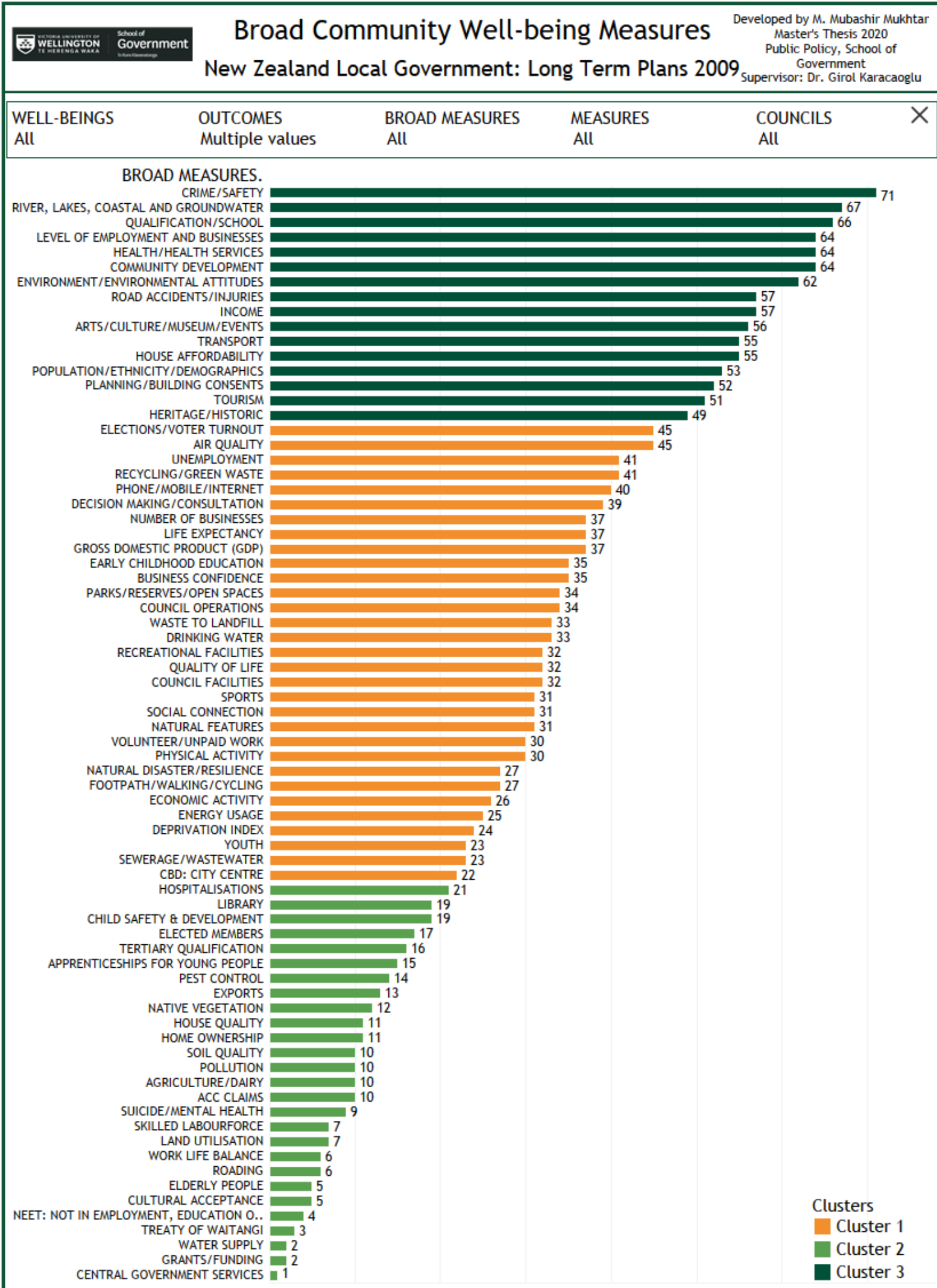

Citation: Mukhtar, M. (2020). Getting Closer to Communities: The Wramth of Community Well-being in Local Government Act 2002 (Master's thesis). Victoria University of Wellington, New Zealand. 


\section{Chapter 6: Aligning the Community Well-being Measures with LSF and IANZ}

In the previous section we covered community well-beings, in the form of outcomes and measures as published by the local government. We intend to expand this analysis and compare the community well-being measures with the two most influential pieces of work done by the central government agencies of New Zealand. They are the Living Standard Framework (LSF) developed by The New Zealand Treasury and Indicators Aotearoa New Zealand (IANZ) developed by Statistics New Zealand.

The purpose of these two pieces of work is to give sight to the government on shaping the long-term well-being strategy for New Zealand. The LSF was released in Dec 2018 with the main aim to support the cross-government well-being priorities, it "is a high-level framework for measuring and analysing intergenerational wellbeing, covering current wellbeing, future wellbeing, and risk and resilience across a range of economic, social and environmental outcome domains" (Treasury, 2018). It was developed after consultation with the public, domestic and international experts, and informed by OECD's well-being approach. Thus, it can be aligned with international datasets and provide an international comparability.

The LSF comprises of a range of outcome indicators which can be seen in detail through the dashboards developed by The Treasury. There are three intergenerational well-being outcomes (i.e., our country, our people, and our future). In here, our country and our people comprise 12 current well-being domains: civic engagement and governance, cultural identity, environment, health, housing, income and consumption, jobs and earnings, knowledge and skills, time use, safety and security, social connections, and subjective wellbeing. Whereas our future comprises four future well-being capitals: natural capital, social capital, human capital and finance/physical capital (Treasury, 2018). In each respective domain there are a few sets of indicators which help monitor the progress made in that respective domain. In total there are 65 indicators across 16 domains.

As the LSF is developed for a national level representation of well-being, it compels us to compare it with the list of community well-being (CWB) measures that are used at a territorial authority level. We tried to match LSF indicators with the CWB measures as best as possible. 
Our analysis shows that out of 65 indicators listed in LSF there was a comparable measure for $39(60 \%)$ in the CWB measures. The match between LSF and CWB does not mean that the data will be available at that level of granularity, although it can happen in some cases; rather, the important point in here is that the indicators used in LSF are in some way useful and reported on at a much granular level in CWB (i.e., at territorial authority level). Furthermore, we can say that for 17 (26\%) indicators there was a near match, as we could not find an exact or strong match. But in this case of near match, we have identified the most likely measure from CWB. Lastly, we could not find any match for 9 (14\%) indicators. See Figures 12 and 17 for the summary and full analysis dashboard; also visit the dashboard online for the interactive full version of this dashboard. We have also provided a full list of indicators in Appendix C and D.

LSF does not intend to replace the other well-being frameworks or datasets or dashboards; it basically complements the other sector-focused or more granular level information currently used in the public sector (Treasury, 2018). One such other framework in the public domain is IANZ. The purpose of IANZ is to bring in the limelight the diversified aspects of well-being and its progress across the board for all New Zealanders. Additionally it will help in improving the systems for the current and future generations. The IANZ set of indicators encompasses both, a holistic view of well-being and sustainable development; this vision aligns with the internationally growing focus on well-being. It follows the United Nations Sustainable Development Goals (SDGs) reporting standard and also brings in the New Zealand context by incorporating cultural and Te Ao Maori perspectives. As compared to LSF, the IANZ set of indicators were derived after a detailed consultation process with the public and subject matter experts. So, it seems to be more rooted in well-being as perceived by the people of New Zealand. This project was started in mid-2018 with the consultation process, the indicators were finalised by early 2019 and the website was launched by mid-2019.

Altogether, there are 133 indicators across 22 topics and two themes (i.e., well-being indicators and contextual indicators). Each topic has multiple indicators but not all indicators have data associated with them. So out of 133 indicators, 87 (65\%) have data and the rest 46 (35\%) are in the development stage. We tried aligning IANZ indicators with the CWB measures as best as possible. Our analysis shows that out of 133 indicators listed in IANZ there were 75 
(56\%) aligned with CWB measures. The alignment between IANZ and CWB does not mean that there is an exact word to word match between the two, but it is almost the same thing. Furthermore, the alignment does not mean that the data will be available at the same level of granularity, although it can happen in some cases. In the case of IANZ most of the data is available at regional level. As highlighted earlier, the important point in here is that the indicators used in IANZ are in some way useful and reported on a much granular level in CWB (i.e., at territorial authority level). For 32 (24\%) indicators there was a near match, as we could not find an exact or strong match. In the case of a near match, we have mentioned the most likely measure from CWB. Lastly, we could not find any match for $26(20 \%)$ indicators. See Figures 12 and 18 for the summary chart of this alignment and the dashboard for the list of indicators respectively. We have also provided a full list of indicators in the Appendix C and D.

From this alignment analysis it can be deduced that most of the indicators used in LSF and IANZ are also used in CWB at a territorial authority level. Which means that the well-being measurement work in the majority of cases can be aligned at a granular level with nationallevel indicators. In the same way, the measures which are available in CWB but not present in LSF or IANZ ${ }^{25}$, and that which are used by majority (cluster 1 and 3 as discussed in previous section) of the Councils, can be built into these two frameworks to give a more in depth view on well-being.

\footnotetext{
${ }^{25}$ See IANZ website for an alignment between LSF and IANZ. 53 (81\%) indicators of LSF match with IANZ. https://wellbeingindicators.stats.govt.nz/en/aligning-with-living-standards-framework/
} 
Figure 17: Coverage of Community Well-being Measures in Living Standard Framework

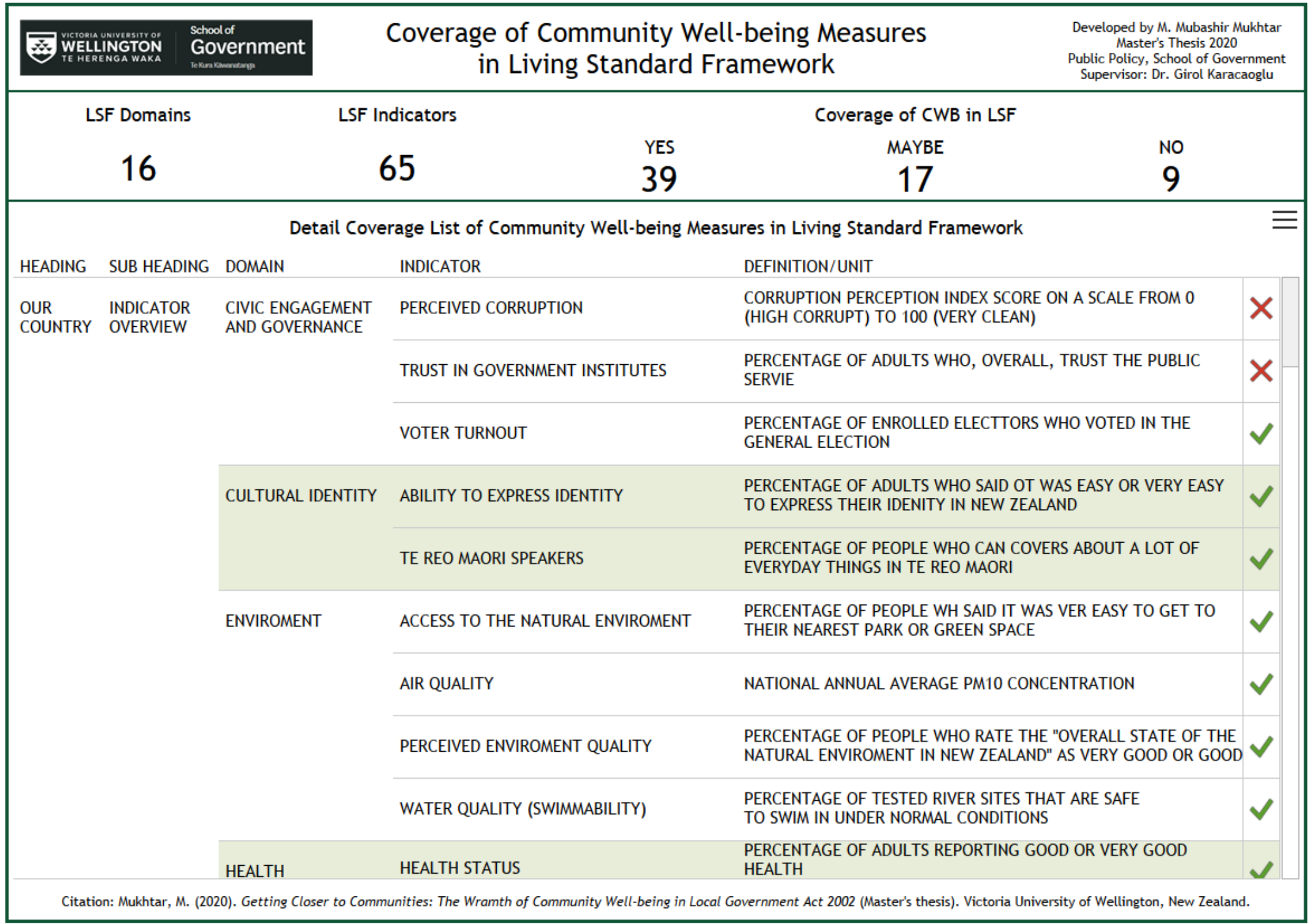


Figure 18: Coverage of Community Well-being Measures in Indicators Aotearoa New Zealand

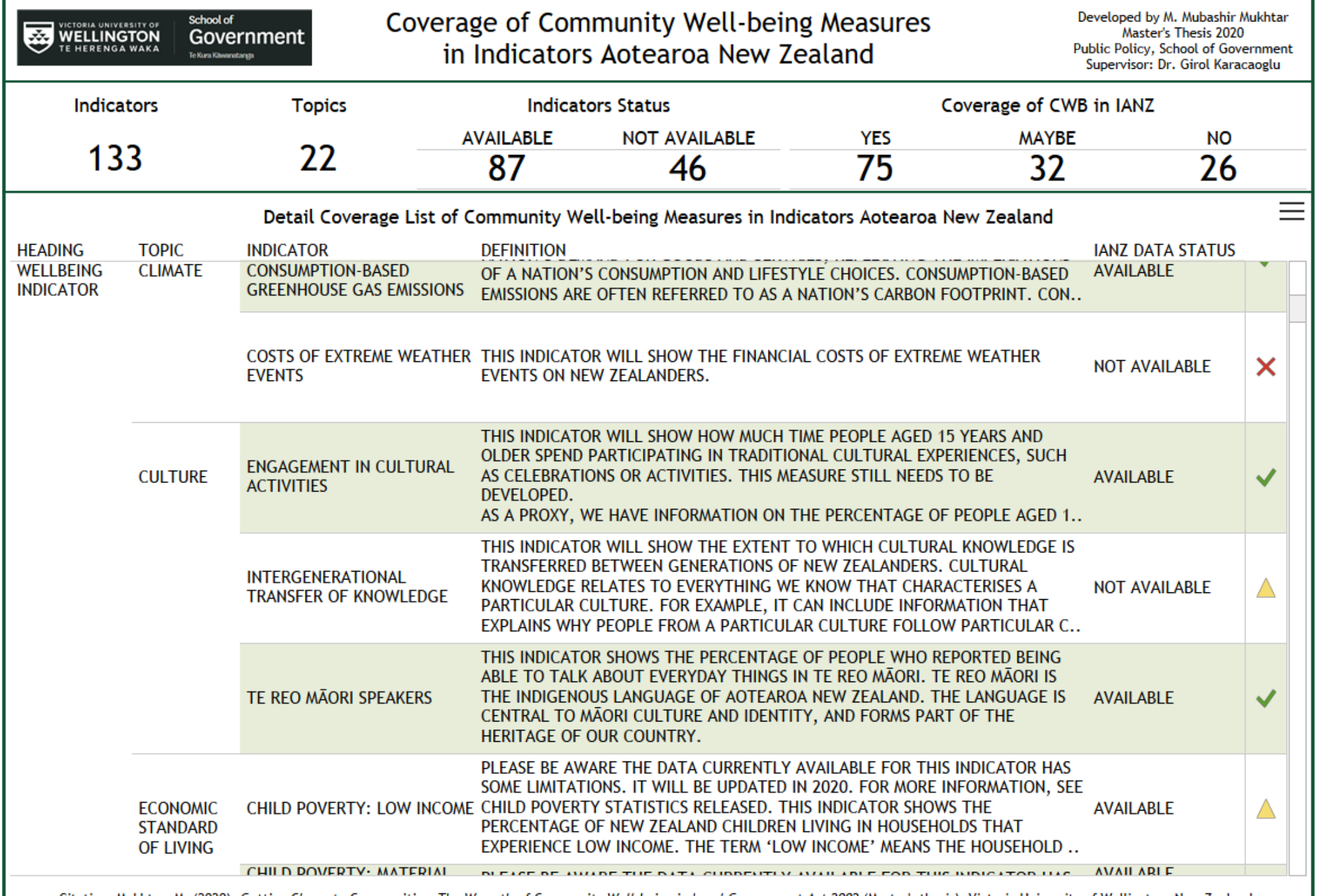

Citation: Mukhtar, M. (2020). Getting Closer to Communities: The Wramth of Community Well-being in Local Government Act 2002 (Master's thesis). Victoria University of Wellington, New Zealand. 


\section{Chapter 7: Case Study: Community Well-beings in Ashburton District Council}

In this chapter we intend to conduct a case study of utilising community well-beings for the Ashburton District Council $(A D C)^{26}$. There is no specific reason for selecting ADC apart from the authors own knowledge about the district and the Council. This case study was done as a desktop exercise in which various documents issued by the Council were used to frame the case.

Ashburton District is located within Canterbury Region, in the South Island of New Zealand. It is mainly an agriculture economy with a major focus on dairy farming (MBIE, 2020). With a population of 35,400 (Stats NZ, 2020) it is one of the fastest growing rural districts (ADC, 2018). The population increase occurred in both urban and rural areas, but it was mainly due to economic growth in rural parts. With an average house price of $\$ 384,998$ in Oct 2020 (Property Value, 2020) and mean weekly rent of \$335 in 2019 (MBIE(a), 2020), Ashburton District is a great family place. At the same time ADC (2018) have mentioned some key issues for the district which are due to growth in population, household structure change, becoming more ethnically diverse, planning for natural disasters (e.g., earthquakes), lack of NZTA subsidy funding, climate change, and management of surface water.

Based on the state of the community report published in 2009 (ADC, 2009a), there were seven community outcomes identified through a consultative process with the community during the year 2005. All round efforts were put in by the Council staff members and the elected members (mayor and councillors) to hear from the community. A series of nine workshops were conducted in which over 100 people and around 70 different groups and agencies attended. A survey was also conducted in which over 600 people responded reflecting on a wide range of issues relating to the community. Apart from these, previous survey responses (undertaken between 2003 and 2005) which were held by the Council were also reviewed. Through this extensive exercise the Council was able to focus on seven community outcomes.

\footnotetext{
${ }^{26}$ The author has worked at Ashburton District Council from Aug 2015 to Aug 2018 as a Research and Monitoring Advisor within the Community Relations Team.
} 
These outcomes related to: economy, environment, identity \& heritage, education, health \& well-being, recreation and democracy.

Further explanations on these outcomes are available in the shape of community statements. They basically outline what the community specifically wants to see for their current and future well-being. In addition, there were a number of measures associated with each of the outcomes so that the achievement of those outcomes could be tracked over time. In total, there were 58 measures across the seven outcomes. These measures were selected on the basis of being SMART (i.e., specific, measurable, achievable, relevant and timebound). Another consideration was that the measures have linkages with regional and national datasets so that a sound comparison could be made. This paragraph summarises the whole approach of community well-being for ADC "if we, as a community, are to make progress to achieving our community outcome goals we need to work together and to play to our individual and collective strengths. This is likely to include working collaboratively and exploring new ways of working that perhaps have not been considered in the past" (ADC, 2009a). See Table 9 for the list of community well-being outcomes, statements and measures as developed by the Ashburton District Council.

In its LTP 2009-19 the Council has clearly sketched out the link between the community outcomes and the Council activities (see Figure 19). Starting from the community outcomes (as discussed above) there were strategic objectives also provided for each of the outcomes and community statements. These strategic objectives were marked as a priority objective where they were so deemed by the community (see Table 10 for the complete list). Council also came up with its strategic vision which connected to the community outcomes. The broad vision of the Council for its community was, "Ashburton: District of Choice for Lifestyle and Opportunity" (ADC, 2009). There were five strategic vision statements that showed what ADC will do, how they will achieve it, and which Council activities are going to contribute towards the community plan ${ }^{27}$. Thus, there was a connection being made between the community outcomes and the Council's strategic vision. To make it even clearer, the Council's

\footnotetext{
27 The author intends to discuss these parts in detail in his PhD thesis (to be started in April 2021). The research area is going to be on local government decision-making process and budgeting.
} 
contributions to achieving community outcomes were also listed. This list showed the Council's group of activities across the board, as well as the key district and regional strategic planning documents which are going to fulfil the community outcomes strategic objectives. It was also noted that while community outcomes are the main drivers for setting up the directions of the Council working, there are two other things which also influences these workplans, and they are: legislative requirement (activities which Council has to undertake irrespective of its impact on community outcomes) and the political mandate of the elected members (with regard to Council activities and which may not exactly match with the community outcomes) (ADC, 2009).

Collaborative working with the community and other organisations was also given a strong emphasis. Because in many cases Council have no major role to play at all in achieving the community outcomes, it has to work along with other agencies, community groups, and the private sector. Council has a number of partnerships with local organisations like Ashburton Safer Community Council, District Health Committee, Ashburton Community Pool Board, and the Ashburton Performing Arts Theatre Trust. A strong working relationship was also identified with Te Runaka o Arowhenua who have mana whenua over Ashburton District. The vision of Te Runaka o Arowhenua also aligns with the Council's vision: "nurturing our people through generations, guardians of the environments we live in, progressing our future locally and globally" (ADC, 2009). Hakatere Marae Komiti, a maata waka organisation based within Ashburton at Hakatere Marae was identified by Te Runaka o Arowhenua to liaise with the Council on local initiatives and contribute towards achieving the community outcomes.

In the LTP 2009-19, ADC was reporting on the progress made on the community well-being through the outcomes framework (measures), and also reporting on the progress made on council activities through its strategic vision framework. Thus, we can say that it was utilising external and internal data to achieve the community well-beings as stipulated in the Local Government Act 2002. This type of reporting and monitoring structure shows a very clear progress on the commitments made by the Council and also helps the community to further decide on the path they intend to walk for their own current and future well-beings. 


\begin{tabular}{|c|c|c|c|}
\hline \multicolumn{4}{|c|}{ Table 9: Community Outcomes and Measures from Ashburton District Council 2009} \\
\hline S.NO & OUTCOMES & $\begin{array}{l}\text { COMMUNITY } \\
\text { STATEMENTS }\end{array}$ & MEASURES \\
\hline 1 & \multirow[t]{12}{*}{ “ECONOMY } & \multirow{12}{*}{$\begin{array}{l}\text { A THRIVING AND DIVERSE } \\
\text { LOCAL ECONOMY THAT } \\
\text { PROVIDES THE } \\
\text { FOUNDATION FOR A } \\
\text { QUALITY LIFESTYLE }\end{array}$} & ASHBURTON DISTRICT POPULATION GROWTH \\
\hline 2 & & & $\begin{array}{l}\text { PROPORTION OF WORKING AGED POPULATION } \\
\text { UNDERTAKING INDUSTRY TRAINING OR } \\
\text { MODERN APPRENTICESHIPS IN THE DISTRICT }\end{array}$ \\
\hline 3 & & & MEDIAN ANNUAL PERSONAL INCOME \\
\hline 4 & & & MEDIAN ANNUAL HOUSEHOLD INCOME \\
\hline 5 & & & WORKFORCE PARTICIPATION RATE \\
\hline 6 & & & HOME OWNERSHIP RATE \\
\hline 7 & & & $\begin{array}{l}\text { RESIDENT SATISFACTION WITH THE QUALITY OF } \\
\text { COUNCIL-PROVIDED INFRASTRUCTURE }\end{array}$ \\
\hline 8 & & & RESIDENT ACCESS TO INTERNET SERVICES \\
\hline 9 & & & $\begin{array}{l}\text { ANNUAL VALUE, TYPE AND NUMBER OF } \\
\text { BUILDING CONSENTS ISSUED }\end{array}$ \\
\hline 10 & & & $\begin{array}{l}\text { RESIDENT SATISFACTION WITH COUNCILS ROLE } \\
\text { IN THE MANAGEMENT OF THE DISTRICTS } \\
\text { WATER RESOURCES }\end{array}$ \\
\hline 11 & & & TOURISM GUEST NIGHT NUMBERS \\
\hline 12 & & & $\begin{array}{l}\text { RESIDENTS SATISFACTION WITH THEIR QUALITY } \\
\text { OF LIFE IN THE DISTRICT }\end{array}$ \\
\hline 13 & \multirow{8}{*}{$\begin{array}{l}\text { ENVIRONM } \\
\text { ENT }\end{array}$} & \multirow{8}{*}{$\begin{array}{l}\text { NATURAL AND } \\
\text { DEVELOPED } \\
\text { ENVIRONMENTS ARE } \\
\text { SUSTAINED FOR THE } \\
\text { ENJOYMENT OF CURRENT } \\
\text { AND FUTURE } \\
\text { GENERATIONS }\end{array}$} & AIR QUALITY - PM10 LEVELS IN ASHBURTON \\
\hline 14 & & & $\begin{array}{l}\text { GROUNDWATER NITRATE LEVELS IN } \\
\text { MONITORED DRINKING WATER WELLS }\end{array}$ \\
\hline 15 & & & $\begin{array}{l}\text { SWIMMING WATER QUALITY IN THE DISTRICTS } \\
\text { RIVERS AND LAKES }\end{array}$ \\
\hline 16 & & & $\begin{array}{l}\text { WATER CONSUMPTION PER HOUSEHOLD } \\
\text { CONNECTION ON COUNCIL DRINKING WATER } \\
\text { SCHEMES }\end{array}$ \\
\hline 17 & & & RATIO OF OPEN SPACE \\
\hline 18 & & & $\begin{array}{l}\text { AMOUNT OF REUSABLE /RECYCLABLE } \\
\text { MATERIAL DIVERTED FROM THE WASTE } \\
\text { STREAM }\end{array}$ \\
\hline 19 & & & $\begin{array}{l}\text { RESIDENT SATISFACTION WITH KERBSIDE } \\
\text { RECYCLING AND RUBBISH COLLECTION } \\
\text { SERVICES PROVIDED BY COUNCIL }\end{array}$ \\
\hline 20 & & & $\begin{array}{l}\text { RESIDENT SATISFACTION THAT CURRENT } \\
\text { ENVIRONMENTAL PROTECTION WILL PRESERVE } \\
\text { AND SUSTAIN THE DISTRICT FOR FUTURE } \\
\text { GENERATIONS }\end{array}$ \\
\hline 21 & \multirow[t]{4}{*}{$\begin{array}{l}\text { IDENTITY \& } \\
\text { HERITAGE }\end{array}$} & \multirow{4}{*}{$\begin{array}{l}\text { A COMMUNITY WITH A } \\
\text { STRONG SENSE OF } \\
\text { IDENTITY AND HERITAGE } \\
\text { WHICH WELCOMES NEW } \\
\text { RESIDENTS AND } \\
\text { ENCOURAGES DIVERSITY }\end{array}$} & $\begin{array}{l}\text { RESIDENTS SATISFACTION WITH COMMUNITY } \\
\text { SPIRIT WITHIN ASHBURTON DISTRICT }\end{array}$ \\
\hline 22 & & & $\begin{array}{l}\text { PERCENTAGE OF MAORI SPEAKERS IN THE } \\
\text { DISTRICT }\end{array}$ \\
\hline 23 & & & $\begin{array}{l}\text { SATISFACTION WITH PRESERVATION OF } \\
\text { HERITAGE FEATURES }\end{array}$ \\
\hline 24 & & & $\begin{array}{l}\text { PROPORTION OF POPULATION PARTICIPATING } \\
\text { IN VOLUNTARY WORK }\end{array}$ \\
\hline
\end{tabular}




\begin{tabular}{|c|c|c|c|}
\hline \multicolumn{4}{|c|}{ Table 9: Community Outcomes and Measures from Ashburton District Council 2009} \\
\hline S.NO & OUTCOMES & $\begin{array}{l}\text { COMMUNITY } \\
\text { STATEMENTS }\end{array}$ & MEASURES \\
\hline 25 & & & RESIDENT PERCEPTIONS OF DIVERSITY \\
\hline 26 & & & $\begin{array}{l}\text { RESIDENT PERCEPTIONS OF THE LEVEL OF } \\
\text { SUPPORT GIVEN TO NEW RESIDENTS AND } \\
\text { MIGRANTS }\end{array}$ \\
\hline 27 & \multirow[t]{7}{*}{$\begin{array}{l}\text { EDUCATIO } \\
\mathrm{N}\end{array}$} & \multirow{7}{*}{$\begin{array}{l}\text { A COMMUNITY WITH } \\
\text { ACCESS TO QUALITY } \\
\text { EDUCATION AND LIFE } \\
\text { LONG LEARNING }\end{array}$} & $\begin{array}{l}\text { NEW SCHOOL ENTRANT PARTICIPATION IN } \\
\text { EARLY CHILDHOOD EDUCATION (ECE) }\end{array}$ \\
\hline 28 & & & $\begin{array}{l}\text { RETENTION RATES OF STUDENTS AT SCHOOL TO } \\
\text { AGE YEARS }\end{array}$ \\
\hline 29 & & & $\begin{array}{l}\text { YOUTH WHO ARE NOT ENGAGED IN } \\
\text { EMPLOYMENT, EDUCATION OR TRAINING } \\
\text { (NEET) }\end{array}$ \\
\hline 30 & & & SUSPENSION AND EXCLUSION RATES \\
\hline 31 & & & HIGHEST QUALIFICATION GAINED \\
\hline 32 & & & $\begin{array}{l}\text { STUDENTS LEAVING SCHOOL WITH NCEA LEVEL } \\
2 \text { AND HIGHER }\end{array}$ \\
\hline 33 & & & $\begin{array}{l}\text { RESIDENT SATISFACTION WITH EDUCATION } \\
\text { SERVICES IN THE DISTRICT }\end{array}$ \\
\hline 34 & \multirow{11}{*}{$\begin{array}{l}\text { HEALTH } \\
\text { AND WELL- } \\
\text { BEING }\end{array}$} & \multirow{11}{*}{$\begin{array}{l}\text { HEALTHY ACTIVE PEOPLE } \\
\text { ENJOYING A GOOD } \\
\text { QUALITY OF LIFE IN A } \\
\text { CARING AND SAFE } \\
\text { ENVIRONMENT }\end{array}$} & $\begin{array}{l}\text { NEW ZEALAND DEPRIVATION INDEX (SOCIAL } \\
\text { DEPRIVATION INDEX) }\end{array}$ \\
\hline 35 & & & $\begin{array}{l}\text { PROPORTION AND DISTRIBUTION OF ALL } \\
\text { MEANS TESTED BENEFI TS BEING RECEIVED }\end{array}$ \\
\hline 36 & & & RENT TO INCOME RATIO \\
\hline 37 & & & SMOKING RATES \\
\hline 38 & & & FREQUENCY OF PHYSICAL ACTIVITY \\
\hline 39 & & & SATISFACTION WITH LOCAL HEALTH SERVICES \\
\hline 40 & & & $\begin{array}{l}\text { SATISFACTION WITH ACCESS TO HEALTH } \\
\text { SERVICES }\end{array}$ \\
\hline 41 & & & $\begin{array}{l}\text { RECORDED CRIME OFFENCES AND } \\
\text { APPREHENSIONS IN THE MID-SOUTH } \\
\text { CANTERBURY POLICE DISTRICT }\end{array}$ \\
\hline 42 & & & SATISFACTION WITH COMMUNITY SAFETY \\
\hline 43 & & & $\begin{array}{l}\text { ROAD CRASH INJURY AND FATALITY RATES FOR } \\
\text { ASHBURTON DISTRICT }\end{array}$ \\
\hline 44 & & & WORKPLACE ACCIDENT AND INJURY RATES \\
\hline 45 & \multirow{7}{*}{$\begin{array}{l}\text { RECREATIO } \\
\mathrm{N}\end{array}$} & \multirow{7}{*}{$\begin{array}{l}\text { A COMMUNITY WITH } \\
\text { ACCESS TO A VARIETY OF } \\
\text { CULTURAL } \\
\text { RECREATIONAL AND } \\
\text { HERITAGE EXPERIENCES } \\
\text { AND FACILITIES THAT } \\
\text { ENHANCE OUR QUALITY } \\
\text { OF LIFE }\end{array}$} & USE OF THE DISTRICT LIBRARY \\
\hline 46 & & & PATRONAGE OF THE ART GALLERY \\
\hline 47 & & & PATRONAGE OF THE MUSEUM \\
\hline 48 & & & $\begin{array}{l}\text { SATISFACTION WITH COUNCILS INVOLVEMENT } \\
\text { WITH ART GALLERY AND MUSEUM }\end{array}$ \\
\hline 49 & & & USE OF THE COMMUNITY POOL \\
\hline 50 & & & $\begin{array}{l}\text { USE OF COUNCIL PROVIDED SPORT AND } \\
\text { RECREATION FACILITIES }\end{array}$ \\
\hline 51 & & & $\begin{array}{l}\text { SATISFACTION WITH COUNCIL PROVIDED SPORT } \\
\text { AND RECREATION FACILITIES }\end{array}$ \\
\hline
\end{tabular}




\begin{tabular}{|c|c|c|c|}
\hline \multicolumn{4}{|c|}{ Table 9: Community Outcomes and Measures from Ashburton District Council 2009} \\
\hline S.NO & OUTCOMES & $\begin{array}{l}\text { COMMUNITY } \\
\text { STATEMENTS }\end{array}$ & MEASURES \\
\hline 52 & & & $\begin{array}{l}\text { SATISFACTION WITH COUNCILS ROLE IN } \\
\text { COMMUNITY EVENTS }\end{array}$ \\
\hline 53 & \multirow[t]{6}{*}{$\begin{array}{l}\text { DEMOCRA } \\
\mathrm{CY}\end{array}$} & \multirow{6}{*}{$\begin{array}{l}\text { A COMMUNITY THAT } \\
\text { CONTRIBUTES TO } \\
\text { IMPROVING OUR } \\
\text { QUALITY OF LIFE } \\
\text { THROUGH DEMOCRATIC } \\
\text { PARTICIPATION }\end{array}$} & $\begin{array}{l}\text { VOTER TURNOUT IN LOCAL AND CENTRAL } \\
\text { GOVERNMENT ELECTIONS }\end{array}$ \\
\hline 54 & & & $\begin{array}{l}\text { SATISFACTION WITH THE WAY COUNCIL } \\
\text { INVOLVES THE PUBLIC IN THE DECISIONS IT } \\
\text { MAKES }\end{array}$ \\
\hline 55 & & & $\begin{array}{l}\text { SATISFACTION WITH THE AVAILABILITY OF } \\
\text { COUNCIL INFORMATION }\end{array}$ \\
\hline 56 & & & $\begin{array}{l}\text { SATISFACTION WITH THE PERFORMANCE OF } \\
\text { THE MAYOR AND COUNCILLORS }\end{array}$ \\
\hline 57 & & & $\begin{array}{l}\text { SATISFACTION WITH CONTACT WITH COUNCIL } \\
\text { STAFF }\end{array}$ \\
\hline 58 & & & $\begin{array}{l}\text { SATISFACTION WITH COUNCILS ADVOCACY } \\
\text { ROLE FOR THE DISTRICT" }\end{array}$ \\
\hline
\end{tabular}

Figure 19: ADC Community Outcomes and Strategic Planning

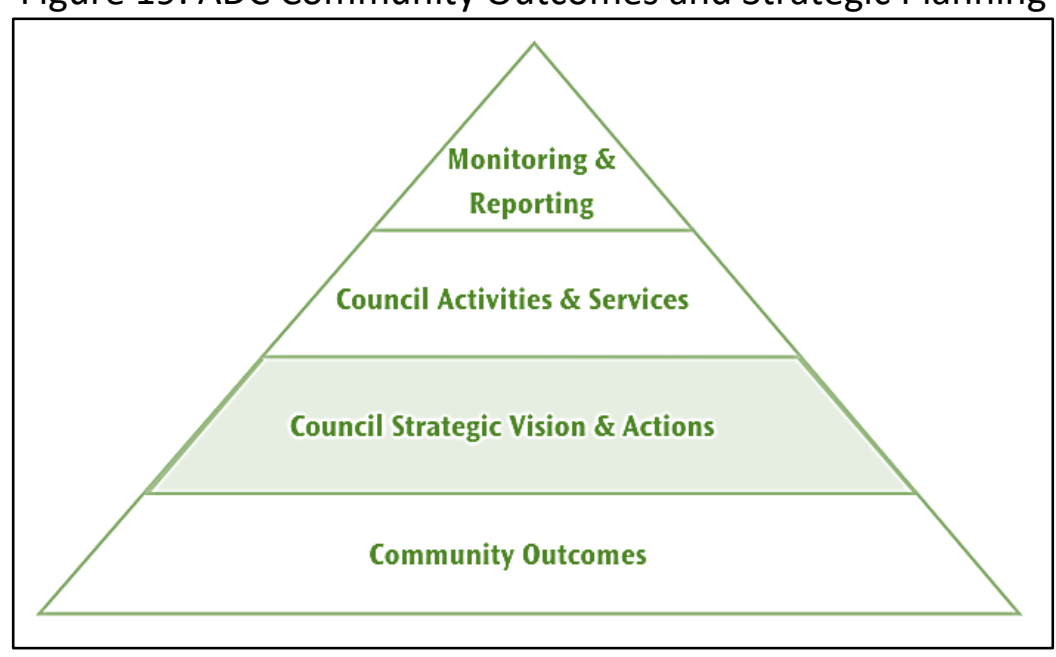

Table 10: Community Outcomes and Strategic Objectives from Ashburton District Council 2009

\section{Outcome}

“Outcome 1 (Economy): A thriving and diverse local economy that provides the
Strategic Objectives

- Our community has access to quality infrastructure (roading, water, footpaths, wastewater and storm water) that is efficient and reliable. (Priority Objective) 


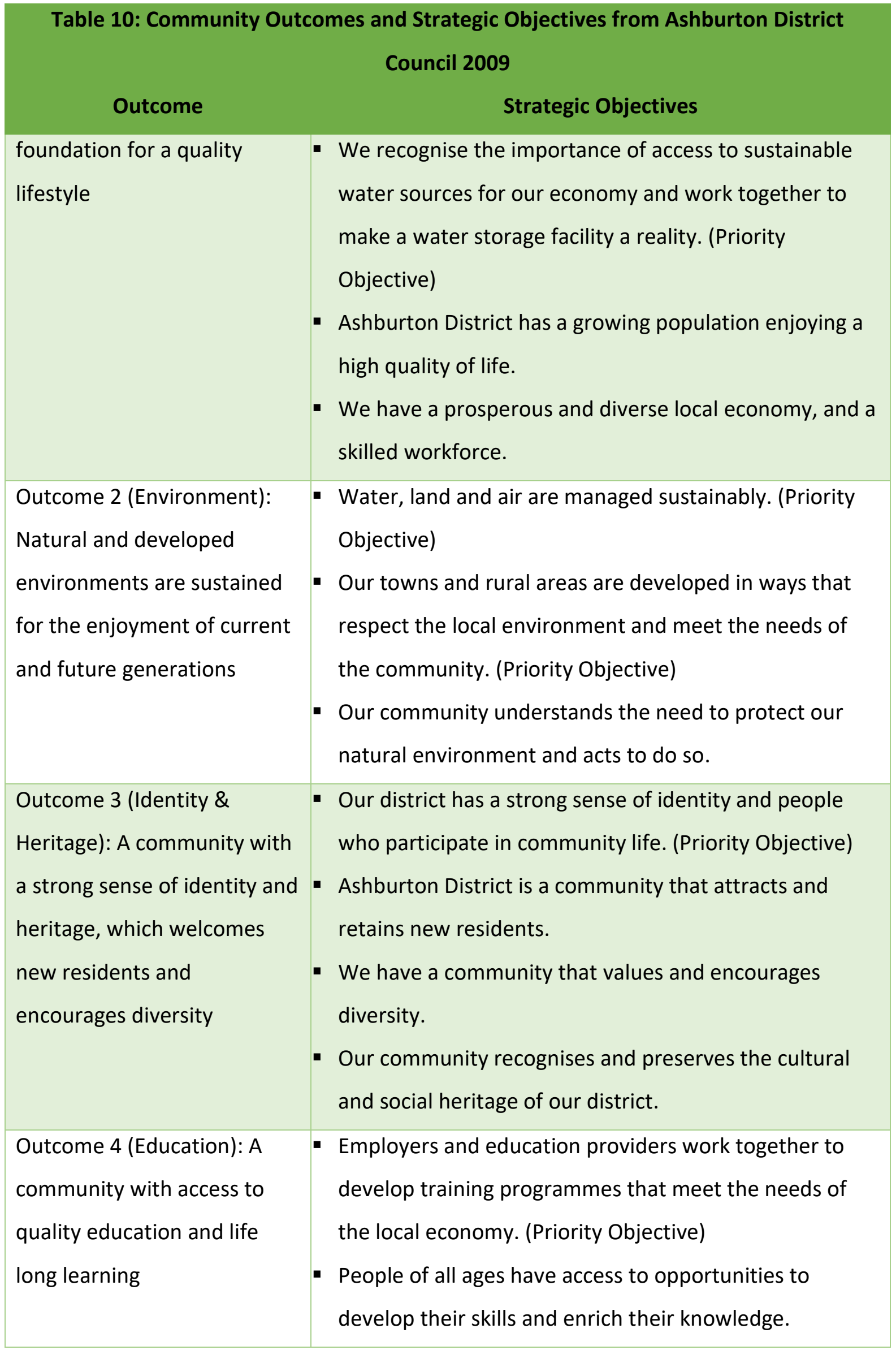


Table 10: Community Outcomes and Strategic Objectives from Ashburton District

Council 2009

Outcome

Strategic Objectives

- Education providers have the resources they require to meet community needs.

Outcome 5 (Health \& Wellbeing): Healthy, active people enjoying a good quality of life in a caring and safe community

Outcome 6 (Recreation): A community with access to a variety of cultural, recreational and heritage experiences and facilities that enrich our quality of life

Outcome 7 (Democracy): A community that contributes to improving our quality of life through democratic participation
- We have healthy, active people with good access to health services. (Priority Objective)

- A range of agencies work together to create a safe environment.

- Good systems are in place to plan for, and respond, to emergencies.

- Young people are supported to lead healthy and safe lives and are active participants in community life.

- We have a caring community that supports the elderly and helps others in need.

- Cultural, recreational and heritage facilities are maintained and developed to meet community needs. (Priority Objective)

- Cultural, recreational and heritage experiences and facilities are accessible to the community and actively used.

- Advocacy by community leaders for identified local concerns and issues at a local, regional and national level. (Priority Objective)

- The community is provided with opportunities to participate in local decision-making.

- There is active participation in elections and consultation processes." 
For the Long Term Plan 2018-28, ADC adopted a unique approach to consult with its community. They utilised the Delphi Study approach in which three rounds of survey were conducted with the community in order to identify the priorities which the community cared about (ADC, 2018a). The consultation process project was named 'Our Place' and was conducted over a span of six months. This round of LTP was on the basis of the amendments in LGA 2002 in which the community well-beings were repealed. Despite that the Council came up with four community outcomes. These community outcomes were different from the ones used in LTP 2009-19. The four community outcomes were (ADC, 2018):

- "Residents are included and have a voice

- A district of great spaces and places

- A balanced and sustainable environment

- A prosperous economy based on innovation and opportunity"

Apart from these community outcomes, Council has its own four strategic priorities which helps in achieving these outcomes. The 'Our Place' ${ }^{28}$ consultation project helped in identifying eight ideas which the community wanted the Council to focus on over the next ten years. There was tremendous response from the community on the survey. In total ADC received over 2,300 responses from all over the district. In the first round ADC received 660 responses, in the second round there were 863 , and in the final (third) round there were 801 responses. The Delphi study enables staff to identify a manageable number of options from a vast range of possibilities. The unique thing which ADC did was to ask open-ended questions in the first round in which the community could give any three ideas which they think are important. Then in the second round they were asked to rank a more refined set of ideas which were developed in the first round. And finally, in the third round, the community was asked to rank the selected ideas from the second round. All of these rankings were based on the number of submissions (or the majority). The top eight ideas which were finalised from the third round were (ADC, 2018):

- "Roading (including sealed and unsealed road maintenance)

- Community safety (including street lighting, road safety, security patrols)

${ }^{28}$ https://www.itsourplace.com/ 
- Environment (including freshwater management and banning plastic bags)

- Town centre development (specifically the Ashburton CBD)

- Second bridge development in Ashburton

- Business development (including retail shops, restaurants and industry)

- EA Networks Centre development (including hydro slides, pool/stadium growth, sports fields development)

- New entertainment centres."

These eight priorities, along with the community outcomes and Council strategic priorities helped in forming the focus of major projects for the LTP 2018-28. The five major projects were (ADC, 2018):

- Our Roading Network

- Our Transportation Upgrades - Second Urban Bridge

- Our Water - Future Planning

- Our Recreation - EA Networks Centre Development

- Our Economy - Economic Development Strategy.

As this LTP was developed under the amended LGA 2002, the Council had a focus on Council activities. There were a range of Council services and activities, and all of them are connected with the community outcomes, Council strategic priorities and community priorities. Progress reporting was done on the basis of level of services and the targets set for achievement. These were monitored through performance measures ${ }^{29}$. And the data for these performance measures are all coming from internal sources, which comprise annual resident surveys and Councils' own data ${ }^{30}$. These datasets are mostly on the output side rather than outcome side. And that is the main difference which we can see between the LTP 2009-19 and LTP 2018-28. Reporting on the external data is missing in the latter LTP whereas it was a significant part of the former LTP. We are hoping that with the reintroduction of community well-being in LGA 2002 this data gap will be addressed by the Council in the LTP 2021-31 and subsequently.

\footnotetext{
${ }^{29}$ The level of services and performance measures are the reflection of the Council's intention on service delivery and the performance to its community.

${ }^{30} \mathrm{CRM}$ or asset management data.
} 
The community consultation project 'Our Place' won a "highly commended citation in The Giblin Group Award for Innovation in Council Community Relations at the [2018] SOLGM Excellence Awards" (SOLGM, 2018b). It was due to the fact that the survey had a more open engagement style and relied on the community to suggest the ideas and rank them as well. The other important thing to note in here is that it was a pre-engagement consultation project which aimed to get some consensus from the community and give a direction for setting the LTP 2018-28. Rather than consulting with the community after writing the LTP, this project aimed to get the community involved beforehand. Keeping the same strategy, ADC have opened the Our Place project and conducted a survey from the community in the last quarter of 2020 . They referred back to the priorities set back in 2018 , and explored if the community wanted to change them, and if so, what would that be. The results of the survey will be shared in the new year and will drive the direction for LTP 2021-31.

In this case study we learned that how Ashburton District Council engaged with its community to identify the needs and priorities of the community. These identified points were translated into community outcomes and measures. Along with it, the Council have developed its strategic vision and aligned the community outcomes accordingly so that the Council service delivery could all go hand in hand with the expectations of the community. Utilisation of different external and internal datasets was a big deal back in 2009, whereas now it is focusing more on the internally collected data and to some extent external demographic and economic datasets, though this might change again in near future. 


\section{Chapter 8: Conclusion and Further Research Directions}

In this last chapter of thesis, we will be discussing what we have learned from the previous chapters and list down the possible future research directions. The initial chapters highlighted the continuous changes in the Local Government Act, reflecting evolving government objectives. These continuous changes disrupt the processes in place for the local government. The long-term plan is a substantive piece of work which the Councils undertake every three years. At the same time, it is a plan which contain ten-year forward-looking objectives. Some minor changes due to different circumstances are somewhat easy to handle but to incorporate any major change, especially at a legislative level, is quite challenging for the Councils.

In the case of community well-being, we saw that Councils had written their initial two ${ }^{31}$ long term plans with a view to improving community well-beings, whereas the next three ${ }^{32}$ long term plans were focused on Council group of activities and level of services. The next upcoming long term plan in 2021 is going to be based again on community well-being. There was a bit of uncertainty about what would happen in case of change of government in the 2020 national election. Any kind of major change from central government impacts on the Council's delivery timetable and puts extra pressure on the Council staff members. It will be a lot better to see some concrete and stable directions from the central government around the utilisation of community well-beings in the local government sector; especially when the current government has a very strong emphasis on well-beings across the board. These uncertainties have a real impact. As we highlighted in chapter four, covering the Audit New Zealand findings that it took a little bit of time for the Councils to get their head around the linkages between the community outcomes and the Council activities. These learning curves have some resources tied to them which is ultimately paid by the ratepayers.

Community well-being is a powerful concept and the planning exercises around it takes significant Council resources. There is no out of the box solution because each place is

\footnotetext{
${ }^{31}$ Year 2006 and 2009. Known as Long Term Council Community Plans (LTCCP)

32 Year 2012, 2015 and 2018
} 
different from the other and engaging with local community takes time and efforts. The thing that we highlighted in chapter five during our analysis of measures common across the Councils, is the lack of a standardised format for developing this whole framework. Community outcomes, their statements, indicators, and measures can be different according to the local community. Even the targets set for Council level of services can be different to the other Councils because it depends on the resources, capacity, and capabilities which the Councils have. But one thing which can be done is to standardise the mechanism of incorporating community well-being into Council activities to a certain level, so that it would be easier to monitor and compare progress across the Councils. In the new reformed LGA 2002, there was very detailed guidance on how to implement the wellbeing framework, whereas in this new amended Act of 2019 there is a very light touch on the implementation side. So a somewhat middle ground will be helpful to the Councils. It will also remove the hesitancy from the Councils regarding what to add and what not to when they are incorporating community well-being. This same point is also evident from our analysis that shows how many councils have reported on the community well-beings i.e., community outcomes, outcome statements, indicators, measures, targets and data sources. A standardised format from Department of Internal Affairs (DIA) will go a long way towards sorting these weaknesses out. We also know that some organisations within the sector (e.g., New Zealand Society of Local Government Managers (SOLGM) are providing resources and guidance in this regard, which is quite helpful and also acknowledged as such by the Councils.

In the chapter six on comparisons between Community Well-being Measures, Living Standard Framework (LSF), and Indicators Aotearoa New Zealand (IANZ) we saw that there was a significant alignment between the three. Thus, it can be said that there is a very strong possibility of developing a set of measures which could tell the story of well-being from the most granular level to the national level, and even compare it internationally where possible. At the same time, this would help in identifying major data gaps. In this alignment of three sets of measures, the important point is the availability of datasets at the most granular level. From our readings of the long-term plans, any dataset available on territorial authority level is good enough, but if it is available on a slightly more granular level that will be really helpful. The next level of granularity is statistical area 2 (SA2) which was previously known as area units (AU). Availability of datasets at SA2 level will not only help the Councils but also the local 
community groups, charities, trusts, boards and agencies who work closely with the Councils in delivering the desired community outcomes.

We identified a major data gap on the outcomes front. Currently, Statistics New Zealand conducts its General Social Survey (GSS) every second year, which comprises outcomesrelated measures. But this survey is conducted at regional level and is of little use for the territorial authorities or local government. A similar survey is conducted by a group of Councils, mainly City Councils, known as Quality of Life Survey ${ }^{33}$, and is conducted by a private research company, basically outsourced by participating Councils. This survey is quite useful as this specific group of Councils can have some more in-depth data on their communities' well-being. Nevertheless, it is only available for the larger Councils; district level Councils are left out. There can be a possibility of tackling this situation by widening the scope of Stats NZ GSS survey to include the territorial authority level of data. A second option could be to develop a standardised outcomes-based survey and conduct it through the private research firm or through Councils own resources. At the moment, all of the Councils do conduct an Annual Resident Survey in which they mostly ask outputs-based questions which are around Councils' activities and levels of service.

One of the limitations of this study is that we have not provided a final list of specific measures. Rather we have provided a list of broad groups of measures. These broad measures can be broken down by Councils into more detailed/granular measures. The reason for not providing this level of detail in the current study is because, although the individual measures have a similar theme they were nevertheless slightly different to each other. This slight difference meant a lot when seen in the context of the city or district. By way of examples, here are couple of measures from different Councils around crime/safety theme:

- Community safety is supported and improved

- Safe community: retain our safe community

- How safe do you feel in your community?

- Perceptions of Rotorua as a safe place

- Perceptions of personal safety

${ }^{33}$ http://qualityoflifeproject.govt.nz/ 
The above example although have the same theme or grouping but they are slightly different. It depends on the community itself what kind of measure they want to develop and keep track of. The suggestion from our side is that all of the Councils should have a core set of measures which are common across them and offer the potential to connect with the national wellbeing frameworks (e.g., Living Standard Framework (LSF) and Indicators Aotearoa New Zealand (IANZ)). At the same time Councils can have a special set of distinct measures which specifically track the well-being progress for their individual communities as they deem. In this approach, we have the flexibility of connecting the Councils to the national level, while also providing data on the well-beings that are very specific to individual Councils. This type of connectivity will also ensure that underpinning there is a degree of alignment between local, regional and national well-being priorities and measures; although a separate piece of work is required to investigate this alignment.

We have provided a full list of measures in the Appendix A, B, C and D. We have also created a set of dashboards and hosted it in Tableau Public. There are eight dashboards hosted online. They are fully interactive with different filtering options. The dashboards can be viewed from: https://public.tableau.com/profile/data.n.dashboards\#!/vizhome/CommunityWellbeings/DataOverview

There are a couple of suggestions for further research directions. First, to create a specific list of common measures from the current 4,337 measures. We think that a common list of around 300 measures will be workable, because there will be some measures which could take on the role of core measures. This exercise should be complemented with compilation of the metadata (i.e., identifying the level of granularity of each dataset), the sources from where it can be gained, any alternate data, and the frequency with which it is released from the source.

The other major further research direction will be to connect these community well-beings with the Council decision-making process. With the limited resources which Councils have access to, it becomes paramount to make sound decisions so that the Council could keep its focus and prioritise the projects. In this regard, the decision-making process goes hand in 
hand with the budgetary aspects of the Council. So, developing an overall framework which would encompass the community well-beings, the Council budget and the decision-making process will be really helpful for the local government sector.

As for identifying and defining the well-being priorities there is a need to further investigate the relative roles of citizens, council staff members and local politicians. Which would also look into the possible correlation with how priorities are defined and measured.

Finally, we would like to comment that the utilisation of community well-being in the local government sector and its support from the central government is a unique step taken by New Zealand. It is quite rare to see these kinds of processes when compared to other countries across the world. We hope that the effort put into this work is maintained, bringing ongoing benefits to the people of New Zealand, while also providing an exemplary case for other countries to follow. 


\section{References}

Archive.org. 2020. Internet Archive: Wayback Machine. [online] Available at:

https://archive.org/web [Accessed 6 December 2020].

Ashburton District Council, 2009. Long Term Council Community Plan 2009-2019. Ashburton District Council.

Ashburton District Council, 2009a. State of the Community Report. Ashburton District Council.

Ashburton District Council, 2018. Long Term Plan 2018-28. Ashburton District Council.

Viewed on 01 December 2020 at https://www.ashburtondc.govt.nz/our-council/plans-andstrategies/Pages/long-term-plan.aspx

Ashburton District Council. (2018a, April 19). 'Our Place' campaign takes out Local Government Excellence award. District Diary, 3.

https://www.ashburtondc.govt.nz/SiteCollectionDocuments/News/District\%20Diary/Issue\% 20121\%20April\%202018.pdf

Audit New Zealand, 2020. Long-Term Plans And Consultation Documents. Wellington: Audit New Zealand.

Carter, H. C. (2002, December 18). Local Government Bill: Second Reading

Speech [Parliament]. https://www.dia.govt.nz/diawebsite.nsf/wpg URL/Legislative-

Reviews-Local-Government-Act-Review-Local-Government-Bill-Second-Reading-

Speech?OpenDocument

Central Hawke's Bay District Council, 2009. Long Term Council Community Plan 2009-2019. Central Hawke's Bay District Council. 
Department of Internal Affairs, 2001. Reviewing The Local Government Act 1974. Have Your Say. Consultation Document. Wellington, New Zealand.

Department of Internal Affairs, 2001b. Review Of The Local Government Act 1974: Synopsis Of Submissions. Wellington, New Zealand.

Department of Internal Affairs. (2012). Better Local Government Fact Sheet: Refocus the purpose of local government. DIA. Viewed on 10 October 2020 at https://www.dia.govt.nz/Fact-sheets-for-2012-Act

Department of Internal Affairs. (2013). Better Local Government Decisions Document: Decisions about the content of a Local Government Act 2002 Amendment Bill. Viewed on 10 October 2020 at https://www.dia.govt.nz/Fact-sheets-for-2012-Act

Department of Internal Affairs. (2018). Regulatory Impact Summary: Local Government (Community Well-being) Amendment Bill.

Department of the Prime Minister and Cabinet, 2019. Guide For Central Government Engagement With Local Government. Wellington: Department of the Prime Minister and Cabinet. Viewed on 15 September 2020 at https://dpmc.govt.nz/sites/default/files/201906/Engagement\%20guidance\%20final\%2020.6 1.pdf

DIA, LGNZ and SOLGM, 2003. The Know How Guide To Decision Making: Under The Local Government Act 2002.

Far North District Council (2018). Long Term Plan 2018-28: Te Pae Tawhiti. Grey District Council, 2009. Long Term Council Community Plan 2009-2019. Grey District Council.

Hamilton City Council, 2009. Long Term Council Community Plan 2009-2019. Hamilton City Council. 
Hide, R. (2010). Decisions for better transparency, accountability and financial management of local government. Retrieved from https://www.dia.govt.nz/diawebsite.nsf/Files/LGTAFMDecisionsdocumentNov2010/\$̧file/LG TAFMDecisionsdocumentNov2010.pdf

Hide, R. (2010a). Local Government Act 2002 Amendment Bill — First Reading - New Zealand Parliament. Retrieved 10 October 2020, from

https://www.parliament.nz/en/pb/hansard-

debates/rhr/document/49HansD $2010050400000914 /$ local-government-act-2002amendment-bill-first-reading

Karacaoglu, G. and Reid, C., 2019. Addressing equity in intergenerational well-being: Valuing community perspectives. Deloitte: State of the State, [online] (Article 2). Available at: https://www2.deloitte.com/nz/en/pages/public-sector/articles/addressing-equity-inintergenerational-well-being.html [Accessed 15 September 2020].

Lee, S., (2001) First Reading Speech of the Local Government Amendment Bill, Hansard, Wellington.

Local Government (Community Well-being) Amendment Act 2019 (2019).

Local Government (Community Well-being) Amendment Bill, BILL_77941 (2018).

Local Government (Community Well-being) Amendment Bill: Explanatory Bill (2018).

Local Government Act 2002 Amendment Act 2010 (2010).

Local Government Act 2002, as at 01 August 2020.

Local Government Act 2002, as at 03 September 2007.

Local Government Act, (1974). 
Lower Hutt City Council, 2009. Long Term Council Community Plan. Lower Hutt City Council.

Mahuta, N. (2018). Local Government (Community Well-being) Amendment Bill - First Reading. New Zealand Parliamentary Debates. Retrieved December 17, 2018, from https://www.parliament.nz/en/pb/hansard-

debates/rhr/combined/HansDeb 201804112018041144

Manawatu District Council, 2009. Long Term Council Community Plan. Manawatu District Council.

Masterton District Council, 2009. Shaping Our Future: Masterton District Community Outcomes (Volume One). Masterton District Council.

Ministry of Business, Innovation \& Employment, 2020. “Modelled Territorial Authority GDP 2020 Release." Ministry of Business, Innovation and Employment, 30 June 2020, www.mbie.govt.nz/business-and-employment/economic-development/regional-economicdevelopment/modelled-territorial-authority-gross-domestic-product/modelled-territorialauthority-gdp-2020-release/. Accessed 1 Dec. 2020.

Ministry of Business, Innovation \& Employment, 2020a. Regional Economic Activity Tools, www.mbie.govt.nz/business-and-employment/economic-development/regional-economicdevelopment/activity-tools/. Accessed 1 Dec. 2020.

Mukhtar, M.M., 2020. Community Well-beings in Long Term Plans 2009. [online] Tableau Public. Available at:

https://public.tableau.com/profile/data.n.dashboards\#!/vizhome/CommunityWellbeings/DataOverview [Accessed 7 December 2020].

New Zealand Government, 2001. Local Government Bill: Explanatory Note. Wellington. 
New Zealand Labour Party, (1999) Local Government Election Manifesto, New Zealand Labour Party, Wellington.

New Zealand Parliament. (2018). Local Government (Community Well-being) Amendment Bill - First Reading. New Zealand Parliament.

New Zealand Parliament. (2018a). Local Government (Community Well-being) Amendment Bill - Second Reading. New Zealand Parliament. Retrieved October 10, 2020, from https://www.parliament.nz/en/pb/hansarddebates/rhr/combined/HansDeb 201904092019040928

New Zealand Society of Local Government Managers, (2019). The Community Well-Being Service.

New Zealand Society of Local Government Managers, 2020. Your Side of The Deal 2021: Performance Management in The Long Term Plan. Wellington: SOLGM.

Office of the Auditor-General New Zealand, 2007. Matters Arising From The 2006-16 LongTerm Council Community Plans. Wellington: Office of the Auditor-General New Zealand.

Office of the Auditor-General New Zealand, 2010. Matters Arising From The 2009-19 LongTerm Council Community Plans. Wellington: Office of the Auditor-General New Zealand.

Office of the Auditor-General New Zealand, 2019. Matters Arising From The 2018-28 LongTerm Council Community Plans. Wellington: Office of the Auditor-General New Zealand.

Queenstown-Lake District Council (2018). Ten Year Plan 2018-2028: He Mahere Kahurutaka 2018-2028.

Rangitikei District Council, 2009. Long Term Council Community Plan. Rangitikei District Council. 
Reid, M., 2010. Strengthening Local Government And Community Governance In New Zealand. Ph.D. Victoria University of Wellington.

Residential House Values. (2020). Property Value.

https://www.propertyvalue.co.nz/property-trends/residential-house-values

Richardson, M. (1999) Community Governance: Resource Kit, viewed on 14 Sep 2020 at http://www.gdrc.org/u-gov/ResourceKit.pdf

Sheard, C. and McDonald, K., 2013. Tightening Local Government's Belt: Will the New LGA Purpose Statement Achieve the Government's Objectives?. Resource Management Journal, (April), pp.6-10.

SOLGM. (2018b, November). Communication and Engagement Forum. New Zealand Society of Local Government Managers.

https://www.solgm.org.nz/Event?Action=View\&Event id=494

Statistics New Zealand, 2020. "Indicators Aotearoa New Zealand.", Statistics New Zealand, www.wellbeingindicators.stats.govt.nz. Accessed 1 Dec. 2020.

Stewart, J., \& Clarke M. (1996) Developments in Local Government, Institute of Local Government Studies Discussion Paper, University of Birmingham, Birmingham.

Tableau, 2020. "Find Clusters in Data." Tableau.com, Tableau, help.tableau.com/v2020.3/pro/desktop/en-gb/clustering.htm . Accessed 1 Dec. 2020.

Tauranga City Council, 2009. Long Term Council Community Plan 2009-2019. Tauranga City Council.

The Treasury, 2019. "Measuring Wellbeing: The LSF Dashboard." The Treasury New Zealand, Dec. 2019, www.treasury.govt.nz/information-and-services/nz-economy/higher-livingstandards/measuring-wellbeing-Isf-dashboard . Accessed 1 Dec. 2020. 
Treasury, The. Living Standards Framework: Background and Future Work. The Treasury, Dec. 2018.

Walters, L. (2018, February 01). NZ Government to lead world in measuring success with well-being measures. Retrieved December 17, 2018, from

https://www.stuff.co.nz/national/politics/101066981/nz-government-to-lead-world-inmeasuring-success-with-well-being-measures 
Appendix A: List of Community Well-beings Outcomes and Measures by Councils

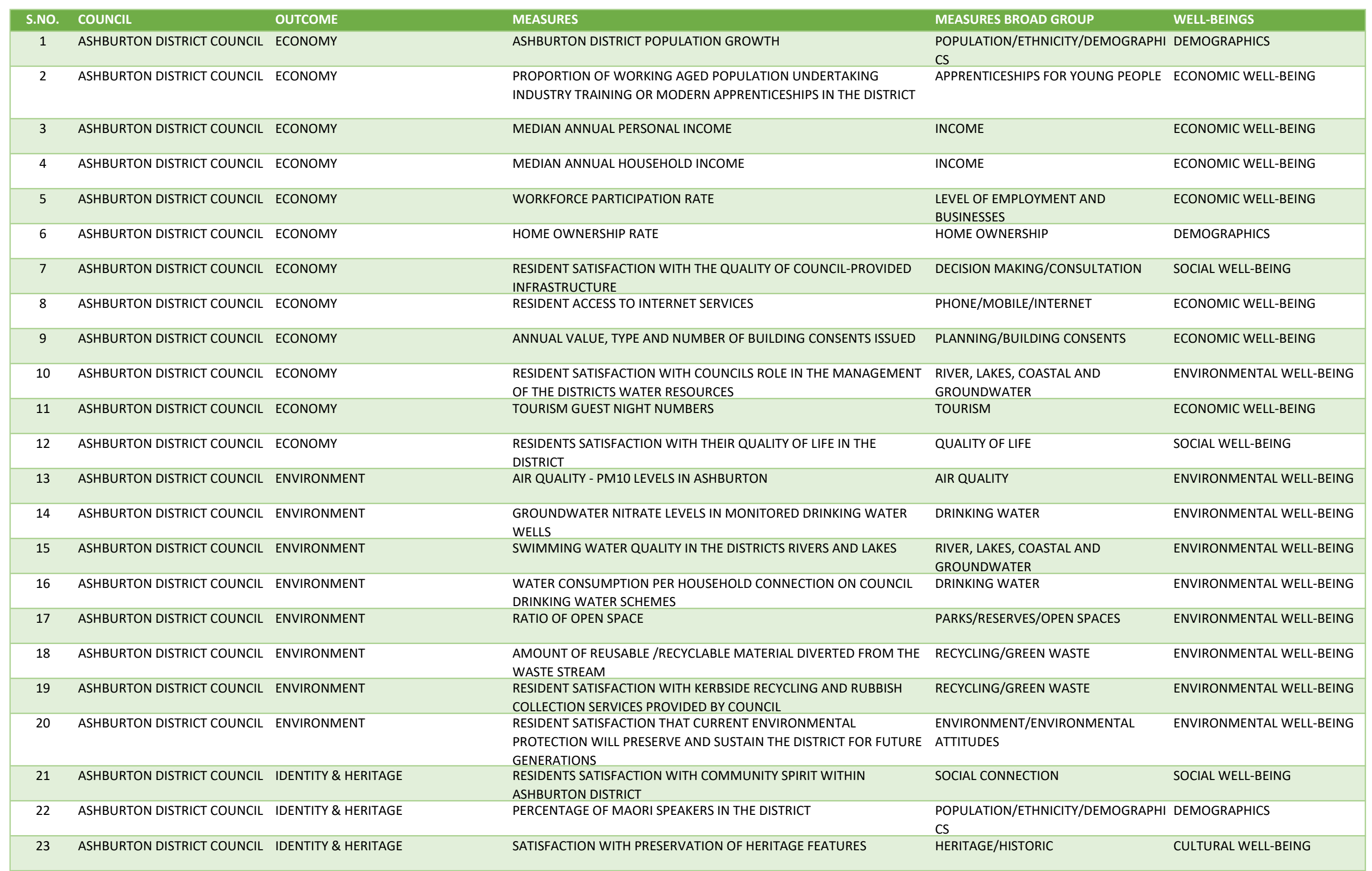


Appendix A: List of Community Well-beings Outcomes and Measures by Councils

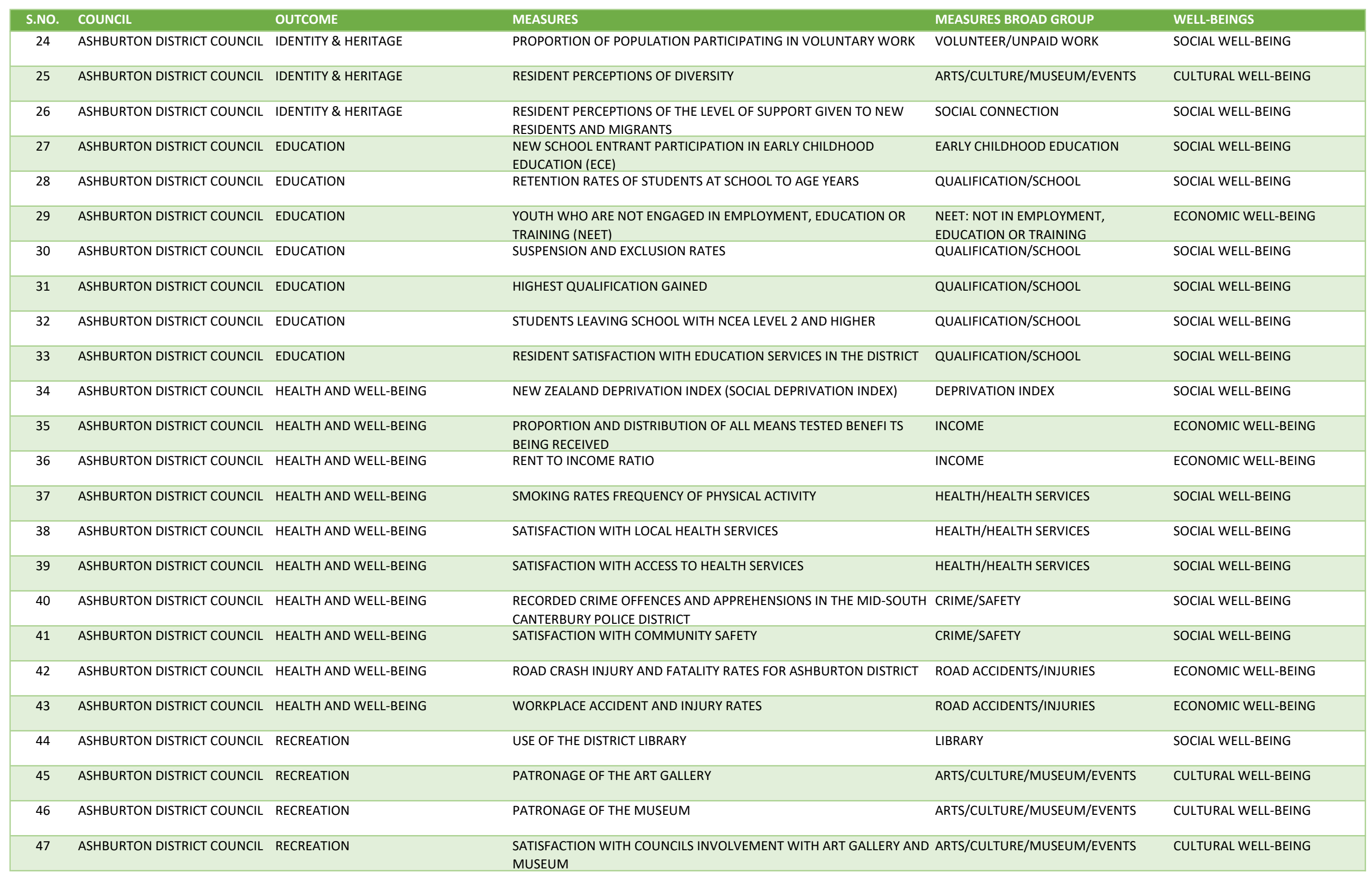


Appendix A: List of Community Well-beings Outcomes and Measures by Councils

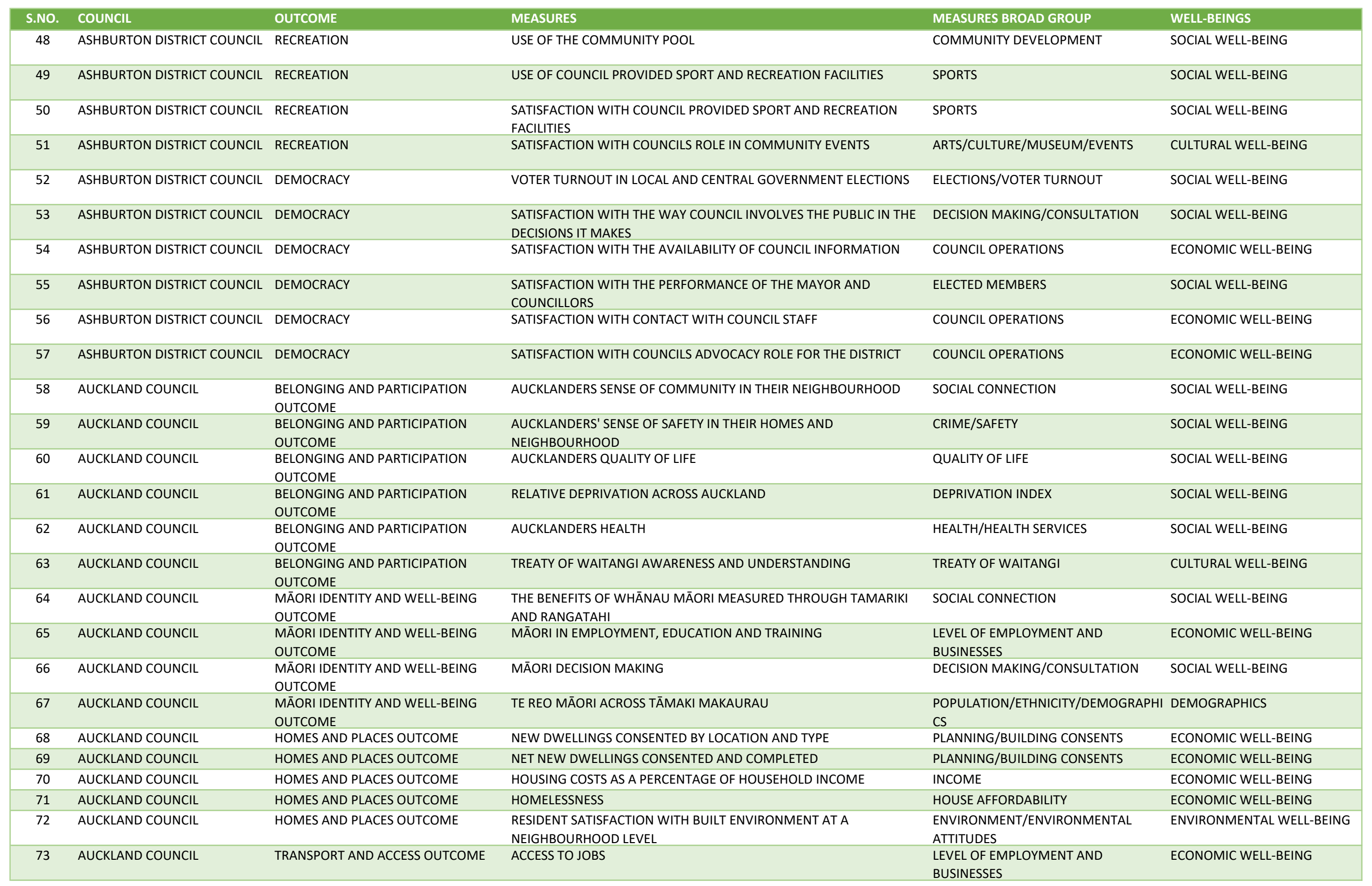


Appendix A: List of Community Well-beings Outcomes and Measures by Councils

\begin{tabular}{|c|c|c|c|c|c|}
\hline S.NO. & COUNCIL & OUTCOME & MEASURES & MEASURES BROAD GROUP & WELL-BEINGS \\
\hline 74 & AUCKLAND COUNCIL & TRANSPORT AND ACCESS OUTCOME & DELAY FROM CONGESTION & TRANSPORT & ECONOMIC WELL-BEING \\
\hline 75 & AUCKLAND COUNCIL & TRANSPORT AND ACCESS OUTCOME & USE OF PUBLIC TRANSPORT, WALKING AND CYCLING & TRANSPORT & ECONOMIC WELL-BEING \\
\hline 76 & AUCKLAND COUNCIL & TRANSPORT AND ACCESS OUTCOME & HOUSEHOLD TRANSPORT COSTS & TRANSPORT & ECONOMIC WELL-BEING \\
\hline 77 & AUCKLAND COUNCIL & TRANSPORT AND ACCESS OUTCOME & DEATHS AND INJURIES FROM TRANSPORT NETWORK & ROAD ACCIDENTS/INJURIES & ECONOMIC WELL-BEING \\
\hline 78 & AUCKLAND COUNCIL & $\begin{array}{l}\text { ENVIRONMENT AND CULTURAL } \\
\text { HERITAGE OUTCOME }\end{array}$ & $\begin{array}{l}\text { STATE AND QUALITY OF LOCALLY, REGIONALLY AND NATIONALLY } \\
\text { SIGNIFICANT ENVIRONMENTS }\end{array}$ & $\begin{array}{l}\text { ENVIRONMENT/ENVIRONMENTAL } \\
\text { ATTITUDES }\end{array}$ & ENVIRONMENTAL WELL-BEING \\
\hline 79 & AUCKLAND COUNCIL & $\begin{array}{l}\text { ENVIRONMENT AND CULTURAL } \\
\text { HERITAGE OUTCOME }\end{array}$ & MARINE AND FRESH WATER QUALITY & $\begin{array}{l}\text { RIVER, LAKES, COASTAL AND } \\
\text { GROUNDWATER }\end{array}$ & ENVIRONMENTAL WELL-BEING \\
\hline 80 & AUCKLAND COUNCIL & $\begin{array}{l}\text { ENVIRONMENT AND CULTURAL } \\
\text { HERITAGE OUTCOME }\end{array}$ & AIR QUALITY AND GREENHOUSE GAS EMISSIONS & AIR QUALITY & ENVIRONMENTAL WELL-BEING \\
\hline 81 & AUCKLAND COUNCIL & $\begin{array}{l}\text { ENVIRONMENT AND CULTURAL } \\
\text { HERITAGE OUTCOME }\end{array}$ & PROTECTION OF THE ENVIRONMENT & $\begin{array}{l}\text { ENVIRONMENT/ENVIRONMENTAL } \\
\text { ATTITUDES }\end{array}$ & ENVIRONMENTAL WELL-BEING \\
\hline 82 & AUCKLAND COUNCIL & $\begin{array}{l}\text { ENVIRONMENT AND CULTURAL } \\
\text { HERITAGE OUTCOME }\end{array}$ & RESILIENCE TO NATURAL THREATS & NATURAL DISASTER/RESILIENCE & ENVIRONMENTAL WELL-BEING \\
\hline 83 & AUCKLAND COUNCIL & $\begin{array}{l}\text { ENVIRONMENT AND CULTURAL } \\
\text { HERITAGE OUTCOME }\end{array}$ & TREASURING OF THE ENVIRONMENT & $\begin{array}{l}\text { ENVIRONMENT/ENVIRONMENTAL } \\
\text { ATTITUDES }\end{array}$ & ENVIRONMENTAL WELL-BEING \\
\hline 84 & AUCKLAND COUNCIL & $\begin{array}{l}\text { OPPORTUNITY AND PROSPERITY } \\
\text { OUTCOME }\end{array}$ & LABOUR PRODUCTIVITY & $\begin{array}{l}\text { LEVEL OF EMPLOYMENT AND } \\
\text { BUSINESSES }\end{array}$ & ECONOMIC WELL-BEING \\
\hline 85 & AUCKLAND COUNCIL & $\begin{array}{l}\text { OPPORTUNITY AND PROSPERITY } \\
\text { OUTCOME }\end{array}$ & AUCKLANDERS AVERAGE WAGES & INCOME & ECONOMIC WELL-BEING \\
\hline 87 & AUCKLAND COUNCIL & $\begin{array}{l}\text { OPPORTUNITY AND PROSPERITY } \\
\text { OUTCOME }\end{array}$ & ZONED INDUSTRIAL LAND & PLANNING/BUILDING CONSENTS & ECONOMIC WELL-BEING \\
\hline 88 & AUCKLAND COUNCIL & $\begin{array}{l}\text { OPPORTUNITY AND PROSPERITY } \\
\text { OUTCOME }\end{array}$ & LEVEL OF UNEMPLOYMENT & UNEMPLOYMENT & ECONOMIC WELL-BEING \\
\hline 89 & AUCKLAND COUNCIL & $\begin{array}{l}\text { OPPORTUNITY AND PROSPERITY } \\
\text { OUTCOME }\end{array}$ & INTERNET USAGE BASED ON INCOME & PHONE/MOBILE/INTERNET & ECONOMIC WELL-BEING \\
\hline 90 & AUCKLAND COUNCIL & $\begin{array}{l}\text { OPPORTUNITY AND PROSPERITY } \\
\text { OUTCOME }\end{array}$ & EDUCATIONAL ACHIEVEMENT OF YOUNG PEOPLE & YOUTH & SOCIAL WELL-BEING \\
\hline 91 & $\begin{array}{l}\text { BAY OF PLENTY REGIONAL } \\
\text { COUNCIL }\end{array}$ & $\begin{array}{l}\text { A CLEAN AND PROTECTED } \\
\text { ENVIRONMENT }\end{array}$ & AIR QUALITY & AIR QUALITY & ENVIRONMENTAL WELL-BEING \\
\hline 92 & $\begin{array}{l}\text { BAY OF PLENTY REGIONAL } \\
\text { COUNCIL }\end{array}$ & $\begin{array}{l}\text { A CLEAN AND PROTECTED } \\
\text { ENVIRONMENT }\end{array}$ & ECOLOGICAL HEALTH OF STREAM WATER & $\begin{array}{l}\text { RIVER, LAKES, COASTAL AND } \\
\text { GROUNDWATER }\end{array}$ & ENVIRONMENTAL WELL-BEING \\
\hline 93 & $\begin{array}{l}\text { BAY OF PLENTY REGIONAL } \\
\text { COUNCIL }\end{array}$ & $\begin{array}{l}\text { A CLEAN AND PROTECTED } \\
\text { ENVIRONMENT }\end{array}$ & RECREATION AND BATHING WATER QUALITY & $\begin{array}{l}\text { RIVER, LAKES, COASTAL AND } \\
\text { GROUNDWATER }\end{array}$ & ENVIRONMENTAL WELL-BEING \\
\hline 94 & $\begin{array}{l}\text { BAY OF PLENTY REGIONAL } \\
\text { COUNCIL }\end{array}$ & $\begin{array}{l}\text { A CLEAN AND PROTECTED } \\
\text { ENVIRONMENT }\end{array}$ & COASTAL HABITAT HEALTH & GROSS DOMESTIC PRODUCT (GDP) & ECONOMIC WELL-BEING \\
\hline 95 & $\begin{array}{l}\text { BAY OF PLENTY REGIONAL } \\
\text { COUNCIL }\end{array}$ & $\begin{array}{l}\text { A CLEAN AND PROTECTED } \\
\text { ENVIRONMENT }\end{array}$ & HARBOUR, FORESHORE AND WATERWAY HEALTH & $\begin{array}{l}\text { RIVER, LAKES, COASTAL AND } \\
\text { GROUNDWATER }\end{array}$ & ENVIRONMENTAL WELL-BEING \\
\hline 96 & $\begin{array}{l}\text { BAY OF PLENTY REGIONAL } \\
\text { COUNCIL }\end{array}$ & $\begin{array}{l}\text { A CLEAN AND PROTECTED } \\
\text { ENVIRONMENT }\end{array}$ & ENVIRONMENTAL ENFORCEMENT & $\begin{array}{l}\text { ENVIRONMENT/ENVIRONMENTAL } \\
\text { ATTITUDES }\end{array}$ & ENVIRONMENTAL WELL-BEING \\
\hline 98 & $\begin{array}{l}\text { BAY OF PLENTY REGIONAL } \\
\text { COUNCIL }\end{array}$ & $\begin{array}{l}\text { A CLEAN AND PROTECTED } \\
\text { ENVIRONMENT }\end{array}$ & PROTECTION OF BIODIVERSITY AREAS & $\begin{array}{l}\text { ENVIRONMENT/ENVIRONMENTAL } \\
\text { ATTITUDES }\end{array}$ & ENVIRONMENTAL WELL-BEING \\
\hline 99 & $\begin{array}{l}\text { BAY OF PLENTY REGIONAL } \\
\text { COUNCIL }\end{array}$ & $\begin{array}{l}\text { A CLEAN AND PROTECTED } \\
\text { ENVIRONMENT }\end{array}$ & PROTECTION OF RARE AND ENDANGERED SPECIES & $\begin{array}{l}\text { ENVIRONMENT/ENVIRONMENTAL } \\
\text { ATTITUDES }\end{array}$ & ENVIRONMENTAL WELL-BEING \\
\hline
\end{tabular}


Appendix A: List of Community Well-beings Outcomes and Measures by Councils

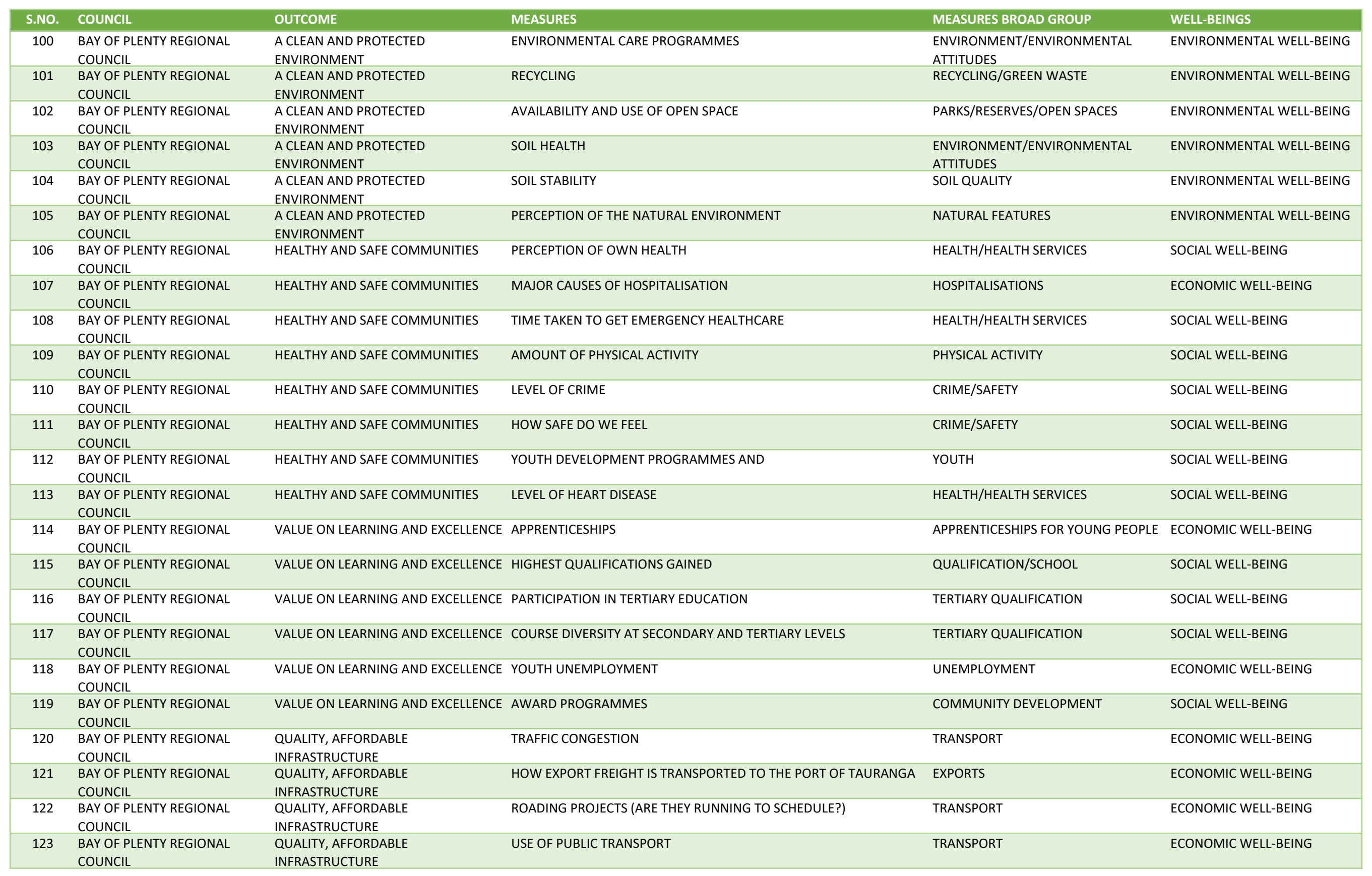


Appendix A: List of Community Well-beings Outcomes and Measures by Councils

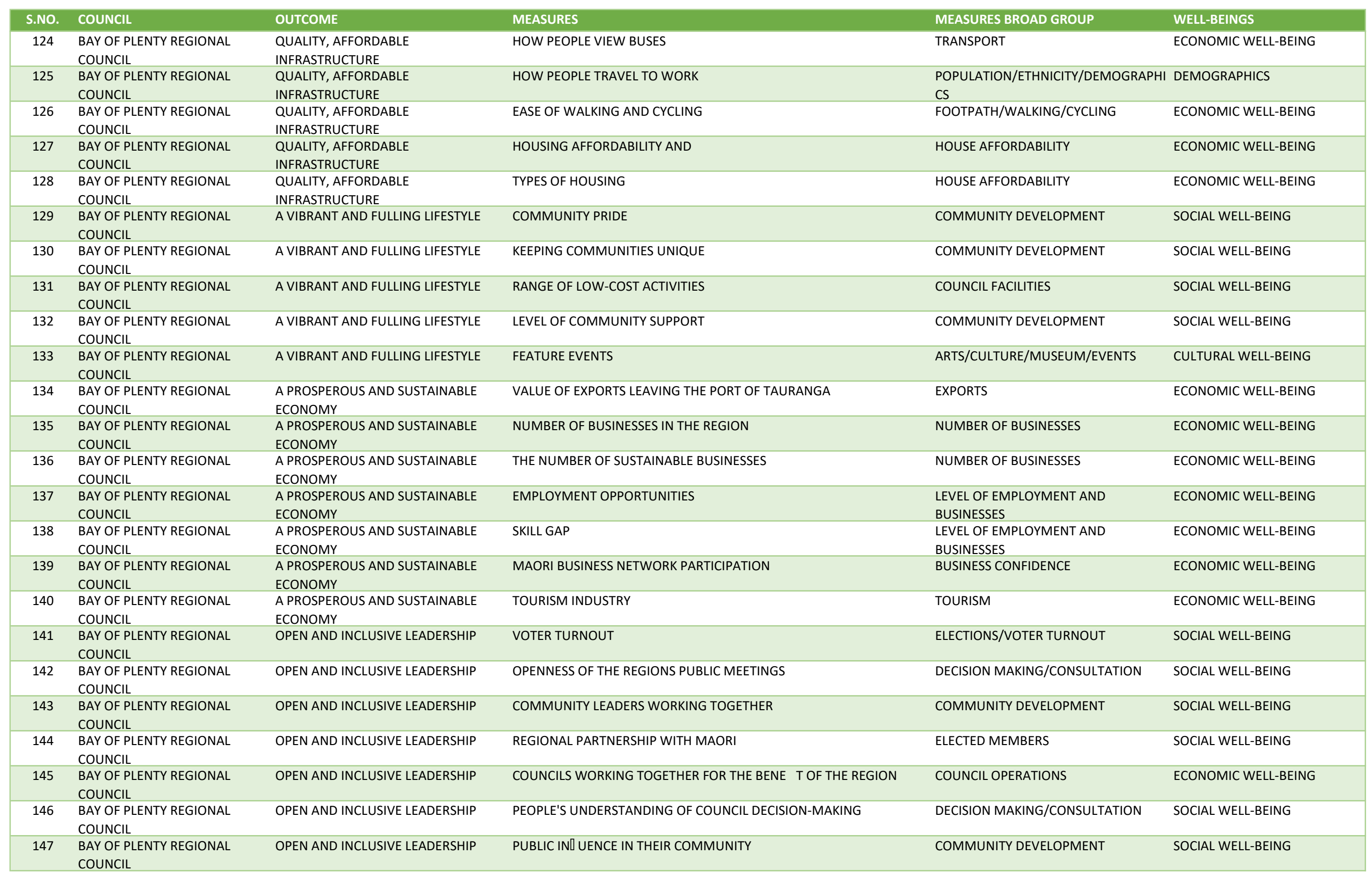


Appendix A: List of Community Well-beings Outcomes and Measures by Councils

\begin{tabular}{|c|c|c|c|c|c|}
\hline S.NO. & COUNCIL & OUTCOME & MEASURES & MEASURES BROAD GROUP & WELL-BEINGS \\
\hline 148 & $\begin{array}{l}\text { BAY OF PLENTY REGIONAL } \\
\text { COUNCIL }\end{array}$ & OPEN AND INCLUSIVE LEADERSHIP & RECOGNITION OF COMMUNITY LEADERS & COMMUNITY DEVELOPMENT & SOCIAL WELL-BEING \\
\hline 149 & $\begin{array}{l}\text { BAY OF PLENTY REGIONAL } \\
\text { COUNCIL }\end{array}$ & OPEN AND INCLUSIVE LEADERSHIP & PUBLIC CONDENCE IN THE REGIONAL COUNCIL'S DECISION-MAKING & DECISION MAKING/CONSULTATION & SOCIAL WELL-BEING \\
\hline 150 & $\begin{array}{l}\text { BAY OF PLENTY REGIONAL } \\
\text { COUNCIL }\end{array}$ & RESPECTED CULTURE AND HERITAGE & DAMAGE TO HISTORIC PLACES & HERITAGE/HISTORIC & CULTURAL WELL-BEING \\
\hline 151 & $\begin{array}{l}\text { BAY OF PLENTY REGIONAL } \\
\text { COUNCIL }\end{array}$ & RESPECTED CULTURE AND HERITAGE & IMPACT OF CULTURAL DIVERSITY & ARTS/CULTURE/MUSEUM/EVENTS & CULTURAL WELL-BEING \\
\hline 152 & $\begin{array}{l}\text { BAY OF PLENTY REGIONAL } \\
\text { COUNCIL }\end{array}$ & RESPECTED CULTURE AND HERITAGE & RANGE OF LANGUAGES SPOKEN & $\begin{array}{l}\text { POPULATION/ETHNICITY/DEMOGRAPHI } \\
\text { CS }\end{array}$ & DEMOGRAPHICS \\
\hline 153 & $\begin{array}{l}\text { BAY OF PLENTY REGIONAL } \\
\text { COUNCIL }\end{array}$ & RESPECTED CULTURE AND HERITAGE & THE RELIGIONS TO WHICH PEOPLE AFFILIATE & $\begin{array}{l}\text { POPULATION/ETHNICITY/DEMOGRAPHI } \\
\text { CS }\end{array}$ & DEMOGRAPHICS \\
\hline 154 & $\begin{array}{l}\text { BAY OF PLENTY REGIONAL } \\
\text { COUNCIL }\end{array}$ & RESPECTED CULTURE AND HERITAGE & PROTECTION OF MAORI CULTURE AND & ARTS/CULTURE/MUSEUM/EVENTS & CULTURAL WELL-BEING \\
\hline 155 & $\begin{array}{l}\text { BAY OF PLENTY REGIONAL } \\
\text { COUNCIL }\end{array}$ & RESPECTED CULTURE AND HERITAGE & NUMBER OF TE REO MAORI SPEAKERS & $\begin{array}{l}\text { POPULATION/ETHNICITY/DEMOGRAPHI } \\
\text { CS }\end{array}$ & DEMOGRAPHICS \\
\hline 156 & BULLER DISTRICT COUNCIL & HEALTH & LIFE EXPECTANCY & LIFE EXPECTANCY & DEMOGRAPHICS \\
\hline 157 & BULLER DISTRICT COUNCIL & HEALTH & ACCESS TO HEALTH CARE (SERVICES AND PROGRAMS) & HEALTH/HEALTH SERVICES & SOCIAL WELL-BEING \\
\hline 158 & BULLER DISTRICT COUNCIL & HEALTH & NEW HEALTH INITIATIVES THAT HAVE BEEN INTRODUCED & HEALTH/HEALTH SERVICES & SOCIAL WELL-BEING \\
\hline 159 & BULLER DISTRICT COUNCIL & HEALTH & DRINKING WATER QUALITY & DRINKING WATER & ENVIRONMENTAL WELL-BEING \\
\hline 161 & BULLER DISTRICT COUNCIL & EDUCATION & SCHOOL ROLLS AND DECILE RATINGS & QUALIFICATION/SCHOOL & SOCIAL WELL-BEING \\
\hline 162 & BULLER DISTRICT COUNCIL & EDUCATION & LEVELS OF QUALIFICATION & QUALIFICATION/SCHOOL & SOCIAL WELL-BEING \\
\hline 163 & BULLER DISTRICT COUNCIL & EDUCATION & NUMBER OF EDUCATIONAL INSTITUTIONS & QUALIFICATION/SCHOOL & SOCIAL WELL-BEING \\
\hline 164 & BULLER DISTRICT COUNCIL & SAFETY & YOUTH OFFENDING RATES & YOUTH & SOCIAL WELL-BEING \\
\hline 165 & BULLER DISTRICT COUNCIL & SAFETY & CRIME AND CRIME RESOLUTION RATES & CRIME/SAFETY & SOCIAL WELL-BEING \\
\hline 166 & BULLER DISTRICT COUNCIL & SAFETY & NUMBERS OF TRAFFI C ACCIDENTS & ROAD ACCIDENTS/INJURIES & ECONOMIC WELL-BEING \\
\hline 167 & BULLER DISTRICT COUNCIL & SAFETY & $\begin{array}{l}\text { IMPROVED SAFETY IN PUBLIC PLACES (DOG ATTACKS, LIQUOR BANS, } \\
\text { SECURITY CAMERAS) }\end{array}$ & CRIME/SAFETY & SOCIAL WELL-BEING \\
\hline 168 & BULLER DISTRICT COUNCIL & SAFETY & NATURAL HAZARD EVENT PREPAREDNESS/PROGRAMMES & NATURAL DISASTER/RESILIENCE & ENVIRONMENTAL WELL-BEING \\
\hline 169 & BULLER DISTRICT COUNCIL & SAFETY & HAZARD IDENTIFI CATION & NATURAL DISASTER/RESILIENCE & ENVIRONMENTAL WELL-BEING \\
\hline 170 & BULLER DISTRICT COUNCIL & ENVIRONMENT & FRESHWATER QUALITY & $\begin{array}{l}\text { RIVER, LAKES, COASTAL AND } \\
\text { GROUNDWATER }\end{array}$ & ENVIRONMENTAL WELL-BEING \\
\hline 171 & BULLER DISTRICT COUNCIL & ENVIRONMENT & VISITOR LEVEL OF APPRECIATION & TOURISM & ECONOMIC WELL-BEING \\
\hline 172 & BULLER DISTRICT COUNCIL & ENVIRONMENT & LEVEL OF VISITOR WASTE MINIMISATION ACTIVITY & TOURISM & ECONOMIC WELL-BEING \\
\hline 173 & BULLER DISTRICT COUNCIL & ENVIRONMENT & ACCESS TO NATURAL FEATURES & NATURAL FEATURES & ENVIRONMENTAL WELL-BEING \\
\hline 174 & BULLER DISTRICT COUNCIL & ECONOMY & EMPLOYMENT LEVELS & $\begin{array}{l}\text { LEVEL OF EMPLOYMENT AND } \\
\text { BUSINESSES }\end{array}$ & ECONOMIC WELL-BEING \\
\hline 175 & BULLER DISTRICT COUNCIL & ECONOMY & AVERAGE INCOMES & INCOME & ECONOMIC WELL-BEING \\
\hline 178 & BULLER DISTRICT COUNCIL & IDENTITY & NUMBER OF COMMUNITY ACTIVITIES AND DISTRICT EVENTS & ARTS/CULTURE/MUSEUM/EVENTS & CULTURAL WELL-BEING \\
\hline 179 & BULLER DISTRICT COUNCIL & IDENTITY & NUMBER OF COMMUNITY FACILITIES & COMMUNITY DEVELOPMENT & SOCIAL WELL-BEING \\
\hline 180 & BULLER DISTRICT COUNCIL & IDENTITY & $\begin{array}{l}\text { NUMBER OF PEOPLE INVOLVED IN SPORTING, CULTURAL AND } \\
\text { RECREATIONAL ACTIVITIES }\end{array}$ & SPORTS & SOCIAL WELL-BEING \\
\hline
\end{tabular}


Appendix A: List of Community Well-beings Outcomes and Measures by Councils

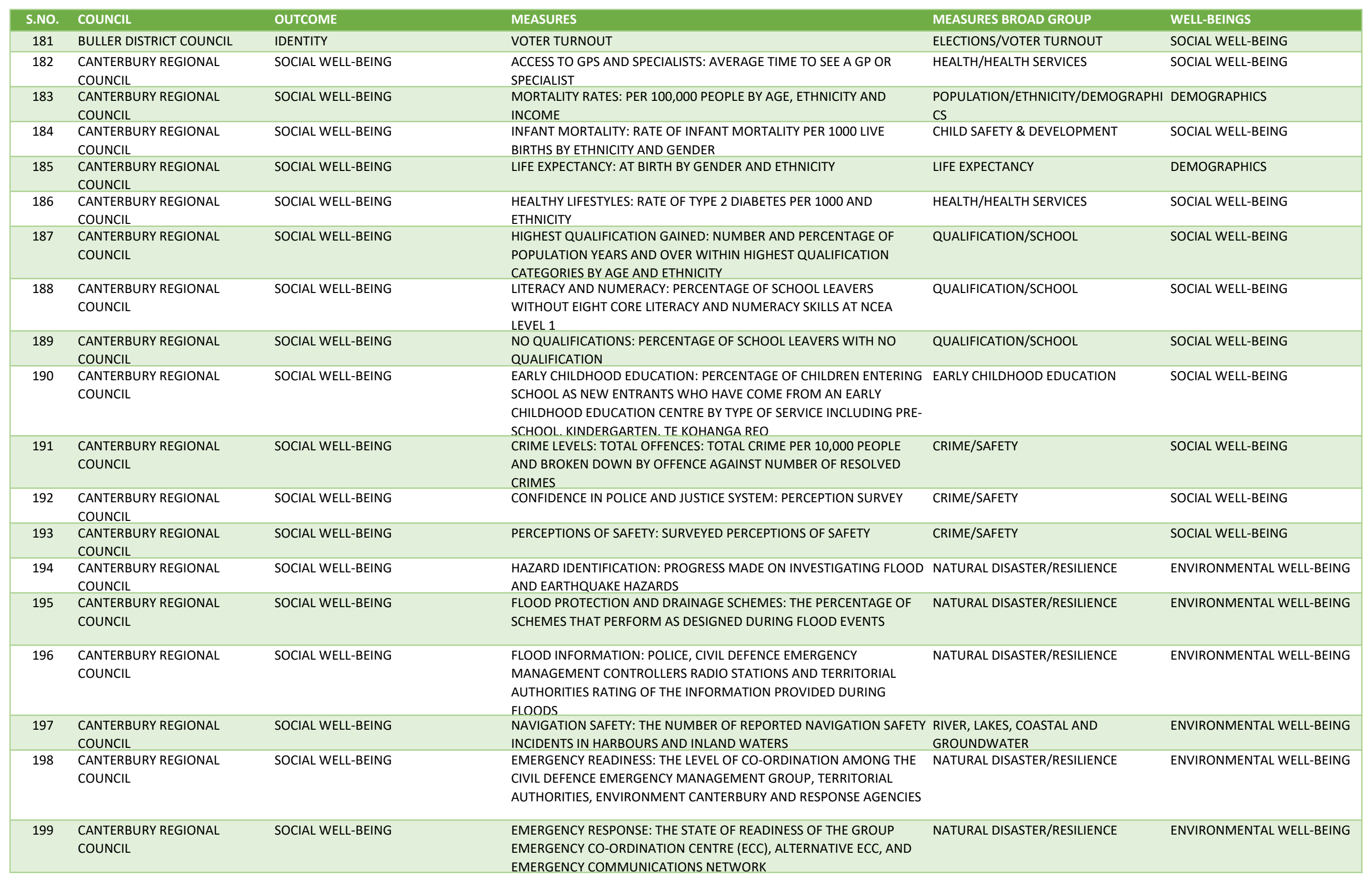


Appendix A: List of Community Well-beings Outcomes and Measures by Councils

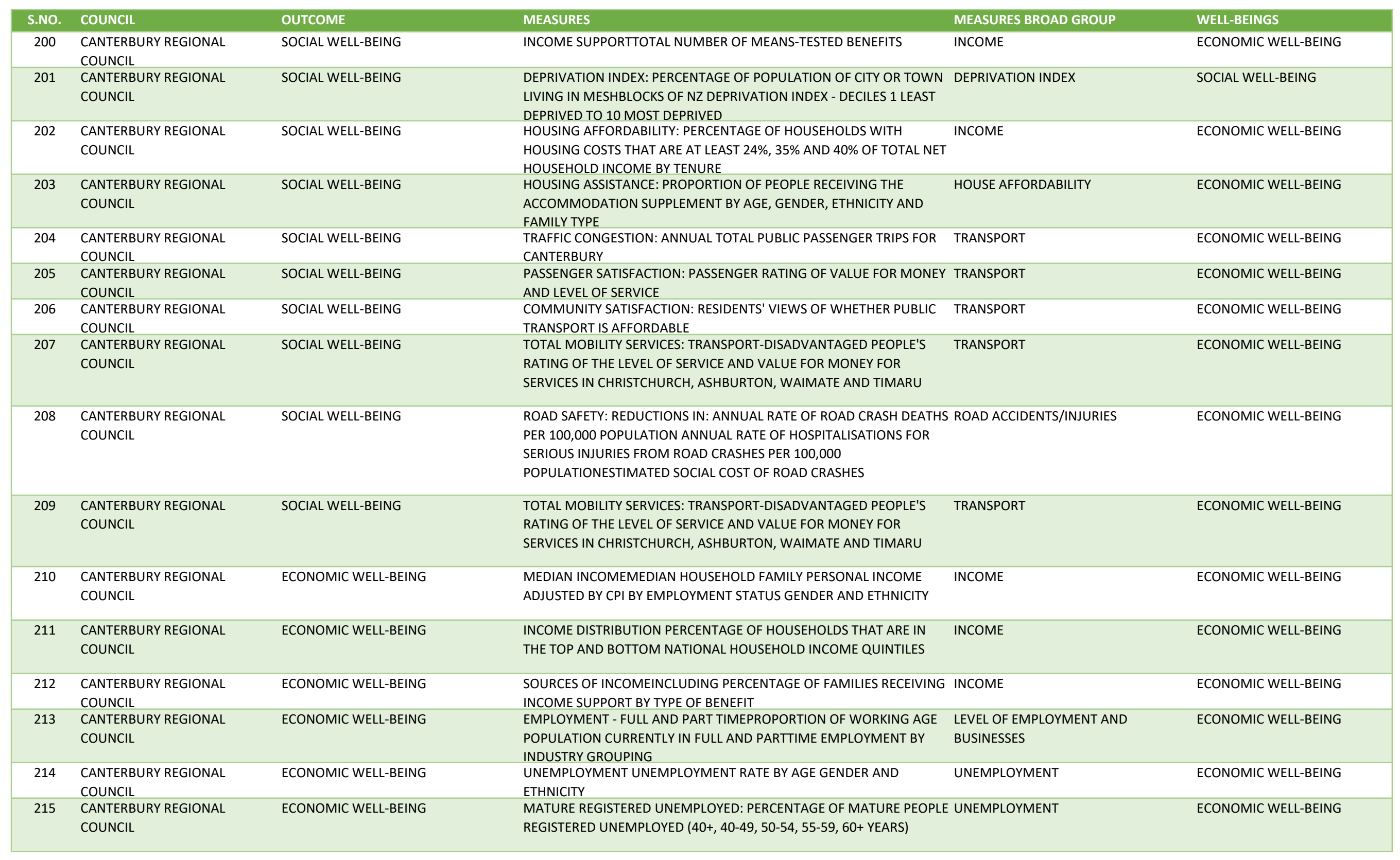




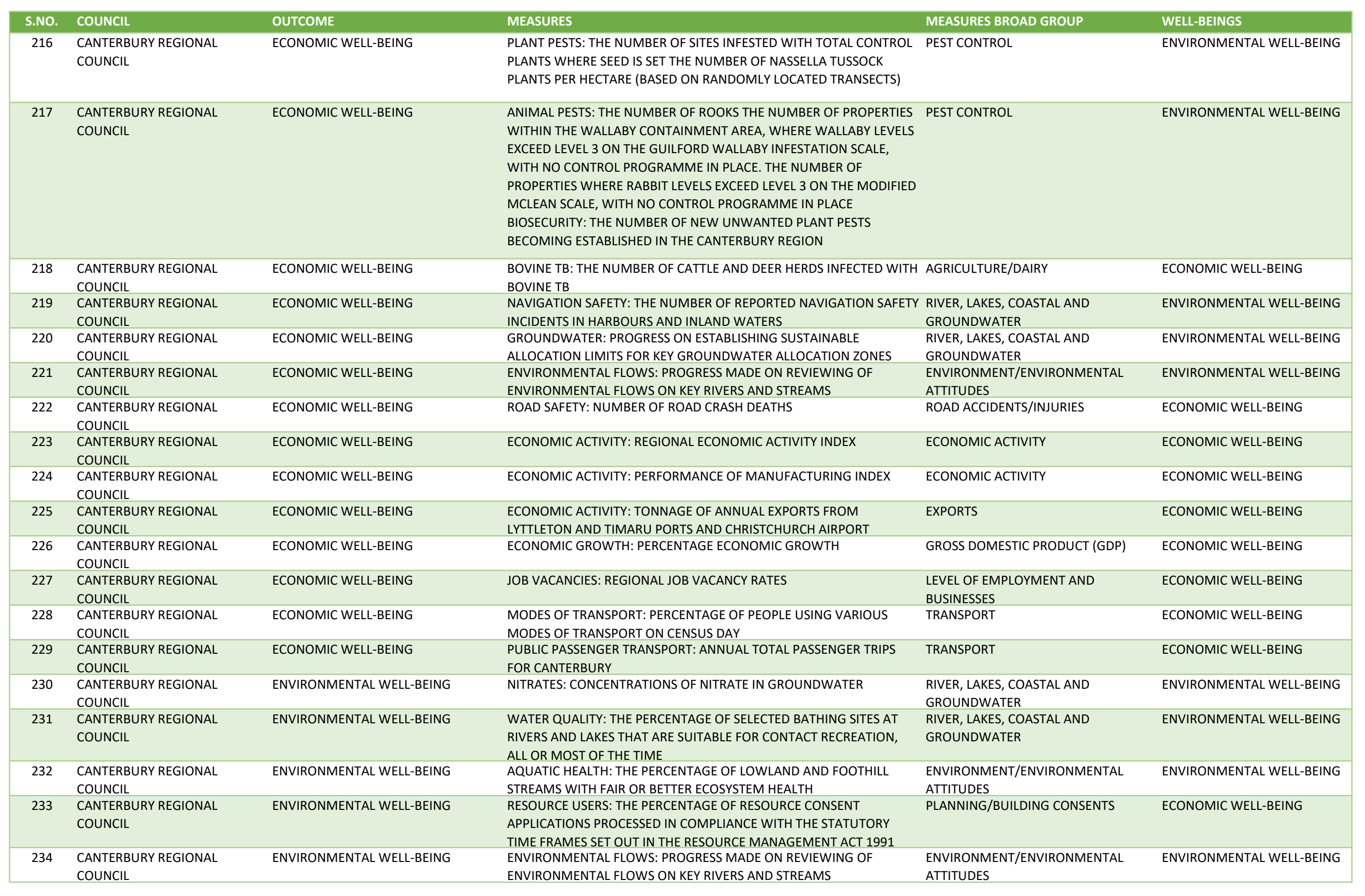




\begin{tabular}{|c|c|c|c|c|c|}
\hline S.NO. & COUNCIL & OUTCOME & MEASURES & MEASURES BROAD GROUP & WELL-BEINGS \\
\hline 235 & $\begin{array}{l}\text { CANTERBURY REGIONAL } \\
\text { COUNCIL }\end{array}$ & ENVIRONMENTAL WELL-BEING & $\begin{array}{l}\text { AIR QUALITY: MEETING NATIONAL ENVIRONMENT STANDARDS FOR } \\
\text { PM10 CONCENTRATIONS IN CHRISTCHURCH, KAIAPOI, RANGIORA, } \\
\text { TIMARU, ASHBURTON, GERALDINE, AND WAIMATE TO ENSURE } \\
\text { COMPLIANCE WITH NATIONAL ENVIRONMENTAL STANDARDS } \\
\text { MEASURING CO, NO2, SO2 CONCENTRATIONS TO ENSURE } \\
\text { COMPIIANICF WIITH NATIONAI FNVIRONMMFNTAI STANIARAS }\end{array}$ & AIR QUALITY & ENVIRONMENTAL WELL-BEING \\
\hline 236 & $\begin{array}{l}\text { CANTERBURY REGIONAL } \\
\text { COUNCIL }\end{array}$ & ENVIRONMENTAL WELL-BEING & $\begin{array}{l}\text { CLEAN HEAT: THE TOTAL NUMBER OF HOMES IN CHRISTCHURCH, } \\
\text { TIMARU, KAIAPOI, RANGIORA, ASHBURTON, GERALDINE AND } \\
\text { WAIMATE AIRSHEDS CONVERTING TO CLEANER FORMS OF HOME }\end{array}$ & HOUSE QUALITY & ECONOMIC WELL-BEING \\
\hline 238 & $\begin{array}{l}\text { CANTERBURY REGIONAL } \\
\text { COUNCIL }\end{array}$ & ENVIRONMENTAL WELL-BEING & $\begin{array}{l}\text { LAND: SOIL QUALITY FACTORS FOR CANTERBURY LAND USES AREA OF } \\
\text { LAND RECEIVING EFFECTIVE PROTECTION FROM WIND EROSION }\end{array}$ & SOIL QUALITY & ENVIRONMENTAL WELL-BEING \\
\hline 239 & $\begin{array}{l}\text { CANTERBURY REGIONAL } \\
\text { COUNCIL }\end{array}$ & ENVIRONMENTAL WELL-BEING & $\begin{array}{l}\text { RESOURCE CONSENTS: THE PERCENTAGE OF RESOURCE CONSENT } \\
\text { APPLICATIONS PROCESSED IN COMPLIANCE WITH THE STATUTORY } \\
\text { TIME FRAMES SET DOWN IN THE RESOURCE MANAGEMENT ACT } 1991 . \\
\text { THE PERCENTAGE OF SIGNIFICANT NONCOMPLIANCES RESOLVED }\end{array}$ & PLANNING/BUILDING CONSENTS & ECONOMIC WELL-BEING \\
\hline 240 & $\begin{array}{l}\text { CANTERBURY REGIONAL } \\
\text { COUNCIL }\end{array}$ & ENVIRONMENTAL WELL-BEING & $\begin{array}{l}\text { HAZARDOUS SUBSTANCES: THE NUMBER OF SITES THAT RECEIVE A SITE } \\
\text { ASSESSMENT AND GUIDANCE ON POLLUTION PREVENTION EACH YEAR. } \\
\text { THE NUMBER OF TERRITORIAL AUTHORITIES THAT HAVE COMPLETED } \\
\text { THE CANTERBURY TARGETED AGRICHEMICAL COLLECTION } \\
\text { PROGRAMMF }\end{array}$ & POLLUTION & ENVIRONMENTAL WELL-BEING \\
\hline 241 & $\begin{array}{l}\text { CANTERBURY REGIONAL } \\
\text { COUNCIL }\end{array}$ & ENVIRONMENTAL WELL-BEING & $\begin{array}{l}\text { CONTAMINATED SITES: THE NUMBER OF TERRITORIAL AUTHORITIES } \\
\text { WHERE ALL LAND WITH ACTIVITIES LISTED ON THE HAZARDOUS } \\
\text { ACTIVITIES AND INDUSTRIES LIST HAS BEEN IDENTIFIED AS REGISTERED } \\
\text { AND SCREENED FOR RISK }\end{array}$ & $\begin{array}{l}\text { ENVIRONMENT/ENVIRONMENTAL } \\
\text { ATTITUDES }\end{array}$ & ENVIRONMENTAL WELL-BEING \\
\hline 242 & $\begin{array}{l}\text { CANTERBURY REGIONAL } \\
\text { COUNCIL }\end{array}$ & ENVIRONMENTAL WELL-BEING & $\begin{array}{l}\text { BONESEED: THE AREA OUTSIDE THE PORT HILLS ZONE INFESTED WITH } \\
\text { BONESEED SUBJECT TO CONTROL PLAN TO PREVENT SEED SETTING }\end{array}$ & ARTS/CULTURE/MUSEUM/EVENTS & CULTURAL WELL-BEING \\
\hline 243 & $\begin{array}{l}\text { CANTERBURY REGIONAL } \\
\text { COUNCIL }\end{array}$ & ENVIRONMENTAL WELL-BEING & $\begin{array}{l}\text { WALLABIES: THE NUMBER OF PROPERTIES WITHIN THE WALLABY } \\
\text { CONTAINMENT AREA, WHERE WALLABY LEVELS EXCEED LEVEL ON THE } \\
\text { GUILFORD WALLABY INFESTATION SCALE, WITH NO CONTROL } \\
\text { PROGRAMME IN PLACE. THE NUMBER OF WALLABY POPULATIONS } \\
\text { REPORTED ESTABLISHING OUTSIDE THE WALLABY CONTAINMENT AREA }\end{array}$ & $\begin{array}{l}\text { ENVIRONMENT/ENVIRONMENTAL } \\
\text { ATTITUDES }\end{array}$ & ENVIRONMENTAL WELL-BEING \\
\hline 244 & $\begin{array}{l}\text { CANTERBURY REGIONAL } \\
\text { COUNCIL }\end{array}$ & ENVIRONMENTAL WELL-BEING & $\begin{array}{l}\text { HAZARDOUS SUBSTANCES: THE NUMBER OF BUSINESSES THAT RECEIVE } \\
\text { A SITE ASSESSMENT AND GUIDANCE ON POLLUTION PREVENTION EACH } \\
\text { YEAR THE NUMBER OF TERRITORIAL AUTHORITIES THAT HAVE } \\
\text { COMPLETED THE CANTERBURY TARGETED AGRICHEMICAL COLLECTION } \\
\text { PROGRAMMF }\end{array}$ & WASTE TO LANDFILL & ENVIRONMENTAL WELL-BEING \\
\hline 245 & $\begin{array}{l}\text { CANTERBURY REGIONAL } \\
\text { COUNCIL }\end{array}$ & ENVIRONMENTAL WELL-BEING & $\begin{array}{l}\text { CONTAMINATED SITES: THE NUMBER OF TERRITORIAL AUTHORITIES } \\
\text { WHERE ALL LAND WITH ACTIVITIES LISTED ON THE HAZARDOUS } \\
\text { ACTIVITIES AND INDUSTRIES LIST HAS BEEN IDENTIFIED AS REGISTERED } \\
\text { AND SCREENED FOR RISK }\end{array}$ & $\begin{array}{l}\text { ENVIRONMENT/ENVIRONMENTAL } \\
\text { ATTITUDES }\end{array}$ & ENVIRONMENTAL WELL-BEING \\
\hline
\end{tabular}




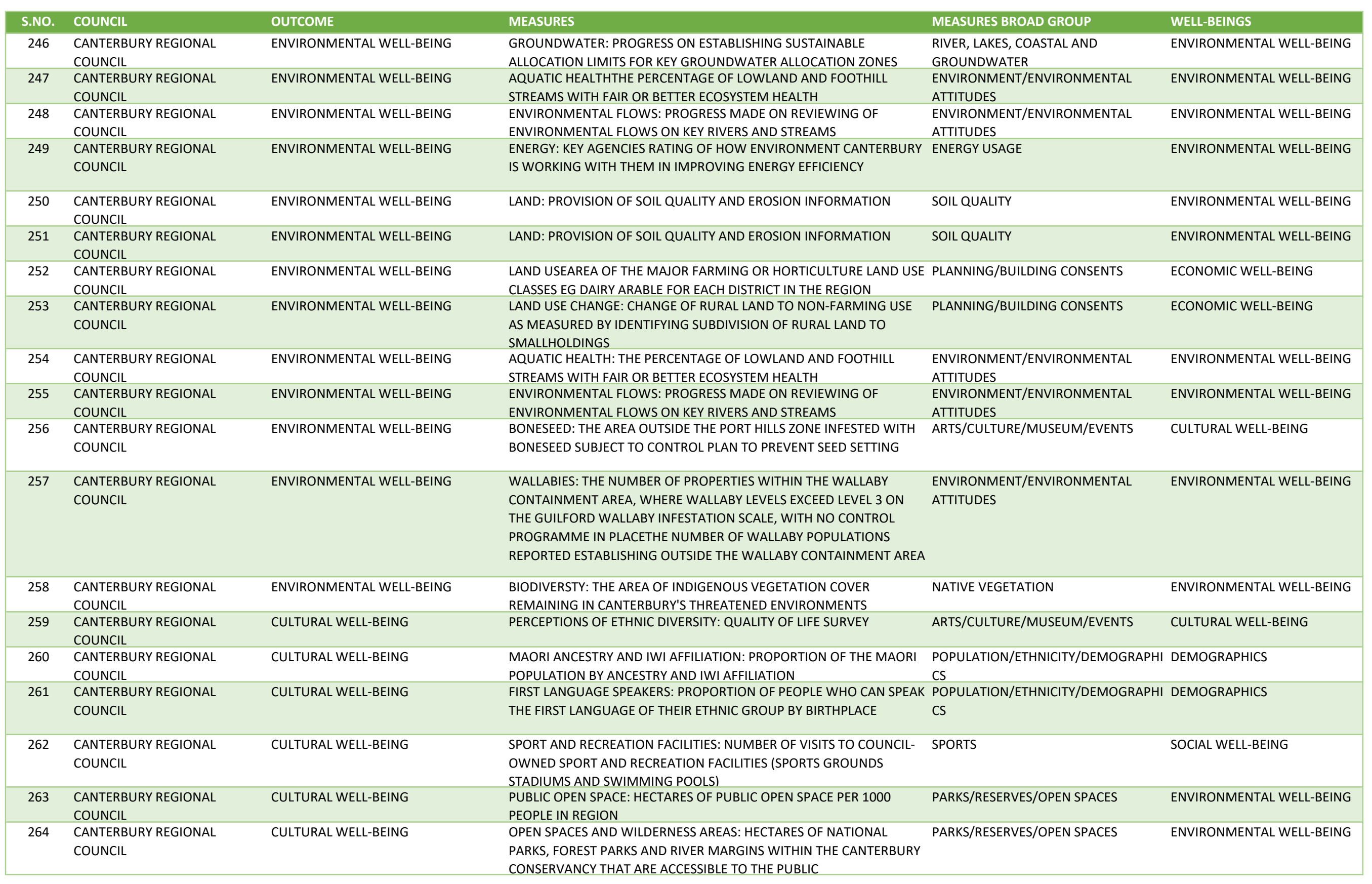


Appendix A: List of Community Well-beings Outcomes and Measures by Councils

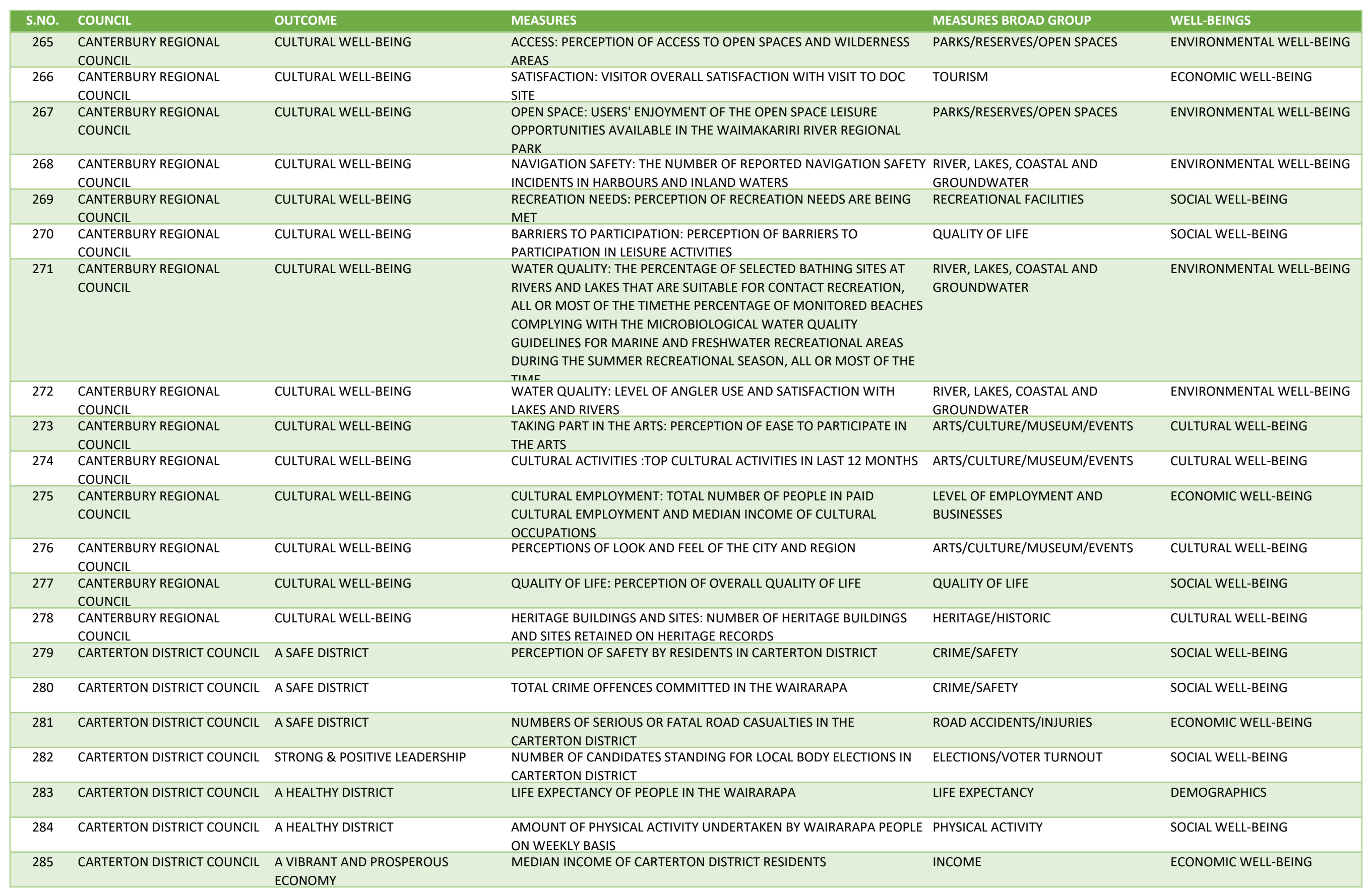


Appendix A: List of Community Well-beings Outcomes and Measures by Councils

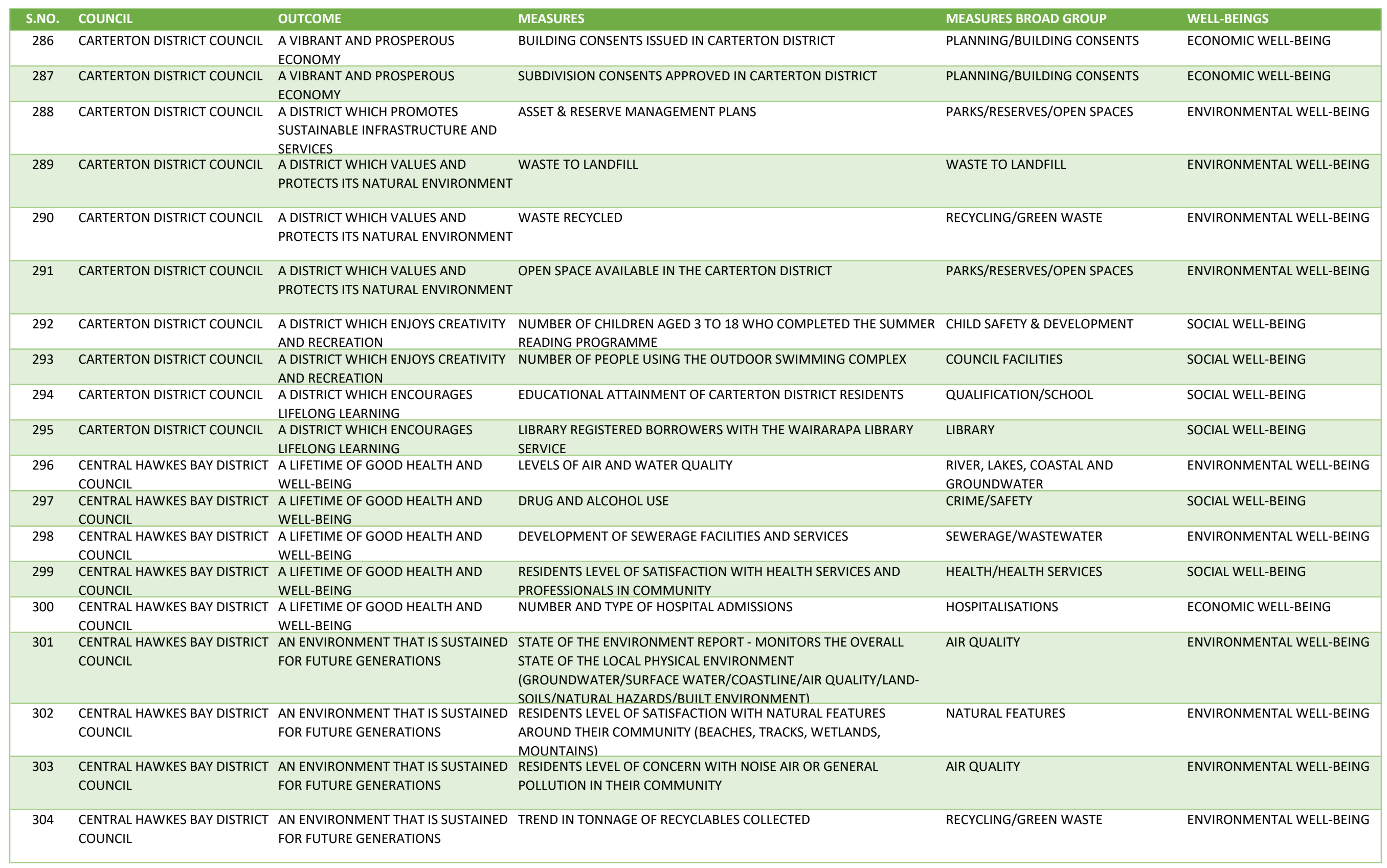


Appendix A: List of Community Well-beings Outcomes and Measures by Councils

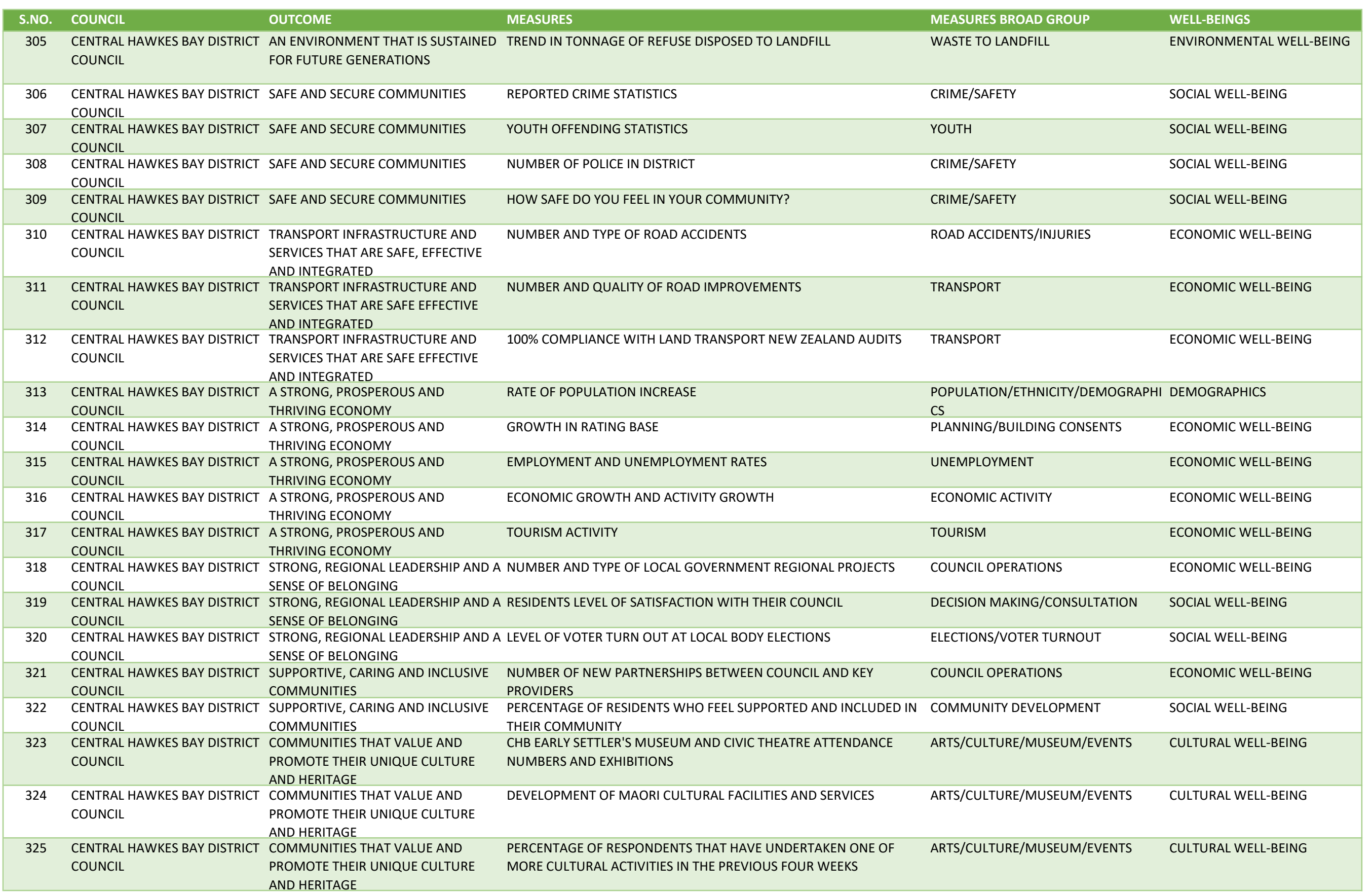









\begin{tabular}{|c|c|c|c|c|c|}
\hline S.NO. & COUNCIL & OUTCOME & MEASURES & MEASURES BROAD GROUP & WELL-BEINGS \\
\hline 343 & $\begin{array}{l}\text { CENTRAL OTAGO DISTRICT } \\
\text { COUNCIL }\end{array}$ & A SAFE AND HEALTHY COMMUNITY & $\begin{array}{l}\text { MAORI: CONTINUED SUPPORT TO THE LOCAL MAORI COMMUNITY } \\
\text { THROUGH A MAORI COMMUNITY WORKER, USE OF THE LOCAL WHARE } \\
\text { AND HAVING AN INCREASED APPRECIATION OF LOCAL MAORI STORIES }\end{array}$ & CULTURAL ACCEPTANCE & CULTURAL WELL-BEING \\
\hline 344 & $\begin{array}{l}\text { CENTRAL OTAGO DISTRICT } \\
\text { COUNCIL }\end{array}$ & A SAFE AND HEALTHY COMMUNITY & $\begin{array}{l}\text { HERITAGE: CLEAR GUIDELINES FOR ACCESSING, MANAGING AND } \\
\text { PRESERVING HERITAGE WITHIN CENTRAL OTAGO WHILE ALSO } \\
\text { IDENTIFYING TOURISM OPPORTUNITIES }\end{array}$ & HERITAGE/HISTORIC & CULTURAL WELL-BEING \\
\hline 345 & $\begin{array}{l}\text { CENTRAL OTAGO DISTRICT } \\
\text { COUNCIL }\end{array}$ & A SUSTAINABLE ENVIRONMENT & $\begin{array}{l}\text { WATER: ENSURING THERE IS AN APPROPRIATE ALLOCATION OF WATER } \\
\text { FOR IRRIGATION WHILE ENSURING SUSTAINABLE WATERWAYS } \\
\text { PROVIDE CERTAINTY IN THE QUALITY AND AVAILABILITY OF } \\
\text { RESIDENTIAL WATER SUPPLIES, AS WELL AS EDUCATION ON WATER } \\
\text { CONSFRVATION }\end{array}$ & $\begin{array}{l}\text { RIVER, LAKES, COASTAL AND } \\
\text { GROUNDWATER }\end{array}$ & ENVIRONMENTAL WELL-BEING \\
\hline 346 & $\begin{array}{l}\text { CENTRAL OTAGO DISTRICT } \\
\text { COUNCIL }\end{array}$ & A SUSTAINABLE ENVIRONMENT & $\begin{array}{l}\text { AIR: ENCOURAGE CLEAN AIR IN OUR LOCAL COMMUNITIES THROUGH } \\
\text { CONSULTATION PROVIDING INFORMATION AND TAKING A BALANCED } \\
\text { APPROACH TO ADDRESSING AIR POLLUTION }\end{array}$ & AIR QUALITY & ENVIRONMENTAL WELL-BEING \\
\hline 347 & $\begin{array}{l}\text { CENTRAL OTAGO DISTRICT } \\
\text { COUNCIL }\end{array}$ & A SUSTAINABLE ENVIRONMENT & $\begin{array}{l}\text { WASTE: MINIMISATION REDUCE THE WASTE WE GENERATE AND } \\
\text { INCREASE RECYCLING }\end{array}$ & RECYCLING/GREEN WASTE & ENVIRONMENTAL WELL-BEING \\
\hline 348 & $\begin{array}{l}\text { CENTRAL OTAGO DISTRICT } \\
\text { COUNCIL }\end{array}$ & A SUSTAINABLE ENVIRONMENT & $\begin{array}{l}\text { MANAGING DEVELOPMENT IMPACTS ON LANDSCAPE AND NATURAL } \\
\text { ECOSYSTEMS: UNDERSTAND AND PROTECT THE INHERENT VALUES OF } \\
\text { OUR LANDSCAPE AND NATURAL ECOSYSTEMS WHILE HAVING WELL } \\
\text { DEFINED AREAS FOR GROWTH }\end{array}$ & $\begin{array}{l}\text { RIVER, LAKES, COASTAL AND } \\
\text { GROUNDWATER }\end{array}$ & ENVIRONMENTAL WELL-BEING \\
\hline 349 & CHATHAM ISLAND COUNCIL & SOCIAL WELL-BEING & $\begin{array}{l}\text { HOUSING AFFORDABILITY: PERCENTAGE OF HOUSEHOLDS IN PRIVATE } \\
\text { DWELLINGS WITH OR WITHOUT A MORTGAGE }\end{array}$ & HOUSE AFFORDABILITY & ECONOMIC WELL-BEING \\
\hline 350 & CHATHAM ISLAND COUNCIL & SOCIAL WELL-BEING & $\begin{array}{l}\text { ACCESS TO QUALITY HOUSING PROGRESS MADE ON INVESTIGATING } \\
\text { AND IMPLEMENTING THE ESTABLISHMENT OF QUALITY HOUSING ON } \\
\text { THE CHATHAM ISLANDS }\end{array}$ & HOUSE QUALITY & ECONOMIC WELL-BEING \\
\hline 351 & CHATHAM ISLAND COUNCIL & SOCIAL WELL-BEING & $\begin{array}{l}\text { ACCESS TO HEALTH PROVIDERS: NUMBER OF HEALTH PROVIDERS } \\
\text { AVAILABLE TO MEET THE NEEDS OF THE COMMUNITY }\end{array}$ & HEALTH/HEALTH SERVICES & SOCIAL WELL-BEING \\
\hline 352 & CHATHAM ISLAND COUNCIL & SOCIAL WELL-BEING & $\begin{array}{l}\text { ACCESS TO EDUCATION OPPORTUNITIES: THE NUMBER OF EDUCATION } \\
\text { PROVIDERS AVAILABLE TO THE COMMUNITY }\end{array}$ & QUALIFICATION/SCHOOL & SOCIAL WELL-BEING \\
\hline 353 & CHATHAM ISLAND COUNCIL & SOCIAL WELL-BEING & $\begin{array}{l}\text { COMMUNITY SATISFACTION ON WHETHER THE EXISTING } \\
\text { INFRASTRUCTURE MEETS THE NEEDS OF THE COMMUNITY }\end{array}$ & COMMUNITY DEVELOPMENT & SOCIAL WELL-BEING \\
\hline 354 & CHATHAM ISLAND COUNCIL & SOCIAL WELL-BEING & $\begin{array}{l}\text { NUMBER OF ON-ISLAND OFFENCES RESULTING IN CONVICTIONS IN THE } \\
\text { LAST } 12 \text { MONTHS }\end{array}$ & CRIME/SAFETY & SOCIAL WELL-BEING \\
\hline 355 & CHATHAM ISLAND COUNCIL & SOCIAL WELL-BEING & SURVEYED PERCEPTIONS ON SAFETY & CRIME/SAFETY & SOCIAL WELL-BEING \\
\hline 356 & CHATHAM ISLAND COUNCIL & SOCIAL WELL-BEING & NUMBER OF ROAD ACCIDENTS & ROAD ACCIDENTS/INJURIES & ECONOMIC WELL-BEING \\
\hline 357 & CHATHAM ISLAND COUNCIL & SOCIAL WELL-BEING & $\begin{array}{l}\text { NUMBER OF EMERGENCY SERVICES AVAILABLE TO THE PUBLIC } \\
\text { INCLUDING VOLUNTEER GROUPS }\end{array}$ & VOLUNTEER/UNPAID WORK & SOCIAL WELL-BEING \\
\hline 358 & CHATHAM ISLAND COUNCIL & SOCIAL WELL-BEING & PROGRESS MADE ON REDUCING NATURAL HAZARDS & NATURAL DISASTER/RESILIENCE & ENVIRONMENTAL WELL-BEING \\
\hline 359 & CHATHAM ISLAND COUNCIL & SOCIAL WELL-BEING & TOTAL NUMBER OF LIBRARY USERS, PERCENTAGE OF POPULATION & LIBRARY & SOCIAL WELL-BEING \\
\hline 360 & CHATHAM ISLAND COUNCIL & SOCIAL WELL-BEING & $\begin{array}{l}\text { COMMUNITY SATISFACTION WITH ACCESS TO SPORTS AND } \\
\text { RECREATION FACILITIES IE WOMEN'S DARTS, NETBALL, TOUCH RUGBY, } \\
\text { YOUTH RUGBY, SQUASH COURTS }\end{array}$ & SPORTS & SOCIAL WELL-BEING \\
\hline 362 & CHATHAM ISLAND COUNCIL & SOCIAL WELL-BEING & $\begin{array}{l}\text { MEDIAN HOUSEHOLD, FAMILY OR PERSONAL INCOME FOR PEOPLE } 15 \\
\text { YEARS AND OVER BY ETHNICITY }\end{array}$ & INCOME & ECONOMIC WELL-BEING \\
\hline
\end{tabular}


Appendix A: List of Community Well-beings Outcomes and Measures by Councils

\begin{tabular}{|c|c|c|c|c|c|}
\hline S.NO. & COUNCIL & OUTCOME & MEASURES & MEASURES BROAD GROUP & WELL-BEINGS \\
\hline 363 & CHATHAM ISLAND COUNCIL & SOCIAL WELL-BEING & $\begin{array}{l}\text { PERCENTAGE OF HOUSEHOLDS THAT ARE IN THE TOP AND BOTTOM } \\
\text { NATIONAL HOUSEHOLD INCOME }\end{array}$ & INCOME & ECONOMIC WELL-BEING \\
\hline 364 & CHATHAM ISLAND COUNCIL & SOCIAL WELL-BEING & PERCENTAGE OF PEOPLE IN EMPLOYMENT BY OCCUPATION & $\begin{array}{l}\text { LEVEL OF EMPLOYMENT AND } \\
\text { BUSINESSES }\end{array}$ & ECONOMIC WELL-BEING \\
\hline 365 & CHATHAM ISLAND COUNCIL & ECONOMIC WELL-BEING & TOURISM -- AMOUNT OF ACCOMMODATION AVAILABLE FOR TOURISM & TOURISM & ECONOMIC WELL-BEING \\
\hline 366 & CHATHAM ISLAND COUNCIL & ECONOMIC WELL-BEING & NUMBER OF NEW ONISLAND BUSINESSES IN THE LAST THREE YEARS & NUMBER OF BUSINESSES & ECONOMIC WELL-BEING \\
\hline 367 & CHATHAM ISLAND COUNCIL & ECONOMIC WELL-BEING & NUMBER OF ON-ISLAND COMMERCIAL FISHING VESSELS & $\begin{array}{l}\text { LEVEL OF EMPLOYMENT AND } \\
\text { BUSINESSES }\end{array}$ & ECONOMIC WELL-BEING \\
\hline 368 & CHATHAM ISLAND COUNCIL & ECONOMIC WELL-BEING & PERCENTAGE OF POPULATION CONNECTED TO THE INTERNET & PHONE/MOBILE/INTERNET & ECONOMIC WELL-BEING \\
\hline 369 & CHATHAM ISLAND COUNCIL & ECONOMIC WELL-BEING & $\begin{array}{l}\text { PERCENTAGE OF WORKFORCE ATTENDING PRACTICAL SKILLS AND } \\
\text { WORK-BASED TRAINING PROGRAMMES UNDERTAKEN ON-ISLAND }\end{array}$ & QUALIFICATION/SCHOOL & SOCIAL WELL-BEING \\
\hline 370 & CHATHAM ISLAND COUNCIL & ECONOMIC WELL-BEING & NUMBER OF SHIPPING SERVICING TO THE ISLANDS & EXPORTS & ECONOMIC WELL-BEING \\
\hline 371 & CHATHAM ISLAND COUNCIL & ECONOMIC WELL-BEING & NUMBER OF AIR SERVICES TO THE ISLANDS & TOURISM & ECONOMIC WELL-BEING \\
\hline 372 & CHATHAM ISLAND COUNCIL & ECONOMIC WELL-BEING & $\begin{array}{l}\text { NUMBER OF PARTNERSHIP PROGRAMMES WITH GOVERNMENT } \\
\text { DEPARTMENTS OR AGENCIES }\end{array}$ & COUNCIL OPERATIONS & SOCIAL WELL-BEING \\
\hline 373 & CHATHAM ISLAND COUNCIL & ENVIRONMENTAL WELL-BEING & $\begin{array}{l}\text { NUMBER OF CONSERVATION BOARD INITIATIVES IN THE } 12 \text { LAST } \\
\text { MONTHS }\end{array}$ & $\begin{array}{l}\text { ENVIRONMENT/ENVIRONMENTAL } \\
\text { ATTITUDES }\end{array}$ & ENVIRONMENTAL WELL-BEING \\
\hline 374 & CHATHAM ISLAND COUNCIL & ENVIRONMENTAL WELL-BEING & NUMBER OF DOC COMMUNITY INITIATIVES IN THE LAST 12 MONTHS & COMMUNITY DEVELOPMENT & SOCIAL WELL-BEING \\
\hline 375 & CHATHAM ISLAND COUNCIL & ENVIRONMENTAL WELL-BEING & $\begin{array}{l}\text { NUMBER OF COUNCIL ENVIRONMENTAL PROGRAMMES WATER } \\
\text { QUALITY, PEST MANAGEMENT, WASTE MANAGEMENT, BIODIVERSITY }\end{array}$ & $\begin{array}{l}\text { RIVER, LAKES, COASTAL AND } \\
\text { GROUNDWATER }\end{array}$ & ENVIRONMENTAL WELL-BEING \\
\hline 376 & CHATHAM ISLAND COUNCIL & ENVIRONMENTAL WELL-BEING & $\begin{array}{l}\text { NUMBER OF INITIATIVES PROMOTING MARINE PROTECTION IN THE } \\
\text { LAST } 12 \text { MONTHS }\end{array}$ & $\begin{array}{l}\text { ENVIRONMENT/ENVIRONMENTAL } \\
\text { ATTITUDES }\end{array}$ & ENVIRONMENTAL WELL-BEING \\
\hline 377 & CHATHAM ISLAND COUNCIL & ENVIRONMENTAL WELL-BEING & NUMBER OF PUBLIC ACCESSES TO HISTORIC PARKS AND RESERVES & PARKS/RESERVES/OPEN SPACES & ENVIRONMENTAL WELL-BEING \\
\hline 378 & CHATHAM ISLAND COUNCIL & ENVIRONMENTAL WELL-BEING & NUMBER OF HISTORIC PLACES THAT ARE REGISTERED & HERITAGE/HISTORIC & CULTURAL WELL-BEING \\
\hline 379 & CHATHAM ISLAND COUNCIL & ENVIRONMENTAL WELL-BEING & NUMBER OF PRIVATE PROTECTED SITES & HERITAGE/HISTORIC & CULTURAL WELL-BEING \\
\hline 380 & CHATHAM ISLAND COUNCIL & ENVIRONMENTAL WELL-BEING & $\begin{array}{l}\text { PERCENTAGE OF THREATENED BIRDS, PLANTS AND FISH ON THE } \\
\text { CHATHAM ISLANDS }\end{array}$ & $\begin{array}{l}\text { ENVIRONMENT/ENVIRONMENTAL } \\
\text { ATTITUDES }\end{array}$ & ENVIRONMENTAL WELL-BEING \\
\hline 381 & CHATHAM ISLAND COUNCIL & CULTURAL WELL-BEING & $\begin{array}{l}\text { NUMBER OF RECOGNISED SITES THAT ARE PROTECTED WHERE } \\
\text { TRADITIONAL FOOD GATHERING IS UNDERTAKEN }\end{array}$ & HERITAGE/HISTORIC & CULTURAL WELL-BEING \\
\hline 382 & CHATHAM ISLAND COUNCIL & CULTURAL WELL-BEING & NUMBER OF RAHUI AREAS SET ASIDE FOR RECREATIONAL USE & RECREATIONAL FACILITIES & SOCIAL WELL-BEING \\
\hline 383 & CHATHAM ISLAND COUNCIL & CULTURAL WELL-BEING & $\begin{array}{l}\text { PERCENTAGE OF PEOPLE WHO IDENTIFIED AS BELONGING TO A MAORI } \\
\text { OR MORIORI ETHNIC GROUP }\end{array}$ & $\begin{array}{l}\text { POPULATION/ETHNICITY/DEMOGRAPHI } \\
\text { CS }\end{array}$ & DEMOGRAPHICS \\
\hline 384 & CHATHAM ISLAND COUNCIL & CULTURAL WELL-BEING & $\begin{array}{l}\text { PERCENTAGE OF PEOPLE WHO SPEAK MAORI AND MORIORI } \\
\text { LANGUAGE }\end{array}$ & $\begin{array}{l}\text { POPULATION/ETHNICITY/DEMOGRAPHI } \\
\text { CS }\end{array}$ & DEMOGRAPHICS \\
\hline 385 & CHATHAM ISLAND COUNCIL & CULTURAL WELL-BEING & $\begin{array}{l}\text { TOTAL NUMBER OF FACILITIES WHERE ART IS DISPLAYED AND } \\
\text { AVAILABLE TO THE PUBLIC }\end{array}$ & ARTS/CULTURE/MUSEUM/EVENTS & CULTURAL WELL-BEING \\
\hline 386 & CHATHAM ISLAND COUNCIL & CULTURAL WELL-BEING & NUMBER OF CULTURAL ACTIVITIES INVOLVING THE ARTS & ARTS/CULTURE/MUSEUM/EVENTS & CULTURAL WELL-BEING \\
\hline 389 & CHRISTCHURCH CITY COUNCIL & STRONG COMMUNITIES & A SENSE OF COMMUNITY IN PEOPLES NEIGHBOURHOODS & SOCIAL CONNECTION & SOCIAL WELL-BEING \\
\hline 390 & CHRISTCHURCH CITY COUNCIL & STRONG COMMUNITIES & LEVELS OF VOLUNTEERING OR UNPAID WORK & VOLUNTEER/UNPAID WORK & SOCIAL WELL-BEING \\
\hline 391 & CHRISTCHURCH CITY COUNCIL & STRONG COMMUNITIES & SOMEONE TO TURN TO IN TIMES OF NEED & SOCIAL CONNECTION & SOCIAL WELL-BEING \\
\hline
\end{tabular}


Appendix A: List of Community Well-beings Outcomes and Measures by Councils

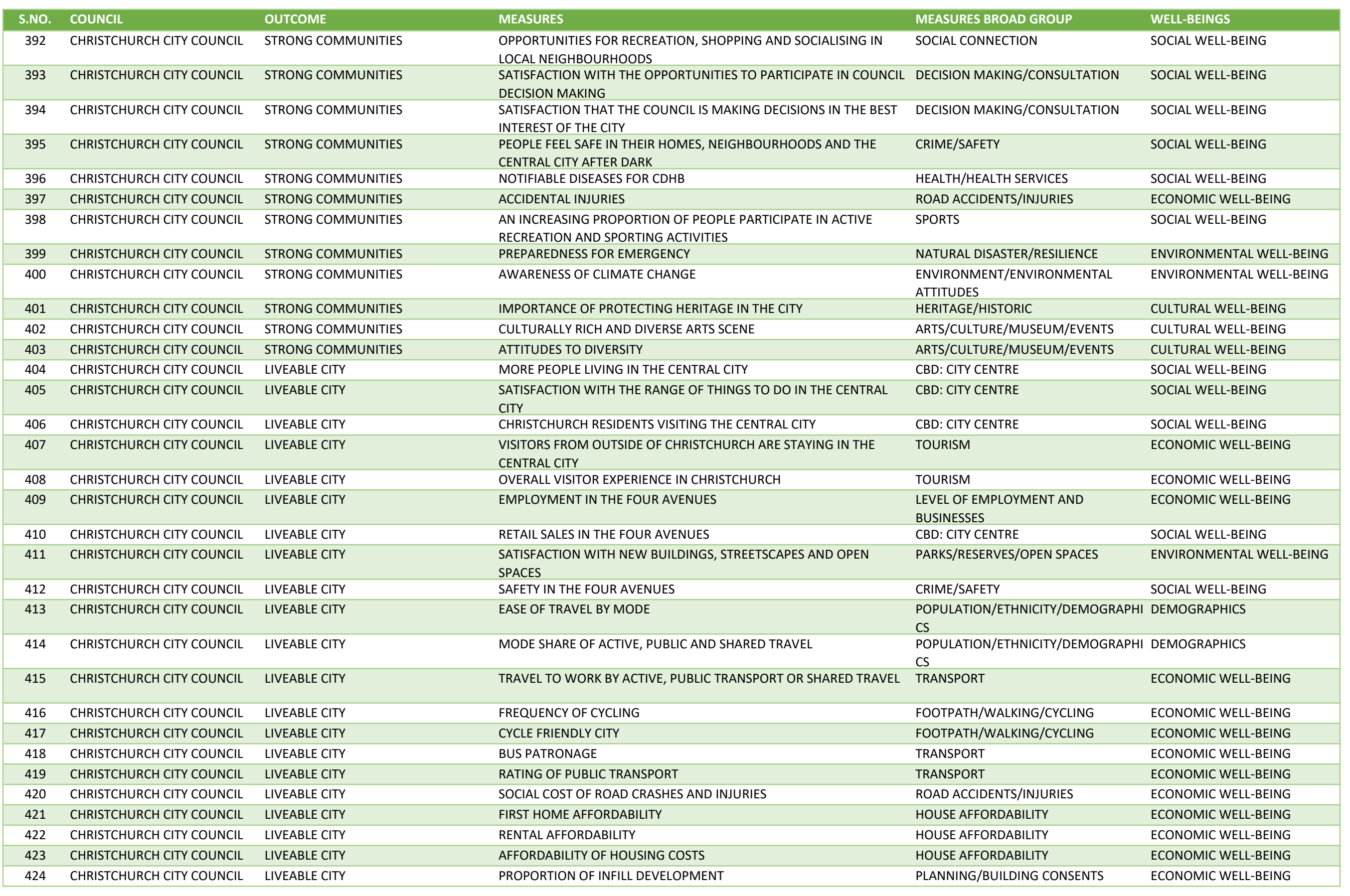


Appendix A: List of Community Well-beings Outcomes and Measures by Councils

\begin{tabular}{|c|c|c|c|c|c|}
\hline S.NO. & COUNCIL & OUTCOME & MEASURES & MEASURES BROAD GROUP & WELL-BEINGS \\
\hline 425 & CHRISTCHURCH CITY COUNCIL & LIVEABLE CITY & HOUSING QUALITY - PROBLEMS WITH DAMP AND MOULD & HOUSE QUALITY & ECONOMIC WELL-BEING \\
\hline 426 & CHRISTCHURCH CITY COUNCIL & LIVEABLE CITY & $\begin{array}{l}\text { SATISFACTION WITH THE RANGE OF HOUSING TO MEET CURRENT } \\
\text { NEEDS }\end{array}$ & HOUSE AFFORDABILITY & ECONOMIC WELL-BEING \\
\hline 427 & CHRISTCHURCH CITY COUNCIL & LIVEABLE CITY & SUITABILITY OF DWELLING TYPE & HOUSE QUALITY & ECONOMIC WELL-BEING \\
\hline 428 & CHRISTCHURCH CITY COUNCIL & LIVEABLE CITY & SUITABILITY OF LOCATION OF HOME & HOUSE AFFORDABILITY & ECONOMIC WELL-BEING \\
\hline 429 & CHRISTCHURCH CITY COUNCIL & LIVEABLE CITY & SENSE OF PRIDE IN CHRISTCHURCH & COMMUNITY DEVELOPMENT & SOCIAL WELL-BEING \\
\hline 430 & CHRISTCHURCH CITY COUNCIL & LIVEABLE CITY & CHRISTCHURCH AS A PLACE TO LIVE, WORK, PLAY AND VISIT & TOURISM & ECONOMIC WELL-BEING \\
\hline 431 & CHRISTCHURCH CITY COUNCIL & LIVEABLE CITY & IMPORTANCE OF THE GARDEN CITY IMAGE & $\begin{array}{l}\text { ENVIRONMENT/ENVIRONMENTAL } \\
\text { ATTITUDES }\end{array}$ & ENVIRONMENTAL WELL-BEING \\
\hline 432 & CHRISTCHURCH CITY COUNCIL & HEALTHY ENVIRONMENT & QUALITY OF CHRISTCHURCH WATERWAYS & $\begin{array}{l}\text { RIVER, LAKES, COASTAL AND } \\
\text { GROUNDWATER }\end{array}$ & ENVIRONMENTAL WELL-BEING \\
\hline 433 & CHRISTCHURCH CITY COUNCIL & HEALTHY ENVIRONMENT & AVON HEATHCOTE ESTUARY /IHUTAI WATER QUALITY INDEX & $\begin{array}{l}\text { RIVER, LAKES, COASTAL AND } \\
\text { GROUNDWATER }\end{array}$ & ENVIRONMENTAL WELL-BEING \\
\hline 434 & CHRISTCHURCH CITY COUNCIL & HEALTHY ENVIRONMENT & $\begin{array}{l}\text { LAKE FORSYTH/TE ROTO O WAIREWA AND LAKE ELLESMERE/TE } \\
\text { WAIHORA WATER QUALITY }\end{array}$ & $\begin{array}{l}\text { RIVER, LAKES, COASTAL AND } \\
\text { GROUNDWATER }\end{array}$ & ENVIRONMENTAL WELL-BEING \\
\hline 435 & CHRISTCHURCH CITY COUNCIL & HEALTHY ENVIRONMENT & $\begin{array}{l}\text { CONTACT RECREATION AT CHRISTCHURCH AND BANKS PENINSULA } \\
\text { RIVERS AND BEACHES }\end{array}$ & RECREATIONAL FACILITIES & SOCIAL WELL-BEING \\
\hline 436 & CHRISTCHURCH CITY COUNCIL & HEALTHY ENVIRONMENT & $\begin{array}{l}\text { SATISFACTION WITH THE OVERALL QUALITY OF THE WATER SUPPLY IN } \\
\text { CHRISTCHURCH }\end{array}$ & $\begin{array}{l}\text { RIVER, LAKES, COASTAL AND } \\
\text { GROUNDWATER }\end{array}$ & ENVIRONMENTAL WELL-BEING \\
\hline 438 & CHRISTCHURCH CITY COUNCIL & HEALTHY ENVIRONMENT & GROUNDWATER QUALITY & $\begin{array}{l}\text { RIVER, LAKES, COASTAL AND } \\
\text { GROUNDWATER }\end{array}$ & ENVIRONMENTAL WELL-BEING \\
\hline 439 & CHRISTCHURCH CITY COUNCIL & HEALTHY ENVIRONMENT & WATER SUPPLY ABSTRACTION AND ABSTRACTION PER PERSON & $\begin{array}{l}\text { RIVER, LAKES, COASTAL AND } \\
\text { GROUNDWATER }\end{array}$ & ENVIRONMENTAL WELL-BEING \\
\hline 440 & CHRISTCHURCH CITY COUNCIL & HEALTHY ENVIRONMENT & QE COVENANTS & $\begin{array}{l}\text { ENVIRONMENT/ENVIRONMENTAL } \\
\text { ATTITUDES }\end{array}$ & ENVIRONMENTAL WELL-BEING \\
\hline 441 & CHRISTCHURCH CITY COUNCIL & HEALTHY ENVIRONMENT & STATE OF NATIVE BUSH, FORESTS, NATURE RESERVES AND OPEN SPACE & PARKS/RESERVES/OPEN SPACES & ENVIRONMENTAL WELL-BEING \\
\hline 442 & CHRISTCHURCH CITY COUNCIL & HEALTHY ENVIRONMENT & $\begin{array}{l}\text { STATE OF THE WETLANDS SURFAC, E WATER, GROUNDWATER AND } \\
\text { COASTLINES }\end{array}$ & $\begin{array}{l}\text { RIVER, LAKES, COASTAL AND } \\
\text { GROUNDWATER }\end{array}$ & ENVIRONMENTAL WELL-BEING \\
\hline 443 & CHRISTCHURCH CITY COUNCIL & HEALTHY ENVIRONMENT & SATISFACTION WITH CONDITION OF WATERWAYS & $\begin{array}{l}\text { RIVER, LAKES, COASTAL AND } \\
\text { GROUNDWATER }\end{array}$ & ENVIRONMENTAL WELL-BEING \\
\hline 444 & CHRISTCHURCH CITY COUNCIL & HEALTHY ENVIRONMENT & $\begin{array}{l}\text { RESIDENTS ACTIONS TO PROTECT AND ENHANCE THE LOCAL } \\
\text { ENVIRONMENT }\end{array}$ & $\begin{array}{l}\text { ENVIRONMENT/ENVIRONMENTAL } \\
\text { ATTITUDES }\end{array}$ & ENVIRONMENTAL WELL-BEING \\
\hline 445 & CHRISTCHURCH CITY COUNCIL & HEALTHY ENVIRONMENT & $\begin{array}{l}\text { RESIDENTS DECISIONS TO REDUCE THEIR IMPACT ON THE } \\
\text { ENVIRONMENT }\end{array}$ & $\begin{array}{l}\text { ENVIRONMENT/ENVIRONMENTAL } \\
\text { ATTITUDES }\end{array}$ & ENVIRONMENTAL WELL-BEING \\
\hline 446 & CHRISTCHURCH CITY COUNCIL & HEALTHY ENVIRONMENT & VOLUNTEERING OR DONATING TO NATURAL ENVIRONMENT CAUSES & VOLUNTEER/UNPAID WORK & SOCIAL WELL-BEING \\
\hline 447 & CHRISTCHURCH CITY COUNCIL & HEALTHY ENVIRONMENT & $\begin{array}{l}\text { IMPORTANCE OF LANDSCAPE TO IDENTITY OF CHRISTCHURCH AND } \\
\text { BANKS PENINSULA }\end{array}$ & NATURAL FEATURES & ENVIRONMENTAL WELL-BEING \\
\hline 450 & CHRISTCHURCH CITY COUNCIL & HEALTHY ENVIRONMENT & TOTAL ENERGY CONSUMPTION & ENERGY USAGE & ENVIRONMENTAL WELL-BEING \\
\hline 451 & CHRISTCHURCH CITY COUNCIL & HEALTHY ENVIRONMENT & RENEWABLE VERSUS NON-RENEWABLE ENERGY CONSUMPTION & ENERGY USAGE & ENVIRONMENTAL WELL-BEING \\
\hline 452 & CHRISTCHURCH CITY COUNCIL & HEALTHY ENVIRONMENT & TOTAL WASTE TO LANDFILL & WASTE TO LANDFILL & ENVIRONMENTAL WELL-BEING \\
\hline
\end{tabular}


Appendix A: List of Community Well-beings Outcomes and Measures by Councils

\begin{tabular}{|c|c|c|c|c|c|}
\hline S.NO. & COUNCIL & OUTCOME & MEASURES & MEASURES BROAD GROUP & WELL-BEINGS \\
\hline 453 & CHRISTCHURCH CITY COUNCIL & PROSPEROUS ECONOMY & OVERALL QUALITY OF LIFE & QUALITY OF LIFE & SOCIAL WELL-BEING \\
\hline 454 & CHRISTCHURCH CITY COUNCIL & PROSPEROUS ECONOMY & HIGHEST QUALIFICATION & QUALIFICATION/SCHOOL & SOCIAL WELL-BEING \\
\hline 455 & CHRISTCHURCH CITY COUNCIL & PROSPEROUS ECONOMY & SKILLED MIGRANT APPLICATIONS & $\begin{array}{l}\text { LEVEL OF EMPLOYMENT AND } \\
\text { BUSINESSES }\end{array}$ & ECONOMIC WELL-BEING \\
\hline 456 & CHRISTCHURCH CITY COUNCIL & PROSPEROUS ECONOMY & INNOVATION CITIES INDEX & ARTS/CULTURE/MUSEUM/EVENTS & CULTURAL WELL-BEING \\
\hline 457 & CHRISTCHURCH CITY COUNCIL & PROSPEROUS ECONOMY & BUSINESS BIRTHS AS A PERCENTAGE OF TURNOVER & NUMBER OF BUSINESSES & ECONOMIC WELL-BEING \\
\hline 458 & CHRISTCHURCH CITY COUNCIL & PROSPEROUS ECONOMY & ABILITY TO MEET THE COST OF EVERYDAY NEEDS & INCOME & ECONOMIC WELL-BEING \\
\hline 459 & CHRISTCHURCH CITY COUNCIL & PROSPEROUS ECONOMY & HOUSEHOLD INCOME DISPARITY & INCOME & ECONOMIC WELL-BEING \\
\hline 461 & CHRISTCHURCH CITY COUNCIL & PROSPEROUS ECONOMY & $\begin{array}{l}\text { NOT IN EMPLOYMENT, EDUCATION OR TRAINING (NEET) RATE FOR } \\
\text { YOUNG PEOPLE }\end{array}$ & $\begin{array}{l}\text { NEET: NOT IN EMPLOYMENT, } \\
\text { EDUCATION OR TRAINING }\end{array}$ & ECONOMIC WELL-BEING \\
\hline 462 & CHRISTCHURCH CITY COUNCIL & PROSPEROUS ECONOMY & CANTERBURY GROSS DOMESTIC PRODUCT (GDP) & GROSS DOMESTIC PRODUCT (GDP) & ECONOMIC WELL-BEING \\
\hline 463 & CHRISTCHURCH CITY COUNCIL & PROSPEROUS ECONOMY & $\begin{array}{l}\text { VALUE OF EXPORT GOODS FROM LYTTELTON PORT AND } \\
\text { CHRISTCHURCH AIRPORT }\end{array}$ & EXPORTS & ECONOMIC WELL-BEING \\
\hline 464 & CHRISTCHURCH CITY COUNCIL & PROSPEROUS ECONOMY & REGIONAL TOURISM SPEND & TOURISM & ECONOMIC WELL-BEING \\
\hline 465 & CHRISTCHURCH CITY COUNCIL & PROSPEROUS ECONOMY & ACCESS TO ULTRA-FAST BROADBAND & PHONE/MOBILE/INTERNET & ECONOMIC WELL-BEING \\
\hline 466 & CHRISTCHURCH CITY COUNCIL & PROSPEROUS ECONOMY & CAPACITY OF VISITOR ACCOMMODATION & TOURISM & ECONOMIC WELL-BEING \\
\hline 467 & CHRISTCHURCH CITY COUNCIL & PROSPEROUS ECONOMY & SATISFACTION WITH COUNCIL INFRASTRUCTURE & COUNCIL OPERATIONS & ECONOMIC WELL-BEING \\
\hline 469 & CLUTHA DISTRICT COUNCI & $\begin{array}{l}\text { WORKING TOWARDS A SAFER AND } \\
\text { HEALTHIER COMMUNITY }\end{array}$ & LIFE EXPECTANCY & LIFE EXPECTANCY & DEMOGRAPHICS \\
\hline 470 & CLUTHA DISTRICT COUNCI & $\begin{array}{l}\text { WORKING TOWARDS A SAFER AND } \\
\text { HEALTHIER COMMUNITY }\end{array}$ & PERCEPTION OF SAFETY & CRIME/SAFETY & SOCIAL WELL-BEING \\
\hline 471 & CLUTHA DISTRICT COUNCI & $\begin{array}{l}\text { WORKING TOWARDS A SAFER AND } \\
\text { HEALTHIER COMMUNITY }\end{array}$ & REPORTED OFFENCES & CRIME/SAFETY & SOCIAL WELL-BEING \\
\hline 472 & CLUTHA DISTRICT COUNCI & $\begin{array}{l}\text { WORKING TOWARDS A SAFER AND } \\
\text { HEALTHIER COMMUNITY }\end{array}$ & PERCEPTION OF AVAILABLE RECREATION OPPORTUNITIES & RECREATIONAL FACILITIES & SOCIAL WELL-BEING \\
\hline 473 & CLUTHA DISTRICT COUNCI & $\begin{array}{l}\text { WORKING TOWARDS A SAFER AND } \\
\text { HEALTHIER COMMUNITY }\end{array}$ & PERCEPTION OF THE LEVEL OF SPORTING GOODS AND OPPORTUNITIES & SPORTS & SOCIAL WELL-BEING \\
\hline 474 & CLUTHA DISTRICT COUNCI & $\begin{array}{l}\text { WORKING TOWARDS A SAFER AND } \\
\text { HEALTHIER COMMUNITY }\end{array}$ & DEATH RATES & $\begin{array}{l}\text { POPULATION/ETHNICITY/DEMOGRAPHI } \\
\text { CS }\end{array}$ & DEMOGRAPHICS \\
\hline 475 & CLUTHA DISTRICT COUNCI & $\begin{array}{l}\text { WORKING TOWARDS A SAFER AND } \\
\text { HEALTHIER COMMUNITY }\end{array}$ & ACCESS TO GP/'S/DOCTORS & HEALTH/HEALTH SERVICES & SOCIAL WELL-BEING \\
\hline 476 & CLUTHA DISTRICT COUNCI & $\begin{array}{l}\text { WORKING TOWARDS A SAFER AND } \\
\text { HEALTHIER COMMUNITY }\end{array}$ & VOLUNTEER FIRE BRIGADE & VOLUNTEER/UNPAID WORK & SOCIAL WELL-BEING \\
\hline 477 & CLUTHA DISTRICT COUNCI & $\begin{array}{l}\text { WORKING TOWARDS A SAFER AND } \\
\text { HEALTHIER COMMUNITY }\end{array}$ & ST JOHN EMERGENCY SERVICES & HOSPITALISATIONS & ECONOMIC WELL-BEING \\
\hline 478 & CLUTHA DISTRICT COUNCI & $\begin{array}{l}\text { WORKING TOWARDS A SAFER AND } \\
\text { HEALTHIER COMMUNITY }\end{array}$ & CRASH STATISTICS & ROAD ACCIDENTS/INJURIES & ECONOMIC WELL-BEING \\
\hline 480 & CLUTHA DISTRICT COUNCI & $\begin{array}{l}\text { WORKING TOWARDS A SAFER AND } \\
\text { HEALTHIER COMMUNITY }\end{array}$ & ACC ENTITLEMENT CLAIMS & ACC CLAIMS & ECONOMIC WELL-BEING \\
\hline 481 & CLUTHA DISTRICT COUNCI & $\begin{array}{l}\text { WORKING TOWARDS A SAFER AND } \\
\text { HEALTHIER COMMUNITY }\end{array}$ & RESIDENTS PREPARED FOR A DISASTER & NATURAL DISASTER/RESILIENCE & ENVIRONMENTAL WELL-BEING \\
\hline
\end{tabular}


Appendix A: List of Community Well-beings Outcomes and Measures by Councils

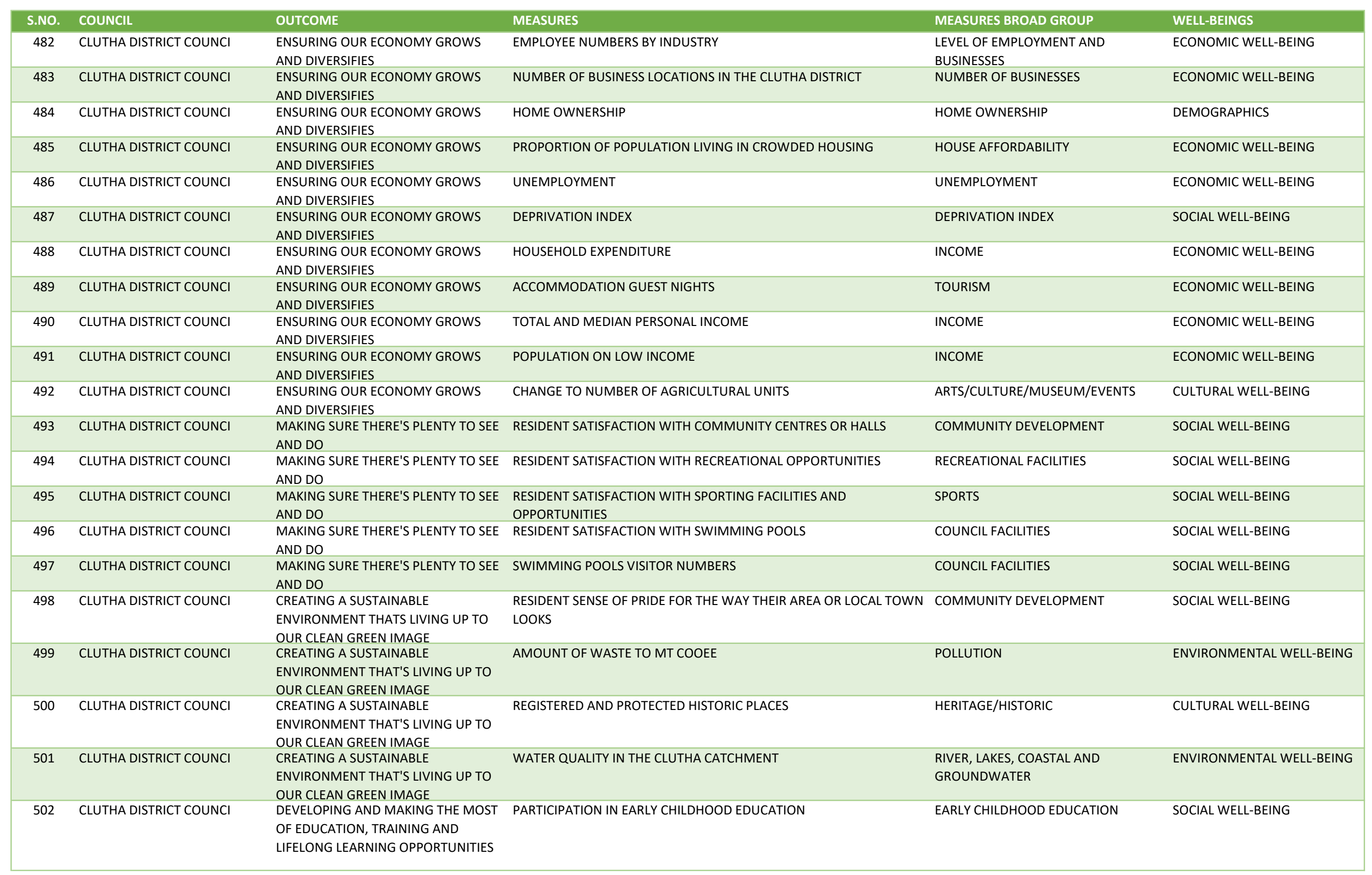




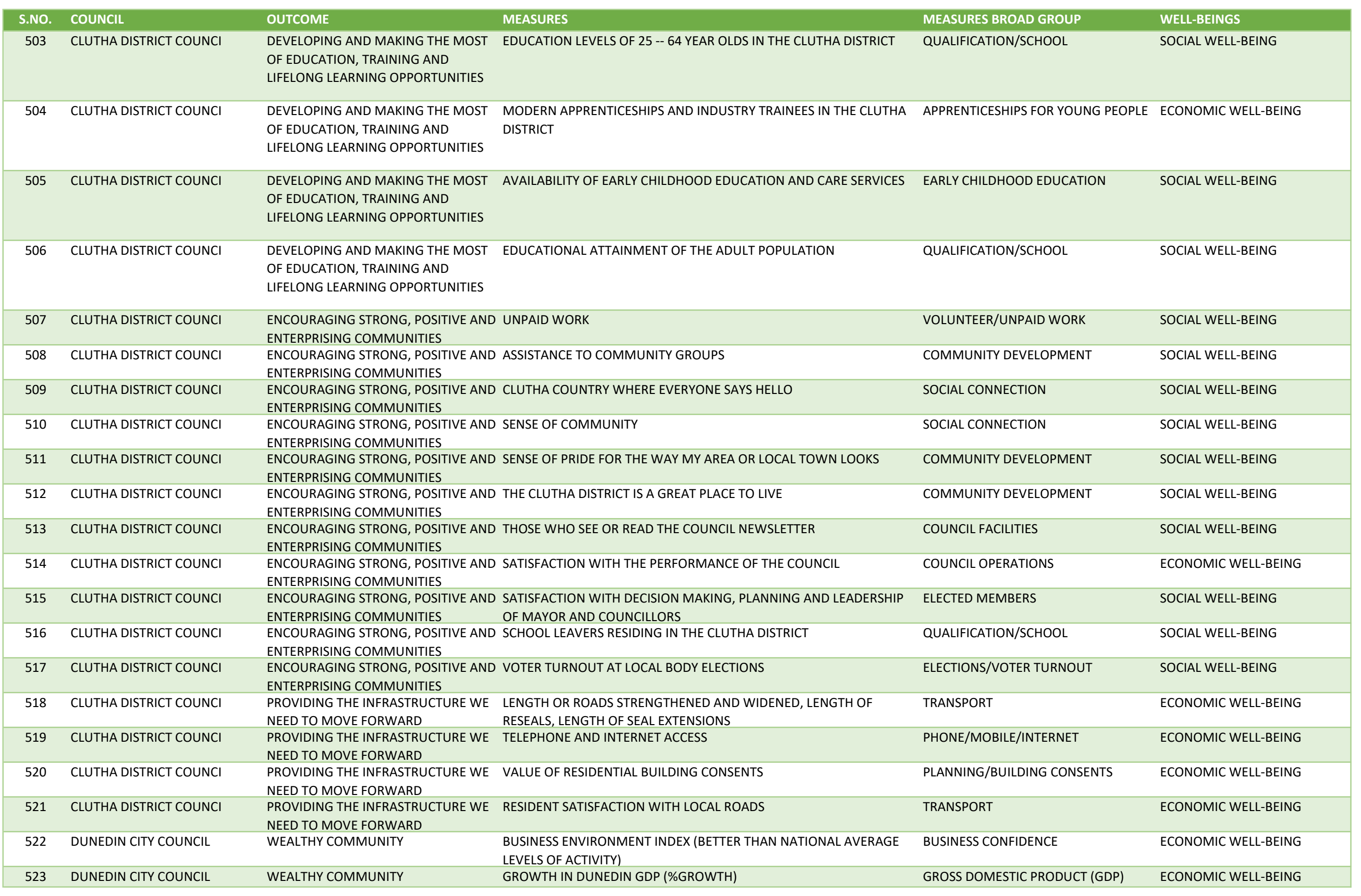


Appendix A: List of Community Well-beings Outcomes and Measures by Councils

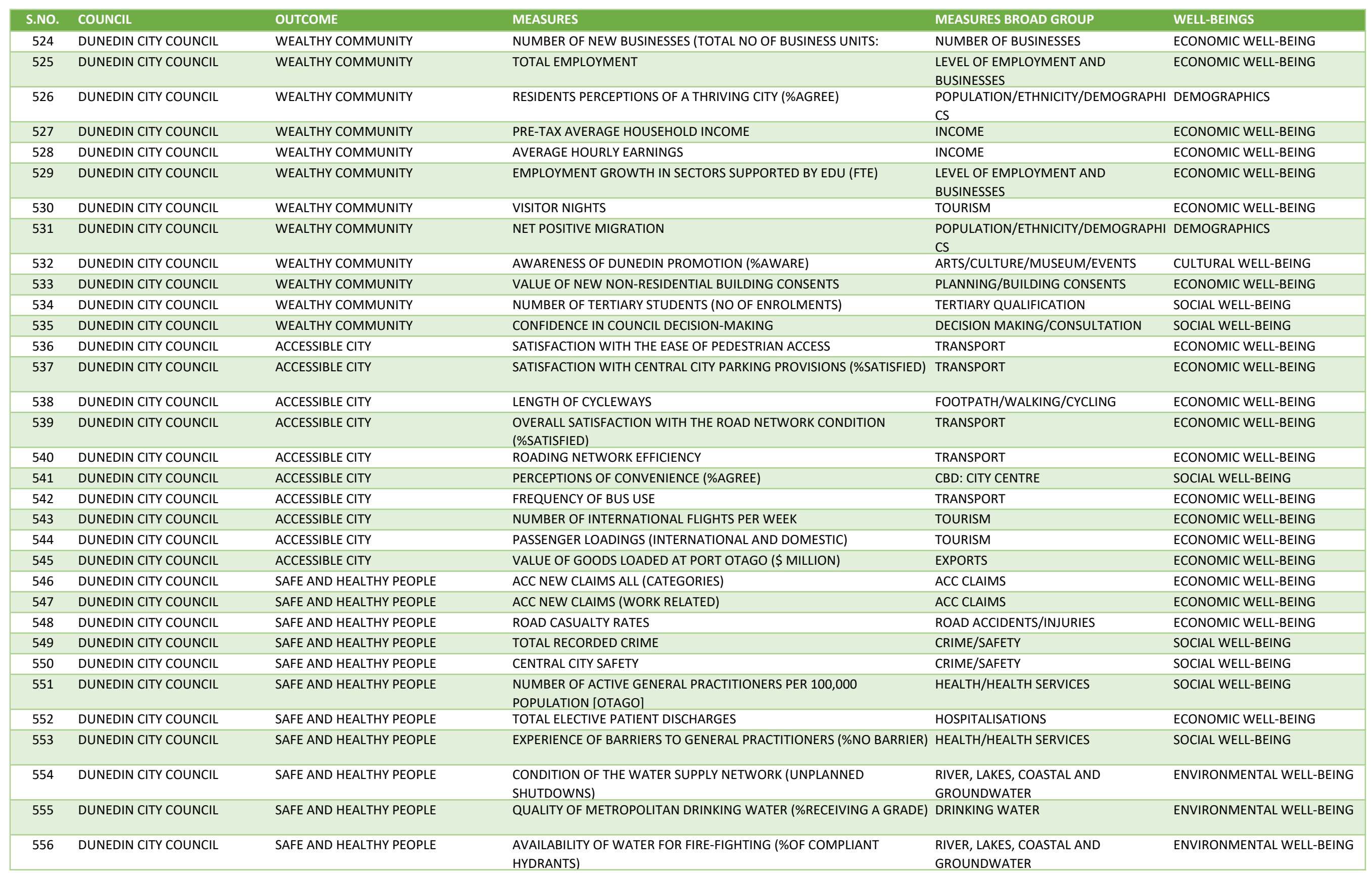


Appendix A: List of Community Well-beings Outcomes and Measures by Councils

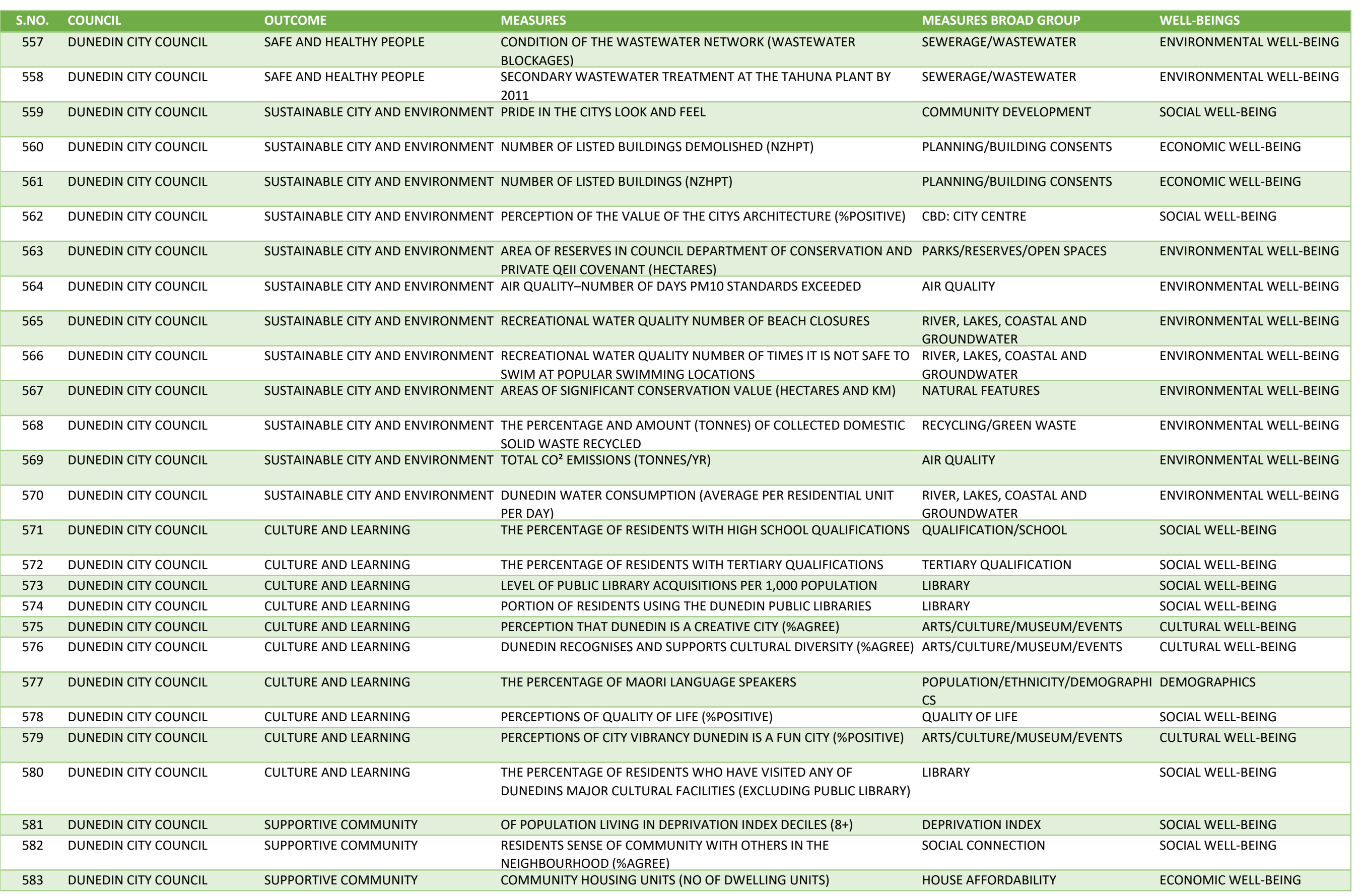


Appendix A: List of Community Well-beings Outcomes and Measures by Councils

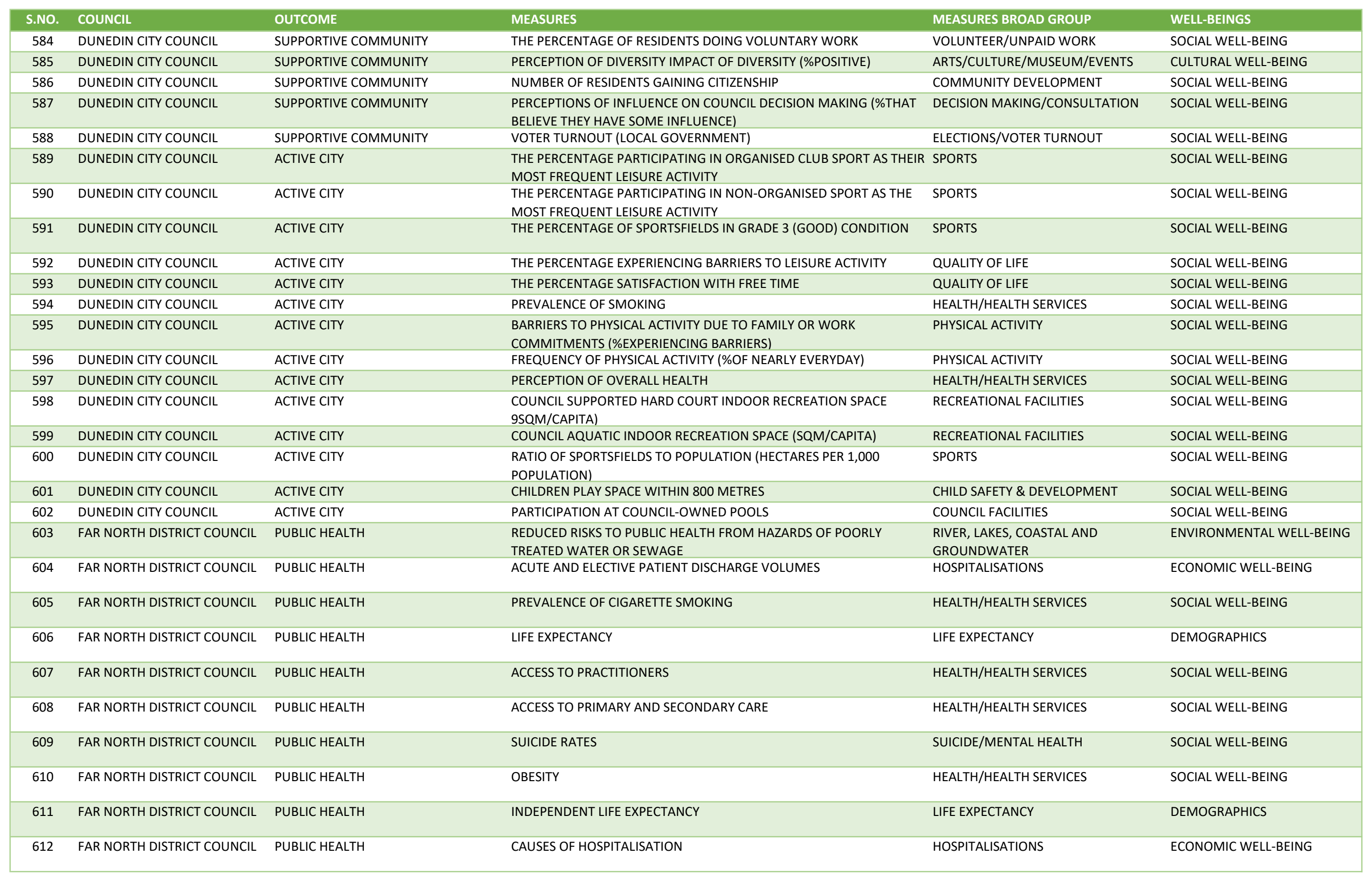


Appendix A: List of Community Well-beings Outcomes and Measures by Councils

\begin{tabular}{|c|c|c|c|c|c|}
\hline S.NO. & COUNCIL & OUTCOME & MEASURES & MEASURES BROAD GROUP & WELL-BEINGS \\
\hline 613 & FAR NORTH DISTRICT COUNCIL & PUBLIC SAFETY & $\begin{array}{l}\text { REDUCTION IN THE NUMBER OF OVERFLOWS AND SPILLS FROM PUBLIC } \\
\text { SYSTEMS IN THE DISTRICT }\end{array}$ & SEWERAGE/WASTEWATER & ENVIRONMENTAL WELL-BEING \\
\hline 614 & FAR NORTH DISTRICT COUNCIL & PUBLIC SAFETY & NUMBER OF RECORDED OFFENCES PER 10,000 PEOPLE & CRIME/SAFETY & SOCIAL WELL-BEING \\
\hline 616 & FAR NORTH DISTRICT COUNCIL & PUBLIC SAFETY & PERCEPTIONS OF SAFETY & CRIME/SAFETY & SOCIAL WELL-BEING \\
\hline 617 & FAR NORTH DISTRICT COUNCIL & PUBLIC SAFETY & $\begin{array}{l}\text { ADDRESSING ISSUES SURROUNDING INTENTIONAL INJURY AND CHILD } \\
\text { MORTALITY }\end{array}$ & CHILD SAFETY \& DEVELOPMENT & SOCIAL WELL-BEING \\
\hline 618 & FAR NORTH DISTRICT COUNCIL & TRANSPORTATION & $\begin{array}{l}\text { REDUCTIONS IN NUMBER OF PEOPLE KILLED OR INJURED IN MOTOR } \\
\text { VEHICLE CRASHES AS A PROPORTION OF THE TOTAL POPULATION }\end{array}$ & ROAD ACCIDENTS/INJURIES & ECONOMIC WELL-BEING \\
\hline 619 & FAR NORTH DISTRICT COUNCIL & TRANSPORTATION & $\begin{array}{l}\text { THE PROPORTION OF SEALED ROADS AS COMPARED TO UNSEALED } \\
\text { ROADS }\end{array}$ & TRANSPORT & ECONOMIC WELL-BEING \\
\hline 620 & FAR NORTH DISTRICT COUNCIL & ENVIRONMENT & DRINKING WATER QUALITY & DRINKING WATER & ENVIRONMENTAL WELL-BEING \\
\hline 621 & FAR NORTH DISTRICT COUNCIL & ENVIRONMENT & NUMBER OF HOUSEHOLDS ON RETICULATED SEWERAGE & SEWERAGE/WASTEWATER & ENVIRONMENTAL WELL-BEING \\
\hline 622 & FAR NORTH DISTRICT COUNCIL & ENVIRONMENT & SOLID WASTE MANAGEMENT AND RECYCLING & RECYCLING/GREEN WASTE & ENVIRONMENTAL WELL-BEING \\
\hline 624 & FAR NORTH DISTRICT COUNCIL & ENVIRONMENT & AIR QUALITY & AIR QUALITY & ENVIRONMENTAL WELL-BEING \\
\hline 625 & FAR NORTH DISTRICT COUNCIL & ENVIRONMENT & BEACH AND STREAM LAKE / WATER QUALITY & $\begin{array}{l}\text { RIVER, LAKES, COASTAL AND } \\
\text { GROUNDWATER }\end{array}$ & ENVIRONMENTAL WELL-BEING \\
\hline 626 & FAR NORTH DISTRICT COUNCIL & ENVIRONMENT & BIODIVERSITY & $\begin{array}{l}\text { ENVIRONMENT/ENVIRONMENTAL } \\
\text { ATTITUDES }\end{array}$ & ENVIRONMENTAL WELL-BEING \\
\hline 627 & FAR NORTH DISTRICT COUNCIL & $\begin{array}{l}\text { EDUCATION, TRAINING AND BUSINESS } \\
\text { OPPORTUNITIES }\end{array}$ & PARTICIPATION IN EARLY CHILDHOOD EDUCATION & EARLY CHILDHOOD EDUCATION & SOCIAL WELL-BEING \\
\hline 628 & FAR NORTH DISTRICT COUNCIL & $\begin{array}{l}\text { EDUCATION, TRAINING AND BUSINESS } \\
\text { OPPORTUNITIES }\end{array}$ & SCHOOL LEAVERS WITH HIGHER QUALIFICATIONS & QUALIFICATION/SCHOOL & SOCIAL WELL-BEING \\
\hline 629 & FAR NORTH DISTRICT COUNCIL & $\begin{array}{l}\text { EDUCATION, TRAINING AND BUSINESS } \\
\text { OPPORTUNITIES }\end{array}$ & EDUCATIONAL ATTAINMENT OF ADULT POPULATION & QUALIFICATION/SCHOOL & SOCIAL WELL-BEING \\
\hline 630 & FAR NORTH DISTRICT COUNCIL & $\begin{array}{l}\text { EDUCATION, TRAINING AND BUSINESS } \\
\text { OPPORTUNITIES }\end{array}$ & ADULT LITERACY SKILLS IN ENGLISH & QUALIFICATION/SCHOOL & SOCIAL WELL-BEING \\
\hline 631 & FAR NORTH DISTRICT COUNCIL & $\begin{array}{l}\text { EDUCATION, TRAINING AND BUSINESS } \\
\text { OPPORTUNITIES }\end{array}$ & GROWTH IN BUSINESS & NUMBER OF BUSINESSES & ECONOMIC WELL-BEING \\
\hline 632 & FAR NORTH DISTRICT COUNCIL & $\begin{array}{l}\text { EDUCATION, TRAINING AND BUSINESS } \\
\text { OPPORTUNITIES }\end{array}$ & NUMBER OF BUSINESSES AND NUMBER OF EMPLOYEES & $\begin{array}{l}\text { LEVEL OF EMPLOYMENT AND } \\
\text { BUSINESSES }\end{array}$ & ECONOMIC WELL-BEING \\
\hline 633 & FAR NORTH DISTRICT COUNCIL & $\begin{array}{l}\text { EDUCATION, TRAINING AND BUSINESS } \\
\text { OPPORTUNITIES }\end{array}$ & UNEMPLOYMENT RATE & UNEMPLOYMENT & ECONOMIC WELL-BEING \\
\hline 635 & FAR NORTH DISTRICT COUNCIL & CULTURE AND HERITAGE & $\begin{array}{l}\text { LANGUAGE RETENTION -- PROPORTION OF PEOPLE WHO CAN SPEAK A } \\
\text { FIRST LANGUAGE OF THEIR ETHNIC GROUP }\end{array}$ & $\begin{array}{l}\text { POPULATION/ETHNICITY/DEMOGRAPHI } \\
\text { CS }\end{array}$ & DEMOGRAPHICS \\
\hline 636 & FAR NORTH DISTRICT COUNCIL & CULTURE AND HERITAGE & MÃORI LANGUAGE SPEAKERS & $\begin{array}{l}\text { POPULATION/ETHNICITY/DEMOGRAPHI } \\
\text { CS }\end{array}$ & DEMOGRAPHICS \\
\hline
\end{tabular}


Appendix A: List of Community Well-beings Outcomes and Measures by Councils

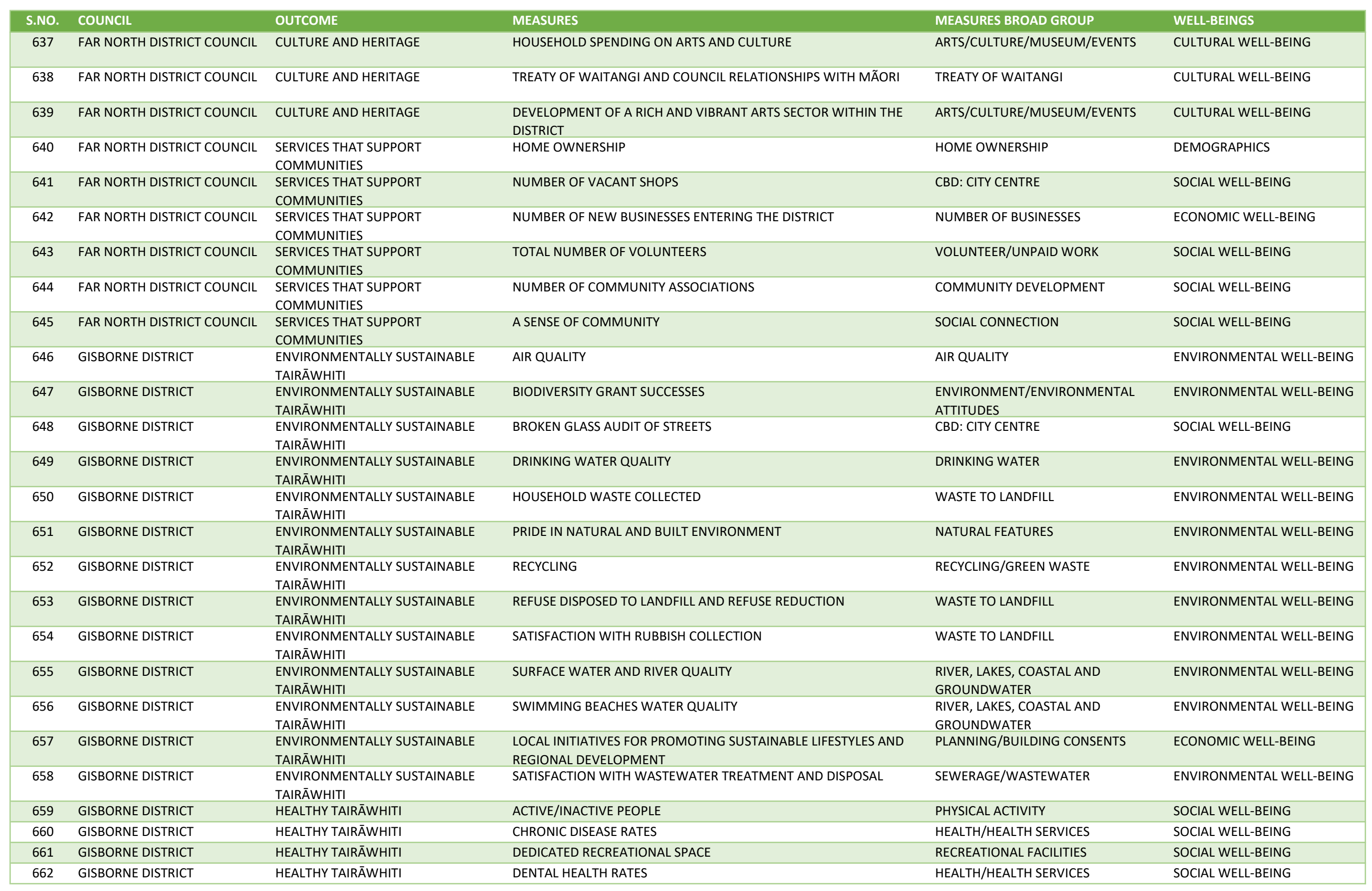


Appendix A: List of Community Well-beings Outcomes and Measures by Councils

\begin{tabular}{|c|c|c|c|c|c|}
\hline S.NO. & COUNCIL & OUTCOME & MEASURES & MEASURES BROAD GROUP & WELL-BEINGS \\
\hline 663 & GISBORNE DISTRICT & HEALTHY TAIRĀWHITI & HOSPITALISATION ADMISSIONS & HOSPITALISATIONS & ECONOMIC WELL-BEING \\
\hline 664 & GISBORNE DISTRICT & HEALTHY TAIRĀWHITI & IMMUNISATION COVERAGE & HEALTH/HEALTH SERVICES & SOCIAL WELL-BEING \\
\hline 665 & GISBORNE DISTRICT & HEALTHY TAIRĀWHITI & LIFE EXPECTANCY & LIFE EXPECTANCY & DEMOGRAPHICS \\
\hline 666 & GISBORNE DISTRICT & HEALTHY TAIRĀWHITI & MORTALITY RATE & $\begin{array}{l}\text { POPULATION/ETHNICITY/DEMOGRAPHI } \\
\text { CS }\end{array}$ & DEMOGRAPHICS \\
\hline 667 & GISBORNE DISTRICT & HEALTHY TAIRĀWHITI & NUMBER OF REGISTERED HEALTH-CARE PROFESSIONALS & HEALTH/HEALTH SERVICES & SOCIAL WELL-BEING \\
\hline 668 & GISBORNE DISTRICT & HEALTHY TAIRĀWHITI & $\begin{array}{l}\text { NUMBER OF HOMES RETROFITTED THROUGH THE HEALTHY HOMES } \\
\text { COMMUNITY PARTNERSHIP }\end{array}$ & HOUSE QUALITY & ECONOMIC WELL-BEING \\
\hline 669 & GISBORNE DISTRICT & HEALTHY TAIRĀWHITI & SECONDARY MENTAL HEALTH SERVICES & SUICIDE/MENTAL HEALTH & SOCIAL WELL-BEING \\
\hline 670 & GISBORNE DISTRICT & HEALTHY TAIRĀWHITI & SMOKING RATES & HEALTH/HEALTH SERVICES & SOCIAL WELL-BEING \\
\hline 671 & GISBORNE DISTRICT & HEALTHY TAIRĀWHITI & SEXUALLY TRANSMITTED INFECTION RATES & HEALTH/HEALTH SERVICES & SOCIAL WELL-BEING \\
\hline 672 & GISBORNE DISTRICT & CONNECTED TAIRĀWHITI & AIRPORT PASSENGER NUMBERS & TOURISM & ECONOMIC WELL-BEING \\
\hline 673 & GISBORNE DISTRICT & CONNECTED TAIRĀWHITI & BUS PASSENGER KM TRAVELLED & TRANSPORT & ECONOMIC WELL-BEING \\
\hline 674 & GISBORNE DISTRICT & CONNECTED TAIRĀWHITI & BUS TICKETS SOLD & TRANSPORT & ECONOMIC WELL-BEING \\
\hline 675 & GISBORNE DISTRICT & CONNECTED TAIRĀWHITI & HOUSEHOLD ACCESS TO THE INTERNET & PHONE/MOBILE/INTERNET & ECONOMIC WELL-BEING \\
\hline 676 & GISBORNE DISTRICT & CONNECTED TAIRĀWHITI & INCREASE IN CYCLE LANES AND WALKWAYS & FOOTPATH/WALKING/CYCLING & ECONOMIC WELL-BEING \\
\hline 677 & GISBORNE DISTRICT & CONNECTED TAIRĀWHITI & PEDESTRIAN AND CYCLE INJURIES & FOOTPATH/WALKING/CYCLING & ECONOMIC WELL-BEING \\
\hline 678 & GISBORNE DISTRICT & CONNECTED TAIRĀWHITI & RESIDENT SATISFACTION WITH ROADS & TRANSPORT & ECONOMIC WELL-BEING \\
\hline 680 & GISBORNE DISTRICT & PROSPEROUS TAIRĀWHITI & BUSINESS AND PUBLIC CONFIDENCE & BUSINESS CONFIDENCE & ECONOMIC WELL-BEING \\
\hline 681 & GISBORNE DISTRICT & PROSPEROUS TAIRĀWHITI & BUSINESS GROWTH & NUMBER OF BUSINESSES & ECONOMIC WELL-BEING \\
\hline 682 & GISBORNE DISTRICT & PROSPEROUS TAIRĀWHITI & HOME AFFORDABILITY & HOUSE AFFORDABILITY & ECONOMIC WELL-BEING \\
\hline 683 & GISBORNE DISTRICT & PROSPEROUS TAIRĀWHITI & INCOME FROM TOURISM & TOURISM & ECONOMIC WELL-BEING \\
\hline 684 & GISBORNE DISTRICT & PROSPEROUS TAIRĀWHITI & MEDIAN INCOME & INCOME & ECONOMIC WELL-BEING \\
\hline 685 & GISBORNE DISTRICT & SKILLED AND EDUCATED TAIRĀWHITI & ATTENDANCE STATISTICS & HOSPITALISATIONS & ECONOMIC WELL-BEING \\
\hline 686 & GISBORNE DISTRICT & SKILLED AND EDUCATED TAIRĀWHITI & $\begin{array}{l}\text { ERO REPORT STANDARDS FOR EARLY CHILDHOOD PRIMARY AND } \\
\text { SECONDARY SCHOOLS }\end{array}$ & EARLY CHILDHOOD EDUCATION & SOCIAL WELL-BEING \\
\hline 687 & GISBORNE DISTRICT & SKILLED AND EDUCATED TAIRĀWHITI & HIGHEST QUALIFICATION GAINED & QUALIFICATION/SCHOOL & SOCIAL WELL-BEING \\
\hline 688 & GISBORNE DISTRICT & SKILLED AND EDUCATED TAIRĀWHITI & LITERACY AND NUMERACY RATES & QUALIFICATION/SCHOOL & SOCIAL WELL-BEING \\
\hline 689 & GISBORNE DISTRICT & SKILLED AND EDUCATED TAIRĀWHITI & NUMBER OF YOUTH LEAVING SCHOOL WITH NO QUALIFICATIONS & QUALIFICATION/SCHOOL & SOCIAL WELL-BEING \\
\hline 690 & GISBORNE DISTRICT & SKILLED AND EDUCATED TAIRĀWHITI & TERTIARY STUDY & TERTIARY QUALIFICATION & SOCIAL WELL-BEING \\
\hline 691 & GISBORNE DISTRICT & VIBRANT TAIRĀWHITI & NUMBER OF EXHIBITIONS & ARTS/CULTURE/MUSEUM/EVENTS & CULTURAL WELL-BEING \\
\hline 692 & GISBORNE DISTRICT & VIBRANT TAIRĀWHITI & FUNDING COMING INTO THE REGION & ECONOMIC ACTIVITY & ECONOMIC WELL-BEING \\
\hline 693 & GISBORNE DISTRICT & VIBRANT TAIRĀWHITI & CURRICULUM THEMES FOR LOCAL SCHOOLS & QUALIFICATION/SCHOOL & SOCIAL WELL-BEING \\
\hline 694 & GISBORNE DISTRICT & VIBRANT TAIRĀWHITI & $\begin{array}{l}\text { NUMBER OF EVENTS HELD AT THE LAWSON FIELD THEATRE, WAR } \\
\text { MEMORIAL THEATRE AND THE OUTDOOR THEATRE }\end{array}$ & ARTS/CULTURE/MUSEUM/EVENTS & CULTURAL WELL-BEING \\
\hline 695 & GISBORNE DISTRICT & VIBRANT TAIRĀWHITI & $\begin{array}{l}\text { NUMBER OF BUILDINGS AND PLACES INCLUDING WAHI TAPU SITES IN } \\
\text { TAIRĀWHITI }\end{array}$ & PLANNING/BUILDING CONSENTS & ECONOMIC WELL-BEING \\
\hline 698 & GISBORNE DISTRICT & VIBRANT TAIRĀWHITI & TAIRĀWHITI MUSEUM VISITOR NUMBERS & TOURISM & ECONOMIC WELL-BEING \\
\hline 699 & GISBORNE DISTRICT & VIBRANT TAIRĀWHITI & NUMBER OF LOCAL EVENTS (GENERALLY) & ARTS/CULTURE/MUSEUM/EVENTS & CULTURAL WELL-BEING \\
\hline
\end{tabular}


Appendix A: List of Community Well-beings Outcomes and Measures by Councils

\begin{tabular}{|c|c|c|c|c|c|}
\hline S.NO. & COUNCIL & OUTCOME & MEASURES & MEASURES BROAD GROUP & WELL-BEINGS \\
\hline 700 & GISBORNE DISTRICT & SAFE TAIRĀWHITI & ACC, OSH, WAGE AND OTHER WORKPLACE BENEFIT LEVELS & ACC CLAIMS & ECONOMIC WELL-BEING \\
\hline 701 & GISBORNE DISTRICT & SAFE TAIRĀWHITI & CRIME STATISTICS & CRIME/SAFETY & SOCIAL WELL-BEING \\
\hline 702 & GISBORNE DISTRICT & SAFE TAIRĀWHITI & FIRES BY TYPE & NATURAL DISASTER/RESILIENCE & ENVIRONMENTAL WELL-BEING \\
\hline 704 & GISBORNE DISTRICT & SAFE TAIRĀWHITI & PERCEPTION OF SAFETY IN PUBLIC PLACES & CRIME/SAFETY & SOCIAL WELL-BEING \\
\hline 705 & GISBORNE DISTRICT & EMPOWERED TAIRĀWHITI & $\begin{array}{l}\text { DIVERSITY AND NUMBER OF AWARDS PRESENTED TO PEOPLE OF } \\
\text { TAIRĀWHITI }\end{array}$ & ARTS/CULTURE/MUSEUM/EVENTS & CULTURAL WELL-BEING \\
\hline 706 & GISBORNE DISTRICT & EMPOWERED TAIRĀWHITI & ELECTED MEMBERS APPROACHABILITY & ELECTED MEMBERS & SOCIAL WELL-BEING \\
\hline 708 & GISBORNE DISTRICT & EMPOWERED TAIRĀWHITI & PERFORMANCE RATING OF MAYOR AND COUNCILLORS & ELECTED MEMBERS & SOCIAL WELL-BEING \\
\hline 709 & GISBORNE DISTRICT & EMPOWERED TAIRĀWHITI & READERSHIP OF INFORMATION PUBLISHED BY COUNCIL & COUNCIL OPERATIONS & ECONOMIC WELL-BEING \\
\hline 710 & GISBORNE DISTRICT & EMPOWERED TAIRĀWHITI & VOTER TURNOUT AT ELECTIONS & ELECTIONS/VOTER TURNOUT & SOCIAL WELL-BEING \\
\hline 711 & GISBORNE DISTRICT & EMPOWERED TAIRĀWHITI & NUMBER OF CANDIDATES FOR RUNANGA & ELECTED MEMBERS & SOCIAL WELL-BEING \\
\hline 712 & GISBORNE DISTRICT & EMPOWERED TAIRĀWHITI & CONTRIBUTION TO LEADERSHIP & COMMUNITY DEVELOPMENT & SOCIAL WELL-BEING \\
\hline 713 & GORE DISTRICT COUNCIL & SOUTHLAND IS A GREAT PLACE TO LIVE & $\begin{array}{l}\text { NEW ZEALAND HISTORIC PLACES TRUST REGISTRATIONS OF CATEGORY } \\
\text { I AND II BUILDINGS AND HISTORIC AREAS IN THE SOUTHLAND REGION } \\
\text { BY TERRITORIAL LOCAL AUTHORITY }\end{array}$ & HERITAGE/HISTORIC & CULTURAL WELL-BEING \\
\hline 715 & GORE DISTRICT COUNCIL & SOUTHLAND IS A GREAT PLACE TO LIVE & $\begin{array}{l}\text { HERITAGE SITES WITHIN THE SOUTHLAND REGION HAVE BEEN } \\
\text { PROGRESSIVELY MADE AVAILABLE TO THE LOCAL COMMUNITY AND } \\
\text { VISITORS ALIKE WITH THE DEVELOPMENT OF HERITAGE TRAILS }\end{array}$ & HERITAGE/HISTORIC & CULTURAL WELL-BEING \\
\hline 716 & GORE DISTRICT COUNCIL & SOUTHLAND IS A GREAT PLACE TO LIVE & $\begin{array}{l}\text { MUSEUM DEVELOPMENTS WITHIN THE SOUTHLAND REGION ENSURE } \\
\text { THAT LOCAL AND REGIONAL HISTORY IS PRESERVED AND PRESENTED } \\
\text { FOR THE EDUCATION AND ENJOYMENT OF LOCAL RESIDENTS AND } \\
\text { VISITORS ALIKE MUSEUM DEVELOPMENTS WITHIN THE AUGUST } 2005 \text { - } \\
\text { AUGUUST } 2008 \text { RFPORTING PFRIOD }\end{array}$ & ARTS/CULTURE/MUSEUM/EVENTS & CULTURAL WELL-BEING \\
\hline 717 & GORE DISTRICT COUNCIL & SOUTHLAND IS A GREAT PLACE TO LIVE & $\begin{array}{l}\text { HERITAGE ACTIVITIES WITHIN THE SOUTHLAND REGION: SOUTHLAND } \\
\text { 150TH CELEBRATIONS } 2006\end{array}$ & HERITAGE/HISTORIC & CULTURAL WELL-BEING \\
\hline 718 & GORE DISTRICT COUNCIL & SOUTHLAND IS A GREAT PLACE TO LIVE & COMMUNITY FUNDING WITHIN THE SOUTHLAND REGION & COMMUNITY DEVELOPMENT & ECONOMIC WELL-BEING \\
\hline 719 & GORE DISTRICT COUNCIL & SOUTHLAND IS A GREAT PLACE TO LIVE & QUALITY OF LIFE SURVEY 2008 & QUALITY OF LIFE & SOCIAL WELL-BEING \\
\hline 720 & GORE DISTRICT COUNCIL & $\begin{array}{l}\text { A DIVERSE ECONOMY BUILT FROM OUR } \\
\text { STRENGTHS FOR GROWTH AND } \\
\text { PROSPERITY }\end{array}$ & REGIONAL GST (\$M) & INCOME & ECONOMIC WELL-BEING \\
\hline 721 & GORE DISTRICT COUNCIL & $\begin{array}{l}\text { A DIVERSE ECONOMY BUILT FROM OUR } \\
\text { STRENGTHS FOR GROWTH AND } \\
\text { PROSPERITY }\end{array}$ & ECONOMIC ACTIVITY & GROSS DOMESTIC PRODUCT (GDP) & ECONOMIC WELL-BEING \\
\hline 723 & GORE DISTRICT COUNCIL & $\begin{array}{l}\text { A DIVERSE ECONOMY BUILT FROM OUR } \\
\text { STRENGTHS FOR GROWTH AND } \\
\text { PROSPERITY }\end{array}$ & & UNEMPLOYMENT & ECONOMIC WELL-BEING \\
\hline
\end{tabular}


Appendix A: List of Community Well-beings Outcomes and Measures by Councils

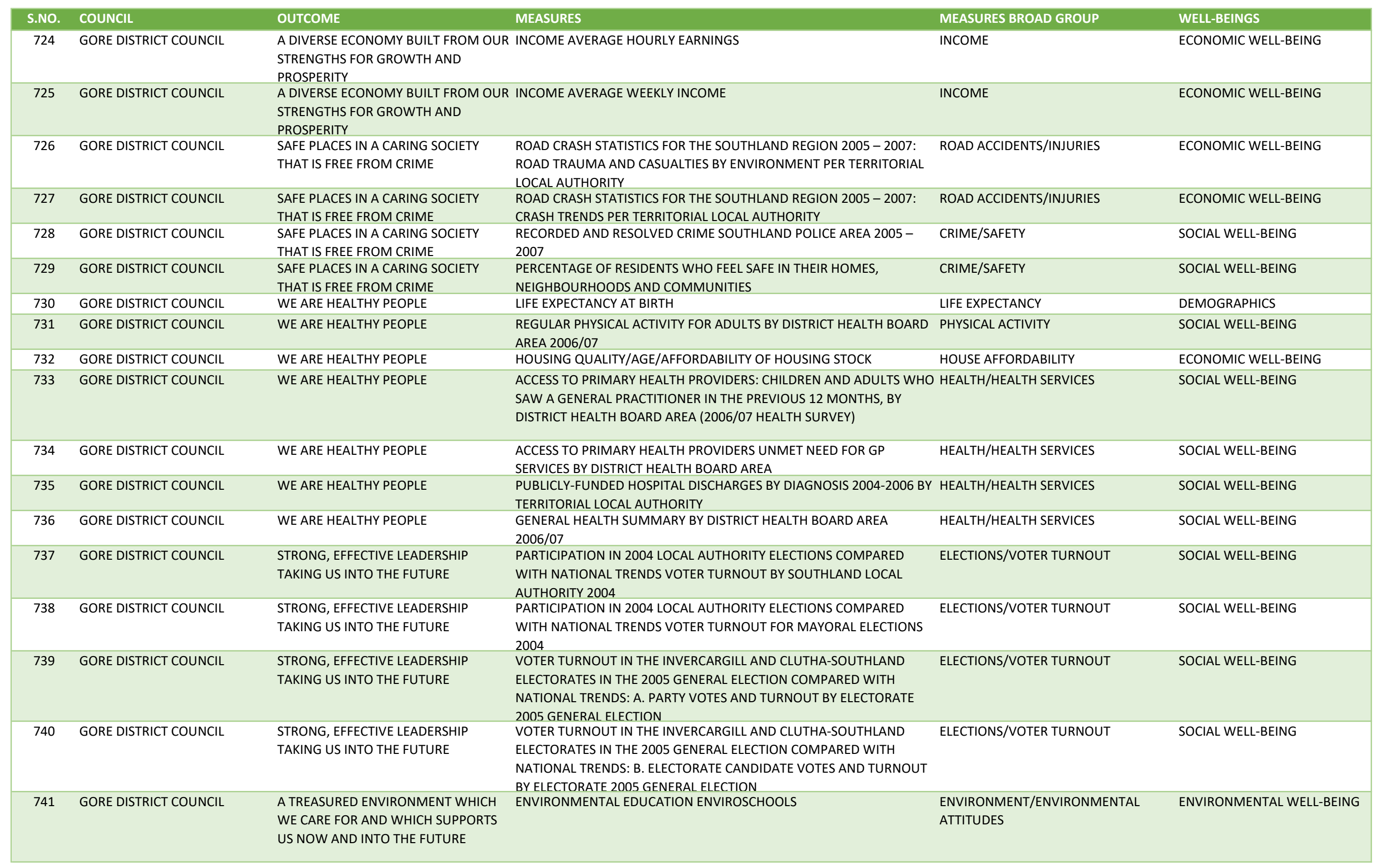




\begin{tabular}{|c|c|c|c|c|c|}
\hline S.NO. & COUNCIL & OUTCOME & MEASURES & MEASURES BROAD GROUP & WELL-BEINGS \\
\hline 742 & GORE DISTRICT COUNCIL & $\begin{array}{l}\text { A TREASURED ENVIRONMENT WHICH } \\
\text { WE CARE FOR AND WHICH SUPPORTS } \\
\text { US NOW AND INTO THE FUTURE }\end{array}$ & $\begin{array}{l}\text { AIR QUALITY: SUMMARY OF THE NATIONAL ENVIRONMENTAL } \\
\text { STANDARD FOR AIR QUALITY FOR AMBIENT FINE PARTICLES - } \\
\text { PARTICULATE MATTER } 10 \text { MICRONS MEASURED IN INVERCARGILL AND } \\
\text { GORE FROM TO } 2003 \text { to } 2007\end{array}$ & AIR QUALITY & ENVIRONMENTAL WELL-BEING \\
\hline 743 & GORE DISTRICT COUNCIL & $\begin{array}{l}\text { A TREASURED ENVIRONMENT WHICH } \\
\text { WE CARE FOR AND WHICH SUPPORTS } \\
\text { US NOW AND INTO THE FUTURE }\end{array}$ & DRINKING WATER & DRINKING WATER & ENVIRONMENTAL WELL-BEING \\
\hline 744 & GORE DISTRICT COUNCIL & $\begin{array}{l}\text { A TREASURED ENVIRONMENT WHICH } \\
\text { WE CARE FOR AND WHICH SUPPORTS } \\
\text { US NOW AND INTO THE FUTURE }\end{array}$ & SOUTHLANDS MARINE RESERVES & $\begin{array}{l}\text { RIVER, LAKES, COASTAL AND } \\
\text { GROUNDWATER }\end{array}$ & ENVIRONMENTAL WELL-BEING \\
\hline 745 & GORE DISTRICT COUNCIL & $\begin{array}{l}\text { A TREASURED ENVIRONMENT WHICH } \\
\text { WE CARE FOR AND WHICH SUPPORTS } \\
\text { US NOW AND INTO THE FUTURE }\end{array}$ & RECYCLING DEPOTS SOUTHLAND & RECYCLING/GREEN WASTE & ENVIRONMENTAL WELL-BEING \\
\hline 746 & GORE DISTRICT COUNCIL & $\begin{array}{l}\text { A WELLEDUCATED AND SKILLED } \\
\text { COMMUNITY CONTINUALLY SEEKING } \\
\text { FURTHER OPPORTUNITIES TO LEARN }\end{array}$ & $\begin{array}{l}\text { EDUCATION PARTICIPATION RATES IN THE SOUTHLAND REGION 2006: } \\
\text { NUMBER OF ENROLMENTS IN EARLY CHILDHOOD EDUCATION SERVICES } \\
\text { BY TYPE OF SERVICE AT } 1 \text { JULY } 2006\end{array}$ & EARLY CHILDHOOD EDUCATION & SOCIAL WELL-BEING \\
\hline 747 & GORE DISTRICT COUNCIL & $\begin{array}{l}\text { A WELLEDUCATED AND SKILLED } \\
\text { COMMUNITY CONTINUALLY SEEKING } \\
\text { FURTHER OPPORTUNITIES TO LEARN }\end{array}$ & $\begin{array}{l}\text { EDUCATION PARTICIPATION RATES IN THE SOUTHLAND REGION } 2006 \text { : } \\
\text { NUMBER OF STUDENTS ENROLLED IN SOUTHLAND BY GOVERNING } \\
\text { AUTHORITY AND SCHOOL TYPE AT } 1 \text { JULY } 2006\end{array}$ & QUALIFICATION/SCHOOL & SOCIAL WELL-BEING \\
\hline 748 & GORE DISTRICT COUNCIL & $\begin{array}{l}\text { A WELLEDUCATED AND SKILLED } \\
\text { COMMUNITY CONTINUALLY SEEKING } \\
\text { FURTHER OPPORTUNITIES TO LEARN }\end{array}$ & $\begin{array}{l}\text { STUDENTS LEAVING SOUTHLAND SECONDARY SCHOOLS DURING } 2005 \\
\text { BY LEVEL OF HIGHEST ATTAINMENT: STUDENTS LEAVING SOUTHLAND } \\
\text { SECONDARY SCHOOLS DURING } 2005 \text { BY LEVEL OF HIGHEST } \\
\text { ATTAINMENT }\end{array}$ & QUALIFICATION/SCHOOL & SOCIAL WELL-BEING \\
\hline 749 & GORE DISTRICT COUNCIL & $\begin{array}{l}\text { A WELLEDUCATED AND SKILLED } \\
\text { COMMUNITY CONTINUALLY SEEKING } \\
\text { FURTHER OPPORTUNITIES TO LEARN }\end{array}$ & $\begin{array}{l}\text { STUDENTS LEAVING SOUTHLAND SECONDARY SCHOOLS DURING } 2005 \\
\text { BY LEVEL OF HIGHEST ATTAINMENT: STUDENTS LEAVING SOUTHLAND } \\
\text { SECONDARY SCHOOLS DURING BY } 2006 \text { LEVEL OF HIGHEST } \\
\text { ATTAINMENT }\end{array}$ & QUALIFICATION/SCHOOL & SOCIAL WELL-BEING \\
\hline 750 & GORE DISTRICT COUNCIL & $\begin{array}{l}\text { A WELLEDUCATED AND SKILLED } \\
\text { COMMUNITY CONTINUALLY SEEKING } \\
\text { FURTHER OPPORTUNITIES TO LEARN }\end{array}$ & $\begin{array}{l}\text { HIGHEST QUALIFICATION FOR PEOPLE AGED } 15 \text { YEARS AND OVER, } \\
\text { SOUTHLAND REGION AND NEW ZEALAND, } 2006\end{array}$ & QUALIFICATION/SCHOOL & SOCIAL WELL-BEING \\
\hline 751 & GORE DISTRICT COUNCIL & $\begin{array}{l}\text { A WELLEDUCATED AND SKILLED } \\
\text { COMMUNITY CONTINUALLY SEEKING } \\
\text { FURTHER OPPORTUNITIES TO LEARN }\end{array}$ & NUMBER OF PEOPLE PARTICIPATING IN CONTINUING EDUCATION & QUALIFICATION/SCHOOL & SOCIAL WELL-BEING \\
\hline 752 & GREY DISTRICT COUNCIL & ENVIRONMENT & $\begin{array}{l}\text { SITES WHERE DEVELOPMENT IS MANAGED TO RETAIN NATURAL } \\
\text { CHARACTER }\end{array}$ & NATURAL FEATURES & ENVIRONMENTAL WELL-BEING \\
\hline 753 & GREY DISTRICT COUNCIL & ENVIRONMENT & VISITOR LEVEL OF APPRECIATION & TOURISM & ECONOMIC WELL-BEING \\
\hline 754 & GREY DISTRICT COUNCIL & ENVIRONMENT & ACCESS TO NATURAL FEATURES & NATURAL FEATURES & ENVIRONMENTAL WELL-BEING \\
\hline 755 & GREY DISTRICT COUNCIL & ENVIRONMENT & SURFACE WATER QUALITY MONITORING & $\begin{array}{l}\text { RIVER, LAKES, COASTAL AND } \\
\text { GROUNDWATER }\end{array}$ & ENVIRONMENTAL WELL-BEING \\
\hline 758 & GREY DISTRICT COUNCIL & ECONOMY & GST TURNOVERGDP & GROSS DOMESTIC PRODUCT (GDP) & ECONOMIC WELL-BEING \\
\hline 759 & GREY DISTRICT COUNCIL & ECONOMY & NUMBER OF BUSINESSES & NUMBER OF BUSINESSES & ECONOMIC WELL-BEING \\
\hline 760 & GREY DISTRICT COUNCIL & ECONOMY & EMPLOYMENT & $\begin{array}{l}\text { LEVEL OF EMPLOYMENT AND } \\
\text { BUSINESSES }\end{array}$ & ECONOMIC WELL-BEING \\
\hline
\end{tabular}


Appendix A: List of Community Well-beings Outcomes and Measures by Councils

\begin{tabular}{|c|c|c|c|c|c|}
\hline S.NO. & COUNCIL & OUTCOME & MEASURES & MEASURES BROAD GROUP & WELL-BEINGS \\
\hline 761 & GREY DISTRICT COUNCIL & HEALTH & LIFE EXPECTANCY & LIFE EXPECTANCY & DEMOGRAPHICS \\
\hline 762 & GREY DISTRICT COUNCIL & HEALTH & ACCESS TO HEALTH CARE OUTSIDE REFERRA & HEALTH/HEALTH SERVICES & SOCIAL WELL-BEING \\
\hline 763 & GREY DISTRICT COUNCIL & HEALTH & RATIO OF HEALTH PROVIDERS PER POPULATION & HEALTH/HEALTH SERVICES & SOCIAL WELL-BEING \\
\hline 764 & GREY DISTRICT COUNCIL & HEALTH & POTABLE WATER SUPPLYQUALITY & $\begin{array}{l}\text { RIVER, LAKES, COASTAL AND } \\
\text { GROUNDWATER }\end{array}$ & ENVIRONMENTAL WELL-BEING \\
\hline 765 & GREY DISTRICT COUNCIL & HEALTH & AIR QUALITY & AIR QUALITY & ENVIRONMENTAL WELL-BEING \\
\hline 766 & GREY DISTRICT COUNCIL & EDUCATION & LEVEL OF QUALIFICATION & QUALIFICATION/SCHOOL & SOCIAL WELL-BEING \\
\hline 767 & GREY DISTRICT COUNCIL & EDUCATION & NUMBER OF EDUCATIONAL INSTITUTIONS & QUALIFICATION/SCHOOL & SOCIAL WELL-BEING \\
\hline 769 & GREY DISTRICT COUNCIL & EDUCATION & APPRENTICESHIPS & APPRENTICESHIPS FOR YOUNG PEOPLE & ECONOMIC WELL-BEING \\
\hline 770 & GREY DISTRICT COUNCIL & SAFETY & OVERALL CRIME RATE (COMPARE AGST NATIONAL AVERAGE) & CRIME/SAFETY & SOCIAL WELL-BEING \\
\hline 771 & GREY DISTRICT COUNCIL & SAFETY & YOUTH OFFENDING & YOUTH & SOCIAL WELL-BEING \\
\hline 772 & GREY DISTRICT COUNCIL & SAFETY & $\begin{array}{l}\text { NUMBER OF MOTOR VEHICLE CRASHES (COMPARE AGST NAT } \\
\text { AVERAGE) }\end{array}$ & ROAD ACCIDENTS/INJURIES & ECONOMIC WELL-BEING \\
\hline 773 & GREY DISTRICT COUNCIL & SAFETY & IMPROVEMENTS IN PREPAREDNESS FOR NATURAL HAZARD EVENTS & NATURAL DISASTER/RESILIENCE & ENVIRONMENTAL WELL-BEING \\
\hline 774 & GREY DISTRICT COUNCIL & SAFETY & $\begin{array}{l}\text { PROGRESS TOWARDS IDENTIFYING HAZARD AREAS WHERE GREATER } \\
\text { MANAGEMENT IS REQUIRED }\end{array}$ & NATURAL DISASTER/RESILIENCE & ENVIRONMENTAL WELL-BEING \\
\hline 777 & GREY DISTRICT COUNCIL & IDENTITY & $\begin{array}{l}\text { USE OF COMMUNITY FACILITIES IE MUSEUMS THEATRES SWIMMING } \\
\text { POOLS }\end{array}$ & ARTS/CULTURE/MUSEUM/EVENTS & CULTURAL WELL-BEING \\
\hline 778 & HAMILTON CITY COUNCIL & SUSTAINABLE AND WELL-PLANNED & TRAVEL TIMES ON KEY ROUTES & TRANSPORT & ECONOMIC WELL-BEING \\
\hline 779 & HAMILTON CITY COUNCIL & SUSTAINABLE AND WELL-PLANNED & ROAD TRAFFIC VOLUMES & TRANSPORT & ECONOMIC WELL-BEING \\
\hline 780 & HAMILTON CITY COUNCIL & SUSTAINABLE AND WELL-PLANNED & MEANS OF TRAVEL TO WORK & TRANSPORT & ECONOMIC WELL-BEING \\
\hline 781 & HAMILTON CITY COUNCIL & SUSTAINABLE AND WELL-PLANNED & CAR OWNERSHIP PER HOUSEHOLD & $\begin{array}{l}\text { POPULATION/ETHNICITY/DEMOGRAPHI } \\
\text { CS }\end{array}$ & DEMOGRAPHICS \\
\hline 782 & HAMILTON CITY COUNCIL & SUSTAINABLE AND WELL-PLANNED & TOTAL PASSENGER NUMBERS USING PUBLIC TRANSPORT & TRANSPORT & ECONOMIC WELL-BEING \\
\hline 783 & HAMILTON CITY COUNCIL & SUSTAINABLE AND WELL-PLANNED & RESIDENTS RATING OF PUBLIC TRANSPORT IN THEIR CITY & TRANSPORT & ECONOMIC WELL-BEING \\
\hline 784 & HAMILTON CITY COUNCIL & SUSTAINABLE AND WELL-PLANNED & $\begin{array}{l}\text { RESIDENTS SATISFACTION WITH COUNCIL PROVIDED CYCLING } \\
\text { FACILITIES }\end{array}$ & FOOTPATH/WALKING/CYCLING & ECONOMIC WELL-BEING \\
\hline 785 & HAMILTON CITY COUNCIL & SUSTAINABLE AND WELL-PLANNED & $\begin{array}{l}\text { RESIDENTS' SATISFACTION WITH COUNCIL PROVIDED } \\
\text { WALKWAYSFOOTPATHS }\end{array}$ & FOOTPATH/WALKING/CYCLING & ECONOMIC WELL-BEING \\
\hline 786 & HAMILTON CITY COUNCIL & SUSTAINABLE AND WELL-PLANNED & $\begin{array}{l}\text { ROAD CRASHES AND CASUALTIES INVOLVING PEDESTRIANS AND } \\
\text { CYCLISTS }\end{array}$ & FOOTPATH/WALKING/CYCLING & ECONOMIC WELL-BEING \\
\hline 787 & HAMILTON CITY COUNCIL & SUSTAINABLE AND WELL-PLANNED & NUMBER OF SCHOOLS AND CHILDREN USING WALKING SCHOOL BUSES & CHILD SAFETY \& DEVELOPMENT & SOCIAL WELL-BEING \\
\hline 788 & HAMILTON CITY COUNCIL & SUSTAINABLE AND WELL-PLANNED & $\begin{array}{l}\text { AIR POLLUTION (INCLUDING PARTICULATE MATTER (PM10) LEVELS, } \\
\text { BENZENE, LEVELS RESIDENTS' PERCEPTION OF AIR POLLUTION) }\end{array}$ & AIR QUALITY & ENVIRONMENTAL WELL-BEING \\
\hline 791 & HAMILTON CITY COUNCIL & SUSTAINABLE AND WELL-PLANNED & BIRD COUNTS & $\begin{array}{l}\text { ENVIRONMENT/ENVIRONMENTAL } \\
\text { ATTITUDES }\end{array}$ & ENVIRONMENTAL WELL-BEING \\
\hline 792 & HAMILTON CITY COUNCIL & SUSTAINABLE AND WELL-PLANNED & AREA OF GREEN SPACE IN THE CITY PER RESIDENT & PARKS/RESERVES/OPEN SPACES & ENVIRONMENTAL WELL-BEING \\
\hline 793 & HAMILTON CITY COUNCIL & SUSTAINABLE AND WELLPLANNED & RATIO OF GREENFIELD TO INFILL DEVELOPMENT & PARKS/RESERVES/OPEN SPACES & ENVIRONMENTAL WELL-BEING \\
\hline
\end{tabular}




\begin{tabular}{|c|c|c|c|c|c|}
\hline S.NO. & COUNCIL & OUTCOME & MEASURES & MEASURES BROAD GROUP & WELL-BEINGS \\
\hline 794 & HAMILTON CITY COUNCIL & SUSTAINABLE AND WELL-PLANNED & $\begin{array}{l}\text { PERCENTAGE OF RESOURCE CONSENT APPLICATIONS THAT RECEIVED } \\
\text { PRE-APPLICATION URBAN DESIGN COMMENTS AND PERCENTAGE OF } \\
\text { THOSE COMMENTS ACTIONED }\end{array}$ & PLANNING/BUILDING CONSENTS & ECONOMIC WELL-BEING \\
\hline 795 & HAMILTON CITY COUNCIL & SUSTAINABLE AND WELL-PLANNED & ECOLOGICAL HEALTH OF THE WAIKATO RIVER & $\begin{array}{l}\text { ENVIRONMENT/ENVIRONMENTAL } \\
\text { ATTITUDES }\end{array}$ & ENVIRONMENTAL WELL-BEING \\
\hline 796 & HAMILTON CITY COUNCIL & SUSTAINABLE AND WELL-PLANNED & ECOLOGICAL HEALTH OF LAKE ROTOROA (HAMILTON LAKE) & $\begin{array}{l}\text { ENVIRONMENT/ENVIRONMENTAL } \\
\text { ATTITUDES }\end{array}$ & ENVIRONMENTAL WELL-BEING \\
\hline 797 & HAMILTON CITY COUNCIL & SUSTAINABLE AND WELL-PLANNED & AVERAGE DAILY WATER USAGE PER PERSON & $\begin{array}{l}\text { RIVER, LAKES, COASTAL AND } \\
\text { GROUNDWATER }\end{array}$ & ENVIRONMENTAL WELL-BEING \\
\hline 798 & HAMILTON CITY COUNCIL & SUSTAINABLE AND WELL-PLANNED & HAMILTONS RESIDENTIAL ENERGY CONSUMPTION & ENERGY USAGE & ENVIRONMENTAL WELL-BEING \\
\hline 799 & HAMILTON CITY COUNCIL & SUSTAINABLE AND WELL-PLANNED & WASTE PER PERSON FROM THE HOUSEHOLD KERBSIDE COLLECTION & WASTE TO LANDFILL & ENVIRONMENTAL WELL-BEING \\
\hline 800 & HAMILTON CITY COUNCIL & SUSTAINABLE AND WELL-PLANNED & $\begin{array}{l}\text { RECYCLABLES PER PERSON FROM THE HOUSEHOLD KERBSIDE } \\
\text { COLLECTION }\end{array}$ & RECYCLING/GREEN WASTE & ENVIRONMENTAL WELL-BEING \\
\hline 801 & HAMILTON CITY COUNCIL & SUSTAINABLE AND WELL-PLANNED & $\begin{array}{l}\text { MATERIALS COLLECTED FOR REUSERECYCLING AT HAMILTON'S REFUSE } \\
\text { TRANSFER STATION }\end{array}$ & RECYCLING/GREEN WASTE & ENVIRONMENTAL WELL-BEING \\
\hline 802 & HAMILTON CITY COUNCIL & SUSTAINABLE AND WELL-PLANNED & $\begin{array}{l}\text { WASTE DIVERTED FROM LANDFILL VIA THE HAMILTON ORGANIC } \\
\text { CENTRE }\end{array}$ & RECYCLING/GREEN WASTE & ENVIRONMENTAL WELL-BEING \\
\hline 803 & HAMILTON CITY COUNCIL & VIBRANT AND CREATIVE & VISITOR GUEST NIGHTS AND HOTELMOTEL OCCUPANCY RATES & TOURISM & ECONOMIC WELL-BEING \\
\hline 804 & HAMILTON CITY COUNCIL & VIBRANT AND CREATIVE & NUMBER OF CONFERENCES HELD & ARTS/CULTURE/MUSEUM/EVENTS & CULTURAL WELL-BEING \\
\hline 806 & HAMILTON CITY COUNCIL & VIBRANT AND CREATIVE & RESIDENTS USE OF COUNCILS ARTS AND CULTURE FACILITIES & ARTS/CULTURE/MUSEUM/EVENTS & CULTURAL WELL-BEING \\
\hline 807 & HAMILTON CITY COUNCIL & VIBRANT AND CREATIVE & $\begin{array}{l}\text { RESIDENTS PERCEPTION THAT THEIR CITY IS A PLACE THAT HAS A } \\
\text { CULTURALLY RICH AND DIVERSE ARTS SCENE }\end{array}$ & ARTS/CULTURE/MUSEUM/EVENTS & CULTURAL WELL-BEING \\
\hline 808 & HAMILTON CITY COUNCIL & VIBRANT AND CREATIVE & CULTURAL INDUSTRIES & $\begin{array}{l}\text { LEVEL OF EMPLOYMENT AND } \\
\text { BUSINESSES }\end{array}$ & ECONOMIC WELL-BEING \\
\hline 809 & HAMILTON CITY COUNCIL & VIBRANT AND CREATIVE & $\begin{array}{l}\text { RESIDENTS PERCEPTIONS OF THE EFFECT OF INCREASED DIVERSITY IN } \\
\text { LIFESTYLES AND CULTURES ON THEIR CITY }\end{array}$ & ARTS/CULTURE/MUSEUM/EVENTS & CULTURAL WELL-BEING \\
\hline 810 & HAMILTON CITY COUNCIL & VIBRANT AND CREATIVE & $\begin{array}{l}\text { NUMBER OF BUILT HERITAGE AND SITES OF ARCHAEOLOGICAL, } \\
\text { HISTORIC AND CULTURAL SIGNIFICANCE IN THE CURRENT DISTRICT } \\
\text { PLAN }\end{array}$ & HERITAGE/HISTORIC & CULTURAL WELL-BEING \\
\hline 811 & HAMILTON CITY COUNCIL & VIBRANT AND CREATIVE & $\begin{array}{l}\text { RESIDENTS RATING OF FEELING SAFE IN HAMILTON'S CENTRAL CITY AT } \\
\text { NIGHT }\end{array}$ & CRIME/SAFETY & SOCIAL WELL-BEING \\
\hline 812 & HAMILTON CITY COUNCIL & VIBRANT AND CREATIVE & NUMBER OF PEOPLE LIVING IN THE CENTRAL CITY AREA & CBD: CITY CENTRE & SOCIAL WELL-BEING \\
\hline 813 & HAMILTON CITY COUNCIL & VIBRANT AND CREATIVE & $\begin{array}{l}\text { VALUE OF BUILDING CONSENTS ISSUED FOR THE CBD (NEW, } \\
\text { ALTERATIONS, ADDITIONS) }\end{array}$ & CBD: CITY CENTRE & SOCIAL WELL-BEING \\
\hline 814 & HAMILTON CITY COUNCIL & UNIQUE IDENTITY & RECREATIONAL HEALTH OF THE WAIKATO RIVER & $\begin{array}{l}\text { ENVIRONMENT/ENVIRONMENTAL } \\
\text { ATTITUDES }\end{array}$ & ENVIRONMENTAL WELL-BEING \\
\hline 815 & HAMILTON CITY COUNCIL & UNIQUE IDENTITY & MAAORI INVOLVEMENT IN PUBLIC DECISION-MAKING & DECISION MAKING/CONSULTATION & SOCIAL WELL-BEING \\
\hline 816 & HAMILTON CITY COUNCIL & UNIQUE IDENTITY & PERCENTAGE OF PEOPLE IN HAMILTON WHO CAN SPEAK MAAORI & $\begin{array}{l}\text { POPULATION/ETHNICITY/DEMOGRAPHI } \\
\text { CS }\end{array}$ & DEMOGRAPHICS \\
\hline 818 & HAMILTON CITY COUNCIL & UNIQUE IDENTITY & $\begin{array}{l}\text { NUMBER OF STREET TREES PLANTED AND NUMBER OF TREES } \\
\text { PROTECTED }\end{array}$ & $\begin{array}{l}\text { ENVIRONMENT/ENVIRONMENTAL } \\
\text { ATTITUDES }\end{array}$ & ENVIRONMENTAL WELL-BEING \\
\hline 819 & HAMILTON CITY COUNCIL & UNIQUE IDENTITY & PARTICIPATION IN NATIVE TREE RESTORATION PROJECTS & SOCIAL CONNECTION & SOCIAL WELL-BEING \\
\hline
\end{tabular}


Appendix A: List of Community Well-beings Outcomes and Measures by Councils

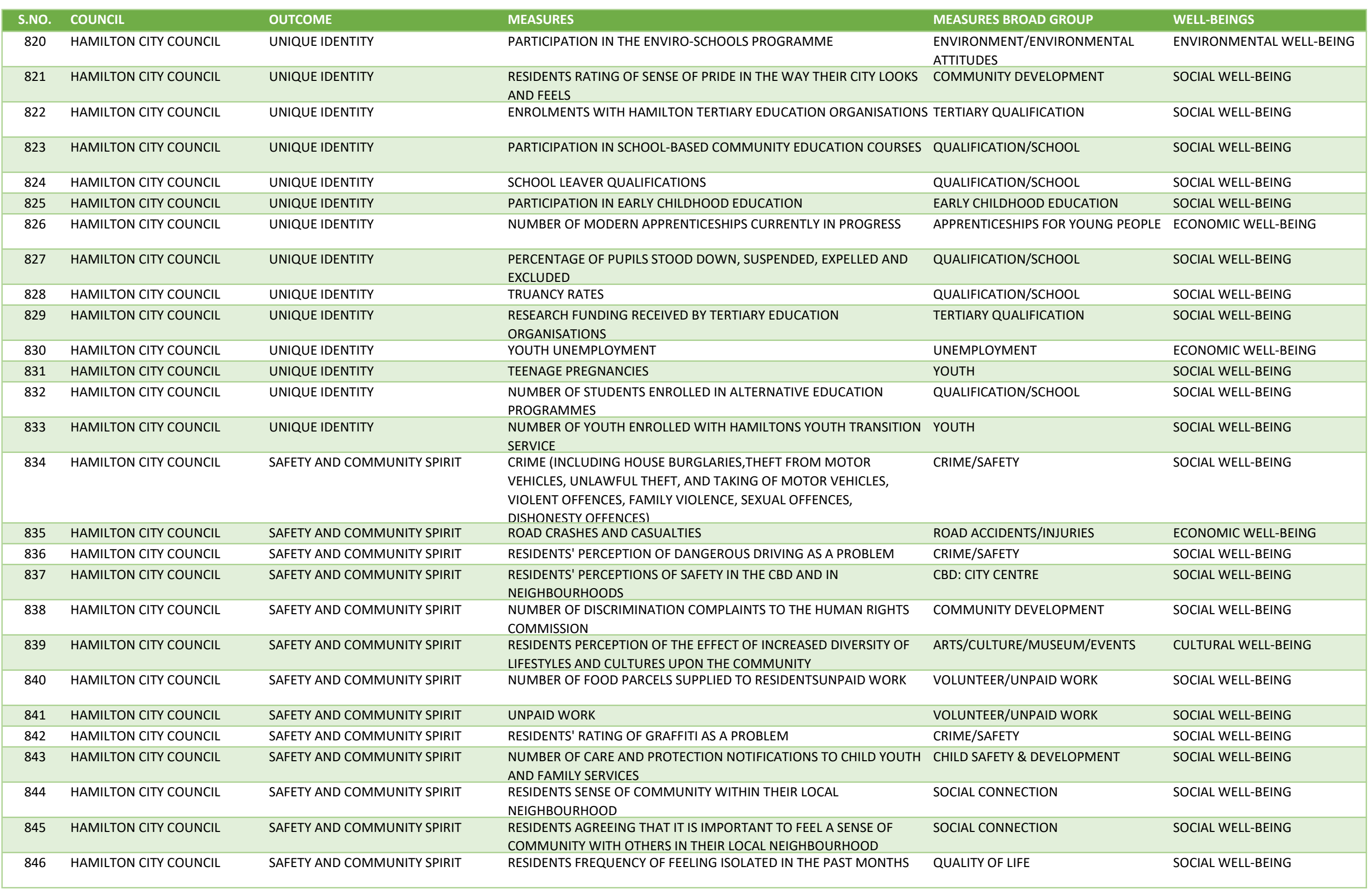


Appendix A: List of Community Well-beings Outcomes and Measures by Councils

\begin{tabular}{|c|c|c|c|c|c|}
\hline S.NO. & COUNCIL & OUTCOME & MEASURES & MEASURES BROAD GROUP & WELL-BEINGS \\
\hline 847 & HAMILTON CITY COUNCIL & SAFETY AND COMMUNITY SPIRIT & RESIDENTS INVOLVEMENT IN SOCIAL NETWORKS AND GROUPS & SOCIAL CONNECTION & SOCIAL WELL-BEING \\
\hline 848 & HAMILTON CITY COUNCIL & HEALTHY AND HAPPY & DRINKING WATER STANDARD & DRINKING WATER & ENVIRONMENTAL WELL-BEING \\
\hline 849 & HAMILTON CITY COUNCIL & HEALTHY AND HAPPY & LIFE EXPECTANCY & LIFE EXPECTANCY & DEMOGRAPHICS \\
\hline 851 & HAMILTON CITY COUNCIL & HEALTHY AND HAPPY & PREVALENCE OF CIGARETTE SMOKING & HEALTH/HEALTH SERVICES & SOCIAL WELL-BEING \\
\hline 852 & HAMILTON CITY COUNCIL & HEALTHY AND HAPPY & LOW BIRTH WEIGHT BABIES & CHILD SAFETY \& DEVELOPMENT & SOCIAL WELL-BEING \\
\hline 853 & HAMILTON CITY COUNCIL & HEALTHY AND HAPPY & HOSPITALISATIONS FOR ATTEMPTED SUICIDEDEATH BY SUICIDE & SUICIDE/MENTAL HEALTH & SOCIAL WELL-BEING \\
\hline 854 & HAMILTON CITY COUNCIL & HEALTHY AND HAPPY & RESIDENTS RATING OF HOW HAPPY THEY ARE & COMMUNITY DEVELOPMENT & SOCIAL WELL-BEING \\
\hline 856 & HAMILTON CITY COUNCIL & HEALTHY AND HAPPY & RESIDENTS FREQUENCY OF PHYSICAL ACTIVITY & PHYSICAL ACTIVITY & SOCIAL WELL-BEING \\
\hline 857 & HAMILTON CITY COUNCIL & HEALTHY AND HAPPY & RESIDENTS USE OF COUNCILS SPORTS AND RECREATIONAL FACILITIES & SPORTS & SOCIAL WELL-BEING \\
\hline 858 & HAMILTON CITY COUNCIL & HEALTHY AND HAPPY & $\begin{array}{l}\text { RESIDENTS SATISFACTION WITH QUALITY AND QUANTITY OF LEISURE } \\
\text { TIME }\end{array}$ & QUALITY OF LIFE & SOCIAL WELL-BEING \\
\hline 859 & HAMILTON CITY COUNCIL & HEALTHY AND HAPPY & NUMBER OF DOCTORS (GP'S) PER HEAD OF THE POPULATION & HEALTH/HEALTH SERVICES & SOCIAL WELL-BEING \\
\hline 860 & HAMILTON CITY COUNCIL & HEALTHY AND HAPPY & RESIDENTS RATING OF ACCESS TO MEDICAL SERVICES & HEALTH/HEALTH SERVICES & SOCIAL WELL-BEING \\
\hline 861 & HAMILTON CITY COUNCIL & HEALTHY AND HAPPY & NUMBER OF CONSENTS ISSUED FOR NEW HOUSES & PLANNING/BUILDING CONSENTS & ECONOMIC WELL-BEING \\
\hline 862 & HAMILTON CITY COUNCIL & HEALTHY AND HAPPY & PROPORTION OF HOUSES OWNED OR RENTED BY THE OCCUPANTS & $\begin{array}{l}\text { POPULATION/ETHNICITY/DEMOGRAPHI } \\
\text { CS }\end{array}$ & DEMOGRAPHICS \\
\hline 863 & HAMILTON CITY COUNCIL & HEALTHY AND HAPPY & HOUSE SALE PRICE INDEX & HOUSE AFFORDABILITY & ECONOMIC WELL-BEING \\
\hline 864 & HAMILTON CITY COUNCIL & HEALTHY AND HAPPY & HOME MORTGAGE AFFORDABILITY (WAIKATO REGION) & HOUSE AFFORDABILITY & ECONOMIC WELL-BEING \\
\hline 865 & HAMILTON CITY COUNCIL & HEALTHY AND HAPPY & MEDIAN RENT & HOUSE AFFORDABILITY & ECONOMIC WELL-BEING \\
\hline 866 & HAMILTON CITY COUNCIL & HEALTHY AND HAPPY & $\begin{array}{l}\text { PERCENTAGE OF WEEKLY HOUSEHOLD EXPENDITURE SPENT ON } \\
\text { HOUSING COSTS }\end{array}$ & HOUSE AFFORDABILITY & ECONOMIC WELL-BEING \\
\hline 867 & HAMILTON CITY COUNCIL & HEALTHY AND HAPPY & $\begin{array}{l}\text { NUMBER AND PRIORITY STATUS OF APPLICANTS ON THE WAITING LIST } \\
\text { OF HOUSING NEW ZEALAND HOUSING }\end{array}$ & HOUSE AFFORDABILITY & ECONOMIC WELL-BEING \\
\hline 868 & HAMILTON CITY COUNCIL & HEALTHY AND HAPPY & $\begin{array}{l}\text { PERCENTAGE OF PEOPLE IN PRIVATE HOUSES LIVING IN CROWDED } \\
\text { HOUSEHOLDS }\end{array}$ & HOUSE AFFORDABILITY & ECONOMIC WELL-BEING \\
\hline 869 & HAMILTON CITY COUNCIL & HEALTHY AND HAPPY & RESIDENTS PERCEPTION THAT THEIR INCOME MEETS EVERYDAY NEEDS & INCOME & ECONOMIC WELL-BEING \\
\hline 870 & HAMILTON CITY COUNCIL & INTELLIGENT AND PROGRESSIVE CITY & NUMBER OF BUSINESSES AND TOTAL EMPLOYMENT & $\begin{array}{l}\text { LEVEL OF EMPLOYMENT AND } \\
\text { BUSINESSES }\end{array}$ & ECONOMIC WELL-BEING \\
\hline 871 & HAMILTON CITY COUNCIL & INTELLIGENT AND PROGRESSIVE CITY & NUMBER OF BUSINESSES OF DIFFERENT SIZES & NUMBER OF BUSINESSES & ECONOMIC WELL-BEING \\
\hline 872 & HAMILTON CITY COUNCIL & INTELLIGENT AND PROGRESSIVE CITY & $\begin{array}{l}\text { RETAIL SALES IN HAMILTON AS A PERCENTAGE OF RETAIL SALES IN THE } \\
\text { WAIKATO REGION }\end{array}$ & CBD: CITY CENTRE & SOCIAL WELL-BEING \\
\hline 873 & HAMILTON CITY COUNCIL & INTELLIGENT AND PROGRESSIVE CITY & $\begin{array}{l}\text { PROPORTION OF EMPLOYEES IN HAMILTON TO THAT OF THE WAIKATO } \\
\text { REGION }\end{array}$ & $\begin{array}{l}\text { LEVEL OF EMPLOYMENT AND } \\
\text { BUSINESSES }\end{array}$ & ECONOMIC WELL-BEING \\
\hline 874 & HAMILTON CITY COUNCIL & INTELLIGENT AND PROGRESSIVE CITY & BUSINESS GAINS, LOSSES AND SURVIVAL RATES & NUMBER OF BUSINESSES & ECONOMIC WELL-BEING \\
\hline 875 & HAMILTON CITY COUNCIL & INTELLIGENT AND PROGRESSIVE CITY & RESEARCH AND DEVELOPMENT UNDERTAKEN BY BUSINESSES & BUSINESS CONFIDENCE & ECONOMIC WELL-BEING \\
\hline 878 & HAMILTON CITY COUNCIL & INTELLIGENT AND PROGRESSIVE CITY & NUMBER OF FILLED JOBS & $\begin{array}{l}\text { LEVEL OF EMPLOYMENT AND } \\
\text { BUSINESSES }\end{array}$ & ECONOMIC WELL-BEING \\
\hline 879 & HAMILTON CITY COUNCIL & INTELLIGENT AND PROGRESSIVE CITY & AVERAGE HOURLY EARNINGS & INCOME & ECONOMIC WELL-BEING \\
\hline
\end{tabular}


Appendix A: List of Community Well-beings Outcomes and Measures by Councils

\begin{tabular}{|c|c|c|c|c|c|}
\hline S.NO. & COUNCIL & OUTCOME & MEASURES & MEASURES BROAD GROUP & WELL-BEINGS \\
\hline 880 & HAMILTON CITY COUNCIL & INTELLIGENT AND PROGRESSIVE CITY & DISTRIBUTION OF INCOME & INCOME & ECONOMIC WELL-BEING \\
\hline 881 & HAMILTON CITY COUNCIL & INTELLIGENT AND PROGRESSIVE CITY & $\begin{array}{l}\text { RESIDENTS RATING OF HOW WELL THEIR SKILLS ARE UTILISED IN THEIR } \\
\text { CURRENT JOB }\end{array}$ & HOUSE AFFORDABILITY & ECONOMIC WELL-BEING \\
\hline 882 & HAMILTON CITY COUNCIL & INTELLIGENT AND PROGRESSIVE CITY & VALUE OF CONSENTS FOR NEW NON-RESIDENTIAL BUILDINGS & PLANNING/BUILDING CONSENTS & ECONOMIC WELL-BEING \\
\hline 883 & HAMILTON CITY COUNCIL & INTELLIGENT AND PROGRESSIVE CITY & $\begin{array}{l}\text { TELECOMMUNICATIONS INFRASTRUCTURE (AWAITING THE } \\
\text { AVAILABILITY OF DATA FOR THIS INDICATOR) }\end{array}$ & PHONE/MOBILE/INTERNET & ECONOMIC WELL-BEING \\
\hline 884 & HAMILTON CITY COUNCIL & INTELLIGENT AND PROGRESSIVE CITY & $\begin{array}{l}\text { NUMBER OF EMPLOYEES BY KEY ECONOMIC DEVELOPMENT STRATEGY } \\
\text { CLUSTERS }\end{array}$ & $\begin{array}{l}\text { LEVEL OF EMPLOYMENT AND } \\
\text { BUSINESSES }\end{array}$ & ECONOMIC WELL-BEING \\
\hline 885 & HAMILTON CITY COUNCIL & INTELLIGENT AND PROGRESSIVE CITY & $\begin{array}{l}\text { PERSONAL BANKRUPTCIES AND INVOLUNTARY COMPANY } \\
\text { LIQUIDATIONS AND RECEIVERSHIPS }\end{array}$ & INCOME & ECONOMIC WELL-BEING \\
\hline 886 & HAMILTON CITY COUNCIL & WORKING TOGETHER & $\begin{array}{l}\text { COMMUNITY SATISFACTION WITH COUNCIL'S PROVISION OF } \\
\text { OPPORTUNITIES FOR COMMUNITY INVOLVEMENT IN DECISION- } \\
\text { MAKING }\end{array}$ & DECISION MAKING/CONSULTATION & SOCIAL WELL-BEING \\
\hline 887 & HAMILTON CITY COUNCIL & WORKING TOGETHER & $\begin{array}{l}\text { RESIDENTS' RATING OF THE EXTENT OF PUBLIC INFLUENCE ON } \\
\text { COUNCIL DECISION-MAKING }\end{array}$ & DECISION MAKING/CONSULTATION & SOCIAL WELL-BEING \\
\hline 888 & HAMILTON CITY COUNCIL & WORKING TOGETHER & $\begin{array}{l}\text { RESIDENTS RATING OF AGREEMENT THAT DECISIONS MADE BY } \\
\text { COUNCIL ARE IN THE BEST INTERESTS OF THE CITY }\end{array}$ & DECISION MAKING/CONSULTATION & SOCIAL WELL-BEING \\
\hline 889 & HAMILTON CITY COUNCIL & WORKING TOGETHER & $\begin{array}{l}\text { PERCENTAGE OF VOTER TURNOUT AT GENERALLOCAL AUTHORITY } \\
\text { ELECTIONS }\end{array}$ & ELECTIONS/VOTER TURNOUT & SOCIAL WELL-BEING \\
\hline 890 & HAMILTON CITY COUNCIL & WORKING TOGETHER & $\begin{array}{l}\text { MAAORI RESIDENTS' SATISFACTION WITH COUNCIL'S PROVISION OF } \\
\text { OPPORTUNITIES FOR COMMUNITY INVOLVEMENT IN DECISION- } \\
\text { MAKING }\end{array}$ & DECISION MAKING/CC & LL-BEING \\
\hline 891 & HAMILTON CITY COUNCIL & WORKING TOGETHER & $\begin{array}{l}\text { MAAORI RESIDENTS' RATING OF THE EXTENT OF PUBLIC INFLUENCE ON } \\
\text { COUNCIL DECISION-MAKING }\end{array}$ & DECISION MAKING/CONSULTATION & SOCIAL WELL-BEING \\
\hline 892 & HAMILTON CITY COUNCIL & WORKING TOGETHER & $\begin{array}{l}\text { MAAORI RESIDENTS RATING OF AGREEMENT THAT DECISIONS MADE BY } \\
\text { COUNCIL ARE IN THE BEST INTERESTS OF THE CITY }\end{array}$ & DECISION MAKING/CONSULTATION & SOCIAL WELL-BEING \\
\hline 893 & HAMILTON CITY COUNCIL & WORKING TOGETHER & $\begin{array}{l}\text { NUMBER OF ORGANISATIONS WORKING COLLABORATIVELY ON } \\
\text { COUNCIL-LED CITY WIDE STRATEGIES }\end{array}$ & DECISION MAKING/CONSULTATION & SOCIAL WELL-BEING \\
\hline 894 & HAMILTON CITY COUNCIL & WORKING TOGETHER & $\begin{array}{l}\text { WRITTEN DESCRIPTION OF FORMAL RELATIONSHIPS BETWEEN MAAORI } \\
\text { ORGANISATIONS AND LOCALENTRAL GOVERNMENT IN THE CITY }\end{array}$ & DECISION MAKING/CONSULTATION & SOCIAL WELL-BEING \\
\hline 895 & HASTINGS DISTRICT COUNCIL & RESOURCES WE NEED & PROTECTION OF THE PRODUCTIVE CAPACITY OF LAND RESOURCES & LAND UTILISATION & ECONOMIC WELL-BEING \\
\hline 896 & HASTINGS DISTRICT COUNCIL & RESOURCES WE NEED & $\begin{array}{l}\text { SUSTAINABLE MANAGEMENT OF NATURAL AND PHYSICAL RESOURCES } \\
\text { THROUGH INTEGRATED LAND USE MANAGEMENT }\end{array}$ & LAND UTILISATION & ENVIRONMENTAL WELL-BEING \\
\hline 897 & HASTINGS DISTRICT COUNCIL & RESOURCES WE NEED & MAINTAIN HEALTHY SOIL & SOIL QUALITY & ENVIRONMENTAL WELL-BEING \\
\hline 898 & HASTINGS DISTRICT COUNCIL & RESOURCES WE NEED & HEALTHY DRINKING WATER & DRINKING WATER & ENVIRONMENTAL WELL-BEING \\
\hline 899 & HASTINGS DISTRICT COUNCIL & RESOURCES WE NEED & BEST USE OF WATER RESOURCES & WATER SUPPLY & ENVIRONMENTAL WELL-BEING \\
\hline 900 & HASTINGS DISTRICT COUNCIL & RESOURCES WE NEED & IMPROVEMENT IN AIR QUALITY & AIR QUALITY & ENVIRONMENTAL WELL-BEING \\
\hline 901 & HASTINGS DISTRICT COUNCIL & RESOURCES WE NEED & MITIGATION OF ADVERSE IMPACTS ON PEOPLE, LAND AND WATER & LAND UTILISATION & ENVIRONMENTAL WELL-BEING \\
\hline 902 & HASTINGS DISTRICT COUNCIL & RESOURCES WE NEED & HARNESS OUR SOLAR ENERGY POTENTIAL & ENERGY USAGE & ENVIRONMENTAL WELL-BEING \\
\hline 905 & HASTINGS DISTRICT COUNCIL & ZERO WASTE & FOSTER WASTE MINIMISATION PARTNERSHIPS & RECYCLING/GREEN WASTE & ENVIRONMENTAL WELL-BEING \\
\hline 906 & HASTINGS DISTRICT COUNCIL & OUR ECONOMY & PARTNER WITH MAORI FOR ECONOMIC DEVELOPMENT & BUSINESS CONFIDENCE & ECONOMIC WELL-BEING \\
\hline 907 & HASTINGS DISTRICT COUNCIL & OUR ECONOMY & GROWING NEW BUSINESS IN RESEARCH AND DEVELOPMENT & $\begin{array}{l}\text { LEVEL OF EMPLOYMENT AND } \\
\text { BUSINESSES }\end{array}$ & ECONOMIC WELL-BEING \\
\hline
\end{tabular}


Appendix A: List of Community Well-beings Outcomes and Measures by Councils

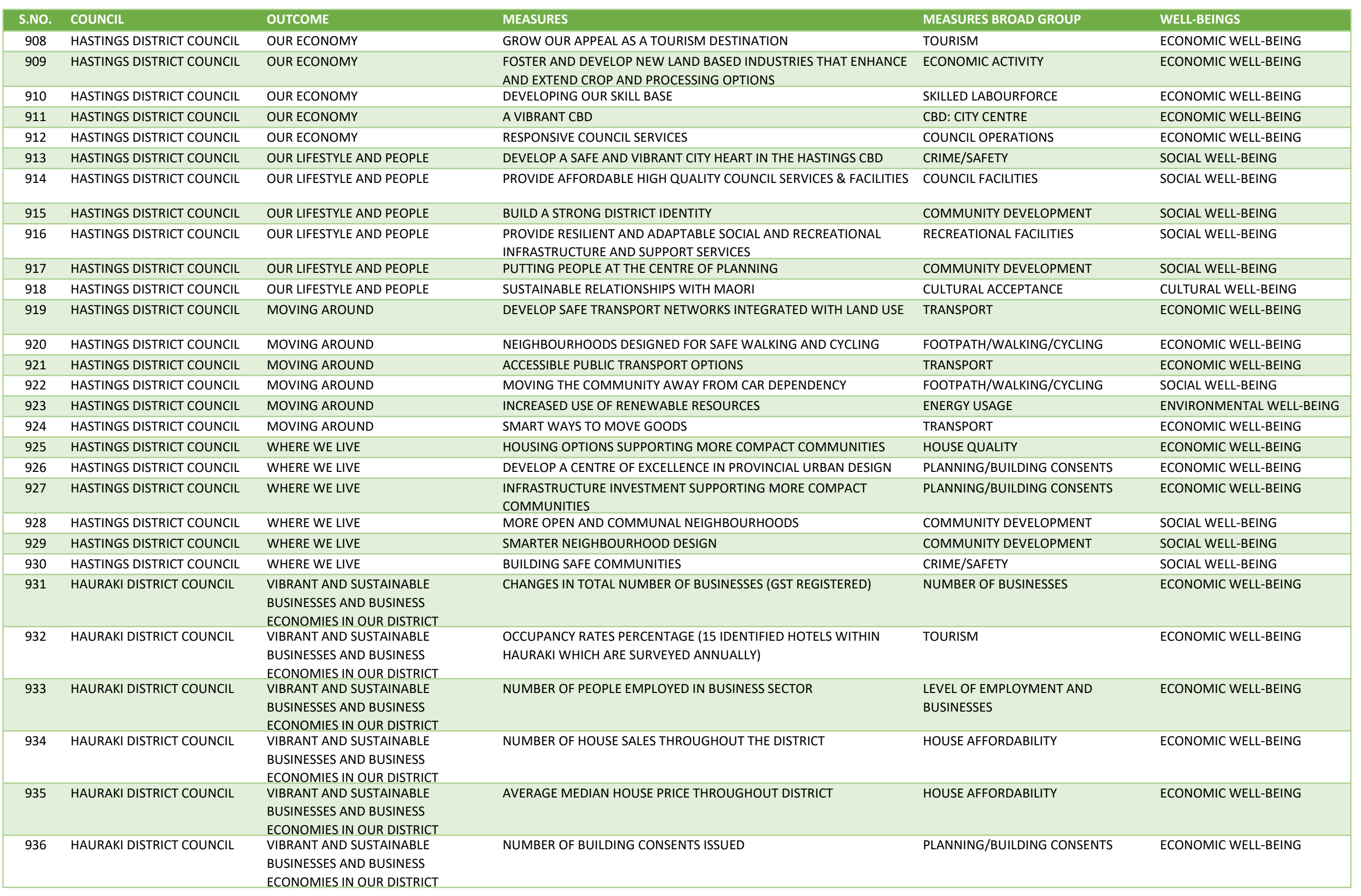


Appendix A: List of Community Well-beings Outcomes and Measures by Councils

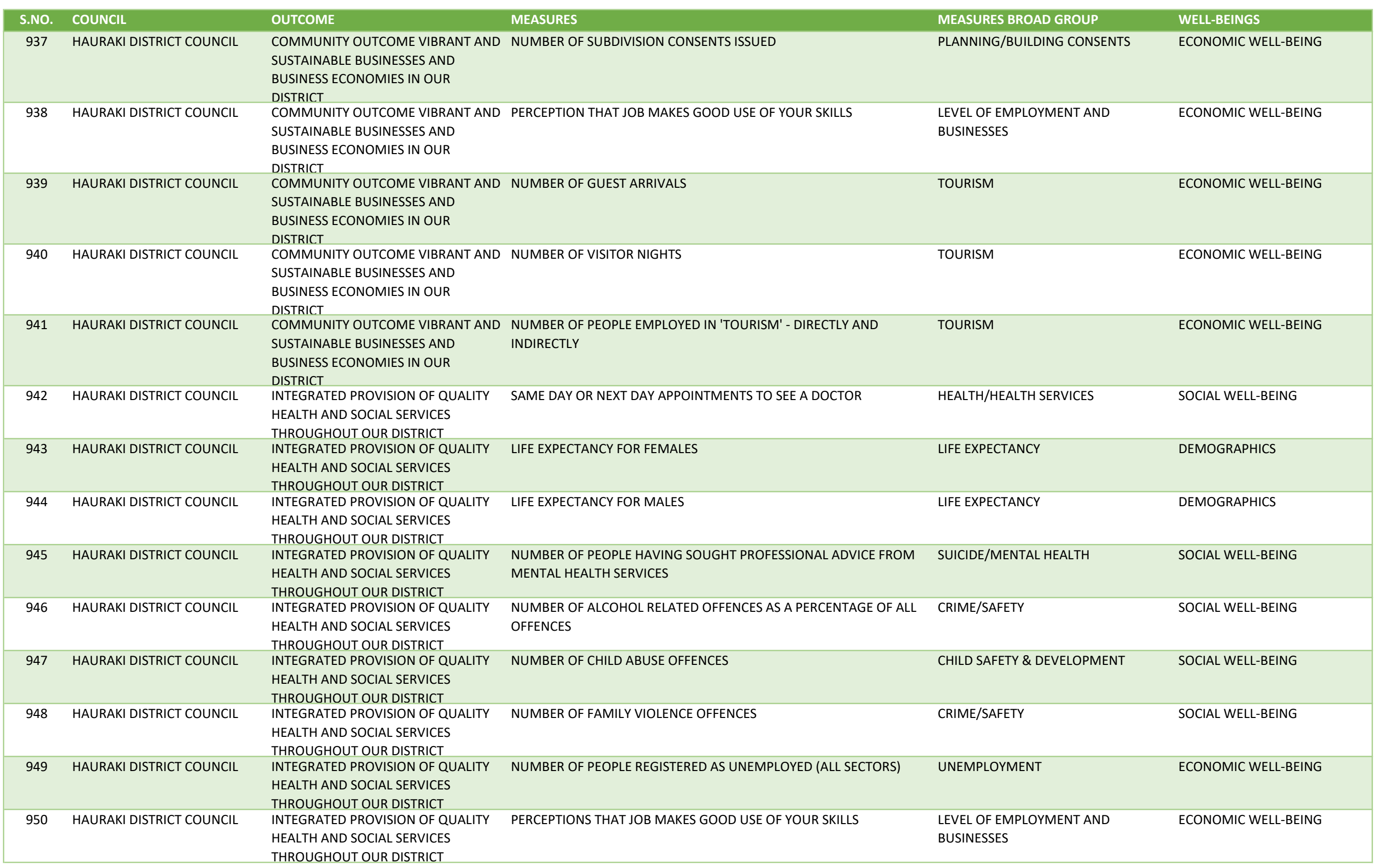


Appendix A: List of Community Well-beings Outcomes and Measures by Councils

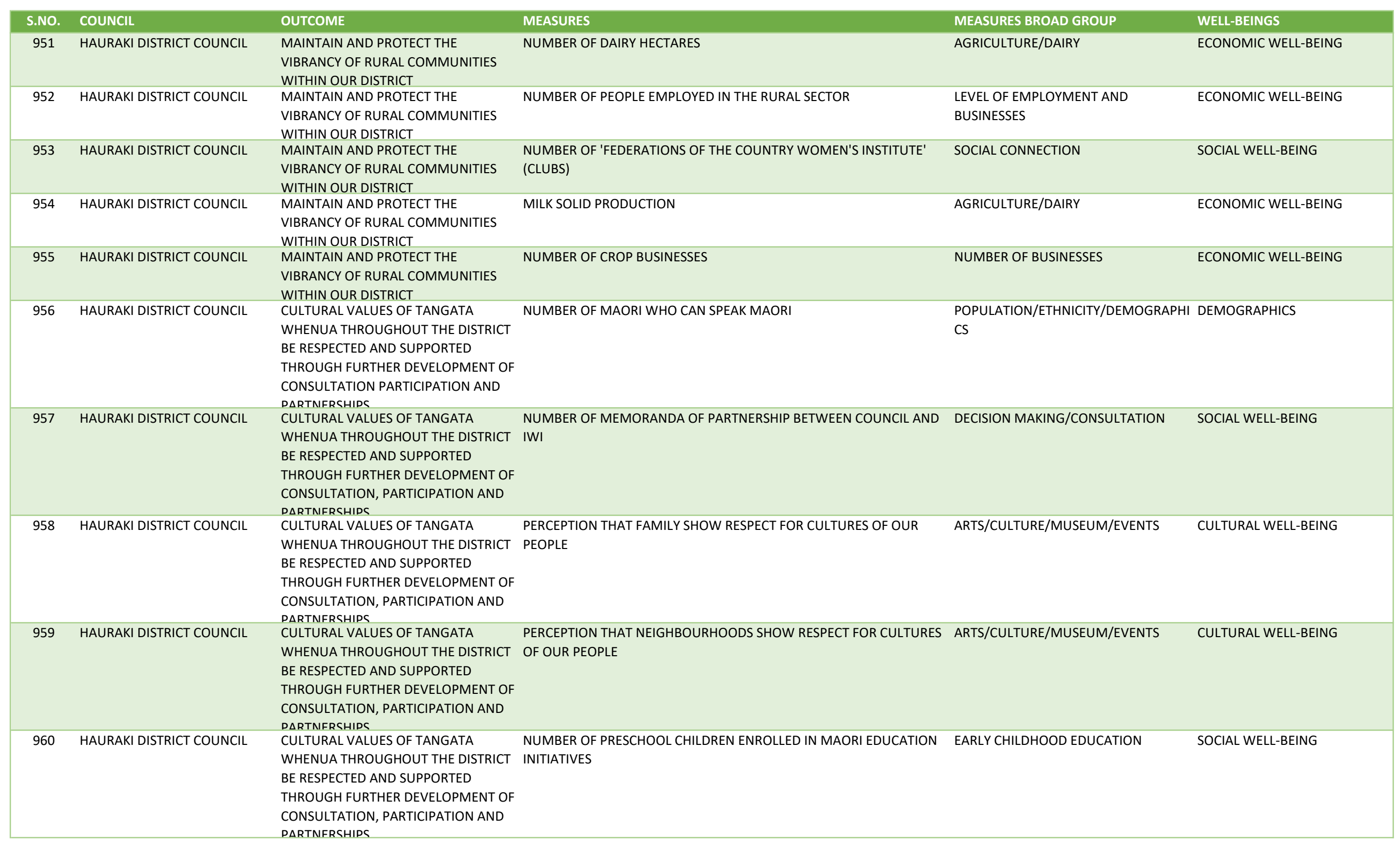




\begin{tabular}{|c|c|c|c|c|c|}
\hline S.NO. & COUNCIL & OUTCOME & MEASURES & MEASURES BROAD GROUP & WELL-BEINGS \\
\hline 961 & HAURAKI DISTRICT COUNCIL & $\begin{array}{l}\text { CULTURAL VALUES OF TANGATA } \\
\text { WHENUA THROUGHOUT THE DISTRICT } \\
\text { BE RESPECTED AND SUPPORTED } \\
\text { THROUGH FURTHER DEVELOPMENT OF } \\
\text { CONSULTATION, PARTICIPATION AND } \\
\text { PARTNFRSHIPS }\end{array}$ & NUMBER OF MAORI SCHOOL LEAVERS WITH LEVEL 2 OR HIGHER NCEA & QUALIFICATION/SCHOOL & SOCIAL WELL-BEING \\
\hline 962 & HAURAKI DISTRICT COUNCIL & $\begin{array}{l}\text { CULTURAL VALUES OF TANGATA } \\
\text { WHENUA THROUGHOUT THE DISTRICT } \\
\text { BE RESPECTED AND SUPPORTED } \\
\text { THROUGH FURTHER DEVELOPMENT OF } \\
\text { CONSULTATION, PARTICIPATION AND } \\
\text { PARTNFRSHIPS }\end{array}$ & $\begin{array}{l}\text { GROWTH IN GENERAL HEALTH SERVICES FOR MAORI BY TE KOROWAI } \\
\text { HAUORA }\end{array}$ & HEALTH/HEALTH SERVICES & SOCIAL WELL-BEING \\
\hline 963 & HAURAKI DISTRICT COUNCIL & $\begin{array}{l}\text { CULTURAL VALUES OF TANGATA } \\
\text { WHENUA THROUGHOUT THE DISTRICT } \\
\text { BE RESPECTED AND SUPPORTED } \\
\text { THROUGH FURTHER DEVELOPMENT OF } \\
\text { CONSULTATION, PARTICIPATION AND } \\
\text { PARTNFRSHIPS }\end{array}$ & PERCENT OF MAORI REGISTERED AS UNEMPLOYED & UNEMPLOYMENT & ECONOMIC WELL-BEING \\
\hline 964 & HAURAKI DISTRICT COUNCIL & $\begin{array}{l}\text { OUR HAURAKI YOUTH BE PROVIDED } \\
\text { WITH GREATER OPPORTUNITIES TO } \\
\text { PARTICIPATE IN THE DECISION-MAKING } \\
\text { PROCESSES PERTAINING TO THE } \\
\text { DEVELOPMENT OF OUR COMMUNITIES }\end{array}$ & PERCENTAGE OF YOUTH WHO HAVE ACCESS TO THE INTERNET & PHONE/MOBILE/INTERNET & ECONOMIC WELL-BEING \\
\hline 965 & HAURAKI DISTRICT COUNCIL & $\begin{array}{l}\text { OUR HAURAKI YOUTH BE PROVIDED } \\
\text { WITH GREATER OPPORTUNITIES TO } \\
\text { PARTICIPATE IN THE DECISION-MAKING } \\
\text { PROCESSES PERTAINING TO THE } \\
\text { DEVELOPMENT OF OUR COMMUNITIES }\end{array}$ & PERCENTAGE OF YOUTH WHO HAVE ACCESS TO A MOTOR VEHICLE & YOUTH & SOCIAL WELL-BEING \\
\hline 966 & HAURAKI DISTRICT COUNCIL & $\begin{array}{l}\text { OUR HAURAKI YOUTH BE PROVIDED } \\
\text { WITH GREATER OPPORTUNITIES TO } \\
\text { PARTICIPATE IN THE DECISION-MAKING } \\
\text { PROCESSES PERTAINING TO THE } \\
\text { DEVELOPMENT OF OUR COMMUNITIES }\end{array}$ & NUMBER OF YOUTH APPREHENSIONS FOR CRIMINAL OFFENCES & YOUTH & SOCIAL WELL-BEING \\
\hline 967 & HAURAKI DISTRICT COUNCIL & $\begin{array}{l}\text { OUR HAURAKI YOUTH BE PROVIDED } \\
\text { WITH GREATER OPPORTUNITIES TO } \\
\text { PARTICIPATE IN THE DECISION-MAKING } \\
\text { PROCESSES PERTAINING TO THE } \\
\text { DEVELOPMENT OF OUR COMMUNITIES }\end{array}$ & OVERALL YOUTH POPULATION OF DISTRICT & $\begin{array}{l}\text { POPULATION/ETHNICITY/DEMOGRAPHI } \\
\text { CS }\end{array}$ & DEMOGRAPHICS \\
\hline
\end{tabular}


Appendix A: List of Community Well-beings Outcomes and Measures by Councils

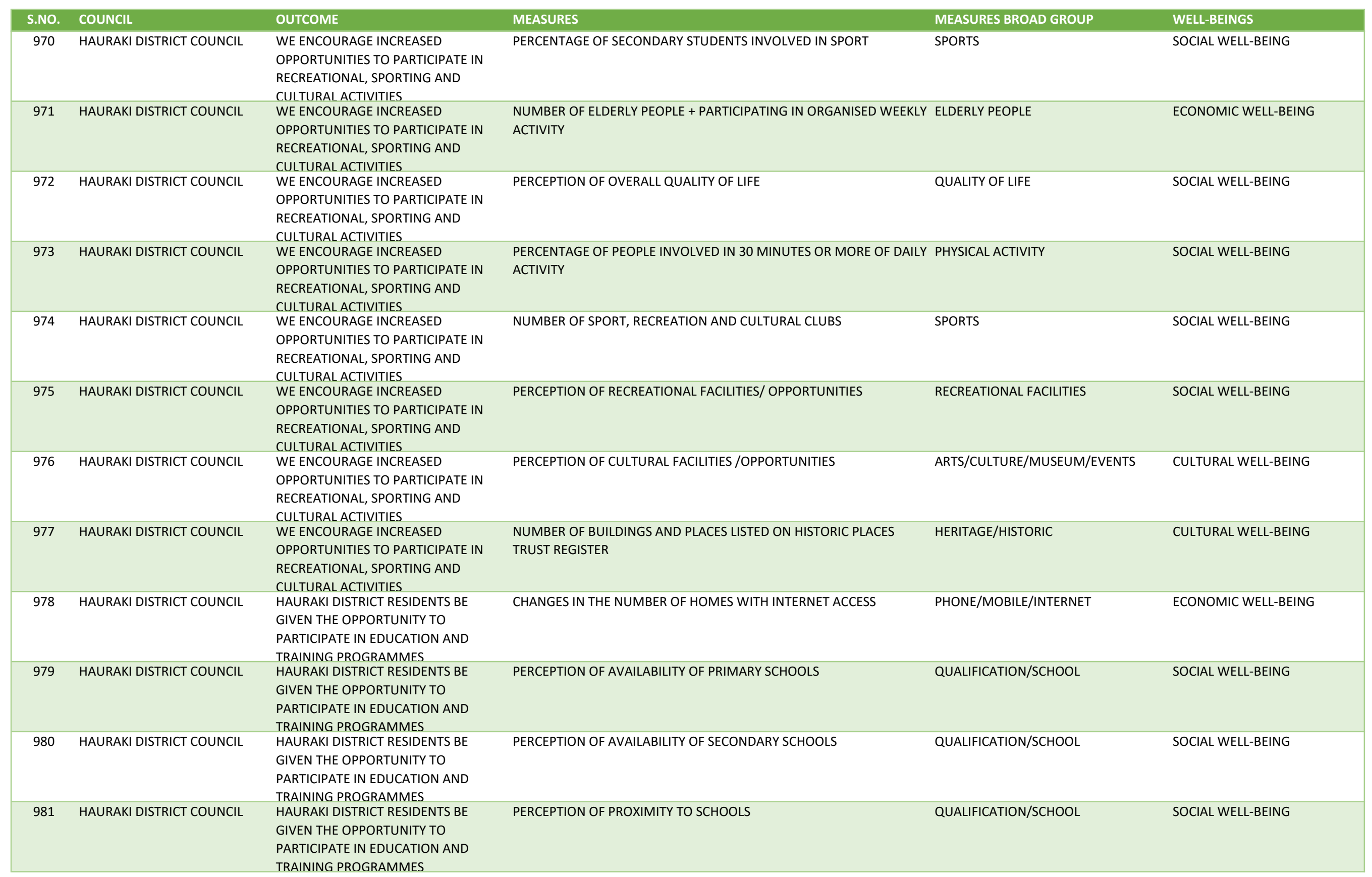


Appendix A: List of Community Well-beings Outcomes and Measures by Councils

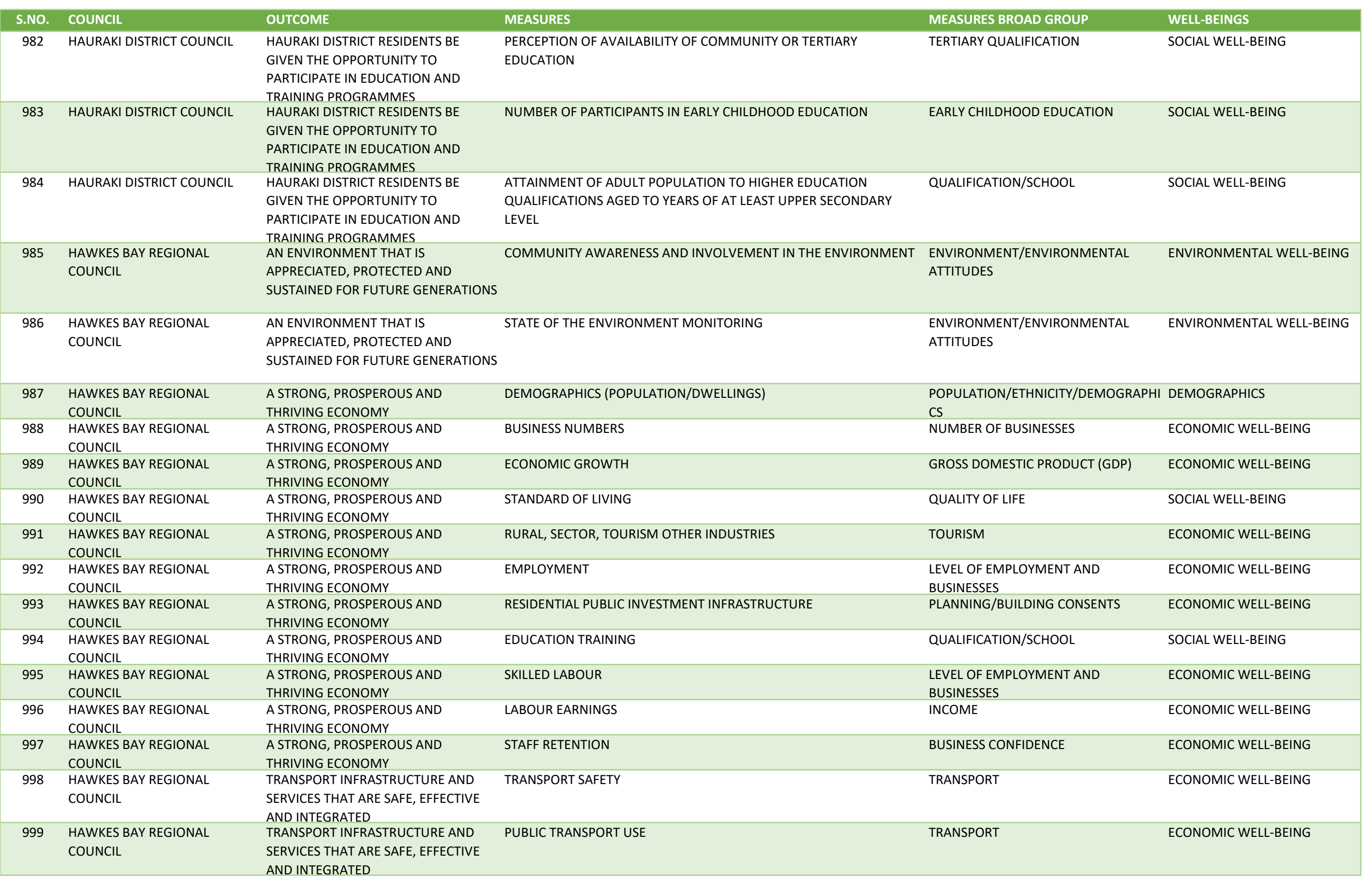


Appendix A: List of Community Well-beings Outcomes and Measures by Councils

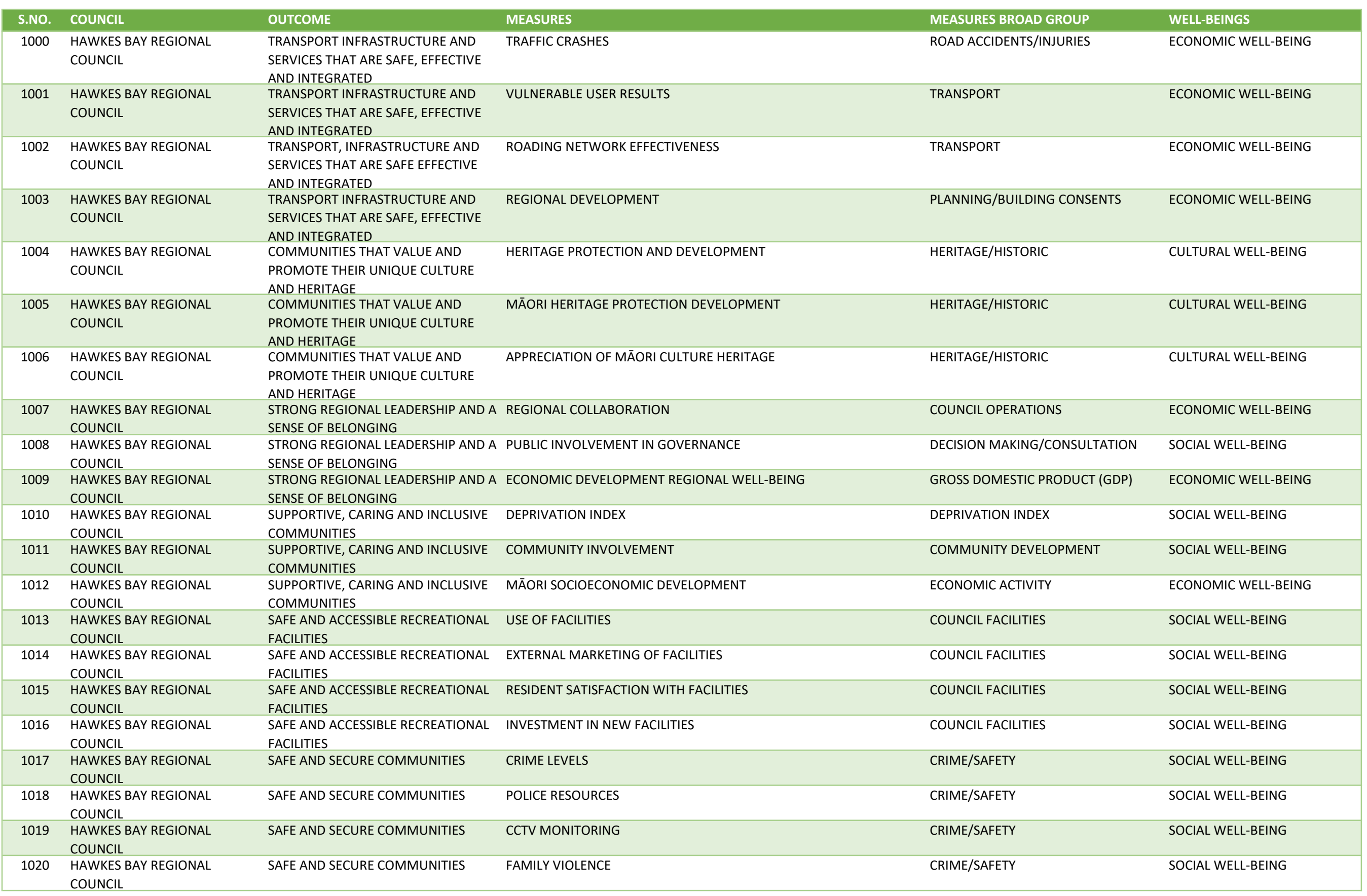


Appendix A: List of Community Well-beings Outcomes and Measures by Councils

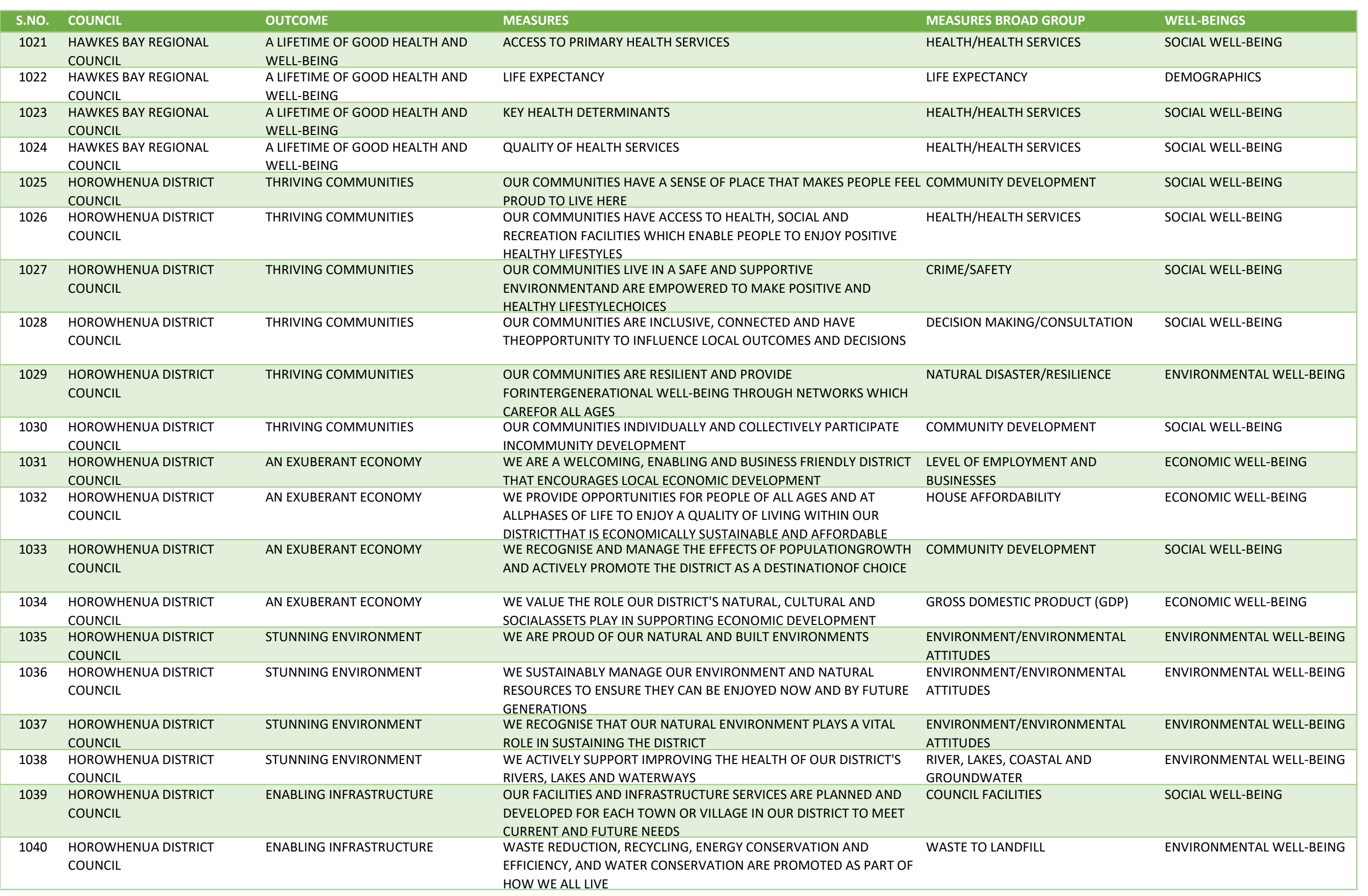


Appendix A: List of Community Well-beings Outcomes and Measures by Councils

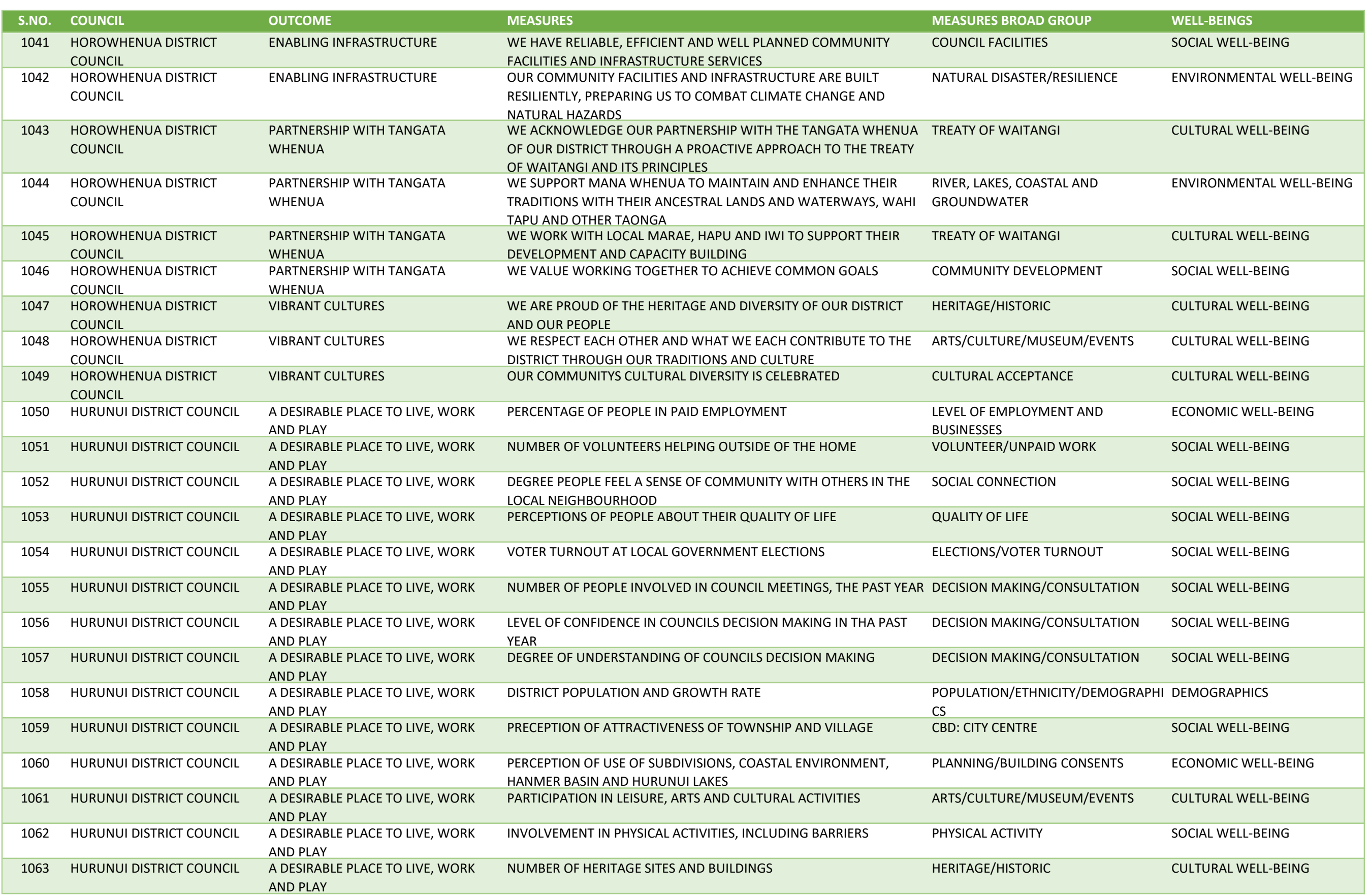




\begin{tabular}{|c|c|c|c|c|c|}
\hline S.NO. & COUNCIL & OUTCOME & MEASURES & MEASURES BROAD GROUP & WELL-BEINGS \\
\hline 1064 & HURUNUI DISTRICT COUNCIL & A HEALTHY, SAFE PLACE TO LIVE & NUMBER AND PERCENTAGE OF PEOPLE ENROLLED IN PHO & HEALTH/HEALTH SERVICES & SOCIAL WELL-BEING \\
\hline 1065 & HURUNUI DISTRICT COUNCIL & A HEALTHY, SAFE PLACE TO LIVE & NUMBER OF GPS PER HEAD OF POPULATION & HEALTH/HEALTH SERVICES & SOCIAL WELL-BEING \\
\hline 1066 & HURUNUI DISTRICT COUNCIL & A HEALTHY, SAFE PLACE TO LIVE & $\begin{array}{l}\text { HOURS SPENT IN THE HURUNUI DISTRICT BY THE DISTRICT NURSING } \\
\text { SERVICE }\end{array}$ & HEALTH/HEALTH SERVICES & SOCIAL WELL-BEING \\
\hline 1067 & HURUNUI DISTRICT COUNCIL & A HEALTHY, SAFE PLACE TO LIVE & $\begin{array}{l}\text { REASONS WHY PEOPLE DID NOT GO TO THE DOCTOR WHEN THEY } \\
\text { WANTED TO }\end{array}$ & HEALTH/HEALTH SERVICES & SOCIAL WELL-BEING \\
\hline 1068 & HURUNUI DISTRICT COUNCIL & A HEALTHY, SAFE PLACE TO LIVE & PEOPLE'S PERCEPTION OF ACCESSIBILITY OF HEALTH SERVICES & HEALTH/HEALTH SERVICES & SOCIAL WELL-BEING \\
\hline 1069 & HURUNUI DISTRICT COUNCIL & A HEALTHY, SAFE PLACE TO LIVE & NUMBER OF NZ FIRE SERVICE CALL-OUTS & NATURAL DISASTER/RESILIENCE & ENVIRONMENTAL WELL-BEING \\
\hline 1070 & HURUNUI DISTRICT COUNCIL & A HEALTHY, SAFE PLACE TO LIVE & $\begin{array}{l}\text { NUMBERS OF VOLUNTEERS IN ESSENTIAL SERVICES (IN COMPARISON } \\
\text { TO NUMBERS REQUIRED) }\end{array}$ & VOLUNTEER/UNPAID WORK & SOCIAL WELL-BEING \\
\hline 1071 & HURUNUI DISTRICT COUNCIL & A HEALTHY, SAFE PLACE TO LIVE & NUMBER OF HOSPITAL ADMISSIONS AND DEATHS FOR INJURIES & HOSPITALISATIONS & ECONOMIC WELL-BEING \\
\hline 1072 & HURUNUI DISTRICT COUNCIL & A HEALTHY, SAFE PLACE TO LIVE & NUMBER OF WORKPLACE FATALITIES AND INJURIES & ROAD ACCIDENTS/INJURIES & ECONOMIC WELL-BEING \\
\hline 1073 & HURUNUI DISTRICT COUNCIL & A HEALTHY, SAFE PLACE TO LIVE & ROAD CRASH FATALITIES AND INJURIES BY CAUSE OF CRASH & ROAD ACCIDENTS/INJURIES & ECONOMIC WELL-BEING \\
\hline 1074 & HURUNUI DISTRICT COUNCIL & A HEALTHY, SAFE PLACE TO LIVE & NUMBER AND TYPES OF CRIME OFFENCES & CRIME/SAFETY & SOCIAL WELL-BEING \\
\hline 1075 & HURUNUI DISTRICT COUNCIL & A HEALTHY, SAFE PLACE TO LIVE & PERCEPTIONS OF SAFETY AT HOME AND IN THE NEIGHBOURHOOD & CRIME/SAFETY & SOCIAL WELL-BEING \\
\hline 1076 & HURUNUI DISTRICT COUNCIL & A HEALTHY, SAFE PLACE TO LIVE & HOME OWNERSHIP STATISTICS & HOME OWNERSHIP & DEMOGRAPHICS \\
\hline 1077 & HURUNUI DISTRICT COUNCIL & A HEALTHY, SAFE PLACE TO LIVE & MEDIAN DWELLING SALE PRICES & HOUSE AFFORDABILITY & ECONOMIC WELL-BEING \\
\hline 1078 & HURUNUI DISTRICT COUNCIL & A HEALTHY, SAFE PLACE TO LIVE & SIZE OF WAITING LIST FOR COUNCIL IN GOVERNMENT HOUSING & HOUSE AFFORDABILITY & ECONOMIC WELL-BEING \\
\hline 1080 & HURUNUI DISTRICT COUNCIL & A HEALTHY, SAFE PLACE TO LIVE & STATUS OF CIVIL DEFENCE RESOURCES AND PLANS & NATURAL DISASTER/RESILIENCE & ENVIRONMENTAL WELL-BEING \\
\hline 1081 & HURUNUI DISTRICT COUNCIL & A THRIVING LOCAL ECONOMY & $\begin{array}{l}\text { PERCENTAGE OF PEOPLE IN FULL AND PART TIME EMPLOYMENT BY } \\
\text { INDUSTRY AN APPLICATION GROUPING }\end{array}$ & $\begin{array}{l}\text { LEVEL OF EMPLOYMENT AND } \\
\text { BUSINESSES }\end{array}$ & ECONOMIC WELL-BEING \\
\hline 1082 & HURUNUI DISTRICT COUNCIL & A THRIVING LOCAL ECONOMY & PERCENTAGE OF PEOPLE IN LABOR FORCE & $\begin{array}{l}\text { LEVEL OF EMPLOYMENT AND } \\
\text { BUSINESSES }\end{array}$ & ECONOMIC WELL-BEING \\
\hline 1083 & HURUNUI DISTRICT COUNCIL & A THRIVING LOCAL ECONOMY & LONG-TERM (6 MONTHS OR ONE YEAR PLUS) UNEMPLOYMENT RATES & UNEMPLOYMENT & ECONOMIC WELL-BEING \\
\hline 1084 & HURUNUI DISTRICT COUNCIL & A THRIVING LOCAL ECONOMY & PERCENTAGE OF PEOPLE REGISTERED UNEMPLOYED & UNEMPLOYMENT & ECONOMIC WELL-BEING \\
\hline 1085 & HURUNUI DISTRICT COUNCIL & A THRIVING LOCAL ECONOMY & $\begin{array}{l}\text { DOLLAR VALUE OF COMMERCIAL AND RESIDENTIAL BUILDING } \\
\text { CONSENTS ISSUED }\end{array}$ & PLANNING/BUILDING CONSENTS & ECONOMIC WELL-BEING \\
\hline 1086 & HURUNUI DISTRICT COUNCIL & A THRIVING LOCAL ECONOMY & AVERAGE WEEKLY EARNINGS AND MEDIAN ANNUAL INCOME & INCOME & ECONOMIC WELL-BEING \\
\hline 1087 & HURUNUI DISTRICT COUNCIL & A THRIVING LOCAL ECONOMY & TOTAL NUMBER OF PEOPLE ON MAIN BENEFITS & INCOME & ECONOMIC WELL-BEING \\
\hline 1088 & HURUNUI DISTRICT COUNCIL & A THRIVING LOCAL ECONOMY & PERCENTAGE AND AVERAGE NUMBERS OF EMPLOYEES & $\begin{array}{l}\text { LEVEL OF EMPLOYMENT AND } \\
\text { BUSINESSES }\end{array}$ & ECONOMIC WELL-BEING \\
\hline 1089 & HURUNUI DISTRICT COUNCIL & A THRIVING LOCAL ECONOMY & NUMBER OF BUSINESSES & NUMBER OF BUSINESSES & ECONOMIC WELL-BEING \\
\hline 1090 & HURUNUI DISTRICT COUNCIL & A THRIVING LOCAL ECONOMY & $\begin{array}{l}\text { DISTRIBUTION OF INDUSTRY BY TYPE (BASED ON STATS NZ BUSINESS } \\
\text { FRAME) }\end{array}$ & $\begin{array}{l}\text { LEVEL OF EMPLOYMENT AND } \\
\text { BUSINESSES }\end{array}$ & ECONOMIC WELL-BEING \\
\hline 1091 & HURUNUI DISTRICT COUNCIL & A THRIVING LOCAL ECONOMY & ACCESS TO TELECOMMUNICATION SERVICES & PHONE/MOBILE/INTERNET & ECONOMIC WELL-BEING \\
\hline 1092 & HURUNUI DISTRICT COUNCIL & A THRIVING LOCAL ECONOMY & TELECOM AND VODAFONE MOBILE COVERAGE AREAS & PHONE/MOBILE/INTERNET & ECONOMIC WELL-BEING \\
\hline 1095 & HURUNUI DISTRICT COUNCIL & ESSENTIAL INFRASTRUCTURE & RATE OF CONSUMPTION VERSUS SUPPLY OF WATER & $\begin{array}{l}\text { RIVER, LAKES, COASTAL AND } \\
\text { GROUNDWATER }\end{array}$ & ENVIRONMENTAL WELL-BEING \\
\hline 1096 & HURUNUI DISTRICT COUNCIL & ESSENTIAL INFRASTRUCTURE & $\begin{array}{l}\text { PEOPLES PERCEPTION OF THE QUALITY AND QUANTITY OF WATER } \\
\text { AVAILABLE }\end{array}$ & $\begin{array}{l}\text { RIVER, LAKES, COASTAL AND } \\
\text { GROUNDWATER }\end{array}$ & ENVIRONMENTAL WELL-BEING \\
\hline
\end{tabular}


Appendix A: List of Community Well-beings Outcomes and Measures by Councils

\begin{tabular}{|c|c|c|c|c|c|}
\hline S.NO. & COUNCIL & OUTCOME & MEASURES & MEASURES BROAD GROUP & WELL-BEINGS \\
\hline 1097 & HURUNUI DISTRICT COUNCIL & ESSENTIAL INFRASTRUCTURE & $\begin{array}{l}\text { PEOPLE'S PERCEPTION OF THE SAFETY AND MAINTENANCE OF ROADS } \\
\text { IN THE DISTRICT }\end{array}$ & CRIME/SAFETY & SOCIAL WELL-BEING \\
\hline 1098 & HURUNUI DISTRICT COUNCIL & ESSENTIAL INFRASTRUCTURE & ROAD ROUGHNESS IN THE DISTRICT & TRANSPORT & ECONOMIC WELL-BEING \\
\hline 1099 & HURUNUI DISTRICT COUNCIL & ESSENTIAL INFRASTRUCTURE & COMPLIANCE OF DISCHARGE CONCERNS FOR WASTEWATER & SEWERAGE/WASTEWATER & ENVIRONMENTAL WELL-BEING \\
\hline 1100 & HURUNUI DISTRICT COUNCIL & ESSENTIAL INFRASTRUCTURE & $\begin{array}{l}\text { PEOPLES PERCEPTION OF THE QUALITY AND QUANTITY OF PUBLIC } \\
\text { TOILET IN THE DISTRICT }\end{array}$ & COUNCIL FACILITIES & SOCIAL WELL-BEING \\
\hline 1101 & HURUNUI DISTRICT COUNCIL & ENVIRONMENTAL RESPONSIBILITY & SWIMMING WATER QUALITY & $\begin{array}{l}\text { RIVER, LAKES, COASTAL AND } \\
\text { GROUNDWATER }\end{array}$ & ENVIRONMENTAL WELL-BEING \\
\hline 1102 & HURUNUI DISTRICT COUNCIL & ENVIRONMENTAL RESPONSIBILITY & $\begin{array}{l}\text { NO OF DAYS THAT MONITORED RIVERS DROP BELOW ECAN MINIMUM } \\
\text { FLOW LEVELS }\end{array}$ & $\begin{array}{l}\text { RIVER, LAKES, COASTAL AND } \\
\text { GROUNDWATER }\end{array}$ & ENVIRONMENTAL WELL-BEING \\
\hline 1103 & HURUNUI DISTRICT COUNCIL & ENVIRONMENTAL RESPONSIBILITY & $\begin{array}{l}\text { LEVEL OF WASTE TO LANDFILL AND PERCENTAGE AND TYPE OF WASTE } \\
\text { TO RECYCLE }\end{array}$ & WASTE TO LANDFILL & ENVIRONMENTAL WELL-BEING \\
\hline 1104 & HURUNUI DISTRICT COUNCIL & ENVIRONMENTAL RESPONSIBILITY & DOLLARS INVESTED IN ENVIRONMENTAL AWARDS & $\begin{array}{l}\text { ENVIRONMENT/ENVIRONMENTAL } \\
\text { ATTITUDES }\end{array}$ & ENVIRONMENTAL WELL-BEING \\
\hline 1105 & HURUNUI DISTRICT COUNCIL & ENVIRONMENTAL RESPONSIBILITY & HECTORS PROTECTED UNDER QEII COVENANTS & $\begin{array}{l}\text { ENVIRONMENT/ENVIRONMENTAL } \\
\text { ATTITUDES }\end{array}$ & ENVIRONMENTAL WELL-BEING \\
\hline 1106 & HURUNUI DISTRICT COUNCIL & ENVIRONMENTAL RESPONSIBILITY & AREA OF NATIVE VEGETATION & NATIVE VEGETATION & ENVIRONMENTAL WELL-BEING \\
\hline 1107 & HURUNUI DISTRICT COUNCIL & ENVIRONMENTAL RESPONSIBILITY & SPREAD OF WILDING TREES & $\begin{array}{l}\text { ENVIRONMENT/ENVIRONMENTAL } \\
\text { ATTITUDES }\end{array}$ & ENVIRONMENTAL WELL-BEING \\
\hline 1108 & HURUNUI DISTRICT COUNCIL & ENVIRONMENTAL RESPONSIBILITY & NUMBER OF NASSELLA PER HECTARE & $\begin{array}{l}\text { ENVIRONMENT/ENVIRONMENTAL } \\
\text { ATTITUDES }\end{array}$ & ENVIRONMENTAL WELL-BEING \\
\hline 1109 & HURUNUI DISTRICT COUNCIL & ENVIRONMENTAL RESPONSIBILITY & LEVEL OF POSSUM FERRETS AND RABBITS & $\begin{array}{l}\text { ENVIRONMENT/ENVIRONMENTAL } \\
\text { ATTITUDES }\end{array}$ & ENVIRONMENTAL WELL-BEING \\
\hline 1110 & HURUNUI DISTRICT COUNCIL & ENVIRONMENTAL RESPONSIBILITY & $\begin{array}{l}\text { PEOPLES PERCEPTION OF ENVIRONMENTAL ISSUES THAT NEED TO BE } \\
\text { FURTHER INVESTIGATED OR BETTER MANAGED IN THE DISTRICT }\end{array}$ & $\begin{array}{l}\text { ENVIRONMENT/ENVIRONMENTAL } \\
\text { ATTITUDES }\end{array}$ & ENVIRONMENTAL WELL-BEING \\
\hline 1111 & HURUNUI DISTRICT COUNCIL & $\begin{array}{l}\text { SKILLS AND EDUCATION FOR WORK } \\
\text { AND LIFE }\end{array}$ & $\begin{array}{l}\text { PERCENTAGE OF SCHOOL ENTRANTS WHO HAVE PARTICIPATED IN } \\
\text { EARLY CHILDHOOD EDUCATION }\end{array}$ & EARLY CHILDHOOD EDUCATION & SOCIAL WELL-BEING \\
\hline 1112 & HURUNUI DISTRICT COUNCIL & $\begin{array}{l}\text { SKILLS AND EDUCATION FOR WORK } \\
\text { AND LIFE }\end{array}$ & NUMBER OF ENROLLMENTS IN EARLY CHILDHOOD EDUCATION & EARLY CHILDHOOD EDUCATION & SOCIAL WELL-BEING \\
\hline 1113 & HURUNUI DISTRICT COUNCIL & $\begin{array}{l}\text { SKILLS AND EDUCATION FOR WORK } \\
\text { AND LIFE }\end{array}$ & HURUNUI SCHOOL ROLL NUMBERS & QUALIFICATION/SCHOOL & SOCIAL WELL-BEING \\
\hline 1114 & HURUNUI DISTRICT COUNCIL & $\begin{array}{l}\text { SKILLS AND EDUCATION FOR WORK } \\
\text { AND LIFE }\end{array}$ & NQF RESULTS FOR LOCAL SCHOOLS & QUALIFICATION/SCHOOL & SOCIAL WELL-BEING \\
\hline 1115 & HURUNUI DISTRICT COUNCIL & $\begin{array}{l}\text { SKILLS AND EDUCATION FOR WORK } \\
\text { AND LIFE }\end{array}$ & DECILE RATING FOR LOCAL SCHOOLS & QUALIFICATION/SCHOOL & SOCIAL WELL-BEING \\
\hline 1116 & HURUNUI DISTRICT COUNCIL & $\begin{array}{l}\text { SKILLS AND EDUCATION FOR WORK } \\
\text { AND LIFE }\end{array}$ & PERCENTAGE OF SCHOOL LEAVERS WITH NO QUALIFICATION & QUALIFICATION/SCHOOL & SOCIAL WELL-BEING \\
\hline 1117 & HURUNUI DISTRICT COUNCIL & $\begin{array}{l}\text { SKILLS AND EDUCATION FOR WORK } \\
\text { AND LIFE }\end{array}$ & NUMBER OF MODERN APPRENTICESHIPS & APPRENTICESHIPS FOR YOUNG PEOPLE & ECONOMIC WELL-BEING \\
\hline 1118 & HURUNUI DISTRICT COUNCIL & $\begin{array}{l}\text { SKILLS AND EDUCATION FOR WORK } \\
\text { AND LIFE }\end{array}$ & NUMBER OF PEOPLE UNDERTAKING INDUSTRY TRAINING & APPRENTICESHIPS FOR YOUNG PEOPLE & ECONOMIC WELL-BEING \\
\hline 1119 & HURUNUI DISTRICT COUNCIL & $\begin{array}{l}\text { SKILLS AND EDUCATION FOR WORK } \\
\text { AND LIFE }\end{array}$ & NUMBER OF ACTIVE MEMBERS OF DISTRICT LIBRARIES & LIBRARY & SOCIAL WELL-BEING \\
\hline 1121 & $\begin{array}{l}\text { INVERCARGILL DISTRICT } \\
\text { COUNCIL }\end{array}$ & SOUTHLAND IS A GREAT PLACE TO LIVE & $\begin{array}{l}\text { IDENTIFICATION AND PRESERVATION OF LOCAL HERITAGE SITES A NEW } \\
\text { ZEALAND HISTORIC PLACES TRUST REGISTRATIONS OF CATEGORY I AND } \\
\text { II BUILDINGS AND HISTORIC AREAS IN THE SOUTHLAND REGION BY } \\
\text { TERRITORIAL LOCAL AUTHORITY }\end{array}$ & HERITAGE/HISTORIC & CULTURAL WELL-BEING \\
\hline
\end{tabular}




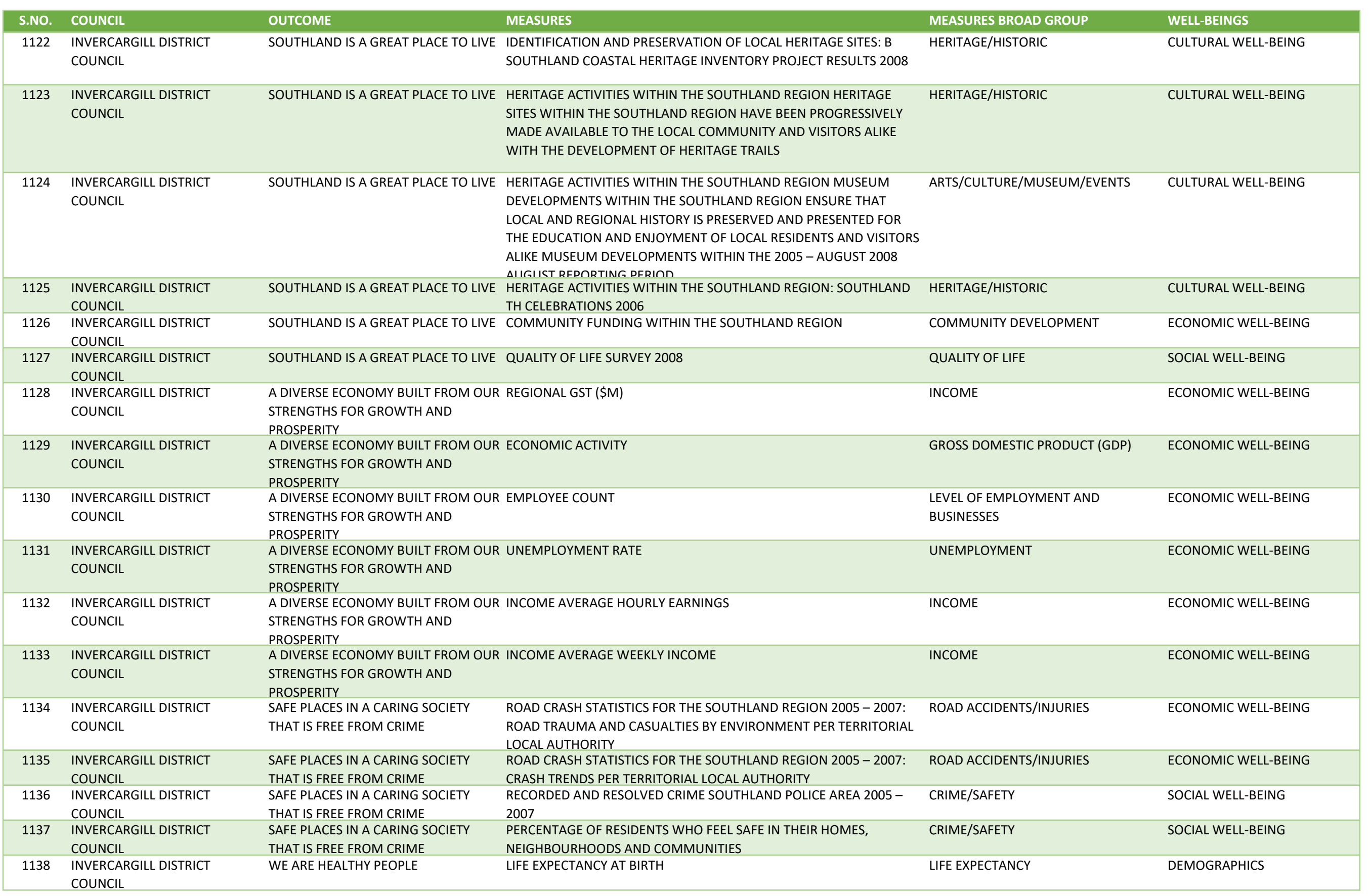


Appendix A: List of Community Well-beings Outcomes and Measures by Councils

\begin{tabular}{|c|c|c|c|c|c|}
\hline S.NO. & COUNCIL & OUTCOME & MEASURES & MEASURES BROAD GROUP & WELL-BEINGS \\
\hline 1139 & $\begin{array}{l}\text { INVERCARGILL DISTRICT } \\
\text { COUNCIL }\end{array}$ & WE ARE HEALTHY PEOPLE & $\begin{array}{l}\text { REGULAR PHYSICAL ACTIVITY FOR ADULTS BY DISTRICT HEALTH BOARD } \\
\text { AREA 2006/07 }\end{array}$ & PHYSICAL ACTIVITY & SOCIAL WELL-BEING \\
\hline 1140 & $\begin{array}{l}\text { INVERCARGILL DISTRICT } \\
\text { COUNCIL }\end{array}$ & WE ARE HEALTHY PEOPLE & HOUSING -- QUALITY/AGE/AFFORDABILITY OF HOUSING STOCK & HOUSE AFFORDABILITY & ECONOMIC WELL-BEING \\
\hline 1141 & $\begin{array}{l}\text { INVERCARGILL DISTRICT } \\
\text { COUNCIL }\end{array}$ & WE ARE HEALTHY PEOPLE & ACCESS TO PRIMARY HEALTH PROVIDERS & HEALTH/HEALTH SERVICES & SOCIAL WELL-BEING \\
\hline 1142 & $\begin{array}{l}\text { INVERCARGILL DISTRICT } \\
\text { COUNCIL }\end{array}$ & WE ARE HEALTHY PEOPLE & $\begin{array}{l}\text { ACCESS TO PRIMARY HEALTH PROVIDERS: CHILDREN AND ADULTS WHO } \\
\text { SAW A GENERAL PRACTITIONER IN THE PREVIOUS } 12 \text { MONTHS, BY } \\
\text { DISTRICT HEALTH BOARD AREA (2006/07 HEALTH SURVEY) }\end{array}$ & HEALTH/HEALTH SERVICES & SOCIAL WELL-BEING \\
\hline 1143 & $\begin{array}{l}\text { INVERCARGILL DISTRICT } \\
\text { COUNCIL }\end{array}$ & WE ARE HEALTHY PEOPLE & $\begin{array}{l}\text { ACCESS TO PRIMARY HEALTH PROVIDERS UNMET NEED FOR GP } \\
\text { SERVICES BY DISTRICT HEALTH BOARD AREA }\end{array}$ & HEALTH/HEALTH SERVICES & SOCIAL WELL-BEING \\
\hline 1144 & $\begin{array}{l}\text { INVERCARGILL DISTRICT } \\
\text { COUNCIL }\end{array}$ & WE ARE HEALTHY PEOPLE & $\begin{array}{l}\text { PUBLICLY-FUNDED HOSPITAL DISCHARGES BY DIAGNOSIS 2004-2006 BY } \\
\text { TERRITORIAL LOCAL AUTHORITY }\end{array}$ & HEALTH/HEALTH SERVICES & SOCIAL WELL-BEING \\
\hline 1145 & $\begin{array}{l}\text { INVERCARGILL DISTRICT } \\
\text { COUNCIL }\end{array}$ & WE ARE HEALTHY PEOPLE & $\begin{array}{l}\text { GENERAL HEALTH SUMMARY BY DISTRICT HEALTH BOARD AREA } \\
\text { 2006/07 }\end{array}$ & HEALTH/HEALTH SERVICES & SOCIAL WELL-BEING \\
\hline 1146 & $\begin{array}{l}\text { INVERCARGILL DISTRICT } \\
\text { COUNCIL }\end{array}$ & $\begin{array}{l}\text { STRONG, EFFECTIVE LEADERSHIP } \\
\text { TAKING US INTO THE FUTURE }\end{array}$ & $\begin{array}{l}\text { PARTICIPATION IN } 2004 \text { LOCAL AUTHORITY ELECTIONS COMPARED } \\
\text { WITH NATIONAL TRENDS VOTER TURNOUT BY SOUTHLAND LOCAL } \\
\text { AUTHORITY } 2004\end{array}$ & ELECTIONS/VOTER TURNOUT & SOCIAL WELL-BEING \\
\hline 1147 & $\begin{array}{l}\text { INVERCARGILL DISTRICT } \\
\text { COUNCIL }\end{array}$ & $\begin{array}{l}\text { STRONG, EFFECTIVE LEADERSHIP } \\
\text { TAKING US INTO THE FUTURE }\end{array}$ & $\begin{array}{l}\text { PARTICIPATION IN } 2004 \text { LOCAL AUTHORITY ELECTIONS COMPARED } \\
\text { WITH NATIONAL TRENDS VOTER TURNOUT FOR MAYORAL ELECTIONS } \\
2004\end{array}$ & ELECTIONS/VOTER TURNOUT & SOCIAL WELL-BEING \\
\hline 1148 & $\begin{array}{l}\text { INVERCARGILL DISTRICT } \\
\text { COUNCIL }\end{array}$ & $\begin{array}{l}\text { STRONG, EFFECTIVE LEADERSHIP } \\
\text { TAKING US INTO THE FUTURE }\end{array}$ & $\begin{array}{l}\text { VOTER TURNOUT IN THE INVERCARGILL AND CLUTHA-SOUTHLAND } \\
\text { ELECTORATES IN THE } 2005 \text { GENERAL ELECTION COMPARED WITH } \\
\text { NATIONAL TRENDS: A PARTY VOTES AND TURNOUT BY ELECTORATE } \\
2005 \text { GENERAL ELECTION }\end{array}$ & ELECTIONS/VOTER TURNOUT & SOCIAL WELL-BEING \\
\hline 1149 & $\begin{array}{l}\text { INVERCARGILL DISTRICT } \\
\text { COUNCIL }\end{array}$ & $\begin{array}{l}\text { STRONG, EFFECTIVE LEADERSHIP } \\
\text { TAKING US INTO THE FUTURE }\end{array}$ & $\begin{array}{l}\text { VOTER TURNOUT IN THE INVERCARGILL AND CLUTHA-SOUTHLAND } \\
\text { ELECTORATES IN THE GENERAL ELECTION COMPARED WITH NATIONAL } \\
\text { TRENDS: B. ELECTORATE CANDIDATE VOTES AND TURNOUT BY } \\
\text { ELECTORATE } 2005 \text { GENERAL ELECTION }\end{array}$ & ELECTIONS/VOTER TURNOUT & SOCIAL WELL-BEING \\
\hline 1150 & $\begin{array}{l}\text { INVERCARGILL DISTRICT } \\
\text { COUNCIL }\end{array}$ & $\begin{array}{l}\text { A TREASURED ENVIRONMENT WHICH } \\
\text { WE CARE FOR AND WHICH SUPPORTS } \\
\text { US NOW AND INTO THE FUTURE }\end{array}$ & ENVIRONMENTAL EDUCATION ENVIROSCHOOLS & $\begin{array}{l}\text { ENVIRONMENT/ENVIRONMENTAL } \\
\text { ATTITUDES }\end{array}$ & ENVIRONMENTAL WELL-BEING \\
\hline 1151 & $\begin{array}{l}\text { INVERCARGILL DISTRICT } \\
\text { COUNCIL }\end{array}$ & $\begin{array}{l}\text { A TREASURED ENVIRONMENT WHICH } \\
\text { WE CARE FOR AND WHICH SUPPORTS } \\
\text { US NOW AND INTO THE FUTURE }\end{array}$ & $\begin{array}{l}\text { AIR QUALITY: SUMMARY OF THE NATIONAL ENVIRONMENTAL } \\
\text { STANDARD FOR AIR QUALITY FOR AMBIENT FINE PARTICLES - } \\
\text { PARTICULATE MATTER } 10 \text { MICRONS MEASURED IN INVERCARGILL AND } \\
\text { GORE FROM TO } 2003 \text { TO } 2007\end{array}$ & AIR QUALITY & ENVIRONMENTAL WELL-BEING \\
\hline 1152 & $\begin{array}{l}\text { INVERCARGILL DISTRICT } \\
\text { COUNCIL }\end{array}$ & $\begin{array}{l}\text { A TREASURED ENVIRONMENT WHICH } \\
\text { WE CARE FOR AND WHICH SUPPORTS } \\
\text { US NOW AND INTO THE FUTURE }\end{array}$ & DRINKING WATER & DRINKING WATER & ENVIRONMENTAL WELL-BEING \\
\hline
\end{tabular}


Appendix A: List of Community Well-beings Outcomes and Measures by Councils

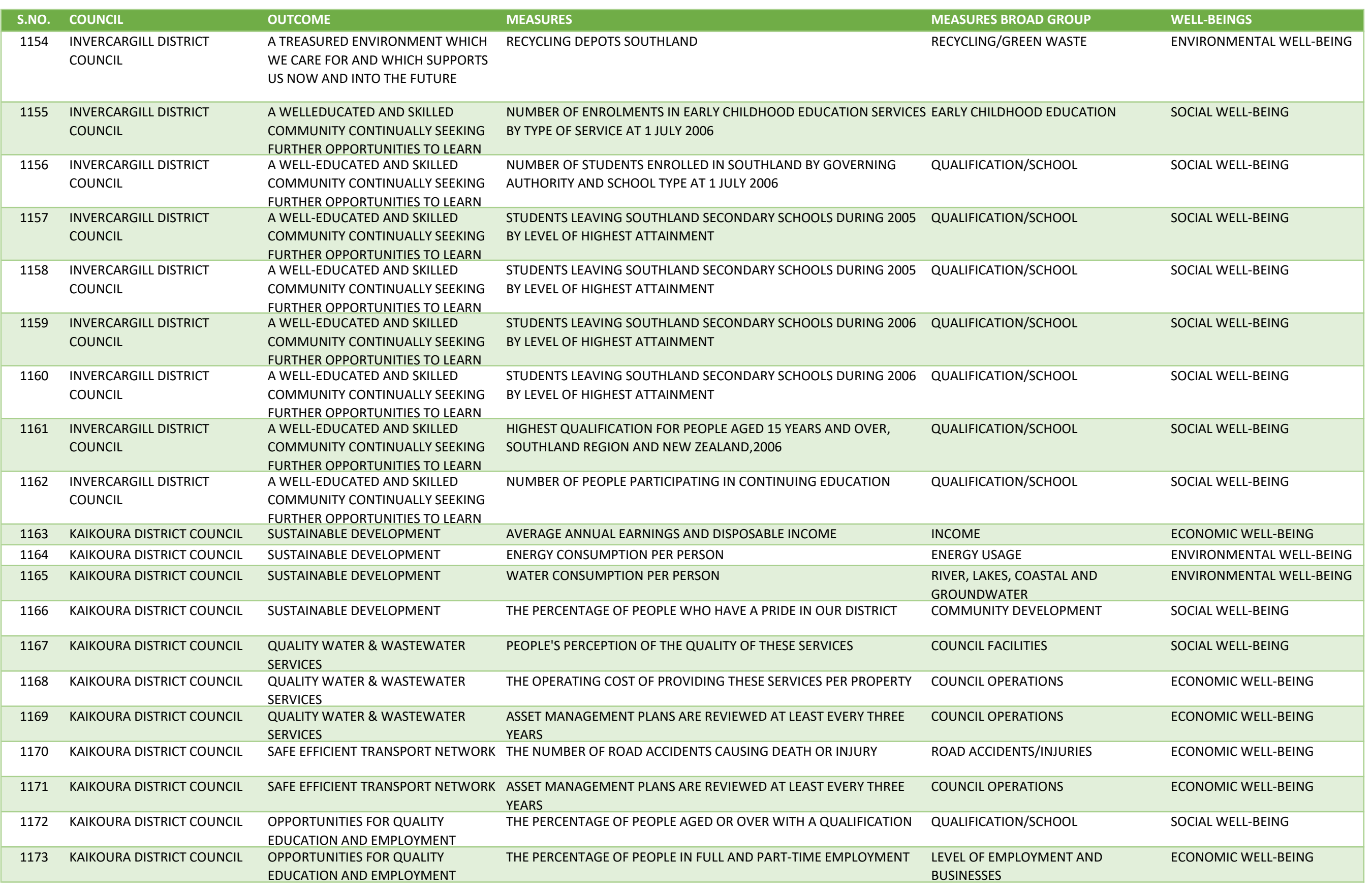


Appendix A: List of Community Well-beings Outcomes and Measures by Councils

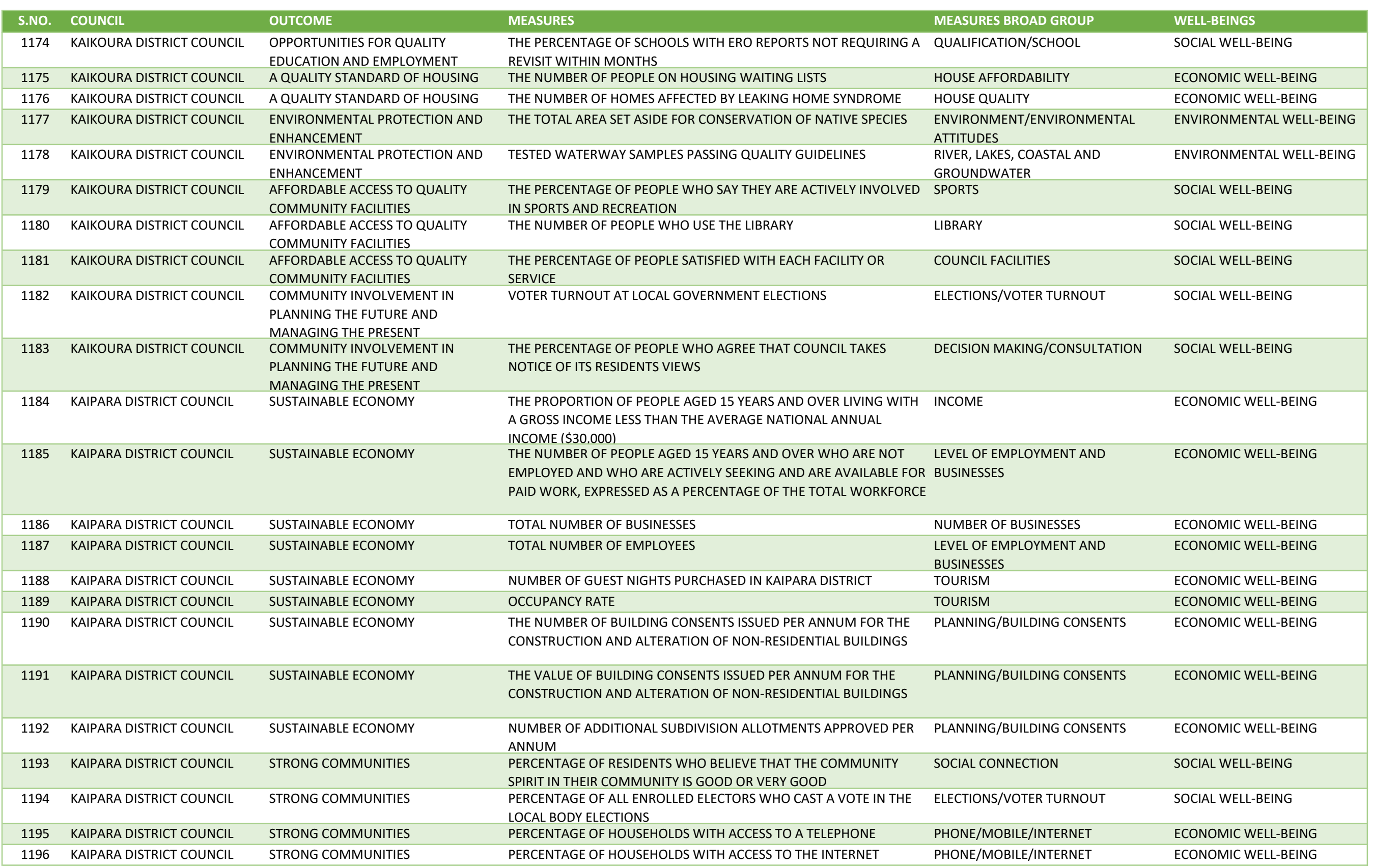




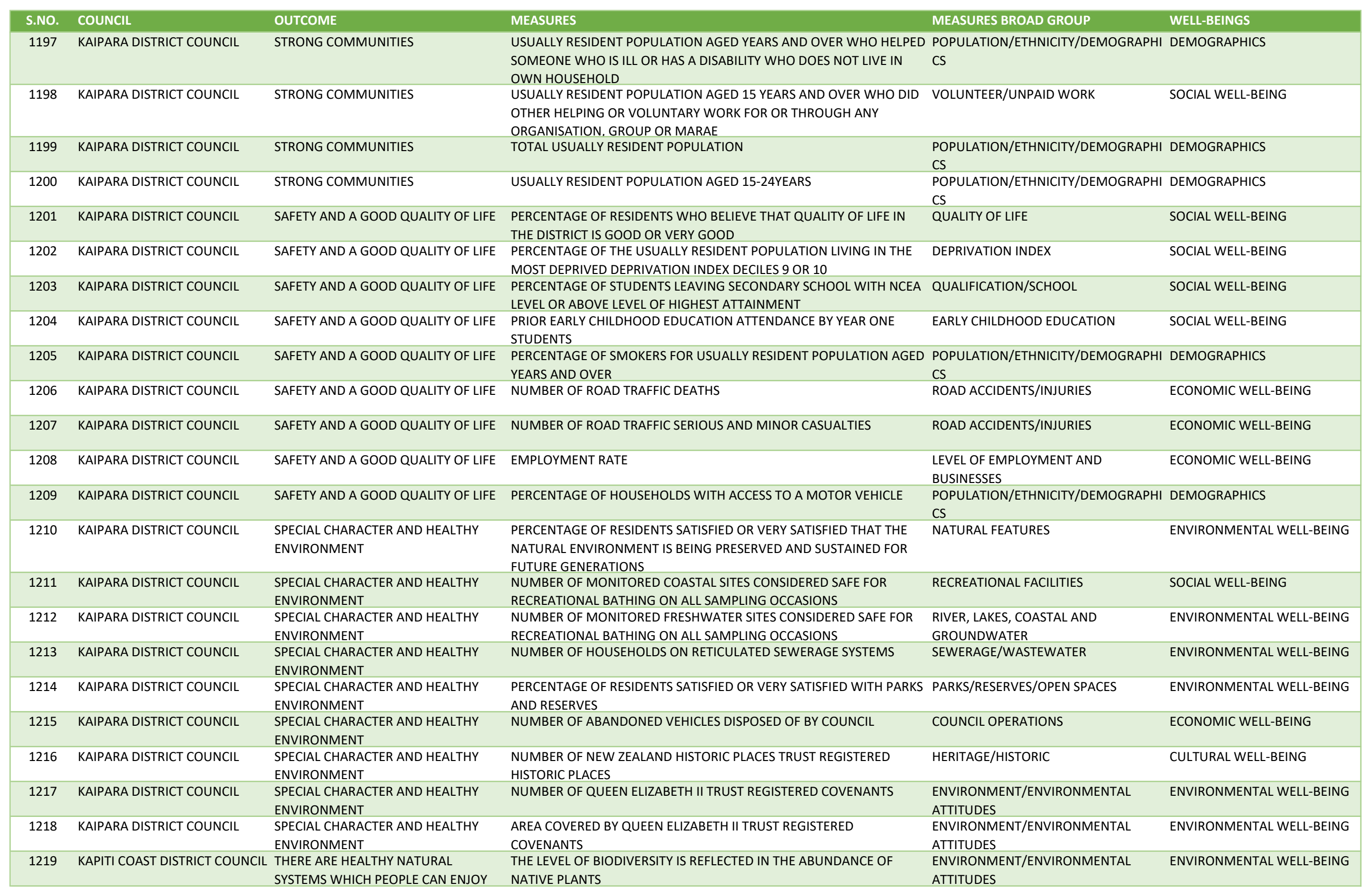




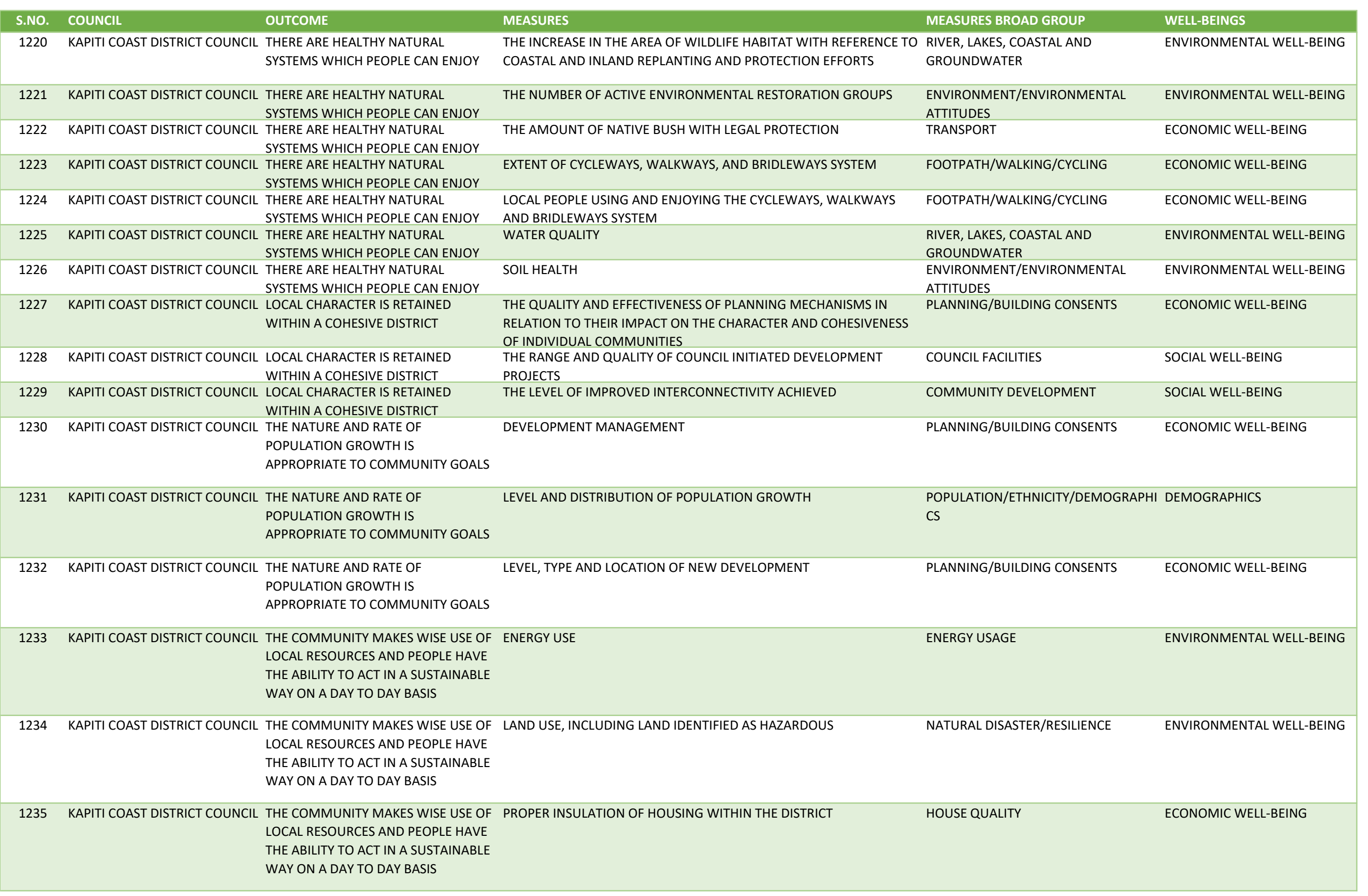




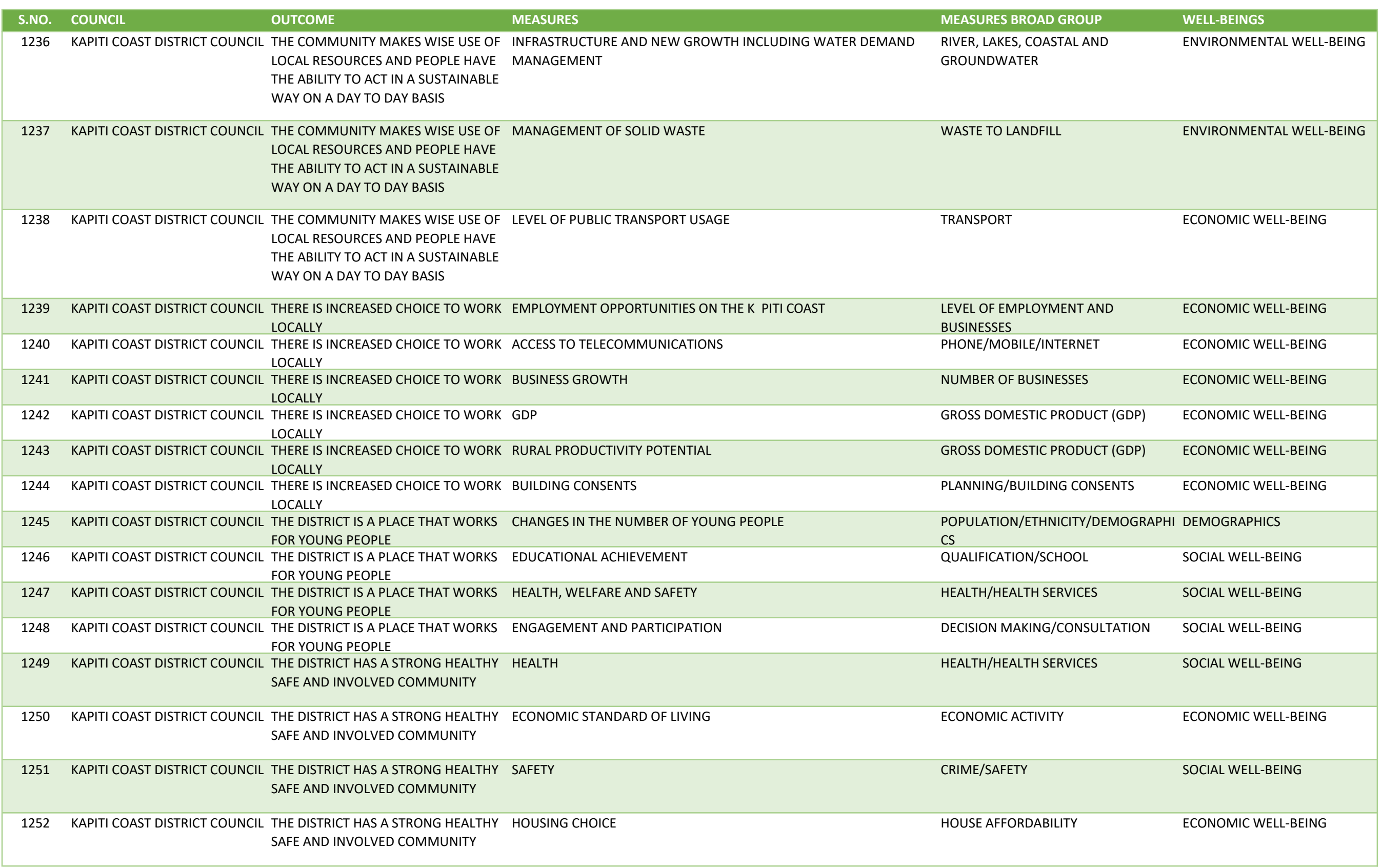




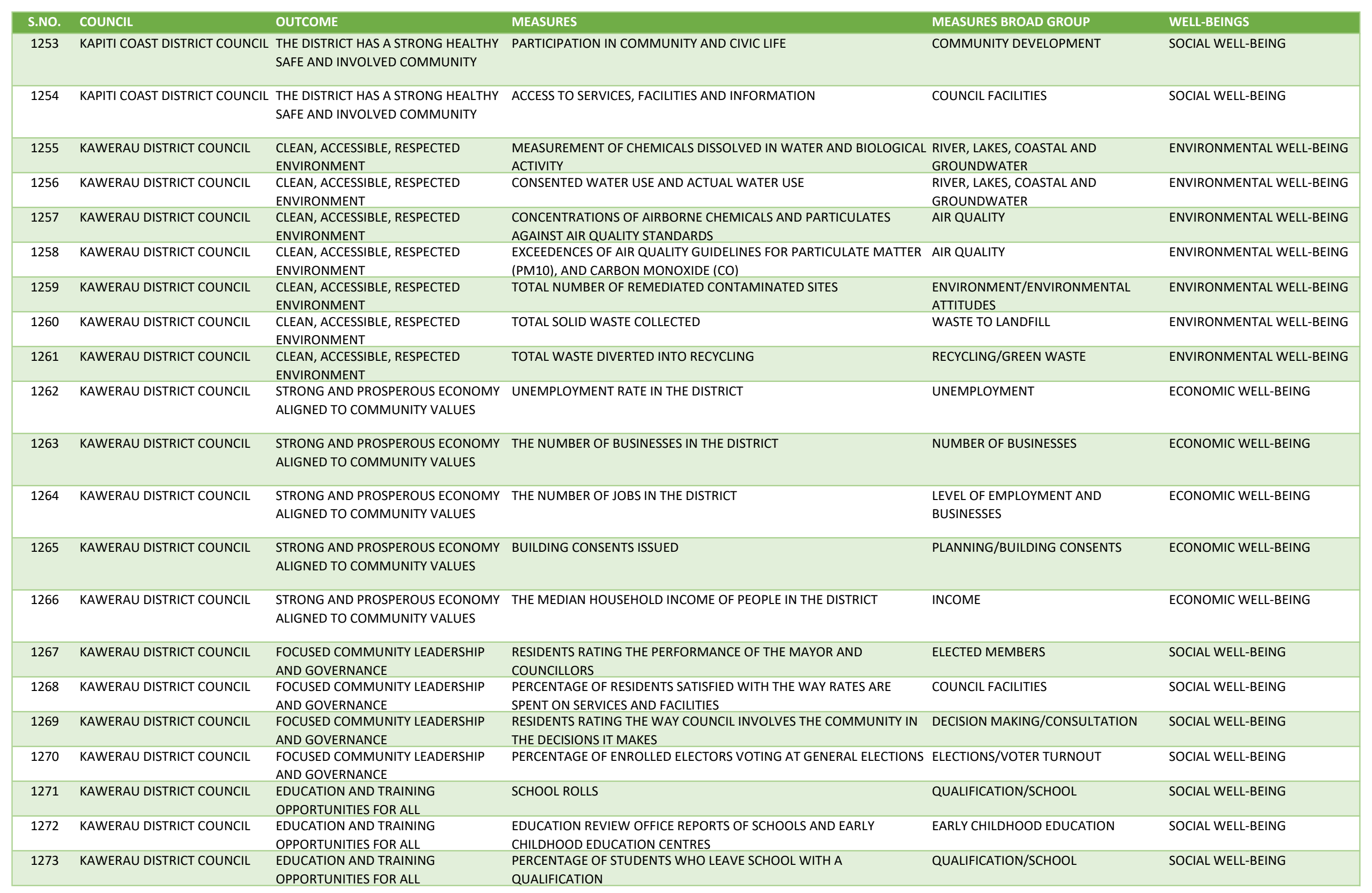


Appendix A: List of Community Well-beings Outcomes and Measures by Councils

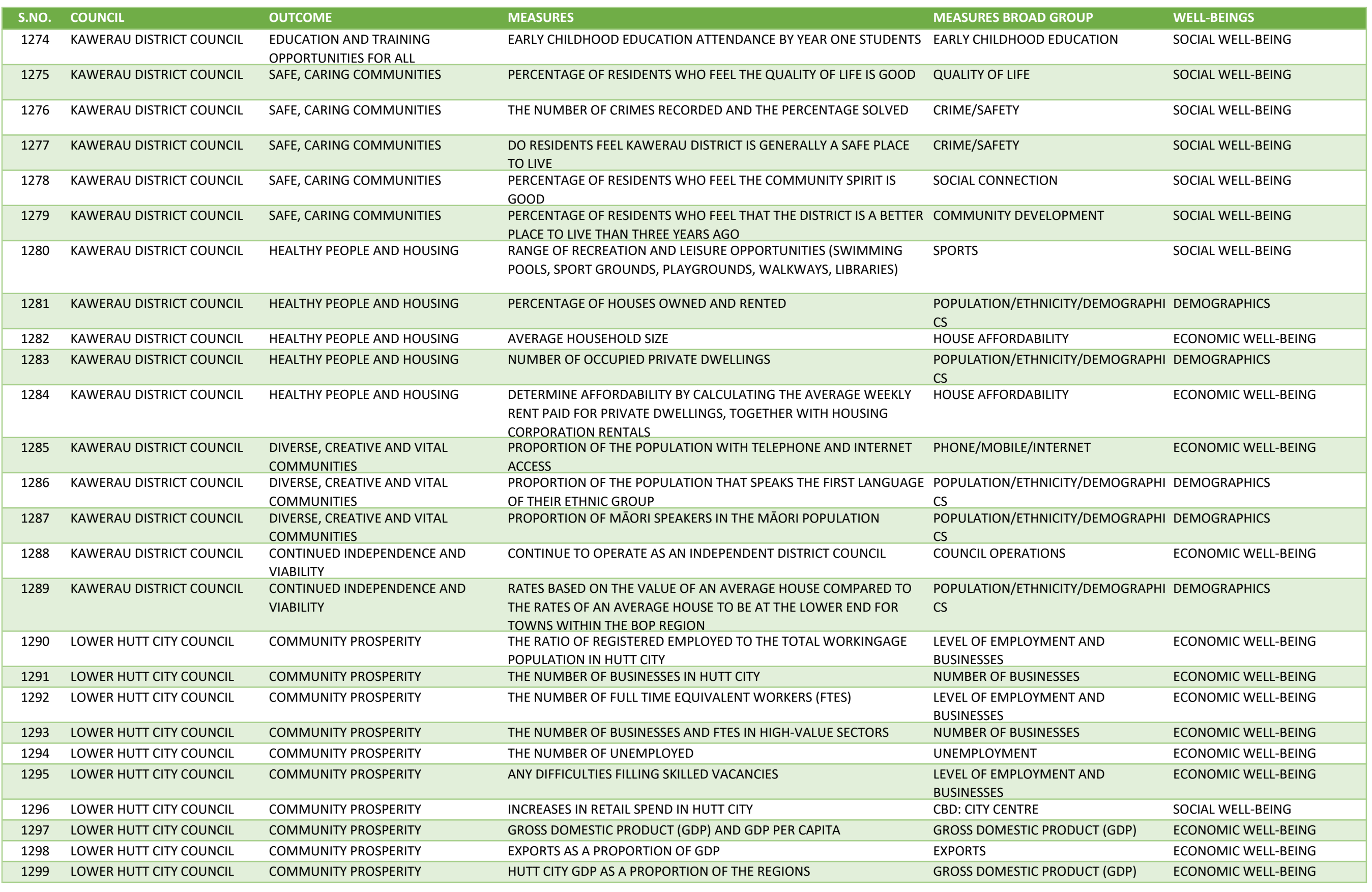


Appendix A: List of Community Well-beings Outcomes and Measures by Councils

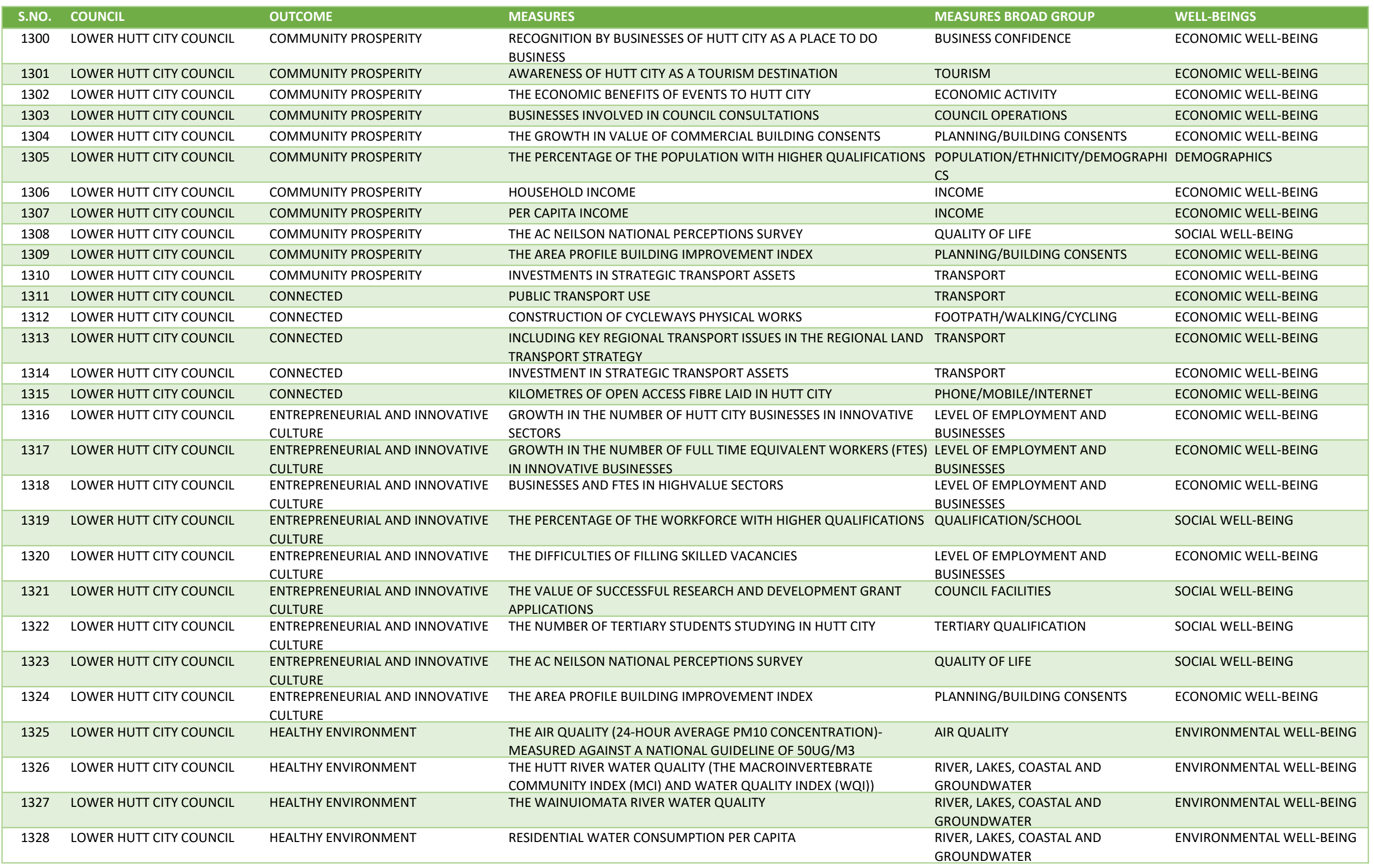




\begin{tabular}{|c|c|c|c|c|c|}
\hline S.NO. & COUNCIL & OUTCOME & MEASURES & MEASURES BROAD GROUP & WELL-BEINGS \\
\hline 1329 & LOWER HUTT CITY COUNCIL & HEALTHY ENVIRONMENT & $\begin{array}{l}\text { SOIL QUALITY -- HAZARDOUS WASTE COLLECTION', A MEASURE OF THE } \\
\text { AMOUNT OF HAZARDOUS WASTE REMOVED PERMANENTLY FROM } \\
\text { SOIL AND THE POTENTIALLY HARMFUL EFFECTS OF SPILLAGE }\end{array}$ & SOIL QUALITY & ENVIRONMENTAL WELL-BEING \\
\hline 1330 & LOWER HUTT CITY COUNCIL & HEALTHY ENVIRONMENT & $\begin{array}{l}\text { WASTE TO LANDFILL PER DOLLAR OF GROSS DOMESTIC PRODUCT } \\
\text { (GDP) }\end{array}$ & WASTE TO LANDFILL & ENVIRONMENTAL WELL-BEING \\
\hline 1331 & LOWER HUTT CITY COUNCIL & HEALTHY ENVIRONMENT & CARBON DIOXIDE EMISSIONS PER DOLLAR OF GDP & GROSS DOMESTIC PRODUCT (GDP) & ECONOMIC WELL-BEING \\
\hline 1332 & LOWER HUTT CITY COUNCIL & HEALTHY ENVIRONMENT & THE VOLUME OF RECYCLING PER CAPITA & RECYCLING/GREEN WASTE & ENVIRONMENTAL WELL-BEING \\
\hline 1333 & LOWER HUTT CITY COUNCIL & HEALTHY ENVIRONMENT & GREEN WASTE DIVERTED FROM LANDFILL & RECYCLING/GREEN WASTE & ENVIRONMENTAL WELL-BEING \\
\hline 1334 & LOWER HUTT CITY COUNCIL & HEALTHY ENVIRONMENT & OTHER WASTE DIVERTED FROM LANDFILL & WASTE TO LANDFILL & ENVIRONMENTAL WELL-BEING \\
\hline 1335 & LOWER HUTT CITY COUNCIL & HEALTHY ENVIRONMENT & $\begin{array}{l}\text { THE PROPORTION OF NEWLY REGISTERED LIGHT VEHICLES IN HUTT } \\
\text { CITY WITH A FUEL ECONOMY OF SIX LITRES PER } 100 \text { KILOMETRES OR } \\
\text { BETTER }\end{array}$ & TRANSPORT & ECONOMIC WELL-BEING \\
\hline 1336 & LOWER HUTT CITY COUNCIL & HEALTHY ENVIRONMENT & FUEL USE PER CAPITA PER ANNUM & ENERGY USAGE & ENVIRONMENTAL WELL-BEING \\
\hline 1337 & LOWER HUTT CITY COUNCIL & HEALTHY ENVIRONMENT & THE PROPORTION OF DRIVER VEHICLE TRIPS MADE TO WORK & $\begin{array}{l}\text { RIVER, LAKES, COASTAL AND } \\
\text { GROUNDWATER }\end{array}$ & ENVIRONMENTAL WELL-BEING \\
\hline 1338 & LOWER HUTT CITY COUNCIL & HEALTHY ENVIRONMENT & KILOMETRES TRAVELLED BY CAR ON COUNCIL BUSINESS & COUNCIL OPERATIONS & ECONOMIC WELL-BEING \\
\hline 1339 & LOWER HUTT CITY COUNCIL & HEALTHY ENVIRONMENT & $\begin{array}{l}\text { THE PROPORTION OF COUNCIL STAFF TRAVELLING TO/FROM WORK IN } \\
\text { SOLE-OCCUPANCY CARS }\end{array}$ & TRANSPORT & ECONOMIC WELL-BEING \\
\hline 1340 & LOWER HUTT CITY COUNCIL & HEALTHY ENVIRONMENT & PUBLIC TRANSPORT PATRONAGE & TRANSPORT & ECONOMIC WELL-BEING \\
\hline 1342 & LOWER HUTT CITY COUNCIL & HEALTHY ENVIRONMENT & THE NUMBER OF CONSENTS FOR SOLAR HOT WATER HEATING & ENERGY USAGE & ENVIRONMENTAL WELL-BEING \\
\hline 1343 & LOWER HUTT CITY COUNCIL & HEALTHY ENVIRONMENT & $\begin{array}{l}\text { THE NUMBER OF CONSENTS FOR SMALL-SCALE LOCAL ELECTRICITY } \\
\text { PRODUCTION PER ANNUM }\end{array}$ & ENERGY USAGE & ENVIRONMENTAL WELL-BEING \\
\hline 1344 & LOWER HUTT CITY COUNCIL & HEALTHY ENVIRONMENT & $\begin{array}{l}\text { THE PROPORTION OF HUTT CITY HOUSING WITH FLOOR AND CEILING } \\
\text { INSULATION }\end{array}$ & HOUSE QUALITY & ECONOMIC WELL-BEING \\
\hline 1345 & LOWER HUTT CITY COUNCIL & HEALTHY ENVIRONMENT & $\begin{array}{l}\text { THE PROPORTION OF HUTT CITY HOUSING WITH EFFICIENT HEATING } \\
\text { (HEAT PUMPS, NATURAL GAS AND PELLET FIRES) }\end{array}$ & ENERGY USAGE & ENVIRONMENTAL WELL-BEING \\
\hline 1346 & LOWER HUTT CITY COUNCIL & HEALTHY ENVIRONMENT & THE COUNCILS ENERGY CONSUMPTION (ELECTRICITY AND GAS) & COUNCIL OPERATIONS & ENVIRONMENTAL WELL-BEING \\
\hline 1347 & LOWER HUTT CITY COUNCIL & HEALTHY ENVIRONMENT & COUNCIL-OWNED AND ADMINISTERED LAND BIOMASS & NATURAL FEATURES & ENVIRONMENTAL WELL-BEING \\
\hline 1348 & LOWER HUTT CITY COUNCIL & HEALTHY ENVIRONMENT & THE URBAN (BUILT-UP AREA) BIOMASS & $\begin{array}{l}\text { ENVIRONMENT/ENVIRONMENTAL } \\
\text { ATTITUDES }\end{array}$ & ENVIRONMENTAL WELL-BEING \\
\hline 1349 & LOWER HUTT CITY COUNCIL & HEALTHY ENVIRONMENT & THE DISTRICT BIOMASS & NATURAL FEATURES & ENVIRONMENTAL WELL-BEING \\
\hline 1350 & LOWER HUTT CITY COUNCIL & HEALTHY ENVIRONMENT & $\begin{array}{l}\text { THE PROPORTION OF NATIVE FOREST TO TOTAL BIOMASS (DISTRICT } \\
\text { WIDE) }\end{array}$ & NATURAL FEATURES & ENVIRONMENTAL WELL-BEING \\
\hline 1351 & LOWER HUTT CITY COUNCIL & HEALTHY ENVIRONMENT & $\begin{array}{l}\text { THE NUMBER OF ENVIRONMENTAL SUSTAINABILITY STRATEGY } \\
\text { ACTIONS COMPLETED }\end{array}$ & $\begin{array}{l}\text { ENVIRONMENT/ENVIRONMENTAL } \\
\text { ATTITUDES }\end{array}$ & ENVIRONMENTAL WELL-BEING \\
\hline 1352 & LOWER HUTT CITY COUNCIL & HEALTHY ENVIRONMENT & OUR ENVIRONMENTAL ACCREDITATION TO ISO 14001 & $\begin{array}{l}\text { ENVIRONMENT/ENVIRONMENTAL } \\
\text { ATTITUDES }\end{array}$ & ENVIRONMENTAL WELL-BEING \\
\hline 1353 & LOWER HUTT CITY COUNCIL & LIFESTYLE & THE PERCENTAGE OF RESIDENTS WHO USE POOLS & COUNCIL FACILITIES & SOCIAL WELL-BEING \\
\hline 1357 & LOWER HUTT CITY COUNCIL & LIFESTYLE & $\begin{array}{l}\text { THE PERCENTAGE OF RESIDENTS WHO ARE SATISFIED WITH THE CITYS } \\
\text { PARKS RESERVES AND GARDENS }\end{array}$ & PARKS/RESERVES/OPEN SPACES & ENVIRONMENTAL WELL-BEING \\
\hline 1358 & LOWER HUTT CITY COUNCIL & LIFESTYLE & $\begin{array}{l}\text { THE PERCENTAGE OF RESIDENTS WHO FEEL SAFE IN THE CITY IN } \\
\text { GENERAL }\end{array}$ & CRIME/SAFETY & SOCIAL WELL-BEING \\
\hline
\end{tabular}


Appendix A: List of Community Well-beings Outcomes and Measures by Councils






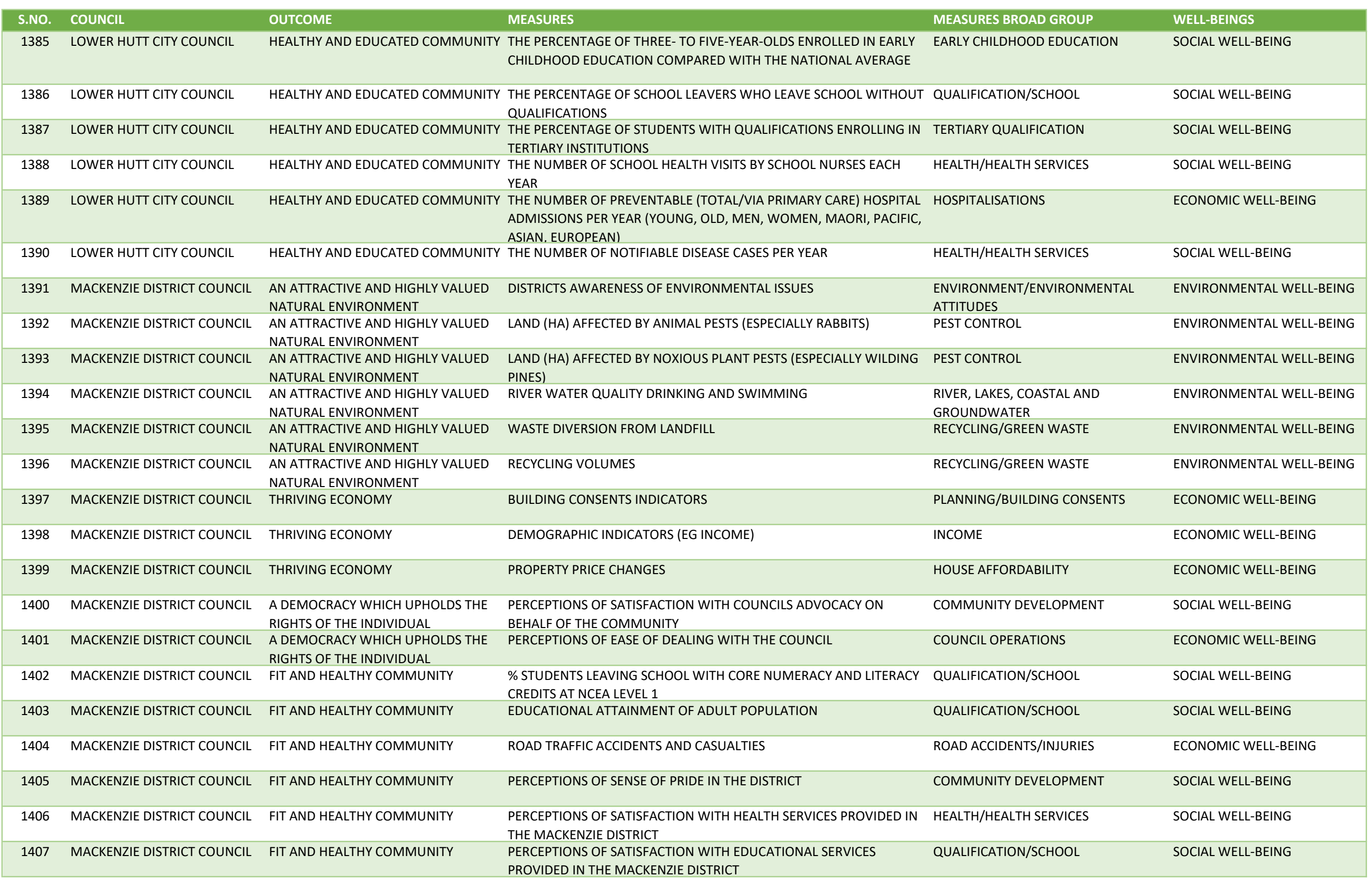


Appendix A: List of Community Well-beings Outcomes and Measures by Councils

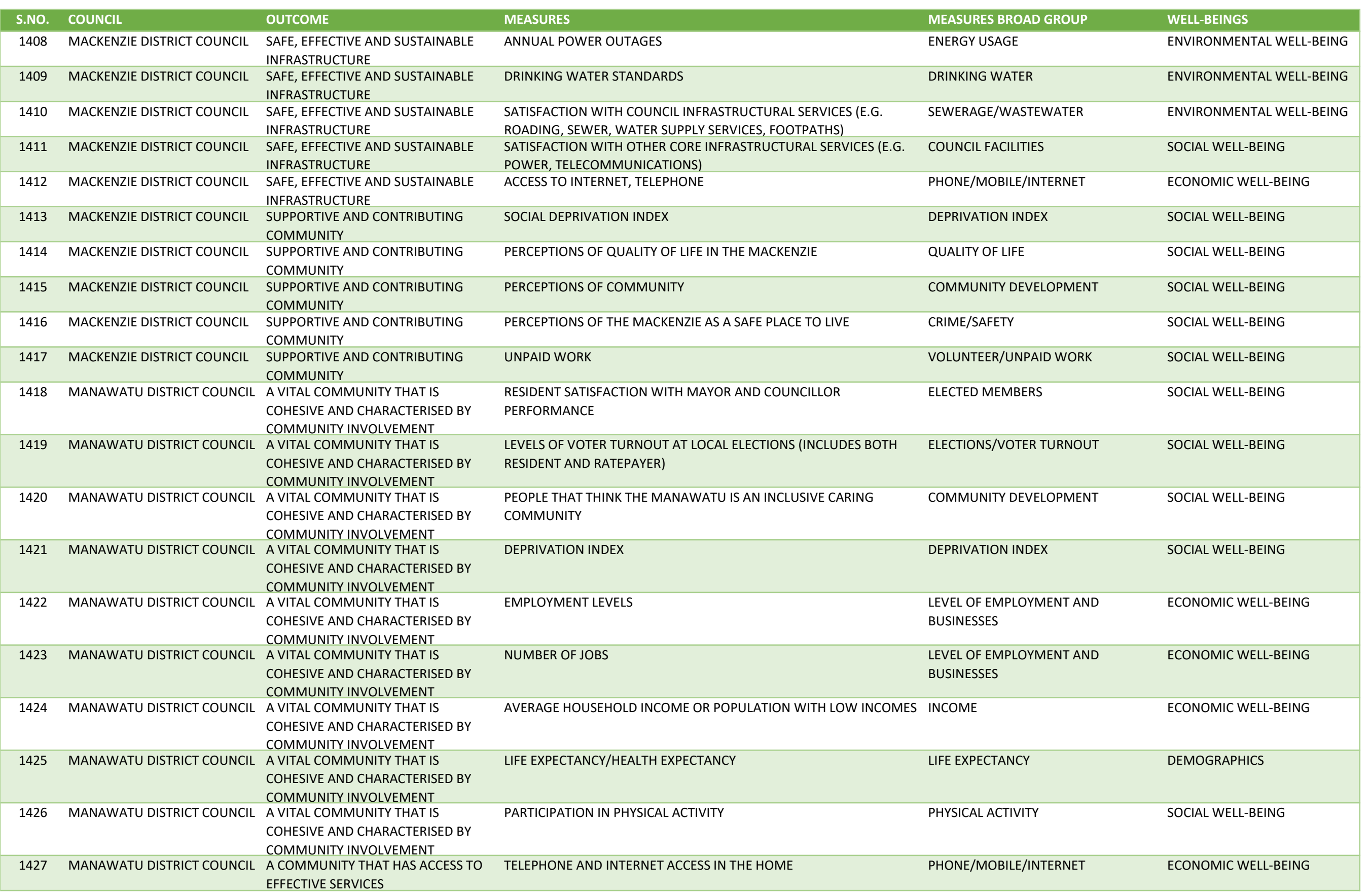




\begin{tabular}{|c|c|c|c|c|c|}
\hline S.NO. & COUNCIL & OUTCOME & MEASURES & MEASURES BROAD GROUP & WELL-BEINGS \\
\hline 1428 & MANAWATU DISTRICT COUNCIL & $\begin{array}{l}\text { A COMMUNITY THAT HAS ACCESS TO } \\
\text { EFFECTIVE SERVICES }\end{array}$ & $\begin{array}{l}\text { COMMUNITY LEVELS OF SATISFACTION WITH COUNCIL SERVICES } \\
\text { INCLUDING ROADS, FOOTPATHS, WATER, STORMWATER, } \\
\text { WASTEWATER AND WASTE }\end{array}$ & SEWERAGE/WASTEWATER & ENVIRONMENTAL WELL-BEING \\
\hline 1429 & MANAWATU DISTRICT COUNCIL & $\begin{array}{l}\text { A COMMUNITY THAT HAS ACCESS TO } \\
\text { EFFECTIVE SERVICES }\end{array}$ & ASSET MANAGEMENT PLANS & COUNCIL OPERATIONS & ECONOMIC WELL-BEING \\
\hline 1430 & MANAWATU DISTRICT COUNCIL & $\begin{array}{l}\text { A SELF-SUFFICIENT ENVIRONMENT } \\
\text { THAT PRESERVES, VALUES AND } \\
\text { DEVELOPS OUR NATURAL } \\
\text { ENVIRONMENT }\end{array}$ & WATER QUALITY & $\begin{array}{l}\text { RIVER, LAKES, COASTAL AND } \\
\text { GROUNDWATER }\end{array}$ & ENVIRONMENTAL WELL-BEING \\
\hline 1431 & MANAWATU DISTRICT COUNCIL & $\begin{array}{l}\text { A SELFSUFFICIENT ENVIRONMENT THAT } \\
\text { PRESERVES VALUES AND DEVELOPS } \\
\text { OUR NATURAL ENVIRONMENT }\end{array}$ & AIR QUALITY & AIR QUALITY & ENVIRONMENTAL WELL-BEING \\
\hline 1432 & MANAWATU DISTRICT COUNCIL & $\begin{array}{l}\text { A SELFSUFFICIENT ENVIRONMENT THAT } \\
\text { PRESERVES VALUES AND DEVELOPS } \\
\text { OUR NATURAL ENVIRONMENT }\end{array}$ & $\begin{array}{l}\text { LEVELS OF COMMUNITY SATISFACTION WITH PARKS, RESERVES AND } \\
\text { SPORTSGROUNDS }\end{array}$ & PARKS/RESERVES/OPEN SPACES & ENVIRONMENTAL WELL-BEING \\
\hline 1433 & MANAWATU DISTRICT COUNCIL & $\begin{array}{l}\text { A SELFSUFFICIENT ENVIRONMENT THAT } \\
\text { PRESERVES VALUES AND DEVELOPS } \\
\text { OUR NATURAL ENVIRONMENT }\end{array}$ & TOURIST NUMBERS OVERNIGHT STAYS WITHIN THE DISTRICT & TOURISM & ECONOMIC WELL-BEING \\
\hline 1435 & MANAWATU DISTRICT COUNCIL & $\begin{array}{l}\text { A SELFSUFFICIENT ENVIRONMENT THAT } \\
\text { PRESERVES VALUES AND DEVELOPS } \\
\text { OUR NATURAL ENVIRONMENT }\end{array}$ & $\begin{array}{l}\text { COMMUNITY LEVELS OF SATISFACTION WITH COUNCIL SERVICES } \\
\text { INCLUDING ROADS, WASTE MANAGEMENT, EMERGENCY SERVICES, } \\
\text { ECONOMIC DEVELOPMENT AND PARKS AND RESERVES }\end{array}$ & COUNCIL OPERATIONS & ECONOMIC WELL-BEING \\
\hline 1436 & MANAWATU DISTRICT COUNCIL & $\begin{array}{l}\text { PEOPLE ARE ABLE TO GO ABOUT THEIR } \\
\text { BUSINESS AND LEISURE ANY TIME OF } \\
\text { THE DAY OR EVENING WITHOUT FEAR } \\
\text { FOR THEIR SAFETY }\end{array}$ & ROAD ACCIDENTS & ROAD ACCIDENTS/INJURIES & ECONOMIC WELL-BEING \\
\hline 1437 & MANAWATU DISTRICT COUNCIL & $\begin{array}{l}\text { PEOPLE ARE ABLE TO GO ABOUT THEIR } \\
\text { BUSINESS AND LEISURE ANY TIME OF } \\
\text { THE DAY OR EVENING WITHOUT FEAR } \\
\text { FOR THEIR SAFETY }\end{array}$ & PERCEPTIONS OF SAFETY (PEOPLE FEEL SAFE) & CRIME/SAFETY & SOCIAL WELL-BEING \\
\hline 1438 & MANAWATU DISTRICT COUNCIL & $\begin{array}{l}\text { PEOPLE ARE ABLE TO GO ABOUT THEIR } \\
\text { BUSINESS AND LEISURE ANY TIME OF } \\
\text { THE DAY OR EVENING WITHOUT FEAR } \\
\text { FOR THEIR SAFETY }\end{array}$ & CRIME FIGURES & CRIME/SAFETY & SOCIAL WELL-BEING \\
\hline 1439 & MANAWATU DISTRICT COUNCIL & $\begin{array}{l}\text { PEOPLE ARE ABLE TO GO ABOUT THEIR } \\
\text { BUSINESS AND LEISURE ANY TIME OF } \\
\text { THE DAY OR EVENING WITHOUT FEAR } \\
\text { FOR THEIR SAFETY }\end{array}$ & INJURY ACCIDENTS & ROAD ACCIDENTS/INJURIES & ECONOMIC WELL-BEING \\
\hline 1440 & $\begin{array}{l}\text { MANAWATUWANGANUI } \\
\text { REGIONAL COUNCIL }\end{array}$ & NATURAL HAZARD RESILIENCE & $\begin{array}{l}\text { REDUCING THE RISKS FROM NATURAL HAZARDS TO ACCEPTABLE } \\
\text { LEVELS THROUGH SUITABLE POLICIES, PLANNING AND } \\
\text { INFRASTRUCTURE }\end{array}$ & NATURAL DISASTER/RESILIENCE & ENVIRONMENTAL WELL-BEING \\
\hline
\end{tabular}




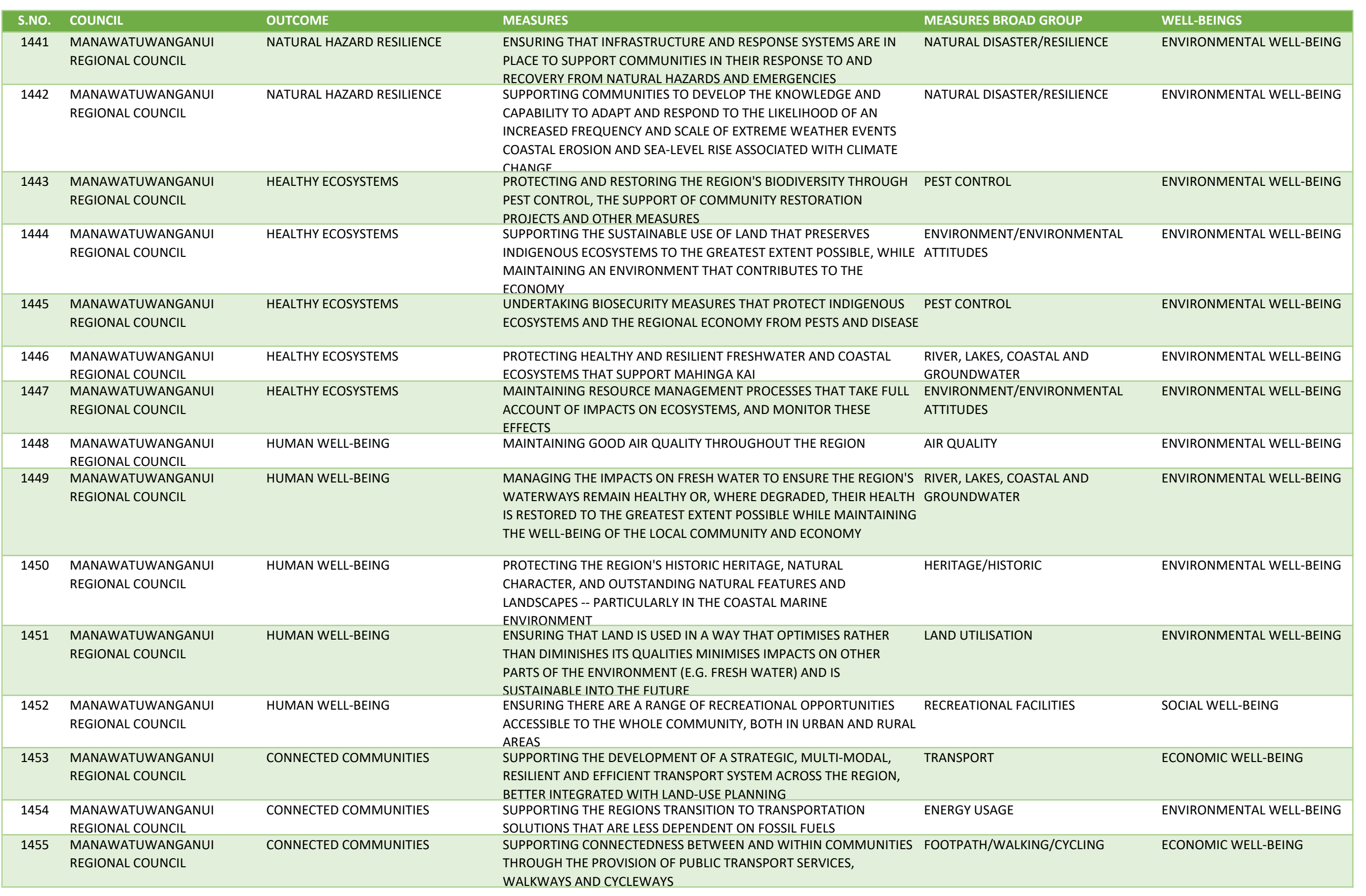




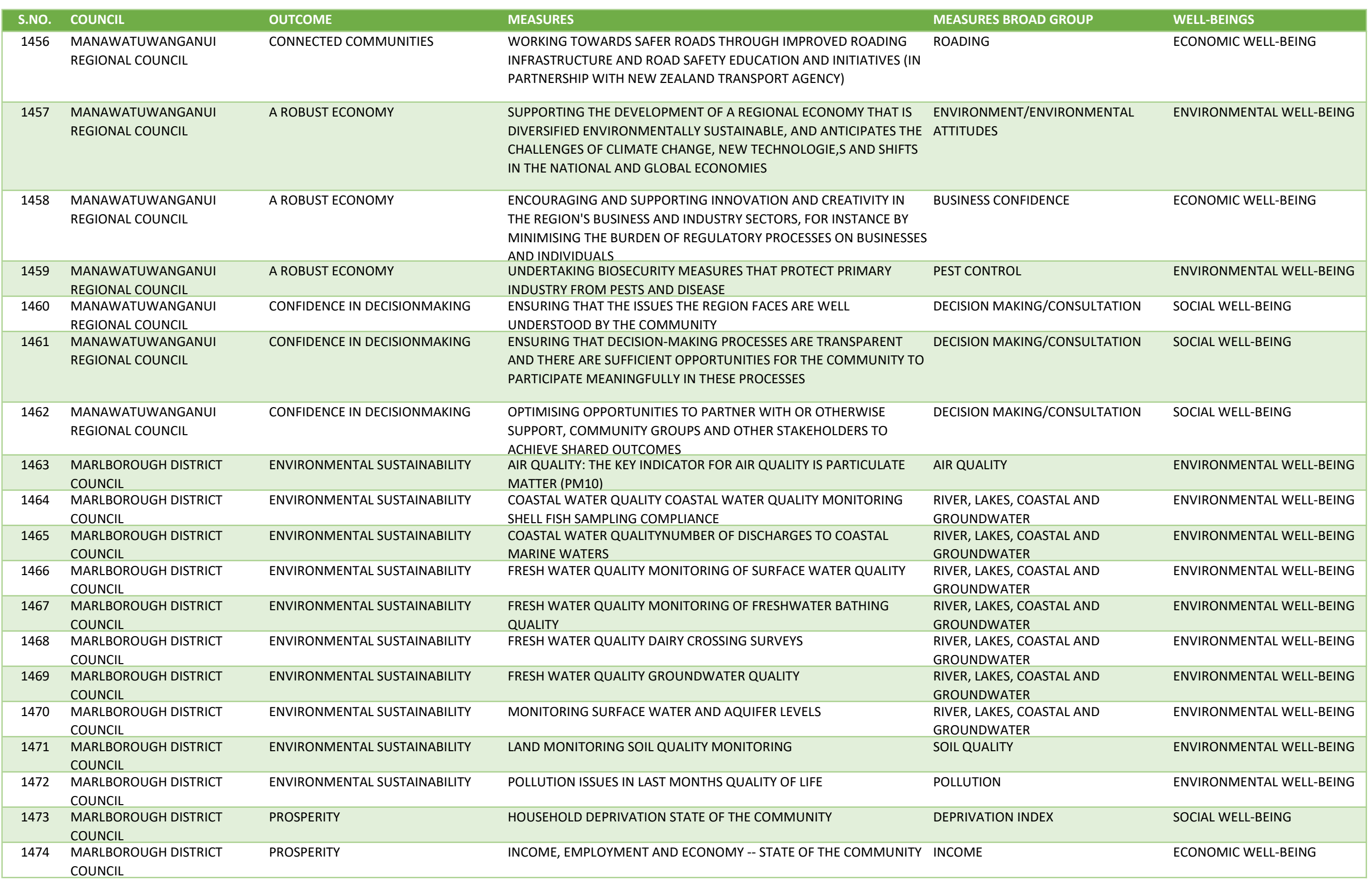


Appendix A: List of Community Well-beings Outcomes and Measures by Councils

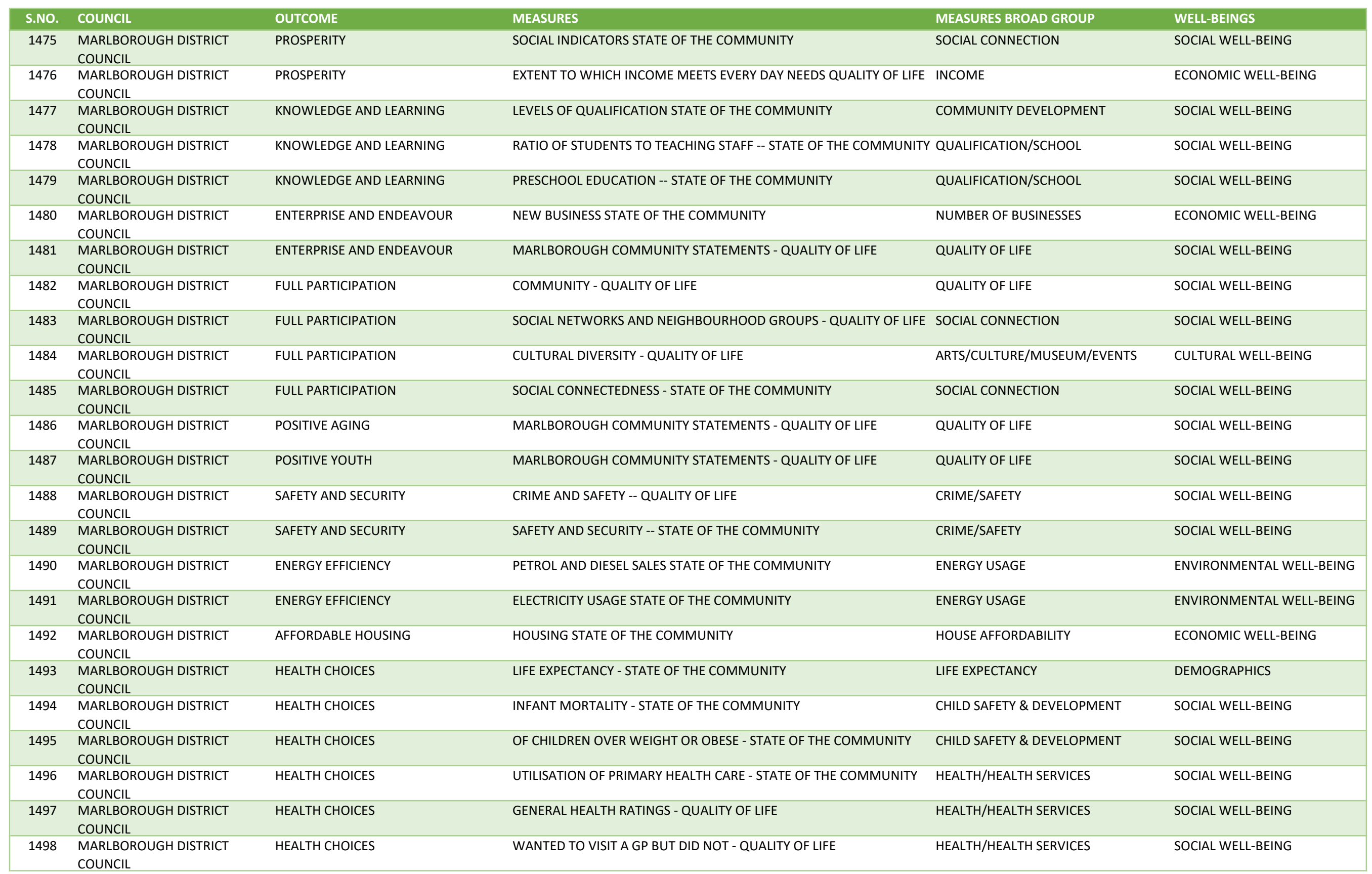


Appendix A: List of Community Well-beings Outcomes and Measures by Councils

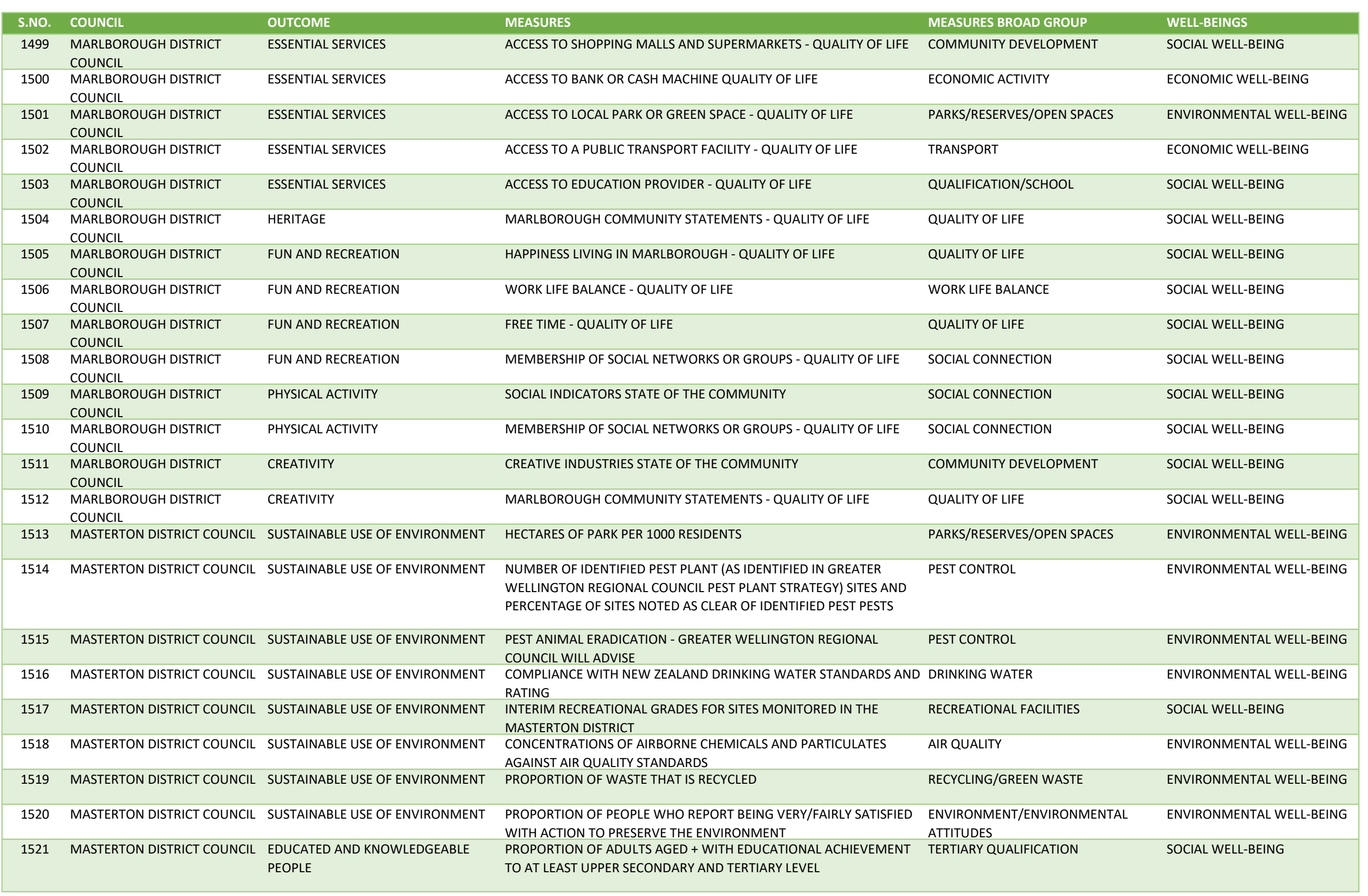


Appendix A: List of Community Well-beings Outcomes and Measures by Councils

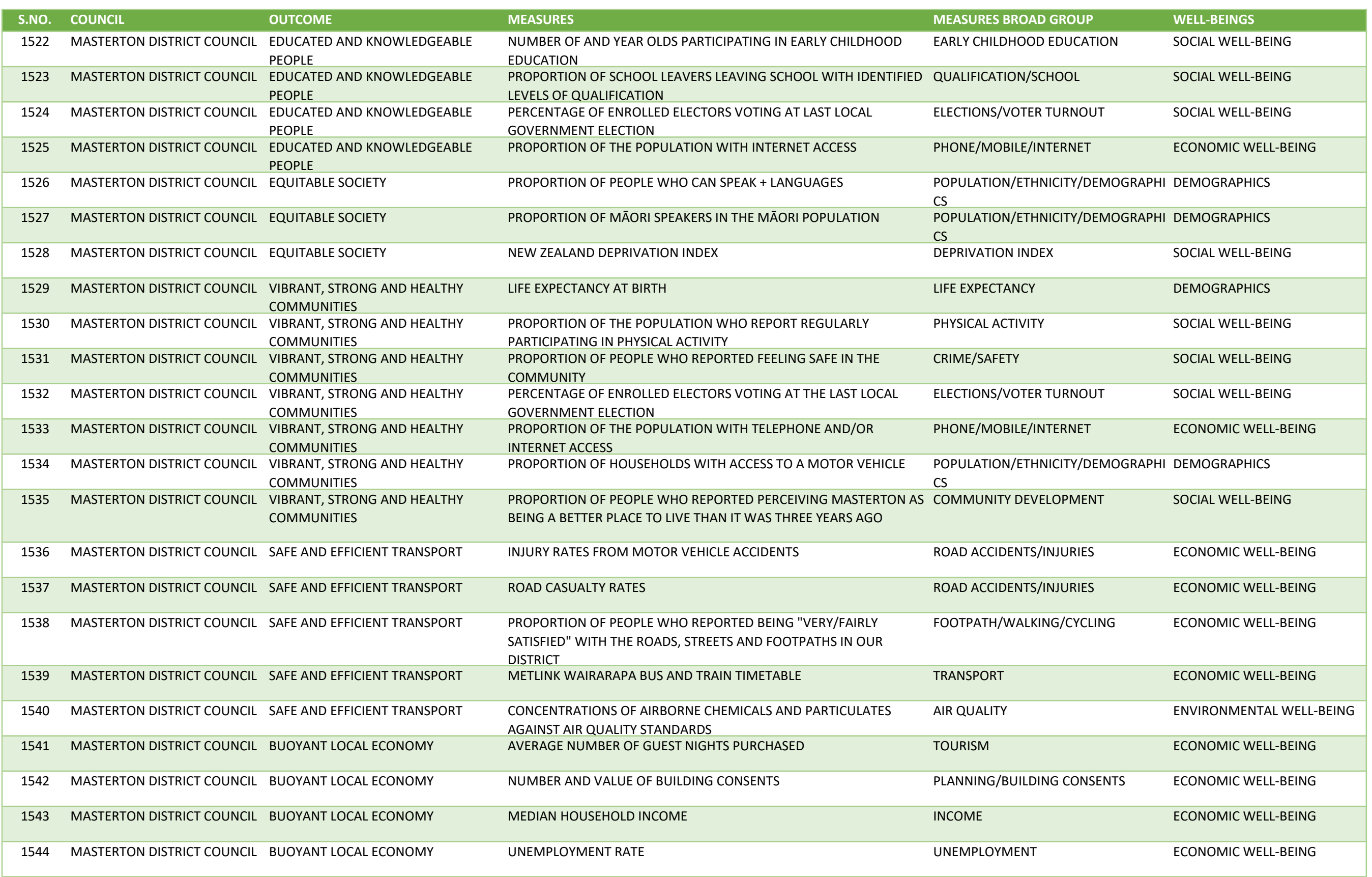


Appendix A: List of Community Well-beings Outcomes and Measures by Councils

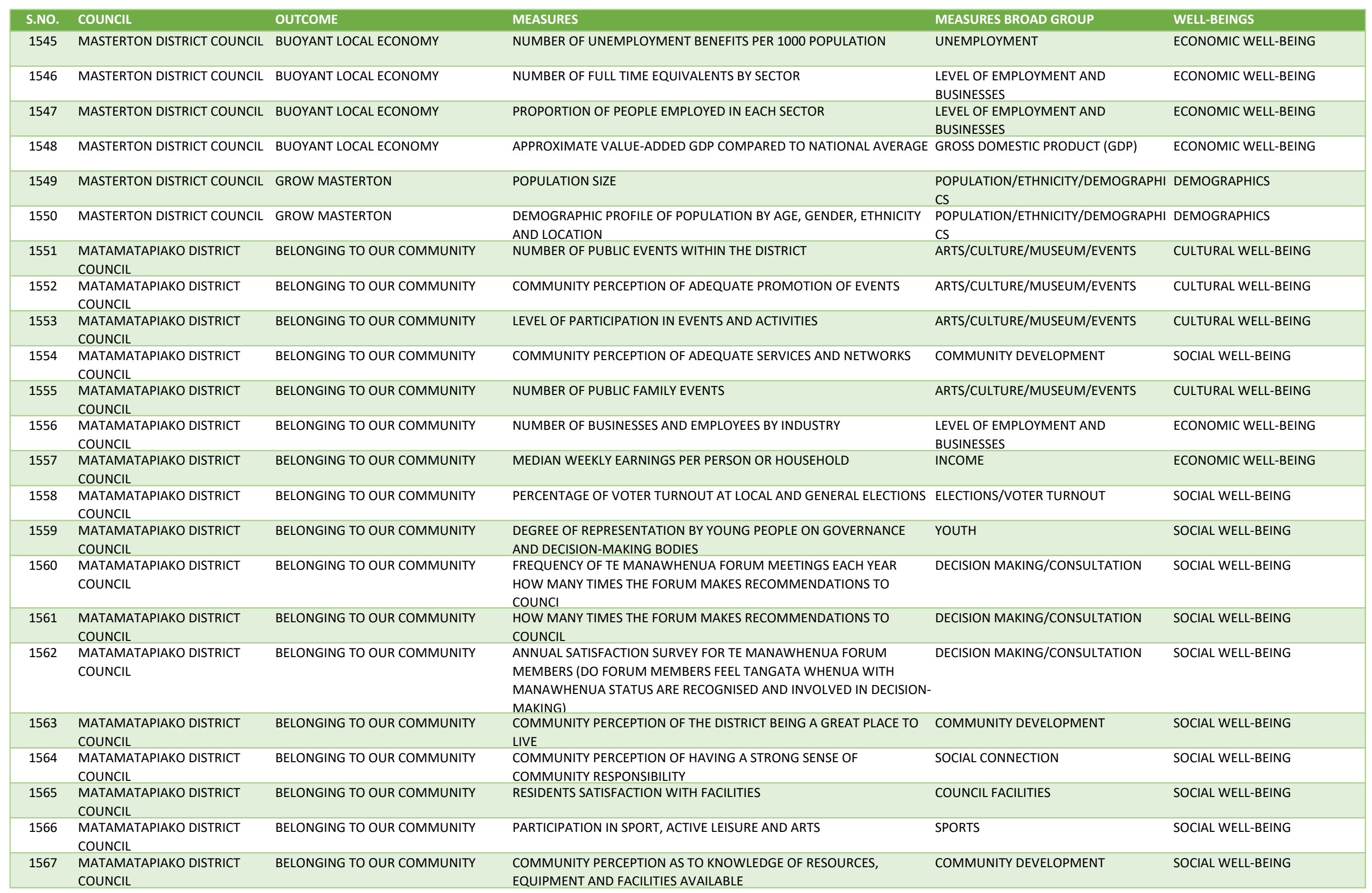


Appendix A: List of Community Well-beings Outcomes and Measures by Councils

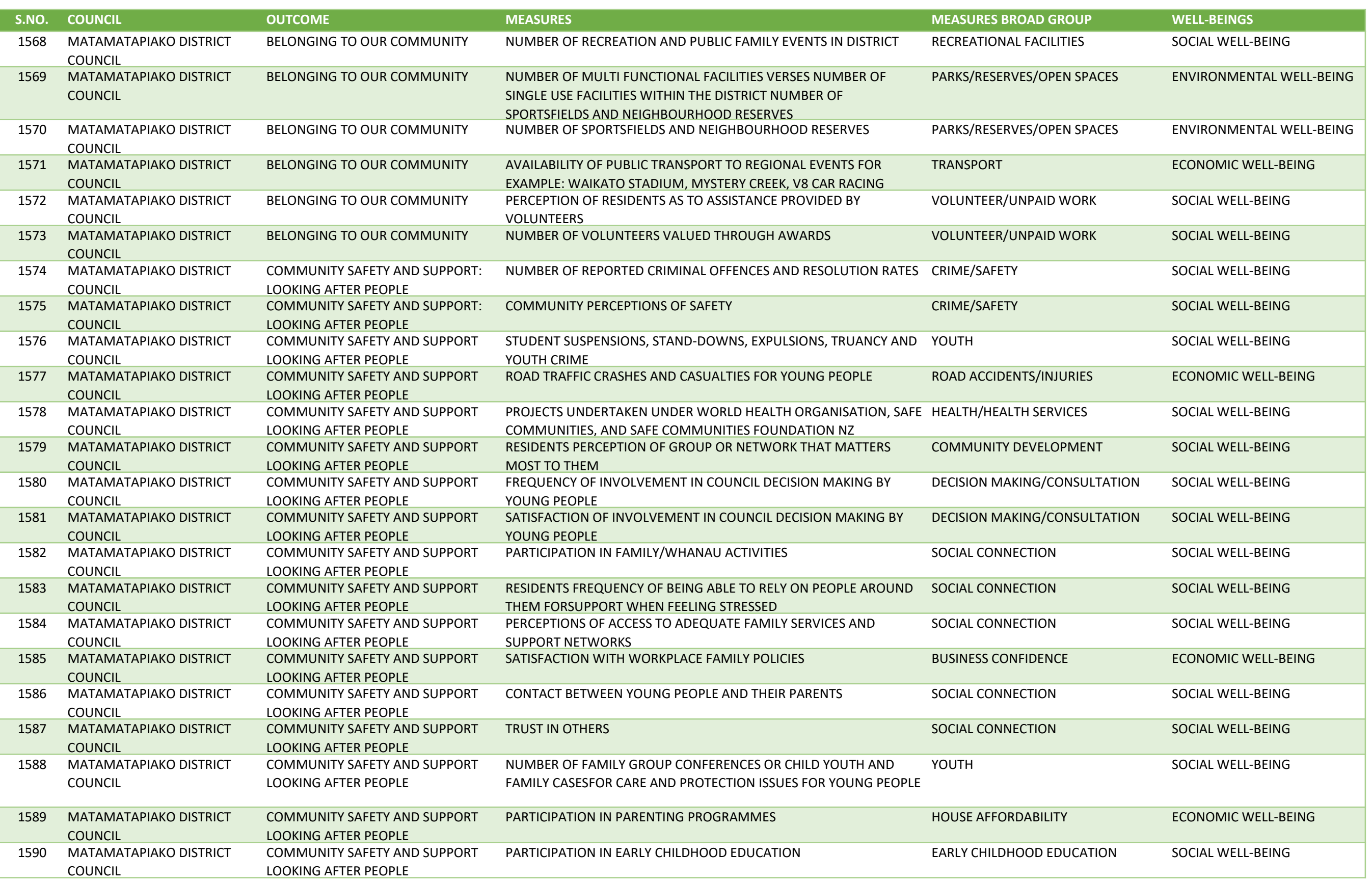


Appendix A: List of Community Well-beings Outcomes and Measures by Councils

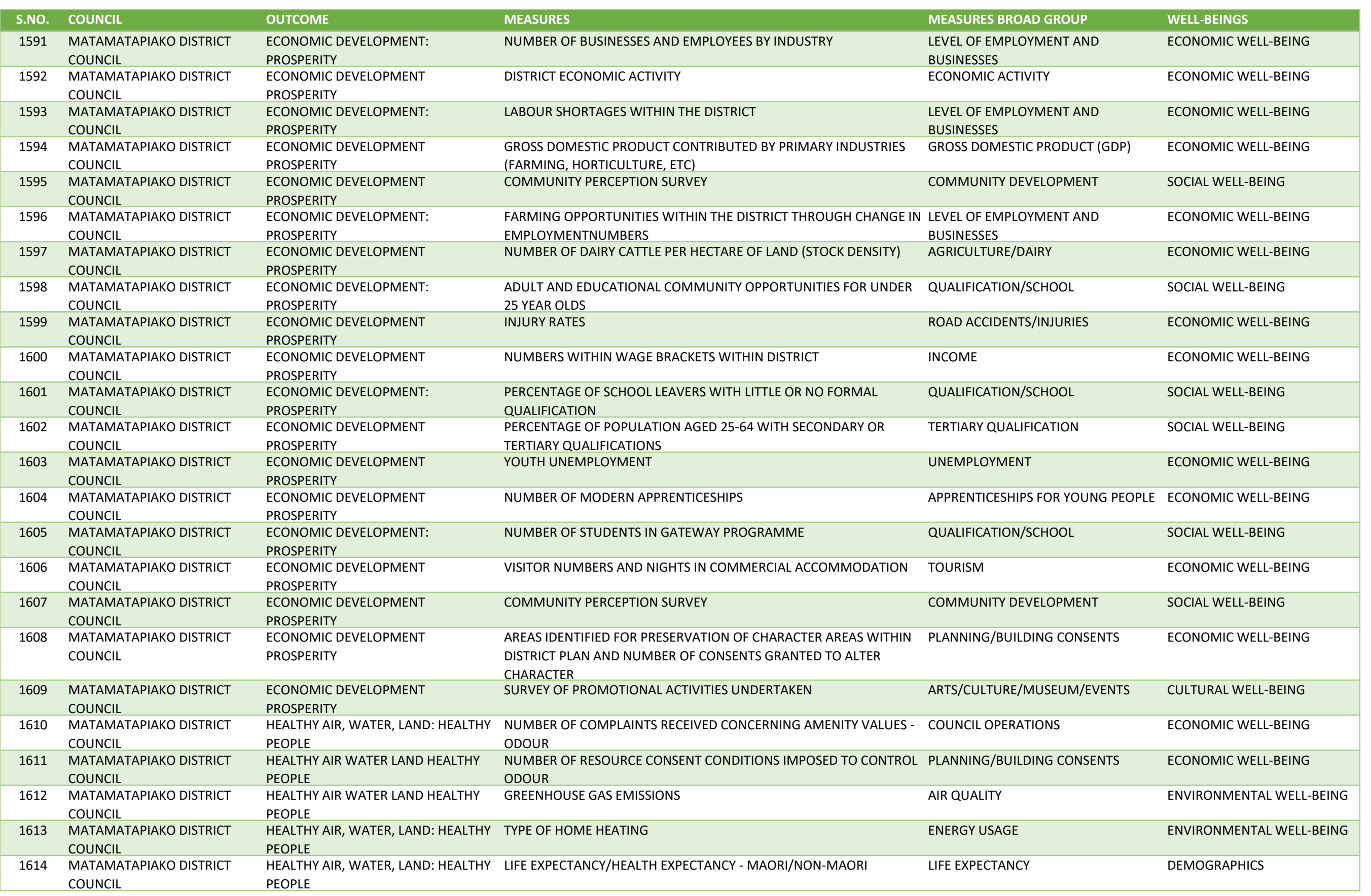




\begin{tabular}{|c|c|c|c|c|c|}
\hline S.NO. & COUNCIL & OUTCOME & MEASURES & MEASURES BROAD GROUP & WELL-BEINGS \\
\hline 1615 & $\begin{array}{l}\text { MATAMATAPIAKO DISTRICT } \\
\text { COUNCIL }\end{array}$ & $\begin{array}{l}\text { HEALTHY AIR, WATER, LAND: HEALTHY } \\
\text { PEOPLE }\end{array}$ & EMOTIONAL WELL-BEING (HAPPINESS) & QUALITY OF LIFE & SOCIAL WELL-BEING \\
\hline 1616 & $\begin{array}{l}\text { MATAMATAPIAKO DISTRICT } \\
\text { COUNCIL }\end{array}$ & $\begin{array}{l}\text { HEALTHY AIR, WATER, LAND: HEALTHY } \\
\text { PEOPLE }\end{array}$ & AVOIDABLE MORTALITY (AND CAUSES) MAORI/NON-MAORI & HOSPITALISATIONS & ECONOMIC WELL-BEING \\
\hline 1617 & $\begin{array}{l}\text { MATAMATAPIAKO DISTRICT } \\
\text { COUNCIL }\end{array}$ & $\begin{array}{l}\text { HEALTHY AIR, WATER, LAND: HEALTHY } \\
\text { PEOPLE }\end{array}$ & INFANT MORTALITY RATE & CHILD SAFETY \& DEVELOPMENT & SOCIAL WELL-BEING \\
\hline 1618 & $\begin{array}{l}\text { MATAMATAPIAKO DISTRICT } \\
\text { COUNCIL }\end{array}$ & $\begin{array}{l}\text { HEALTHY AIR, WATER, LAND: HEALTHY } \\
\text { PEOPLE }\end{array}$ & RATE OF AVOIDABLE HOSPITALISATIONS FOR DIABETES & HOSPITALISATIONS & ECONOMIC WELL-BEING \\
\hline 1619 & $\begin{array}{l}\text { MATAMATAPIAKO DISTRICT } \\
\text { COUNCIL }\end{array}$ & $\begin{array}{l}\text { HEALTHY AIR, WATER, LAND: HEALTHY } \\
\text { PEOPLE }\end{array}$ & PREVALENCE OF CIGARETTE SMOKING - MAORI/NON-MAORI & HEALTH/HEALTH SERVICES & SOCIAL WELL-BEING \\
\hline 1620 & $\begin{array}{l}\text { MATAMATAPIAKO DISTRICT } \\
\text { COUNCIL }\end{array}$ & $\begin{array}{l}\text { HEALTHY AIR, WATER, LAND: HEALTHY } \\
\text { PEOPLE }\end{array}$ & ABUNDANCE AND DISTRIBUTION OF PLANT AND ANIMAL PESTS & PEST CONTROL & ENVIRONMENTAL WELL-BEING \\
\hline 1621 & $\begin{array}{l}\text { MATAMATAPIAKO DISTRICT } \\
\text { COUNCIL }\end{array}$ & $\begin{array}{l}\text { HEALTHY AIR, WATER, LAND: HEALTHY } \\
\text { PEOPLE }\end{array}$ & LOCATION, AREA AND TYPE OF PEST CONTROL OPERATION & PEST CONTROL & ENVIRONMENTAL WELL-BEING \\
\hline 1622 & $\begin{array}{l}\text { MATAMATAPIAKO DISTRICT } \\
\text { COUNCIL }\end{array}$ & $\begin{array}{l}\text { HEALTHY AIR, WATER, LAND: HEALTHY } \\
\text { PEOPLE }\end{array}$ & $\begin{array}{l}\text { QUALITY OF THE DISTRICT WATERWAYS I.E. (TEMP, PH, DO, BOD, } \\
\text { TOTAL N, TURBIDITY, FAECAL COLIFORMS, HEAVY METALS) }\end{array}$ & $\begin{array}{l}\text { RIVER, LAKES, COASTAL AND } \\
\text { GROUNDWATER }\end{array}$ & ENVIRONMENTAL WELL-BEING \\
\hline 1623 & $\begin{array}{l}\text { MATAMATAPIAKO DISTRICT } \\
\text { COUNCIL }\end{array}$ & $\begin{array}{l}\text { HEALTHY AIR, WATER, LAND: HEALTHY } \\
\text { PEOPLE }\end{array}$ & $\begin{array}{l}\text { PERCENTAGE OF DISTRICT'S WATERWAYS THAT COMPLY WITH } \\
\text { BATHING STANDARDGUIDELINES }\end{array}$ & $\begin{array}{l}\text { RIVER, LAKES, COASTAL AND } \\
\text { GROUNDWATER }\end{array}$ & ENVIRONMENTAL WELL-BEING \\
\hline 1624 & $\begin{array}{l}\text { MATAMATAPIAKO DISTRICT } \\
\text { COUNCIL }\end{array}$ & $\begin{array}{l}\text { HEALTHY AIR, WATER, LAND: HEALTHY } \\
\text { PEOPLE }\end{array}$ & NUMBER AND VALUE OF INCENTIVES OFFERED EG RATE RELIEF & COUNCIL OPERATIONS & ECONOMIC WELL-BEING \\
\hline 1626 & $\begin{array}{l}\text { MATAMATAPIAKO DISTRICT } \\
\text { COUNCIL }\end{array}$ & $\begin{array}{l}\text { HEALTHY AIR, WATER, LAND: HEALTHY } \\
\text { PEOPLE }\end{array}$ & $\begin{array}{l}\text { PERCENTAGE OF THE COMMUNITY WHICH RECEIVED EDUCATIONAL } \\
\text { MATERIAL ABOUT RIPARIAN MANAGEMENT }\end{array}$ & QUALIFICATION/SCHOOL & SOCIAL WELL-BEING \\
\hline 1627 & $\begin{array}{l}\text { MATAMATAPIAKO DISTRICT } \\
\text { COUNCIL }\end{array}$ & $\begin{array}{l}\text { HEALTHY AIR, WATER, LAND: HEALTHY } \\
\text { PEOPLE }\end{array}$ & $\begin{array}{l}\text { TOTAL QUANTITY OF HAZARDOUS WASTE DISPOSED TO LANDFILL AND } \\
\text { SEWER }\end{array}$ & SEWERAGE/WASTEWATER & ENVIRONMENTAL WELL-BEING \\
\hline 1628 & $\begin{array}{l}\text { MATAMATAPIAKO DISTRICT } \\
\text { COUNCIL }\end{array}$ & $\begin{array}{l}\text { HEALTHY AIR, WATER, LAND: HEALTHY } \\
\text { PEOPLE }\end{array}$ & NUMBER OF INCIDENCES AND SPILL INVOLVING HAZARDOUS WASTE & POLLUTION & ENVIRONMENTAL WELL-BEING \\
\hline 1629 & $\begin{array}{l}\text { MATAMATAPIAKO DISTRICT } \\
\text { COUNCIL }\end{array}$ & $\begin{array}{l}\text { HEALTHY AIR, WATER, LAND: HEALTHY } \\
\text { PEOPLE }\end{array}$ & $\begin{array}{l}\text { COMPLIANCE OF DISPOSAL FACILITIES WITH RESOURCE CONSENT } \\
\text { CONDITIONS }\end{array}$ & WASTE TO LANDFILL & ENVIRONMENTAL WELL-BEING \\
\hline 1630 & $\begin{array}{l}\text { MATAMATAPIAKO DISTRICT } \\
\text { COUNCIL }\end{array}$ & $\begin{array}{l}\text { HEALTHY AIR, WATER, LAND: HEALTHY } \\
\text { PEOPLE }\end{array}$ & GROUNDWATER QUALITY UPSTREAM AND DOWNSTREAM OF LANDFILL & $\begin{array}{l}\text { RIVER, LAKES, COASTAL AND } \\
\text { GROUNDWATER }\end{array}$ & ENVIRONMENTAL WELL-BEING \\
\hline 1631 & $\begin{array}{l}\text { MATAMATAPIAKO DISTRICT } \\
\text { COUNCIL }\end{array}$ & $\begin{array}{l}\text { HEALTHY AIR, WATER, LAND: HEALTHY } \\
\text { PEOPLE }\end{array}$ & NUMBER OF POTENTIALLY CONTAMINATED SITES & $\begin{array}{l}\text { ENVIRONMENT/ENVIRONMENTAL } \\
\text { ATTITUDES }\end{array}$ & ENVIRONMENTAL WELL-BEING \\
\hline 1632 & $\begin{array}{l}\text { MATAMATAPIAKO DISTRICT } \\
\text { COUNCIL }\end{array}$ & $\begin{array}{l}\text { HEALTHY AIR, WATER, LAND: HEALTHY } \\
\text { PEOPLE }\end{array}$ & QUANTITY OF HAZARDOUS WASTE SAFELY DISPOSED OF & POLLUTION & ENVIRONMENTAL WELL-BEING \\
\hline 1633 & $\begin{array}{l}\text { MATAMATAPIAKO DISTRICT } \\
\text { COUNCIL }\end{array}$ & $\begin{array}{l}\text { HEALTHY AIR, WATER, LAND: HEALTHY } \\
\text { PEOPLE }\end{array}$ & $\begin{array}{l}\text { TOTAL QUANTITY OF WASTE DISPOSED TO LANDFILL FROM } \\
\text { RESIDENTIAL AND BUSINESSSOURCES }\end{array}$ & WASTE TO LANDFILL & ENVIRONMENTAL WELL-BEING \\
\hline 1634 & $\begin{array}{l}\text { MATAMATAPIAKO DISTRICT } \\
\text { COUNCIL }\end{array}$ & $\begin{array}{l}\text { HEALTHY AIR, WATER, LAND: HEALTHY } \\
\text { PEOPLE }\end{array}$ & PEOPLES ENVIRONMENTAL ATTITUDES & $\begin{array}{l}\text { ENVIRONMENT/ENVIRONMENTAL } \\
\text { ATTITUDES }\end{array}$ & ENVIRONMENTAL WELL-BEING \\
\hline 1635 & $\begin{array}{l}\text { MATAMATAPIAKO DISTRICT } \\
\text { COUNCIL }\end{array}$ & $\begin{array}{l}\text { HEALTHY AIR, WATER, LAND: HEALTHY } \\
\text { PEOPLE }\end{array}$ & PUBLIC PERCEPTION OF AMENITY AND LANDSCAPE VALUES & $\begin{array}{l}\text { ENVIRONMENT/ENVIRONMENTAL } \\
\text { ATTITUDES }\end{array}$ & ENVIRONMENTAL WELL-BEING \\
\hline 1637 & $\begin{array}{l}\text { MATAMATAPIAKO DISTRICT } \\
\text { COUNCIL }\end{array}$ & $\begin{array}{l}\text { HEALTHY AIR, WATER, LAND: HEALTHY } \\
\text { PEOPLE }\end{array}$ & AREA OF INDIGENOUS VEGETATION REMOVED EACH YEAR & NATIVE VEGETATION & ENVIRONMENTAL WELL-BEING \\
\hline 1638 & $\begin{array}{l}\text { MATAMATAPIAKO DISTRICT } \\
\text { COUNCIL }\end{array}$ & $\begin{array}{l}\text { HEALTHY AIR, WATER, LAND: HEALTHY } \\
\text { PEOPLE }\end{array}$ & CHANGE IN THE SURFACE AREA OF THE KOPUATAI PEAT DOME & NATURAL FEATURES & ENVIRONMENTAL WELL-BEING \\
\hline
\end{tabular}




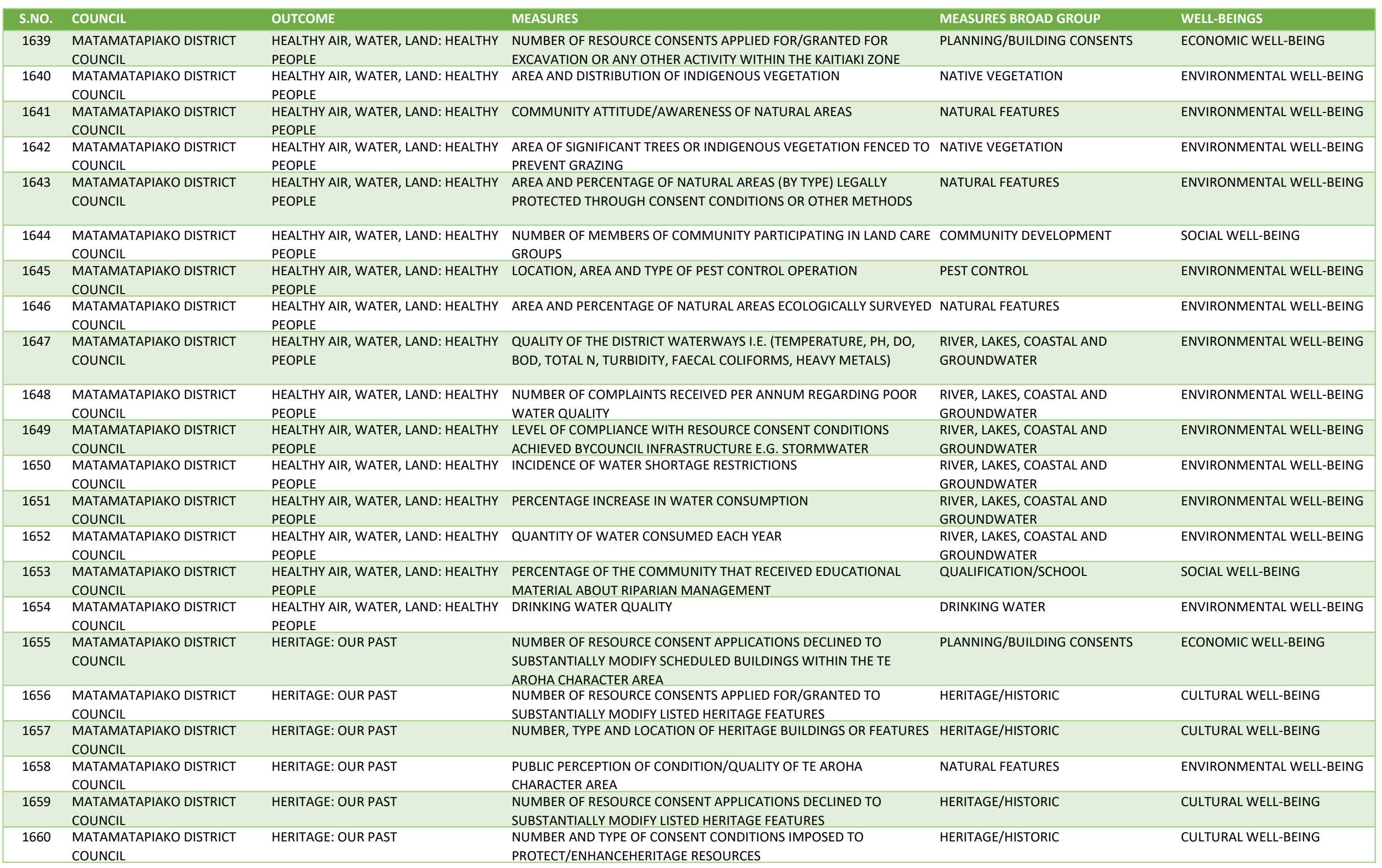


Appendix A: List of Community Well-beings Outcomes and Measures by Councils

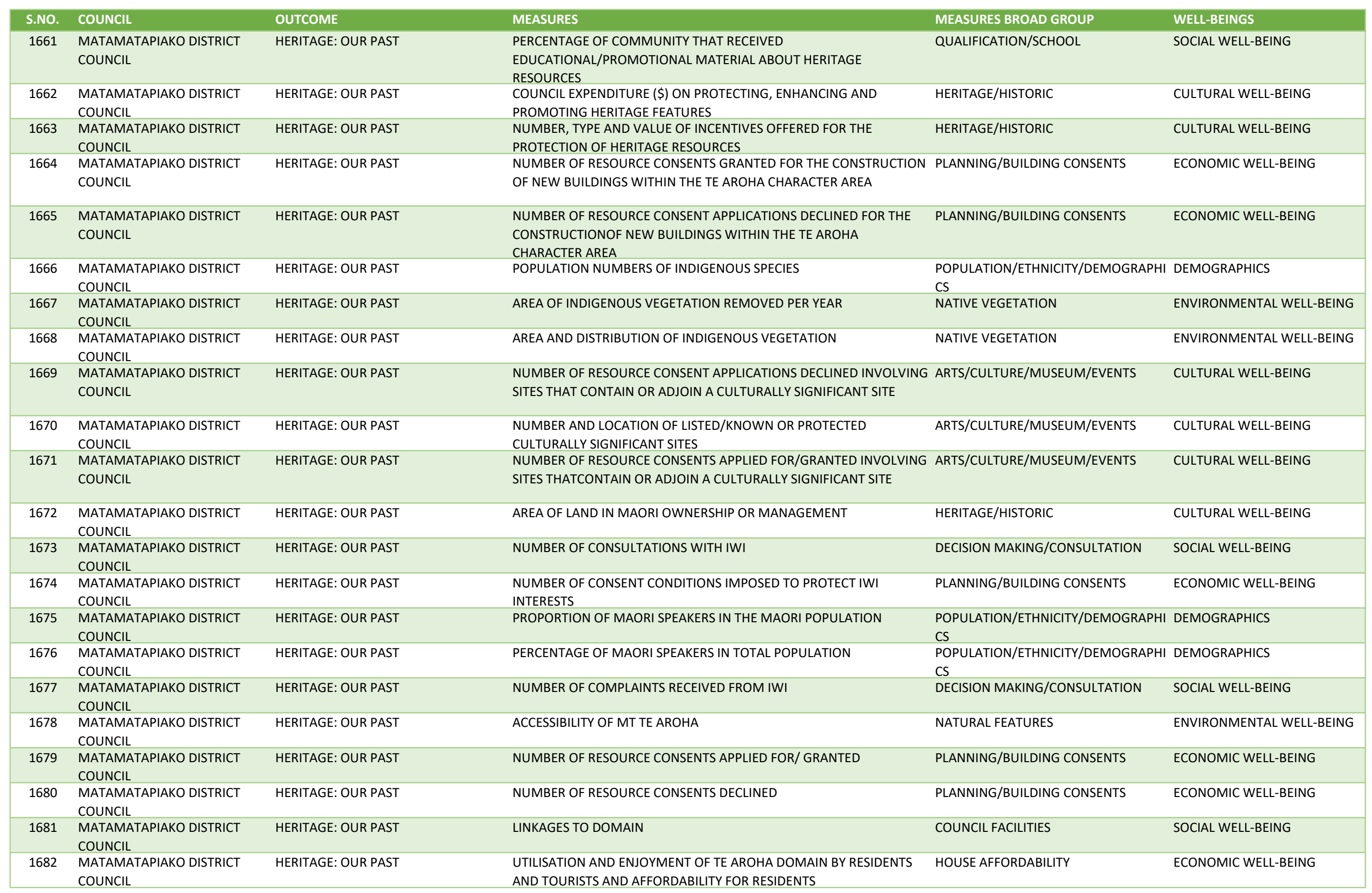


Appendix A: List of Community Well-beings Outcomes and Measures by Councils

\begin{tabular}{|c|c|c|c|c|c|}
\hline S.NO. & COUNCIL & OUTCOME & MEASURES & MEASURES BROAD GROUP & WELL-BEINGS \\
\hline 1683 & $\begin{array}{l}\text { MATAMATAPIAKO DISTRICT } \\
\text { COUNCIL }\end{array}$ & HERITAGE: OUR PAST & AREA OF INDIGENOUS VEGETATION REMOVED PER YEAR & NATIVE VEGETATION & ENVIRONMENTAL WELL-BEING \\
\hline 1684 & $\begin{array}{l}\text { MATAMATAPIAKO DISTRICT } \\
\text { COUNCIL }\end{array}$ & HERITAGE: OUR PAST & CHANGE IN THE SURFACE AREA OF THE KOPUATAI PEAT DOME & NATURAL FEATURES & ENVIRONMENTAL WELL-BEING \\
\hline 1685 & $\begin{array}{l}\text { MATAMATAPIAKO DISTRICT } \\
\text { COUNCIL }\end{array}$ & HERITAGE: OUR PAST & $\begin{array}{l}\text { NUMBER OF RESOURCE CONSENTS APPLIED FOR/GRANTED FOR } \\
\text { EXCAVATION OR ANY OTHER ACTIVITY WITHIN THE KAITIAKI ZONE }\end{array}$ & PLANNING/BUILDING CONSENTS & ECONOMIC WELL-BEING \\
\hline 1686 & $\begin{array}{l}\text { MATAMATAPIAKO DISTRICT } \\
\text { COUNCIL }\end{array}$ & HERITAGE: OUR PAST & AREA AND DISTRIBUTION OF INDIGENOUS VEGETATION & NATIVE VEGETATION & ENVIRONMENTAL WELL-BEING \\
\hline 1687 & $\begin{array}{l}\text { MATAMATAPIAKO DISTRICT } \\
\text { COUNCIL }\end{array}$ & HERITAGE: OUR PAST & COMMUNITY ATTITUDE/AWARENESS OF NATURAL AREAS & NATURAL FEATURES & ENVIRONMENTAL WELL-BEING \\
\hline 1688 & $\begin{array}{l}\text { MATAMATAPIAKO DISTRICT } \\
\text { COUNCIL }\end{array}$ & HERITAGE: OUR PAST & $\begin{array}{l}\text { AREA OF SIGNIFICANT TREES OR INDIGENOUS VEGETATION FENCED TO } \\
\text { PREVENTGRAZING }\end{array}$ & NATIVE VEGETATION & ENVIRONMENTAL WELL-BEING \\
\hline 1689 & $\begin{array}{l}\text { MATAMATAPIAKO DISTRICT } \\
\text { COUNCIL }\end{array}$ & HERITAGE: OUR PAST & $\begin{array}{l}\text { AREA AND PERCENTAGE OF NATURAL AREAS (BY TYPES) LEGALLY } \\
\text { PROTECTED THROUGH CONSENT CONDITIONS OR OTHER METHODS }\end{array}$ & NATURAL FEATURES & ENVIRONMENTAL WELL-BEING \\
\hline 1690 & $\begin{array}{l}\text { MATAMATAPIAKO DISTRICT } \\
\text { COUNCIL }\end{array}$ & HERITAGE: OUR PAST & $\begin{array}{l}\text { NUMBER OF MEMBERS OF COMMUNITY PARTICIPATING IN LAND CARE } \\
\text { GROUPS }\end{array}$ & COMMUNITY DEVELOPMENT & SOCIAL WELL-BEING \\
\hline 1691 & $\begin{array}{l}\text { MATAMATAPIAKO DISTRICT } \\
\text { COUNCIL }\end{array}$ & HERITAGE: OUR PAST & LOCATION AREA AND TYPE OF PEST CONTROL OPERATION & PEST CONTROL & ENVIRONMENTAL WELL-BEING \\
\hline 1692 & $\begin{array}{l}\text { MATAMATAPIAKO DISTRICT } \\
\text { COUNCIL }\end{array}$ & HERITAGE: OUR PAST & AREA AND PERCENT OF NATURAL AREAS ECOLOGICALLY SURVEYED & NATURAL FEATURES & ENVIRONMENTAL WELL-BEING \\
\hline 1694 & $\begin{array}{l}\text { MATAMATAPIAKO DISTRICT } \\
\text { COUNCIL }\end{array}$ & HERITAGE: OUR PAST & $\begin{array}{l}\text { NUMBER AND LOCATION OF LISTEDKNOWN OR PROTECTED } \\
\text { CULTURALLY SIGNIFICANT SITES }\end{array}$ & ARTS/CULTURE/MUSEUM/EVENTS & CULTURAL WELL-BEING \\
\hline 1695 & $\begin{array}{l}\text { MATAMATAPIAKO DISTRICT } \\
\text { COUNCIL }\end{array}$ & HERITAGE: OUR PAST & $\begin{array}{l}\text { NUMBER OF RESOURCE CONSENT APPLICATIONS DECLINED INVOLVING } \\
\text { SITES WHAT CONTAIN OR ADJOIN A CULTURALLY SIGNIFICANT SITE }\end{array}$ & ARTS/CULTURE/MUSEUM/EVENTS & CULTURAL WELL-BEING \\
\hline 1696 & $\begin{array}{l}\text { MATAMATAPIAKO DISTRICT } \\
\text { COUNCIL }\end{array}$ & HERITAGE: OUR PAST & EXTENT OF WETLANDS & $\begin{array}{l}\text { RIVER, LAKES, COASTAL AND } \\
\text { GROUNDWATER }\end{array}$ & ENVIRONMENTAL WELL-BEING \\
\hline 1697 & $\begin{array}{l}\text { MATAMATAPIAKO DISTRICT } \\
\text { COUNCIL }\end{array}$ & OUR SOCIAL INFRASTRUCTURE & PARTICIPATION RATES WITH EXTERNAL AGENCIES & SOCIAL CONNECTION & SOCIAL WELL-BEING \\
\hline 1698 & $\begin{array}{l}\text { MATAMATAPIAKO DISTRICT } \\
\text { COUNCIL }\end{array}$ & OUR SOCIAL INFRASTRUCTURE & $\begin{array}{l}\text { NUMBER OF PROGRAMMES PROTOCOLS AND PARTNERSHIPS IN PLACE } \\
\text { WITH EXTERNAL AGENCIES }\end{array}$ & COUNCIL OPERATIONS & ECONOMIC WELL-BEING \\
\hline 1699 & $\begin{array}{l}\text { MATAMATAPIAKO DISTRICT } \\
\text { COUNCIL }\end{array}$ & OUR SOCIAL INFRASTRUCTURE & CHANGE IN THE NUMBER OF SCHOOLS AND HEALTH SERVICES & HEALTH/HEALTH SERVICES & SOCIAL WELL-BEING \\
\hline 1700 & $\begin{array}{l}\text { MATAMATAPIAKO DISTRICT } \\
\text { COUNCIL }\end{array}$ & OUR SOCIAL INFRASTRUCTURE & BARRIERS TO ACCESSING HEALTH CARE AND EDUCATION & HEALTH/HEALTH SERVICES & SOCIAL WELL-BEING \\
\hline 1701 & $\begin{array}{l}\text { MATAMATAPIAKO DISTRICT } \\
\text { COUNCIL }\end{array}$ & OUR SOCIAL INFRASTRUCTURE & AVOIDABLE MORTALITY & HOSPITALISATIONS & ECONOMIC WELL-BEING \\
\hline 1702 & $\begin{array}{l}\text { MATAMATAPIAKO DISTRICT } \\
\text { COUNCIL }\end{array}$ & OUR SOCIAL INFRASTRUCTURE & SENSE OF EMOTIONAL WELL-BEING (HAPPINESS, QUALITY OF LIFE) & QUALITY OF LIFE & SOCIAL WELL-BEING \\
\hline 1704 & $\begin{array}{l}\text { MATAMATAPIAKO DISTRICT } \\
\text { COUNCIL }\end{array}$ & OUR SOCIAL INFRASTRUCTURE & $\begin{array}{l}\text { LAND USE ZONES WITHIN THE DISTRICT PLAN PROVIDE FOR AT LEAST A } \\
20 \text { YEARGROWTH PERIOD FOR MATAMATA, MORRINSVILLE AND TE } \\
\text { AROHA }\end{array}$ & PLANNING/BUILDING CONSENTS & ECONOMIC WELL-BEING \\
\hline
\end{tabular}


Appendix A: List of Community Well-beings Outcomes and Measures by Councils

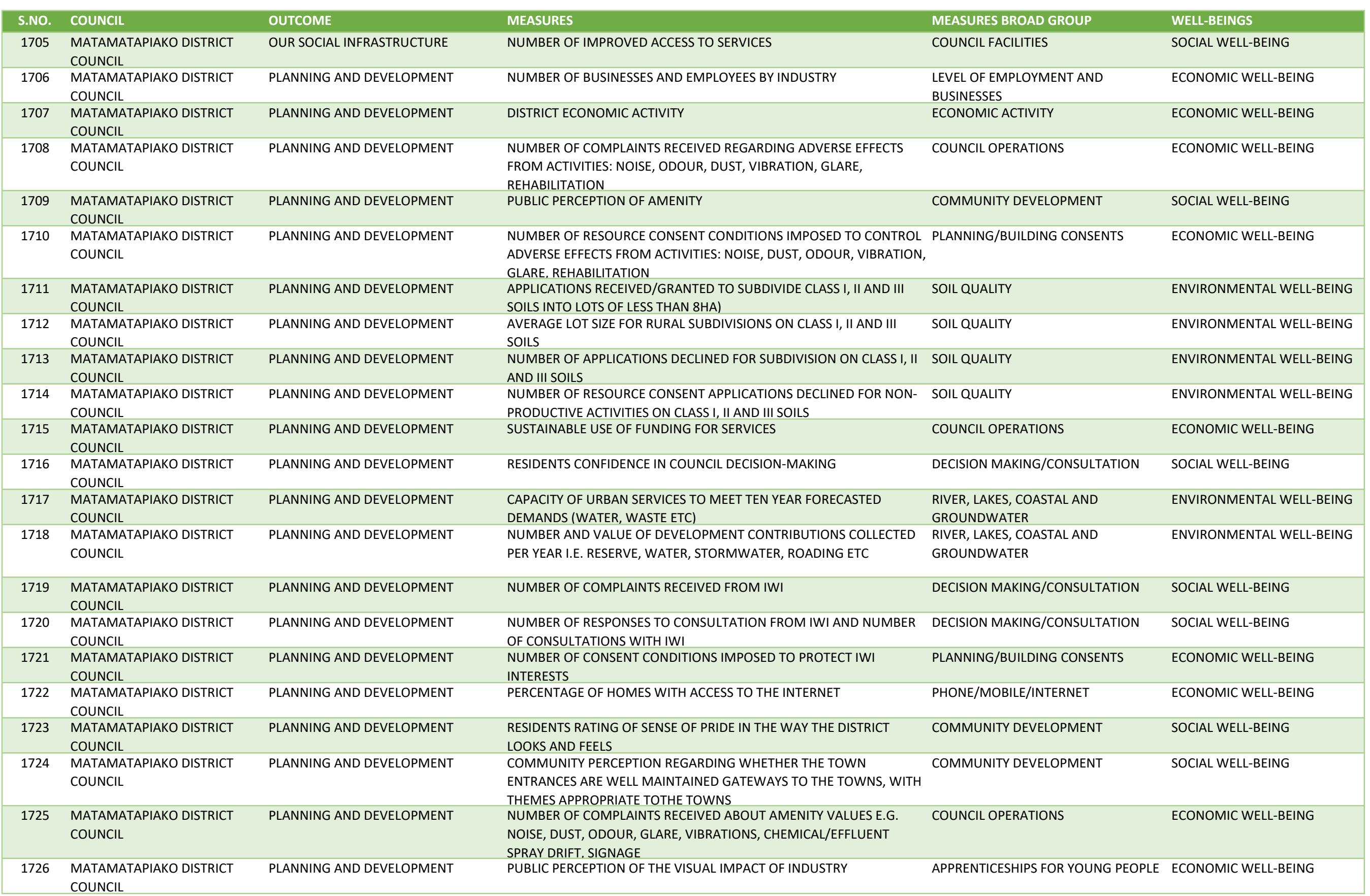




\begin{tabular}{|c|c|c|c|c|c|}
\hline S.NO. & COUNCIL & OUTCOME & MEASURES & MEASURES BROAD GROUP & WELL-BEINGS \\
\hline 1727 & $\begin{array}{l}\text { MATAMATAPIAKO DISTRICT } \\
\text { COUNCIL }\end{array}$ & PLANNING AND DEVELOPMENT & $\begin{array}{l}\text { COMMUNITY PERCEPTION OF WHETHER THE APPEARANCE OF OUR } \\
\text { TOWNS, THE NAMES OF THE ROADS, SITES AND FACILITIES REFLECT THE } \\
\text { DIVERSITY OF THE RESIDENTS }\end{array}$ & COMMUNITY DEVELOPMENT & SOCIAL WELL-BEING \\
\hline 1728 & $\begin{array}{l}\text { MATAMATAPIAKO DISTRICT } \\
\text { COUNCIL }\end{array}$ & PLANNING AND DEVELOPMENT & $\begin{array}{l}\text { LAND USE ZONES WITHIN THE DISTRICT PLAN PROVIDE FOR AT LEAST A } \\
\text { TWENTYYEAR GROWTH PERIOD }\end{array}$ & PLANNING/BUILDING CONSENTS & ECONOMIC WELL-BEING \\
\hline 1729 & $\begin{array}{l}\text { MATAMATAPIAKO DISTRICT } \\
\text { COUNCIL }\end{array}$ & PLANNING AND DEVELOPMENT & $\begin{array}{l}\text { URBAN POPULATION NUMBERS, INCLUDING HOUSEHOLD UNIT, AGE } \\
\text { DEMOGRAPHICS ETC }\end{array}$ & $\begin{array}{l}\text { POPULATION/ETHNICITY/DEMOGRAPHI } \\
\text { CS }\end{array}$ & DEMOGRAPHICS \\
\hline 1730 & $\begin{array}{l}\text { MATAMATAPIAKO DISTRICT } \\
\text { COUNCIL }\end{array}$ & PLANNING AND DEVELOPMENT & HOME OWNERSHIP RATE & HOME OWNERSHIP & DEMOGRAPHICS \\
\hline 1732 & $\begin{array}{l}\text { MATAMATAPIAKO DISTRICT } \\
\text { COUNCIL }\end{array}$ & PLANNING AND DEVELOPMENT & INFLATION ADJUSTED MEDIAN WEEKLY RENT & HOUSE AFFORDABILITY & ECONOMIC WELL-BEING \\
\hline 1733 & $\begin{array}{l}\text { MATAMATAPIAKO DISTRICT } \\
\text { COUNCIL }\end{array}$ & PRIDE AND JUSTICE & PERCEIVED DISCRIMINATION & COMMUNITY DEVELOPMENT & SOCIAL WELL-BEING \\
\hline 1734 & $\begin{array}{l}\text { MATAMATAPIAKO DISTRICT } \\
\text { COUNCIL }\end{array}$ & PRIDE AND JUSTICE & $\begin{array}{l}\text { RESIDENTS RATING OF SENSE OF PRIDE IN THE WAY THE DISTRICT } \\
\text { LOOKS AND FEELS }\end{array}$ & COMMUNITY DEVELOPMENT & SOCIAL WELL-BEING \\
\hline 1735 & $\begin{array}{l}\text { MATAMATAPIAKO DISTRICT } \\
\text { COUNCIL }\end{array}$ & PRIDE AND JUSTICE & CHANGE IN THE NUMBER OF OPPORTUNITIES AVAILABLE & $\begin{array}{l}\text { LEVEL OF EMPLOYMENT AND } \\
\text { BUSINESSES }\end{array}$ & ECONOMIC WELL-BEING \\
\hline 1736 & $\begin{array}{l}\text { MATAMATAPIAKO DISTRICT } \\
\text { COUNCIL }\end{array}$ & PRIDE AND JUSTICE & $\begin{array}{l}\text { A QUESTIONNAIRE WILL BE SENT OUT TO THESE ORGANISATIONS } \\
\text { ASKING HOW HAVE THEY ENSURED THAT THEY ARE INFORMED OF } \\
\text { LOCAL MAORI VALUE WHENMAKING SIGNIFICANT DECISIONS ON } \\
\text { BEHALF OF THE COMMUNITY }\end{array}$ & DECISION MAKING/CONSULTATION & SOCIAL WELL-BEING \\
\hline 1737 & $\begin{array}{l}\text { MATAMATAPIAKO DISTRICT } \\
\text { COUNCIL }\end{array}$ & PRIDE AND JUSTICE & $\begin{array}{l}\text { COMMUNITY SATISFACTION WITH THE WAY THE COUNCIL INVOLVES } \\
\text { THE PUBLICIN THE DECISIONS IT MAKES }\end{array}$ & DECISION MAKING/CONSULTATION & SOCIAL WELL-BEING \\
\hline 1738 & $\begin{array}{l}\text { MATAMATAPIAKO DISTRICT } \\
\text { COUNCIL }\end{array}$ & PRIDE AND JUSTICE & SOCIAL DEPRIVATION INDEX & DEPRIVATION INDEX & SOCIAL WELL-BEING \\
\hline 1739 & $\begin{array}{l}\text { MATAMATAPIAKO DISTRICT } \\
\text { COUNCIL }\end{array}$ & PRIDE AND JUSTICE & SURVEY OF YOUNG PEOPLE & YOUTH & SOCIAL WELL-BEING \\
\hline 1740 & $\begin{array}{l}\text { MATAMATAPIAKO DISTRICT } \\
\text { COUNCIL }\end{array}$ & PRIDE AND JUSTICE & $\begin{array}{l}\text { SEND QUESTIONNAIRE TO DISTRICT SCHOOLS TO SEE IF THEY VISIT/DO } \\
\text { ANYTHINGFOR ELDERLY MEMBERS OF OUR COMMUNITY }\end{array}$ & ELDERLY PEOPLE & ECONOMIC WELL-BEING \\
\hline 1741 & $\begin{array}{l}\text { MATAMATAPIAKO DISTRICT } \\
\text { COUNCIL }\end{array}$ & PRIDE AND JUSTICE & $\begin{array}{l}\text { QUESTIONNAIRE FOR FORUM MEMBERS (HOW SATISFIED ARE YOU } \\
\text { THAT OUR KOROUA AND KUIA (ELDERS) ARE RESPECTED FOR THE } \\
\text { VALUABLE CONTRIBUTION THEY MAKE TO OUR COMMUNITY?) }\end{array}$ & ELDERLY PEOPLE & ECONOMIC WELL-BEING \\
\hline 1742 & $\begin{array}{l}\text { MATAMATAPIAKO DISTRICT } \\
\text { COUNCIL }\end{array}$ & PRIDE AND JUSTICE & $\begin{array}{l}\text { COUNCIL AWARDS (VOLUNTEER AWARDS, BUSINESS AWARDS) - } \\
\text { H1272NUMBER AND PERCENTAGE OF ELDERLY PEOPLE RECOGNISED AT } \\
\text { THESE AWARDS }\end{array}$ & ELDERLY PEOPLE & ECONOMIC WELL-BEING \\
\hline 1743 & $\begin{array}{l}\text { MATAMATAPIAKO DISTRICT } \\
\text { COUNCIL }\end{array}$ & PRIDE AND JUSTICE & $\begin{array}{l}\text { SATISFACTION SURVEY OF TE MANAWHENUA FORUM (HOW } \\
\text { CONFIDENT ARE YOU THAT ALL SETTLEMENTS FOR LANDS AND } \\
\text { PROPERTIES CONFISCATED UNJUSTLYWILL BE NEGOTIATED TO FAIR } \\
\text { AND DURABLE OUTCOMES?) }\end{array}$ & HERITAGE/HISTORIC & CULTURAL WELL-BEING \\
\hline 1744 & $\begin{array}{l}\text { MATAMATAPIAKO DISTRICT } \\
\text { COUNCIL }\end{array}$ & PRIDE AND JUSTICE & $\begin{array}{l}\text { NUMBER OF MAJOR SETTLEMENT CLAIMS SUCCESSFULLY NEGOTIATED } \\
\text { TO REACHAN OUTCOME }\end{array}$ & HERITAGE/HISTORIC & CULTURAL WELL-BEING \\
\hline 1746 & $\begin{array}{l}\text { MATAMATAPIAKO DISTRICT } \\
\text { COUNCIL }\end{array}$ & TRANSPORT: PEOPLE GOING PLACES & COMMUNITY SATISFACTION WITH PARKING AVAILABILITY & TRANSPORT & ECONOMIC WELL-BEING \\
\hline 1747 & $\begin{array}{l}\text { MATAMATAPIAKO DISTRICT } \\
\text { COUNCIL }\end{array}$ & TRANSPORT: PEOPLE GOING PLACES & NUMBER OF ACCIDENTS REPORTED ON DISTRICT ROADS EACH YEAR & ROAD ACCIDENTS/INJURIES & ECONOMIC WELL-BEING \\
\hline
\end{tabular}


Appendix A: List of Community Well-beings Outcomes and Measures by Councils

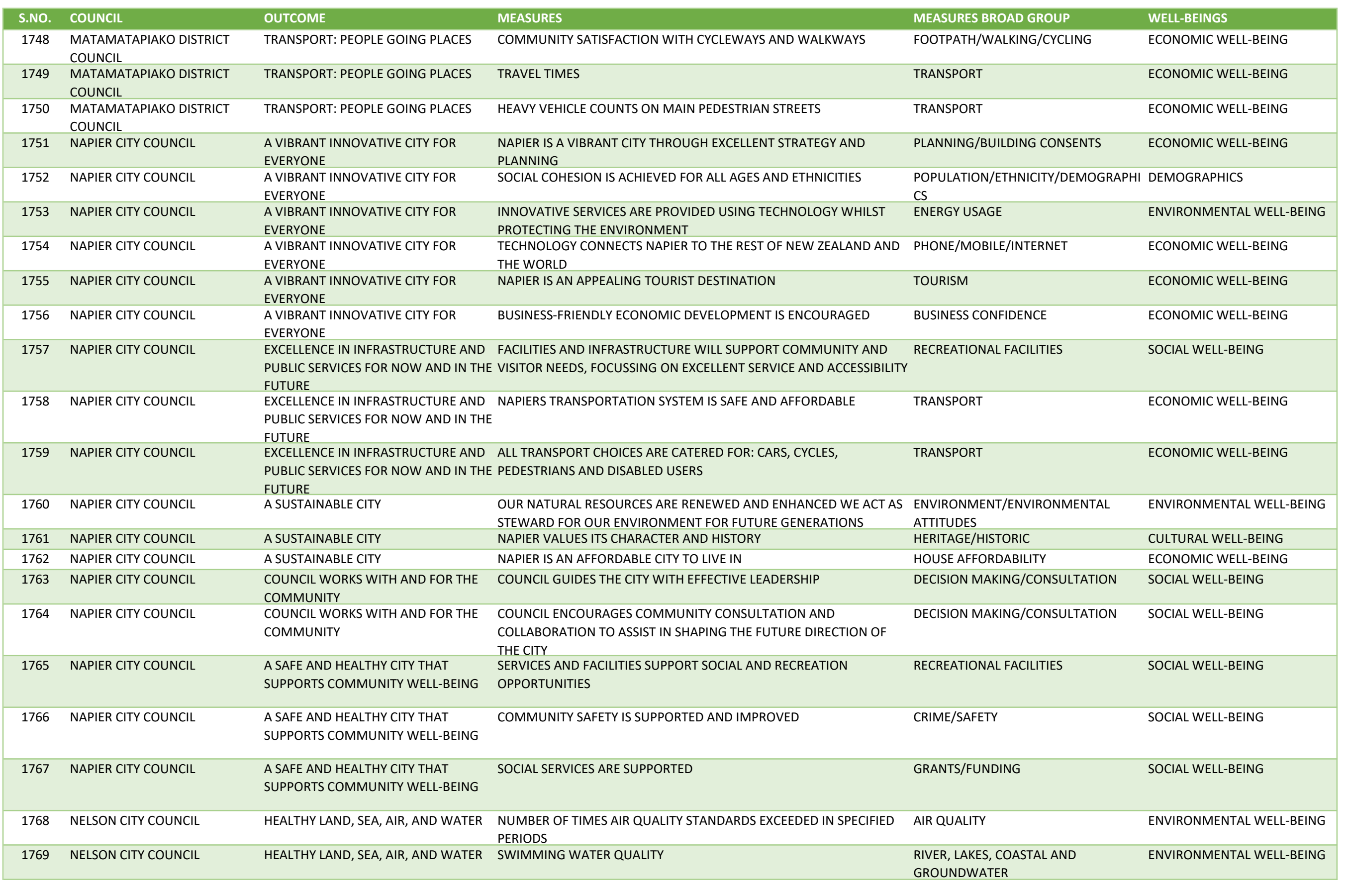


Appendix A: List of Community Well-beings Outcomes and Measures by Councils

\begin{tabular}{|c|c|c|c|c|c|}
\hline S.NO. & COUNCIL & OUTCOME & MEASURES & MEASURES BROAD GROUP & WELL-BEINGS \\
\hline 1770 & NELSON CITY COUNCIL & HEALTHY LAND, SEA, AIR, AND WATER & ECOLOGICAL CONDITION OF FRESH WATER & $\begin{array}{l}\text { RIVER, LAKES, COASTAL AND } \\
\text { GROUNDWATER }\end{array}$ & ENVIRONMENTAL WELL-BEING \\
\hline 1771 & NELSON CITY COUNCIL & HEALTHY LAND, SEA, AIR, AND WATER & $\begin{array}{l}\text { TOTAL AREA OF PARKS, RESERVES AND OPEN SPACE AS PROPORTION } \\
\text { OF LAND AREA (RURAL AND URBAN) }\end{array}$ & PARKS/RESERVES/OPEN SPACES & ENVIRONMENTAL WELL-BEING \\
\hline 1772 & NELSON CITY COUNCIL & HEALTHY LAND, SEA, AIR, AND WATER & TONNES OF WASTE SENT TO LANDFILL ANNUALLY & WASTE TO LANDFILL & ENVIRONMENTAL WELL-BEING \\
\hline 1773 & NELSON CITY COUNCIL & HEALTHY LAND, SEA, AIR, AND WATER & $\begin{array}{l}\text { BIODIVERSITY MEASURES AND PERFORMANCE TARGETS FOR PEST } \\
\text { CONTROL WILL BE DEVELOPED THROUGH IMPLEMENTING THE } \\
\text { BIODIVERSITY STRATEGY }\end{array}$ & PEST CONTROL & ENVIRONMENTAL WELL-BEING \\
\hline 1774 & NELSON CITY COUNCIL & HEALTHY LAND, SEA, AIR, AND WATER & SOIL HEALTH MEASURED BY 500 SOILS SURVEY & $\begin{array}{l}\text { ENVIRONMENT/ENVIRONMENTAL } \\
\text { ATTITUDES }\end{array}$ & ENVIRONMENTAL WELL-BEING \\
\hline 1775 & NELSON CITY COUNCIL & HEALTHY LAND, SEA, AIR, AND WATER & $\begin{array}{l}\text { RESIDENTS SATISFACTION WITH THE QUALITY OF THE NATURAL } \\
\text { ENVIRONMENT }\end{array}$ & NATURAL FEATURES & ENVIRONMENTAL WELL-BEING \\
\hline 1776 & NELSON CITY COUNCIL & HEALTHY LAND, SEA, AIR, AND WATER & $\begin{array}{l}\text { ACTIONS BY NONCOUNCIL GROUPS AND INDIVIDUALS -- COMMUNITY } \\
\text { CONTRIBUTION }\end{array}$ & VOLUNTEER/UNPAID WORK & SOCIAL WELL-BEING \\
\hline 1777 & NELSON CITY COUNCIL & HEALTHY LAND, SEA, AIR, AND WATER & PROPORTION OF HOUSEHOLDS RECYCLING REGULARLY & RECYCLING/GREEN WASTE & ENVIRONMENTAL WELL-BEING \\
\hline 1778 & NELSON CITY COUNCIL & PEOPLE-FRIENDLY PLACES & $\begin{array}{l}\text { NUMBER AND AREA OF OPEN SPACES AVAILABLE TO PUBLIC, } \\
\text { INCLUDING PARKS, CYCLEWAYS AND RESERVES }\end{array}$ & FOOTPATH/WALKING/CYCLING & ECONOMIC WELL-BEING \\
\hline 1779 & NELSON CITY COUNCIL & PEOPLE-FRIENDLY PLACES & $\begin{array}{l}\text { RESIDENT SATISFACTION WITH PUBLIC TRANSPORT (RESIDENT'S } \\
\text { SURVEY) }\end{array}$ & TRANSPORT & ECONOMIC WELL-BEING \\
\hline 1780 & NELSON CITY COUNCIL & PEOPLE-FRIENDLY PLACES & $\begin{array}{l}\text { USER AND RESIDENT SATISFACTION WITH CYCLE NETWORK } \\
\text { (RESIDENT'S SURVEY) }\end{array}$ & FOOTPATH/WALKING/CYCLING & ECONOMIC WELL-BEING \\
\hline 1781 & NELSON CITY COUNCIL & PEOPLE-FRIENDLY PLACES & TRAFFIC COUNTS AND TRAVEL TIMES ON MAJOR ROUTES & TRANSPORT & ECONOMIC WELL-BEING \\
\hline 1782 & NELSON CITY COUNCIL & PEOPLE-FRIENDLY PLACES & HOUSING AFFORDABILITY & HOUSE AFFORDABILITY & ECONOMIC WELL-BEING \\
\hline 1783 & NELSON CITY COUNCIL & PEOPLE-FRIENDLY PLACES & $\begin{array}{l}\text { PROPORTION OF RESIDENTS WALKING AND CYCLING REGULARLY } \\
\text { (RECREATION AND COMMUTING) }\end{array}$ & FOOTPATH/WALKING/CYCLING & ECONOMIC WELL-BEING \\
\hline 1784 & NELSON CITY COUNCIL & PEOPLE-FRIENDLY PLACES & $\begin{array}{l}\text { RESIDENTS SATISFACTION WITH MANAGEMENT OF HERITAGE } \\
\text { (RESIDENT'S SURVEY) }\end{array}$ & HERITAGE/HISTORIC & CULTURAL WELL-BEING \\
\hline 1785 & NELSON CITY COUNCIL & PEOPLE-FRIENDLY PLACES & RESIDENT PERCEPTION OF SAFETY COMPARED WITH CRIME STATISTICS & CRIME/SAFETY & SOCIAL WELL-BEING \\
\hline 1786 & NELSON CITY COUNCIL & PEOPLE-FRIENDLY PLACES & ADDITIONAL MEASURES DEVELOPED FOR HERITAGE STRATEGY & HERITAGE/HISTORIC & CULTURAL WELL-BEING \\
\hline 1787 & NELSON CITY COUNCIL & PEOPLE-FRIENDLY PLACES & $\begin{array}{l}\text { ACTIONS BY NONCOUNCIL GROUPS AND INDIVIDUALS -- COMMUNITY } \\
\text { CONTRIBUTION }\end{array}$ & VOLUNTEER/UNPAID WORK & SOCIAL WELL-BEING \\
\hline 1788 & NELSON CITY COUNCIL & PEOPLE-FRIENDLY PLACES & $\begin{array}{l}\text { PUBLIC SATISFACTION LEVELS WITH QUALITY OF THE BUILT } \\
\text { ENVIRONMENT (RESIDENT'S SURVEY) }\end{array}$ & $\begin{array}{l}\text { ENVIRONMENT/ENVIRONMENTAL } \\
\text { ATTITUDES }\end{array}$ & ENVIRONMENTAL WELL-BEING \\
\hline 1789 & NELSON CITY COUNCIL & A STRONG ECONOMY & NUMBER OF BUILDING PERMITS ISSUED & PLANNING/BUILDING CONSENTS & ECONOMIC WELL-BEING \\
\hline 1790 & NELSON CITY COUNCIL & A STRONG ECONOMY & BUSINESS CONFIDENCE & BUSINESS CONFIDENCE & ECONOMIC WELL-BEING \\
\hline 1791 & NELSON CITY COUNCIL & A STRONG ECONOMY & $\begin{array}{l}\text { INDUSTRY GROSS DOMESTIC PRODUCT (\$) FOR TOP FIVE NELSON } \\
\text { SECTORS }\end{array}$ & GROSS DOMESTIC PRODUCT (GDP) & ECONOMIC WELL-BEING \\
\hline 1792 & NELSON CITY COUNCIL & A STRONG ECONOMY & INTERNET ACCESS, NZ STATISTICS CENSUS DATA & PHONE/MOBILE/INTERNET & ECONOMIC WELL-BEING \\
\hline 1793 & NELSON CITY COUNCIL & A STRONG ECONOMY & MEDIAN HOUSEHOLD INCOME & INCOME & ECONOMIC WELL-BEING \\
\hline 1797 & NELSON CITY COUNCIL & A STRONG ECONOMY & EDUCATIONAL ATTAINMENT OF ADULT POPULATION (FULL RANGE) & QUALIFICATION/SCHOOL & SOCIAL WELL-BEING \\
\hline 1798 & NELSON CITY COUNCIL & A STRONG ECONOMY & $\begin{array}{l}\text { ACTIONS BY NONCOUNCIL GROUPS AND INDIVIDUALS -- COMMUNITY } \\
\text { CONTRIBUTION }\end{array}$ & VOLUNTEER/UNPAID WORK & SOCIAL WELL-BEING \\
\hline
\end{tabular}


Appendix A: List of Community Well-beings Outcomes and Measures by Councils

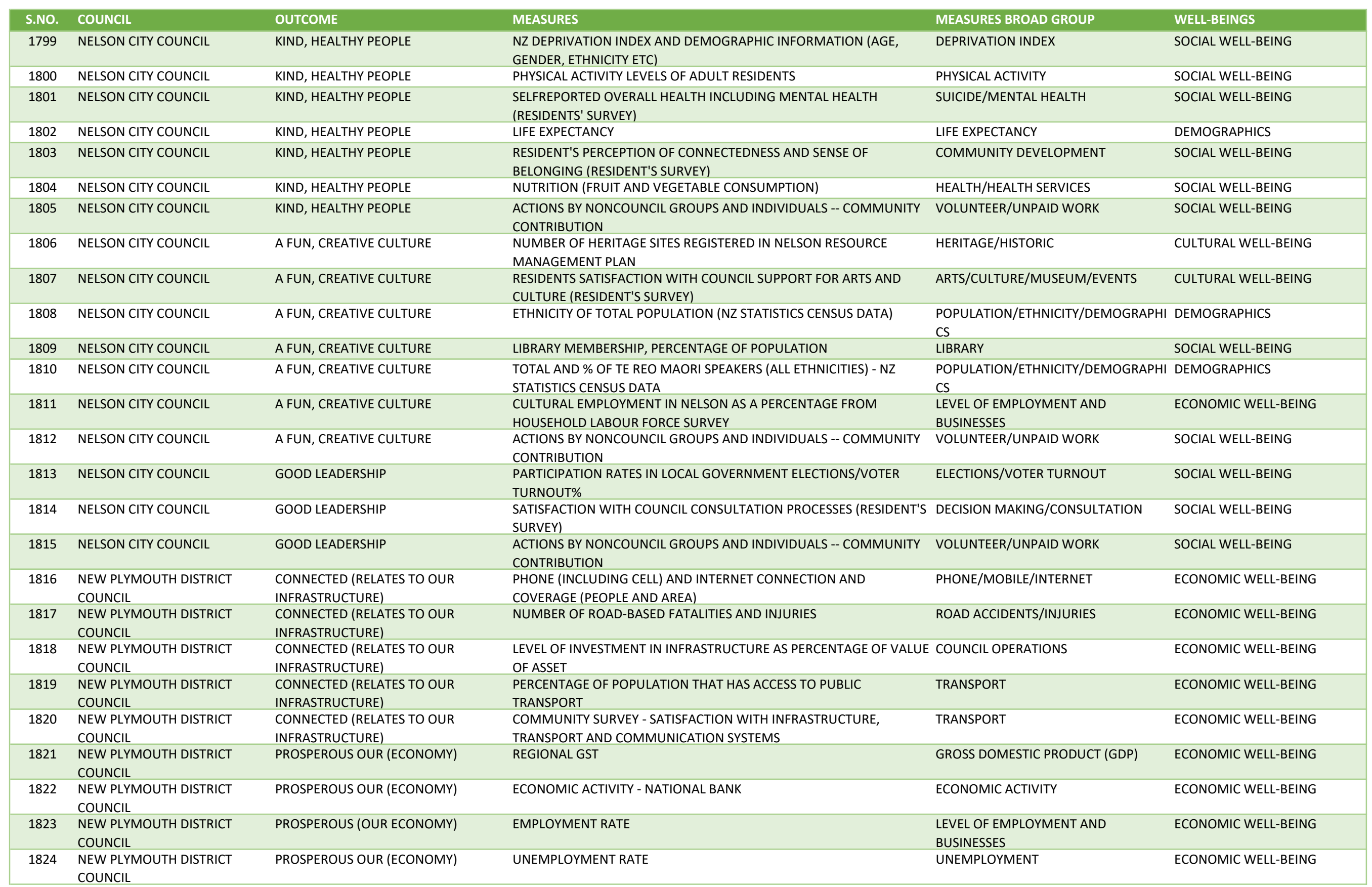


Appendix A: List of Community Well-beings Outcomes and Measures by Councils

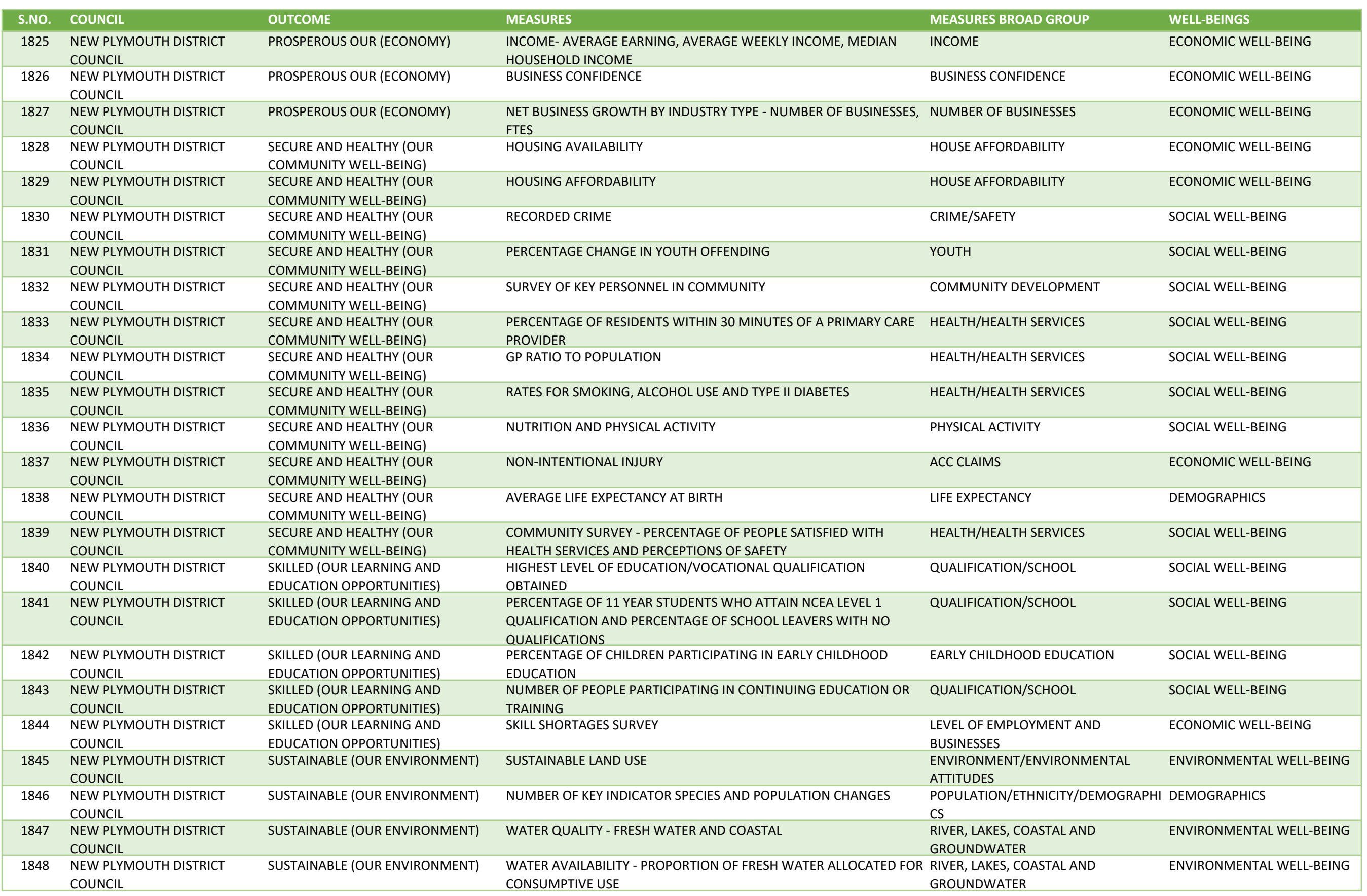


Appendix A: List of Community Well-beings Outcomes and Measures by Councils

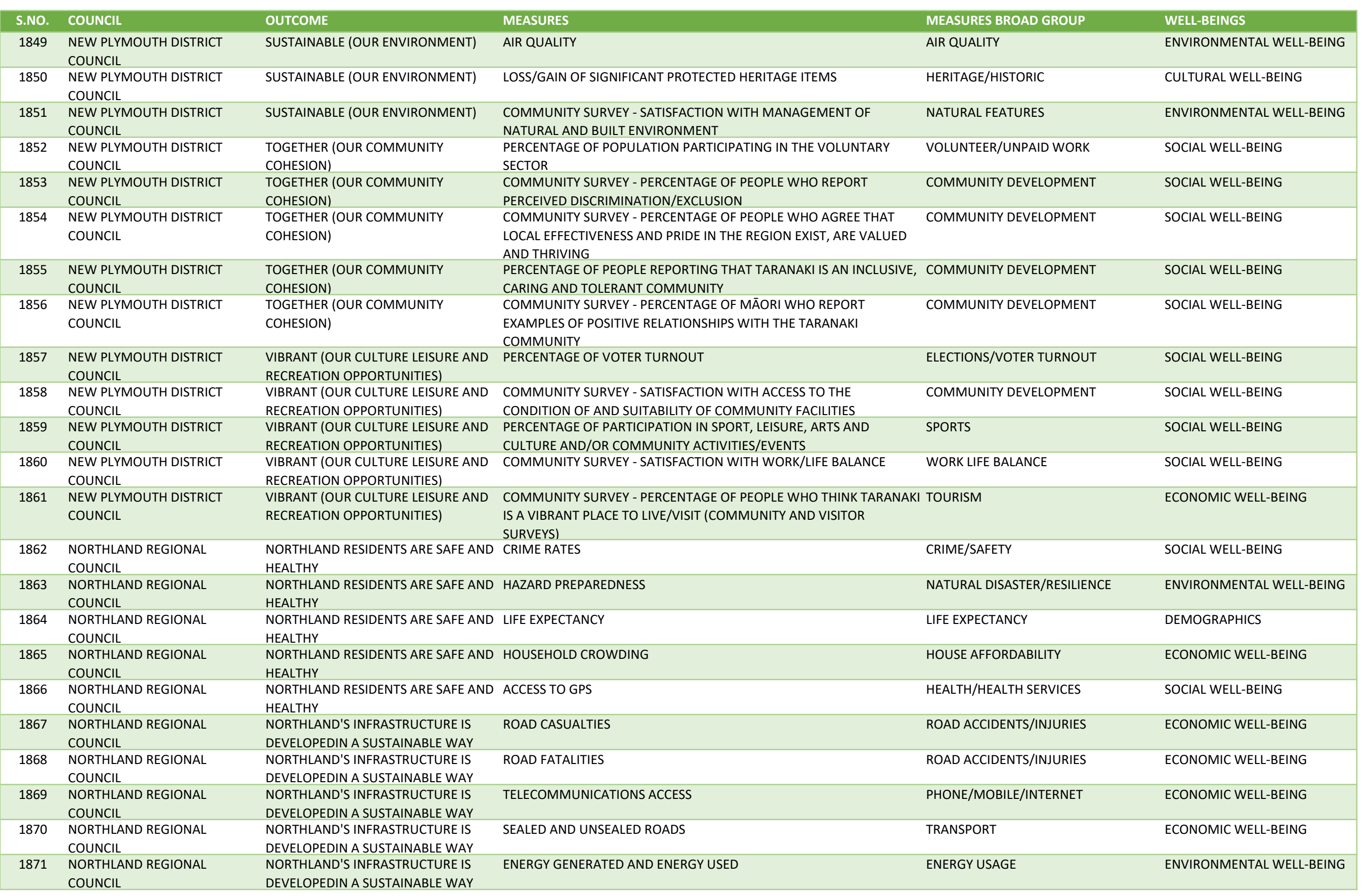


Appendix A: List of Community Well-beings Outcomes and Measures by Councils

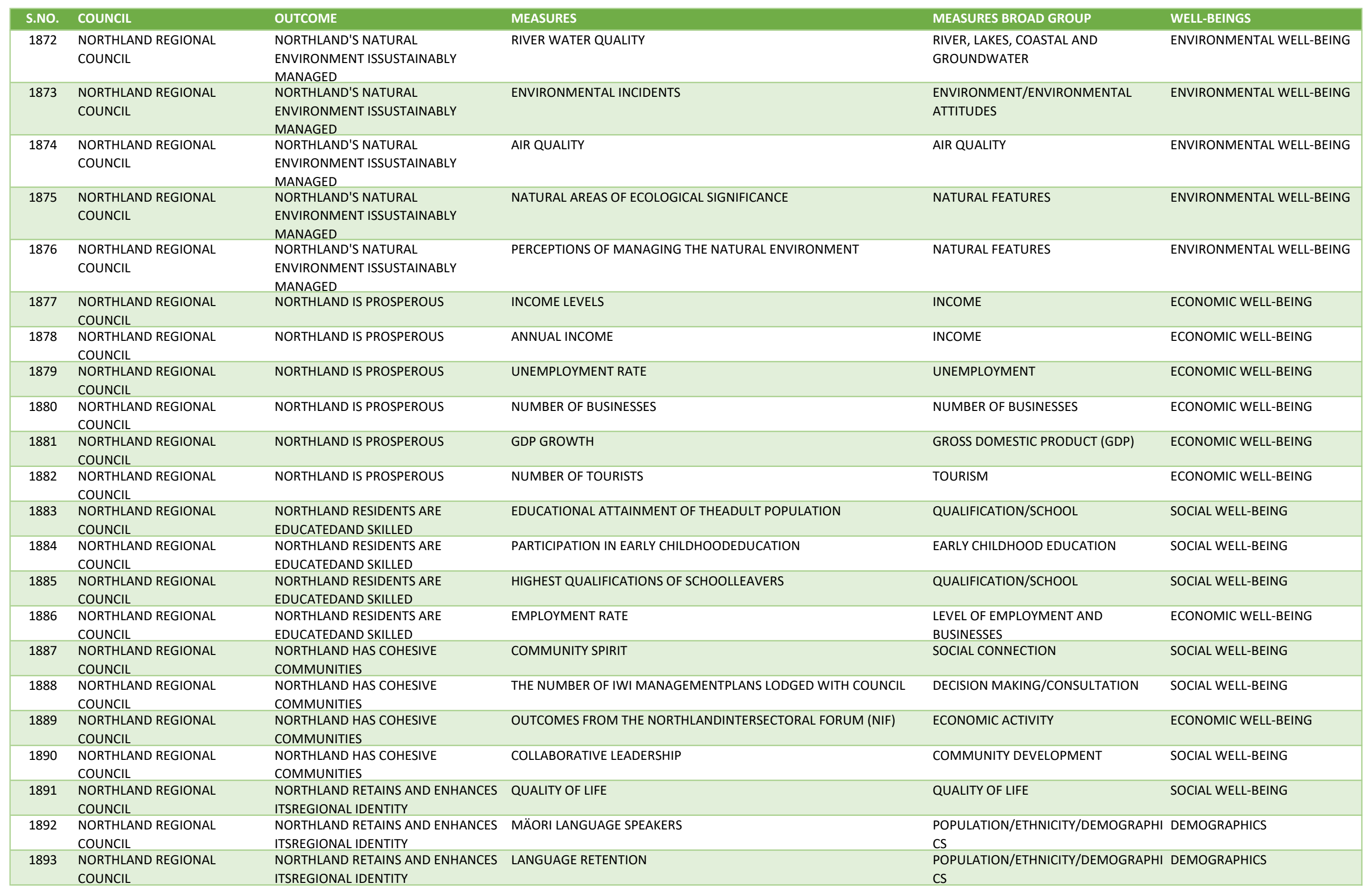


Appendix A: List of Community Well-beings Outcomes and Measures by Councils

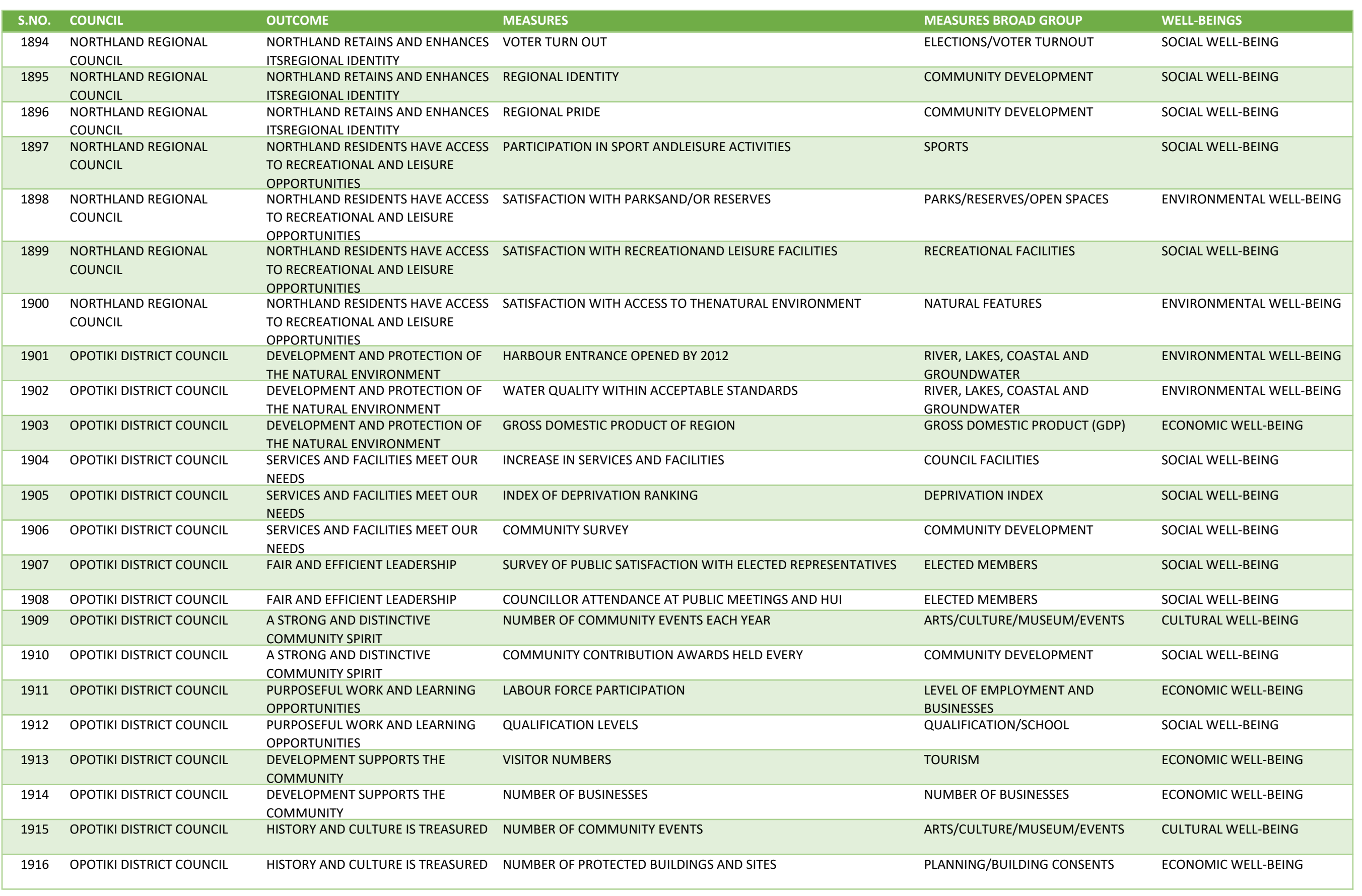


Appendix A: List of Community Well-beings Outcomes and Measures by Councils

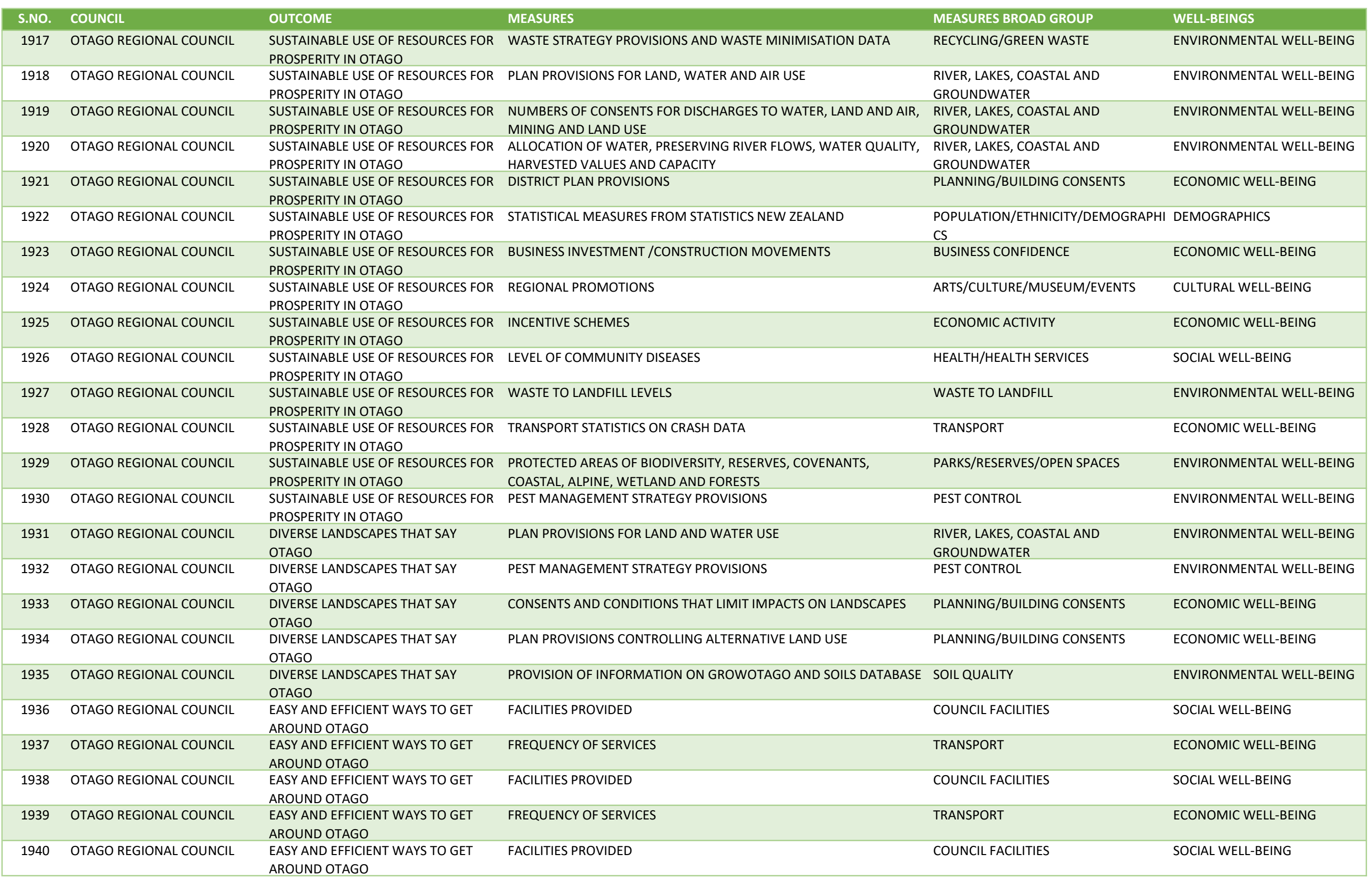


Appendix A: List of Community Well-beings Outcomes and Measures by Councils

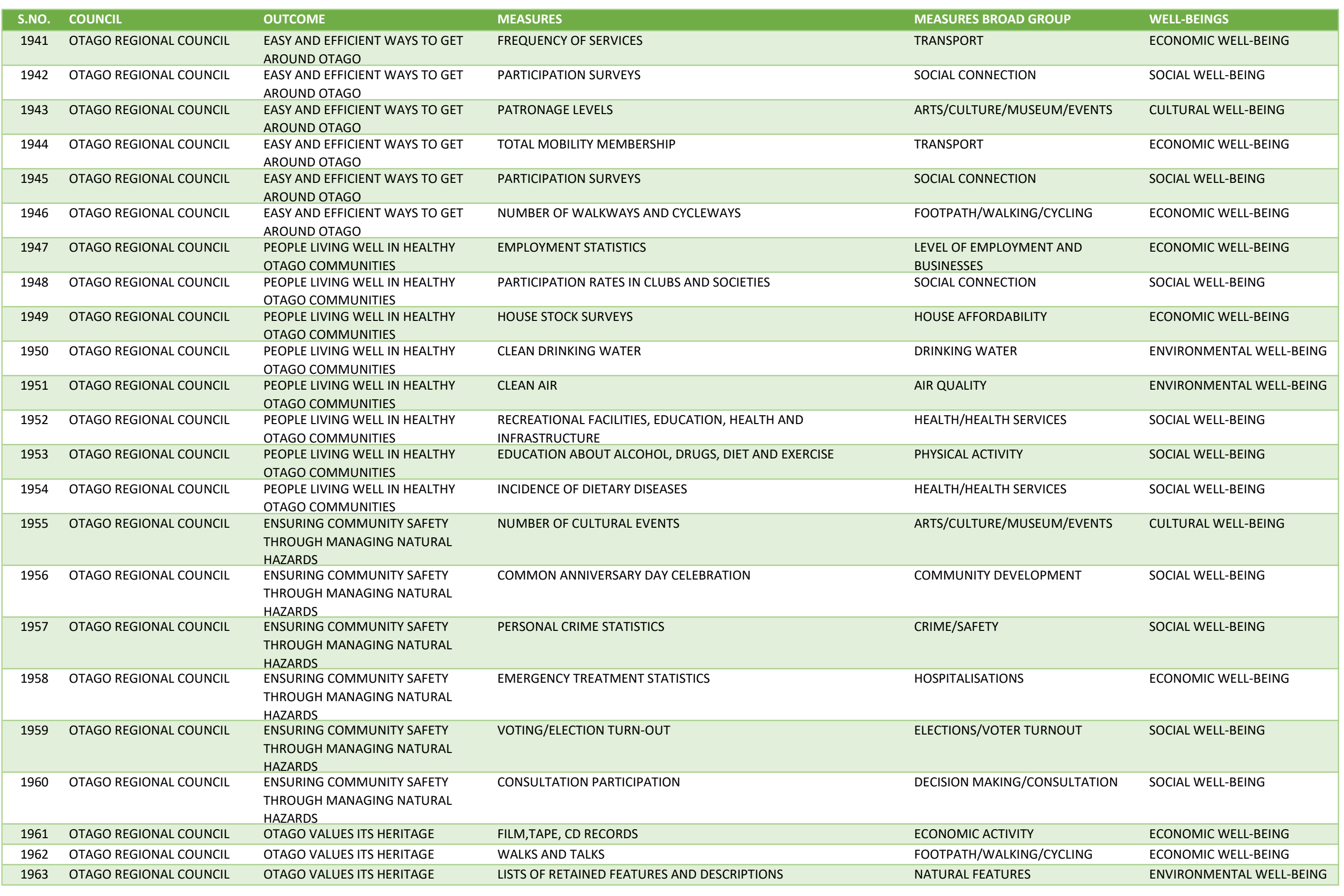


Appendix A: List of Community Well-beings Outcomes and Measures by Councils

\begin{tabular}{|c|c|c|c|c|c|}
\hline S.NO. & COUNCIL & OUTCOME & MEASURES & MEASURES BROAD GROUP & WELL-BEINGS \\
\hline 1964 & OTAGO REGIONAL COUNCIL & OTAGO VALUES ITS HERITAGE & PLAN PROVISIONS FOR HERITAGE PROTECTION & HERITAGE/HISTORIC & CULTURAL WELL-BEING \\
\hline 1965 & OTAGO REGIONAL COUNCIL & OTAGO VALUES ITS HERITAGE & ARCHAEOLOGICAL RECORDS & HERITAGE/HISTORIC & CULTURAL WELL-BEING \\
\hline 1966 & OTAGO REGIONAL COUNCIL & OTAGO VALUES ITS HERITAGE & REGIONAL STRATEGY FOR HERITAGE MANAGEMENT & HERITAGE/HISTORIC & CULTURAL WELL-BEING \\
\hline 1967 & OTAGO REGIONAL COUNCIL & $\begin{array}{l}\text { KNOWLEDGE AND LEARNING FOR } \\
\text { PROSPERITY IN OTAGO }\end{array}$ & WATER AND AIR STANDARDS & $\begin{array}{l}\text { RIVER, LAKES, COASTAL AND } \\
\text { GROUNDWATER }\end{array}$ & ENVIRONMENTAL WELL-BEING \\
\hline 1968 & OTAGO REGIONAL COUNCIL & $\begin{array}{l}\text { KNOWLEDGE AND LEARNING FOR } \\
\text { PROSPERITY IN OTAGO }\end{array}$ & SOIL CONTAMINATION LEVELS & SOIL QUALITY & ENVIRONMENTAL WELL-BEING \\
\hline 1969 & OTAGO REGIONAL COUNCIL & $\begin{array}{l}\text { KNOWLEDGE AND LEARNING FOR } \\
\text { PROSPERITY IN OTAGO }\end{array}$ & ENDANGERED SPECIES & $\begin{array}{l}\text { ENVIRONMENT/ENVIRONMENTAL } \\
\text { ATTITUDES }\end{array}$ & ENVIRONMENTAL WELL-BEING \\
\hline 1971 & OTAGO REGIONAL COUNCIL & $\begin{array}{l}\text { KNOWLEDGE AND LEARNING FOR } \\
\text { PROSPERITY IN OTAGO }\end{array}$ & $\begin{array}{l}\text { ALLOCATION OF WATER, PRESERVING RIVER FLOWS AND WATER } \\
\text { QUALITY, AND PROMOTING HARVESTED VALUES }\end{array}$ & $\begin{array}{l}\text { RIVER, LAKES, COASTAL AND } \\
\text { GROUNDWATER }\end{array}$ & ENVIRONMENTAL WELL-BEING \\
\hline 1972 & OTAGO REGIONAL COUNCIL & $\begin{array}{l}\text { KNOWLEDGE AND LEARNING FOR } \\
\text { PROSPERITY IN OTAGO }\end{array}$ & LEVEL OF SOLID WASTE BEING DISCHARGED TO LAND & WASTE TO LANDFILL & ENVIRONMENTAL WELL-BEING \\
\hline 1973 & OTAGO REGIONAL COUNCIL & $\begin{array}{l}\text { KNOWLEDGE AND LEARNING FOR } \\
\text { PROSPERITY IN OTAGO }\end{array}$ & ENERGY PLANS, EFFICIENCIES AND ALTERNATIVES & ENERGY USAGE & ENVIRONMENTAL WELL-BEING \\
\hline 1974 & OTAGO REGIONAL COUNCIL & $\begin{array}{l}\text { KNOWLEDGE AND LEARNING FOR } \\
\text { PROSPERITY IN OTAGO }\end{array}$ & INVESTMENT IN LOCAL RESEARCH & COUNCIL OPERATIONS & ECONOMIC WELL-BEING \\
\hline 1975 & OTAGO REGIONAL COUNCIL & $\begin{array}{l}\text { KNOWLEDGE AND LEARNING FOR } \\
\text { PROSPERITY IN OTAGO }\end{array}$ & $\begin{array}{l}\text { PROVISION OF DATA AND INFORMATION VIA WEB, BROCHURE AND } \\
\text { SEMINARS }\end{array}$ & COMMUNITY DEVELOPMENT & SOCIAL WELL-BEING \\
\hline 1976 & OTAGO REGIONAL COUNCIL & $\begin{array}{l}\text { KNOWLEDGE AND LEARNING FOR } \\
\text { PROSPERITY IN OTAGO }\end{array}$ & AVAILABILITY OF COMMUNICATION TOOLS AND INFRASTRUCTURE & PHONE/MOBILE/INTERNET & ECONOMIC WELL-BEING \\
\hline 1977 & OTAGO REGIONAL COUNCIL & $\begin{array}{l}\text { KNOWLEDGE AND LEARNING FOR } \\
\text { PROSPERITY IN OTAGO }\end{array}$ & BROCHURES, REPORTS, WORKSHOPS AND FIELD DAYS & ARTS/CULTURE/MUSEUM/EVENTS & CULTURAL WELL-BEING \\
\hline 1978 & OTAGO REGIONAL COUNCIL & $\begin{array}{l}\text { KNOWLEDGE AND LEARNING FOR } \\
\text { PROSPERITY IN OTAGO }\end{array}$ & $\begin{array}{l}\text { STATISTICAL MEASURES FROM COMPANIES OFFICE AND STATISTICS } \\
\text { NEW ZEALAND }\end{array}$ & $\begin{array}{l}\text { POPULATION/ETHNICITY/DEMOGRAPHI } \\
\text { CS }\end{array}$ & DEMOGRAPHICS \\
\hline 1979 & OTAGO REGIONAL COUNCIL & $\begin{array}{l}\text { KNOWLEDGE AND LEARNING FOR } \\
\text { PROSPERITY IN OTAGO }\end{array}$ & EMPLOYMENT AGENCIES & $\begin{array}{l}\text { LEVEL OF EMPLOYMENT AND } \\
\text { BUSINESSES }\end{array}$ & ECONOMIC WELL-BEING \\
\hline 1980 & $\begin{array}{l}\text { OTOROHANGA DISTRICT } \\
\text { COUNCIL }\end{array}$ & OTOROHANGA IS A SAFE PLACE TO LIVE & $\begin{array}{l}\text { PERCENTAGE OF RESIDENTS AND NON-RESIDENT RATEPAYERS } \\
\text { SATISFIED WITH DISTRICT FOOTPATHS }\end{array}$ & FOOTPATH/WALKING/CYCLING & ECONOMIC WELL-BEING \\
\hline 1981 & $\begin{array}{l}\text { OTOROHANGA DISTRICT } \\
\text { COUNCIL }\end{array}$ & OTOROHANGA IS A SAFE PLACE TO LIVE & NUMBER OF REPORTED PEDESTRIAN ACCIDENTS ON FOOTPATHS & ROAD ACCIDENTS/INJURIES & ECONOMIC WELL-BEING \\
\hline 1982 & $\begin{array}{l}\text { OTOROHANGA DISTRICT } \\
\text { COUNCIL }\end{array}$ & OTOROHANGA IS A SAFE PLACE TO LIVE & $\begin{array}{l}\text { NUMBER OF REPORTING PEDESTRIAN AND VEHICULAR ROAD SAFETY } \\
\text { ACCIDENTS ON MAIN STREET }\end{array}$ & ROAD ACCIDENTS/INJURIES & ECONOMIC WELL-BEING \\
\hline 1983 & $\begin{array}{l}\text { OTOROHANGA DISTRICT } \\
\text { COUNCIL }\end{array}$ & OTOROHANGA IS A SAFE PLACE TO LIVE & INCIDENCE OF REPORTED CRIME WITHIN THE DISTRICT & CRIME/SAFETY & SOCIAL WELL-BEING \\
\hline 1984 & $\begin{array}{l}\text { OTOROHANGA DISTRICT } \\
\text { COUNCIL }\end{array}$ & OTOROHANGA IS A SAFE PLACE TO LIVE & $\begin{array}{l}\text { PERCENTAGE OF RESIDENTS PERCEPTION OF SAFETY WITHIN THE } \\
\text { OTOROHANGA DISTRICT }\end{array}$ & CRIME/SAFETY & SOCIAL WELL-BEING \\
\hline 1985 & $\begin{array}{l}\text { OTOROHANGA DISTRICT } \\
\text { COUNCIL }\end{array}$ & OTOROHANGA IS A SAFE PLACE TO LIVE & NUMBER OF DRUG RELATED ARRESTS IN OTOROHANGA & CRIME/SAFETY & SOCIAL WELL-BEING \\
\hline 1988 & $\begin{array}{l}\text { OTOROHANGA DISTRICT } \\
\text { COUNCIL }\end{array}$ & OTOROHANGA IS A SAFE PLACE TO LIVE & $\begin{array}{l}\text { SURVEY OF RURAL COMMUNITIES AWARENESS OF CIVIL DEFENCE } \\
\text { PROCEDURES, EG: LOCAL GATHERING POINTS }\end{array}$ & NATURAL DISASTER/RESILIENCE & ENVIRONMENTAL WELL-BEING \\
\hline
\end{tabular}


Appendix A: List of Community Well-beings Outcomes and Measures by Councils

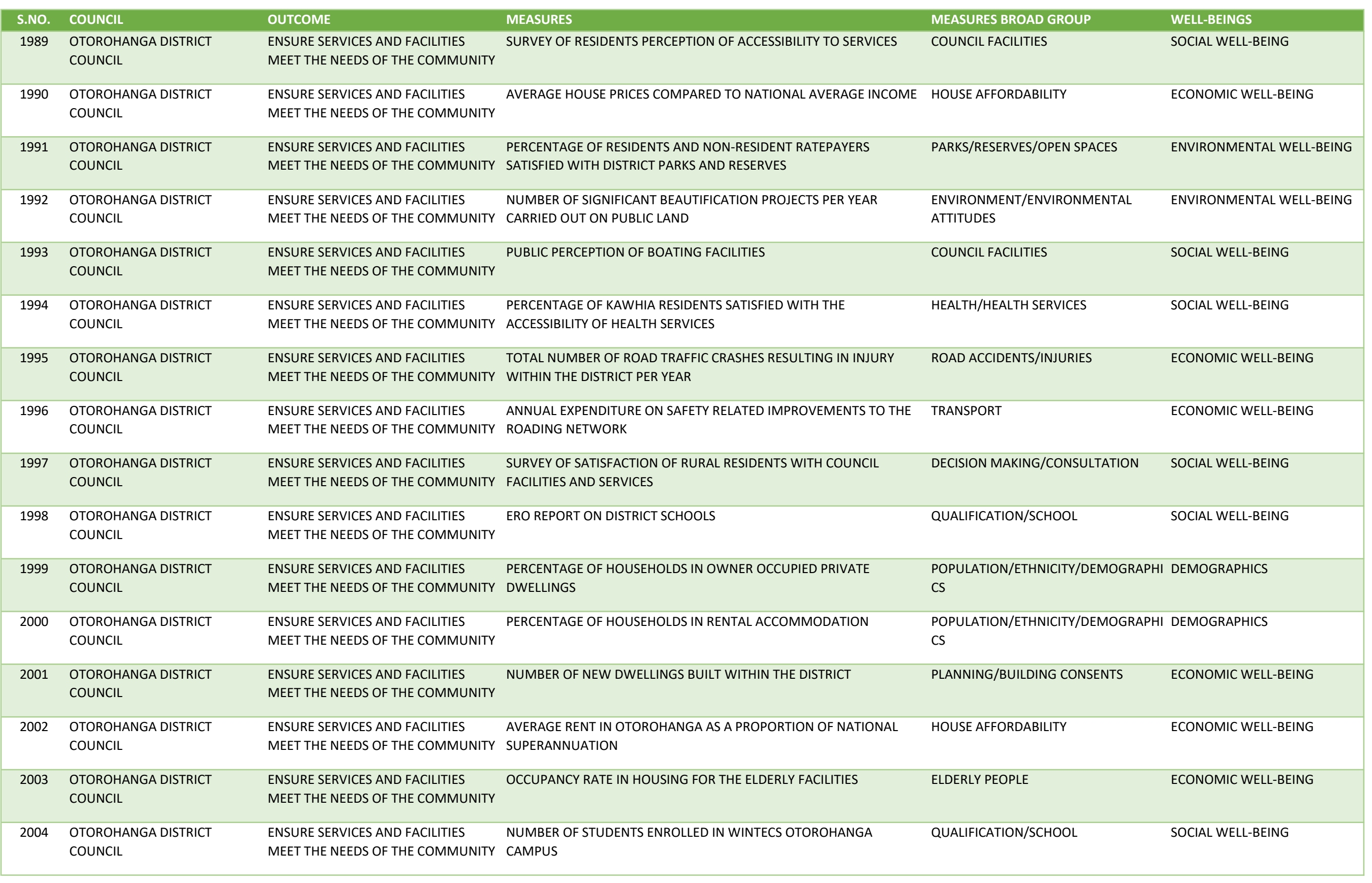


Appendix A: List of Community Well-beings Outcomes and Measures by Councils

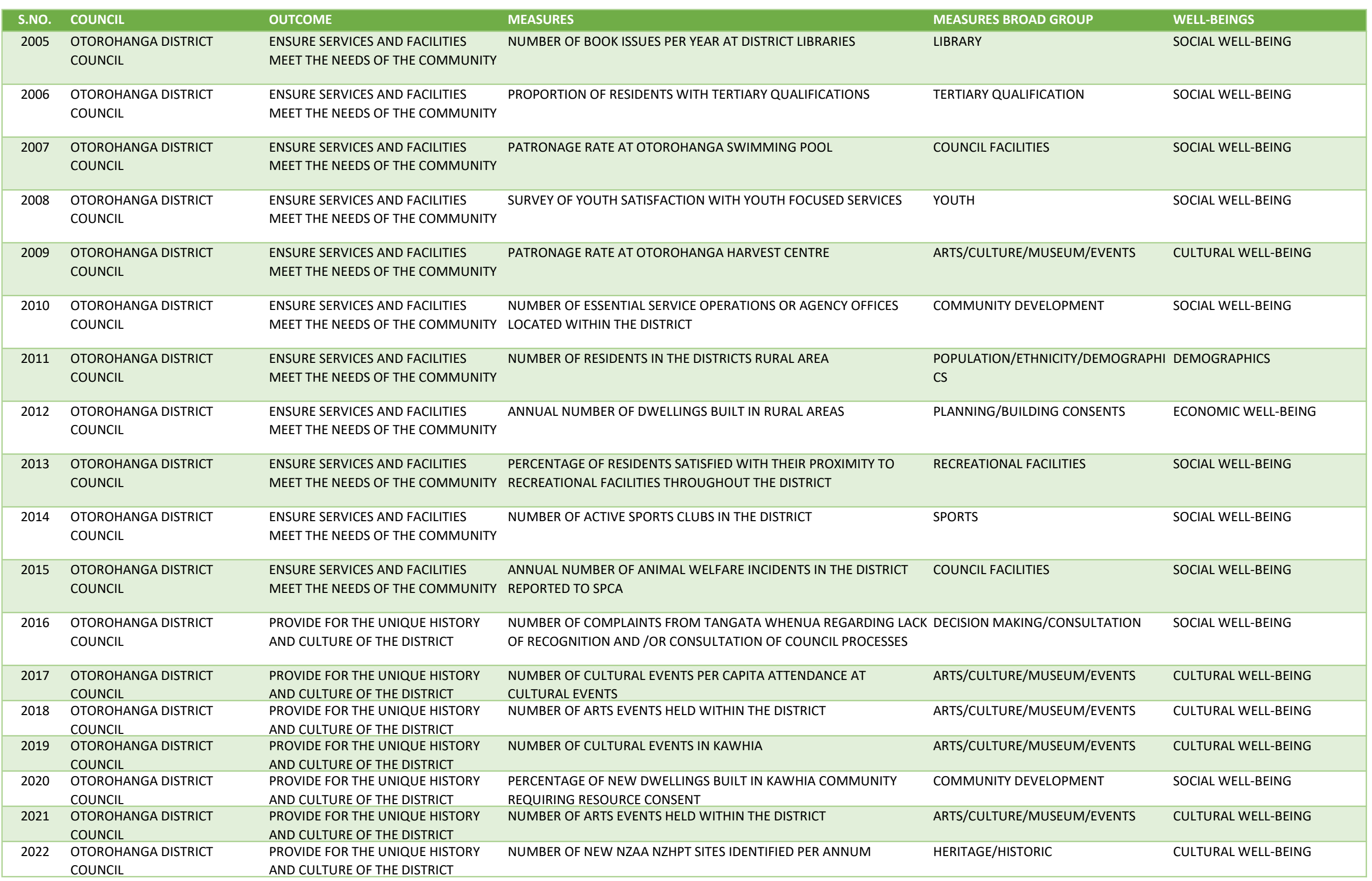


Appendix A: List of Community Well-beings Outcomes and Measures by Councils

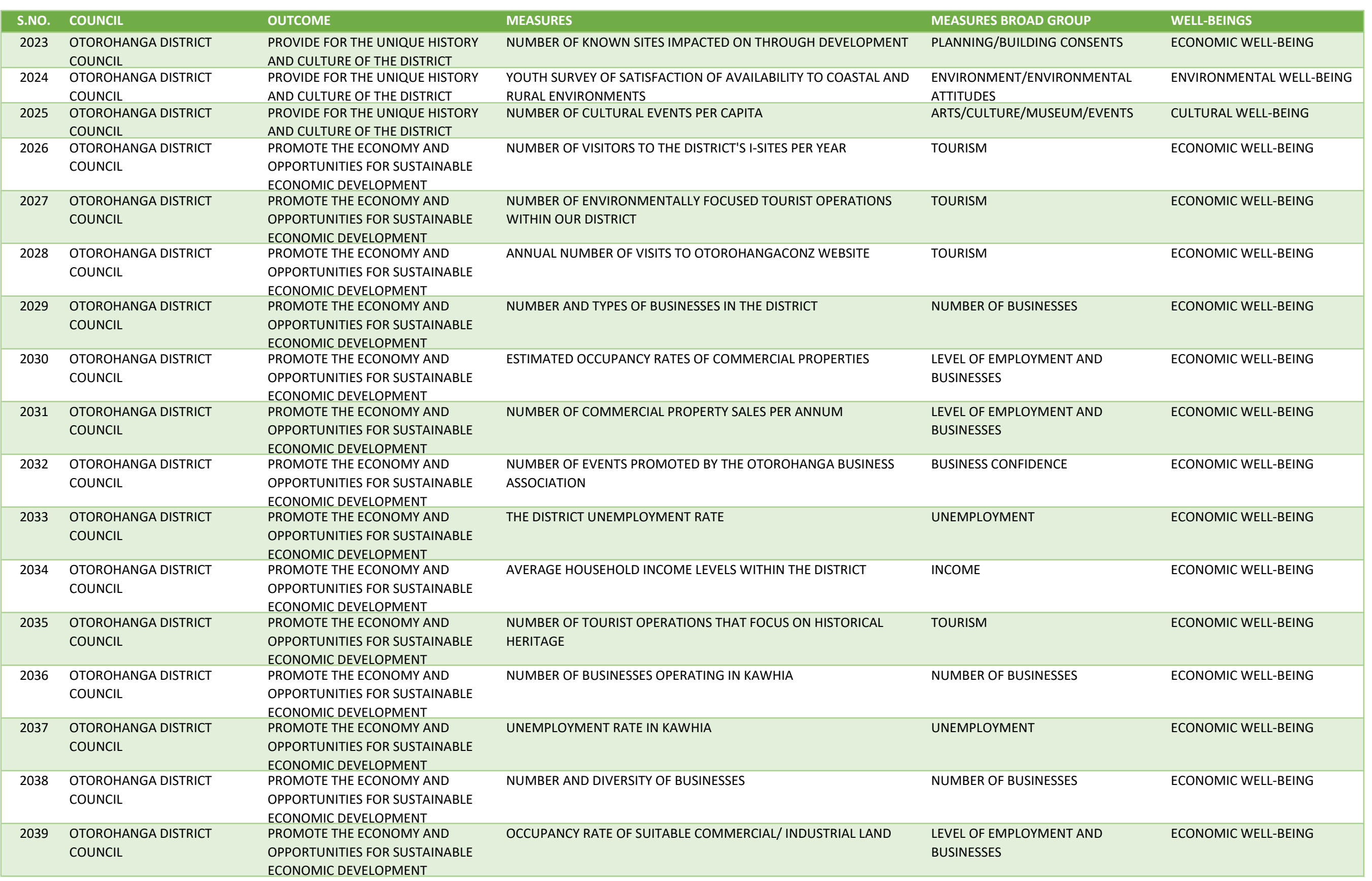


Appendix A: List of Community Well-beings Outcomes and Measures by Councils

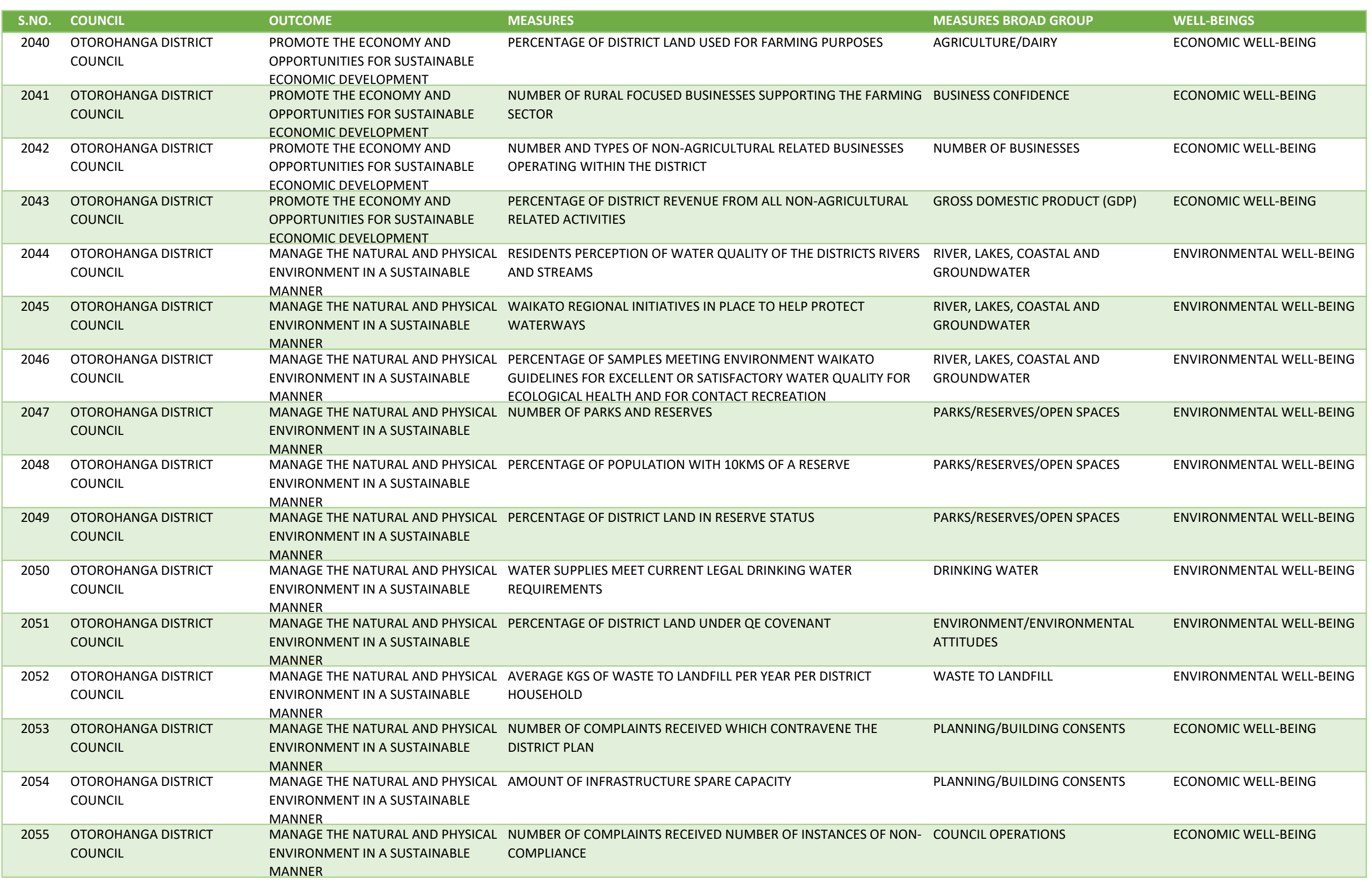




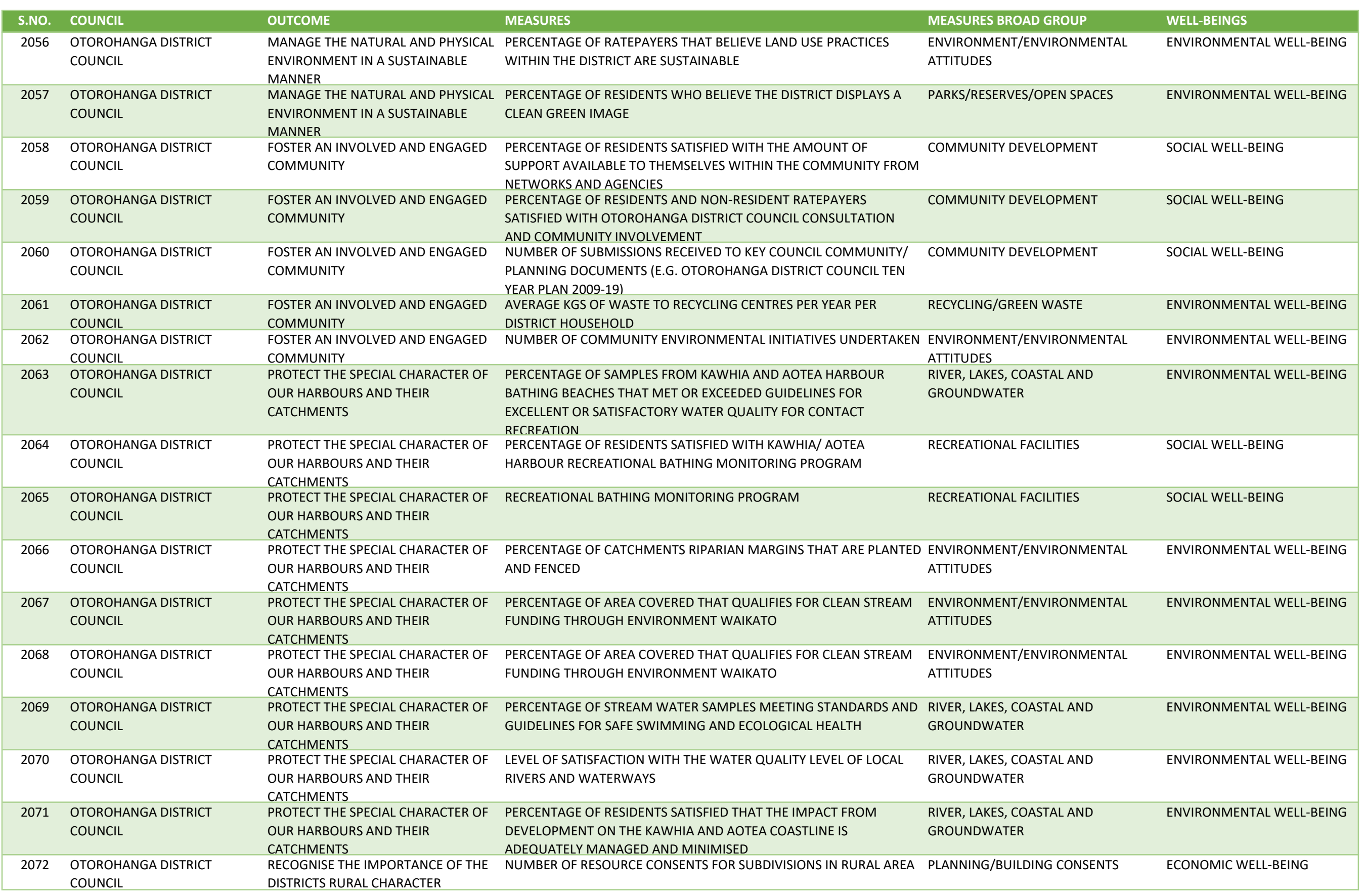




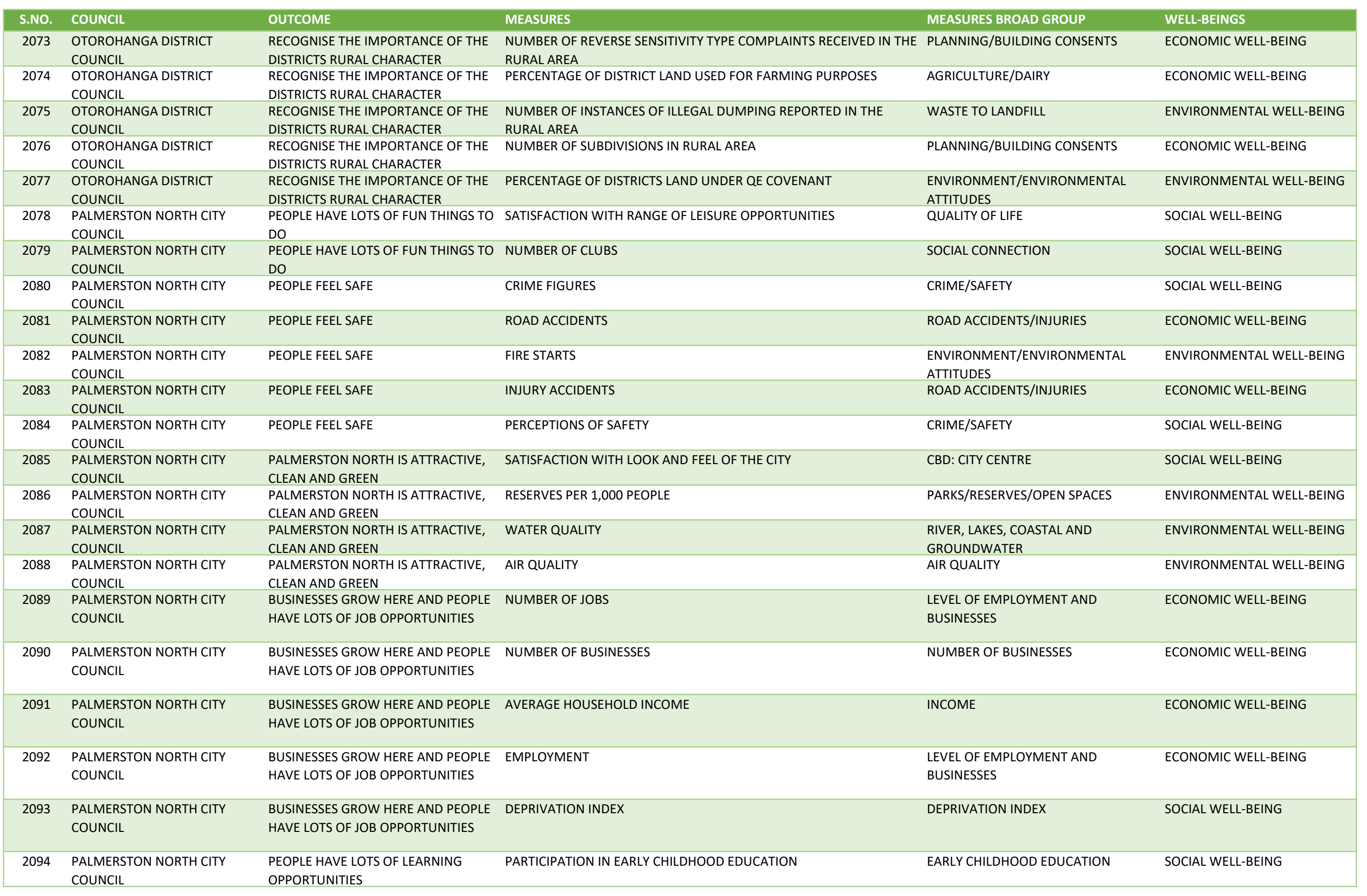


Appendix A: List of Community Well-beings Outcomes and Measures by Councils

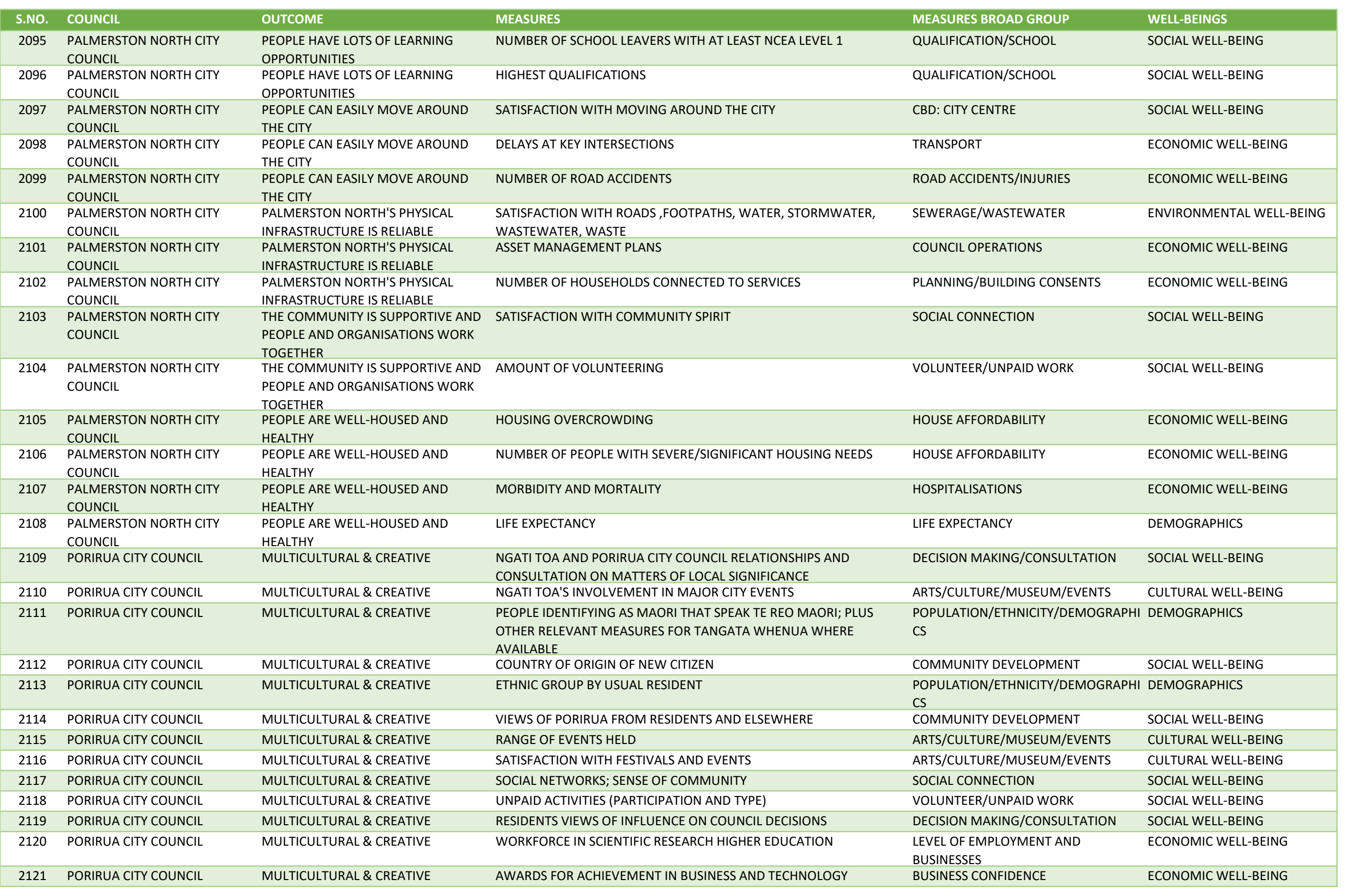


Appendix A: List of Community Well-beings Outcomes and Measures by Councils

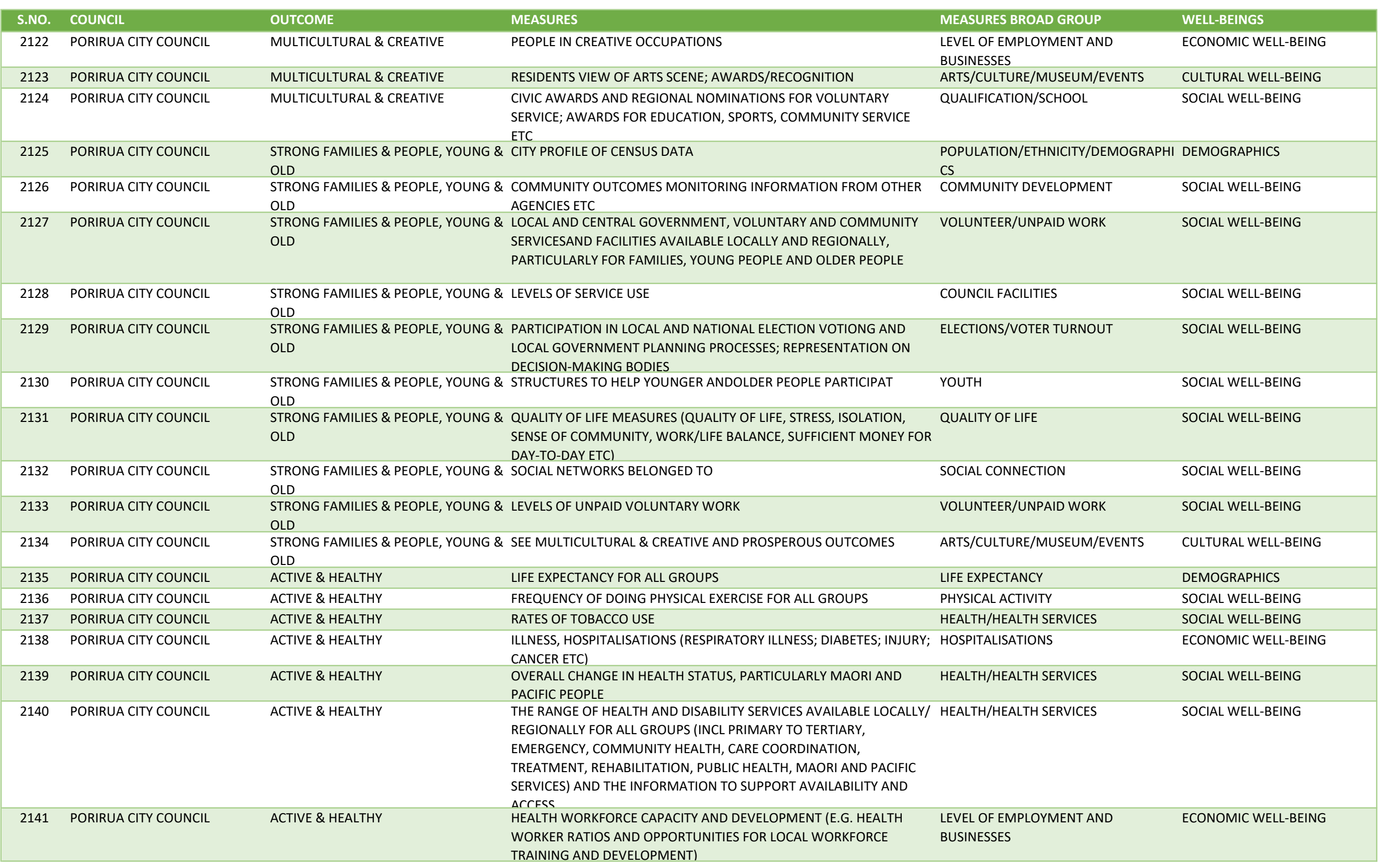


Appendix A: List of Community Well-beings Outcomes and Measures by Councils

\begin{tabular}{|c|c|c|c|c|c|}
\hline S.NO. & COUNCIL & OUTCOME & MEASURES & MEASURES BROAD GROUP & WELL-BEINGS \\
\hline 2142 & PORIRUA CITY COUNCIL & ACTIVE \& HEALTHY & $\begin{array}{l}\text { USE OF / BARRIERS TO HEALTH SERVICES BY ALL GROUPS (EG } \\
\text { ADMISSIONS, ACCIDENT \& MEDICAL PRESENTATIONS, NON- } \\
\text { ATTENDANCE, PRIMARY HEALTH ENROLMENT ETC) }\end{array}$ & HEALTH/HEALTH SERVICES & SOCIAL WELL-BEING \\
\hline 2143 & PORIRUA CITY COUNCIL & LEARNING FOR LIFE & $\begin{array}{l}\text { RANGE OF EDUCATIONAL FACILITIES IN THE CITY AND REGION FOR } \\
\text { DIFFERENT AGES AND PURPOSES }\end{array}$ & QUALIFICATION/SCHOOL & SOCIAL WELL-BEING \\
\hline 2144 & PORIRUA CITY COUNCIL & LEARNING FOR LIFE & PARTICIPATION RATES IN ALL LEVELS AND TYPES OF EDUCATION & QUALIFICATION/SCHOOL & SOCIAL WELL-BEING \\
\hline 2145 & PORIRUA CITY COUNCIL & LEARNING FOR LIFE & SCHOOL ATTENDANCE BY SCHOOL-AGED CHILDREN & QUALIFICATION/SCHOOL & SOCIAL WELL-BEING \\
\hline 2146 & PORIRUA CITY COUNCIL & LEARNING FOR LIFE & EDUCATIONAL ACHIEVEMENT IN PORIRUAS SCHOOLS & QUALIFICATION/SCHOOL & SOCIAL WELL-BEING \\
\hline 2147 & PORIRUA CITY COUNCIL & LEARNING FOR LIFE & $\begin{array}{l}\text { EDUCATIONAL ACHIEVEMENT OF PORIRUA RESIDENTS (I.E. IN PORIRUA } \\
\text { AND IN SCHOOLS OUTSIDE PORIRUA) }\end{array}$ & QUALIFICATION/SCHOOL & SOCIAL WELL-BEING \\
\hline 2148 & PORIRUA CITY COUNCIL & LEARNING FOR LIFE & $\begin{array}{l}\text { EDUCATIONAL RESULTS AND TRENDS OVER TIME, FOR DIFFERENT } \\
\text { GROUPS }\end{array}$ & QUALIFICATION/SCHOOL & SOCIAL WELL-BEING \\
\hline 2149 & PORIRUA CITY COUNCIL & LEARNING FOR LIFE & $\begin{array}{l}\text { NCEA ACHIEVEMENT RATES, PARTICULARLY } 1 \text { LEVEL LITERACY AND } \\
\text { NUMERACY }\end{array}$ & QUALIFICATION/SCHOOL & SOCIAL WELL-BEING \\
\hline 2150 & PORIRUA CITY COUNCIL & LEARNING FOR LIFE & $\begin{array}{l}\text { EVENTS AND OPPORTUNITIES TO ACKNOWLEDGE AND CELEBRATE } \\
\text { EDUCATIONAL ACHIEVEMENT }\end{array}$ & QUALIFICATION/SCHOOL & SOCIAL WELL-BEING \\
\hline 2151 & PORIRUA CITY COUNCIL & PROSPEROUS & BUSINESS NUMBERS BY SECTOR & NUMBER OF BUSINESSES & ECONOMIC WELL-BEING \\
\hline 2152 & PORIRUA CITY COUNCIL & PROSPEROUS & BUSINESS GROWTH & NUMBER OF BUSINESSES & ECONOMIC WELL-BEING \\
\hline 2153 & PORIRUA CITY COUNCIL & PROSPEROUS & MAORI AND PACIFIC ECONOMIC DEVELOPMENT & ECONOMIC ACTIVITY & ECONOMIC WELL-BEING \\
\hline 2156 & PORIRUA CITY COUNCIL & PROSPEROUS & $\begin{array}{l}\text { BUSINESS SATISFACTION WITH BUSINESS RETENTION, EXPANSION AND } \\
\text { SUPPORT }\end{array}$ & BUSINESS CONFIDENCE & ECONOMIC WELL-BEING \\
\hline 2157 & PORIRUA CITY COUNCIL & PROSPEROUS & ABILITY TO COVER COSTS OF EVERYDAY NEEDS & INCOME & ECONOMIC WELL-BEING \\
\hline 2158 & PORIRUA CITY COUNCIL & PROSPEROUS & PERSONAL INCOME & INCOME & ECONOMIC WELL-BEING \\
\hline 2159 & PORIRUA CITY COUNCIL & PROSPEROUS & $\begin{array}{l}\text { VILLAGE BUSINESS REVITALISATION (E.G. WAITANGIRUA MALL } \\
\text { REDEVELOPMENT) }\end{array}$ & CBD: CITY CENTRE & SOCIAL WELL-BEING \\
\hline 2160 & PORIRUA CITY COUNCIL & PROSPEROUS & OF GDP SPENT ON RESEARCH AND DEVELOPMENT & GROSS DOMESTIC PRODUCT (GDP) & ECONOMIC WELL-BEING \\
\hline 2161 & PORIRUA CITY COUNCIL & PROSPEROUS & $\begin{array}{l}\text { TERTIARY EDUCATION INSTITUTIONS AND CROWN RESEARCH } \\
\text { INSTITUTES IN PORIRUA }\end{array}$ & TERTIARY QUALIFICATION & SOCIAL WELL-BEING \\
\hline 2162 & PORIRUA CITY COUNCIL & PROSPEROUS & OF PORIRUA BUSINESSES EXPORTING & EXPORTS & ECONOMIC WELL-BEING \\
\hline 2163 & PORIRUA CITY COUNCIL & PROSPEROUS & EMPLOYMENT GROWTH & $\begin{array}{l}\text { LEVEL OF EMPLOYMENT AND } \\
\text { BUSINESSES }\end{array}$ & ECONOMIC WELL-BEING \\
\hline 2164 & PORIRUA CITY COUNCIL & PROSPEROUS & EMPLOYMENT NUMBERS BY SECTOR & $\begin{array}{l}\text { LEVEL OF EMPLOYMENT AND } \\
\text { BUSINESSES }\end{array}$ & ECONOMIC WELL-BEING \\
\hline 2165 & PORIRUA CITY COUNCIL & A SAFE CITY & $\begin{array}{l}\text { HOSPITALISATIONS FOR ALL TYPES OF INJURY (ACCIDENTAL AND } \\
\text { INTENTIONAL) }\end{array}$ & HOSPITALISATIONS & ECONOMIC WELL-BEING \\
\hline 2166 & PORIRUA CITY COUNCIL & A SAFE CITY & VEHICLE CRASHES (ALL TYPES), DEATHS AND INJURIES & ROAD ACCIDENTS/INJURIES & ECONOMIC WELL-BEING \\
\hline 2167 & PORIRUA CITY COUNCIL & A SAFE CITY & CLAIMS FOR INJURY COMPENSATION (WORK, SPORT, HOME) & ACC CLAIMS & ECONOMIC WELL-BEING \\
\hline 2168 & PORIRUA CITY COUNCIL & A SAFE CITY & $\begin{array}{l}\text { CRIME STATISTICS (BURGLARY, PUBLIC PLACE VIOLENCE, SHOP THEFT } \\
\text { ETC) }\end{array}$ & CRIME/SAFETY & SOCIAL WELL-BEING \\
\hline 2170 & PORIRUA CITY COUNCIL & A SAFE CITY & $\begin{array}{l}\text { VIEWS ON SAFETY IN THE HOME, NEIGHBOURHOOD, CITY CENTRE, } \\
\text { PUBLIC TRANSPORT }\end{array}$ & TRANSPORT & ECONOMIC WELL-BEING \\
\hline 2171 & PORIRUA CITY COUNCIL & A SAFE CITY & $\begin{array}{l}\text { PROGRESS WITH THE PORIRUA: SAFE AS! COMMUNITY SAFETY } \\
\text { STRATEGY }\end{array}$ & CRIME/SAFETY & SOCIAL WELL-BEING \\
\hline
\end{tabular}


Appendix A: List of Community Well-beings Outcomes and Measures by Councils

\begin{tabular}{|c|c|c|c|c|c|}
\hline S.NO. & COUNCIL & OUTCOME & MEASURES & MEASURES BROAD GROUP & WELL-BEINGS \\
\hline 2172 & PORIRUA CITY COUNCIL & A SAFE CITY & MAINTAINING WHO SAFER CITY STATUS & CRIME/SAFETY & SOCIAL WELL-BEING \\
\hline 2173 & PORIRUA CITY COUNCIL & A SAFE CITY & VIEWS ON EMERGENCY MANAGEMENT IN GENERAL & NATURAL DISASTER/RESILIENCE & ENVIRONMENTAL WELL-BEING \\
\hline 2174 & PORIRUA CITY COUNCIL & A SAFE CITY & HOUSEHOLDS PREPARED FOR EMERGENCIES & NATURAL DISASTER/RESILIENCE & ENVIRONMENTAL WELL-BEING \\
\hline 2175 & PORIRUA CITY COUNCIL & A SAFE CITY & $\begin{array}{l}\text { USE OF READYNET (WEB NETWORK FOR EMERGENCY MANAGEMENT } \\
\text { INFORMATION BY ORGANISATIONS AND FAMILIES) }\end{array}$ & NATURAL DISASTER/RESILIENCE & ENVIRONMENTAL WELL-BEING \\
\hline 2176 & PORIRUA CITY COUNCIL & A SAFE CITY & INFORMATION ON EMERGENCY TRAINING AND FACILITIES ETC & NATURAL DISASTER/RESILIENCE & ENVIRONMENTAL WELL-BEING \\
\hline 2177 & PORIRUA CITY COUNCIL & SUSTAINABLY DESIGNED \& BUILT & PORIRUA DEVELOPMENT FRAMEWORK AND DISTRICT PLAN & PLANNING/BUILDING CONSENTS & ECONOMIC WELL-BEING \\
\hline 2178 & PORIRUA CITY COUNCIL & SUSTAINABLY DESIGNED \& BUILT & RESOURCE AND BUILDING CONSENT TYPES AND TRENDS & PLANNING/BUILDING CONSENTS & ECONOMIC WELL-BEING \\
\hline 2180 & PORIRUA CITY COUNCIL & SUSTAINABLY DESIGNED \& BUILT & BUILT HERITAGE SITES & HERITAGE/HISTORIC & CULTURAL WELL-BEING \\
\hline 2181 & PORIRUA CITY COUNCIL & SUSTAINABLY DESIGNED \& BUILT & PRIDE IN CITYS 'LOOK AND FEEL' & COMMUNITY DEVELOPMENT & SOCIAL WELL-BEING \\
\hline 2182 & PORIRUA CITY COUNCIL & SUSTAINABLY DESIGNED \& BUILT & VIEWS ON CITY PLANNING & PLANNING/BUILDING CONSENTS & ECONOMIC WELL-BEING \\
\hline 2183 & PORIRUA CITY COUNCIL & SUSTAINABLY DESIGNED \& BUILT & A HOUSING NEEDS ASSESSMENT & HOUSE AFFORDABILITY & ECONOMIC WELL-BEING \\
\hline 2184 & PORIRUA CITY COUNCIL & SUSTAINABLY DESIGNED \& BUILT & $\begin{array}{l}\text { HOUSING TENURE BY INCOME; HOUSE SALES/ PRICES; LEVELS OF RENT } \\
\text { SOCIAL HOUSING AS A PROPORTION OF PRIVATE RENTED DWELLINGS; } \\
\text { OCCUPANCY LEVELS; (CROWDING ETC); HOME HEATING }\end{array}$ & HOUSE AFFORDABILITY & ECONOMIC WELL-BEING \\
\hline 2185 & PORIRUA CITY COUNCIL & SUSTAINABLY DESIGNED \& BUILT & A SUSTAINABLE HOUSING PLAN FOR PORIRUA CITY & HOUSE AFFORDABILITY & ECONOMIC WELL-BEING \\
\hline 2187 & PORIRUA CITY COUNCIL & SUSTAINABLY DESIGNED \& BUILT & WATER USE PER PERSON CITY PROPORTION OF REGIONAL SUPPLY & $\begin{array}{l}\text { RIVER, LAKES, COASTAL AND } \\
\text { GROUNDWATER }\end{array}$ & ENVIRONMENTAL WELL-BEING \\
\hline 2188 & PORIRUA CITY COUNCIL & SUSTAINABLY DESIGNED \& BUILT & LEVELS OF WASTE TO LANDFILL AND RECYCLING & WASTE TO LANDFILL & ENVIRONMENTAL WELL-BEING \\
\hline 2189 & PORIRUA CITY COUNCIL & SUSTAINABLY DESIGNED \& BUILT & RENEWABLE AND NON-RENEWABLE ENERGY CONSUMPTION & ENERGY USAGE & ENVIRONMENTAL WELL-BEING \\
\hline 2190 & PORIRUA CITY COUNCIL & SUSTAINABLY DESIGNED \& BUILT & KNOWLEDGE AND AWARENESS OF SUSTAINABILITY & $\begin{array}{l}\text { ENVIRONMENT/ENVIRONMENTAL } \\
\text { ATTITUDES }\end{array}$ & ENVIRONMENTAL WELL-BEING \\
\hline 2191 & PORIRUA CITY COUNCIL & SUSTAINABLY DESIGNED \& BUILT & SUSTAINABILITY PROGRAMMES AND ACTIVITIES & COUNCIL FACILITIES & SOCIAL WELL-BEING \\
\hline 2192 & PORIRUA CITY COUNCIL & A VALUED NATURAL ENVIRONMENT & $\begin{array}{l}\text { INFORMATION ON WATER, SOIL, SEDIMENT, AIR, PLANTS AND } \\
\text { WILDLIFE }\end{array}$ & $\begin{array}{l}\text { RIVER, LAKES, COASTAL AND } \\
\text { GROUNDWATER }\end{array}$ & ENVIRONMENTAL WELL-BEING \\
\hline 2193 & PORIRUA CITY COUNCIL & A VALUED NATURAL ENVIRONMENT & LEVELS AND MEASURES OF LAND PROTECTION & PLANNING/BUILDING CONSENTS & ECONOMIC WELL-BEING \\
\hline 2194 & PORIRUA CITY COUNCIL & A VALUED NATURAL ENVIRONMENT & SITES OF ECOLOGICAL HERITAGE AND SPECIAL SIGNIFICANCE & HERITAGE/HISTORIC & CULTURAL WELL-BEING \\
\hline 2195 & PORIRUA CITY COUNCIL & A VALUED NATURAL ENVIRONMENT & VIEWS ON CITY PLANNING AND THE ENVIRONMENT & $\begin{array}{l}\text { ENVIRONMENT/ENVIRONMENTAL } \\
\text { ATTITUDES }\end{array}$ & ENVIRONMENTAL WELL-BEING \\
\hline 2196 & PORIRUA CITY COUNCIL & A VALUED NATURAL ENVIRONMENT & VIEWS ON THE 'LOOK AND FEEL' OF THE CITY & CBD: CITY CENTRE & SOCIAL WELL-BEING \\
\hline 2197 & PORIRUA CITY COUNCIL & A VALUED NATURAL ENVIRONMENT & VIEWS ON RUBBISH AND POLLUTION (WATER, AIR ETC) & $\begin{array}{l}\text { RIVER, LAKES, COASTAL AND } \\
\text { GROUNDWATER }\end{array}$ & ENVIRONMENTAL WELL-BEING \\
\hline 2198 & PORIRUA CITY COUNCIL & A VALUED NATURAL ENVIRONMENT & $\begin{array}{l}\text { VIEWS ON RUBBISH COLLECTION AND RECYCLING SERVICES, FACILITIES } \\
\text { ETC }\end{array}$ & RECYCLING/GREEN WASTE & ENVIRONMENTAL WELL-BEING \\
\hline 2199 & PORIRUA CITY COUNCIL & A VALUED NATURAL ENVIRONMENT & PORIRUA DEVELOPMENT FRAMEWORK & PLANNING/BUILDING CONSENTS & ECONOMIC WELL-BEING \\
\hline 2203 & PORIRUA CITY COUNCIL & A VALUED NATURAL ENVIRONMENT & $\begin{array}{l}\text { DISTRICT PLAN (ALL PARTS: RURAL, URBAN, SUBURBAN, LANDSCAPE } \\
\text { ETC) }\end{array}$ & PLANNING/BUILDING CONSENTS & WELL-BEING \\
\hline
\end{tabular}


Appendix A: List of Community Well-beings Outcomes and Measures by Councils

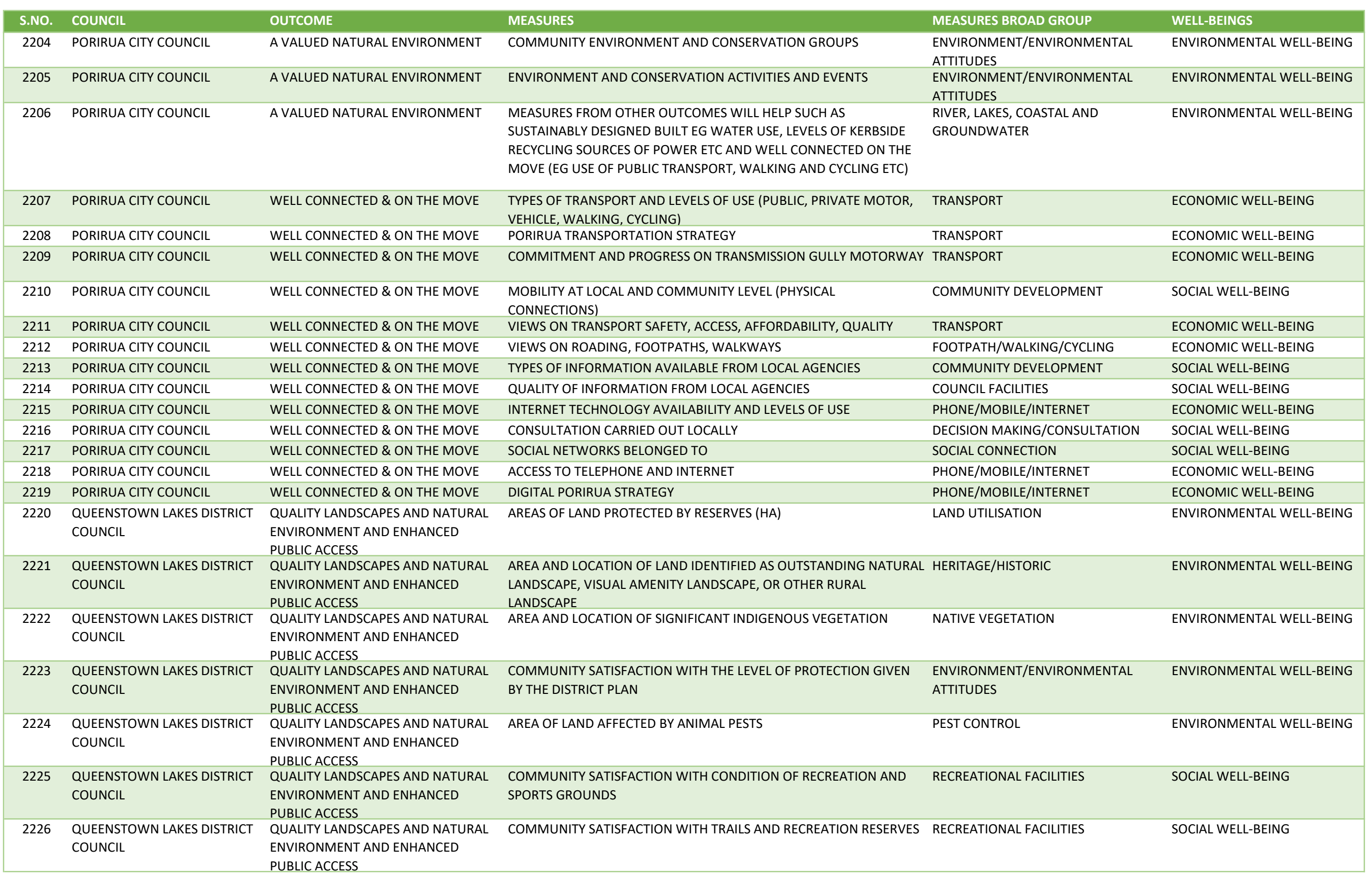


Appendix A: List of Community Well-beings Outcomes and Measures by Councils

\begin{tabular}{|c|c|c|c|c|c|}
\hline S.NO. & COUNCIL & OUTCOME & MEASURES & MEASURES BROAD GROUP & WELL-BEINGS \\
\hline 2227 & $\begin{array}{l}\text { QUEENSTOWN LAKES DISTRICT } \\
\text { COUNCIL }\end{array}$ & $\begin{array}{l}\text { QUALITY LANDSCAPES AND NATURAL } \\
\text { ENVIRONMENT AND ENHANCED } \\
\text { PUBLIC ACCESS }\end{array}$ & COMMUNITY SATISFACTION WITH ACCESS TO WATERWAYS & $\begin{array}{l}\text { RIVER, LAKES, COASTAL AND } \\
\text { GROUNDWATER }\end{array}$ & ENVIRONMENTAL WELL-BEING \\
\hline 2228 & $\begin{array}{l}\text { QUEENSTOWN LAKES DISTRICT } \\
\text { COUNCIL }\end{array}$ & $\begin{array}{l}\text { A SAFE AND HEALTHY COMMUNITY } \\
\text { THAT IS STRONG DIVERSE AND } \\
\text { INCLUSIVE FOR PEOPLE OF ALL AGE } \\
\text { GROUPS AND INCOMES }\end{array}$ & MEDIAN HOUSE SALE PRICES & HOUSE AFFORDABILITY & ECONOMIC WELL-BEING \\
\hline 2229 & $\begin{array}{l}\text { QUEENSTOWN LAKES DISTRICT } \\
\text { COUNCIL }\end{array}$ & $\begin{array}{l}\text { A SAFE AND HEALTHY COMMUNITY } \\
\text { THAT IS STRONG DIVERSE AND } \\
\text { INCLUSIVE FOR PEOPLE OF ALL AGE } \\
\text { GROUPS AND INCOMES }\end{array}$ & TENURE STRUCTURE AND RENTAL PRICE & HOUSE AFFORDABILITY & ECONOMIC WELL-BEING \\
\hline 2230 & $\begin{array}{l}\text { QUEENSTOWN LAKES DISTRICT } \\
\text { COUNCIL }\end{array}$ & $\begin{array}{l}\text { A SAFE AND HEALTHY COMMUNITY } \\
\text { THAT IS STRONG DIVERSE AND } \\
\text { INCLUSIVE FOR PEOPLE OF ALL AGE } \\
\text { GROUPS AND INCOMES }\end{array}$ & AGE & $\begin{array}{l}\text { POPULATION/ETHNICITY/DEMOGRAPHI } \\
\text { CS }\end{array}$ & DEMOGRAPHICS \\
\hline 2231 & $\begin{array}{l}\text { QUEENSTOWN LAKES DISTRICT } \\
\text { COUNCIL }\end{array}$ & $\begin{array}{l}\text { A SAFE AND HEALTHY COMMUNITY } \\
\text { THAT IS STRONG DIVERSE AND } \\
\text { INCLUSIVE FOR PEOPLE OF ALL AGE } \\
\text { GROUPS AND INCOMES }\end{array}$ & ETHNICITY & $\begin{array}{l}\text { POPULATION/ETHNICITY/DEMOGRAPHI } \\
\text { CS }\end{array}$ & DEMOGRAPHICS \\
\hline 2232 & $\begin{array}{l}\text { QUEENSTOWN LAKES DISTRICT } \\
\text { COUNCIL }\end{array}$ & $\begin{array}{l}\text { A SAFE AND HEALTHY COMMUNITY } \\
\text { THAT IS STRONG DIVERSE AND } \\
\text { INCLUSIVE FOR PEOPLE OF ALL AGE } \\
\text { GROUPS AND INCOMES }\end{array}$ & NUMBER OF VOLUNTEERS & VOLUNTEER/UNPAID WORK & SOCIAL WELL-BEING \\
\hline 2233 & $\begin{array}{l}\text { QUEENSTOWN LAKES DISTRICT } \\
\text { COUNCIL }\end{array}$ & $\begin{array}{l}\text { A SAFE AND HEALTHY COMMUNITY } \\
\text { THAT IS STRONG DIVERSE AND } \\
\text { INCLUSIVE FOR PEOPLE OF ALL AGE } \\
\text { GROUPS AND INCOMES }\end{array}$ & EMPLOYMENT BY INDUSTRY TYPE & $\begin{array}{l}\text { LEVEL OF EMPLOYMENT AND } \\
\text { BUSINESSES }\end{array}$ & ECONOMIC WELL-BEING \\
\hline 2234 & $\begin{array}{l}\text { QUEENSTOWN LAKES DISTRICT } \\
\text { COUNCIL }\end{array}$ & $\begin{array}{l}\text { A SAFE AND HEALTHY COMMUNITY } \\
\text { THAT IS STRONG DIVERSE AND } \\
\text { INCLUSIVE FOR PEOPLE OF ALL AGE } \\
\text { GROUPS AND INCOMES }\end{array}$ & INCOME BRACKETS & INCOME & ECONOMIC WELL-BEING \\
\hline 2235 & $\begin{array}{l}\text { QUEENSTOWN LAKES DISTRICT } \\
\text { COUNCIL }\end{array}$ & $\begin{array}{l}\text { A SAFE AND HEALTHY COMMUNITY } \\
\text { THAT IS STRONG DIVERSE AND } \\
\text { INCLUSIVE FOR PEOPLE OF ALL AGE } \\
\text { GROUPS AND INCOMES }\end{array}$ & EDUCATIONAL ATTAINMENT & $\begin{array}{l}\text { POPULATION/ETHNICITY/DEMOGRAPHI } \\
\text { CS }\end{array}$ & DEMOGRAPHICS \\
\hline 2236 & $\begin{array}{l}\text { QUEENSTOWN LAKES DISTRICT } \\
\text { COUNCIL }\end{array}$ & $\begin{array}{l}\text { A SAFE AND HEALTHY COMMUNITY } \\
\text { THAT IS STRONG DIVERSE AND } \\
\text { INCLUSIVE FOR PEOPLE OF ALL AGE } \\
\text { GROUPS AND INCOMES }\end{array}$ & RELIGIOUS AFFILIATION & $\begin{array}{l}\text { POPULATION/ETHNICITY/DEMOGRAPHI } \\
\text { CS }\end{array}$ & DEMOGRAPHICS \\
\hline 2237 & $\begin{array}{l}\text { QUEENSTOWN LAKES DISTRICT } \\
\text { COUNCIL }\end{array}$ & $\begin{array}{l}\text { A SAFE AND HEALTHY COMMUNITY } \\
\text { THAT IS STRONG DIVERSE AND } \\
\text { INCLUSIVE FOR PEOPLE OF ALL AGE } \\
\text { GROUPS AND INCOMES }\end{array}$ & COMMUNITY SATISFACTION WITH RANGE OF HEALTH SERVICES & HEALTH/HEALTH SERVICES & SOCIAL WELL-BEING \\
\hline
\end{tabular}




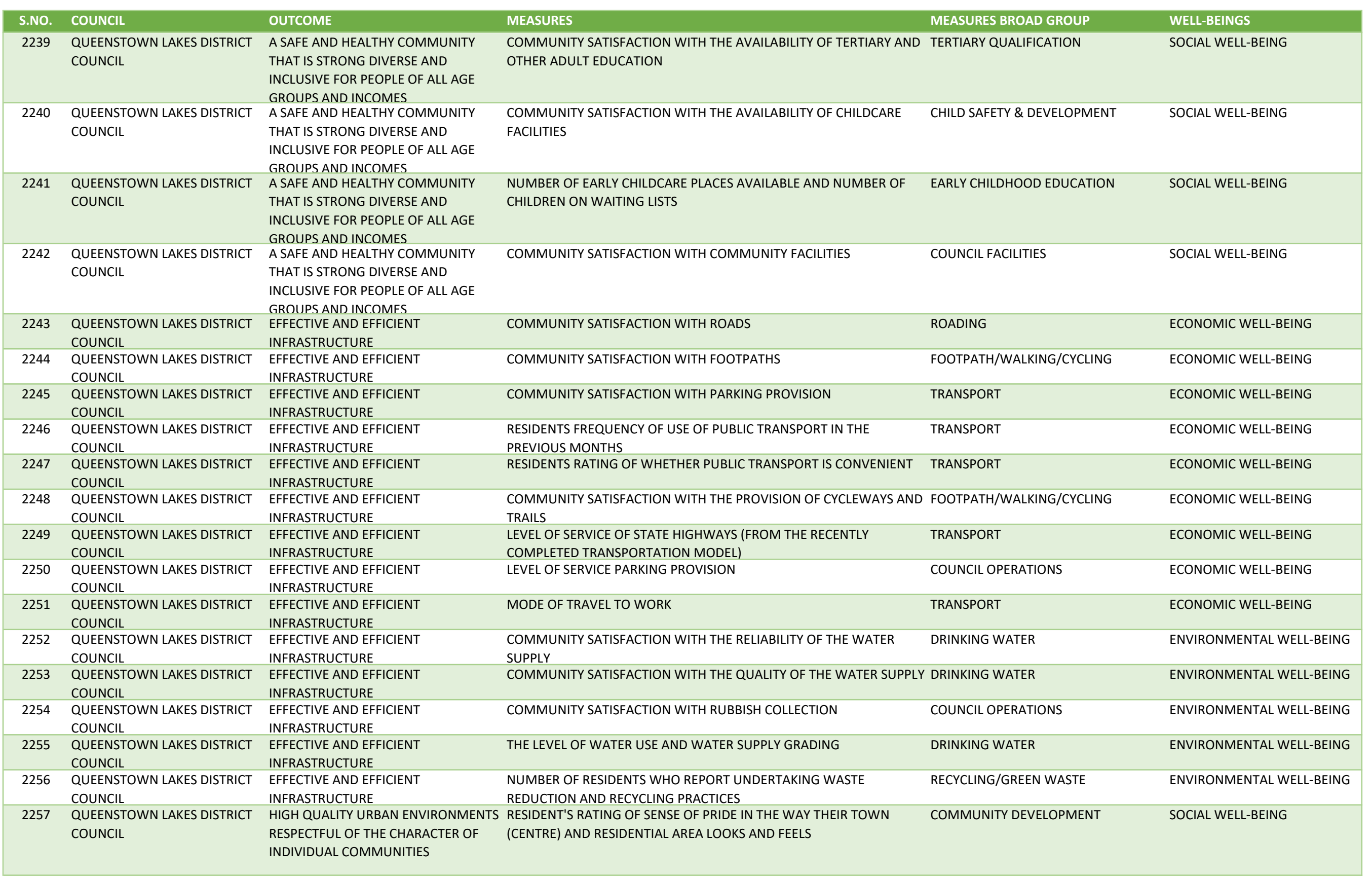




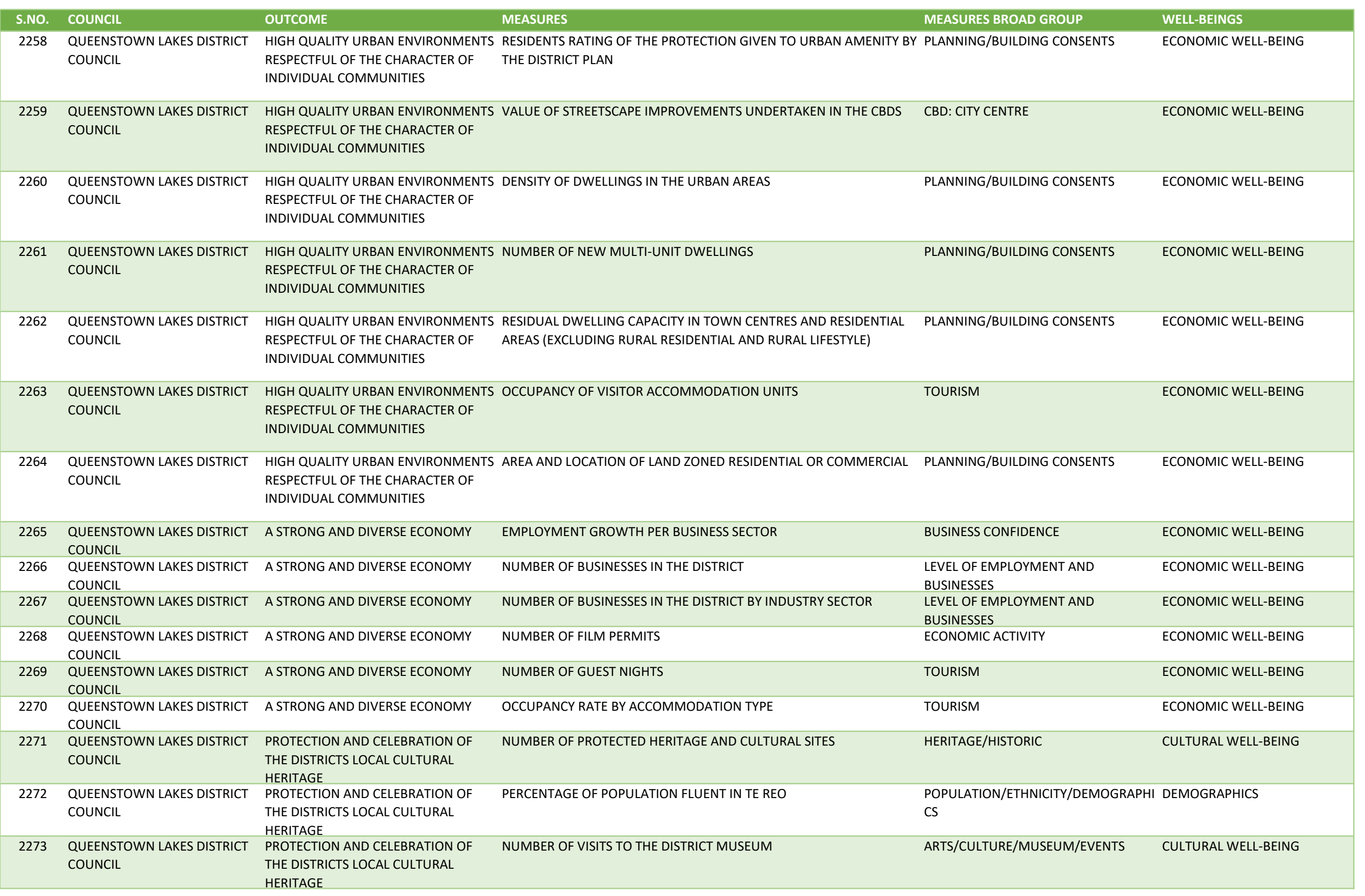


Appendix A: List of Community Well-beings Outcomes and Measures by Councils

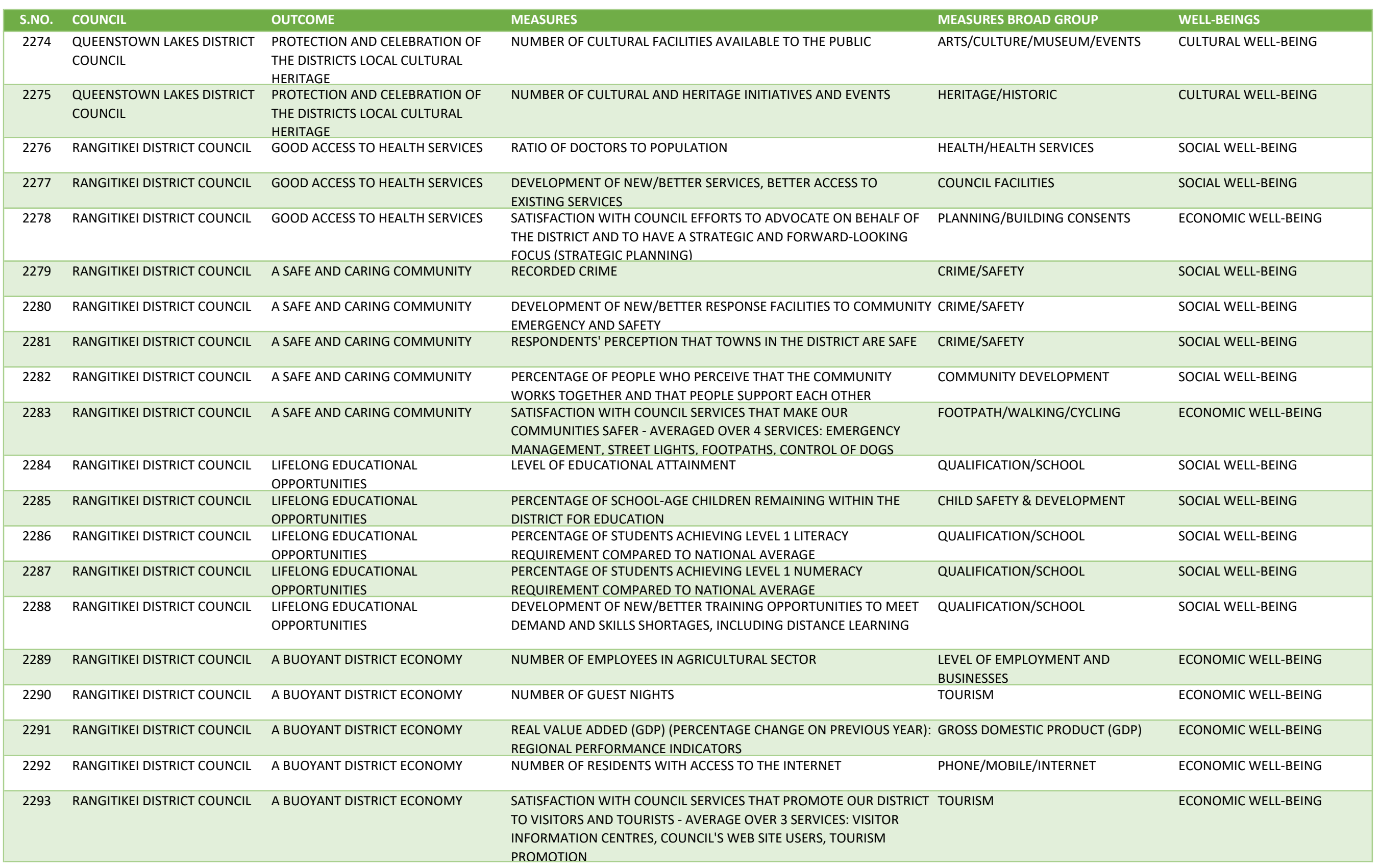




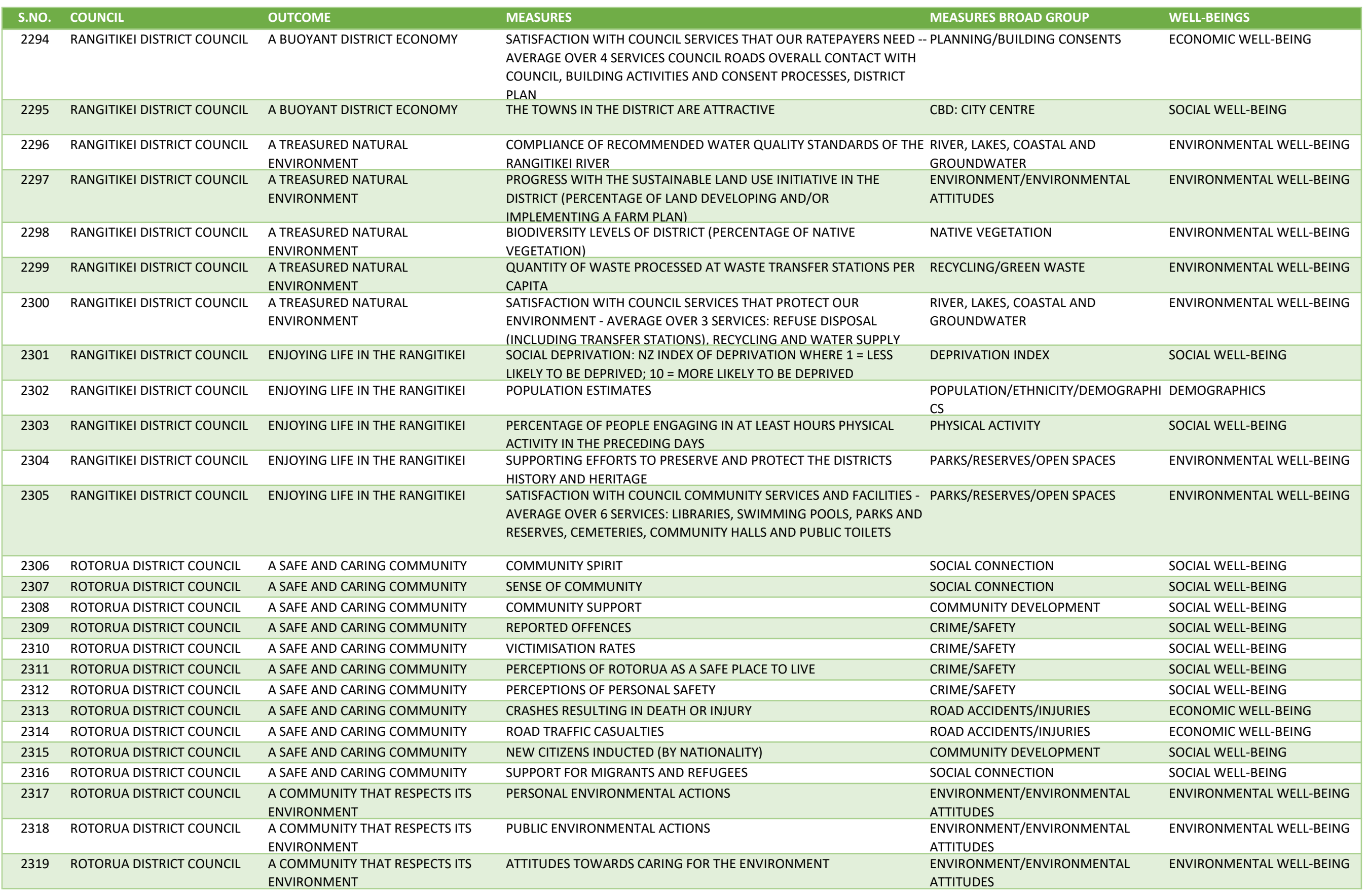


Appendix A: List of Community Well-beings Outcomes and Measures by Councils

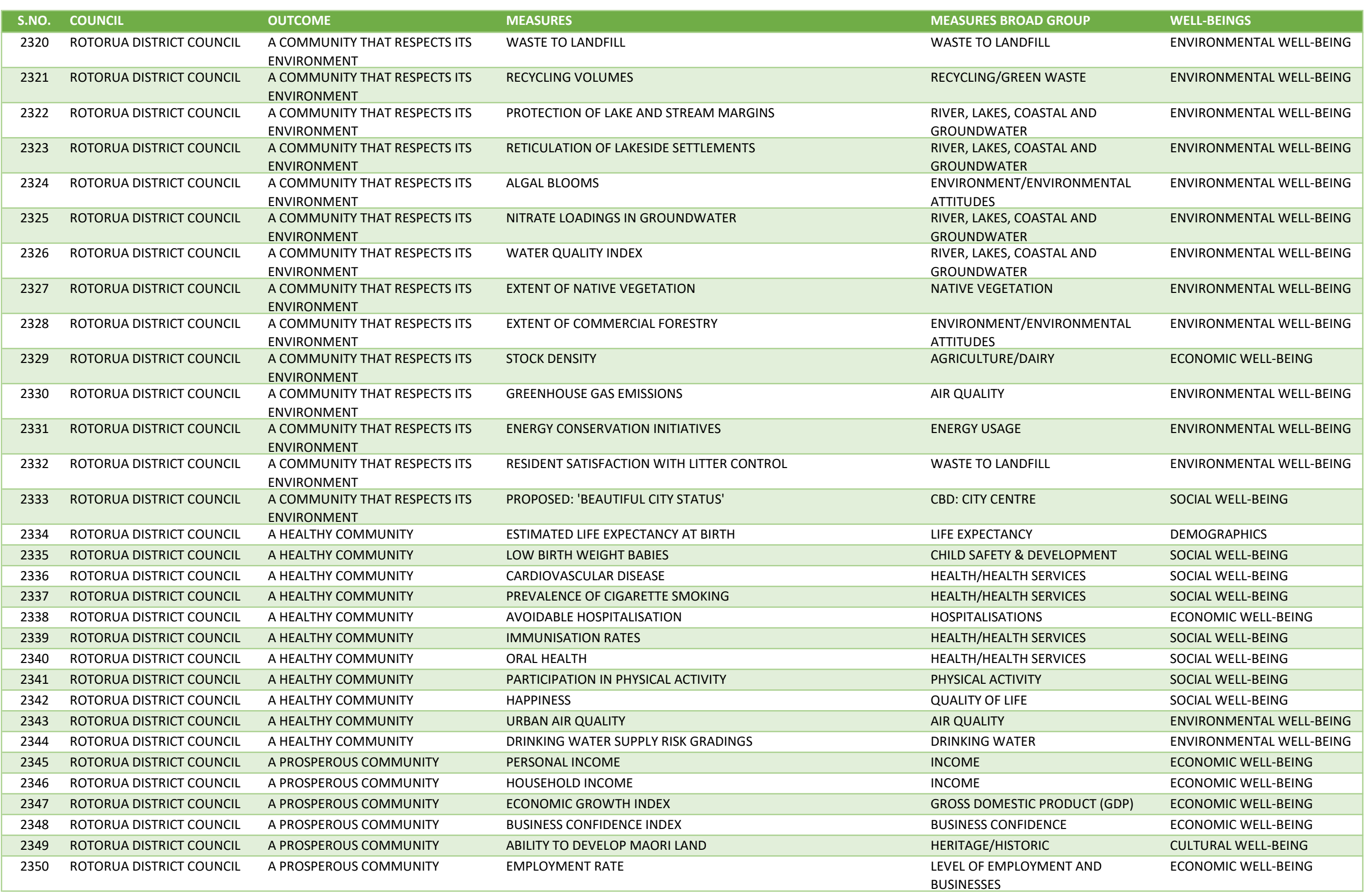


Appendix A: List of Community Well-beings Outcomes and Measures by Councils

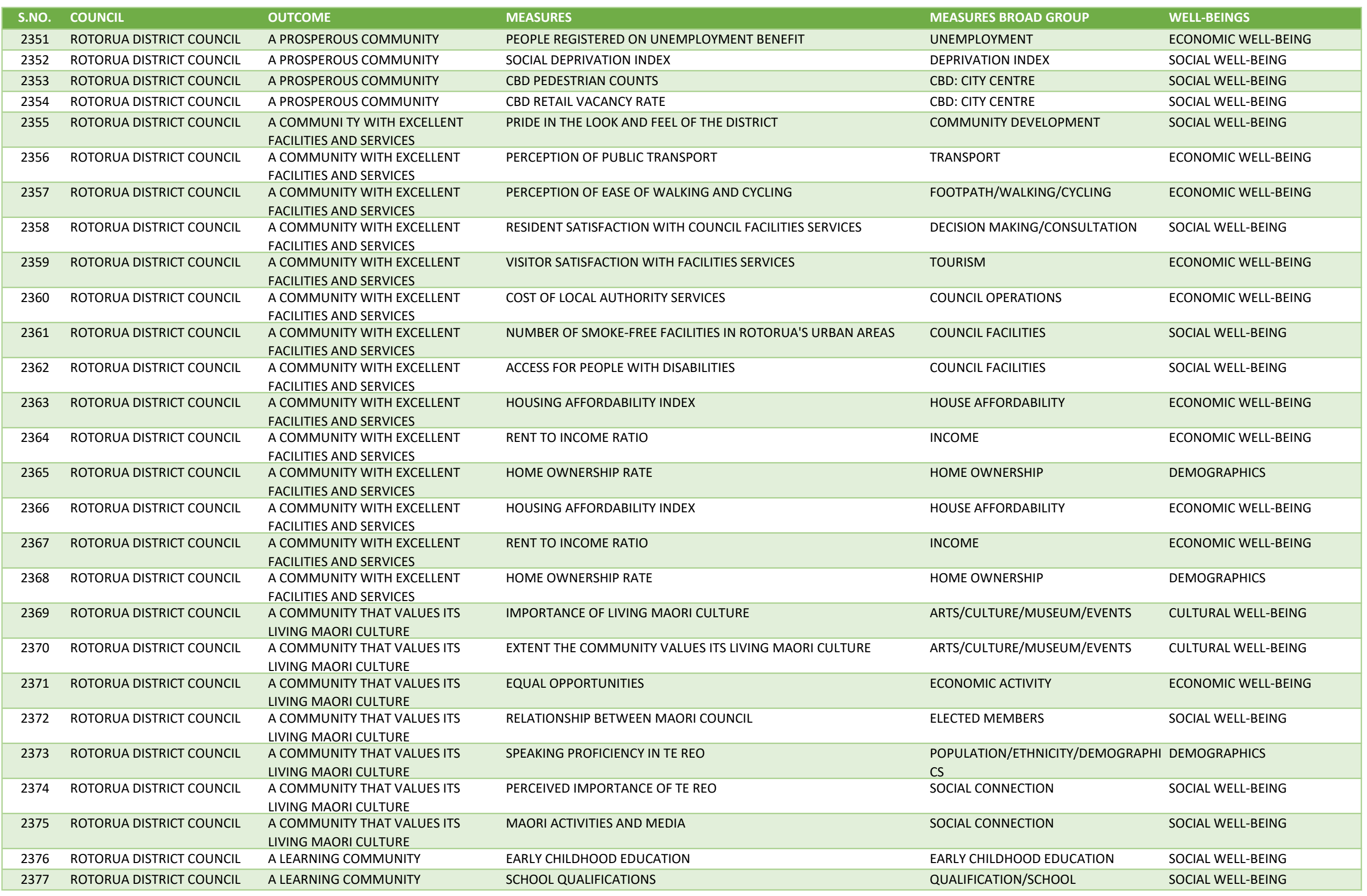


Appendix A: List of Community Well-beings Outcomes and Measures by Councils

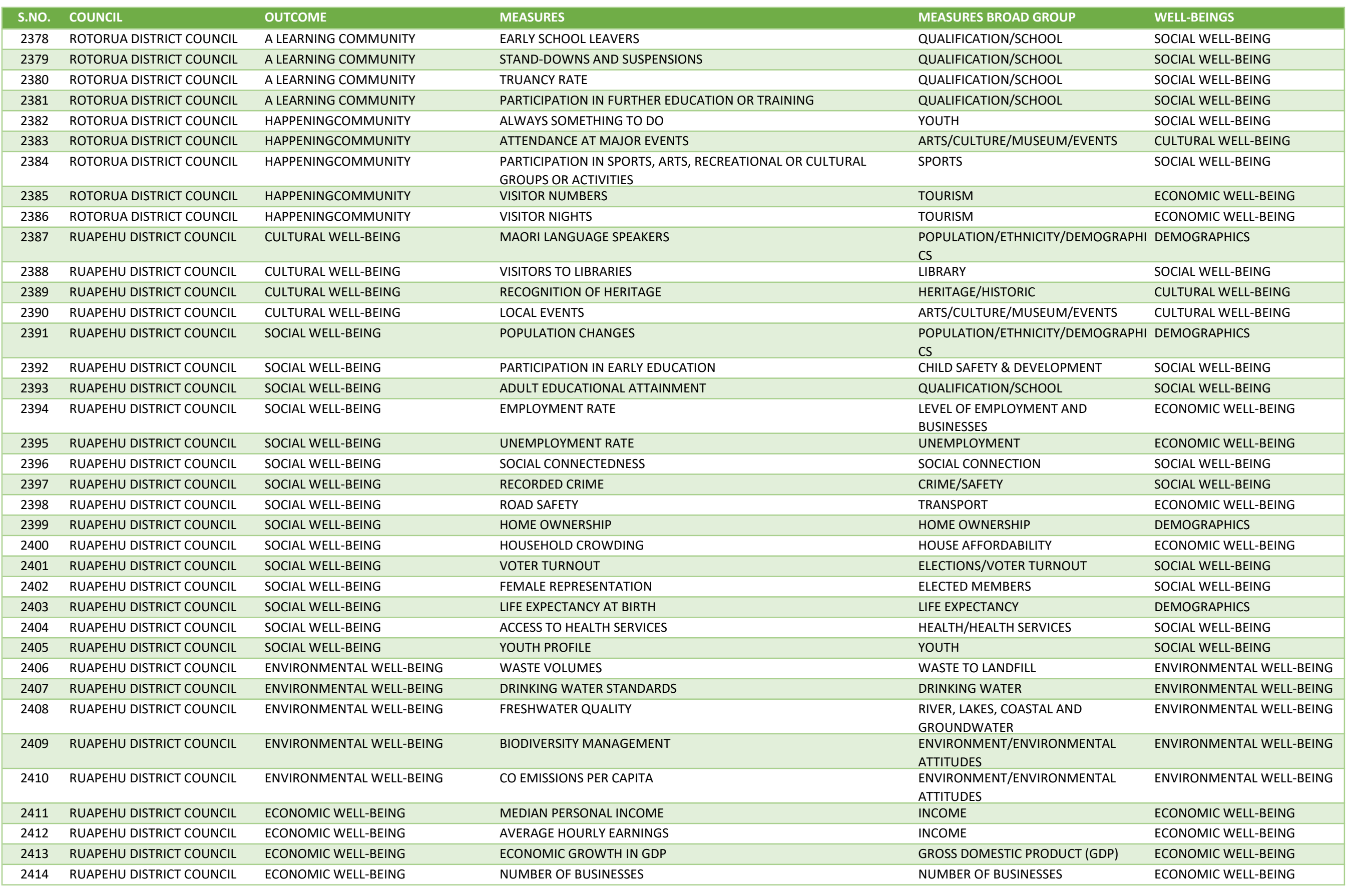


Appendix A: List of Community Well-beings Outcomes and Measures by Councils

\begin{tabular}{|c|c|c|c|c|c|}
\hline S.NO. & COUNCIL & OUTCOME & MEASURES & MEASURES BROAD GROUP & WELL-BEINGS \\
\hline 2415 & RUAPEHU DISTRICT COUNCIL & ECONOMIC WELL-BEING & EMPLOYMENT GROWTH & $\begin{array}{l}\text { LEVEL OF EMPLOYMENT AND } \\
\text { BUSINESSES }\end{array}$ & ECONOMIC WELL-BEING \\
\hline 2416 & RUAPEHU DISTRICT COUNCIL & ECONOMIC WELL-BEING & GROWTH IN TOURIST NUMBERS & TOURISM & ECONOMIC WELL-BEING \\
\hline 2417 & RUAPEHU DISTRICT COUNCIL & ECONOMIC WELL-BEING & RESOURCE CONSENTS & PLANNING/BUILDING CONSENTS & ECONOMIC WELL-BEING \\
\hline 2418 & RUAPEHU DISTRICT COUNCIL & ECONOMIC WELL-BEING & BUILDING CONSENTS & PLANNING/BUILDING CONSENTS & ECONOMIC WELL-BEING \\
\hline 2419 & SELWYN DISTRICT COUNCIL & ENVIRONMENT & $\begin{array}{l}\text { ENVIRONMENT CANTERBURY DO NOT DEEM IT NECESSARY TO } \\
\text { MONITOR AIR QUALITY IN THE DISTRICT }\end{array}$ & AIR QUALITY & ENVIRONMENTAL WELL-BEING \\
\hline 2420 & SELWYN DISTRICT COUNCIL & ENVIRONMENT & $\begin{array}{l}\text { TOTAL HECTARES OF VULNERABLE INDIGENOUS VEGETATION WHICH } \\
\text { ARE PROTECTED UNDER MANAGEMENT AGREEMENTS }\end{array}$ & NATIVE VEGETATION & ENVIRONMENTAL WELL-BEING \\
\hline 2422 & SELWYN DISTRICT COUNCIL & ENVIRONMENT & $\begin{array}{l}\text { COMPLIANCE WITH DWSNZ } 2008 \text { CRITERIA FOR WATER LEAVING } \\
\text { TREATMENT PLANT AS REPORTED BY THE MINISTRY OF HEALTH, FOR } \\
\text { EACH REGISTERED WATER SUPPLY }\end{array}$ & $\begin{array}{l}\text { RIVER, LAKES, COASTAL AND } \\
\text { GROUNDWATER }\end{array}$ & ENVIRONMENTAL WELL-BEING \\
\hline 2423 & SELWYN DISTRICT COUNCIL & ENVIRONMENT & $\begin{array}{l}\text { TOTAL NUMBER OF SEWER AND SEWER PUMP STATION OVERFLOWS } \\
\text { EVENTS (WET AND DRY WEATHER) }\end{array}$ & SEWERAGE/WASTEWATER & ENVIRONMENTAL WELL-BEING \\
\hline 2424 & SELWYN DISTRICT COUNCIL & ENVIRONMENT & $\begin{array}{l}\text { THE PROPOTION OF MONITORED FRESHWATER SITES CONSIDERED } \\
\text { SUITABLE FOR RECREATION }\end{array}$ & $\begin{array}{l}\text { RIVER, LAKES, COASTAL AND } \\
\text { GROUNDWATER }\end{array}$ & ENVIRONMENTAL WELL-BEING \\
\hline 2425 & SELWYN DISTRICT COUNCIL & ENVIRONMENT & $\begin{array}{l}\text { THE PERCENTAGE OF THE COMMUNITY THAT ARE SATISFIED WITH THE } \\
\text { QUALITY OF RURAL ENVIRONMENT, AND SATISFACTION WITH THE } \\
\text { PROTECTION GIVEN TO IT BY THE DISTRICT PLAN AS IDENTIFIED IN THE } \\
\text { RESIDENTS' SURVEY }\end{array}$ & $\begin{array}{l}\text { ENVIRONMENT/ENVIRONMENTAL } \\
\text { ATTITUDES }\end{array}$ & ENVIRONMENTAL WELL-BEING \\
\hline 2426 & SELWYN DISTRICT COUNCIL & ENVIRONMENT & $\begin{array}{l}\text { THE PERCENTAGE OF GROWTH OCCURRING INSIDE METROPOLITAN } \\
\text { URBAN LIMITS OR ON ZONED URBAN LAND }\end{array}$ & PLANNING/BUILDING CONSENTS & ECONOMIC WELL-BEING \\
\hline 2427 & SELWYN DISTRICT COUNCIL & SOCIAL & $\begin{array}{l}\text { INCREASE IN THE NUMBER OF HEALTH AND SOCIAL SERVICE } \\
\text { PROVIDERS PRESENT IN THE DISTRICT }\end{array}$ & HEALTH/HEALTH SERVICES & SOCIAL WELL-BEING \\
\hline 2428 & SELWYN DISTRICT COUNCIL & SOCIAL & $\begin{array}{l}\text { NUMBERS AND PERCENTAGEOF PEOPLE ENROLLED IN PUBLIC HEALTH } \\
\text { ORGANISATIONS }\end{array}$ & HEALTH/HEALTH SERVICES & SOCIAL WELL-BEING \\
\hline 2429 & SELWYN DISTRICT COUNCIL & SOCIAL & GPS PER HEAD OF POPULATION & HEALTH/HEALTH SERVICES & SOCIAL WELL-BEING \\
\hline 2430 & SELWYN DISTRICT COUNCIL & SOCIAL & IMPROVED ACCESS TO GP SERVICES & HEALTH/HEALTH SERVICES & SOCIAL WELL-BEING \\
\hline 2431 & SELWYN DISTRICT COUNCIL & SOCIAL & $\begin{array}{l}\text { INCREASE IN THE NUMBER OF PEOPLE PARTICIPATING IN REGULAR } \\
\text { PHYSICAL ACTIVITY }\end{array}$ & PHYSICAL ACTIVITY & SOCIAL WELL-BEING \\
\hline 2432 & SELWYN DISTRICT COUNCIL & SOCIAL & $\begin{array}{l}\text { INCREASE IN THE NUMBER OF NEIGHBOURHOOD SUPPORT AND OTHER } \\
\text { COMMUNITY SUPPORT GROUPS }\end{array}$ & COMMUNITY DEVELOPMENT & SOCIAL WELL-BEING \\
\hline 2433 & SELWYN DISTRICT COUNCIL & SOCIAL & $\begin{array}{l}\text { INCREASE IN RESIDENTS' PERCEPTION OF SAFETY AT HOME AND IN THE } \\
\text { COMMUNITY }\end{array}$ & CRIME/SAFETY & SOCIAL WELL-BEING \\
\hline 2434 & SELWYN DISTRICT COUNCIL & SOCIAL & REDUCTION IN NUMBER OF INJURIES IN SELWYN & ROAD ACCIDENTS/INJURIES & ECONOMIC WELL-BEING \\
\hline 2435 & SELWYN DISTRICT COUNCIL & SOCIAL & $\begin{array}{l}\text { REQUIREMENTS OF THE CANTERBURY CIVIL DEFENCE EMERGENCY } \\
\text { MANAGEMENT PLAN AND CIVIL DEFENCE EMERGENCY MANAGEMENT } \\
\text { ACT MET }\end{array}$ & NATURAL DISASTER/RESILIENCE & ENVIRONMENTAL WELL-BEING \\
\hline 2436 & SELWYN DISTRICT COUNCIL & SOCIAL & $\begin{array}{l}\text { REQUIREMENTS OF THE FOREST AND RURAL FIRES ACT 1977, THE } \\
\text { FOREST AND RURAL FIRES REGULATIONS } 2005 \text { AND NATIONAL } \\
\text { STANDARDS MET IN TERMS OF BOTH LEGISLATION COMPLIANCE AND } \\
\text { AGREED STANDARDS }\end{array}$ & NATURAL DISASTER/RESILIENCE & ENVIRONMENTAL WELL-BEING \\
\hline 2437 & SELWYN DISTRICT COUNCIL & SOCIAL & $\begin{array}{l}\text { INCREASE IN NUMBER OF VOLUNTEERS AND COMMUNITY GROUPS } \\
\text { INVOLVED IN EMERGENCY MANAGEMENT }\end{array}$ & VOLUNTEER/UNPAID WORK & SOCIAL WELL-BEING \\
\hline 2438 & SELWYN DISTRICT COUNCIL & SOCIAL & NO MORE THAN SERIOUS CASUALTIES ANNUALLY BY 2010 & ROAD ACCIDENTS/INJURIES & ECONOMIC WELL-BEING \\
\hline
\end{tabular}


Appendix A: List of Community Well-beings Outcomes and Measures by Councils

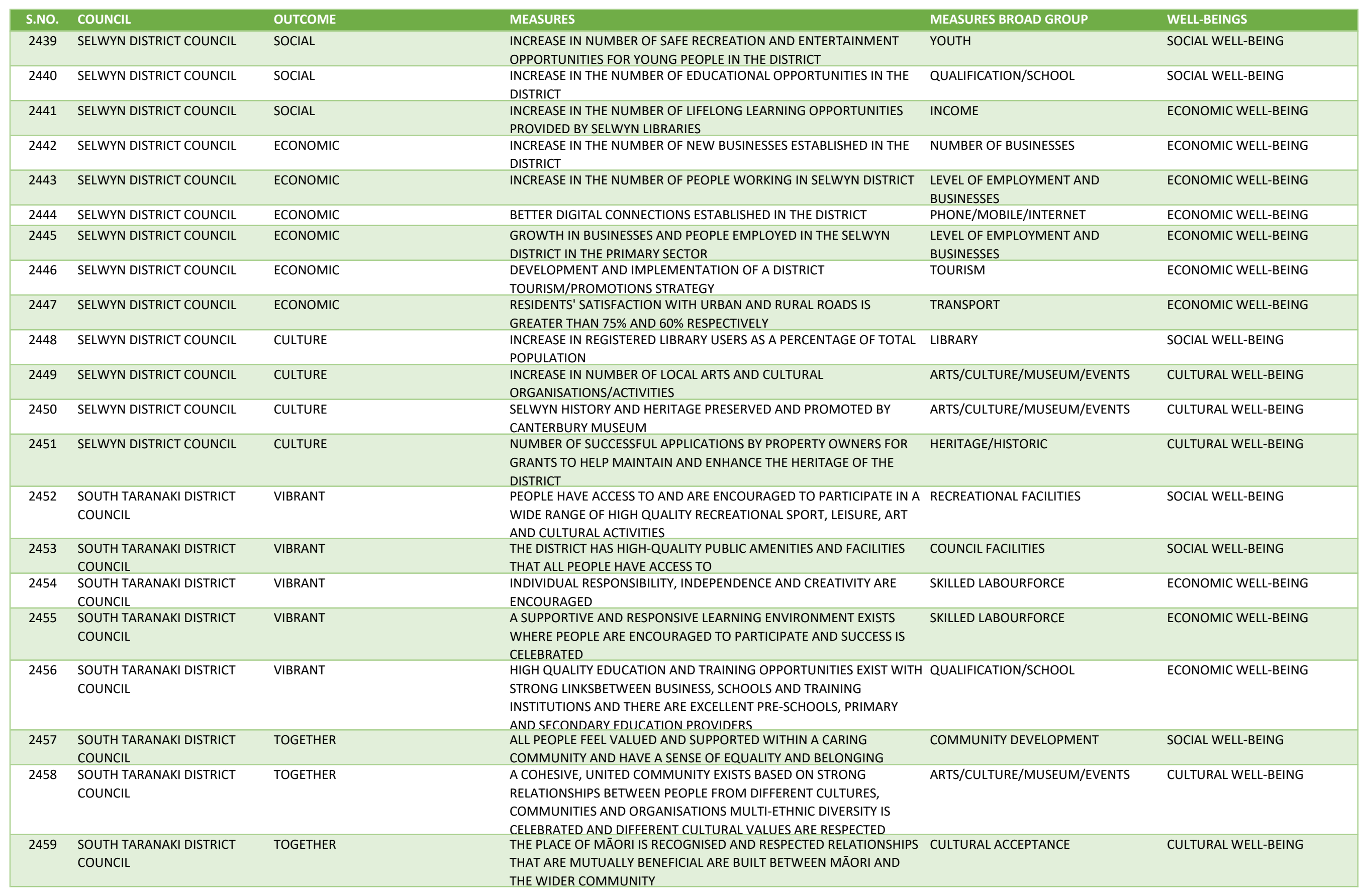




\begin{tabular}{|c|c|c|c|c|c|}
\hline S.NO. & COUNCIL & OUTCOME & MEASURES & MEASURES BROAD GROUP & WELL-BEINGS \\
\hline 2460 & $\begin{array}{l}\text { SOUTH TARANAKI DISTRICT } \\
\text { COUNCIL }\end{array}$ & TOGETHER & $\begin{array}{l}\text { PEOPLE FROM ALL SECTORS OF THE COMMUNITY ARE ABLE AND } \\
\text { ENCOURAGED TO CONTRIBUTE TO THEIR COMMUNITIES AND } \\
\text { OPPORTUNITIES TO PARTICIPATE ARE ENHANCED }\end{array}$ & COMMUNITY DEVELOPMENT & SOCIAL WELL-BEING \\
\hline 2461 & $\begin{array}{l}\text { SOUTH TARANAKI DISTRICT } \\
\text { COUNCIL }\end{array}$ & TOGETHER & $\begin{array}{l}\text { SOUTH TARANAKI PEOPLE HAVE A STRONG SENSE OF PLACE THE } \\
\text { SPECIAL SIGNIFICANCE OF MOUNT TARANAKI TO THE PEOPLE OF } \\
\text { SOUTH TARANAKI AND ITS ROLE IN SOUTH TARANAKIS IDENTITY IS } \\
\text { RECOGNISED AND PROVIDED FOR }\end{array}$ & COMMUNITY DEVELOPMENT & SOCIAL WELL-BEING \\
\hline 2462 & $\begin{array}{l}\text { SOUTH TARANAKI DISTRICT } \\
\text { COUNCIL }\end{array}$ & PROSPEROUS & $\begin{array}{l}\text { SOUTH TARANAKI IS AN ATTRACTIVE PLACE TO WORK DO BUSINESS } \\
\text { AND TO VISIT WHERE THE WORKFORCE HAS THE SKILLS TO MEET THE } \\
\text { NEEDS OF THE DISTRICTS EMPLOYERS }\end{array}$ & BUSINESS CONFIDENCE & ECONOMIC WELL-BEING \\
\hline 2463 & $\begin{array}{l}\text { SOUTH TARANAKI DISTRICT } \\
\text { COUNCIL }\end{array}$ & PROSPEROUS & $\begin{array}{l}\text { THE DISTRICT IS A BIRTHPLACE OF INNOVATION, IDEAS AND TRAINING } \\
\text { OPPORTUNITIES, WHERE TECHNOLOGICAL DEVELOPMENTS AND } \\
\text { OPPORTUNITIES FOR ADDED VALUE ARE CAPITALISED ON }\end{array}$ & BUSINESS CONFIDENCE & ECONOMIC WELL-BEING \\
\hline 2464 & $\begin{array}{l}\text { SOUTH TARANAKI DISTRICT } \\
\text { COUNCIL }\end{array}$ & PROSPEROUS & $\begin{array}{l}\text { SOUTH TARANAKIS STRENGTHS ARE RECOGNISED AND } \\
\text { DIVERSIFICATION OF BUSINESS ANDINDUSTRY IS VALUED AND } \\
\text { ENCOURAGED }\end{array}$ & $\begin{array}{l}\text { LEVEL OF EMPLOYMENT AND } \\
\text { BUSINESSES }\end{array}$ & ECONOMIC WELL-BEING \\
\hline 2465 & $\begin{array}{l}\text { SOUTH TARANAKI DISTRICT } \\
\text { COUNCIL }\end{array}$ & PROSPEROUS & $\begin{array}{l}\text { THERE IS A HIGH LEVEL OF EMPLOYMENT AND ADEQUATE INCOMES } \\
\text { WHERE LEARNING AND THE CREATION OF KNOWLEDGE IS VALUED }\end{array}$ & SKILLED LABOURFORCE & ECONOMIC WELL-BEING \\
\hline 2467 & $\begin{array}{l}\text { SOUTH TARANAKI DISTRICT } \\
\text { COUNCIL }\end{array}$ & PROSPEROUS & PEOPLE ARE CONFIDENT AND ARE HAPPY TO INVEST IN THE FUTURE & BUSINESS CONFIDENCE & ECONOMIC WELL-BEING \\
\hline 2468 & $\begin{array}{l}\text { SOUTH TARANAKI DISTRICT } \\
\text { COUNCIL }\end{array}$ & PROSPEROUS & $\begin{array}{l}\text { EFFECTIVE EFFICIENT SAFE AND RELIABLE INFRASTRUCTURE IS } \\
\text { PROVIDED AND MAINTAINED }\end{array}$ & ROADING & ECONOMIC WELL-BEING \\
\hline 2469 & $\begin{array}{l}\text { SOUTH TARANAKI DISTRICT } \\
\text { COUNCIL }\end{array}$ & SUSTAINABLE & $\begin{array}{l}\text { THERE IS SUSTAINABLE USE, DEVELOPMENT AND PROTECTION OF } \\
\text { RESOURCES SOUTH TARANAKI'S LAND AND SOIL, WATER, AIR AND } \\
\text { COAST, ITS BIODIVERSITY AND ITS NATURAL FEATURES AND } \\
\text { LANDSCAPES ARE UNDERSTOOD, VALUED, MAINTAINED AND } \\
\text { FNHANCFD FOR FIITIIRF GFNFRATIONS }\end{array}$ & LAND UTILISATION & ENVIRONMENTAL WELL-BEING \\
\hline 2470 & $\begin{array}{l}\text { SOUTH TARANAKI DISTRICT } \\
\text { COUNCIL }\end{array}$ & SUSTAINABLE & $\begin{array}{l}\text { SOUTH TARANAKI'S HISTORIC HERITAGE IS IDENTIFIED RECOGNISED } \\
\text { AND PROTECTED }\end{array}$ & ARTS/CULTURE/MUSEUM/EVENTS & CULTURAL WELL-BEING \\
\hline 2471 & $\begin{array}{l}\text { SOUTH TARANAKI DISTRICT } \\
\text { COUNCIL }\end{array}$ & SUSTAINABLE & $\begin{array}{l}\text { BUILT ENVIRONMENTS AND ENVIRONMENTAL AMENITIES ARE OF A } \\
\text { HIGH STANDARD AND CONTRIBUTE SIGNIFICANTLY TO THE WELL- } \\
\text { BEING OF PEOPLE AND COMMUNITIES }\end{array}$ & $\begin{array}{l}\text { ENVIRONMENT/ENVIRONMENTAL } \\
\text { ATTITUDES }\end{array}$ & ENVIRONMENTAL WELL-BEING \\
\hline 2472 & $\begin{array}{l}\text { SOUTH TARANAKI DISTRICT } \\
\text { COUNCIL }\end{array}$ & SUSTAINABLE & $\begin{array}{l}\text { PEOPLE ARE VALUED AND THEIR CONTRIBUTION TO THE ECONOMIC, } \\
\text { SOCIAL, CULTURAL AND ENVIRONMENTAL WELL-BEING OF THE } \\
\text { DISTRICT IS RECOGNISED AND SUPPORTED }\end{array}$ & COMMUNITY DEVELOPMENT & SOCIAL WELL-BEING \\
\hline 2473 & $\begin{array}{l}\text { SOUTH WAIKATO DISTRICT } \\
\text { COUNCIL }\end{array}$ & PROSPERITY FOR ALL & NUMBER OF BUSINESSES AND EMPLOYEES BY INDUSTRY & $\begin{array}{l}\text { LEVEL OF EMPLOYMENT AND } \\
\text { BUSINESSES }\end{array}$ & ECONOMIC WELL-BEING \\
\hline 2476 & $\begin{array}{l}\text { SOUTH WAIKATO DISTRICT } \\
\text { COUNCIL }\end{array}$ & PROSPERITY FOR ALL & INCOME BY ETHNICITY - MĀORI AND PACIFIC ISLAND ETHNIC GROUPS & INCOME & ECONOMIC WELL-BEING \\
\hline
\end{tabular}


Appendix A: List of Community Well-beings Outcomes and Measures by Councils

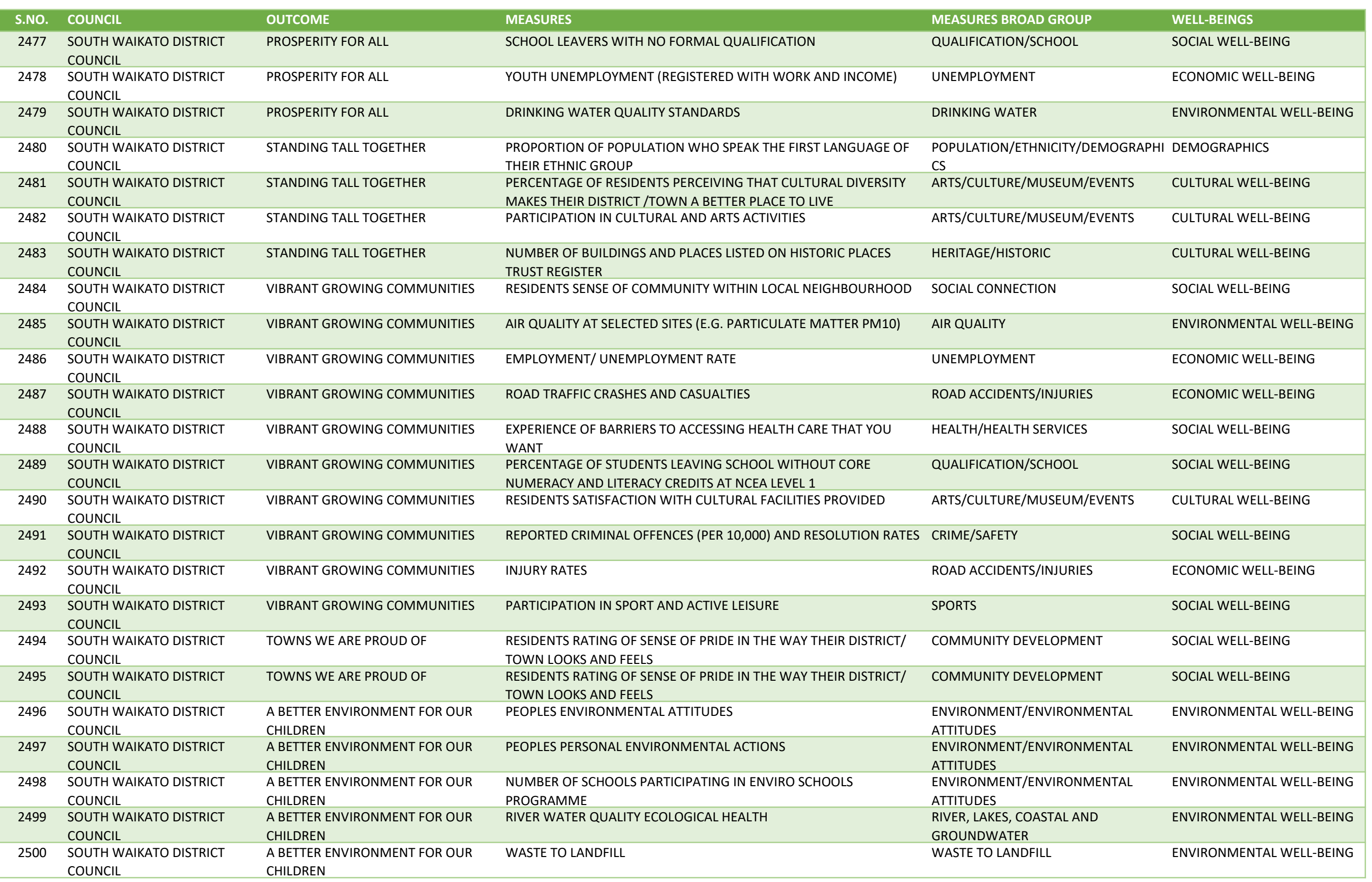


Appendix A: List of Community Well-beings Outcomes and Measures by Councils

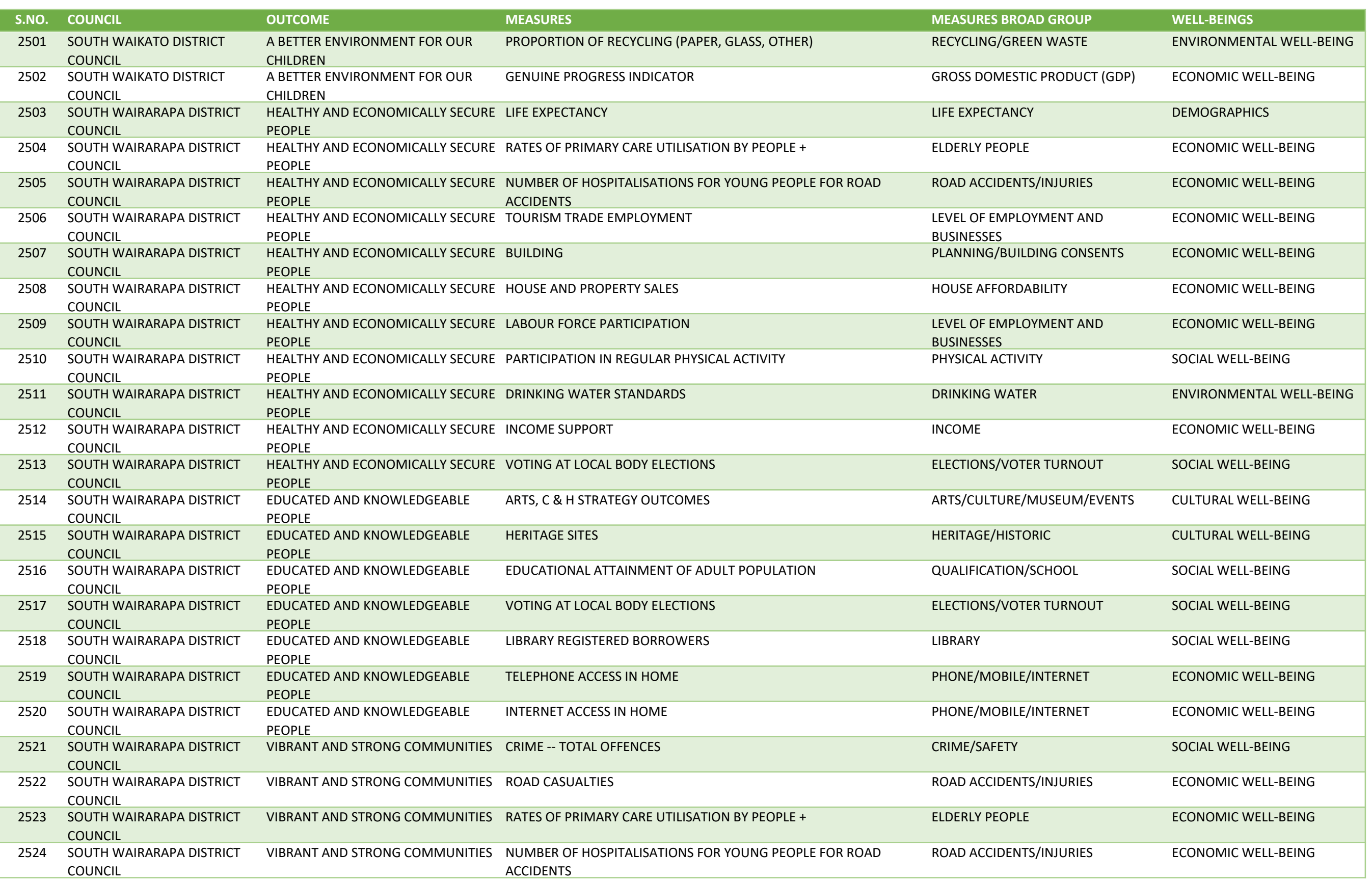


Appendix A: List of Community Well-beings Outcomes and Measures by Councils

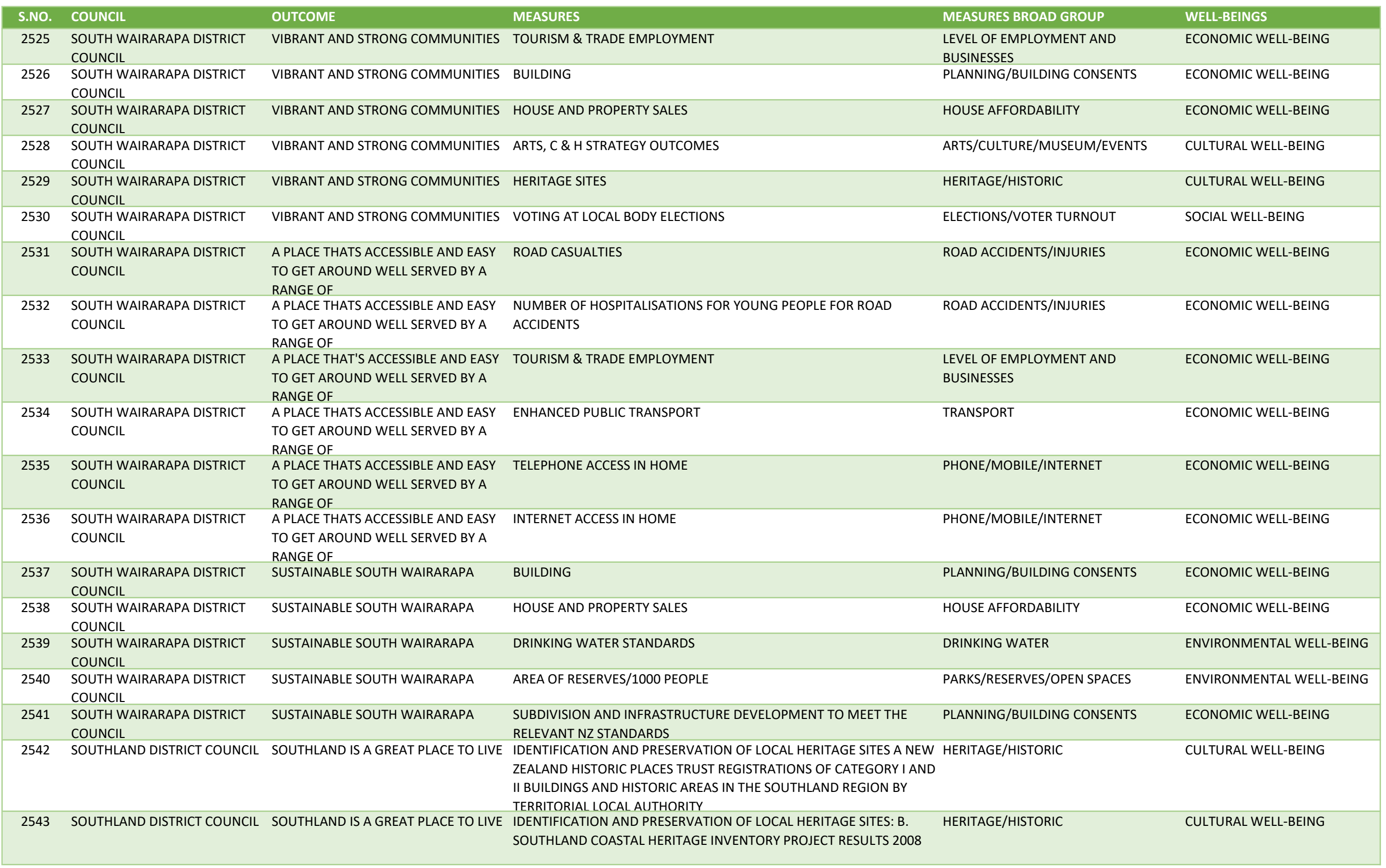




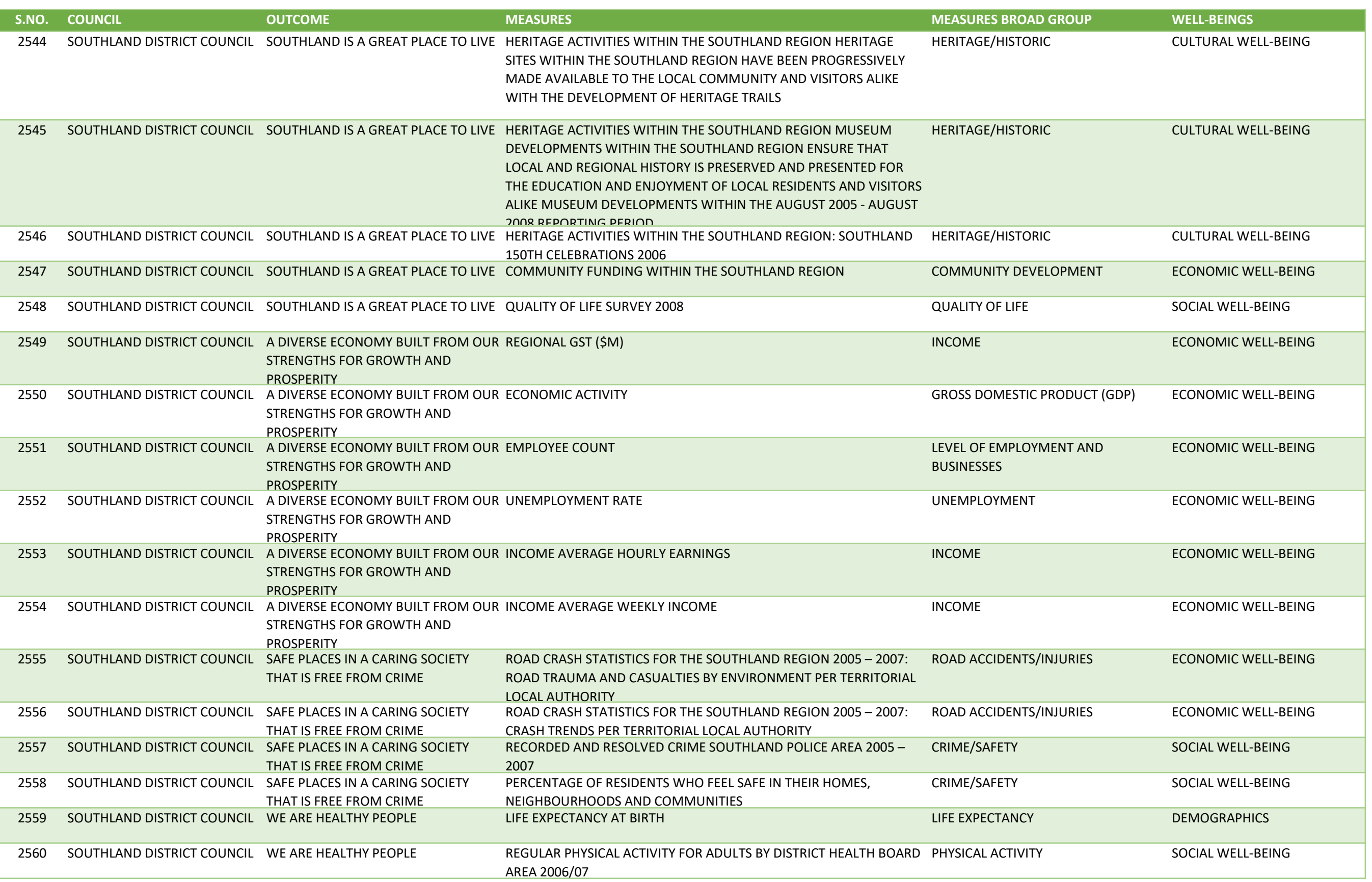


Appendix A: List of Community Well-beings Outcomes and Measures by Councils

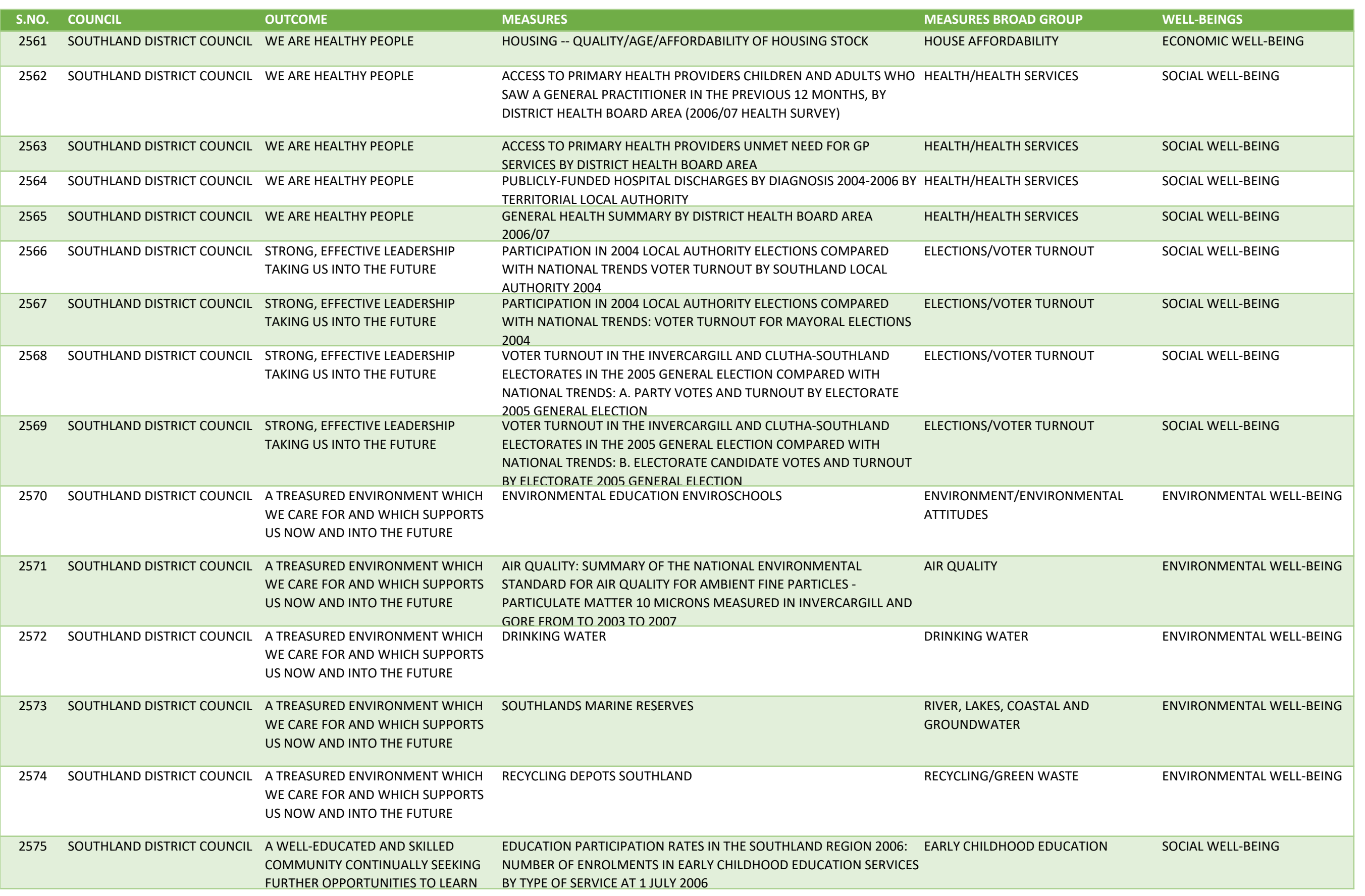




\begin{tabular}{|c|c|c|c|c|c|}
\hline S.NO. & COUNCIL & OUTCOME & MEASURES & MEASURES BROAD GROUP & WELL-BEINGS \\
\hline 2576 & SOUTHLAND DISTRICT COUNCIL & $\begin{array}{l}\text { A WELL-EDUCATED AND SKILLED } \\
\text { COMMUNITY CONTINUALY SEEKING } \\
\text { FURTHER OPPORTUNITIES TO LEARN }\end{array}$ & EDUCATION PARTICIPATION RATES IN THE SOUTHLAND REGION 2006 & QUALIFICATION/SCHOOL & SOCIAL WELL-BEING \\
\hline 2577 & SOUTHLAND DISTRICT COUNCIL & $\begin{array}{l}\text { A WELL-EDUCATED AND SKILLED } \\
\text { COMMUNITY CONTINUALLY SEEKING } \\
\text { FURTHER OPPORTUNITIES TO LEARN }\end{array}$ & $\begin{array}{l}\text { STUDENTS LEAVING SOUTHLAND SECONDARY SCHOOLS DURING } 2005 \\
\text { BY LEVEL OF HIGHEST ATTAINMENT }\end{array}$ & QUALIFICATION/SCHOOL & SOCIAL WELL-BEING \\
\hline 2578 & SOUTHLAND DISTRICT COUNCIL & $\begin{array}{l}\text { A WELL-EDUCATED AND SKILLED } \\
\text { COMMUNITY CONTINUALLY SEEKING } \\
\text { FURTHER OPPORTUNITIES TO LEARN }\end{array}$ & $\begin{array}{l}\text { STUDENTS LEAVING SOUTHLAND SECONDARY SCHOOLS DURING } 2005 \\
\text { BY LEVEL OF HIGHEST ATTAINMENT: STUDENTS LEAVING SOUTHLAND } \\
\text { SECONDARY SCHOOLS DURING } 2006 \text { BY LEVEL OF HIGHEST } \\
\text { ATTAINMENT }\end{array}$ & QUALIFICATION/SCHOOL & SOCIAL WELL-BEING \\
\hline 2579 & SOUTHLAND DISTRICT COUNCIL & $\begin{array}{l}\text { A WELL-EDUCATED AND SKILLED } \\
\text { COMMUNITY CONTINUALLY SEEKING } \\
\text { FURTHER OPPORTUNITIES TO LEARN }\end{array}$ & $\begin{array}{l}\text { HIGHEST QUALIFICATION FOR PEOPLE AGED } 15 \text { YEARS AND OVER, } \\
\text { SOUTHLAND REGION AND NEW ZEALAND, } 2006\end{array}$ & QUALIFICATION/SCHOOL & SOCIAL WELL-BEING \\
\hline 2580 & SOUTHLAND DISTRICT COUNCIL & $\begin{array}{l}\text { A WELL-EDUCATED AND SKILLED } \\
\text { COMMUNITY CONTINUALLY SEEKING } \\
\text { FURTHER OPPORTUNITIES TO LEARN }\end{array}$ & NUMBER OF PEOPLE PARTICIPATING IN CONTINUING EDUCATION & QUALIFICATION/SCHOOL & SOCIAL WELL-BEING \\
\hline 2581 & $\begin{array}{l}\text { SOUTHLAND REGIONAL } \\
\text { COUNCIL }\end{array}$ & SOUTHLAND IS A GREAT PLACE TO LIVE & $\begin{array}{l}\text { NEW ZEALAND HISTORIC PLACES TRUST REGISTRATIONS OF CATEGORY } \\
\text { I AND II BUILDINGS AND HISTORIC AREAS IN THE SOUTHLAND REGION } \\
\text { BY TERRITORIAL LOCAL AUTHORITY }\end{array}$ & HERITAGE/HISTORIC & CULTURAL WELL-BEING \\
\hline 2582 & $\begin{array}{l}\text { SOUTHLAND REGIONAL } \\
\text { COUNCIL }\end{array}$ & SOUTHLAND IS A GREAT PLACE TO LIVE & SOUTHLAND COASTAL HERITAGE INVENTORY PROJECT RESULTS 2008 & HERITAGE/HISTORIC & CULTURAL WELL-BEING \\
\hline 2584 & $\begin{array}{l}\text { SOUTHLAND REGIONAL } \\
\text { COUNCIL }\end{array}$ & SOUTHLAND IS A GREAT PLACE TO LIVE & $\begin{array}{l}\text { MUSEUM DEVELOPMENTS WITHIN THE SOUTHLAND REGION ENSURE } \\
\text { THAT LOCAL AND REGIONAL HISTORY IS PRESERVED AND PRESENTED } \\
\text { FOR THE EDUCATION AND ENJOYMENT OF LOCAL RESIDENTS AND } \\
\text { VISITORS ALIKE MUSEUM DEVELOPMENTS WITHIN THE AUGUST 2005- } \\
\text { AIIGIIST ?חOR RFPORTING PFRION }\end{array}$ & ARTS/CULTURE/MUSEUM/EVENTS & CULTURAL WELL-BEING \\
\hline 2585 & $\begin{array}{l}\text { SOUTHLAND REGIONAL } \\
\text { COUNCIL }\end{array}$ & SOUTHLAND IS A GREAT PLACE TO LIVE & $\begin{array}{l}\text { HERITAGE ACTIVITIES WITHIN THE SOUTHLAND REGION: SOUTHLAND } \\
\text { 150TH CELEBRATIONS } 2006\end{array}$ & HERITAGE/HISTORIC & CULTURAL WELL-BEING \\
\hline 2586 & $\begin{array}{l}\text { SOUTHLAND REGIONAL } \\
\text { COUNCIL }\end{array}$ & SOUTHLAND IS A GREAT PLACE TO LIVE & COMMUNITY FUNDING WITHIN THE SOUTHLAND REGION & COMMUNITY DEVELOPMENT & ECONOMIC WELL-BEING \\
\hline 2587 & $\begin{array}{l}\text { SOUTHLAND REGIONAL } \\
\text { COUNCIL }\end{array}$ & SOUTHLAND IS A GREAT PLACE TO LIVE & QUALITY OF LIFE SURVEY 2008 & QUALITY OF LIFE & SOCIAL WELL-BEING \\
\hline 2588 & $\begin{array}{l}\text { SOUTHLAND REGIONAL } \\
\text { COUNCIL }\end{array}$ & $\begin{array}{l}\text { A DIVERSE ECONOMY BUILT FROM OUR } \\
\text { STRENGTHS FOR GROWTH AND } \\
\text { PROSPERITY }\end{array}$ & REGIONAL GST (\$M) & INCOME & ECONOMIC WELL-BEING \\
\hline 2589 & $\begin{array}{l}\text { SOUTHLAND REGIONAL } \\
\text { COUNCIL }\end{array}$ & $\begin{array}{l}\text { A DIVERSE ECONOMY BUILT FROM OUR } \\
\text { STRENGTHS FOR GROWTH AND } \\
\text { PROSPERITY }\end{array}$ & ECONOMIC ACTIVITY & GROSS DOMESTIC PRODUCT (GDP) & ECONOMIC WELL-BEING \\
\hline 2591 & $\begin{array}{l}\text { SOUTHLAND REGIONAL } \\
\text { COUNCIL }\end{array}$ & $\begin{array}{l}\text { A DIVERSE ECONOMY BUILT FROM OUR } \\
\text { STRENGTHS FOR GROWTH AND } \\
\text { PROSPERITY }\end{array}$ & UNEMPLOYMENT RATE & UNEMPLOYMENT & ECONOMIC WELL-BEING \\
\hline
\end{tabular}


Appendix A: List of Community Well-beings Outcomes and Measures by Councils

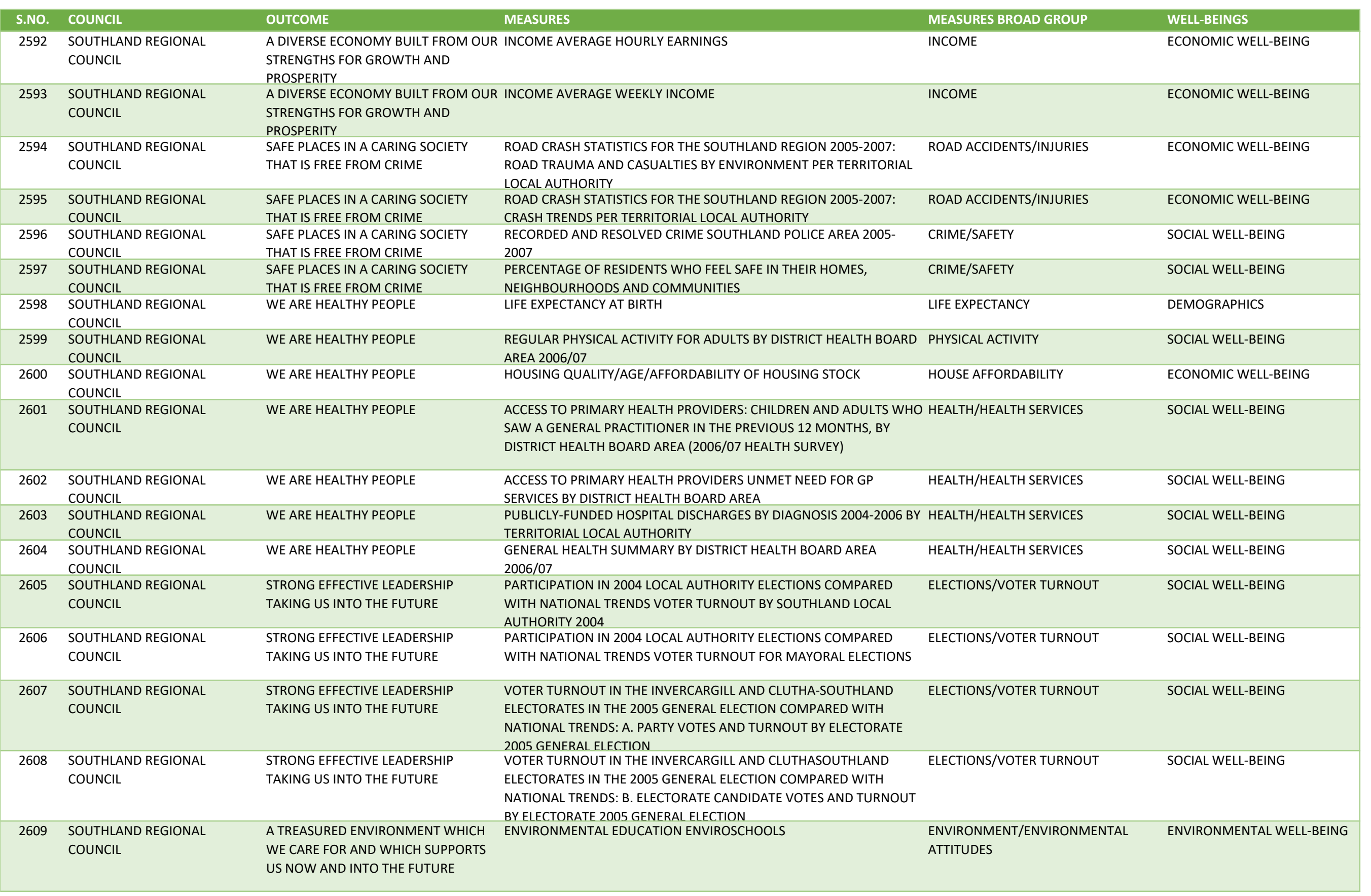




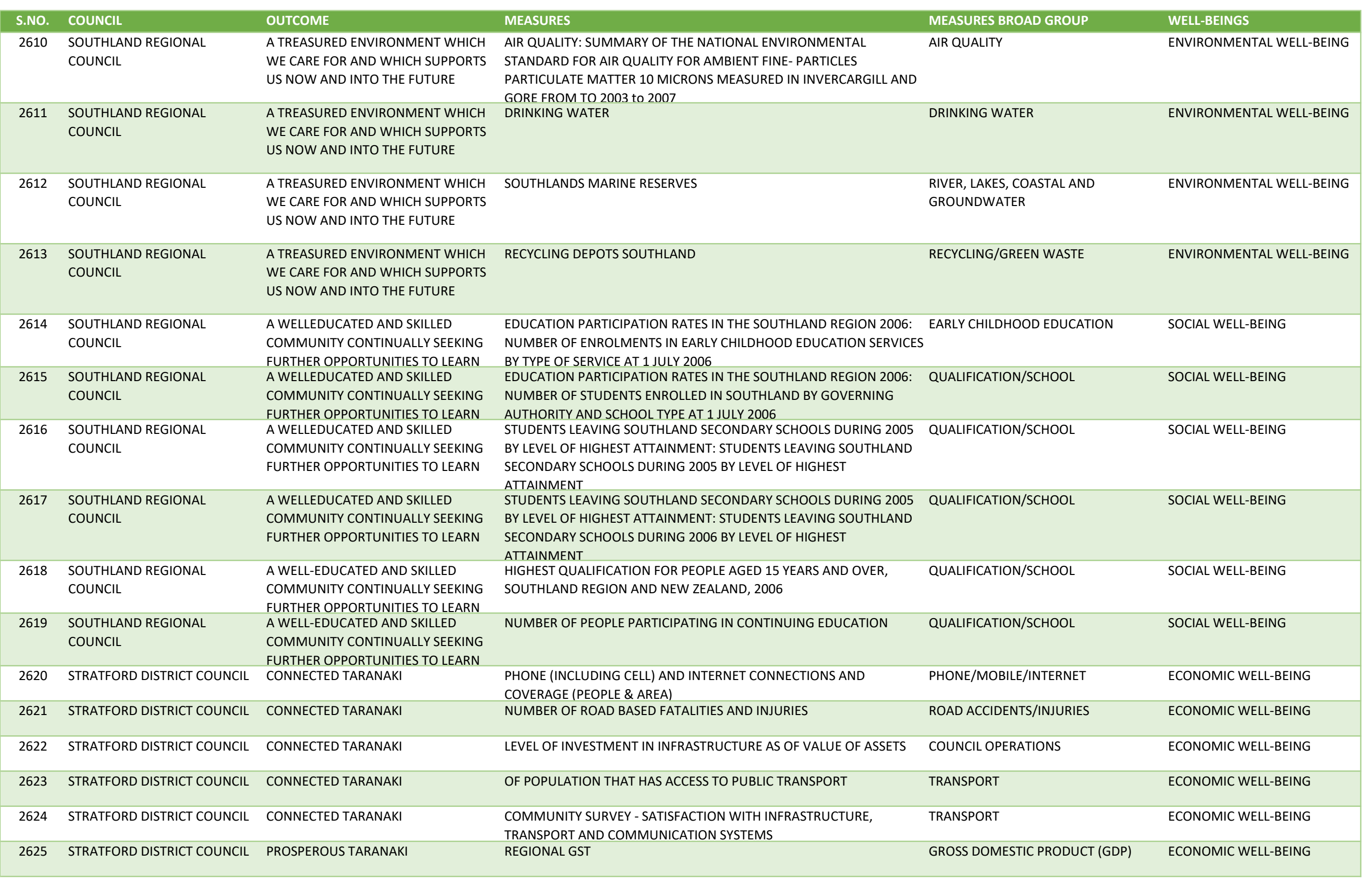


Appendix A: List of Community Well-beings Outcomes and Measures by Councils

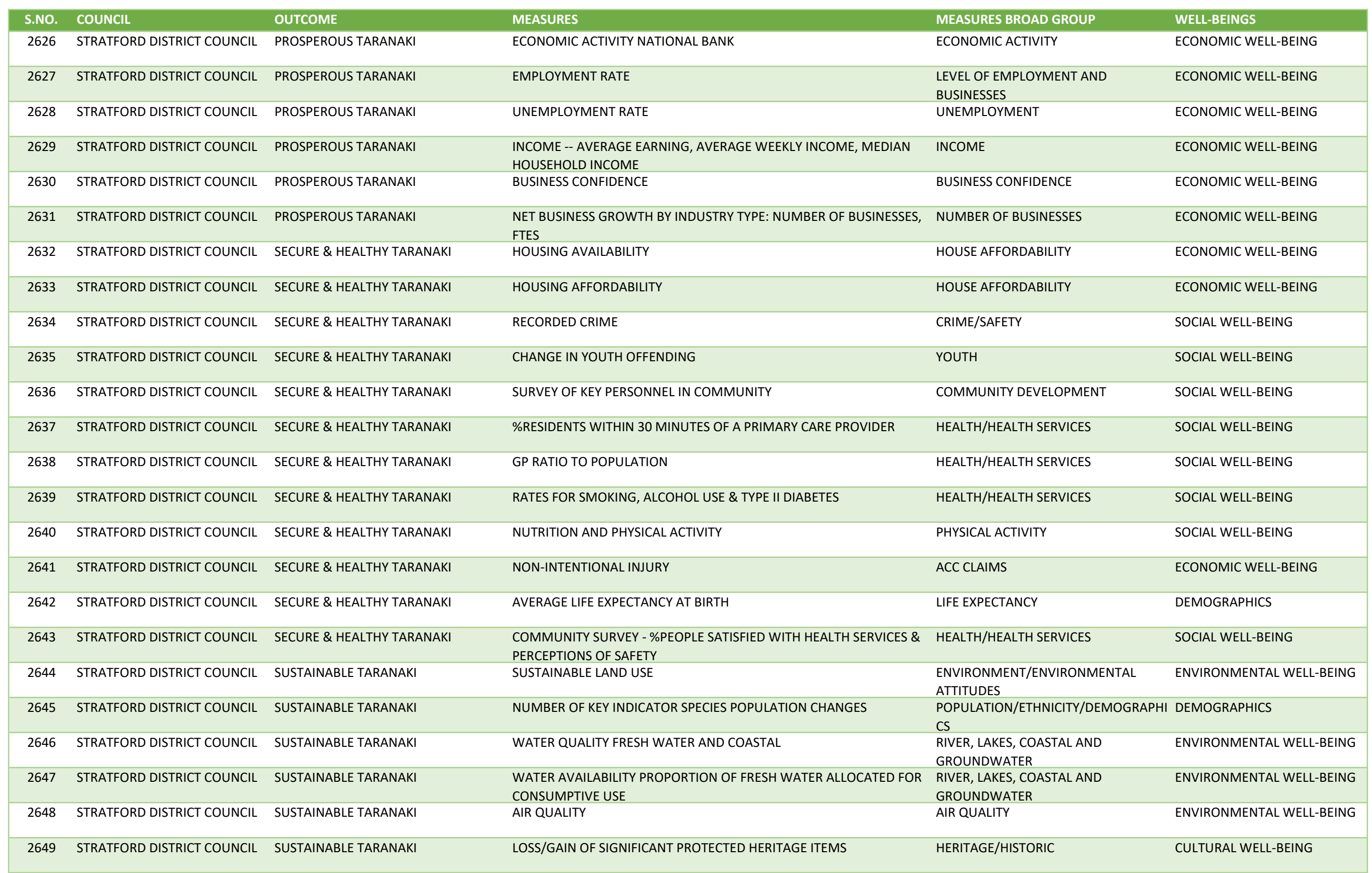


Appendix A: List of Community Well-beings Outcomes and Measures by Councils

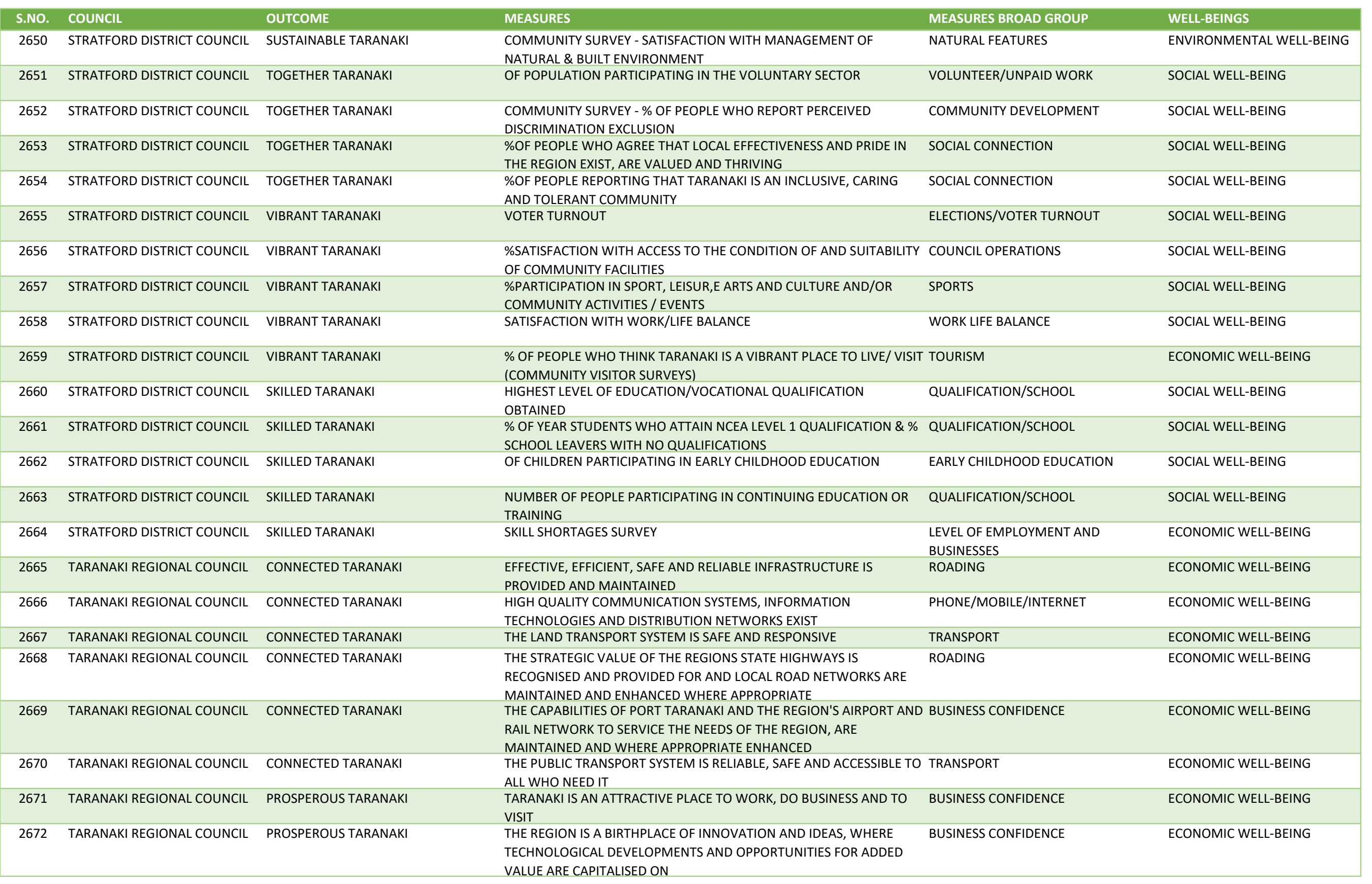


Appendix A: List of Community Well-beings Outcomes and Measures by Councils

\begin{tabular}{|c|c|c|c|c|c|}
\hline S.NO. & COUNCIL & OUTCOME & MEASURES & MEASURES BROAD GROUP & WELL-BEINGS \\
\hline 2673 & TARANAKI REGIONAL COUNCIL & PROSPEROUS TARANAKI & $\begin{array}{l}\text { TARANAKIS STRENGTHS ARE RECOGNISED AND DIVERSIFICATION OF } \\
\text { BUSINESS AND INDUSTRY IS VALUED AND ENCOURAGED }\end{array}$ & BUSINESS CONFIDENCE & ECONOMIC WELL-BEING \\
\hline 2674 & TARANAKI REGIONAL COUNCIL & PROSPEROUS TARANAKI & $\begin{array}{l}\text { THERE IS A HIGH LEVEL OF EMPLOYMENT AND ADEQUATE INCOMES } \\
\text { FOR ALL }\end{array}$ & INCOME & ECONOMIC WELL-BEING \\
\hline 2675 & TARANAKI REGIONAL COUNCIL & PROSPEROUS TARANAKI & $\begin{array}{l}\text { DEVELOPMENT AND POPULATION GROWTH IN THE REGION IS } \\
\text { ENCOURAGED BUT MANAGED IN A MANNER THAT DOES NOT } \\
\text { COMPROMISE THE NATURAL OR SOCIAL ENVIRONMENT }\end{array}$ & $\begin{array}{l}\text { ENVIRONMENT/ENVIRONMENTAL } \\
\text { ATTITUDES }\end{array}$ & ENVIRONMENTAL WELL-BEING \\
\hline 2676 & TARANAKI REGIONAL COUNCIL & PROSPEROUS TARANAKI & PEOPLE ARE CONFIDENT AND ARE HAPPY TO INVEST IN THE FUTURE & BUSINESS CONFIDENCE & ECONOMIC WELL-BEING \\
\hline 2678 & TARANAKI REGIONAL COUNCIL & SECURE AND HEALTHY TARANAKI & $\begin{array}{l}\text { THE ENVIRONMENTAL, PHYSICAL AND MENTAL HEALTH OF THE PEOPLE } \\
\text { OF TARANAKI IS MAINTAINED, ENHANCED, PROMOTED AND } \\
\text { PROTECTED }\end{array}$ & HEALTH/HEALTH SERVICES & SOCIAL WELL-BEING \\
\hline 2679 & TARANAKI REGIONAL COUNCIL & SECURE AND HEALTHY TARANAKI & $\begin{array}{l}\text { A WELL-BEING MODEL FOR HEALTH IS PROMOTED IN THE REGION, } \\
\text { WHEREBY PEOPLE ARE ENCOURAGED TO TAKE RESPONSIBILITY FOR } \\
\text { THEIR OWN HEALTH IN ORDER TO PROMOTE GOOD HEALTH } \\
\text { OUTCOMES }\end{array}$ & HEALTH/HEALTH SERVICES & SOCIAL WELL-BEING \\
\hline 2680 & TARANAKI REGIONAL COUNCIL & SECURE AND HEALTHY TARANAKI & THERE IS ADEQUATE AND AFFORDABLE HOUSING FOR ALL & HOUSE AFFORDABILITY & ECONOMIC WELL-BEING \\
\hline 2681 & TARANAKI REGIONAL COUNCIL & SECURE AND HEALTHY TARANAKI & RESIDENTS AND VISITORS OF ALL AGES FEEL SAFE FROM CRIME & CRIME/SAFETY & SOCIAL WELL-BEING \\
\hline 2683 & TARANAKI REGIONAL COUNCIL & SECURE AND HEALTHY TARANAKI & TARANAKI IS A FRIENDLY AND WELCOMING PLACE & COMMUNITY DEVELOPMENT & SOCIAL WELL-BEING \\
\hline 2684 & TARANAKI REGIONAL COUNCIL & SKILLED TARANAKI & LEARNING AND THE CREATION OF KNOWLEDGE IS VALUED & SKILLED LABOURFORCE & ECONOMIC WELL-BEING \\
\hline 2685 & TARANAKI REGIONAL COUNCIL & SKILLED TARANAKI & $\begin{array}{l}\text { HIGH QUALITY EDUCATION AND TRAINING OPPORTUNITIES EXIST WITH } \\
\text { STRONG LINKS BETWEEN BUSINESS, SCHOOLS AND TRAINING } \\
\text { INSTITUTIONS. THERE IS EXCELLENT PRE-SCHOOL, PRIMARY AND } \\
\text { SECONDARY EDUCATION WITH SUPERB TEACHERS AND LITERARY } \\
\text { PROGRAMMFS }\end{array}$ & QUALIFICATION/SCHOOL & ECONOMIC WELL-BEING \\
\hline 2686 & TARANAKI REGIONAL COUNCIL & SKILLED TARANAKI & $\begin{array}{l}\text { A WIDE RANGE OF INNOVATIVE EDUCATION AND TRAINING } \\
\text { OPPORTUNITIES ARE ACCESSIBLE TO PEOPLE OF ALL AGES }\end{array}$ & SKILLED LABOURFORCE & ECONOMIC WELL-BEING \\
\hline 2687 & TARANAKI REGIONAL COUNCIL & SKILLED TARANAKI & $\begin{array}{l}\text { A SUPPORTIVE AND RESPONSIVE LEARNING ENVIRONMENT EXISTS } \\
\text { WHERE PEOPLE ARE ENCOURAGED TO PARTICIPATE }\end{array}$ & SKILLED LABOURFORCE & ECONOMIC WELL-BEING \\
\hline 2688 & TARANAKI REGIONAL COUNCIL & SKILLED TARANAKI & $\begin{array}{l}\text { THE WORKFORCE HAS THE SKILLS TO MEET THE NEEDS OF THE } \\
\text { REGIONS EMPLOYERS }\end{array}$ & SKILLED LABOURFORCE & ECONOMIC WELL-BEING \\
\hline 2689 & TARANAKI REGIONAL COUNCIL & SUSTAINABLE TARANAKI & $\begin{array}{l}\text { THERE IS SUSTAINABLE USE, DEVELOPMENT AND PROTECTION OF } \\
\text { RESOURCES TARANAKI'S LAND AND SOIL, WATER, AIR AND COAST ITS } \\
\text { BIODIVERSITY AND ITS NATURAL FEATURES AND LANDSCAPES ARE } \\
\text { UNDERSTOOD, VALUED, MAINTAINED AND ENHANCED FOR FUTURE } \\
\text { GFNFRATIONS }\end{array}$ & LAND UTILISATION & ENVIRONMENTAL WELL-BEING \\
\hline 2692 & TARANAKI REGIONAL COUNCIL & SUSTAINABLE TARANAKI & $\begin{array}{l}\text { BUILT ENVIRONMENTS AND ENVIRONMENTAL AMENITIES ARE OF A } \\
\text { HIGH STANDARD AND CONTRIBUTE SIGNIFICANTLY TO THE WELL- } \\
\text { BEING OF PEOPLE AND COMMUNITIES }\end{array}$ & $\begin{array}{l}\text { ENVIRONMENT/ENVIRONMENTAL } \\
\text { ATTITUDES }\end{array}$ & ENVIRONMENTAL WELL-BEING \\
\hline
\end{tabular}


Appendix A: List of Community Well-beings Outcomes and Measures by Councils

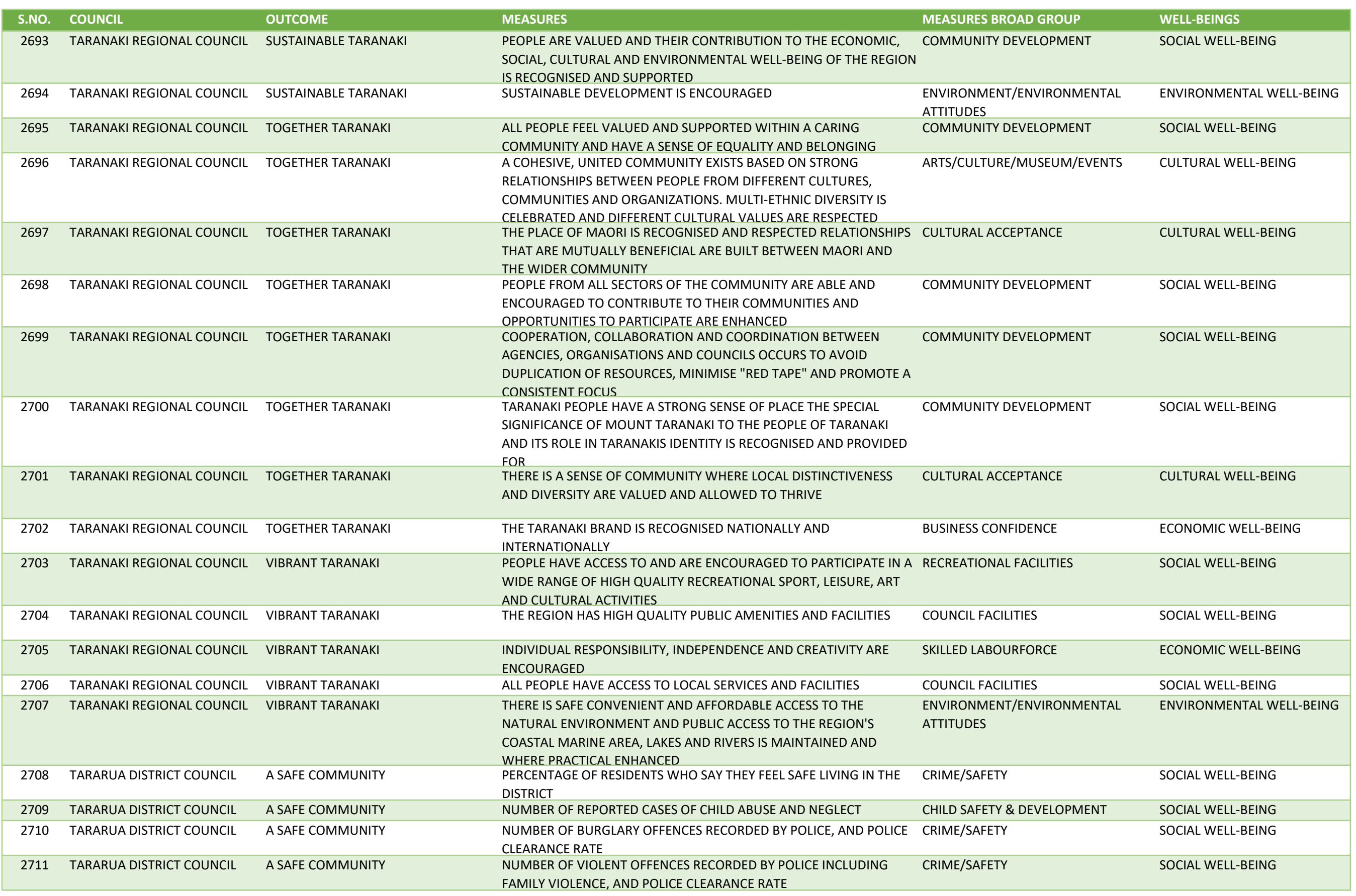


Appendix A: List of Community Well-beings Outcomes and Measures by Councils

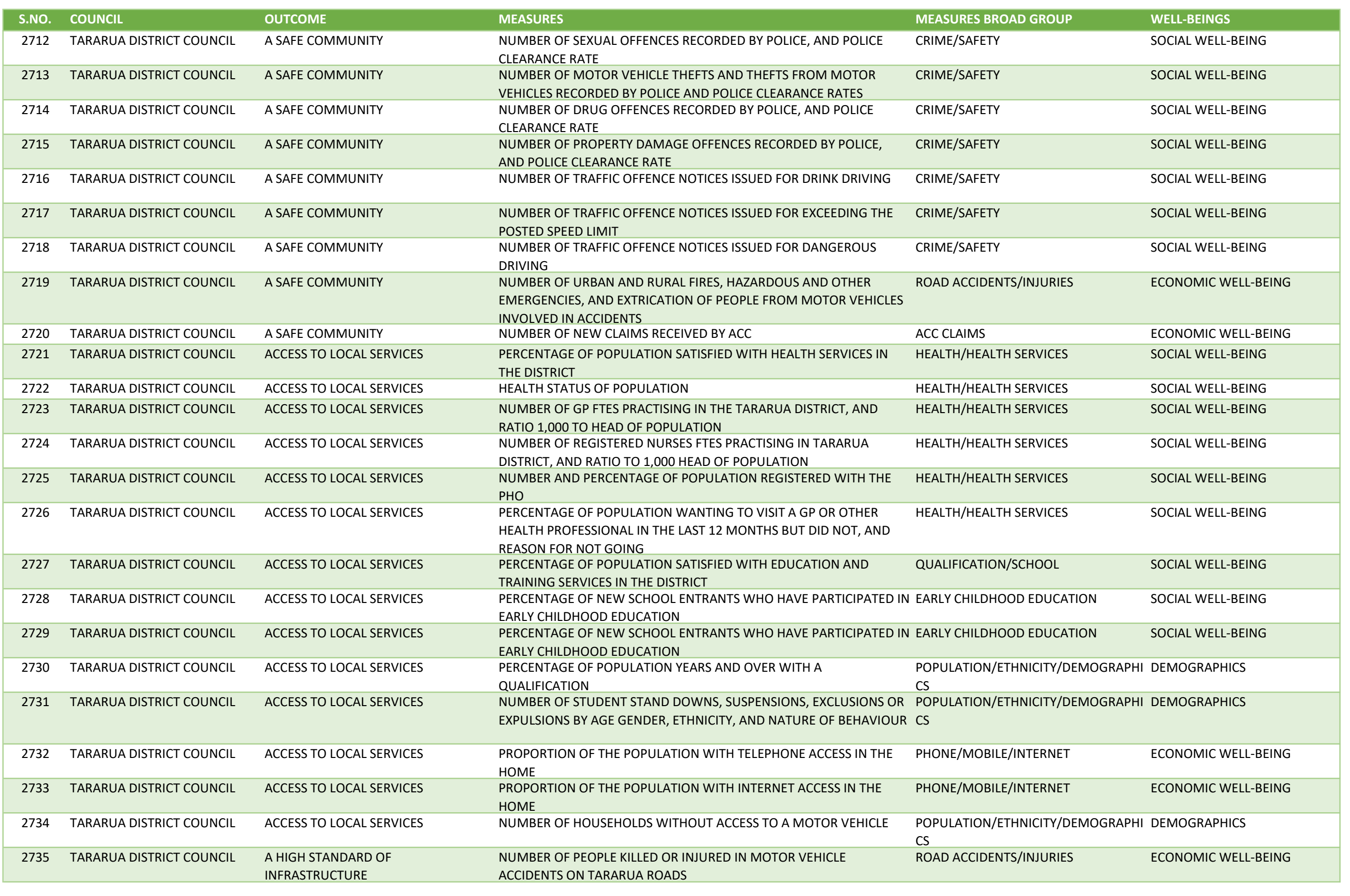


Appendix A: List of Community Well-beings Outcomes and Measures by Councils

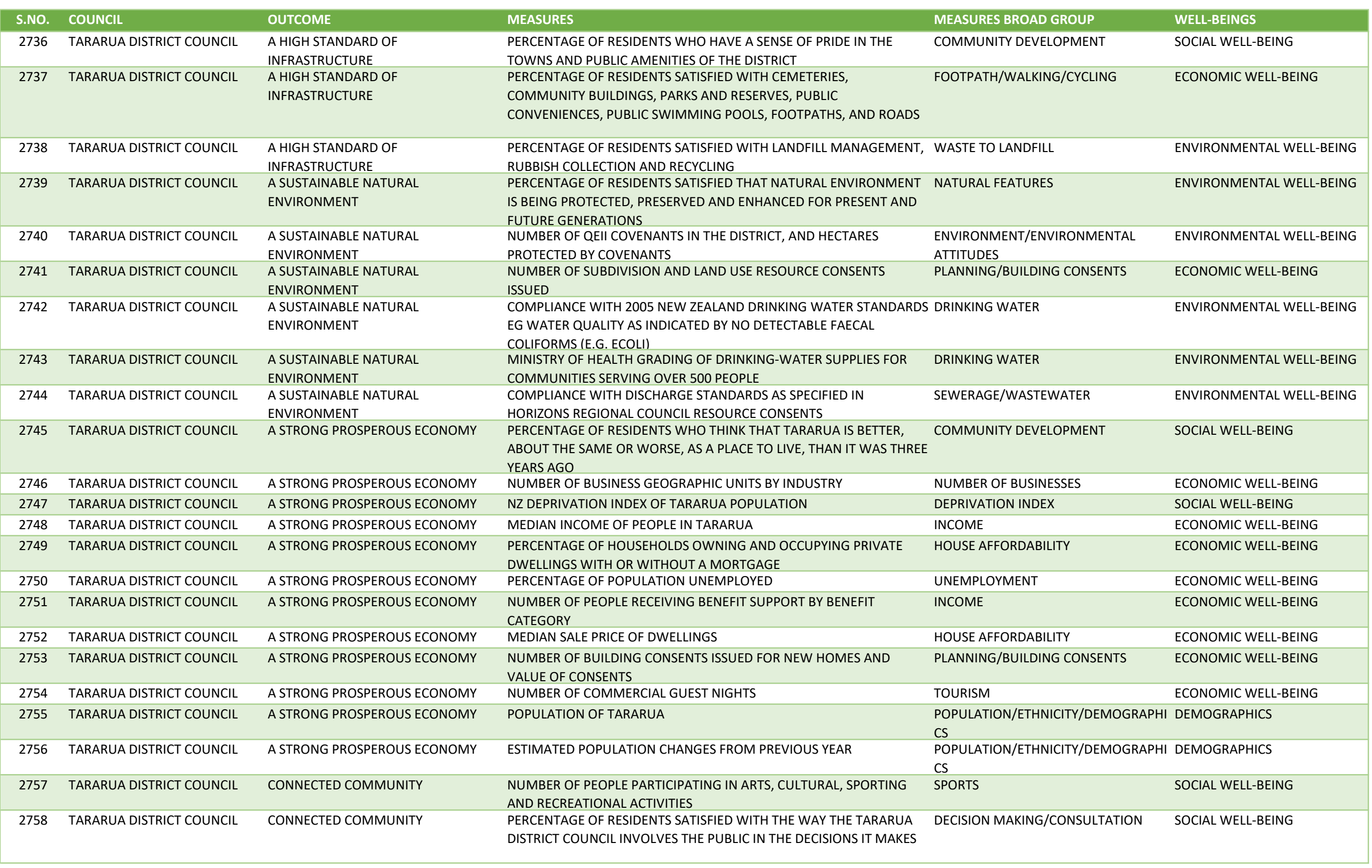




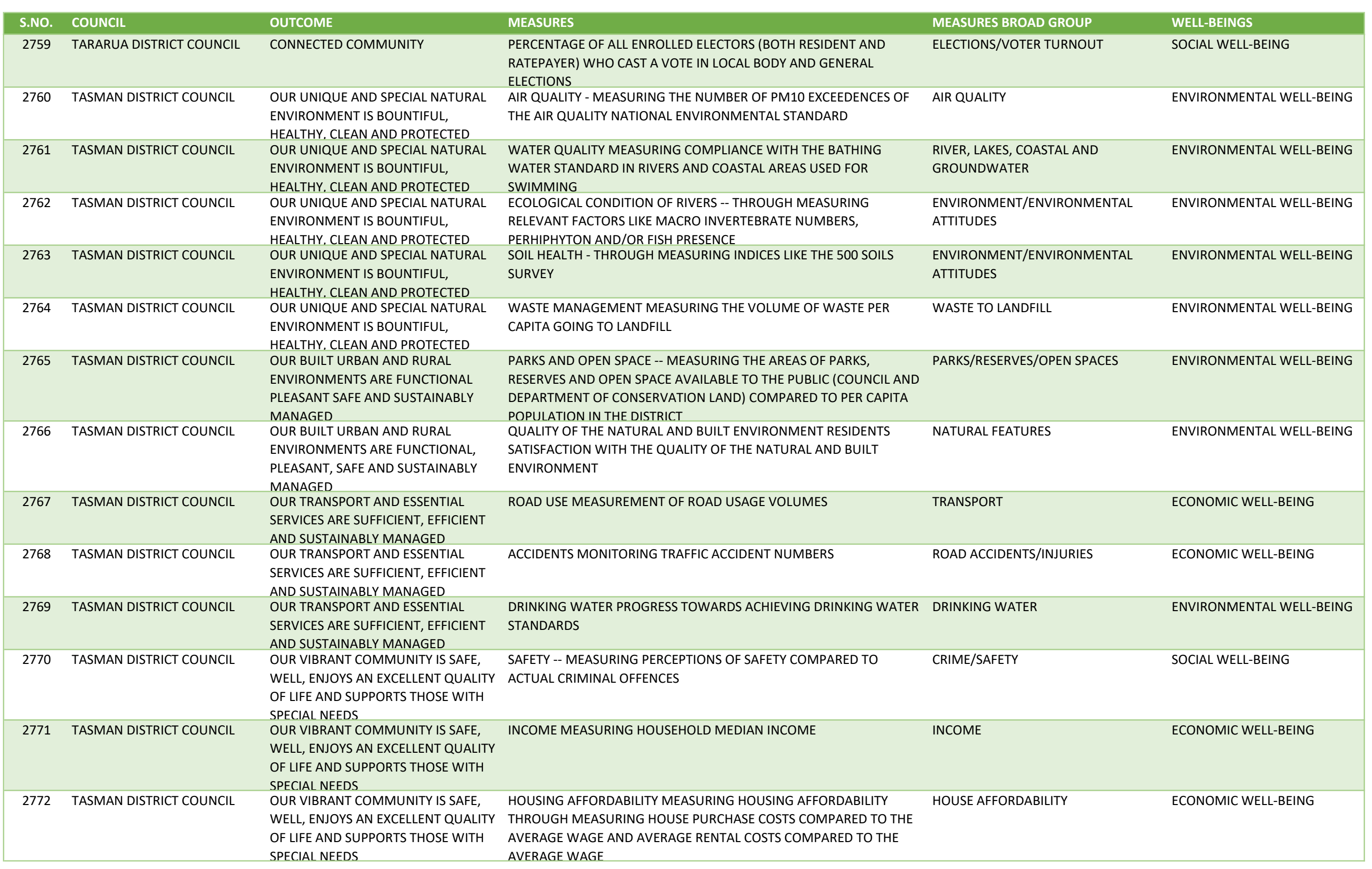


Appendix A: List of Community Well-beings Outcomes and Measures by Councils

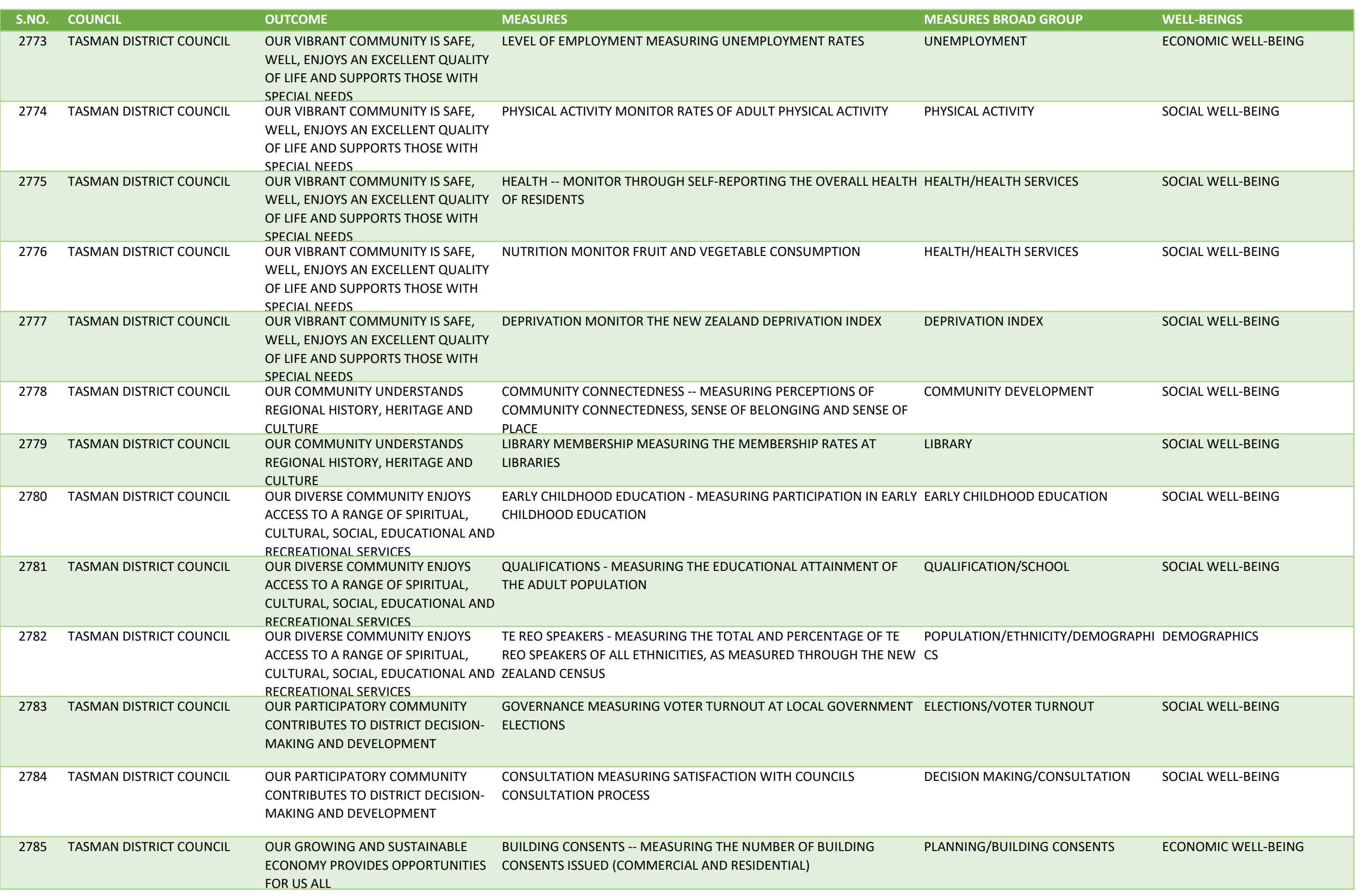


Appendix A: List of Community Well-beings Outcomes and Measures by Councils

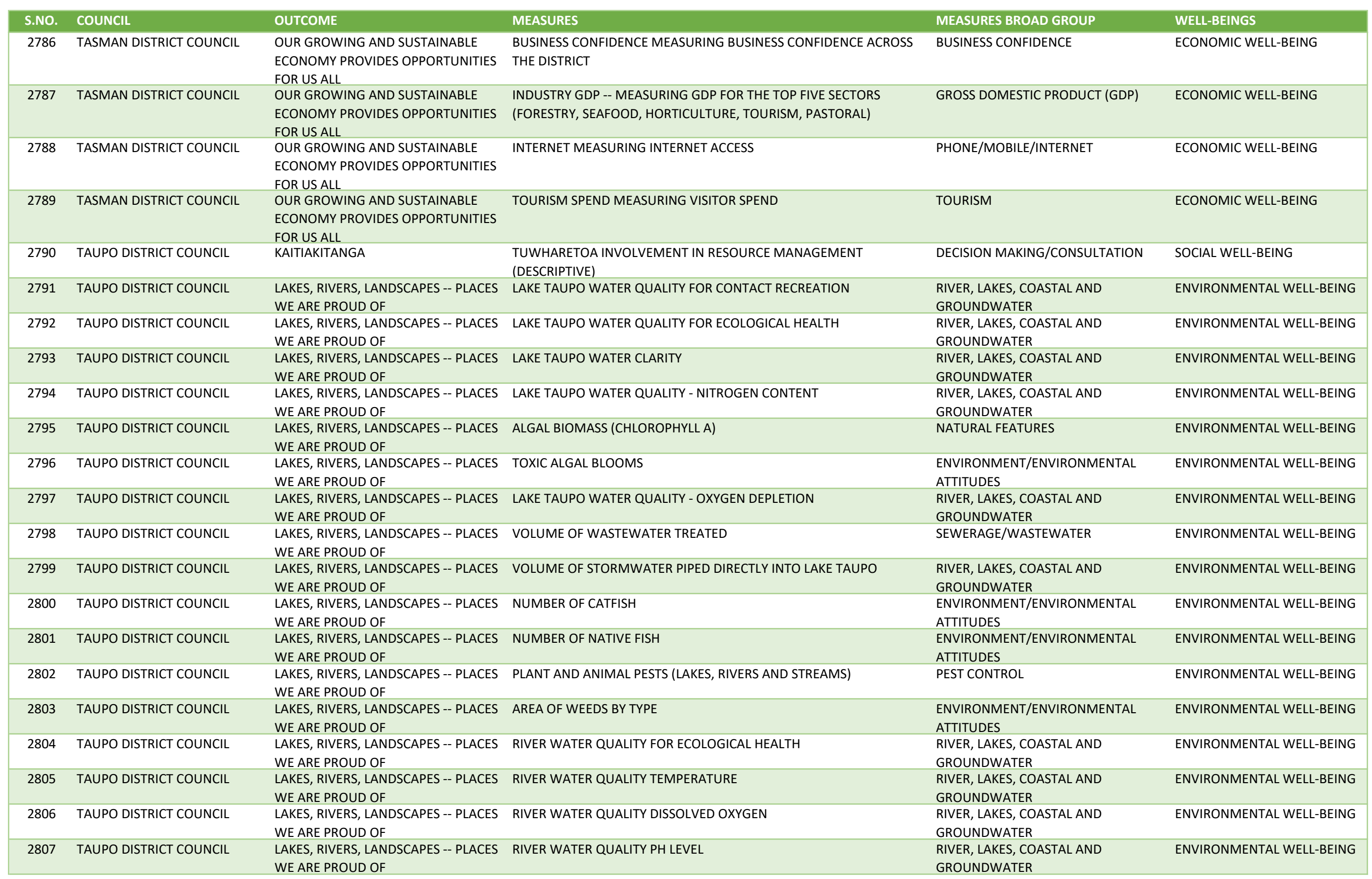


Appendix A: List of Community Well-beings Outcomes and Measures by Councils

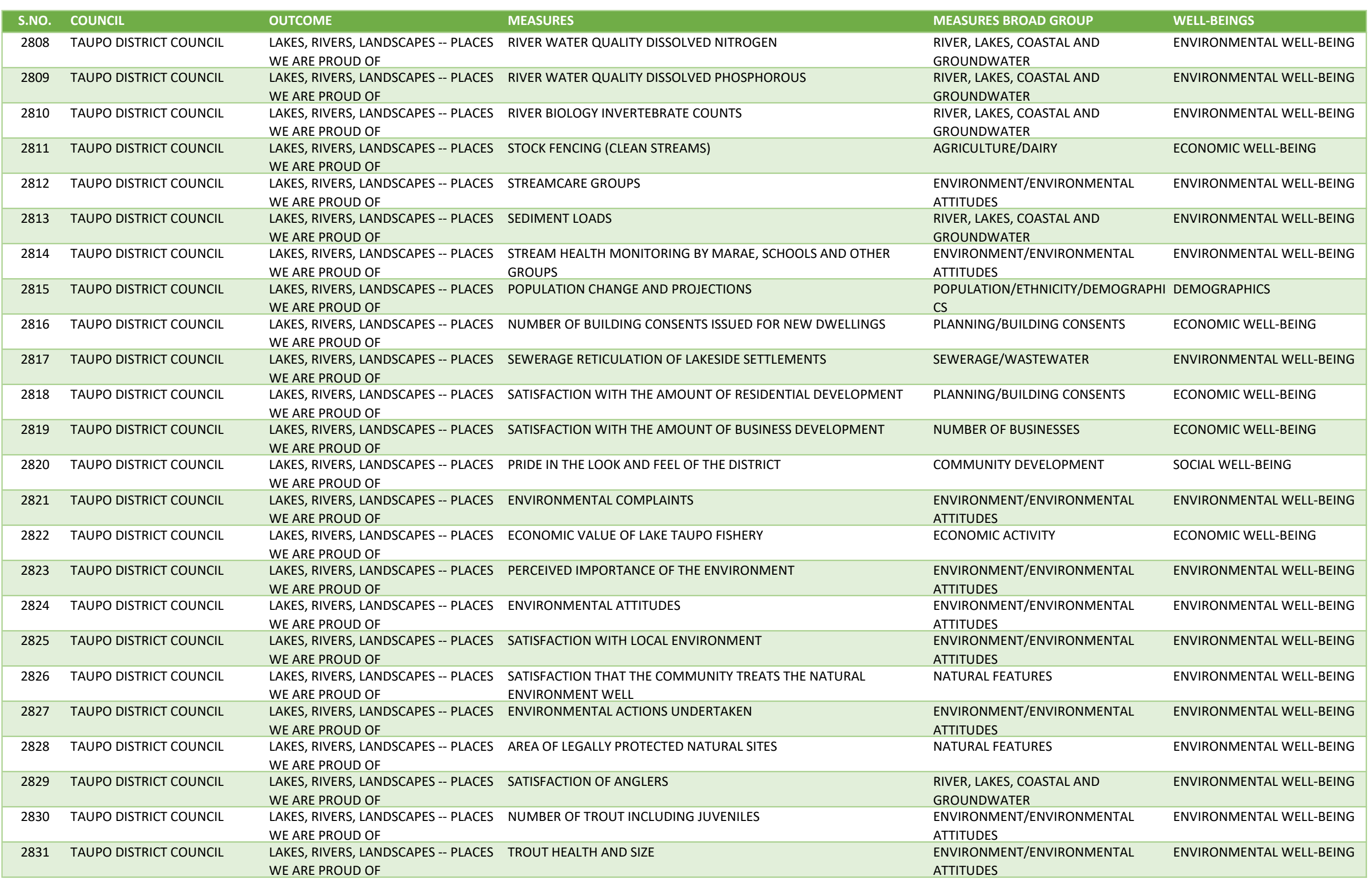


Appendix A: List of Community Well-beings Outcomes and Measures by Councils

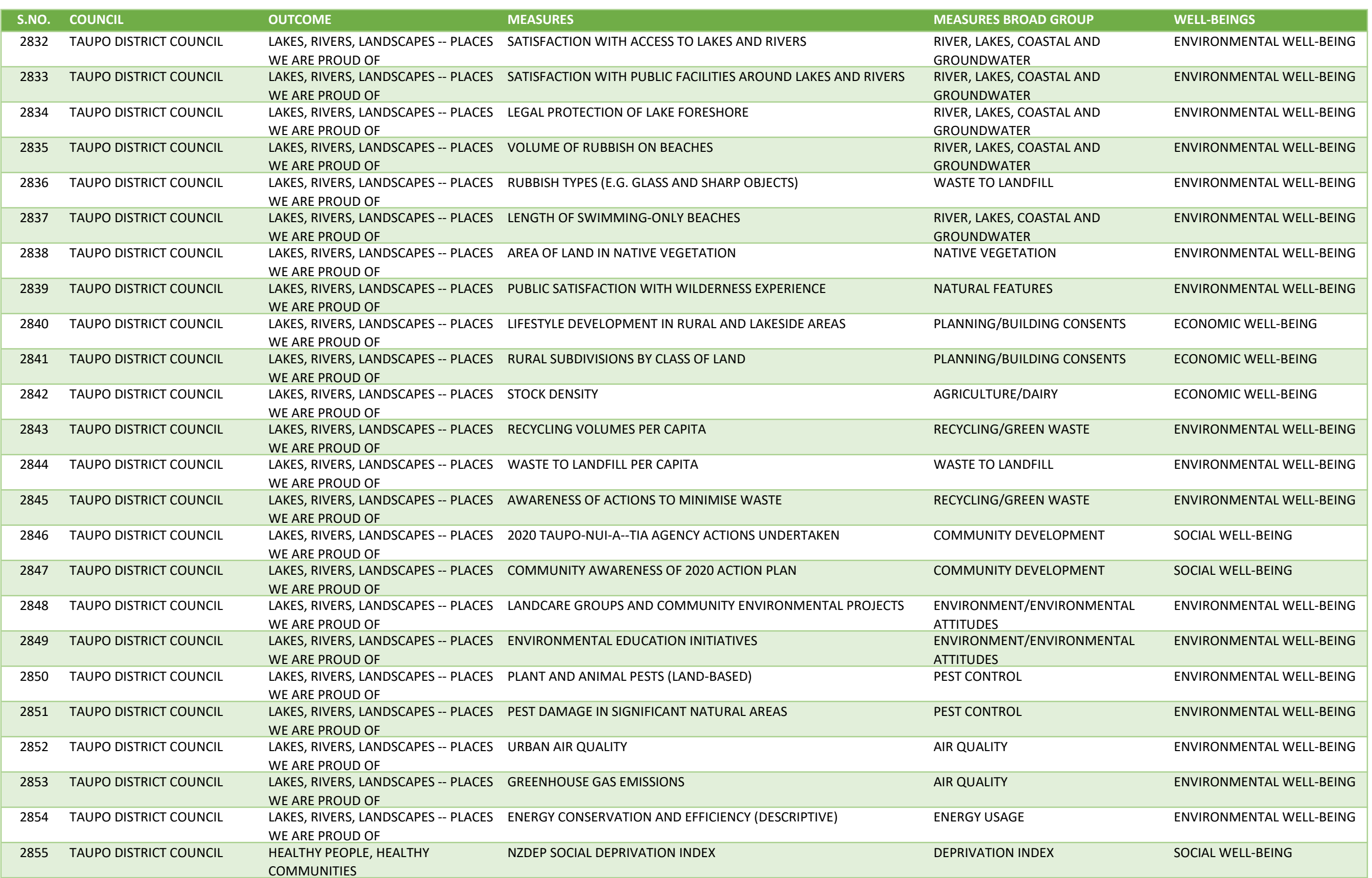


Appendix A: List of Community Well-beings Outcomes and Measures by Councils

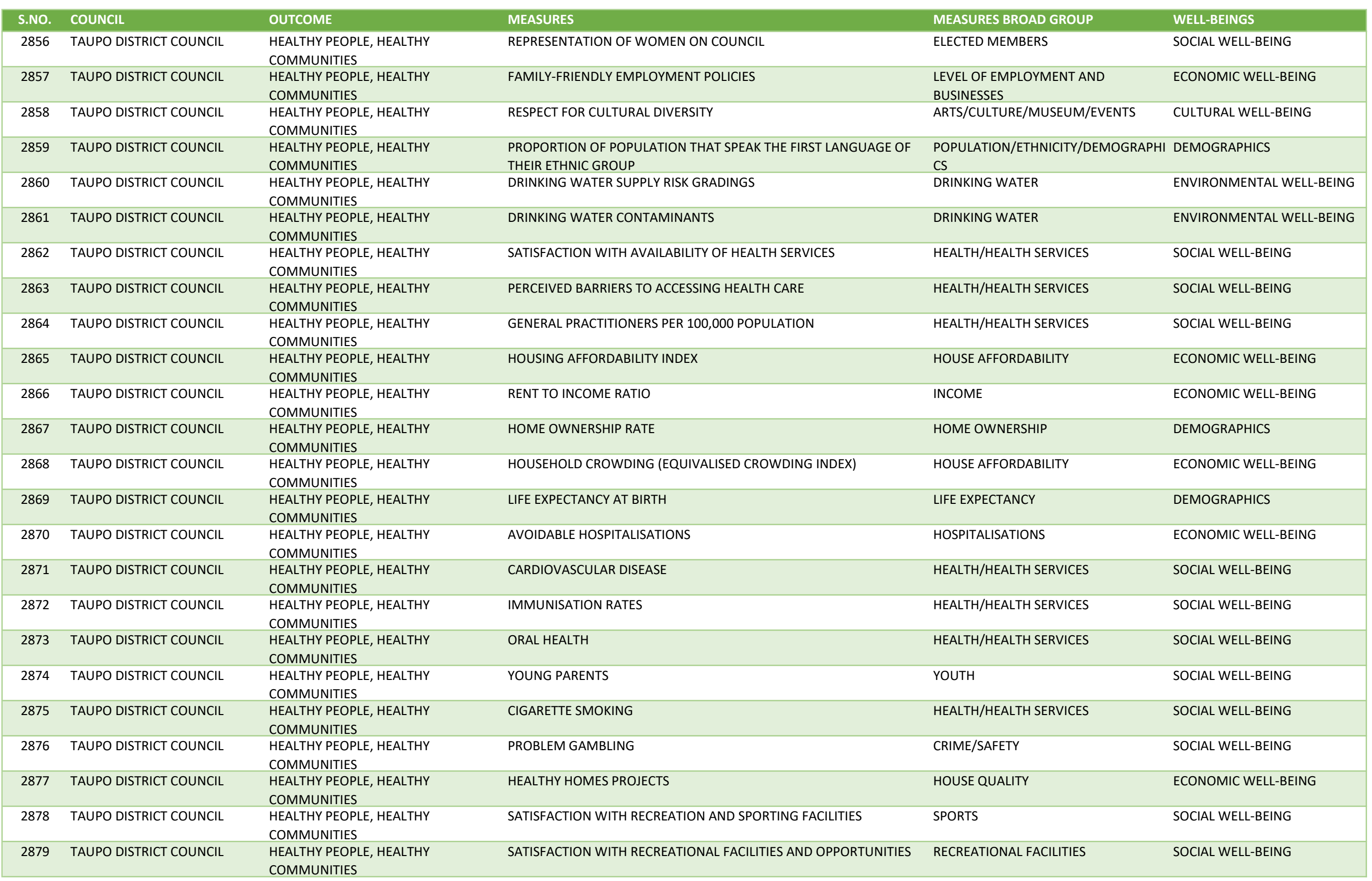


Appendix A: List of Community Well-beings Outcomes and Measures by Councils

\begin{tabular}{|c|c|c|c|c|c|}
\hline S.NO. & COUNCIL & OUTCOME & MEASURES & MEASURES BROAD GROUP & WELL-BEINGS \\
\hline 2880 & TAUPO DISTRICT COUNCIL & $\begin{array}{l}\text { HEALTHY PEOPLE, HEALTHY } \\
\text { COMMUNITIES }\end{array}$ & PARTICIPATION IN PHYSICAL ACTIVITY & PHYSICAL ACTIVITY & SOCIAL WELL-BEING \\
\hline 2881 & TAUPO DISTRICT COUNCIL & $\begin{array}{l}\text { HEALTHY PEOPLE, HEALTHY } \\
\text { COMMUNITIES }\end{array}$ & $\begin{array}{l}\text { USAGE OF COUNCIL RECREATIONAL FACILITIES (PARKS, } \\
\text { SPORTSGROUNDS, AC BATHS) }\end{array}$ & PARKS/RESERVES/OPEN SPACES & ENVIRONMENTAL WELL-BEING \\
\hline 2883 & TAUPO DISTRICT COUNCIL & $\begin{array}{l}\text { HEALTHY PEOPLE, HEALTHY } \\
\text { COMMUNITIES }\end{array}$ & EXERCISE ON PRESCRIPTION & PHYSICAL ACTIVITY & SOCIAL WELL-BEING \\
\hline 2884 & TAUPO DISTRICT COUNCIL & $\begin{array}{l}\text { HEALTHY PEOPLE, HEALTHY } \\
\text { COMMUNITIES }\end{array}$ & SATISFACTION WITH WORK LIFE BALANCE & WORK LIFE BALANCE & SOCIAL WELL-BEING \\
\hline 2885 & TAUPO DISTRICT COUNCIL & $\begin{array}{l}\text { HEALTHY PEOPLE, HEALTHY } \\
\text { COMMUNITIES }\end{array}$ & PERCEIVED LEVEL OF COMMUNITY SPIRIT & SOCIAL CONNECTION & SOCIAL WELL-BEING \\
\hline 2886 & TAUPO DISTRICT COUNCIL & $\begin{array}{l}\text { HEALTHY PEOPLE, HEALTHY } \\
\text { COMMUNITIES }\end{array}$ & PERCEIVED QUALITY OF LIFE & QUALITY OF LIFE & SOCIAL WELL-BEING \\
\hline 2887 & TAUPO DISTRICT COUNCIL & $\begin{array}{l}\text { HEALTHY PEOPLE, HEALTHY } \\
\text { COMMUNITIES }\end{array}$ & PERCENTAGE OF VOTER TURNOUT AT LOCAL ELECTIONS & ELECTIONS/VOTER TURNOUT & SOCIAL WELL-BEING \\
\hline 2888 & TAUPO DISTRICT COUNCIL & $\begin{array}{l}\text { HEALTHY PEOPLE, HEALTHY } \\
\text { COMMUNITIES }\end{array}$ & WELL-BEING THROUGH TIKANGA MAORI & QUALITY OF LIFE & SOCIAL WELL-BEING \\
\hline 2889 & TAUPO DISTRICT COUNCIL & SAFE AND SECURE & $\begin{array}{l}\text { REPORTED OFFENCES PER 10,000 POPULATION AND RESOLUTION } \\
\text { RATES }\end{array}$ & CRIME/SAFETY & SOCIAL WELL-BEING \\
\hline 2892 & TAUPO DISTRICT COUNCIL & SAFE AND SECURE & $\begin{array}{l}\text { NOTIFICATIONS TO CYFS REPORTED AND SUBSTANTIATED CASES OF } \\
\text { CHILD ABUSE AND NEGLECT }\end{array}$ & CHILD SAFETY \& DEVELOPMENT & SOCIAL WELL-BEING \\
\hline 2893 & TAUPO DISTRICT COUNCIL & SAFE AND SECURE & NAVIGATION SAFETY PATROLS & CRIME/SAFETY & SOCIAL WELL-BEING \\
\hline 2894 & TAUPO DISTRICT COUNCIL & SAFE AND SECURE & NAVIGATION INCIDENTS AND INJURIES & ROAD ACCIDENTS/INJURIES & ECONOMIC WELL-BEING \\
\hline 2895 & TAUPO DISTRICT COUNCIL & SAFE AND SECURE & NAVIGATION COMPLAINTS & COUNCIL OPERATIONS & ECONOMIC WELL-BEING \\
\hline 2896 & TAUPO DISTRICT COUNCIL & SAFE AND SECURE & CIVIL DEFENCE AWARENESS & NATURAL DISASTER/RESILIENCE & ENVIRONMENTAL WELL-BEING \\
\hline 2897 & TAUPO DISTRICT COUNCIL & SAFE AND SECURE & VISITOR NIGHTS IN COMMERCIAL ACCOMMODATION & TOURISM & ECONOMIC WELL-BEING \\
\hline 2898 & TAUPO DISTRICT COUNCIL & SAFE AND SECURE & VISITOR EXPENDITURE (INTERNATIONAL AND DOMESTIC) & TOURISM & ECONOMIC WELL-BEING \\
\hline 2899 & TAUPO DISTRICT COUNCIL & SAFE AND SECURE & MOTOR VEHICLE CRASHES & ROAD ACCIDENTS/INJURIES & ECONOMIC WELL-BEING \\
\hline 2900 & TAUPO DISTRICT COUNCIL & SAFE AND SECURE & MOTOR VEHICLE CASUALTIES & ROAD ACCIDENTS/INJURIES & ECONOMIC WELL-BEING \\
\hline 2901 & TAUPO DISTRICT COUNCIL & SAFE AND SECURE & PERCEIVED LEVEL OF SAFETY ON ROADS & TRANSPORT & ECONOMIC WELL-BEING \\
\hline 2902 & TAUPO DISTRICT COUNCIL & SAFE AND SECURE & SATISFACTION WITH AVAILABILITY OF COMMUNITY SUPPORT SERVICES & COMMUNITY DEVELOPMENT & SOCIAL WELL-BEING \\
\hline 2903 & TAUPO DISTRICT COUNCIL & SAFE AND SECURE & UNPAID WORK & VOLUNTEER/UNPAID WORK & SOCIAL WELL-BEING \\
\hline 2904 & TAUPO DISTRICT COUNCIL & THRIVING AND PROSPEROUS & REAL MEDIAN PERSONAL INCOME & INCOME & ECONOMIC WELL-BEING \\
\hline 2905 & TAUPO DISTRICT COUNCIL & THRIVING AND PROSPEROUS & AVERAGE HOURLY EARNINGS & INCOME & ECONOMIC WELL-BEING \\
\hline 2906 & TAUPO DISTRICT COUNCIL & THRIVING AND PROSPEROUS & EMPLOYMENT BY INDUSTRY & $\begin{array}{l}\text { LEVEL OF EMPLOYMENT AND } \\
\text { BUSINESSES }\end{array}$ & ECONOMIC WELL-BEING \\
\hline 2908 & TAUPO DISTRICT COUNCIL & THRIVING AND PROSPEROUS & SATISFACTION WITH AVAILABILITY OF EMPLOYMENT OPPORTUNITIES & $\begin{array}{l}\text { LEVEL OF EMPLOYMENT AND } \\
\text { BUSINESSES }\end{array}$ & ECONOMIC WELL-BEING \\
\hline 2909 & TAUPO DISTRICT COUNCIL & THRIVING AND PROSPEROUS & $\begin{array}{l}\text { SATISFACTION THAT JOB MAKES GOOD USE OF SKILLS, TRAINING AND } \\
\text { EXPERIENCE }\end{array}$ & $\begin{array}{l}\text { LEVEL OF EMPLOYMENT AND } \\
\text { BUSINESSES }\end{array}$ & ECONOMIC WELL-BEING \\
\hline
\end{tabular}


Appendix A: List of Community Well-beings Outcomes and Measures by Councils

\begin{tabular}{|c|c|c|c|c|c|}
\hline S.NO. & COUNCIL & OUTCOME & MEASURES & MEASURES BROAD GROUP & WELL-BEINGS \\
\hline 2910 & TAUPO DISTRICT COUNCIL & THRIVING AND PROSPEROUS & UNEMPLOYMENT RATE & UNEMPLOYMENT & ECONOMIC WELL-BEING \\
\hline 2911 & TAUPO DISTRICT COUNCIL & THRIVING AND PROSPEROUS & UNEMPLOYMENT RATE OF MAORI RESIDENTS & UNEMPLOYMENT & ECONOMIC WELL-BEING \\
\hline 2912 & TAUPO DISTRICT COUNCIL & THRIVING AND PROSPEROUS & REGISTERED UNEMPLOYED & UNEMPLOYMENT & ECONOMIC WELL-BEING \\
\hline 2913 & TAUPO DISTRICT COUNCIL & THRIVING AND PROSPEROUS & REGISTERED UNEMPLOYED - YOUNG PEOPLE & UNEMPLOYMENT & ECONOMIC WELL-BEING \\
\hline 2914 & TAUPO DISTRICT COUNCIL & THRIVING AND PROSPEROUS & $\begin{array}{l}\text { PERCENTAGE OF YOUNG PEOPLE AGED } 15-19 \text { NOT IN SCHOOL, WORK } \\
\text { OR TRAINING }\end{array}$ & $\begin{array}{l}\text { NEET: NOT IN EMPLOYMENT, } \\
\text { EDUCATION OR TRAINING }\end{array}$ & ECONOMIC WELL-BEING \\
\hline 2915 & TAUPO DISTRICT COUNCIL & THRIVING AND PROSPEROUS & REGISTERED UNEMPLOYED - LONG-TERM & UNEMPLOYMENT & ECONOMIC WELL-BEING \\
\hline 2916 & TAUPO DISTRICT COUNCIL & THRIVING AND PROSPEROUS & NUMBER OF BUSINESSES BY INDUSTRY & NUMBER OF BUSINESSES & ECONOMIC WELL-BEING \\
\hline 2917 & TAUPO DISTRICT COUNCIL & THRIVING AND PROSPEROUS & GROSS REGIONAL PRODUCT & GROSS DOMESTIC PRODUCT (GDP) & ECONOMIC WELL-BEING \\
\hline 2918 & TAUPO DISTRICT COUNCIL & THRIVING AND PROSPEROUS & ECONOMIC GROWTH INDEX & GROSS DOMESTIC PRODUCT (GDP) & ECONOMIC WELL-BEING \\
\hline 2919 & TAUPO DISTRICT COUNCIL & THRIVING AND PROSPEROUS & EMPLOYMENT SKILLS SHORTAGES & $\begin{array}{l}\text { LEVEL OF EMPLOYMENT AND } \\
\text { BUSINESSES }\end{array}$ & ECONOMIC WELL-BEING \\
\hline 2920 & TAUPO DISTRICT COUNCIL & THRIVING AND PROSPEROUS & BUSINESS CONFIDENCE & BUSINESS CONFIDENCE & ECONOMIC WELL-BEING \\
\hline 2921 & TAUPO DISTRICT COUNCIL & THRIVING AND PROSPEROUS & RETAIL VACANCIES & CBD: CITY CENTRE & SOCIAL WELL-BEING \\
\hline 2922 & TAUPO DISTRICT COUNCIL & THRIVING AND PROSPEROUS & MAORI BUSINESSES & NUMBER OF BUSINESSES & ECONOMIC WELL-BEING \\
\hline 2923 & TAUPO DISTRICT COUNCIL & THRIVING AND PROSPEROUS & PARTICIPATION IN EARLY CHILDHOOD EDUCATION & EARLY CHILDHOOD EDUCATION & SOCIAL WELL-BEING \\
\hline 2924 & TAUPO DISTRICT COUNCIL & THRIVING AND PROSPEROUS & SCHOOL LEAVERS WITH NO FORMAL QUALIFICATION & QUALIFICATION/SCHOOL & SOCIAL WELL-BEING \\
\hline 2925 & TAUPO DISTRICT COUNCIL & THRIVING AND PROSPEROUS & SCHOOL LEAVERS WITH QUALIFICATIONS HIGHER THAN NCEA LEVEL 1 & QUALIFICATION/SCHOOL & SOCIAL WELL-BEING \\
\hline 2927 & TAUPO DISTRICT COUNCIL & THRIVING AND PROSPEROUS & $\begin{array}{l}\text { NUMBER OF MODERN APPRENTICESHIPS AND INDUSTRY TRAINING } \\
\text { CURRENTLY IN PROGRESS }\end{array}$ & APPRENTICESHIPS FOR YOUNG PEOPLE & ECONOMIC WELL-BEING \\
\hline 2928 & TAUPO DISTRICT COUNCIL & THRIVING AND PROSPEROUS & $\begin{array}{l}\text { NUMBERS OF YOUNG PEOPLE IN GATEWAY PROGRAMME AND OTHER } \\
\text { YOUTH TRANSITION COURSES }\end{array}$ & YOUTH & SOCIAL WELL-BEING \\
\hline 2929 & TAUPO DISTRICT COUNCIL & THRIVING AND PROSPEROUS & SATISFACTION WITH INFRASTRUCTURE AND ESSENTIAL SERVICES & COUNCIL FACILITIES & SOCIAL WELL-BEING \\
\hline 2930 & TAUPO DISTRICT COUNCIL & THRIVING AND PROSPEROUS & SATISFACTION WITH PROXIMITY TO WORK, STUDY AND RECREATION & RECREATIONAL FACILITIES & SOCIAL WELL-BEING \\
\hline 2931 & TAUPO DISTRICT COUNCIL & THRIVING AND PROSPEROUS & SATISFACTION WITH PERFORMANCE OF COUNCIL STAFF & COUNCIL OPERATIONS & ECONOMIC WELL-BEING \\
\hline 2932 & TAUPO DISTRICT COUNCIL & THRIVING AND PROSPEROUS & TELECOMMUNICATIONS CONNECTION & PHONE/MOBILE/INTERNET & ECONOMIC WELL-BEING \\
\hline 2933 & TAUPO DISTRICT COUNCIL & THRIVING AND PROSPEROUS & CONFIDENCE IN COUNCIL DECISION-MAKING & DECISION MAKING/CONSULTATION & SOCIAL WELL-BEING \\
\hline 2934 & TAUPO DISTRICT COUNCIL & THRIVING AND PROSPEROUS & SATISFACTION WITH COUNCIL'S LONG-TERM DIRECTION & COUNCIL OPERATIONS & ECONOMIC WELL-BEING \\
\hline 2935 & TAUPO DISTRICT COUNCIL & THRIVING AND PROSPEROUS & SATISFACTION WITH MAYOR AND COUNCILLORS PERFORMANCE & ELECTED MEMBERS & SOCIAL WELL-BEING \\
\hline 2936 & TAUPO DISTRICT COUNCIL & THRIVING AND PROSPEROUS & SATISFACTION WITH COUNCIL SERVICES AND FACILITIES & COUNCIL FACILITIES & SOCIAL WELL-BEING \\
\hline 2937 & TAUPO DISTRICT COUNCIL & THRIVING AND PROSPEROUS & SATISFACTION WITH LAND TRANSPORT NETWORK & TRANSPORT & ECONOMIC WELL-BEING \\
\hline 2938 & TAUPO DISTRICT COUNCIL & THRIVING AND PROSPEROUS & SATISFACTION WITH FLOOD PROTECTION SYSTEMS & NATURAL DISASTER/RESILIENCE & ENVIRONMENTAL WELL-BEING \\
\hline 2939 & TAUPO DISTRICT COUNCIL & THRIVING AND PROSPEROUS & SATISFACTION WITH AVAILABILITY OF PRIMARY SCHOOLS & QUALIFICATION/SCHOOL & SOCIAL WELL-BEING \\
\hline 2940 & TAUPO DISTRICT COUNCIL & THRIVING AND PROSPEROUS & SATISFACTION WITH AVAILABILITY OF SECONDARY SCHOOLS & QUALIFICATION/SCHOOL & SOCIAL WELL-BEING \\
\hline 2941 & TAUPO DISTRICT COUNCIL & THRIVING AND PROSPEROUS & $\begin{array}{l}\text { SATISFACTION WITH AVAILABILITY OF COMMUNITY OR TERTIARY } \\
\text { EDUCATION }\end{array}$ & TERTIARY QUALIFICATION & SOCIAL WELL-BEING \\
\hline 2943 & TAUPO DISTRICT COUNCIL & THRIVING AND PROSPEROUS & TRUANCY RATE & QUALIFICATION/SCHOOL & SOCIAL WELL-BEING \\
\hline 2944 & TAUPO DISTRICT COUNCIL & THRIVING AND PROSPEROUS & EARLY SCHOOL LEAVERS & QUALIFICATION/SCHOOL & SOCIAL WELL-BEING \\
\hline 2945 & TAUPO DISTRICT COUNCIL & VIBRANT AND DIVERSE & NUMBER OF MAORI SPEAKERS (IN TOTAL AND MAORI POPULATION) & $\begin{array}{l}\text { POPULATION/ETHNICITY/DEMOGRAPHI } \\
\text { CS }\end{array}$ & DEMOGRAPHICS \\
\hline
\end{tabular}


Appendix A: List of Community Well-beings Outcomes and Measures by Councils

\begin{tabular}{|c|c|c|c|c|c|}
\hline S.NO. & COUNCIL & OUTCOME & MEASURES & MEASURES BROAD GROUP & WELL-BEINGS \\
\hline 2946 & TAUPO DISTRICT COUNCIL & VIBRANT AND DIVERSE & LAKE TAUPO MUSEUM AND ART GALLERY VISITOR NUMBERS & TOURISM & ECONOMIC WELL-BEING \\
\hline 2947 & TAUPO DISTRICT COUNCIL & VIBRANT AND DIVERSE & $\begin{array}{l}\text { SATISFACTION THAT THE COMMUNITY DOES A GOOD JOB OF } \\
\text { PROMOTING LOCAL HISTORY }\end{array}$ & HERITAGE/HISTORIC & CULTURAL WELL-BEING \\
\hline 2948 & TAUPO DISTRICT COUNCIL & VIBRANT AND DIVERSE & $\begin{array}{l}\text { NUMBER OF BUILDINGS AND PLACES LISTED ON HISTORIC PLACES } \\
\text { TRUST REGISTER }\end{array}$ & HERITAGE/HISTORIC & CULTURAL WELL-BEING \\
\hline 2949 & TAUPO DISTRICT COUNCIL & VIBRANT AND DIVERSE & TICKETEK SALES TO SHOWS AT THE GREAT LAKE CENTRE & ARTS/CULTURE/MUSEUM/EVENTS & CULTURAL WELL-BEING \\
\hline 2950 & TAUPO DISTRICT COUNCIL & VIBRANT AND DIVERSE & SATISFACTION WITH CULTURAL FACILITIES AND OPPORTUNITIES & ARTS/CULTURE/MUSEUM/EVENTS & CULTURAL WELL-BEING \\
\hline 2951 & TAUPO DISTRICT COUNCIL & VIBRANT AND DIVERSE & ATTENDANCE AT ARTS EVENTS & ARTS/CULTURE/MUSEUM/EVENTS & CULTURAL WELL-BEING \\
\hline 2952 & TAUPO DISTRICT COUNCIL & VIBRANT AND DIVERSE & $\begin{array}{l}\text { USAGE OF COUNCIL CULTURAL FACILITIES (DISTRICT LIBRARIES, GREAT } \\
\text { LAKE CENTRE, TAUPO EVENTS CENTRE, TAUPO MUSEUM AND ART } \\
\text { GALLERY) }\end{array}$ & ARTS/CULTURE/MUSEUM/EVENTS & CULTURAL WELL-BEING \\
\hline 2953 & TAUPO DISTRICT COUNCIL & VIBRANT AND DIVERSE & EVENTS PLANNED BY, FOR AND WITH YOUNG PEOPLE (DESCRIPTIVE) & YOUTH & SOCIAL WELL-BEING \\
\hline 2954 & TAUPO DISTRICT COUNCIL & VIBRANT AND DIVERSE & YOUTH ACTIVITIES AND FACILITIES (STOCK TAKE -- DESCRIPTIVE) & YOUTH & SOCIAL WELL-BEING \\
\hline 2955 & TAURANGA CITY COUNCIL & EASY AND SAFE TO MOVE AROUND & LEVELS OF ROAD ACCIDENTS & ROAD ACCIDENTS/INJURIES & ECONOMIC WELL-BEING \\
\hline 2956 & TAURANGA CITY COUNCIL & EASY AND SAFE TO MOVE AROUND & TRAFFIC VOLUMES AND CONGESTION LEVELS & TRANSPORT & ECONOMIC WELL-BEING \\
\hline 2957 & TAURANGA CITY COUNCIL & EASY AND SAFE TO MOVE AROUND & USE OF PUBLIC TRANSPORT & TRANSPORT & ECONOMIC WELL-BEING \\
\hline 2958 & TAURANGA CITY COUNCIL & EASY AND SAFE TO MOVE AROUND & SAFETY, CONVENIENCE AND AFFORDABILITY OF PUBLIC TRANSPORT & TRANSPORT & ECONOMIC WELL-BEING \\
\hline 2960 & TAURANGA CITY COUNCIL & EASY AND SAFE TO MOVE AROUND & NUMBER OF CHILDREN BIKING AND WALKING TO SCHOOL & CHILD SAFETY \& DEVELOPMENT & SOCIAL WELL-BEING \\
\hline 2961 & TAURANGA CITY COUNCIL & EASY AND SAFE TO MOVE AROUND & NUMBER OF PEOPLE WALKING AND CYCLING ON KEY ROUTES & FOOTPATH/WALKING/CYCLING & ECONOMIC WELL-BEING \\
\hline 2962 & TAURANGA CITY COUNCIL & EASY AND SAFE TO MOVE AROUND & EASE OF WALKING AND CYCLING AROUND THE CITY & FOOTPATH/WALKING/CYCLING & ECONOMIC WELL-BEING \\
\hline 2963 & TAURANGA CITY COUNCIL & EASY AND SAFE TO MOVE AROUND & AIR POLLUTION LEVELS AT KEY SITES & AIR QUALITY & ENVIRONMENTAL WELL-BEING \\
\hline 2964 & TAURANGA CITY COUNCIL & $\begin{array}{l}\text { BUILT TO FIT OUR HILLS, HARBOUR } \\
\text { AND COAST }\end{array}$ & $\begin{array}{l}\text { STRENGTH OF OUR CITY CENTRE -- AS EVIDENCED BY THINGS SUCH AS } \\
\text { PEDESTRIAN COUNTS, NUMBER OF DWELLING UNITS, NUMBER OF } \\
\text { EMPLOYEES AND ACTIVITIES BY TYPE OF BUSINESS }\end{array}$ & CBD: CITY CENTRE & SOCIAL WELL-BEING \\
\hline 2965 & TAURANGA CITY COUNCIL & $\begin{array}{l}\text { BUILT TO FIT OUR HILLS, HARBOUR } \\
\text { AND COAST }\end{array}$ & HOUSING TYPES AND DENSITY & HOUSE AFFORDABILITY & ECONOMIC WELL-BEING \\
\hline 2966 & TAURANGA CITY COUNCIL & $\begin{array}{l}\text { BUILT TO FIT OUR HILLS, HARBOUR } \\
\text { AND COAST }\end{array}$ & $\begin{array}{l}\text { ACCESSIBILITY OF KEY SERVICES SUCH AS SCHOOLS, RESERVES, } \\
\text { PLAYGROUNDS AND LOCAL SHOPS (PERCEPTION AND FACTUAL) }\end{array}$ & QUALIFICATION/SCHOOL & SOCIAL WELL-BEING \\
\hline 2967 & TAURANGA CITY COUNCIL & $\begin{array}{l}\text { BUILT TO FIT OUR HILLS, HARBOUR } \\
\text { AND COAST }\end{array}$ & SENSE OF PRIDE IN THE WAY OUR CITY LOOKS AND FEELS & COMMUNITY DEVELOPMENT & SOCIAL WELL-BEING \\
\hline 2968 & TAURANGA CITY COUNCIL & $\begin{array}{l}\text { BUILT TO FIT OUR HILLS, HARBOUR } \\
\text { AND COAST }\end{array}$ & UNIQUE CHARACTERISTICS OF THE CITY ARE BEING MAINTAINED & CBD: CITY CENTRE & SOCIAL WELL-BEING \\
\hline 2969 & TAURANGA CITY COUNCIL & $\begin{array}{l}\text { BUILT TO FIT OUR HILLS, HARBOUR } \\
\text { AND COAST }\end{array}$ & PROTECTION OF HISTORIC AND ARCHAEOLOGICAL HERITAGE & HERITAGE/HISTORIC & CULTURAL WELL-BEING \\
\hline 2970 & TAURANGA CITY COUNCIL & $\begin{array}{l}\text { BUILT TO FIT OUR HILLS, HARBOUR } \\
\text { AND COAST }\end{array}$ & $\begin{array}{l}\text { RETENTION OF CHARACTER AND HERITAGE IN OUR CITY CENTRE AND } \\
\text { NEIGHBOURHOOD CENTRES }\end{array}$ & HERITAGE/HISTORIC & CULTURAL WELL-BEING \\
\hline 2972 & TAURANGA CITY COUNCIL & $\begin{array}{l}\text { BUILT TO FIT OUR HILLS, HARBOUR } \\
\text { AND COAST }\end{array}$ & HOUSING AFFORDABILITY AND TENURE (OWNING VERSUS RENTING) & HOUSE AFFORDABILITY & ECONOMIC WELL-BEING \\
\hline 2973 & TAURANGA CITY COUNCIL & $\begin{array}{l}\text { BUILT TO FIT OUR HILLS, HARBOUR } \\
\text { AND COAST }\end{array}$ & VIBRANT AND WELL-USED CITY CENTRE & CBD: CITY CENTRE & SOCIAL WELL-BEING \\
\hline
\end{tabular}


Appendix A: List of Community Well-beings Outcomes and Measures by Councils

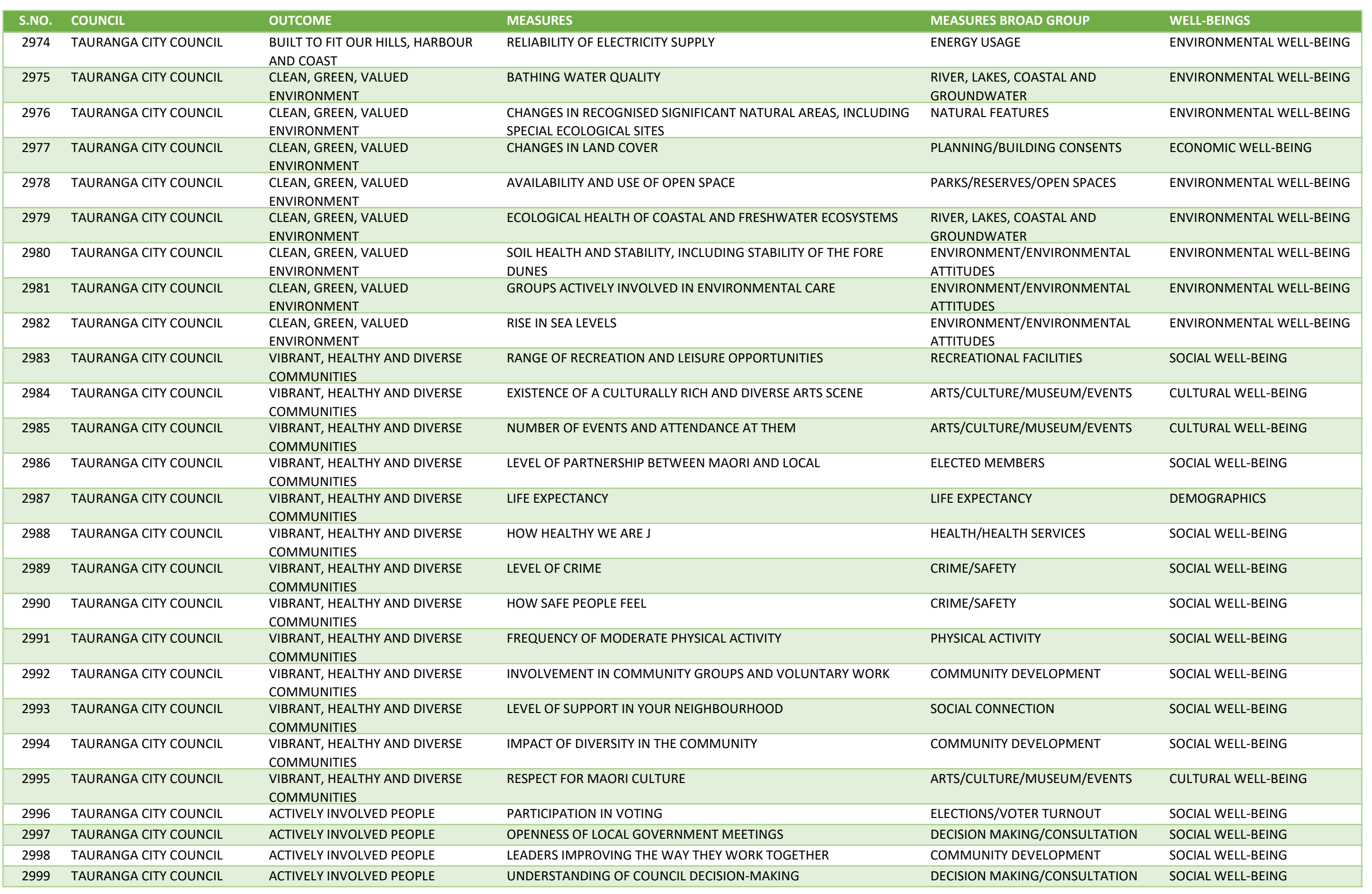


Appendix A: List of Community Well-beings Outcomes and Measures by Councils

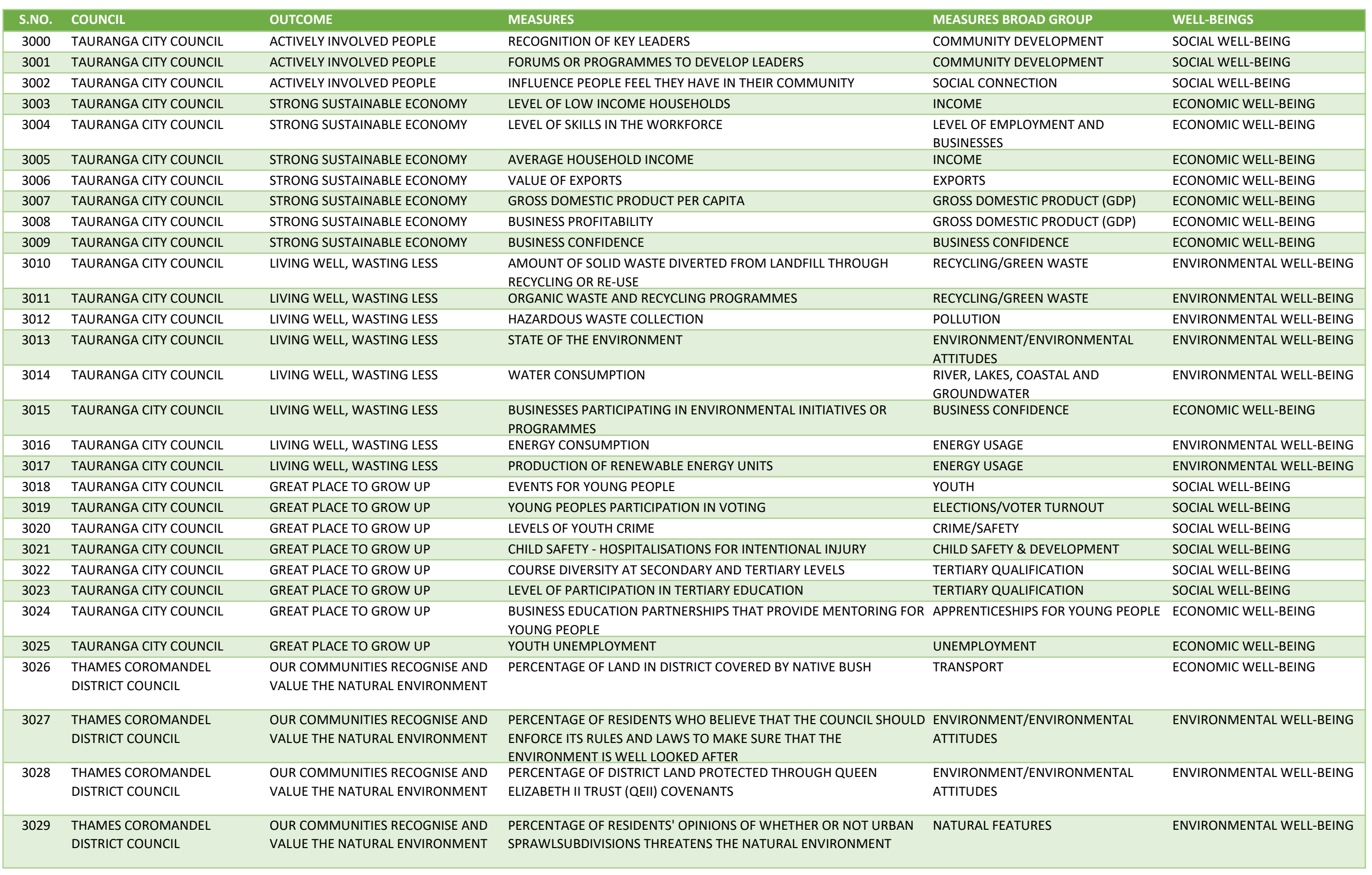




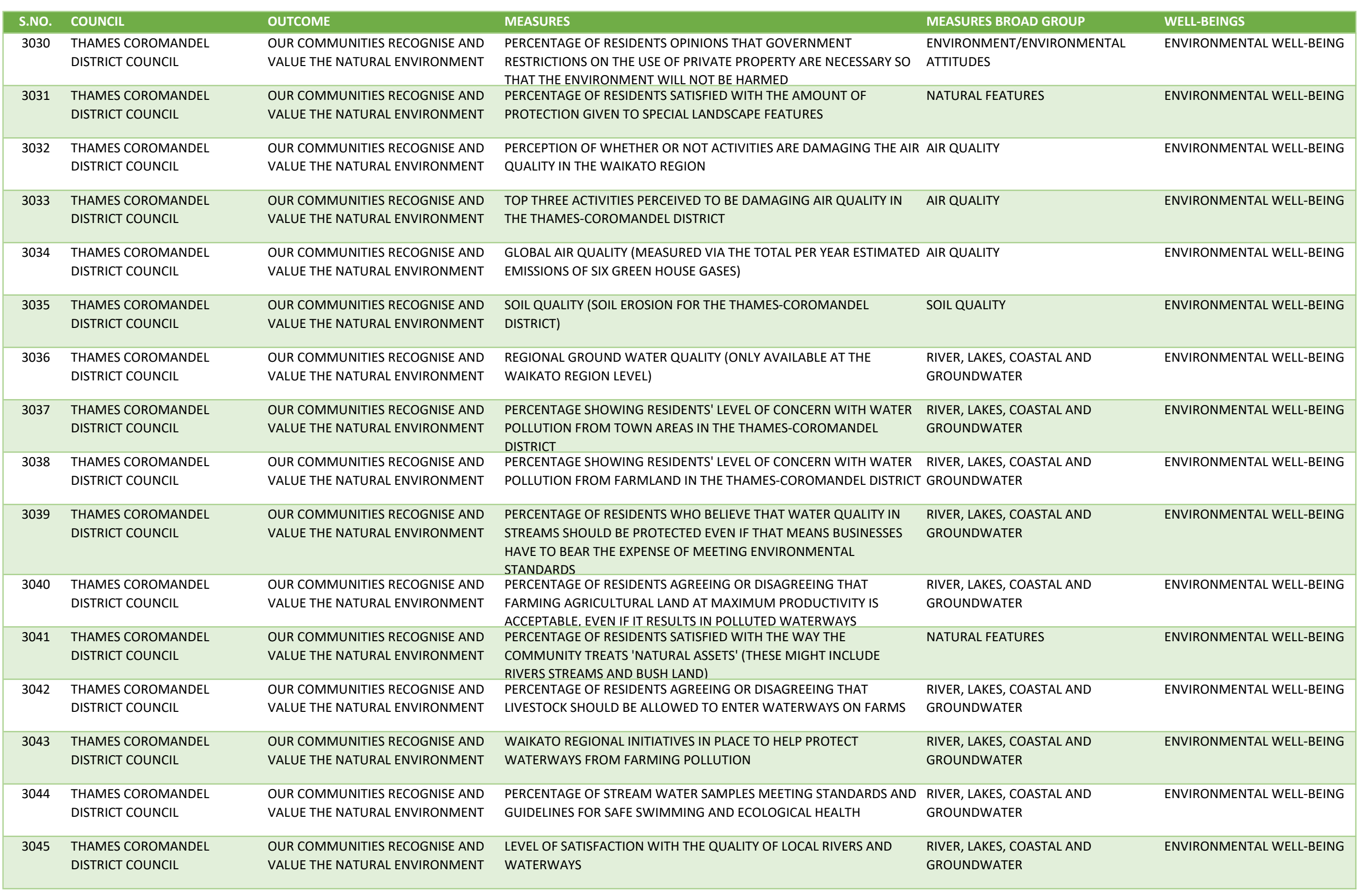




\begin{tabular}{|c|c|c|c|c|c|}
\hline S.NO. & COUNCIL & OUTCOME & MEASURES & MEASURES BROAD GROUP & WELL-BEINGS \\
\hline 3046 & $\begin{array}{l}\text { THAMES COROMANDEL } \\
\text { DISTRICT COUNCIL }\end{array}$ & OUR COMMUNITIES RECOGNISE AND & PERCENTAGE OF RESIDENTS OF THE OPINION THAT TREATED HUMAN & RIVER, LAKES, COASTAL AND & ENVIRONMENTAL WELL-BEING \\
\hline 3047 & $\begin{array}{l}\text { THAMES COROMANDEL } \\
\text { DISTRICT COUNCIL }\end{array}$ & $\begin{array}{l}\text { OUR COMMUNITIES RECOGNISE AND } \\
\text { VALUE THE NATURAL ENVIRONMENT }\end{array}$ & $\begin{array}{l}\text { PERCENTAGE OF RESIDENTS OF THE OPINION THAT POLLUTION IN THE } \\
\text { REGIONS RIVERS AND STREAMS COMES MAINLY FROM FARMLAND }\end{array}$ & POLLUTION & ENVIRONMENTAL WELL-BEING \\
\hline 3048 & $\begin{array}{l}\text { THAMES COROMANDEL } \\
\text { DISTRICT COUNCIL }\end{array}$ & $\begin{array}{l}\text { THE NATURAL VALUES OF OUR COAST } \\
\text { AND BEACHES ARE RESPECTED AND } \\
\text { ENHANCED }\end{array}$ & $\begin{array}{l}\text { PERCENTAGE OF SAMPLES FROM COASTAL BATHING BEACHES THAT } \\
\text { MET OR EXCEEDED GUIDELINES FOR EXCELLENT, SATISFACTORY, OR } \\
\text { UNSATISFACTORY WATER QUALITY FOR CONTACT RECREATION }\end{array}$ & $\begin{array}{l}\text { RIVER, LAKES, COASTAL AND } \\
\text { GROUNDWATER }\end{array}$ & ENVIRONMENTAL WELL-BEING \\
\hline 3049 & $\begin{array}{l}\text { THAMES COROMANDEL } \\
\text { DISTRICT COUNCIL }\end{array}$ & $\begin{array}{l}\text { THE NATURAL VALUES OF OUR COAST } \\
\text { AND BEACHES ARE RESPECTED AND } \\
\text { ENHANCED }\end{array}$ & $\begin{array}{l}\text { PERCENTAGE OF CONCERN REGARDING LOSS OF NATURAL CHARACTER } \\
\text { OF REGIONS BEACHES THROUGH DEVELOPMENT }\end{array}$ & NATURAL FEATURES & ENVIRONMENTAL WELL-BEING \\
\hline 3050 & $\begin{array}{l}\text { THAMES COROMANDEL } \\
\text { DISTRICT COUNCIL }\end{array}$ & $\begin{array}{l}\text { THE NATURAL VALUES OF OUR COAST } \\
\text { AND BEACHES ARE RESPECTED AND } \\
\text { ENHANCED }\end{array}$ & $\begin{array}{l}\text { PERCENTAGE OF RESIDENTS AND RATEPAYERS WHO BELIEVE THAT } \\
\text { DUNES AT THE BEACH ARE IMPORTANT }\end{array}$ & $\begin{array}{l}\text { ENVIRONMENT/ENVIRONMENTAL } \\
\text { ATTITUDES }\end{array}$ & ENVIRONMENTAL WELL-BEING \\
\hline 3051 & $\begin{array}{l}\text { THAMES COROMANDEL } \\
\text { DISTRICT COUNCIL }\end{array}$ & $\begin{array}{l}\text { THE NATURAL VALUES OF OUR COAST } \\
\text { AND BEACHES ARE RESPECTED AND } \\
\text { ENHANCED }\end{array}$ & $\begin{array}{l}\text { PERCENTAGE OF RESIDENTS SATISFIED THAT THE IMPACT FROM } \\
\text { DEVELOPMENT ON THE COASTLINE IS ADEQUATELY MANAGED AND } \\
\text { MINIMISED }\end{array}$ & $\begin{array}{l}\text { RIVER, LAKES, COASTAL AND } \\
\text { GROUNDWATER }\end{array}$ & ENVIRONMENTAL WELL-BEING \\
\hline 3052 & $\begin{array}{l}\text { THAMES COROMANDEL } \\
\text { DISTRICT COUNCIL }\end{array}$ & $\begin{array}{l}\text { THE NATURAL VALUES OF OUR COAST } \\
\text { AND BEACHES ARE RESPECTED AND } \\
\text { ENHANCED }\end{array}$ & $\begin{array}{l}\text { PERCENTAGE OF RESIDENTS SATISFIED WITH THE APPRECIATION OF } \\
\text { THE NATURAL VALUE OF THE COAST }\end{array}$ & NATURAL FEATURES & ENVIRONMENTAL WELL-BEING \\
\hline 3053 & $\begin{array}{l}\text { THAMES COROMANDEL } \\
\text { DISTRICT COUNCIL }\end{array}$ & $\begin{array}{l}\text { THE NATURAL VALUES OF OUR COAST } \\
\text { AND BEACHES ARE RESPECTED AND } \\
\text { ENHANCED }\end{array}$ & $\begin{array}{l}\text { PERCENTAGE OF RESIDENTS AND NON-RESIDENT RATEPAYERS } \\
\text { SATISFIED WITH BOAT RAMPS AND LAUNCHING FACILITIES IN THE } \\
\text { DISTRICT }\end{array}$ & COUNCIL FACILITIES & SOCIAL WELL-BEING \\
\hline 3054 & $\begin{array}{l}\text { THAMES COROMANDEL } \\
\text { DISTRICT COUNCIL }\end{array}$ & $\begin{array}{l}\text { THE NATURAL VALUES OF OUR COAST } \\
\text { AND BEACHES ARE RESPECTED AND } \\
\text { ENHANCED }\end{array}$ & $\begin{array}{l}\text { PERCENTAGE OF RESIDENTS AND RATEPAYERS RATING OF EASY ACCESS } \\
\text { TO THE BEACH IN THE DISTRICT }\end{array}$ & $\begin{array}{l}\text { RIVER, LAKES, COASTAL AND } \\
\text { GROUNDWATER }\end{array}$ & ENVIRONMENTAL WELL-BEING \\
\hline 3055 & $\begin{array}{l}\text { THAMES COROMANDEL } \\
\text { DISTRICT COUNCIL }\end{array}$ & $\begin{array}{l}\text { THE NATURAL VALUES OF OUR COAST } \\
\text { AND BEACHES ARE RESPECTED AND } \\
\text { ENHANCED }\end{array}$ & $\begin{array}{l}\text { PERCENTAGE OF RESIDENTS AND RATEPAYERS VISITING ANY BEACH IN } \\
\text { THE DISTRICT }\end{array}$ & $\begin{array}{l}\text { RIVER, LAKES, COASTAL AND } \\
\text { GROUNDWATER }\end{array}$ & ENVIRONMENTAL WELL-BEING \\
\hline 3056 & $\begin{array}{l}\text { THAMES COROMANDEL } \\
\text { DISTRICT COUNCIL }\end{array}$ & $\begin{array}{l}\text { OUR COMMUNITIES ARE HEALTHY, } \\
\text { COHESIVE, CARING AND SUPPORTIVE }\end{array}$ & $\begin{array}{l}\text { WAIKATO DISTRICT HEALTH BOARD KEY HEALTH ISSUES IN THE THAMES- } \\
\text { COROMANDEL DISTRICT }\end{array}$ & - HEALTH/HEALTH SERVICES & SOCIAL WELL-BEING \\
\hline 3057 & $\begin{array}{l}\text { THAMES COROMANDEL } \\
\text { DISTRICT COUNCIL }\end{array}$ & $\begin{array}{l}\text { OUR COMMUNITIES ARE HEALTHY, } \\
\text { COHESIVE, CARING AND SUPPORTIVE }\end{array}$ & PERCENTAGE OF NEW PROBLEM GAMBLING CLIENTS PER YEAR & CRIME/SAFETY & SOCIAL WELL-BEING \\
\hline 3058 & $\begin{array}{l}\text { THAMES COROMANDEL } \\
\text { DISTRICT COUNCIL }\end{array}$ & $\begin{array}{l}\text { OUR COMMUNITIES ARE HEALTHY, } \\
\text { COHESIVE, CARING AND SUPPORTIVE }\end{array}$ & $\begin{array}{l}\text { REGIONAL CRIME RECORDED PER YEAR (ONLY CURRENTLY AVAILABLE } \\
\text { AT THE WAIKATO REGION LEVEL) }\end{array}$ & CRIME/SAFETY & SOCIAL WELL-BEING \\
\hline 3059 & $\begin{array}{l}\text { THAMES COROMANDEL } \\
\text { DISTRICT COUNCIL }\end{array}$ & $\begin{array}{l}\text { OUR COMMUNITIES ARE HEALTHY, } \\
\text { COHESIVE, CARING AND SUPPORTIVE }\end{array}$ & $\begin{array}{l}\text { PERCENTAGE OF RESIDENTS SATISFIED WITH THE RANGE OF HEALTH } \\
\text { SERVICES PROVIDED IN THE DISTRICT }\end{array}$ & HEALTH/HEALTH SERVICES & SOCIAL WELL-BEING \\
\hline 3060 & $\begin{array}{l}\text { THAMES COROMANDEL } \\
\text { DISTRICT COUNCIL }\end{array}$ & $\begin{array}{l}\text { OUR COMMUNITIES ARE HEALTHY, } \\
\text { COHESIVE, CARING AND SUPPORTIVE }\end{array}$ & AVOIDABLE MORTALITY RATE & HOSPITALISATIONS & ECONOMIC WELL-BEING \\
\hline 3061 & $\begin{array}{l}\text { THAMES COROMANDEL } \\
\text { DISTRICT COUNCIL }\end{array}$ & $\begin{array}{l}\text { OUR COMMUNITIES ARE HEALTHY, } \\
\text { COHESIVE, CARING AND SUPPORTIVE }\end{array}$ & $\begin{array}{l}\text { PERCENTAGE OF RESIDENTS WHO FELT THERE HAD BEEN A TIME IN THE } \\
\text { LAST YEAR WHEN THEY WANTED TO GO TO A GP BUT DIDN'T, AND } \\
\text { WHY }\end{array}$ & HEALTH/HEALTH SERVICES & SOCIAL WELL-BEING \\
\hline 3063 & $\begin{array}{l}\text { THAMES COROMANDEL } \\
\text { DISTRICT COUNCIL }\end{array}$ & $\begin{array}{l}\text { OUR COMMUNITIES ARE HEALTHY, } \\
\text { COHESIVE, CARING AND SUPPORTIVE }\end{array}$ & $\begin{array}{l}\text { NUMBER OF HIGH SCHOOLS IN THE DISTRICT THAT PARTICIPATE IN } \\
\text { CAREER EDUCATION PROGRAMMES }\end{array}$ & QUALIFICATION/SCHOOL & SOCIAL WELL-BEING \\
\hline
\end{tabular}




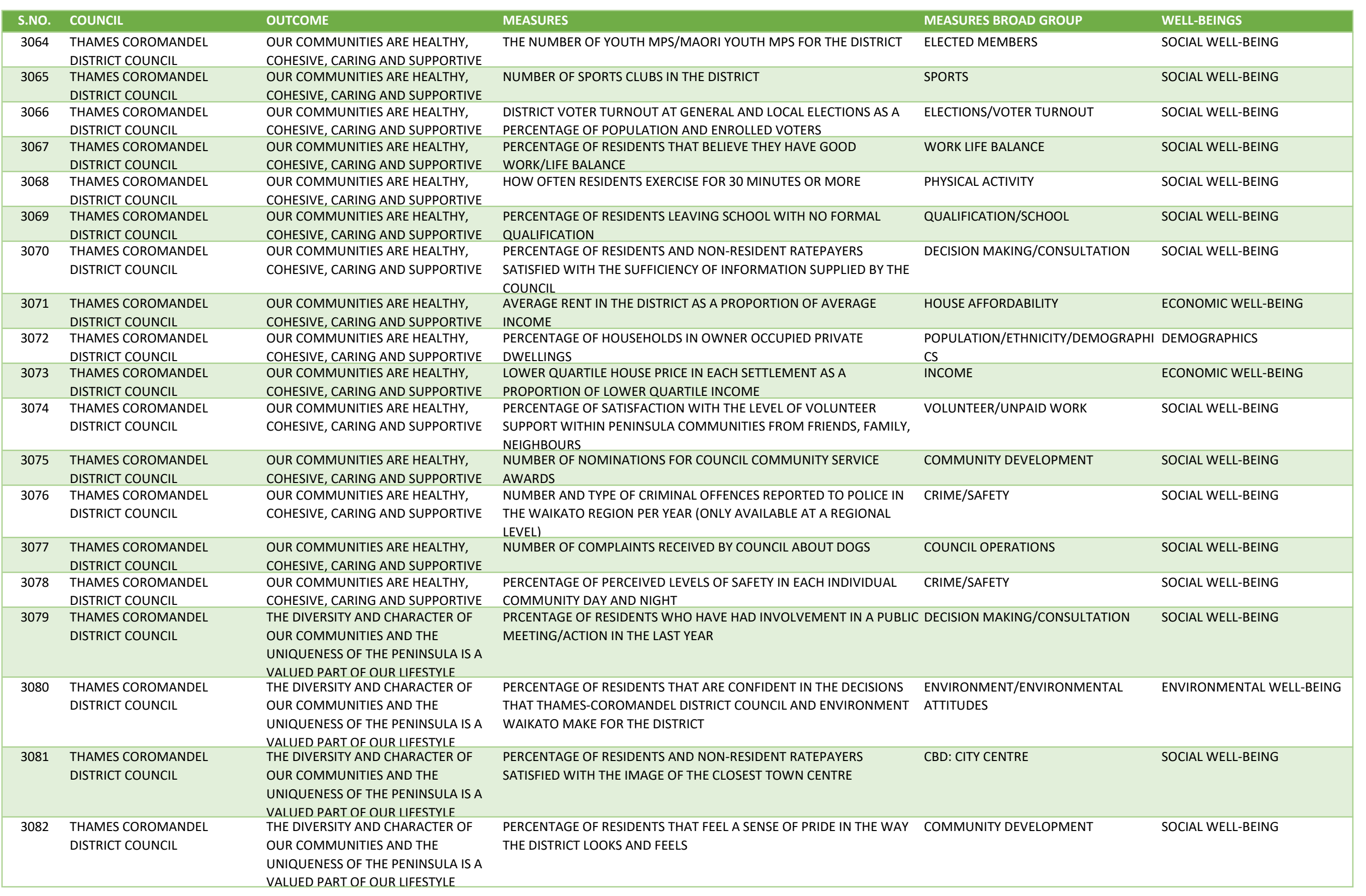




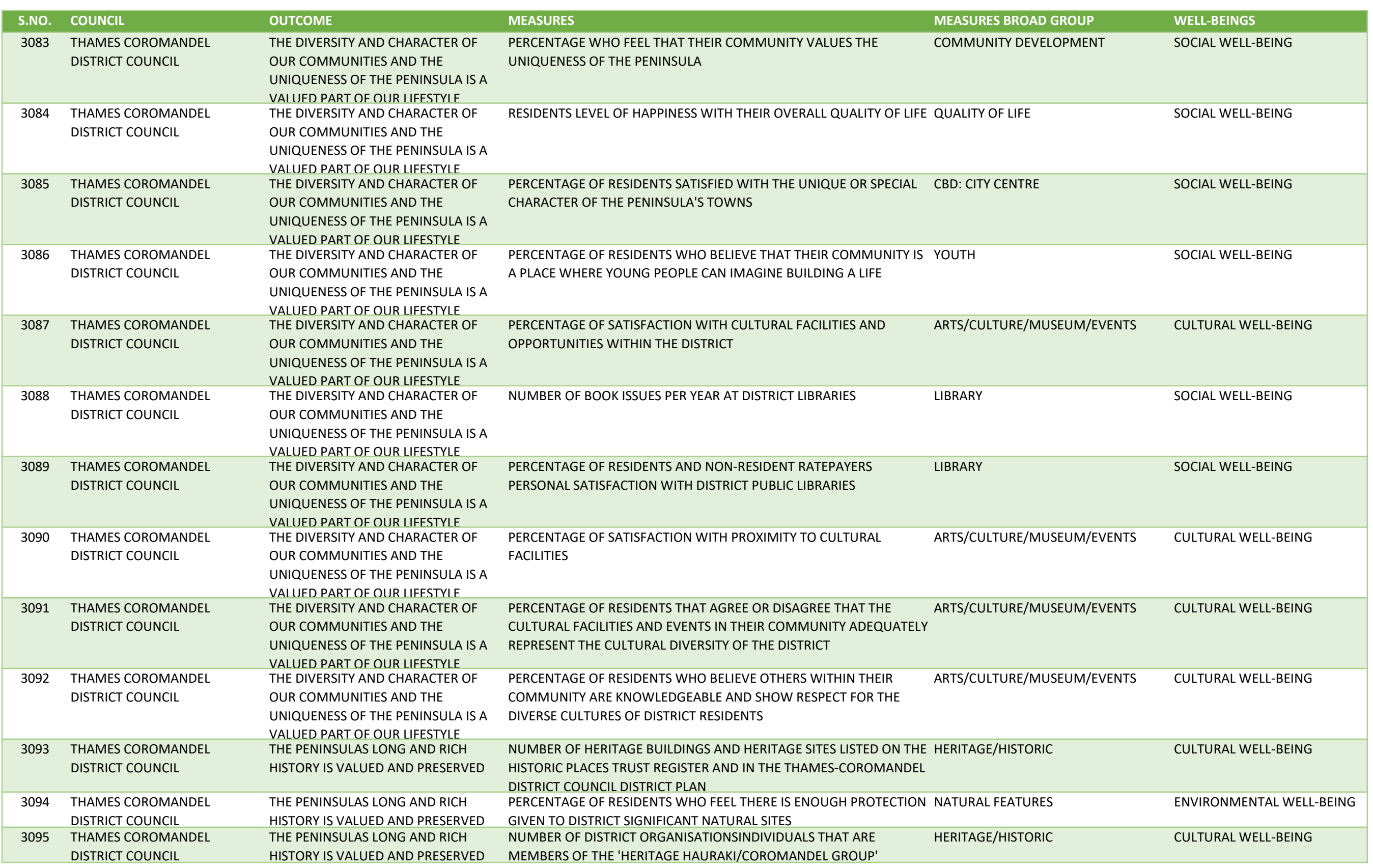




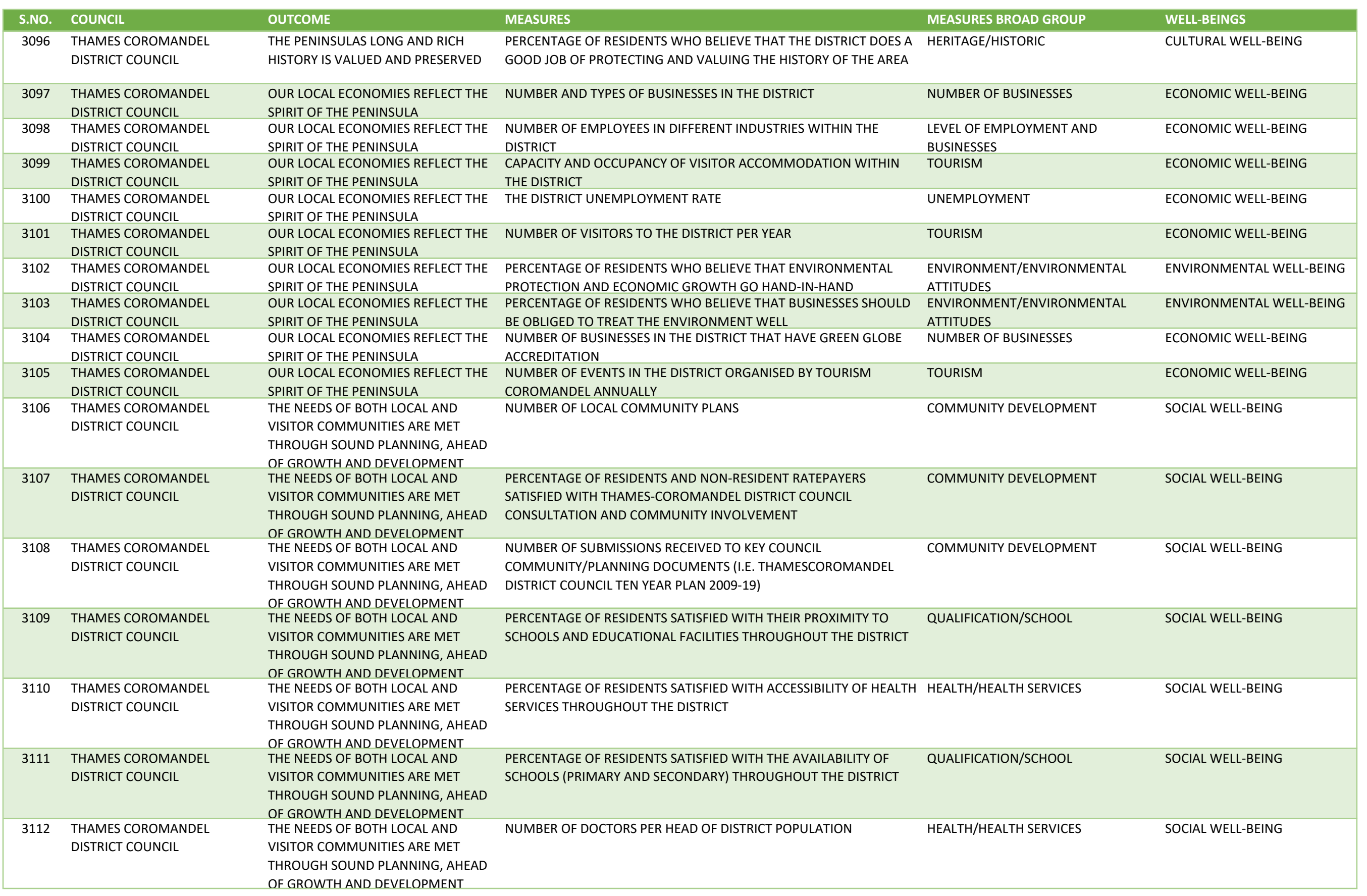




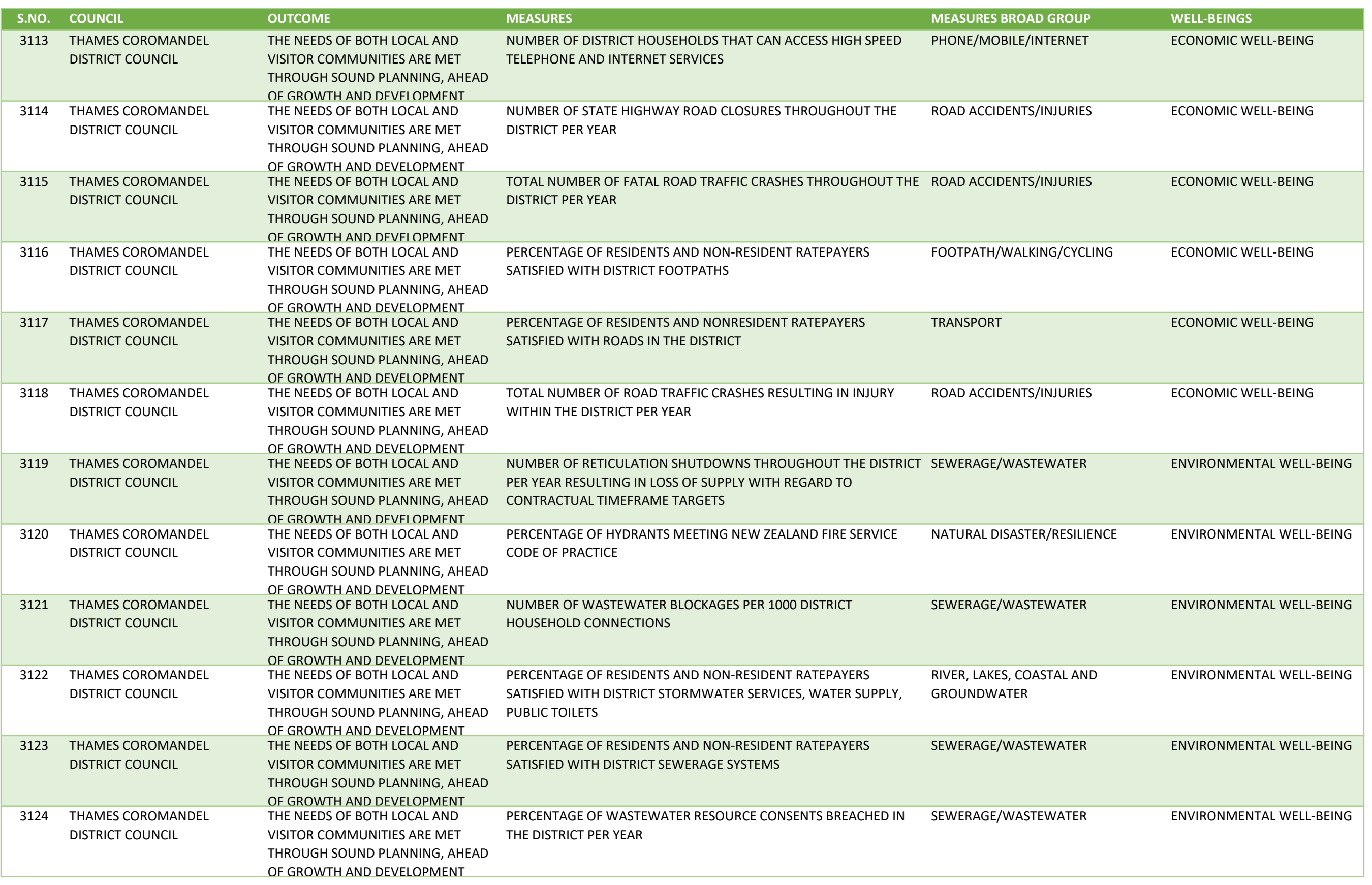




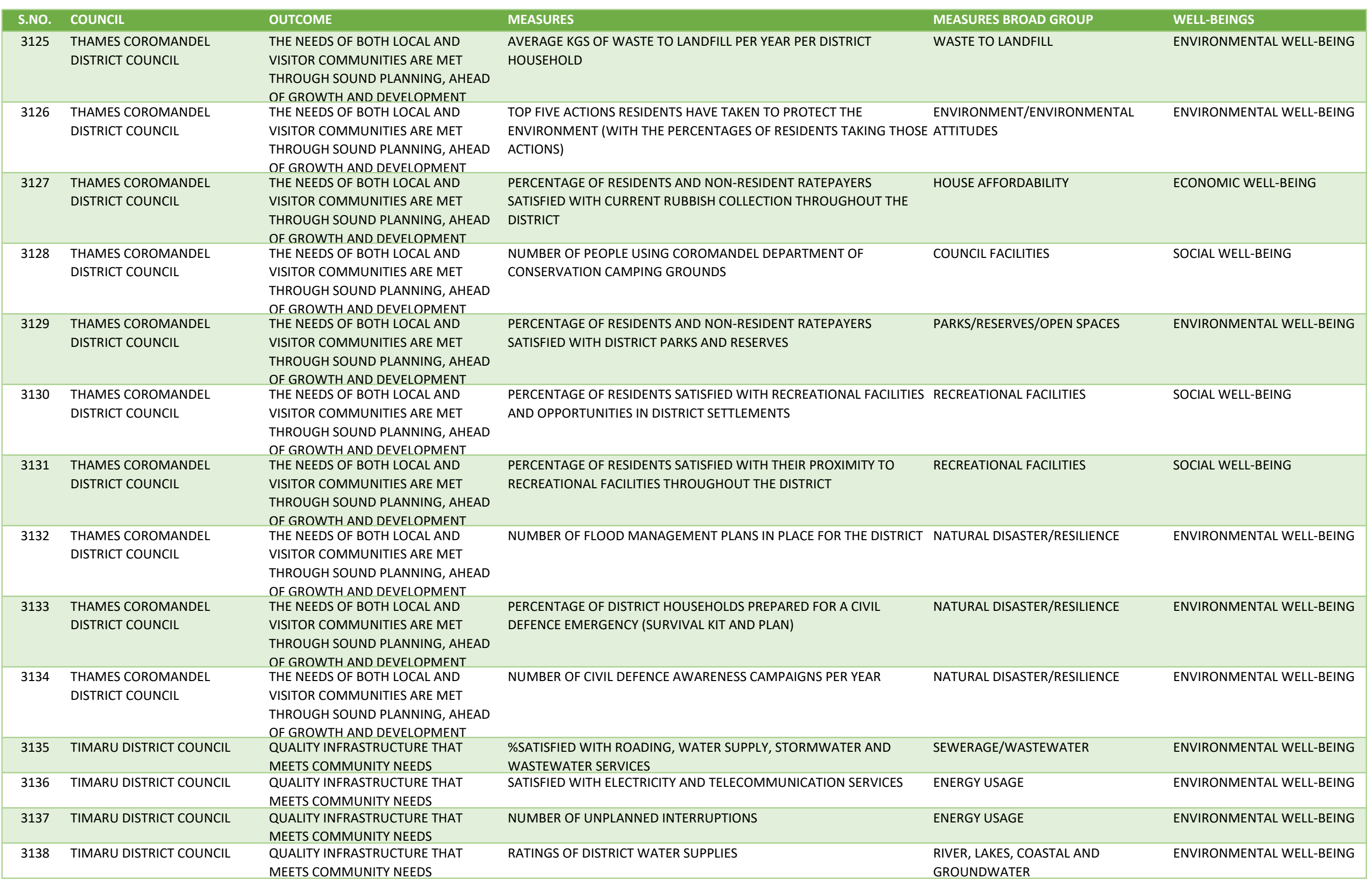


Appendix A: List of Community Well-beings Outcomes and Measures by Councils

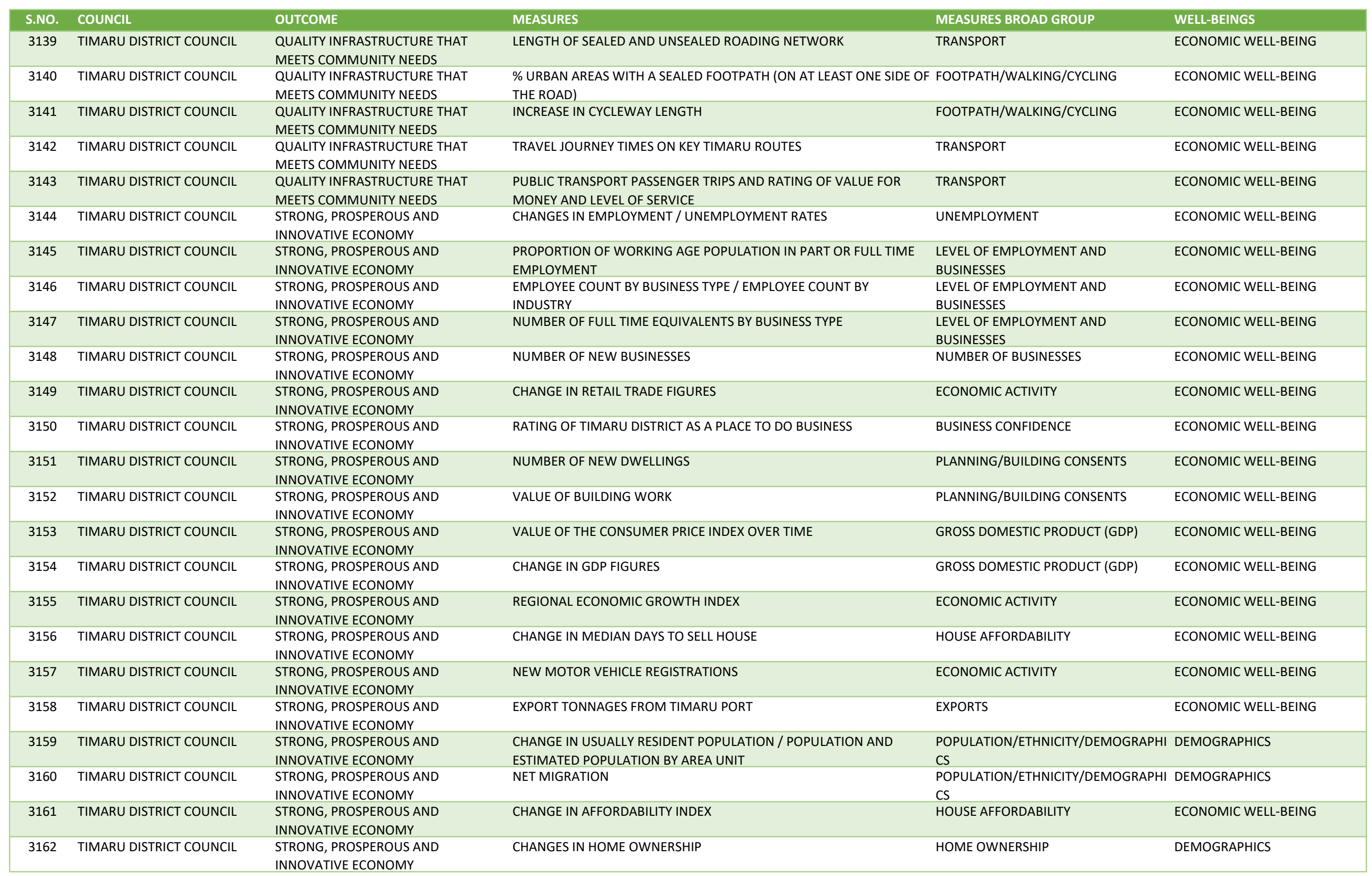


Appendix A: List of Community Well-beings Outcomes and Measures by Councils

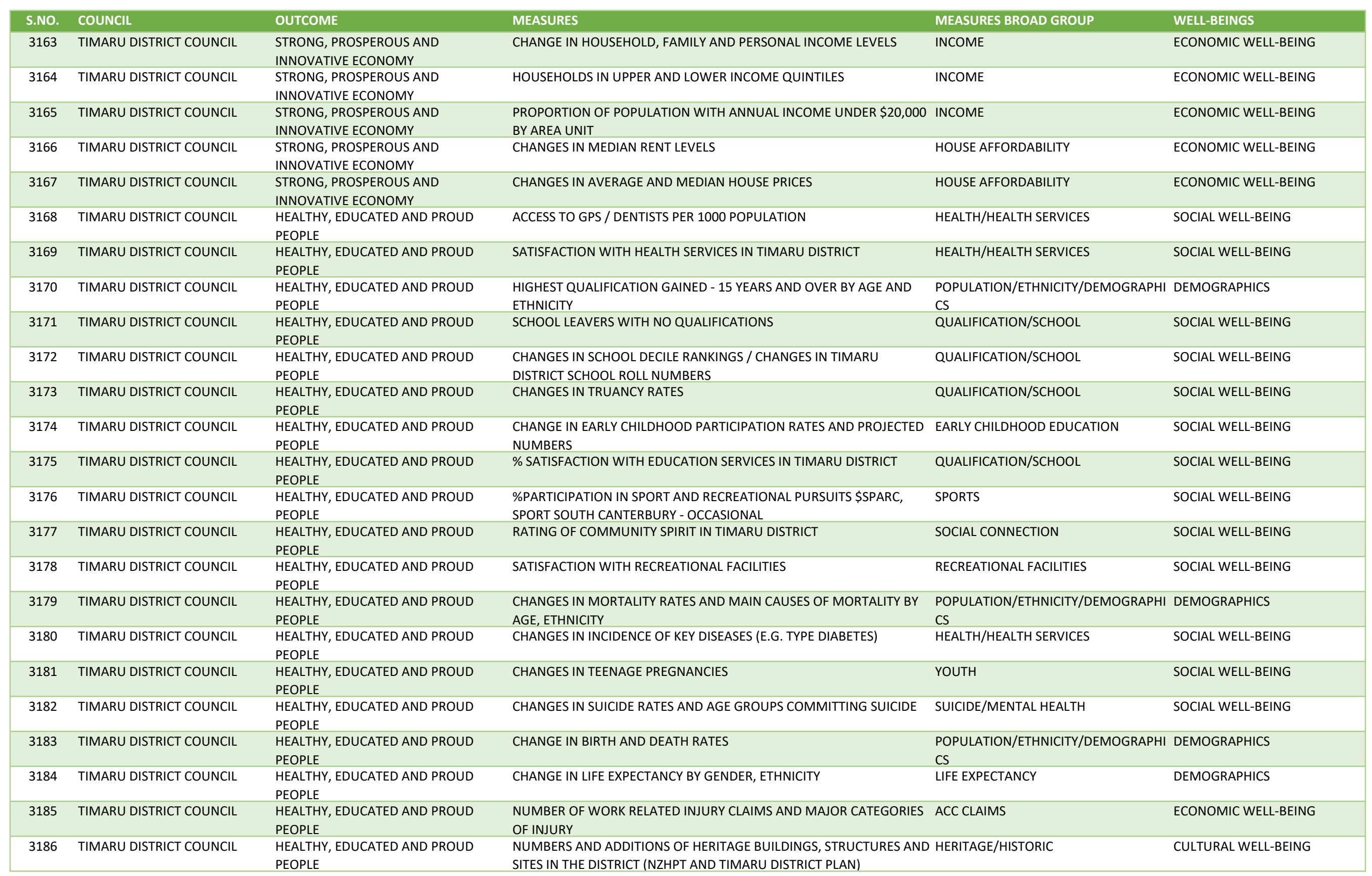




\begin{tabular}{|c|c|c|c|c|c|}
\hline S.NO. & COUNCIL & OUTCOME & MEASURES & MEASURES BROAD GROUP & WELL-BEINGS \\
\hline 3187 & TIMARU DISTRICT COUNCIL & $\begin{array}{l}\text { HEALTHY, VALUED AND ACCESSIBLE } \\
\text { ENVIRONMENT }\end{array}$ & $\begin{array}{l}\text { AIR QUALITY AT SELECTED SITES -- NUMBER OF DAYS WITH PM10 } \\
\text { CONCENTRATIONS OF } 50+\text { IN KEY TOWNSHIPS }\end{array}$ & AIR QUALITY & ENVIRONMENTAL WELL-BEING \\
\hline 3188 & TIMARU DISTRICT COUNCIL & $\begin{array}{l}\text { HEALTHY, VALUED AND ACCESSIBLE } \\
\text { ENVIRONMENT }\end{array}$ & $\begin{array}{l}\text { OVERALL AVERAGE REDUCTION IN PM10 CONCENTRATIONS IN } \\
\text { SELECTED AREAS ON AN ANNUAL BASIS }\end{array}$ & AIR QUALITY & ENVIRONMENTAL WELL-BEING \\
\hline 3189 & TIMARU DISTRICT COUNCIL & $\begin{array}{l}\text { HEALTHY, VALUED AND ACCESSIBLE } \\
\text { ENVIRONMENT }\end{array}$ & $\begin{array}{l}\text { NUMBER OF SPECIES NATIVE TO TIMARU DISTRICT/ SOUTH } \\
\text { CANTERBURY ON DOCS LIST OF THREATENED SPECIES DEPARTMENT OF } \\
\text { CONSERVATION - OCCASIONAL }\end{array}$ & $\begin{array}{l}\text { ENVIRONMENT/ENVIRONMENTAL } \\
\text { ATTITUDES }\end{array}$ & ENVIRONMENTAL WELL-BEING \\
\hline 3190 & TIMARU DISTRICT COUNCIL & $\begin{array}{l}\text { HEALTHY, VALUED AND ACCESSIBLE } \\
\text { ENVIRONMENT }\end{array}$ & COASTAL WATER QUALITY AT VARIOUS SITES & $\begin{array}{l}\text { RIVER, LAKES, COASTAL AND } \\
\text { GROUNDWATER }\end{array}$ & ENVIRONMENTAL WELL-BEING \\
\hline 3191 & TIMARU DISTRICT COUNCIL & $\begin{array}{l}\text { HEALTHY, VALUED AND ACCESSIBLE } \\
\text { ENVIRONMENT }\end{array}$ & RATING OF ACCESSIBILITY TO THE DISTRICTS NATURAL ENVIRONMENT & NATURAL FEATURES & ENVIRONMENTAL WELL-BEING \\
\hline 3192 & TIMARU DISTRICT COUNCIL & $\begin{array}{l}\text { HEALTHY, VALUED AND ACCESSIBLE } \\
\text { ENVIRONMENT }\end{array}$ & INCIDENCE OF PLANT AND ANIMAL PESTS & PEST CONTROL & ENVIRONMENTAL WELL-BEING \\
\hline 3193 & TIMARU DISTRICT COUNCIL & $\begin{array}{l}\text { HEALTHY, VALUED AND ACCESSIBLE } \\
\text { ENVIRONMENT }\end{array}$ & NATURE AND EXTENT OF LAND USE CHANGE & PLANNING/BUILDING CONSENTS & ECONOMIC WELL-BEING \\
\hline 3194 & TIMARU DISTRICT COUNCIL & $\begin{array}{l}\text { HEALTHY, VALUED AND ACCESSIBLE } \\
\text { ENVIRONMENT }\end{array}$ & HECTARES OF BUSH REMNANTS IN THE DISTRICT & PARKS/RESERVES/OPEN SPACES & ENVIRONMENTAL WELL-BEING \\
\hline 3195 & TIMARU DISTRICT COUNCIL & $\begin{array}{l}\text { HEALTHY, VALUED AND ACCESSIBLE } \\
\text { ENVIRONMENT }\end{array}$ & $\begin{array}{l}\text { WASTE DIVERTED FROM LANDFILL TIMARU DISTRICT COUNCIL - } \\
\text { ANNUAL }\end{array}$ & RECYCLING/GREEN WASTE & ENVIRONMENTAL WELL-BEING \\
\hline 3196 & TIMARU DISTRICT COUNCIL & $\begin{array}{l}\text { HEALTHY, VALUED AND ACCESSIBLE } \\
\text { ENVIRONMENT }\end{array}$ & $\begin{array}{l}\text { TOTAL KWH ENERGY USED BY TIMARU DISTRICT RESIDENTS EECA, } \\
\text { ENERGY COMPANIES, ECAN -- ANNUAL }\end{array}$ & ENERGY USAGE & ENVIRONMENTAL WELL-BEING \\
\hline 3198 & TIMARU DISTRICT COUNCIL & $\begin{array}{l}\text { HEALTHY, VALUED AND ACCESSIBLE } \\
\text { ENVIRONMENT }\end{array}$ & SOIL QUALITY AND EROSION INFORMATION IN THE TIMARU DISTRICT & SOIL QUALITY & ENVIRONMENTAL WELL-BEING \\
\hline 3199 & TIMARU DISTRICT COUNCIL & $\begin{array}{l}\text { HEALTHY, VALUED AND ACCESSIBLE } \\
\text { ENVIRONMENT }\end{array}$ & $\begin{array}{l}\text { TRENDS IN PLANT OR ANIMAL PEST ISSUES IN THE TIMARU DISTRICT } \\
\text { ENVIRONMENT CANTERBURY - ANNUAL }\end{array}$ & PEST CONTROL & ENVIRONMENTAL WELL-BEING \\
\hline 3200 & TIMARU DISTRICT COUNCIL & ATTRACTIVE AND DESIRABLE DISTRICT & $\begin{array}{l}\text { CHANGES IN GUEST NIGHTS AND VISITOR SPEND STATISTICS NEW } \\
\text { ZEALAND - MONTHLY }\end{array}$ & TOURISM & ECONOMIC WELL-BEING \\
\hline 3201 & TIMARU DISTRICT COUNCIL & ATTRACTIVE AND DESIRABLE DISTRICT & RATING OF TIMARU AS A PLACE TO LIVE & COMMUNITY DEVELOPMENT & SOCIAL WELL-BEING \\
\hline 3202 & TIMARU DISTRICT COUNCIL & ATTRACTIVE AND DESIRABLE DISTRICT & RATING OF COMMUNITY SPIRIT IN TIMARU DISTRICT & SOCIAL CONNECTION & SOCIAL WELL-BEING \\
\hline 3203 & TIMARU DISTRICT COUNCIL & ATTRACTIVE AND DESIRABLE DISTRICT & VISITOR PERCEPTIONS OF TIMARU DISTRICT AS A PLACE TO VISIT & TOURISM & ECONOMIC WELL-BEING \\
\hline 3204 & TIMARU DISTRICT COUNCIL & ATTRACTIVE AND DESIRABLE DISTRICT & $\begin{array}{l}\text { NUMBER OF TOURISM BUSINESSES IN TIMARU DISTRICT AND } \\
\text { EMPLOYEE COUNTS STATISTICS NEW ZEALAND - ANNUAL }\end{array}$ & TOURISM & ECONOMIC WELL-BEING \\
\hline 3205 & TIMARU DISTRICT COUNCIL & $\begin{array}{l}\text { VIBRANT, SAFE AND CARING } \\
\text { COMMUNITIES }\end{array}$ & CHANGES IN CHILD/OLDER PERSONS RATIO & $\begin{array}{l}\text { POPULATION/ETHNICITY/DEMOGRAPHI } \\
\text { CS }\end{array}$ & DEMOGRAPHICS \\
\hline 3206 & TIMARU DISTRICT COUNCIL & $\begin{array}{l}\text { VIBRANT, SAFE AND CARING } \\
\text { COMMUNITIES }\end{array}$ & CHANGES IN DEPENDENCY RATIO & $\begin{array}{l}\text { POPULATION/ETHNICITY/DEMOGRAPHI } \\
\text { CS }\end{array}$ & DEMOGRAPHICS \\
\hline 3207 & TIMARU DISTRICT COUNCIL & $\begin{array}{l}\text { VIBRANT, SAFE AND CARING } \\
\text { COMMUNITIES }\end{array}$ & $\begin{array}{l}\text { INCOME SUPPORT TOTAL NUMBERS RECEIVING MEANS TESTED } \\
\text { BENEFITS }\end{array}$ & INCOME & ECONOMIC WELL-BEING \\
\hline 3209 & TIMARU DISTRICT COUNCIL & $\begin{array}{l}\text { VIBRANT, SAFE AND CARING } \\
\text { COMMUNITIES }\end{array}$ & CHANGES IN ETHNIC COMPOSITION / ETHNIC AGE-SEX STRUCTURE & $\begin{array}{l}\text { POPULATION/ETHNICITY/DEMOGRAPHI } \\
\text { CS }\end{array}$ & DEMOGRAPHICS \\
\hline 3210 & TIMARU DISTRICT COUNCIL & $\begin{array}{l}\text { VIBRANT, SAFE AND CARING } \\
\text { COMMUNITIES }\end{array}$ & $\begin{array}{l}\text { TOTAL EMPLOYMENT IN CULTURAL INDUSTRIES AND TOP CULTURAL } \\
\text { ACTIVITIES }\end{array}$ & $\begin{array}{l}\text { LEVEL OF EMPLOYMENT AND } \\
\text { BUSINESSES }\end{array}$ & ECONOMIC WELL-BEING \\
\hline
\end{tabular}


Appendix A: List of Community Well-beings Outcomes and Measures by Councils

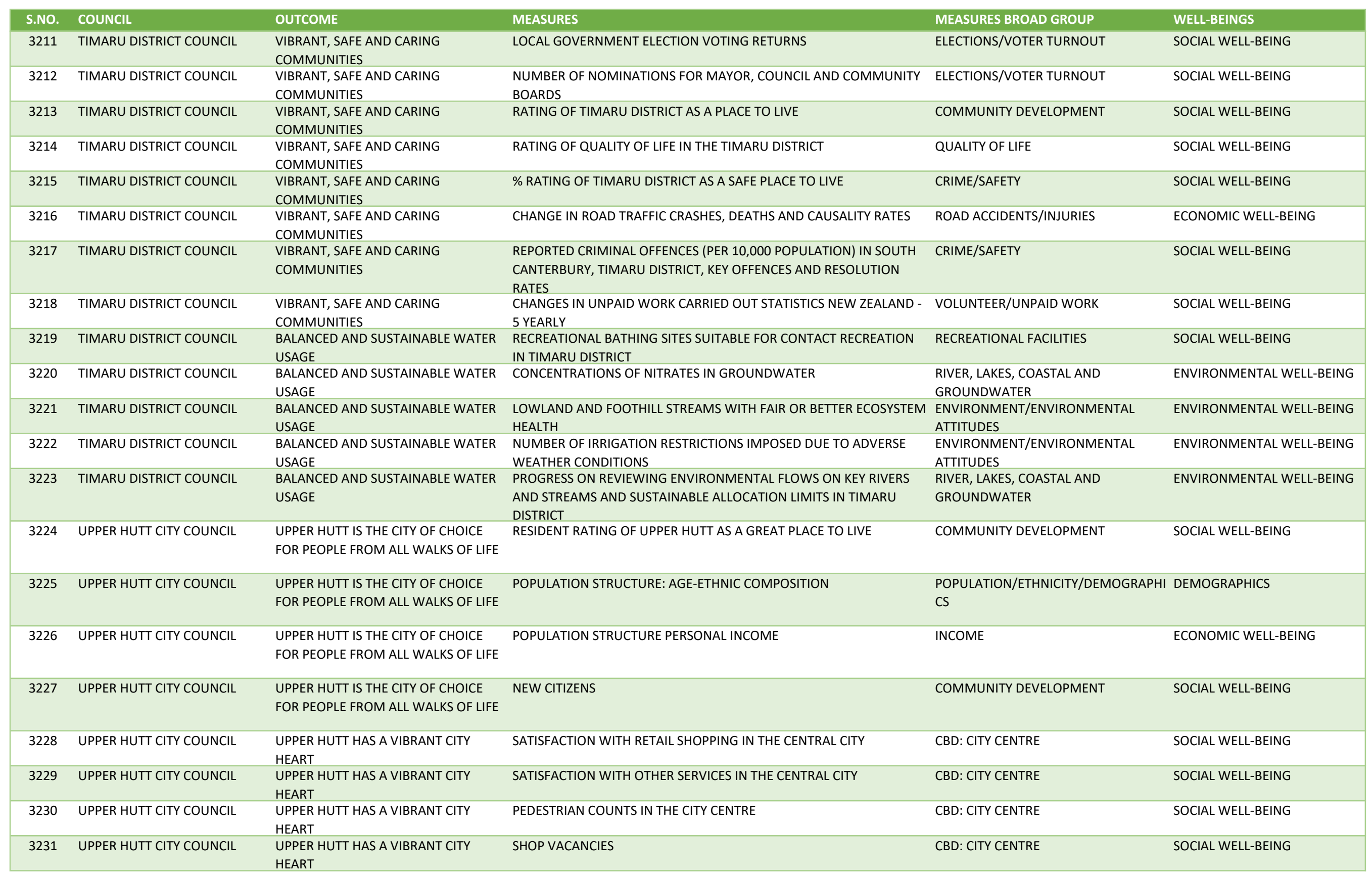


Appendix A: List of Community Well-beings Outcomes and Measures by Councils

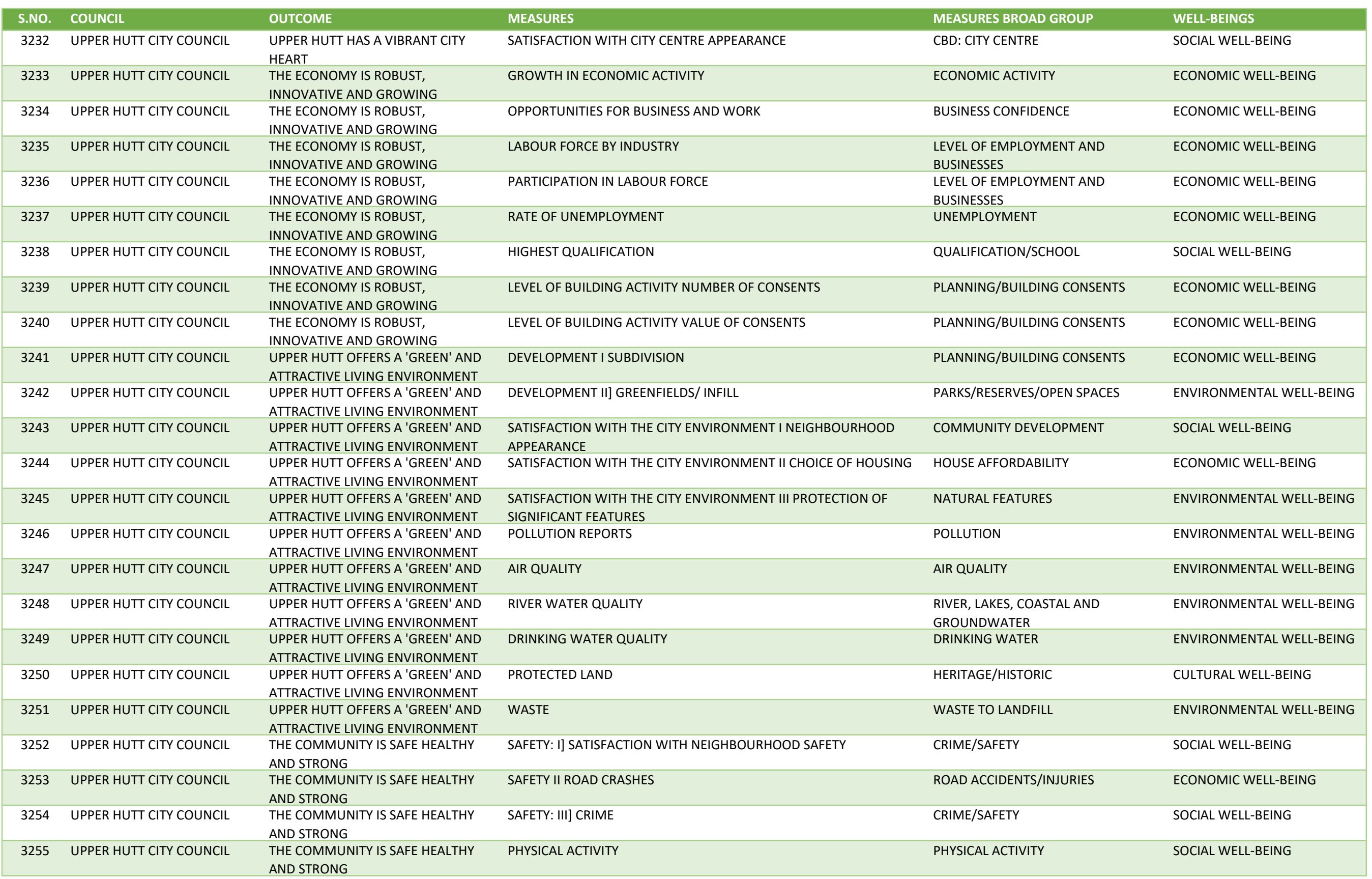


Appendix A: List of Community Well-beings Outcomes and Measures by Councils

\begin{tabular}{|c|c|c|c|c|c|}
\hline S.NO. & COUNCIL & OUTCOME & MEASURES & MEASURES BROAD GROUP & WELL-BEINGS \\
\hline 3256 & UPPER HUTT CITY COUNCIL & $\begin{array}{l}\text { THE COMMUNITY IS SAFE HEALTHY } \\
\text { AND STRONG }\end{array}$ & ACCESS TO HEALTH SERVICES & HEALTH/HEALTH SERVICES & SOCIAL WELL-BEING \\
\hline 3257 & UPPER HUTT CITY COUNCIL & $\begin{array}{l}\text { THE COMMUNITY IS SAFE HEALTHY } \\
\text { AND STRONG }\end{array}$ & ACCESS TO EDUCATION SERVICES & QUALIFICATION/SCHOOL & SOCIAL WELL-BEING \\
\hline 3258 & UPPER HUTT CITY COUNCIL & $\begin{array}{l}\text { THE COMMUNITY IS SAFE, HEALTHY } \\
\text { AND STRONG }\end{array}$ & VOTER TURNOUT & ELECTIONS/VOTER TURNOUT & SOCIAL WELL-BEING \\
\hline 3259 & UPPER HUTT CITY COUNCIL & $\begin{array}{l}\text { LEISURE OPPORTUNITIES ARE } \\
\text { OUTSTANDING }\end{array}$ & HO XTREAM I SATISFACTION WITH THE FACILITY & COUNCIL FACILITIES & SOCIAL WELL-BEING \\
\hline 3260 & UPPER HUTT CITY COUNCIL & $\begin{array}{l}\text { LEISURE OPPORTUNITIES ARE } \\
\text { OUTSTANDING }\end{array}$ & HO XTREAM II REGIONAL USE & ECONOMIC ACTIVITY & ECONOMIC WELL-BEING \\
\hline 3261 & UPPER HUTT CITY COUNCIL & $\begin{array}{l}\text { LEISURE OPPORTUNITIES ARE } \\
\text { OUTSTANDING }\end{array}$ & EXPRESSIONS ARTS AND ENTERTAINMENT CENTRE I SATISFACTION & ARTS/CULTURE/MUSEUM/EVENTS & CULTURAL WELL-BEING \\
\hline 3262 & UPPER HUTT CITY COUNCIL & $\begin{array}{l}\text { LEISURE OPPORTUNITIES ARE } \\
\text { OUTSTANDING }\end{array}$ & EXPRESSIONS ARTS AND ENTERTAINMENT CENTRE II REGIONAL USE & ARTS/CULTURE/MUSEUM/EVENTS & CULTURAL WELL-BEING \\
\hline 3263 & UPPER HUTT CITY COUNCIL & $\begin{array}{l}\text { LEISURE OPPORTUNITIES ARE } \\
\text { OUTSTANDING }\end{array}$ & SATISFACTION WITH PARKS AND RESERVES & PARKS/RESERVES/OPEN SPACES & ENVIRONMENTAL WELL-BEING \\
\hline 3264 & UPPER HUTT CITY COUNCIL & $\begin{array}{l}\text { LEISURE OPPORTUNITIES ARE } \\
\text { OUTSTANDING }\end{array}$ & REGIONAL USE OF PARKS & PARKS/RESERVES/OPEN SPACES & ENVIRONMENTAL WELL-BEING \\
\hline 3265 & UPPER HUTT CITY COUNCIL & $\begin{array}{l}\text { LEISURE OPPORTUNITIES ARE } \\
\text { OUTSTANDING }\end{array}$ & SATISFACTION WITH LIBRARY & LIBRARY & SOCIAL WELL-BEING \\
\hline 3267 & UPPER HUTT CITY COUNCIL & $\begin{array}{l}\text { UPPER HUTT IS CONNECTED WITH THE } \\
\text { WORLD }\end{array}$ & TRAVEL TIME TO WELLINGTON & TRANSPORT & ECONOMIC WELL-BEING \\
\hline 3268 & UPPER HUTT CITY COUNCIL & $\begin{array}{l}\text { UPPER HUTT IS CONNECTED WITH THE } \\
\text { WORLD }\end{array}$ & AVAILABILITY OF NEW TECHNOLOGY I HOMES WITH INTERNET ACCESS & PHONE/MOBILE/INTERNET & ECONOMIC WELL-BEING \\
\hline 3269 & UPPER HUTT CITY COUNCIL & $\begin{array}{l}\text { UPPER HUTT IS CONNECTED WITH THE } \\
\text { WORLD }\end{array}$ & $\begin{array}{l}\text { AVAILABILITY OF NEW TECHNOLOGY II NEW COMMUNICATION } \\
\text { TECHNOLOGIES AVAILABLE }\end{array}$ & PHONE/MOBILE/INTERNET & ECONOMIC WELL-BEING \\
\hline 3270 & UPPER HUTT CITY COUNCIL & $\begin{array}{l}\text { UPPER HUTT IS CONNECTED WITH THE } \\
\text { WORLD }\end{array}$ & MODE OF JOURNEY TO WORK & $\begin{array}{l}\text { POPULATION/ETHNICITY/DEMOGRAPHI } \\
\text { CS }\end{array}$ & DEMOGRAPHICS \\
\hline 3271 & UPPER HUTT CITY COUNCIL & $\begin{array}{l}\text { UPPER HUTT IS CONNECTED WITH THE } \\
\text { WORLD }\end{array}$ & SATISFACTION WITH RANGE OF TRANSPORT OPTIONS AVAILABLE & TRANSPORT & ECONOMIC WELL-BEING \\
\hline 3272 & WAIKATO DISTRICT COUNCIL & ACCESSIBLE WAIKATO & $\begin{array}{l}\text { A DECLINING TREND IN THE NUMBER OF ROAD TRAFFIC CRASHES AND } \\
\text { CASUALTIES IN THE DISTRICT }\end{array}$ & ROAD ACCIDENTS/INJURIES & ECONOMIC WELL-BEING \\
\hline 3273 & WAIKATO DISTRICT COUNCIL & ACCESSIBLE WAIKATO & $\begin{array}{l}\text { AN INCREASING TREND IN THE NUMBER OF HOUSEHOLD TELEPHONE } \\
\text { AND INTERNET CONNECTIONS IN THE DISTRICT }\end{array}$ & PHONE/MOBILE/INTERNET & ECONOMIC WELL-BEING \\
\hline 3274 & WAIKATO DISTRICT COUNCIL & ACCESSIBLE WAIKATO & $\begin{array}{l}\text { CONTINUED PROGRESS TOWARDS THE DEVELOPMENT OF THE } \\
\text { WAIKATO EXPRESSWAY }\end{array}$ & TRANSPORT & ECONOMIC WELL-BEING \\
\hline 3275 & WAIKATO DISTRICT COUNCIL & ACCESSIBLE WAIKATO & $\begin{array}{l}\text { INCREASING TREND IN PERCEPTION OF THE DISTRICTS PROGRESS } \\
\text { TOWARDS THE ACHIEVEMENT OF ACCESSIBLE WAIKATO }\end{array}$ & COUNCIL FACILITIES & SOCIAL WELL-BEING \\
\hline 3276 & WAIKATO DISTRICT COUNCIL & ACTIVE WAIKATO & AN INCREASING TREND IN SATISFACTION WITH DISTRICT LIBRARIES & LIBRARY & SOCIAL WELL-BEING \\
\hline 3278 & WAIKATO DISTRICT COUNCIL & ACTIVE WAIKATO & $\begin{array}{l}\text { AN INCREASING TREND IN PARTICIPATION LEVELS IN RECREATION AND } \\
\text { SPORTING ACTIVITIES }\end{array}$ & SPORTS & SOCIAL WELL-BEING \\
\hline 3279 & WAIKATO DISTRICT COUNCIL & ACTIVE WAIKATO & $\begin{array}{l}\text { AN INCREASING TREND IN PERCEPTION OF THE DISTRICTS PROGRESS } \\
\text { TOWARDS THE ACHIEVEMENT OF ACTIVE WAIKATO }\end{array}$ & PHYSICAL ACTIVITY & SOCIAL WELL-BEING \\
\hline
\end{tabular}


Appendix A: List of Community Well-beings Outcomes and Measures by Councils

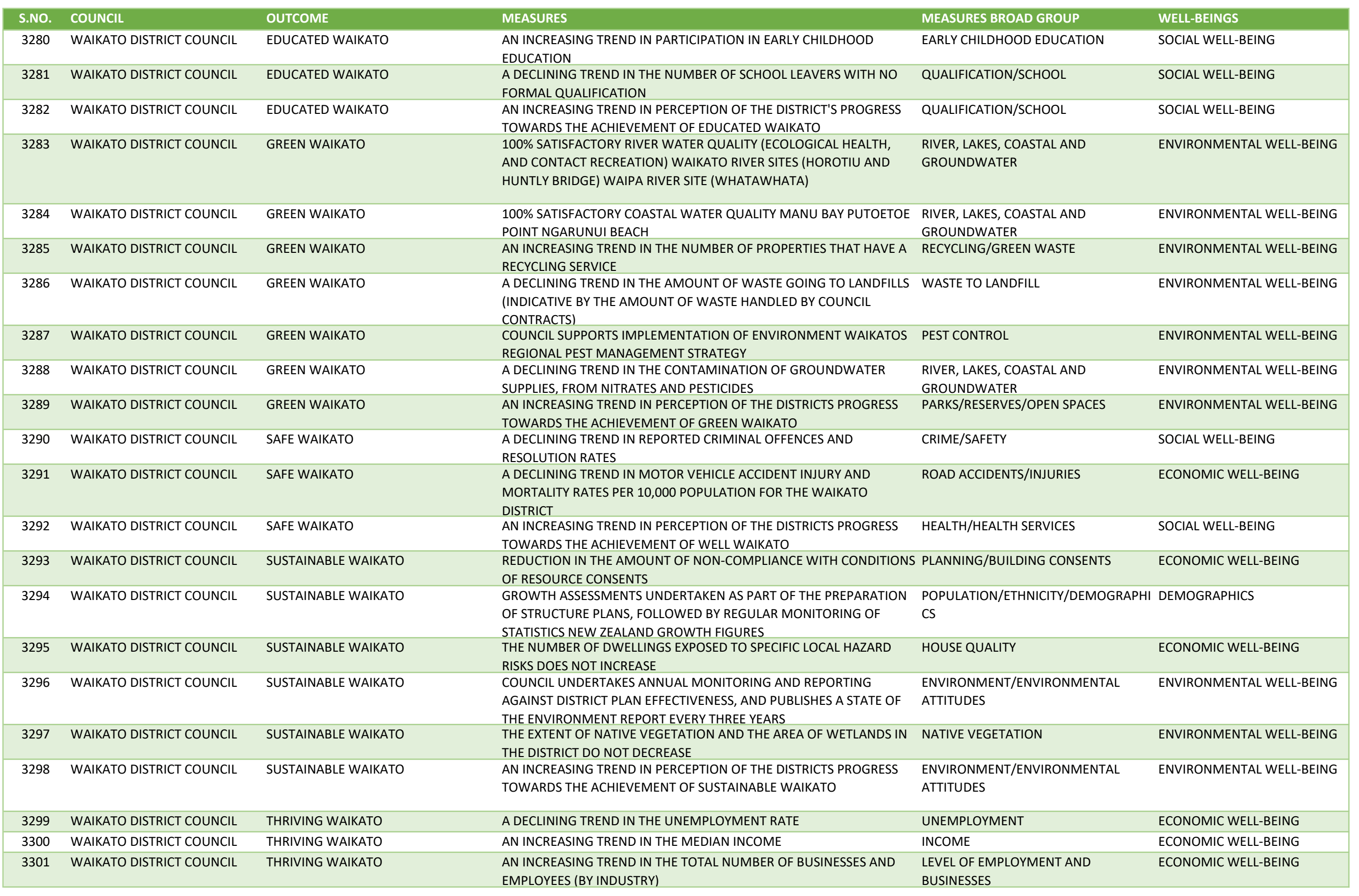


Appendix A: List of Community Well-beings Outcomes and Measures by Councils

\begin{tabular}{|c|c|c|c|c|c|}
\hline S.NO. & COUNCIL & OUTCOME & MEASURES & MEASURES BROAD GROUP & WELL-BEINGS \\
\hline 3302 & WAIKATO DISTRICT COUNCIL & THRIVING WAIKATO & $\begin{array}{l}\text { AN INCREASING TREND IN OCCUPANCY RATES IN COMMERCIAL } \\
\text { ACCOMMODATION WITHIN THE DISTRICT, EVIDENCED BY AN INCREASE }\end{array}$ & TOURISM & ECONOMIC WELL-BEING \\
\hline 3303 & WAIKATO DISTRICT COUNCIL & THRIVING WAIKATO & $\begin{array}{l}\text { AN INCREASING TREND IN PERCEPTION OF THE DISTRICTS PROGRESS } \\
\text { TOWARDS THE ACHIEVEMENT OF THRIVING WAIKATO }\end{array}$ & ECONOMIC ACTIVITY & ECONOMIC WELL-BEING \\
\hline 3304 & WAIKATO DISTRICT COUNCIL & VIBRANT WAIKATO & $\begin{array}{l}\text { COUNCIL PROVIDES FUNDING FOR CULTURAL AND ARTS } \\
\text { EVENTS/ACTIVITIES/ORGANISATIONS }\end{array}$ & ARTS/CULTURE/MUSEUM/EVENTS & CULTURAL WELL-BEING \\
\hline 3305 & WAIKATO DISTRICT COUNCIL & VIBRANT WAIKATO & $\begin{array}{l}\text { A REDUCTION IN THE PROPORTION OF THOSE SITES DESTROYED THAT } \\
\text { ARE REGISTERED WITH THE HISTORIC PLACES TRUST, OR LISTED AS } \\
\text { HERITAGE ITEMS IN THE DISTRICT PLAN }\end{array}$ & HERITAGE/HISTORIC & CULTURAL WELL-BEING \\
\hline 3306 & WAIKATO DISTRICT COUNCIL & VIBRANT WAIKATO & $\begin{array}{l}\text { AN INCREASING TREND IN THE NUMBERS OF VISITORS TO GALLERIES } \\
\text { AND MUSEUMS THAT ARE SUPPORTED BY COUNCIL }\end{array}$ & TOURISM & ECONOMIC WELL-BEING \\
\hline 3307 & WAIKATO DISTRICT COUNCIL & VIBRANT WAIKATO & $\begin{array}{l}\text { AN INCREASING TREND IN THE COMMUNITYS AWARENESS OF ARTS } \\
\text { FACILITIES IN THE DISTRICT }\end{array}$ & ARTS/CULTURE/MUSEUM/EVENTS & CULTURAL WELL-BEING \\
\hline 3308 & WAIKATO DISTRICT COUNCIL & VIBRANT WAIKATO & $\begin{array}{l}\text { AN INCREASING TREND IN PERCEPTION OF THE DISTRICTS PROGRESS } \\
\text { TOWARDS THE ACHIEVEMENT OF VIBRANT WAIKATO }\end{array}$ & COMMUNITY DEVELOPMENT & SOCIAL WELL-BEING \\
\hline 3309 & WAIKATO DISTRICT COUNCIL & WELL WAIKATO & A DECLINING TREND IN AVOIDABLE HOSPITALISATIONS & HOSPITALISATIONS & ECONOMIC WELL-BEING \\
\hline 3310 & WAIKATO DISTRICT COUNCIL & WELL WAIKATO & A DECLINING TREND IN THE PREVALENCE OF CIGARETTE SMOKING & HEALTH/HEALTH SERVICES & SOCIAL WELL-BEING \\
\hline 3311 & WAIKATO DISTRICT COUNCIL & WELL WAIKATO & 100\% COMPLIANCE WITH NZ DRINKING WATER STANDARDS 2005 & DRINKING WATER & ENVIRONMENTAL WELL-BEING \\
\hline 3312 & WAIKATO DISTRICT COUNCIL & WELL WAIKATO & $\begin{array}{l}\text { AN INCREASING TREND IN SATISFACTION LEVELS OF WATER SERVICES } \\
\text { AS PER WDC SATISFACTION SURVEY }\end{array}$ & $\begin{array}{l}\text { RIVER, LAKES, COASTAL AND } \\
\text { GROUNDWATER }\end{array}$ & ENVIRONMENTAL WELL-BEING \\
\hline 3313 & WAIKATO DISTRICT COUNCIL & WELL WAIKATO & $\begin{array}{l}\text { AN INCREASING TREND IN SATISFACTION LEVELS OF WASTEWATER } \\
\text { SERVICES, AS PER WDC SATISFACTION SURVEY }\end{array}$ & SEWERAGE/WASTEWATER & ENVIRONMENTAL WELL-BEING \\
\hline 3314 & WAIKATO DISTRICT COUNCIL & WELL WAIKATO & $\begin{array}{l}\text { AN INCREASING TREND IN PERCEPTION OF THE DISTRICTS PROGRESS } \\
\text { TOWARDS THE ACHIEVEMENT OF WELL WAIKATO }\end{array}$ & HEALTH/HEALTH SERVICES & SOCIAL WELL-BEING \\
\hline 3315 & WAIKATO REGIONAL COUNCIL & SUSTAINABLE ENVIRONMENT & RIVER WATER QUALITY FOR ECOLOGICAL HEALTH & $\begin{array}{l}\text { RIVER, LAKES, COASTAL AND } \\
\text { GROUNDWATER }\end{array}$ & ENVIRONMENTAL WELL-BEING \\
\hline 3316 & WAIKATO REGIONAL COUNCIL & SUSTAINABLE ENVIRONMENT & RIVER WATER QUALITY FOR RECREATION & $\begin{array}{l}\text { RIVER, LAKES, COASTAL AND } \\
\text { GROUNDWATER }\end{array}$ & ENVIRONMENTAL WELL-BEING \\
\hline 3317 & WAIKATO REGIONAL COUNCIL & SUSTAINABLE ENVIRONMENT & LAKES WATER QUALITY FOR ECOLOGICAL HEALTH & $\begin{array}{l}\text { RIVER, LAKES, COASTAL AND } \\
\text { GROUNDWATER }\end{array}$ & ENVIRONMENTAL WELL-BEING \\
\hline 3318 & WAIKATO REGIONAL COUNCIL & SUSTAINABLE ENVIRONMENT & LAKES WATER QUALITY FOR CONTACT RECREATION & $\begin{array}{l}\text { RIVER, LAKES, COASTAL AND } \\
\text { GROUNDWATER }\end{array}$ & ENVIRONMENTAL WELL-BEING \\
\hline 3319 & WAIKATO REGIONAL COUNCIL & SUSTAINABLE ENVIRONMENT & LAND USE & PLANNING/BUILDING CONSENTS & ECONOMIC WELL-BEING \\
\hline 3320 & WAIKATO REGIONAL COUNCIL & SUSTAINABLE ENVIRONMENT & URBAN AIR QUALITY & AIR QUALITY & ENVIRONMENTAL WELL-BEING \\
\hline 3321 & WAIKATO REGIONAL COUNCIL & SUSTAINABLE ENVIRONMENT & GROUNDWATER AVAILABILITY AND USE & $\begin{array}{l}\text { RIVER, LAKES, COASTAL AND } \\
\text { GROUNDWATER }\end{array}$ & ENVIRONMENTAL WELL-BEING \\
\hline 3322 & WAIKATO REGIONAL COUNCIL & SUSTAINABLE ENVIRONMENT & SURFACE WATER AVAILABILITY AND USE & $\begin{array}{l}\text { RIVER, LAKES, COASTAL AND } \\
\text { GROUNDWATER }\end{array}$ & ENVIRONMENTAL WELL-BEING \\
\hline 3323 & WAIKATO REGIONAL COUNCIL & SUSTAINABLE ENVIRONMENT & PROTECTION OF NATURAL HERITAGE AND LANDSCAPES & NATURAL FEATURES & ENVIRONMENTAL WELL-BEING \\
\hline 3324 & WAIKATO REGIONAL COUNCIL & SUSTAINABLE ENVIRONMENT & EXTENT OF NATIVE VEGETATION & NATIVE VEGETATION & ENVIRONMENTAL WELL-BEING \\
\hline 3327 & WAIKATO REGIONAL COUNCIL & SUSTAINABLE ENVIRONMENT & PEOPLES PERSONAL ENVIRONMENTAL ACTIONS & $\begin{array}{l}\text { ENVIRONMENT/ENVIRONMENTAL } \\
\text { ATTITUDES }\end{array}$ & ENVIRONMENTAL WELL-BEING \\
\hline 3328 & WAIKATO REGIONAL COUNCIL & SUSTAINABLE ENVIRONMENT & COASTAL WATER QUALITY FOR RECREATION & $\begin{array}{l}\text { RIVER, LAKES, COASTAL AND } \\
\text { GROUNDWATER }\end{array}$ & ENVIRONMENTAL WELL-BEING \\
\hline
\end{tabular}


Appendix A: List of Community Well-beings Outcomes and Measures by Councils

\begin{tabular}{|c|c|c|c|c|c|}
\hline S.NO. & COUNCIL & OUTCOME & MEASURES & MEASURES BROAD GROUP & WELL-BEINGS \\
\hline 3329 & WAIKATO REGIONAL COUNCIL & SUSTAINABLE ENVIRONMENT & PUBLIC ACCESS TO COAST (COASTLINE OWNERSHIP) & $\begin{array}{l}\text { RIVER, LAKES, COASTAL AND } \\
\text { GROUNDWATER }\end{array}$ & ENVIRONMENTAL WELL-BEING \\
\hline 3330 & WAIKATO REGIONAL COUNCIL & SUSTAINABLE ENVIRONMENT & RURAL SUBDIVISION & PLANNING/BUILDING CONSENTS & ECONOMIC WELL-BEING \\
\hline 3331 & WAIKATO REGIONAL COUNCIL & SUSTAINABLE ENVIRONMENT & STOCK DENSITY & AGRICULTURE/DAIRY & ECONOMIC WELL-BEING \\
\hline 3332 & WAIKATO REGIONAL COUNCIL & SUSTAINABLE ENVIRONMENT & TOTAL ENERGY CONSUMPTION & ENERGY USAGE & ENVIRONMENTAL WELL-BEING \\
\hline 3333 & WAIKATO REGIONAL COUNCIL & SUSTAINABLE ENVIRONMENT & GREENHOUSE GAS EMISSIONS & AIR QUALITY & ENVIRONMENTAL WELL-BEING \\
\hline 3334 & WAIKATO REGIONAL COUNCIL & SUSTAINABLE ENVIRONMENT & ENERGY EFFICIENCY & ENERGY USAGE & ENVIRONMENTAL WELL-BEING \\
\hline 3335 & WAIKATO REGIONAL COUNCIL & SUSTAINABLE ENVIRONMENT & WASTE TO LANDFILLS & WASTE TO LANDFILL & ENVIRONMENTAL WELL-BEING \\
\hline 3336 & WAIKATO REGIONAL COUNCIL & SUSTAINABLE ENVIRONMENT & PROPORTION OF RECYCLING & RECYCLING/GREEN WASTE & ENVIRONMENTAL WELL-BEING \\
\hline 3337 & WAIKATO REGIONAL COUNCIL & QUALITY OF LIFE & LIFE EXPECTANCY AT BIRTH & LIFE EXPECTANCY & DEMOGRAPHICS \\
\hline 3338 & WAIKATO REGIONAL COUNCIL & QUALITY OF LIFE & SOCIAL DEPRIVATION INDEX(*) & DEPRIVATION INDEX & SOCIAL WELL-BEING \\
\hline 3339 & WAIKATO REGIONAL COUNCIL & QUALITY OF LIFE & AVOIDABLE MORTALITY AND HOSPITALISATION RATES(*) & HOSPITALISATIONS & ECONOMIC WELL-BEING \\
\hline 3340 & WAIKATO REGIONAL COUNCIL & QUALITY OF LIFE & OVERALL QUALITY OF LIFE & QUALITY OF LIFE & SOCIAL WELL-BEING \\
\hline 3341 & WAIKATO REGIONAL COUNCIL & QUALITY OF LIFE & BARRIERS TO ACCESSING GENERAL HEALTH PRACTITIONERS & HEALTH/HEALTH SERVICES & SOCIAL WELL-BEING \\
\hline 3342 & WAIKATO REGIONAL COUNCIL & QUALITY OF LIFE & SCHOOL LEAVERS WITH NO FORMAL QUALIFICATION & QUALIFICATION/SCHOOL & SOCIAL WELL-BEING \\
\hline 3343 & WAIKATO REGIONAL COUNCIL & QUALITY OF LIFE & EDUCATIONAL ATTAINMENT OF THE ADULT POPULATION & QUALIFICATION/SCHOOL & SOCIAL WELL-BEING \\
\hline 3344 & WAIKATO REGIONAL COUNCIL & QUALITY OF LIFE & PARTICIPATION IN EARLY CHILDHOOD EDUCATION & EARLY CHILDHOOD EDUCATION & SOCIAL WELL-BEING \\
\hline 3345 & WAIKATO REGIONAL COUNCIL & QUALITY OF LIFE & ADULT AND COMMUNITY EDUCATION & QUALIFICATION/SCHOOL & SOCIAL WELL-BEING \\
\hline 3347 & WAIKATO REGIONAL COUNCIL & QUALITY OF LIFE & RENT TO INCOME RATIO & HOUSE AFFORDABILITY & ECONOMIC WELL-BEING \\
\hline 3348 & WAIKATO REGIONAL COUNCIL & QUALITY OF LIFE & HOUSING AFFORDABILITY & HOUSE AFFORDABILITY & ECONOMIC WELL-BEING \\
\hline 3349 & WAIKATO REGIONAL COUNCIL & QUALITY OF LIFE & HOME OWNERSHIP RATE & HOME OWNERSHIP & DEMOGRAPHICS \\
\hline 3350 & WAIKATO REGIONAL COUNCIL & QUALITY OF LIFE & HOUSEHOLD CROWDING & HOUSE AFFORDABILITY & ECONOMIC WELL-BEING \\
\hline 3351 & WAIKATO REGIONAL COUNCIL & QUALITY OF LIFE & PROXIMITY TO WORK, STUDY, RECREATION & RECREATIONAL FACILITIES & SOCIAL WELL-BEING \\
\hline 3352 & WAIKATO REGIONAL COUNCIL & QUALITY OF LIFE & $\begin{array}{l}\text { CRIMINAL VICTIMISATION RATES (OR REPORTED CRIMINAL OFFENCES } \\
\text { AND RESOLUTION RATES) }\end{array}$ & CRIME/SAFETY & SOCIAL WELL-BEING \\
\hline 3353 & WAIKATO REGIONAL COUNCIL & QUALITY OF LIFE & PERCEPTIONS OF SAFETY & CRIME/SAFETY & SOCIAL WELL-BEING \\
\hline 3354 & WAIKATO REGIONAL COUNCIL & QUALITY OF LIFE & ROAD TRAFFIC CRASHES AND CASULTIES & ROAD ACCIDENTS/INJURIES & ECONOMIC WELL-BEING \\
\hline 3355 & WAIKATO REGIONAL COUNCIL & QUALITY OF LIFE & UNPAID WORK & VOLUNTEER/UNPAID WORK & SOCIAL WELL-BEING \\
\hline 3356 & WAIKATO REGIONAL COUNCIL & QUALITY OF LIFE & PARTICIPATION IN SPORT AND ACTIVE LEISURE & SPORTS & SOCIAL WELL-BEING \\
\hline 3357 & WAIKATO REGIONAL COUNCIL & QUALITY OF LIFE & PARTICIPATION IN SOCIAL NETWORKS AND GROUPS & SOCIAL CONNECTION & SOCIAL WELL-BEING \\
\hline 3358 & WAIKATO REGIONAL COUNCIL & QUALITY OF LIFE & CONTACT BETWEEN YOUNG PEOPLE AND THEIR PARENTS & SOCIAL CONNECTION & SOCIAL WELL-BEING \\
\hline 3359 & WAIKATO REGIONAL COUNCIL & QUALITY OF LIFE & YOUTH AND OLDER PEOPLE'S ENGAGEMENT IN DECISION-MAKING & DECISION MAKING/CONSULTATION & SOCIAL WELL-BEING \\
\hline 3360 & WAIKATO REGIONAL COUNCIL & SUSTAINABLE ECONOMY & GENUINE PROGRESS INDICATOR (OR ECOLOGICAL FOOTPRINT) & $\begin{array}{l}\text { ENVIRONMENT/ENVIRONMENTAL } \\
\text { ATTITUDES }\end{array}$ & ENVIRONMENTAL WELL-BEING \\
\hline 3361 & WAIKATO REGIONAL COUNCIL & SUSTAINABLE ECONOMY & REGIONAL GDP & GROSS DOMESTIC PRODUCT (GDP) & ECONOMIC WELL-BEING \\
\hline 3364 & WAIKATO REGIONAL COUNCIL & SUSTAINABLE ECONOMY & NUMBER IF BUSINESSES AND EMPLOYEES BY INDUSTRY & $\begin{array}{l}\text { LEVEL OF EMPLOYMENT AND } \\
\text { BUSINESSES }\end{array}$ & ECONOMIC WELL-BEING \\
\hline 3365 & WAIKATO REGIONAL COUNCIL & SUSTAINABLE ECONOMY & BUILDING CONSENTS & PLANNING/BUILDING CONSENTS & ECONOMIC WELL-BEING \\
\hline 3366 & WAIKATO REGIONAL COUNCIL & SUSTAINABLE ECONOMY & DRINKING WATER QUALITY & DRINKING WATER & ENVIRONMENTAL WELL-BEING \\
\hline
\end{tabular}


Appendix A: List of Community Well-beings Outcomes and Measures by Councils

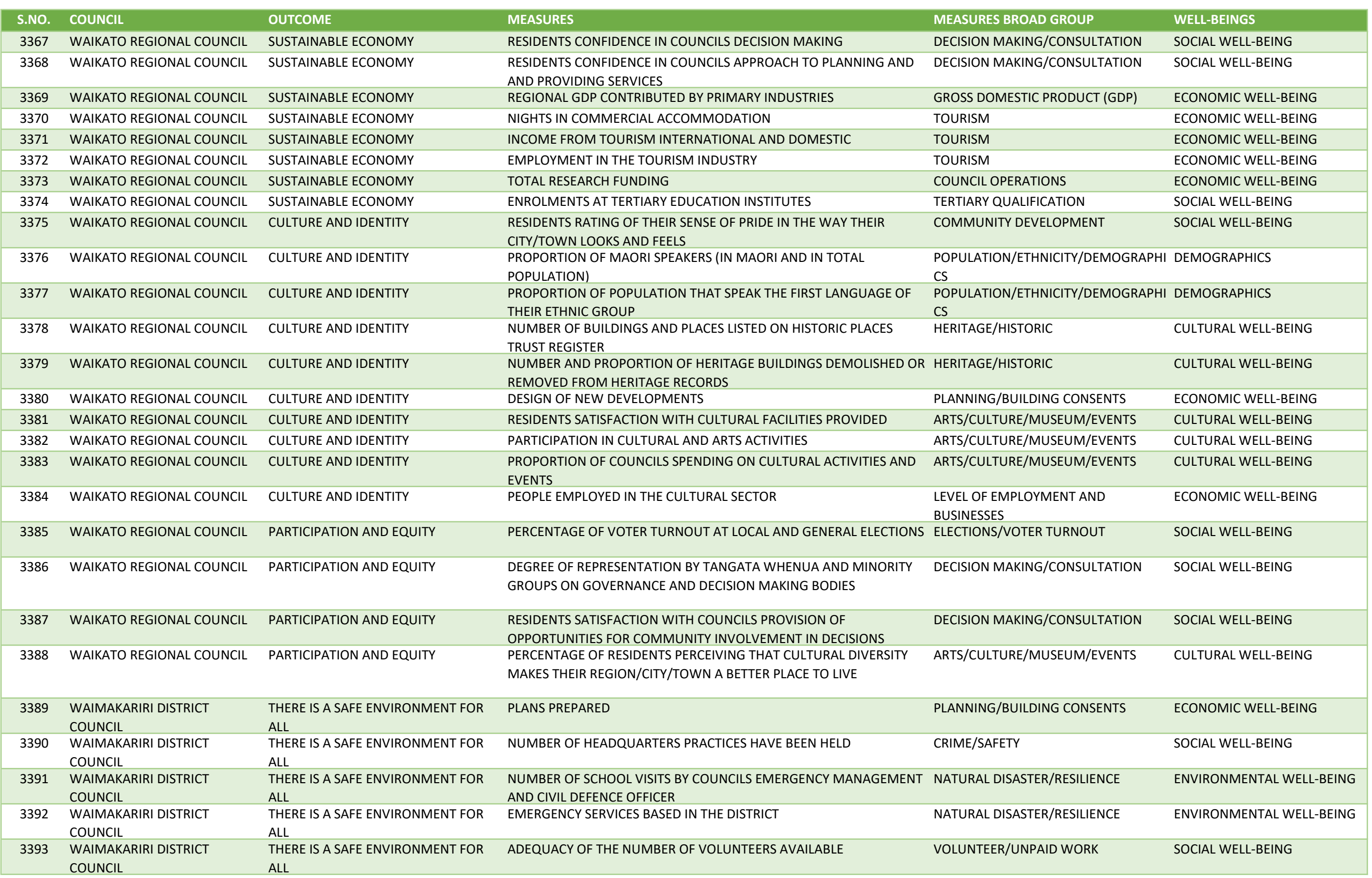




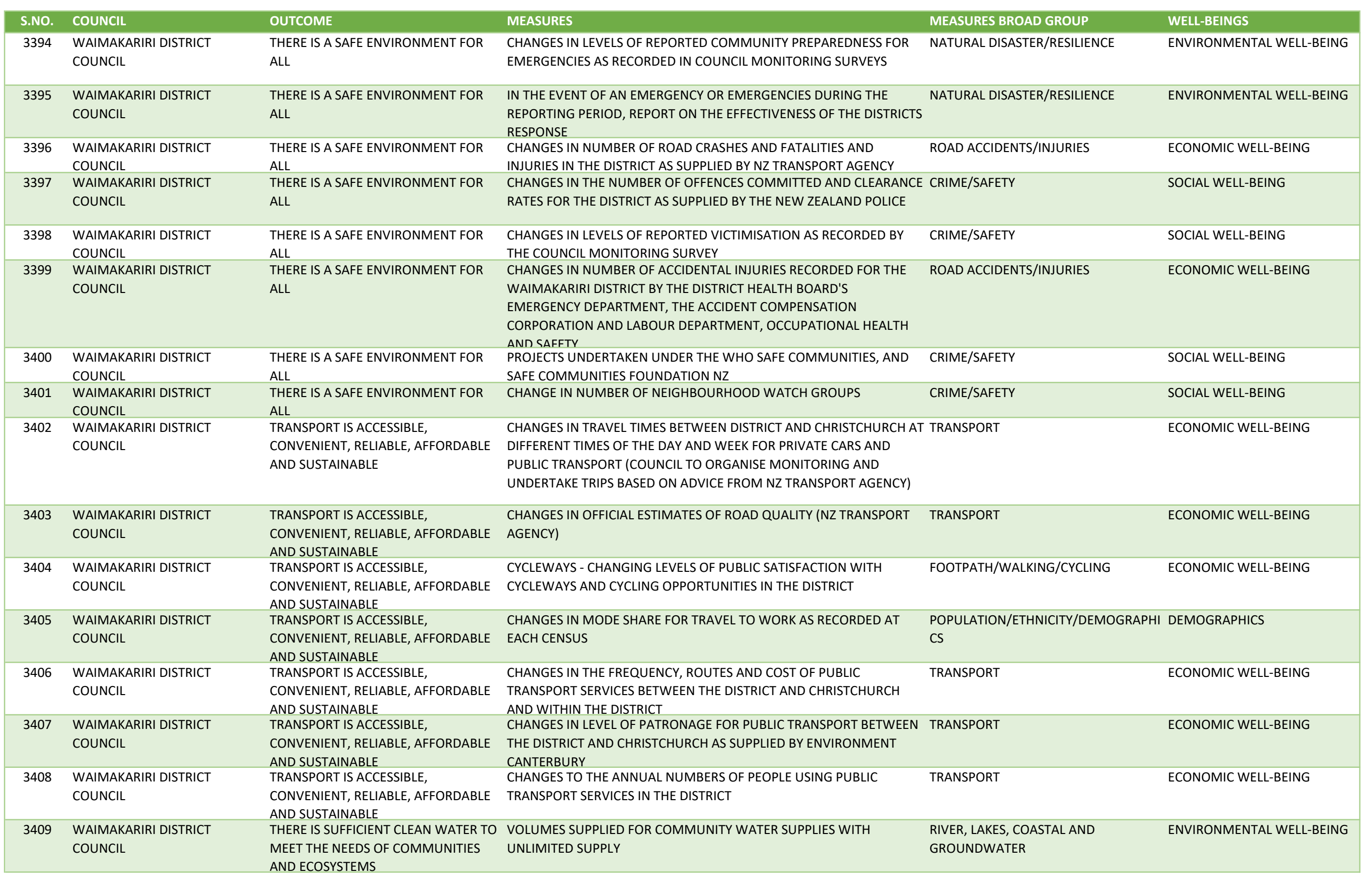




\begin{tabular}{|c|c|c|c|c|c|}
\hline S.NO. & COUNCIL & OUTCOME & MEASURES & MEASURES BROAD GROUP & WELL-BEINGS \\
\hline 3410 & $\begin{array}{l}\text { WAIMAKARIRI DISTRICT } \\
\text { COUNCIL }\end{array}$ & $\begin{array}{l}\text { THERE IS SUFFICIENT CLEAN WATER TO } \\
\text { MEET THE NEEDS OF COMMUNITIES } \\
\text { AND ECOSYSTEMS }\end{array}$ & $\begin{array}{l}\text { NUMBERS OF DAYS THAT CONSTRAINTS ARE APPLIED TO COMMUNITY } \\
\text { WATER IN A SUMMER SEASON }\end{array}$ & $\begin{array}{l}\text { RIVER, LAKES, COASTAL AND } \\
\text { GROUNDWATER }\end{array}$ & ENVIRONMENTAL WELL-BEING \\
\hline 3411 & $\begin{array}{l}\text { WAIMAKARIRI DISTRICT } \\
\text { COUNCIL }\end{array}$ & $\begin{array}{l}\text { THERE IS SUFFICIENT CLEAN WATER TO } \\
\text { MEET THE NEEDS OF COMMUNITIES } \\
\text { AND ECOSYSTEMS }\end{array}$ & $\begin{array}{l}\text { CHANGES IN TOTAL NUMBER OF WELLS AND VOLUME OF AUTHORISED } \\
\text { TAKES FROM GROUNDWATER FOR IRRIGATION }\end{array}$ & $\begin{array}{l}\text { RIVER, LAKES, COASTAL AND } \\
\text { GROUNDWATER }\end{array}$ & ENVIRONMENTAL WELL-BEING \\
\hline 3412 & $\begin{array}{l}\text { WAIMAKARIRI DISTRICT } \\
\text { COUNCIL }\end{array}$ & $\begin{array}{l}\text { THERE IS SUFFICIENT CLEAN WATER TO } \\
\text { MEET THE NEEDS OF COMMUNITIES } \\
\text { AND ECOSYSTEMS }\end{array}$ & $\begin{array}{l}\text { TRENDS IN GROUNDWATER LEVELS ON THE WAIMAKARIRI/ASHLEY } \\
\text { PLAIN }\end{array}$ & $\begin{array}{l}\text { RIVER, LAKES, COASTAL AND } \\
\text { GROUNDWATER }\end{array}$ & ENVIRONMENTAL WELL-BEING \\
\hline 3413 & $\begin{array}{l}\text { WAIMAKARIRI DISTRICT } \\
\text { COUNCIL }\end{array}$ & $\begin{array}{l}\text { THERE IS SUFFICIENT CLEAN WATER TO } \\
\text { MEET THE NEEDS OF COMMUNITIES } \\
\text { AND ECOSYSTEMS }\end{array}$ & $\begin{array}{l}\text { CHANGES IN KEY WATER QUALITY INDICATORS COMMUNITY WATER } \\
\text { SUPPLY WELLS BY THE COUNCIL, AND THE ENVIRONMENT } \\
\text { CANTERBURY MONITOR WELLS IN THE DISTRICT }\end{array}$ & $\begin{array}{l}\text { RIVER, LAKES, COASTAL AND } \\
\text { GROUNDWATER }\end{array}$ & ENVIRONMENTAL WELL-BEING \\
\hline 3414 & $\begin{array}{l}\text { WAIMAKARIRI DISTRICT } \\
\text { COUNCIL }\end{array}$ & $\begin{array}{l}\text { THERE IS SUFFICIENT CLEAN WATER TO } \\
\text { MEET THE NEEDS OF COMMUNITIES } \\
\text { AND ECOSYSTEMS }\end{array}$ & $\begin{array}{l}\text { CHANGES IN THE OVERALL HEALTH OF STREAMS AS MEASURED BY } \\
\text { AQUATIC BIODIVERSITY, STATE OF STREAM BED AND ABUNDANCE AND } \\
\text { DIVERSITY OF BENTHIC FAUNA }\end{array}$ & $\begin{array}{l}\text { ENVIRONMENT/ENVIRONMENTAL } \\
\text { ATTITUDES }\end{array}$ & ENVIRONMENTAL WELL-BEING \\
\hline 3415 & $\begin{array}{l}\text { WAIMAKARIRI DISTRICT } \\
\text { COUNCIL }\end{array}$ & $\begin{array}{l}\text { BUSINESSES IN THE DISTRICT ARE } \\
\text { DIVERSE, ADAPTABLE, AND GROWING }\end{array}$ & $\begin{array}{l}\text { CHANGES IN THE NUMBER OF BUSINESS (EXCLUDING FARMS) AND } \\
\text { EMPLOYEE COUNT FOR THESE BUSINESSES LOCATED IN THE DISTRICT } \\
\text { AS RECORDED BY STATISTICS NEW ZEALAND IN FEBRUARY EACH YEAR }\end{array}$ & $\begin{array}{l}\text { LEVEL OF EMPLOYMENT AND } \\
\text { BUSINESSES }\end{array}$ & ECONOMIC WELL-BEING \\
\hline 3416 & $\begin{array}{l}\text { WAIMAKARIRI DISTRICT } \\
\text { COUNCIL }\end{array}$ & $\begin{array}{l}\text { BUSINESSES IN THE DISTRICT ARE } \\
\text { DIVERSE, ADAPTABLE, AND GROWING }\end{array}$ & $\begin{array}{l}\text { CHANGES IN THE NUMBER OF PEOPLE REPORTING WORKING IN THE } \\
\text { DISTRICT AT EACH CENSUS, AND THE PERCENTAGE OF THE PEOPLE } \\
\text { WORKING IN THE DISTRICT WHO ALSO LIVE IN THE DISTRICT }\end{array}$ & $\begin{array}{l}\text { POPULATION/ETHNICITY/DEMOGRAPHI } \\
\text { CS }\end{array}$ & DEMOGRAPHICS \\
\hline 3417 & $\begin{array}{l}\text { WAIMAKARIRI DISTRICT } \\
\text { COUNCIL }\end{array}$ & $\begin{array}{l}\text { BUSINESSES IN THE DISTRICT ARE } \\
\text { DIVERSE, ADAPTABLE, AND GROWING }\end{array}$ & $\begin{array}{l}\text { CHANGES IN THE NUMBER OF BUSINESSES IN EACH INDUSTRIAL } \\
\text { CATEGORY AS RECORDED BY STATISTIC NEW ZEALAND }\end{array}$ & NUMBER OF BUSINESSES & ECONOMIC WELL-BEING \\
\hline 3418 & $\begin{array}{l}\text { WAIMAKARIRI DISTRICT } \\
\text { COUNCIL }\end{array}$ & $\begin{array}{l}\text { BUSINESSES IN THE DISTRICT ARE } \\
\text { DIVERSE, ADAPTABLE, AND GROWING }\end{array}$ & $\begin{array}{l}\text { CHANGES IN THE NUMBER OF BUSINESS NEW BUSINESSES AND } \\
\text { BUSINESSES THAT CEASE TRADING BASED IN THE DISTRICT }\end{array}$ & NUMBER OF BUSINESSES & ECONOMIC WELL-BEING \\
\hline 3419 & $\begin{array}{l}\text { WAIMAKARIRI DISTRICT } \\
\text { COUNCIL }\end{array}$ & $\begin{array}{l}\text { BUSINESSES IN THE DISTRICT ARE } \\
\text { DIVERSE, ADAPTABLE, AND GROWING }\end{array}$ & $\begin{array}{l}\text { CHANGES IN THE NUMBER OF COURSES BEING OFFERED BY } \\
\text { ENTERPRISE NORTH CANTERBURY AND OTHER TRAINERS }\end{array}$ & BUSINESS CONFIDENCE & ECONOMIC WELL-BEING \\
\hline 3420 & $\begin{array}{l}\text { WAIMAKARIRI DISTRICT } \\
\text { COUNCIL }\end{array}$ & $\begin{array}{l}\text { BUSINESSES IN THE DISTRICT ARE } \\
\text { DIVERSE, ADAPTABLE, AND GROWING }\end{array}$ & $\begin{array}{l}\text { CHANGES IN THE NUMBER OF PEOPLE ATTENDING COURSES IN THE } \\
\text { DISTRICT }\end{array}$ & QUALIFICATION/SCHOOL & SOCIAL WELL-BEING \\
\hline 3421 & $\begin{array}{l}\text { WAIMAKARIRI DISTRICT } \\
\text { COUNCIL }\end{array}$ & $\begin{array}{l}\text { BUSINESSES IN THE DISTRICT ARE } \\
\text { DIVERSE, ADAPTABLE, AND GROWING }\end{array}$ & $\begin{array}{l}\text { CHANGES IN THE NUMBER OF YOUNG PEOPLE ON APPRENTICESHIPS } \\
\text { WORKING IN DISTRICT BASED BUSINESSES }\end{array}$ & APPRENTICESHIPS FOR YOUNG PEOPLE & ECONOMIC WELL-BEING \\
\hline 3422 & $\begin{array}{l}\text { WAIMAKARIRI DISTRICT } \\
\text { COUNCIL }\end{array}$ & $\begin{array}{l}\text { CORE UTILITY SERVICES ARE PROVIDED } \\
\text { IN A TIMELY, SUSTAINABLE AND } \\
\text { AFFORDABLE MANNER }\end{array}$ & $\begin{array}{l}\text { CHANGES IN THE NUMBER OF BREACHES IN CONSENT CONDITIONS FOR } \\
\text { COMMUNITY SEWAGE AND STORMWATER DISCHARGES }\end{array}$ & $\begin{array}{l}\text { ENVIRONMENT/ENVIRONMENTAL } \\
\text { ATTITUDES }\end{array}$ & ENVIRONMENTAL WELL-BEING \\
\hline
\end{tabular}




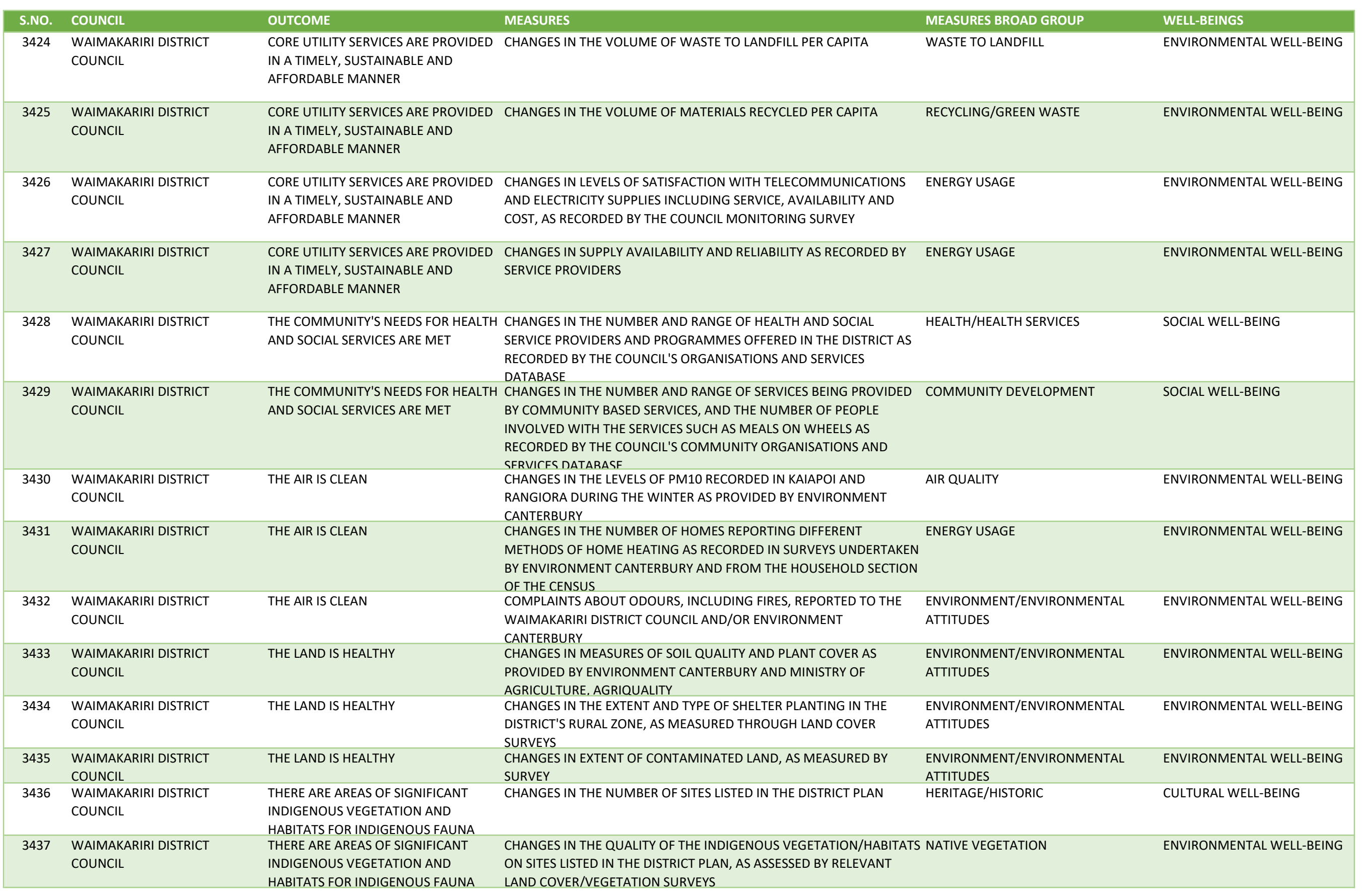




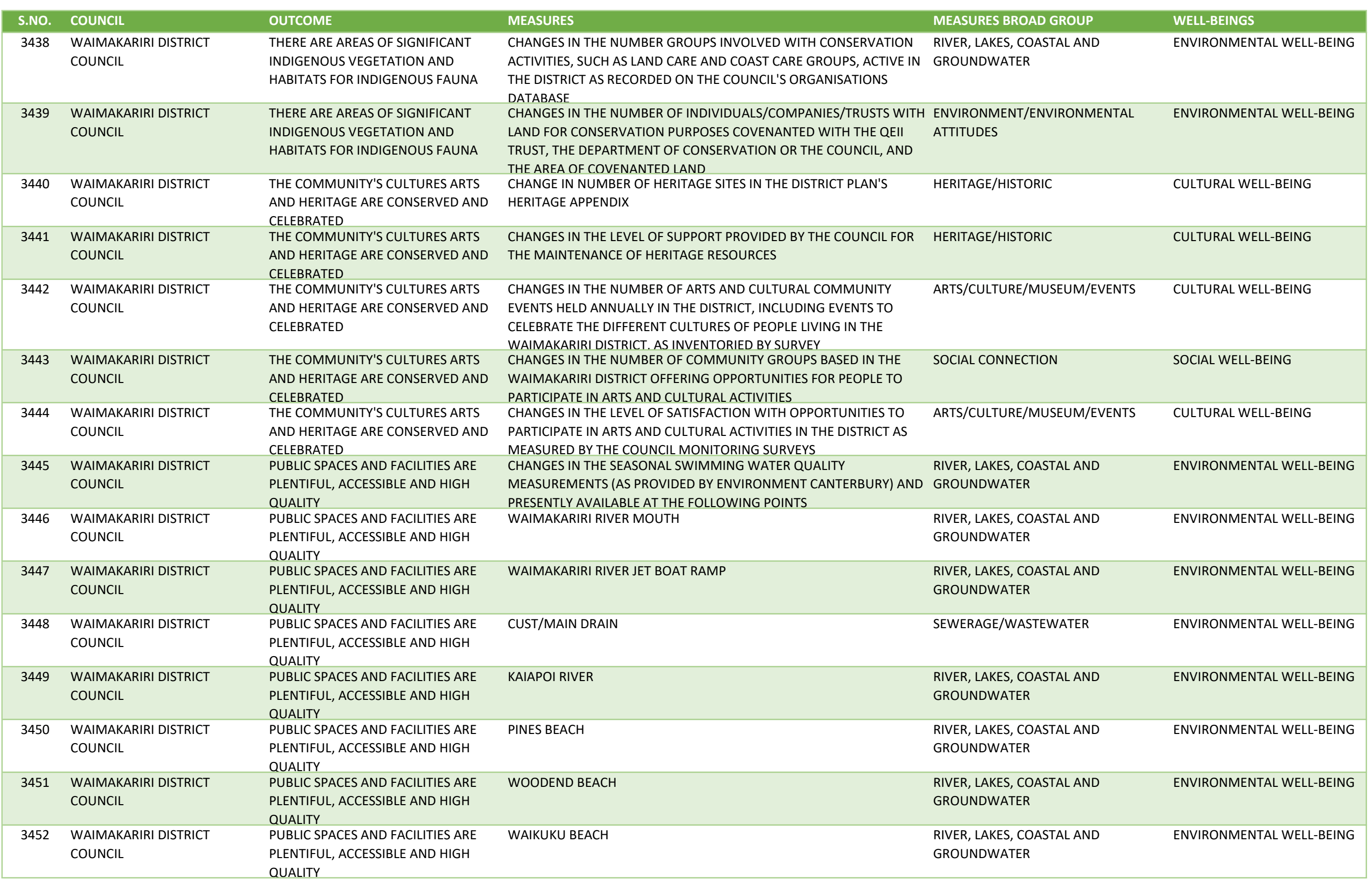


Appendix A: List of Community Well-beings Outcomes and Measures by Councils

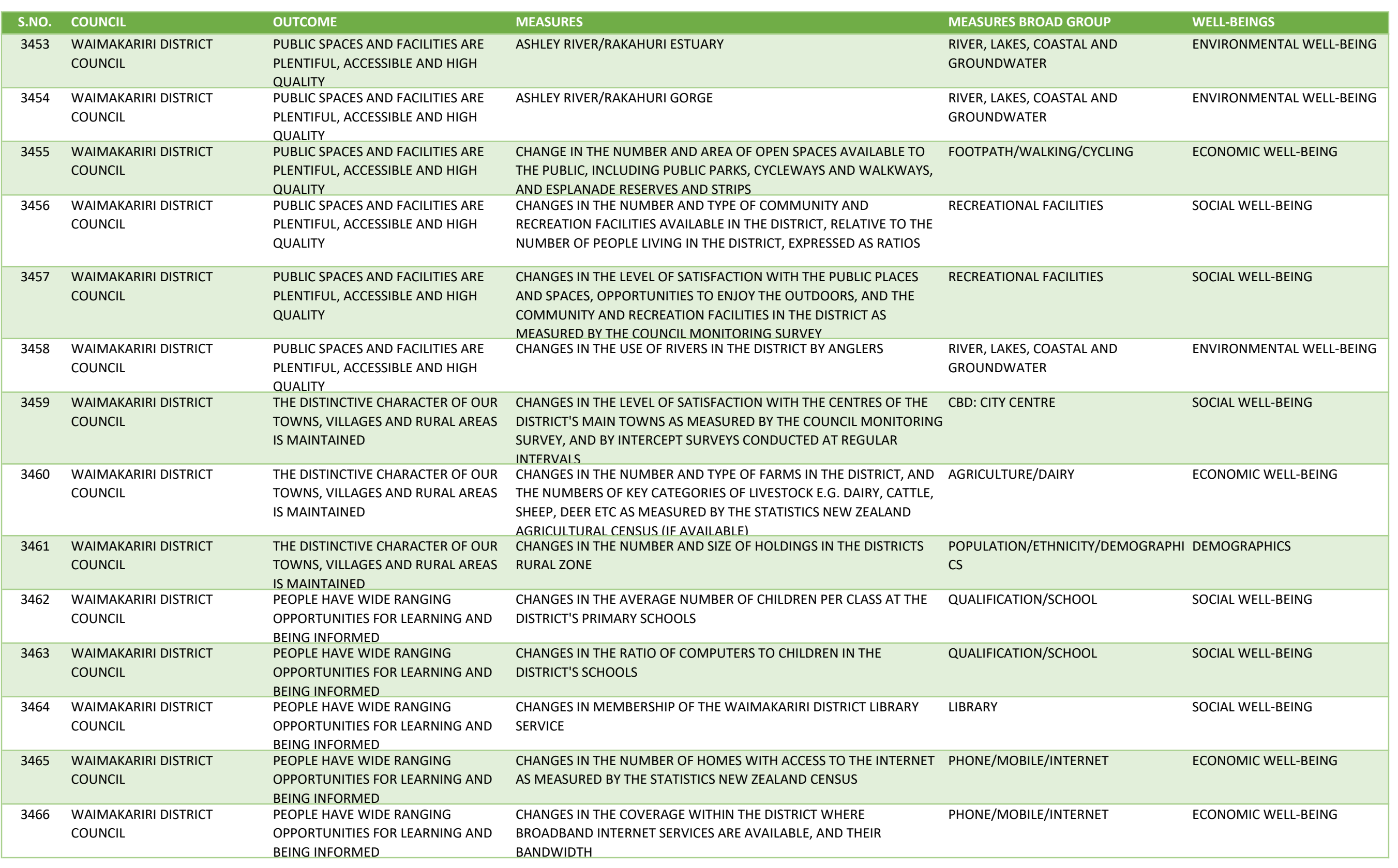




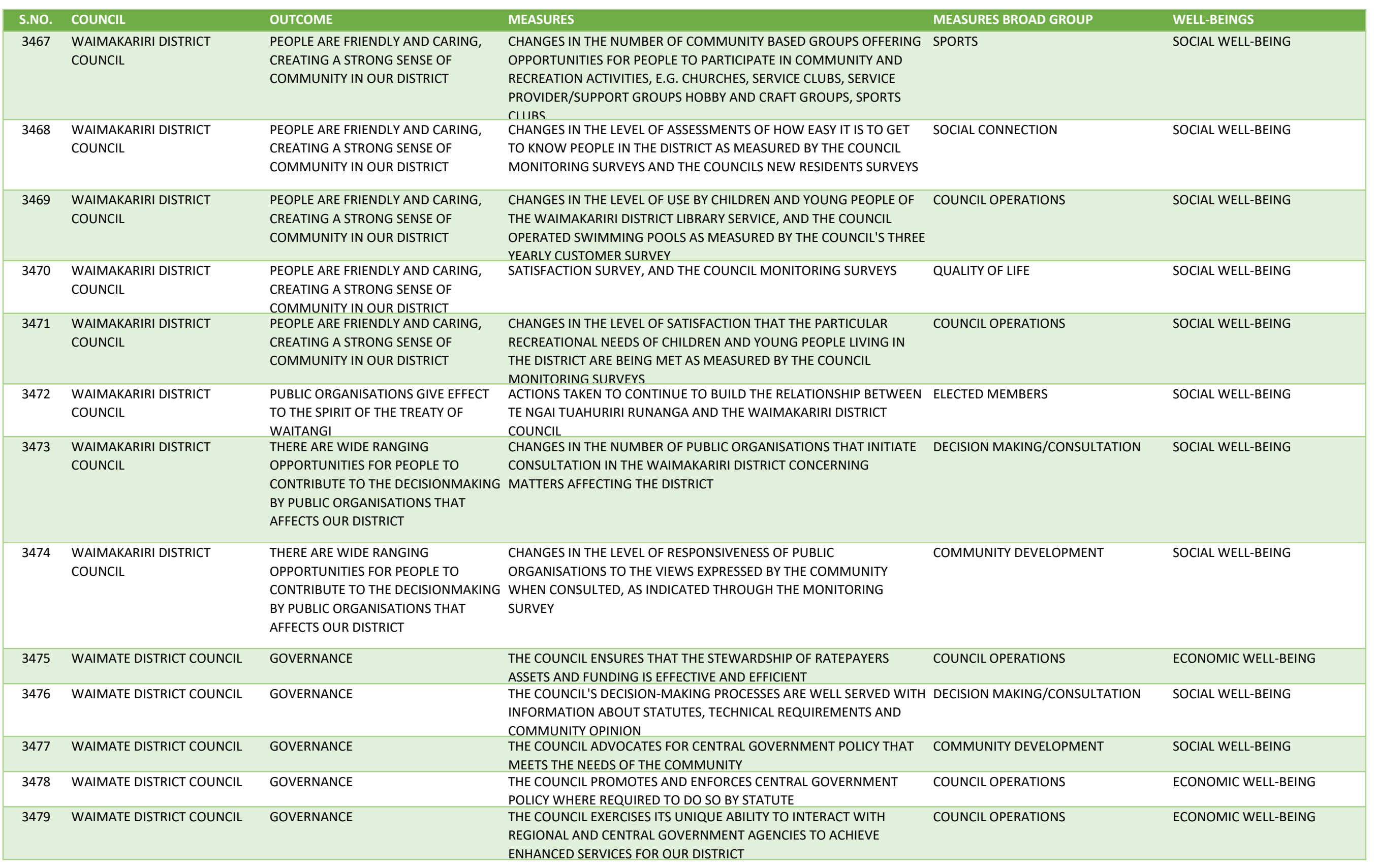




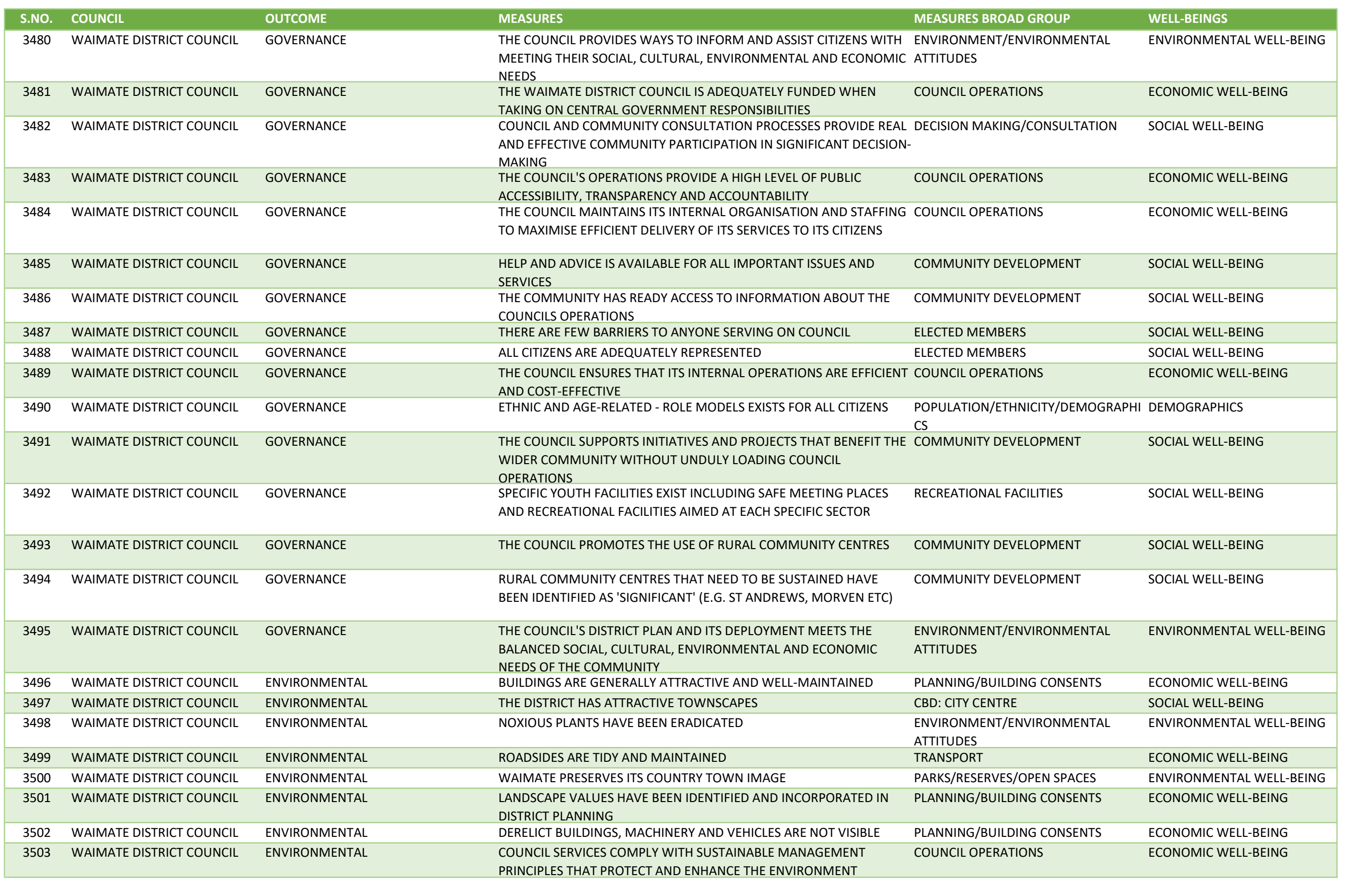


Appendix A: List of Community Well-beings Outcomes and Measures by Councils

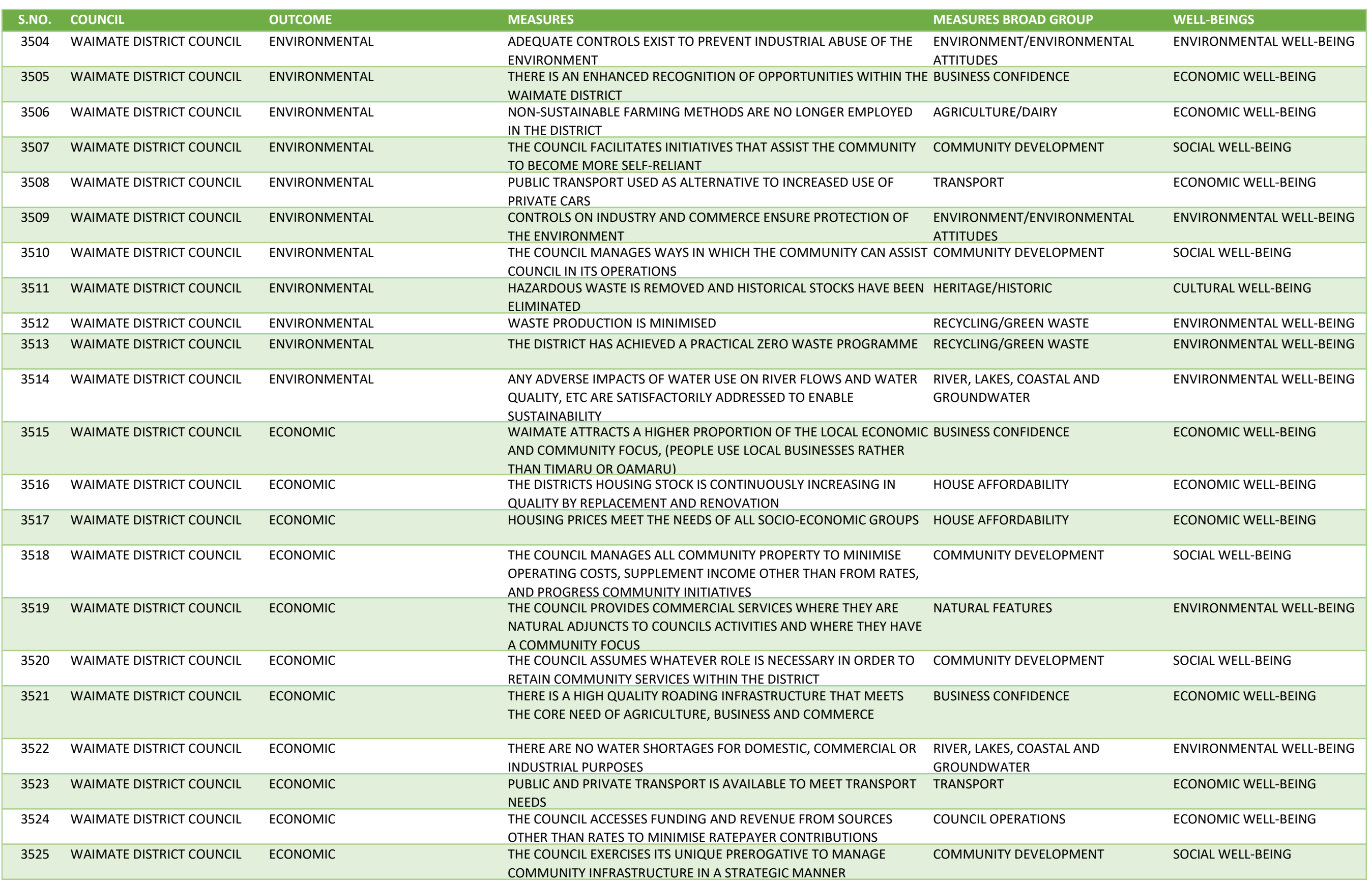


Appendix A: List of Community Well-beings Outcomes and Measures by Councils

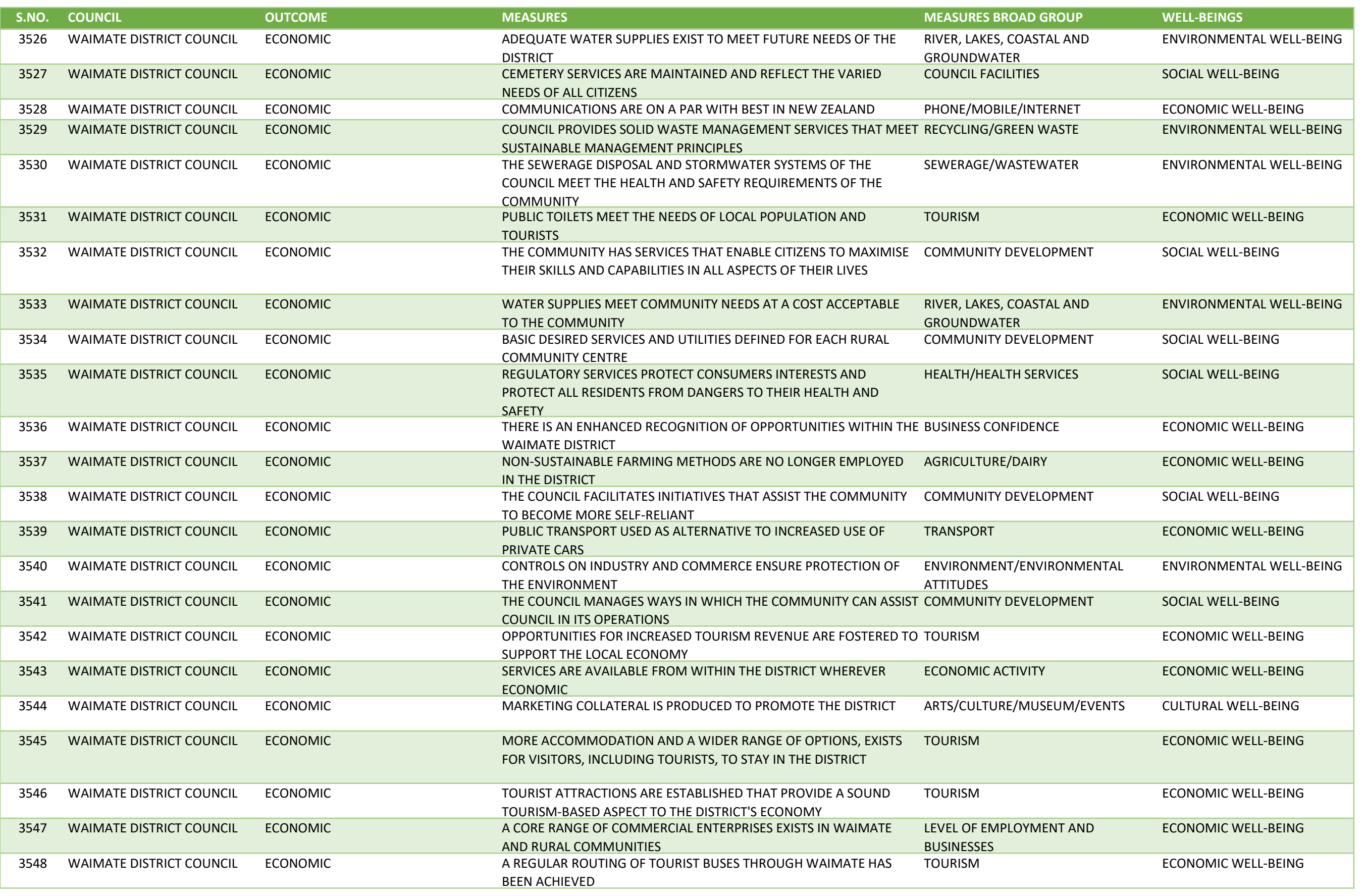


Appendix A: List of Community Well-beings Outcomes and Measures by Councils

\begin{tabular}{|c|c|c|c|c|c|}
\hline S.NO. & COUNCIL & OUTCOME & MEASURES & MEASURES BROAD GROUP & WELL-BEINGS \\
\hline 3549 & WAIMATE DISTRICT COUNCIL & ECONOMIC & $\begin{array}{l}\text { AN EFFECTIVE DISTRICT TOURIST THEME IS RECOGNISED AND ADOPTED } \\
\text { FOR WAIMATE DISTRICT }\end{array}$ & TOURISM & ECONOMIC WELL-BEING \\
\hline 3550 & WAIMATE DISTRICT COUNCIL & ECONOMIC & $\begin{array}{l}\text { TOURISM OPERATORS, RETAIL AND SERVICE BUSINESSES AND VISITOR- } \\
\text { INFORMATION PROVIDERS ARE ORGANISED FOR SEVEN DAY SERVICING }\end{array}$ & TOURISM & ECONOMIC WELL-BEING \\
\hline 3551 & WAIMATE DISTRICT COUNCIL & ECONOMIC & $\begin{array}{l}\text { WAIMATE DISTRICT COUNCILS INVESTMENT AND ECONOMIC } \\
\text { DEVELOPMENT POLICIES ARE STRUCTURED TO ASSIST SECONDARY } \\
\text { INDUSTRIES TO ESTABLISH WITHIN THE DISTRICT }\end{array}$ & ECONOMIC ACTIVITY & ECONOMIC WELL-BEING \\
\hline 3552 & WAIMATE DISTRICT COUNCIL & ECONOMIC & $\begin{array}{l}\text { THERE ARE MORE 'CLUSTERED/VERTICAL' - INDUSTRIES THAT HAVE } \\
\text { LONG-TERM VIABILITY }\end{array}$ & NUMBER OF BUSINESSES & ECONOMIC WELL-BEING \\
\hline 3553 & WAIMATE DISTRICT COUNCIL & ECONOMIC & $\begin{array}{l}\text { SUPPORTING INFRASTRUCTURE AND SERVICE INDUSTRIES DO NOT } \\
\text { PRESENT OBSTACLES FOR POTENTIAL RESIDENTS AND BUSINESSES } \\
\text { ENTERING OUR DISTRICT }\end{array}$ & BUSINESS CONFIDENCE & ECONOMIC WELL-BEING \\
\hline 3554 & WAIMATE DISTRICT COUNCIL & ECONOMIC & $\begin{array}{l}\text { LOCAL PORTS AND AIRPORT ARE EFFICIENT AND MEET THE NEEDS OF } \\
\text { THE LOCAL BUSINESS COMMUNITY }\end{array}$ & BUSINESS CONFIDENCE & ECONOMIC WELL-BEING \\
\hline 3555 & WAIMATE DISTRICT COUNCIL & ECONOMIC & ALL BASIC SERVICES TO SUPPORT AGRICULTURE EXIST LOCALLY & BUSINESS CONFIDENCE & ECONOMIC WELL-BEING \\
\hline 3556 & WAIMATE DISTRICT COUNCIL & ECONOMIC & $\begin{array}{l}\text { FUNDING MECHANISMS FOR COUNCIL OPERATIONS DO NOT UNDULY } \\
\text { LOAD ITS CITIZENS }\end{array}$ & COMMUNITY DEVELOPMENT & SOCIAL WELL-BEING \\
\hline 3557 & WAIMATE DISTRICT COUNCIL & ECONOMIC & $\begin{array}{l}\text { VARIED EMPLOYMENT OPPORTUNITIES EXIST TO SUIT DIFFERING } \\
\text { DEMANDS }\end{array}$ & $\begin{array}{l}\text { LEVEL OF EMPLOYMENT AND } \\
\text { BUSINESSES }\end{array}$ & ECONOMIC WELL-BEING \\
\hline 3559 & WAIMATE DISTRICT COUNCIL & SOCIAL & $\begin{array}{l}\text { THE COUNCIL MAINTAINS SERVICES WHICH PROTECT INDIVIDUALS AND } \\
\text { THE WIDER COMMUNITY FROM AVOIDABLE DANGERS TO THEIR } \\
\text { HEALTH }\end{array}$ & HEALTH/HEALTH SERVICES & SOCIAL WELL-BEING \\
\hline 3560 & WAIMATE DISTRICT COUNCIL & SOCIAL & $\begin{array}{l}\text { ACCESS/TRANSPORT TO HOSPITAL AND SPECIALIST SERVICES MEETS } \\
\text { THE NEEDS OF ALL CITIZENS }\end{array}$ & HOSPITALISATIONS & ECONOMIC WELL-BEING \\
\hline 3561 & WAIMATE DISTRICT COUNCIL & SOCIAL & $\begin{array}{l}\text { WAIMATE HAS APPROPRIATE ACCESS TO ACCIDENT AND EMERGENCY } \\
\text { FACILITIES }\end{array}$ & HOSPITALISATIONS & ECONOMIC WELL-BEING \\
\hline 3562 & WAIMATE DISTRICT COUNCIL & SOCIAL & $\begin{array}{l}\text { OVERALL MEDICAL FACILITIES REGION-WIDE, INCLUDING TIMARU } \\
\text { HOSPITAL MEET OUR NEEDS }\end{array}$ & HOSPITALISATIONS & ECONOMIC WELL-BEING \\
\hline 3563 & WAIMATE DISTRICT COUNCIL & SOCIAL & $\begin{array}{l}\text { TERTIARY EDUCATION PROVIDERS SUCH AS POLYTECHNICS PROVIDE } \\
\text { APPROPRIATE SPECIALISED AND GENERAL COURSES WITHIN THE } \\
\text { DISTRICT COURSES AVAILABLE TO COVER STRATEGIC ECONOMIC } \\
\text { DEVELOPMENT SUBJECTS EG FORESTRY }\end{array}$ & ECONOMIC ACTIVITY & ECONOMIC WELL-BEING \\
\hline 3564 & WAIMATE DISTRICT COUNCIL & SOCIAL & $\begin{array}{l}\text { THE WAIMATE HIGH SCHOOL PROVIDES A COMPREHENSIVE SET OF } \\
\text { COURSES AT ALL AGE LEVELS }\end{array}$ & QUALIFICATION/SCHOOL & SOCIAL WELL-BEING \\
\hline 3565 & WAIMATE DISTRICT COUNCIL & SOCIAL & $\begin{array}{l}\text { LIBRARY FACILITIES PROVIDE KEY RESOURCES FOR } \\
\text { RECREATION/EDUCATION DEVELOPMENT }\end{array}$ & LIBRARY & SOCIAL WELL-BEING \\
\hline 3566 & WAIMATE DISTRICT COUNCIL & SOCIAL & $\begin{array}{l}\text { EDUCATIONAL FACILITIES FOR AGRICULTURAL COURSES ARE IN PLACE } \\
\text { FOR ALL REQUIREMENTS }\end{array}$ & QUALIFICATION/SCHOOL & SOCIAL WELL-BEING \\
\hline 3567 & WAIMATE DISTRICT COUNCIL & SOCIAL & $\begin{array}{l}\text { CHOICES FOR SECONDARY EDUCATION ARE AVAILABLE WITHIN OUR } \\
\text { DISTRICT INCLUDING HOME SCHOOLING }\end{array}$ & QUALIFICATION/SCHOOL & SOCIAL WELL-BEING \\
\hline 3569 & WAIMATE DISTRICT COUNCIL & SOCIAL & $\begin{array}{l}\text { EDUCATIONAL FACILITIES ACCEPT THE NEED FOR MULTI-CULTURAL } \\
\text { EDUCATION AND TRAINING }\end{array}$ & QUALIFICATION/SCHOOL & SOCIAL WELL-BEING \\
\hline
\end{tabular}




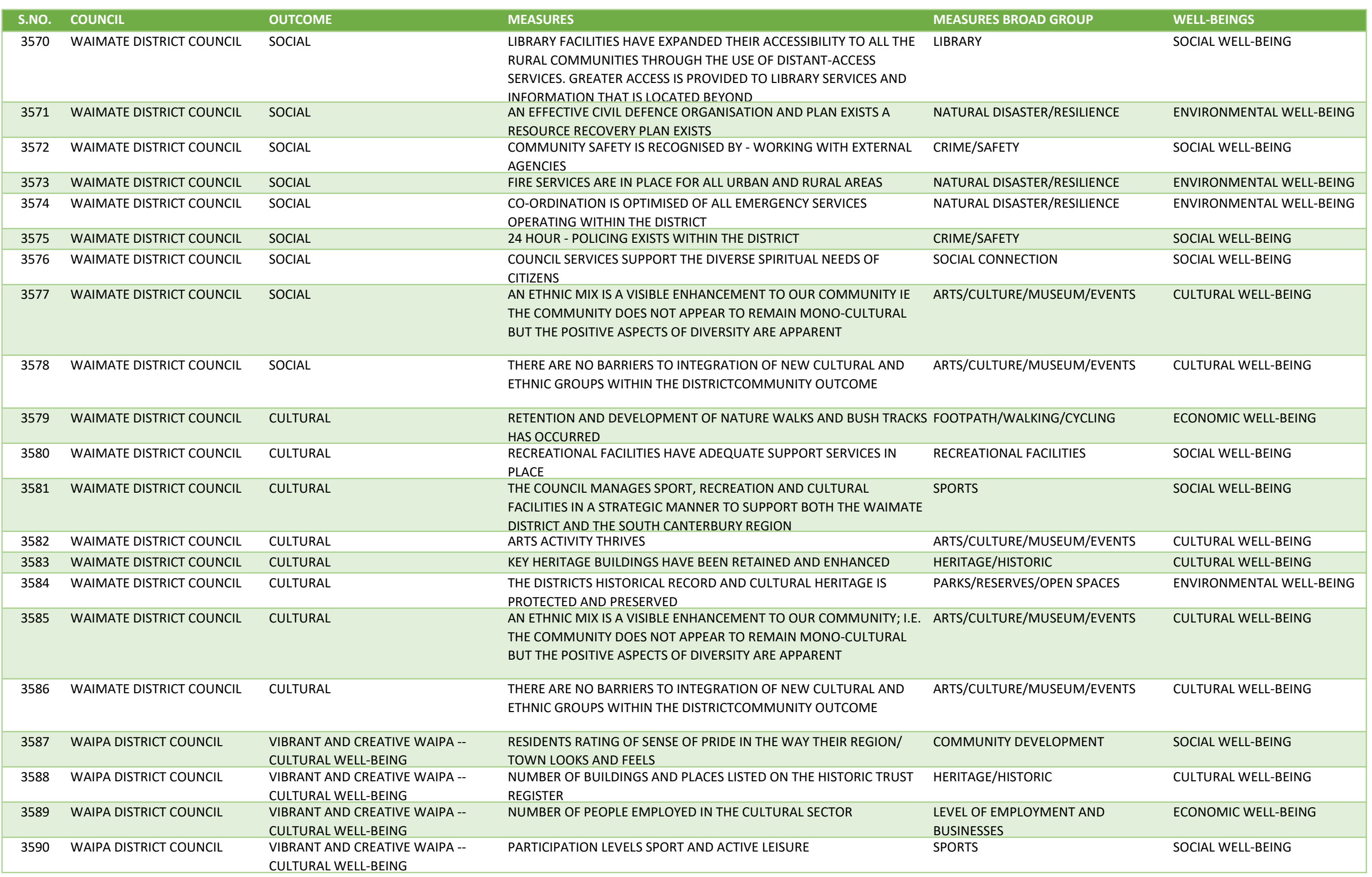


Appendix A: List of Community Well-beings Outcomes and Measures by Councils

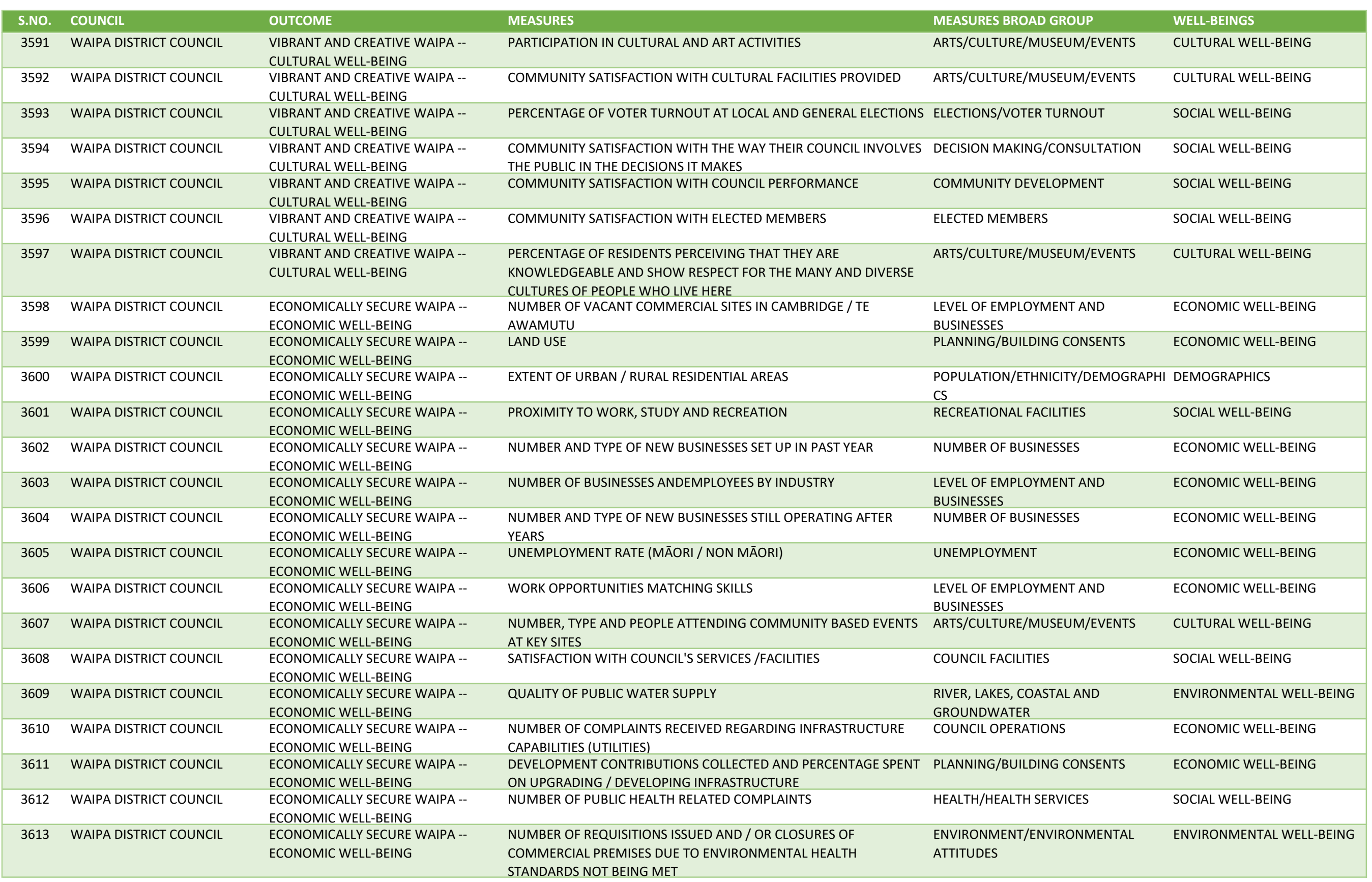


Appendix A: List of Community Well-beings Outcomes and Measures by Councils

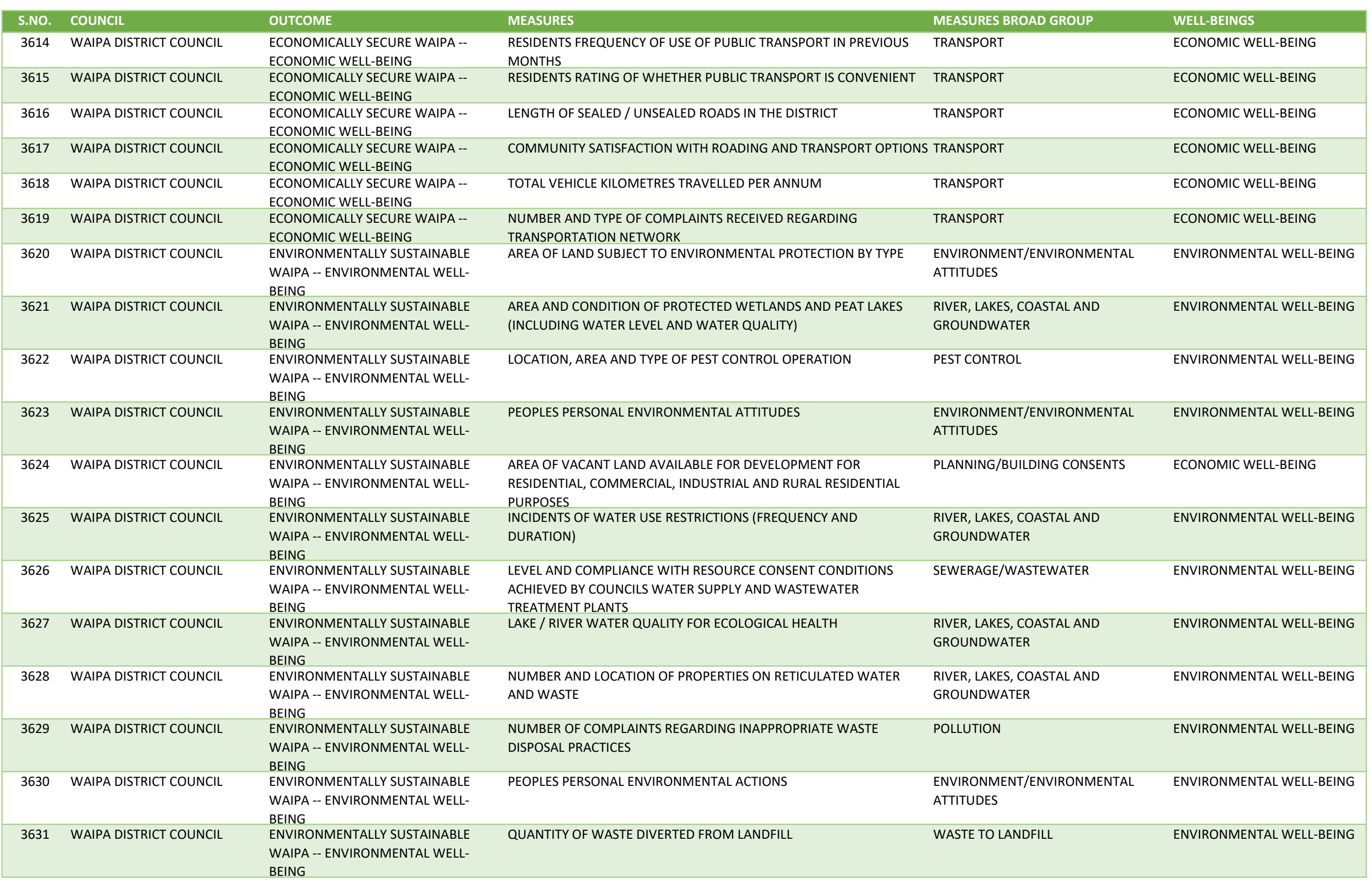


Appendix A: List of Community Well-beings Outcomes and Measures by Councils

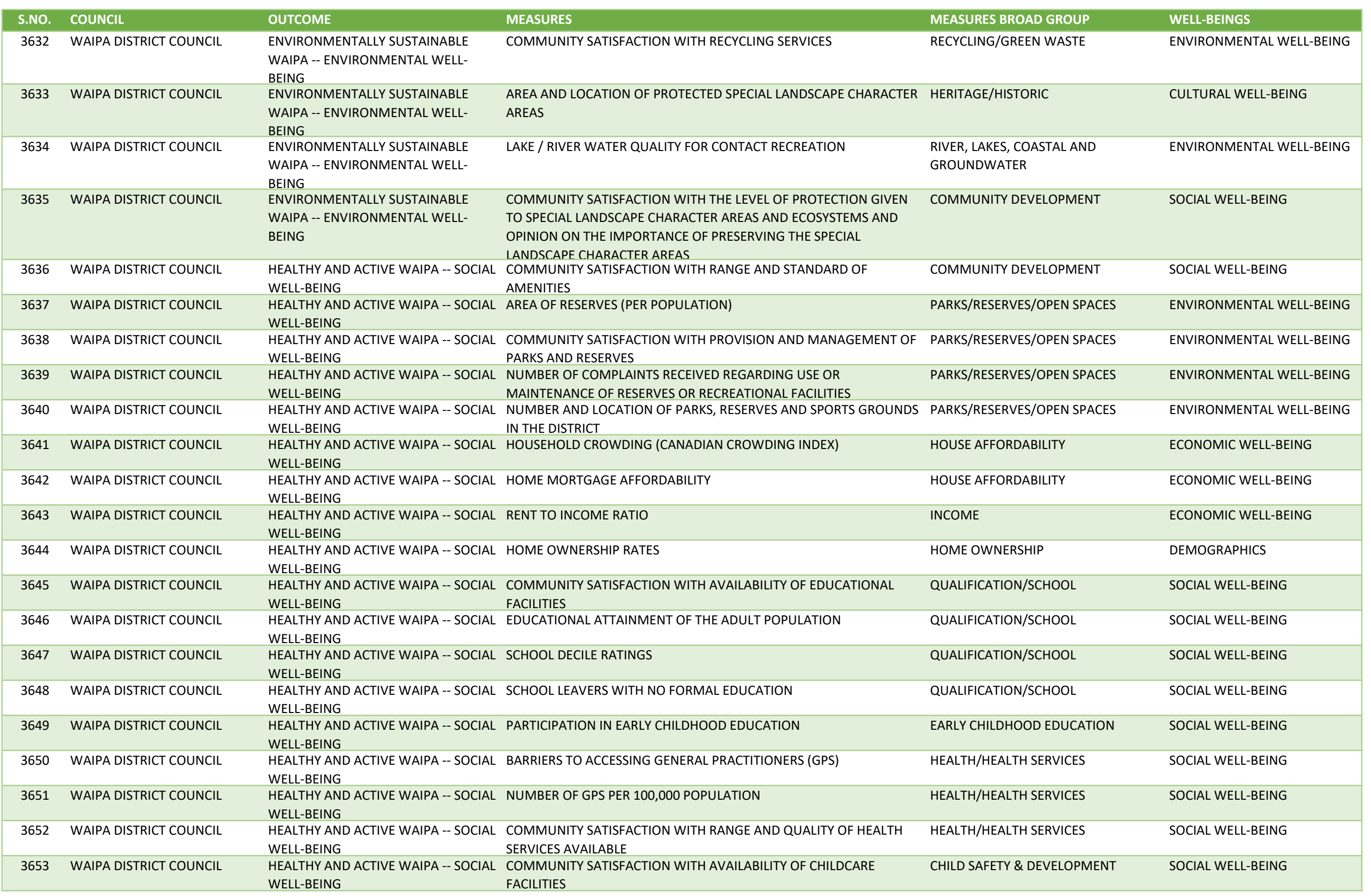


Appendix A: List of Community Well-beings Outcomes and Measures by Councils

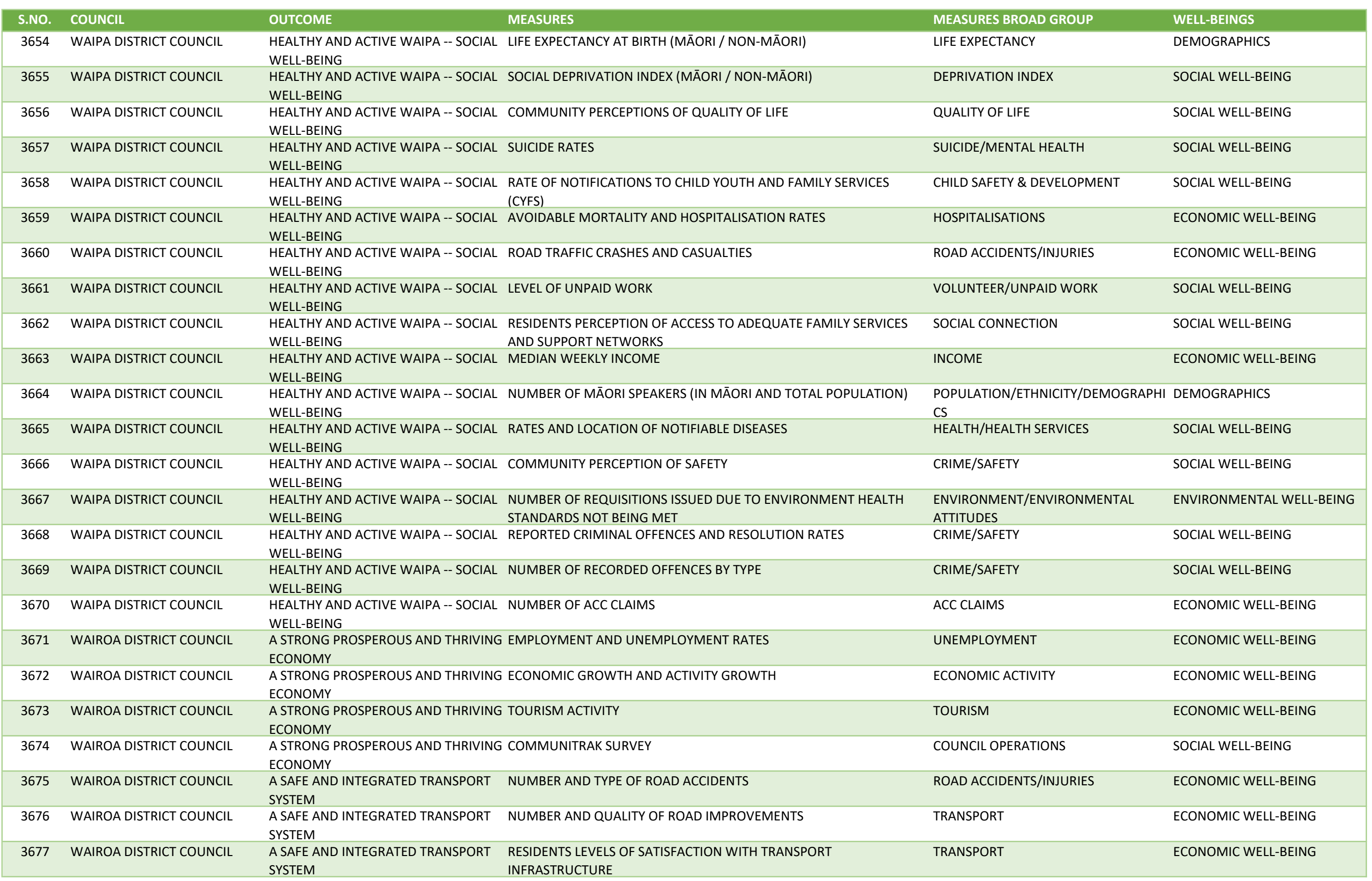


Appendix A: List of Community Well-beings Outcomes and Measures by Councils

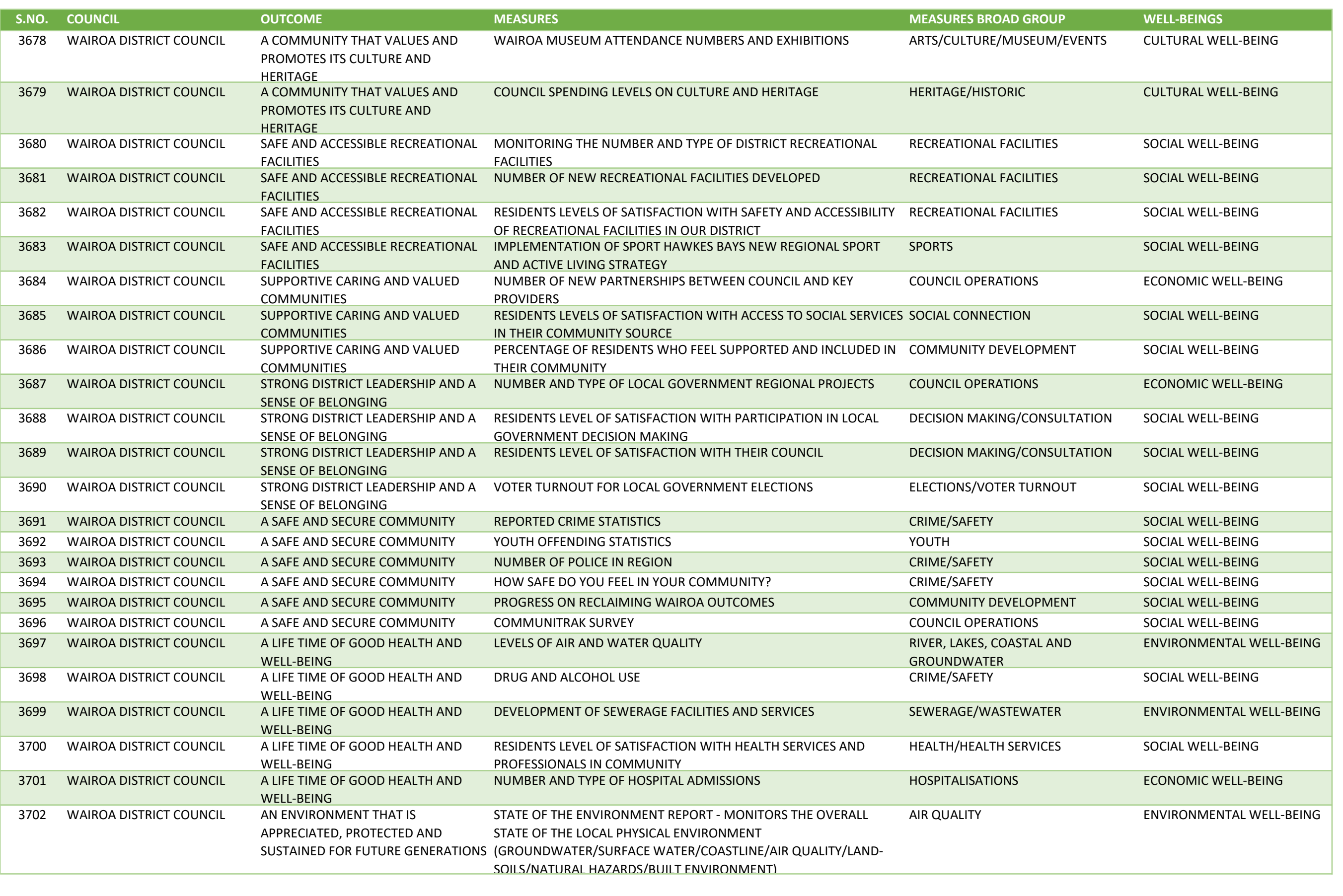




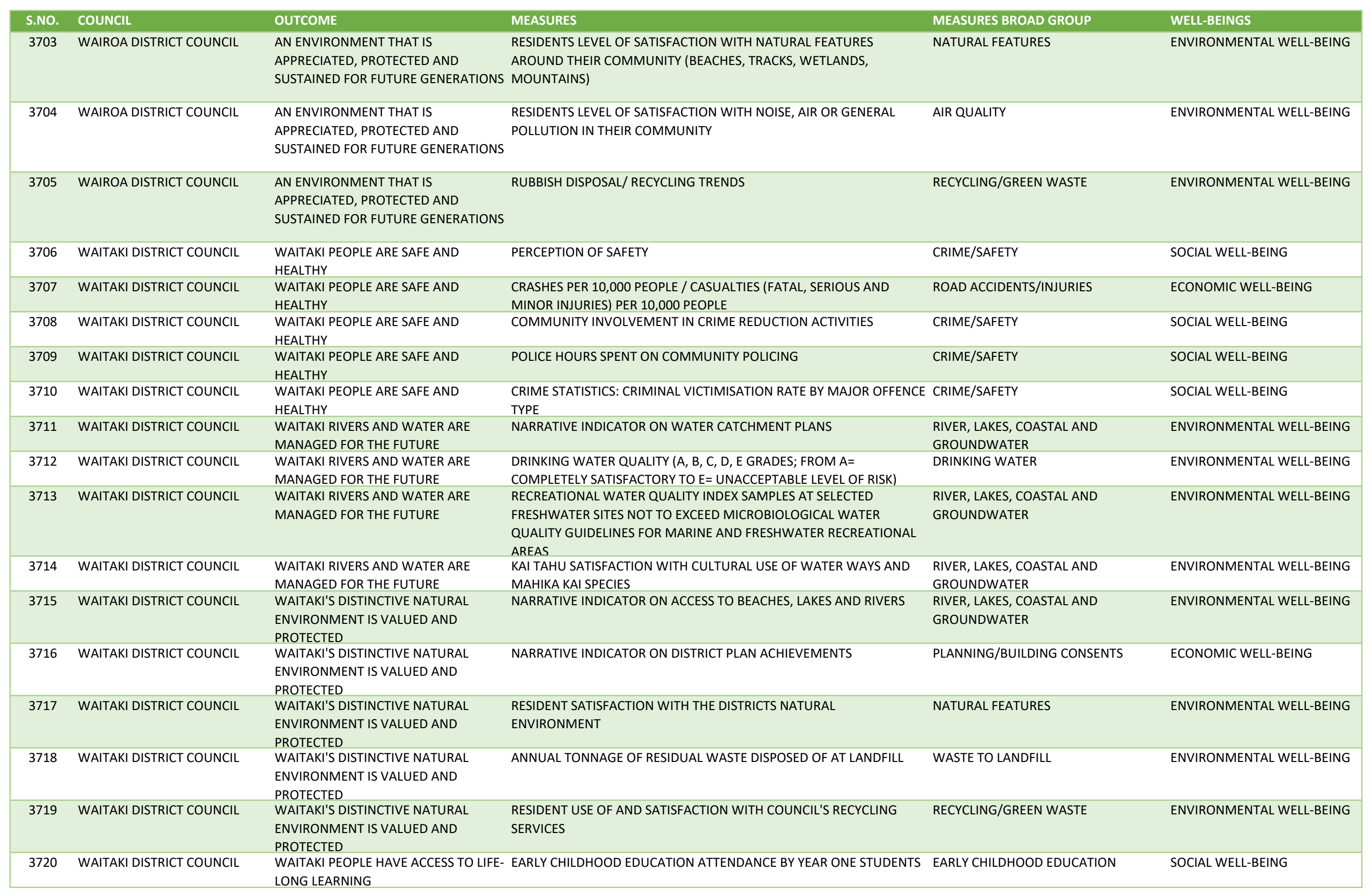




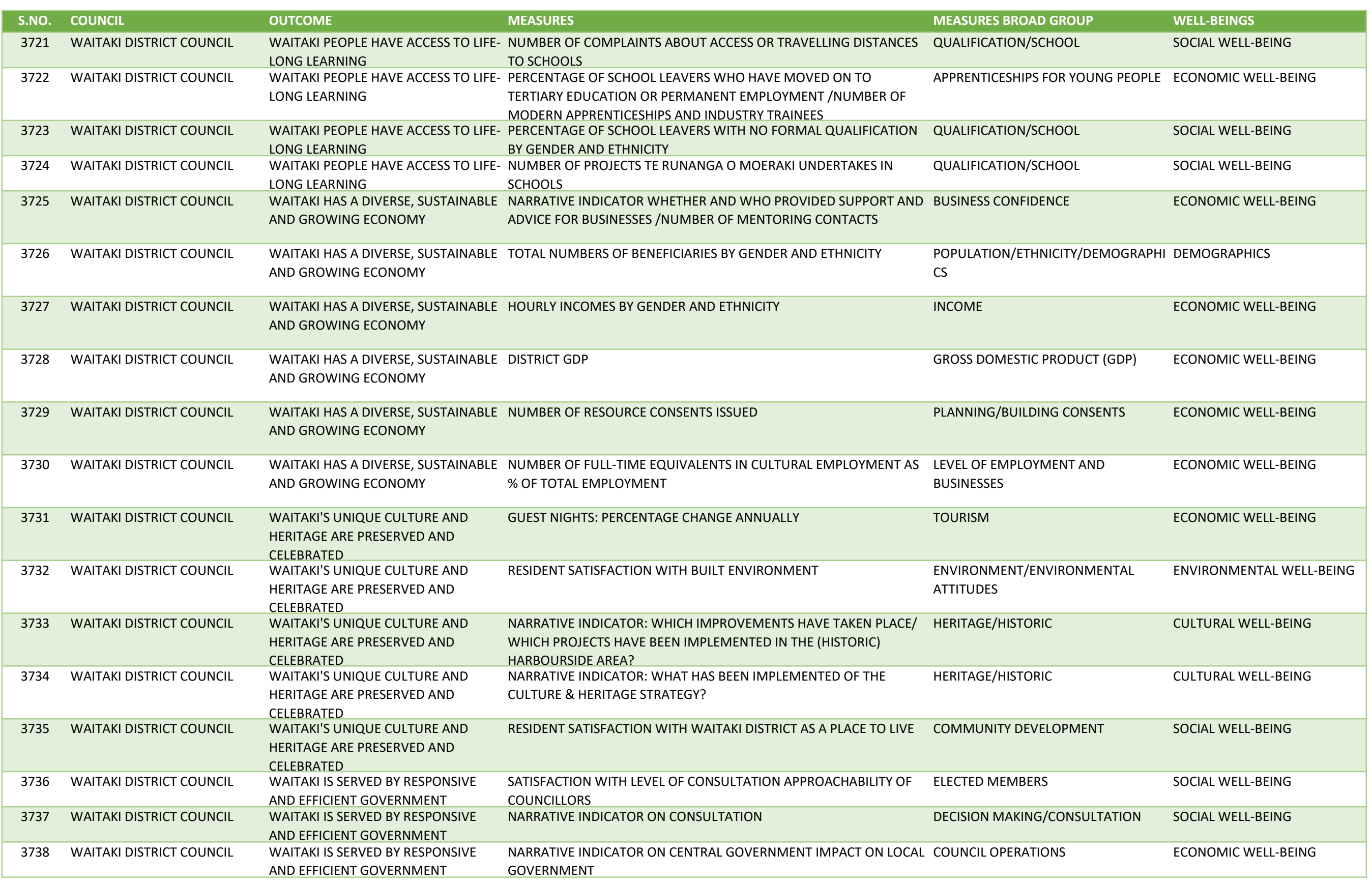




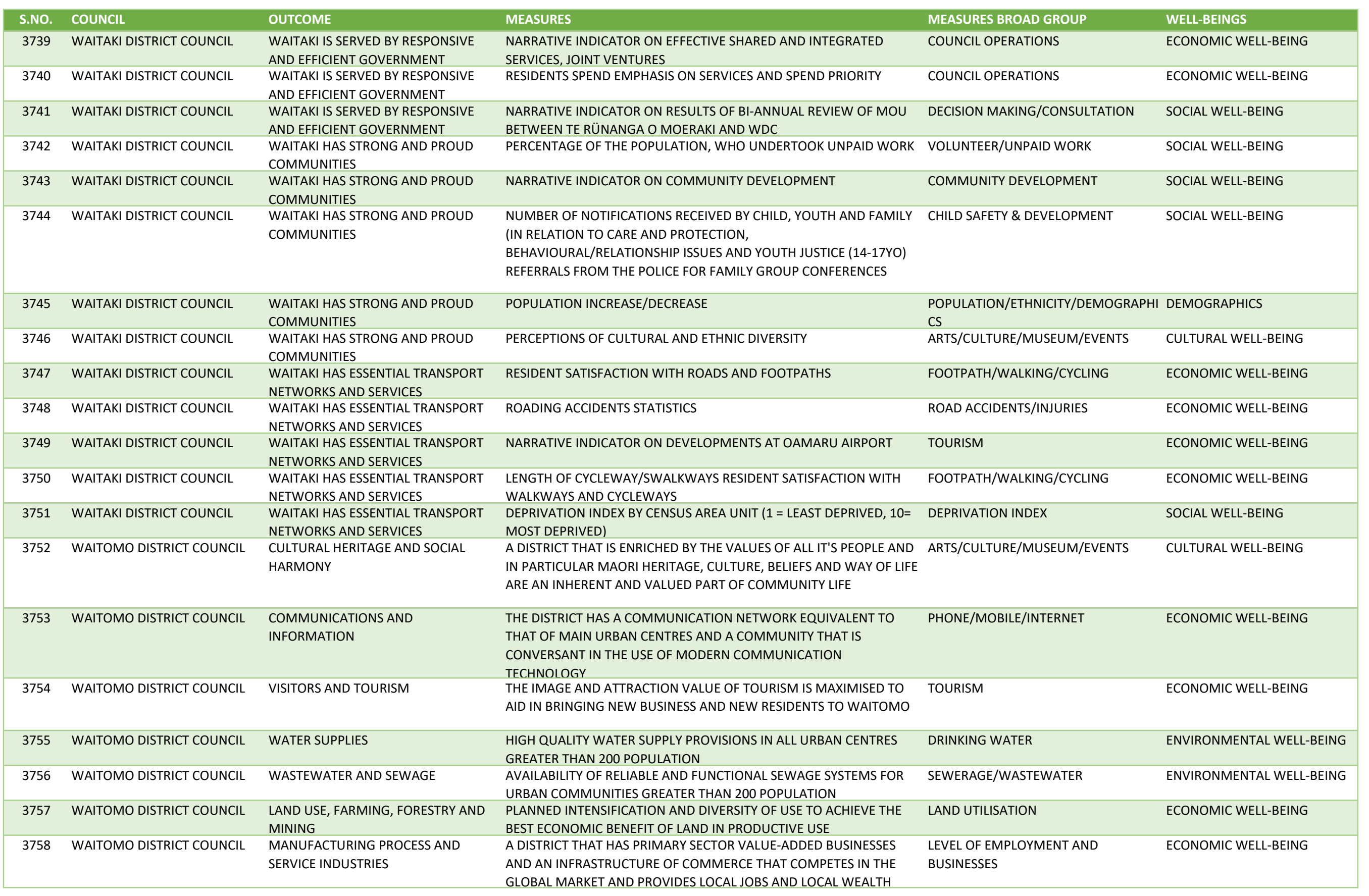




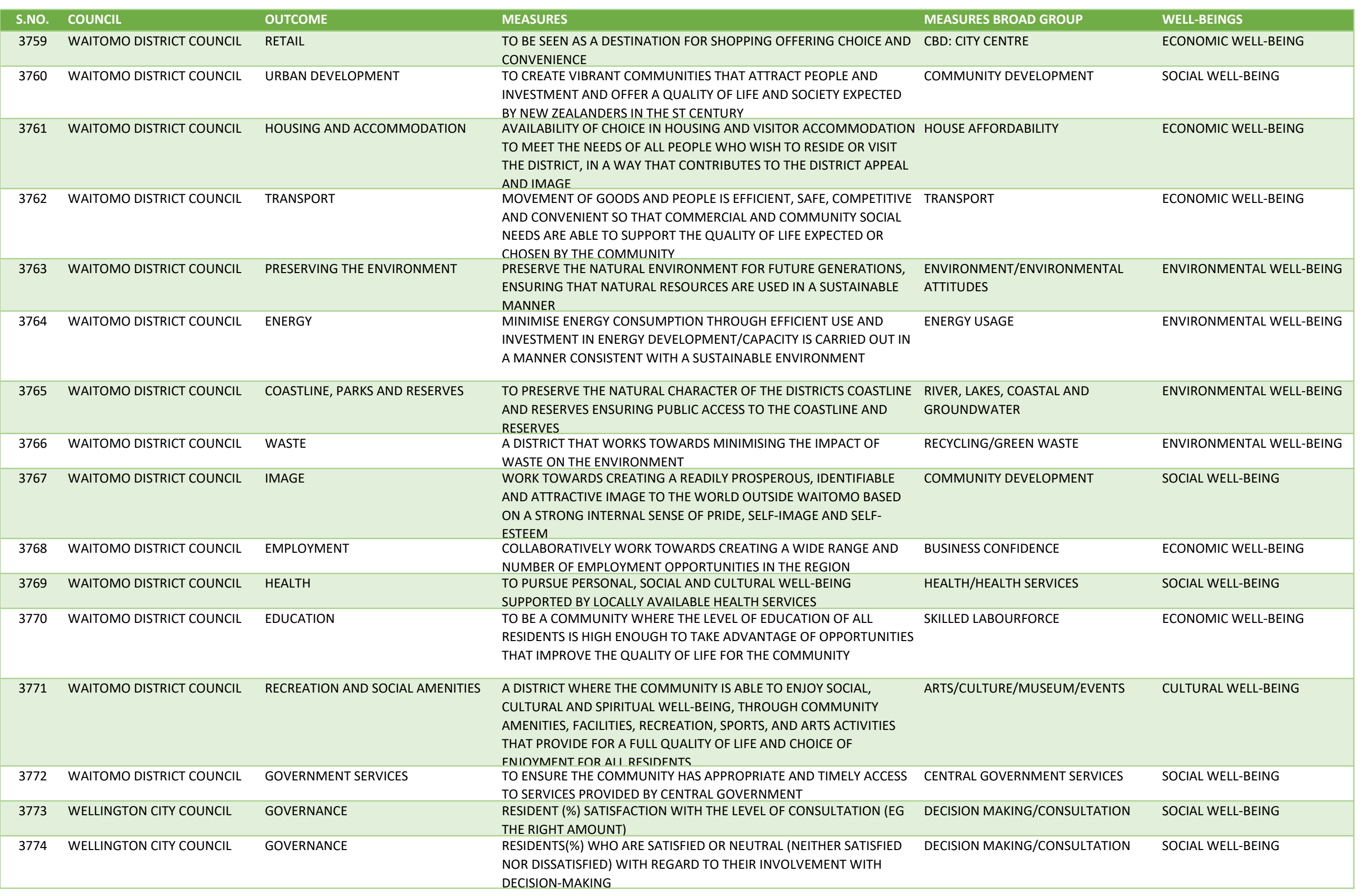


Appendix A: List of Community Well-beings Outcomes and Measures by Councils

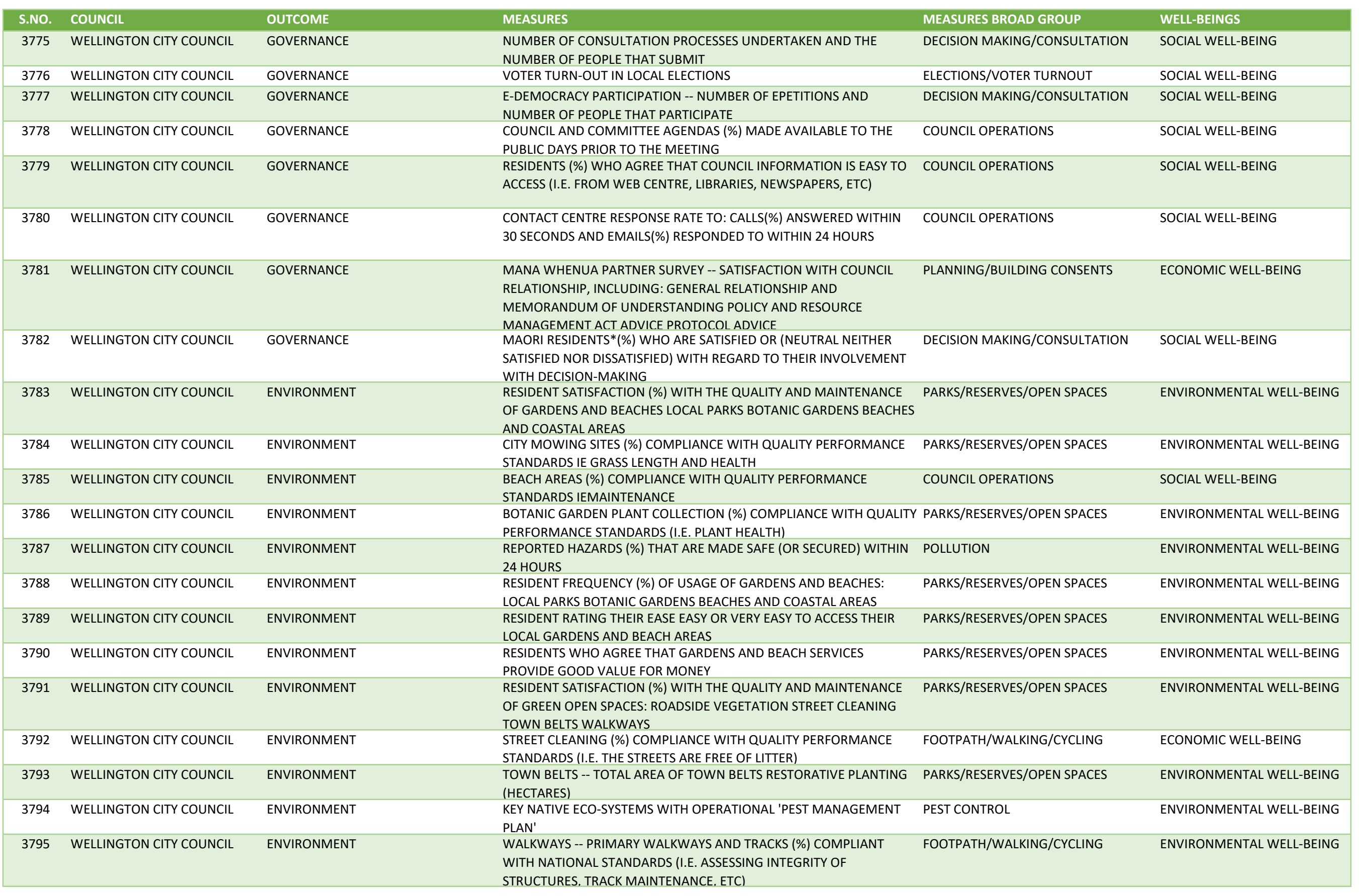


Appendix A: List of Community Well-beings Outcomes and Measures by Councils

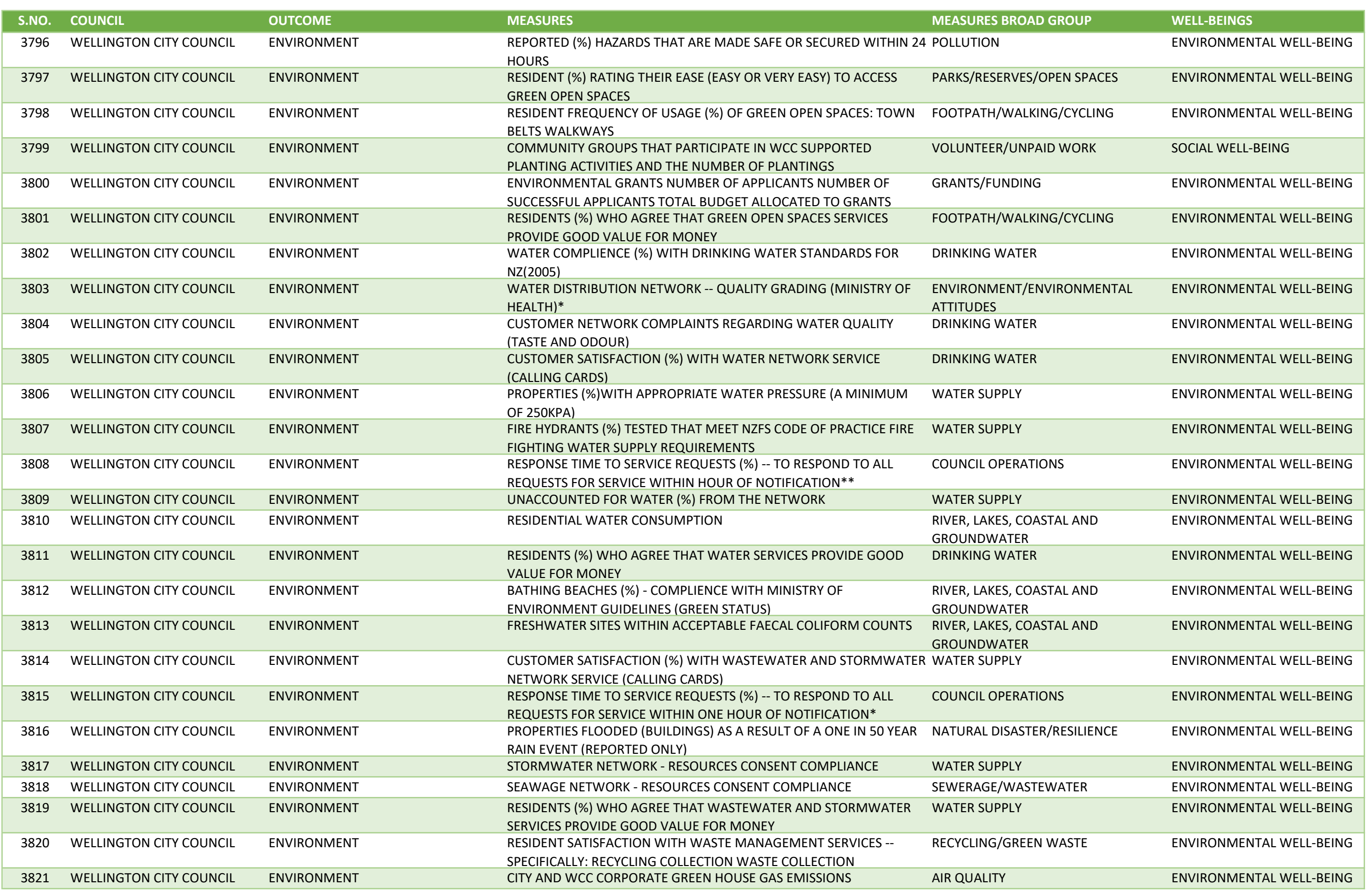


Appendix A: List of Community Well-beings Outcomes and Measures by Councils

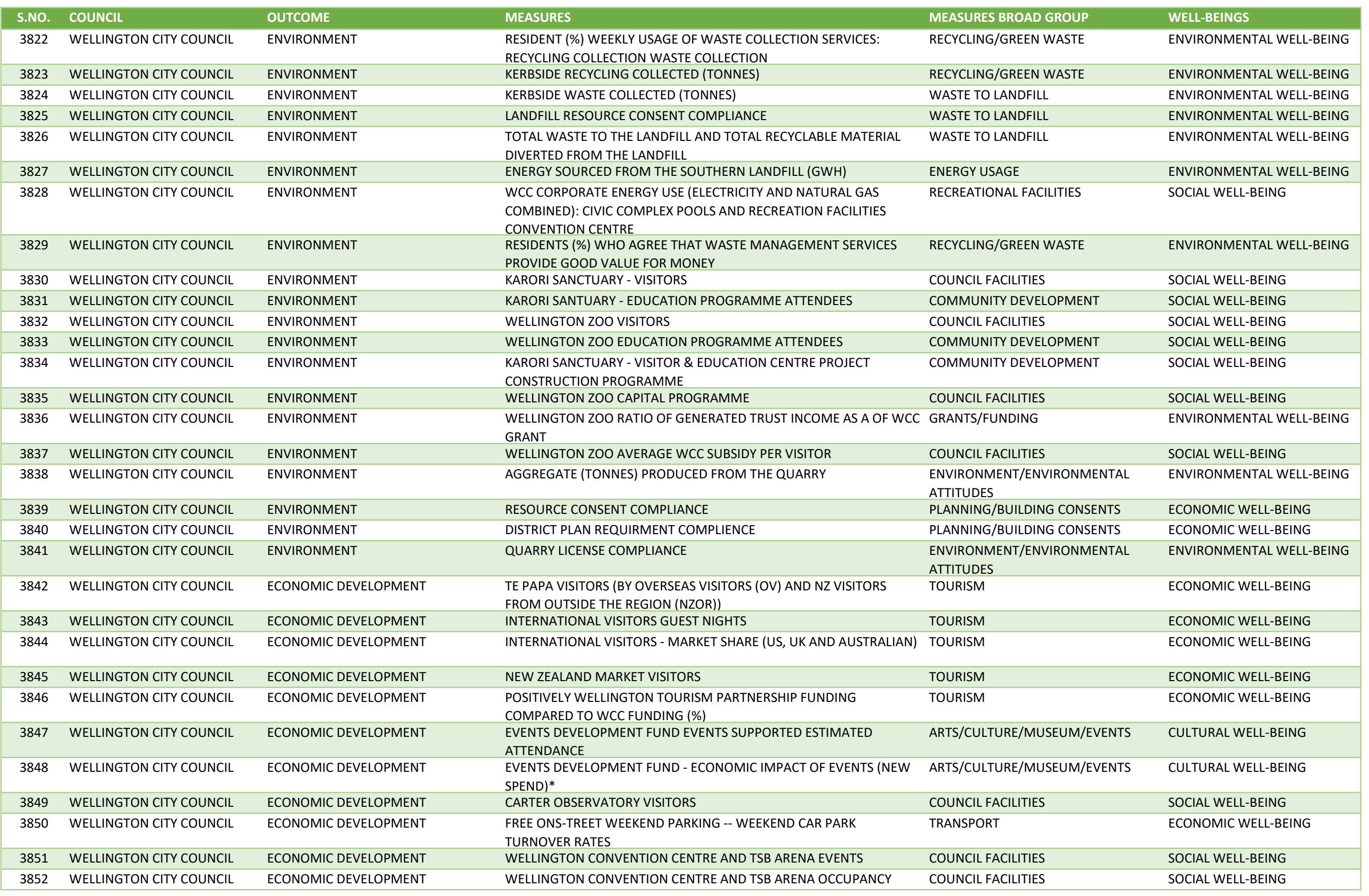




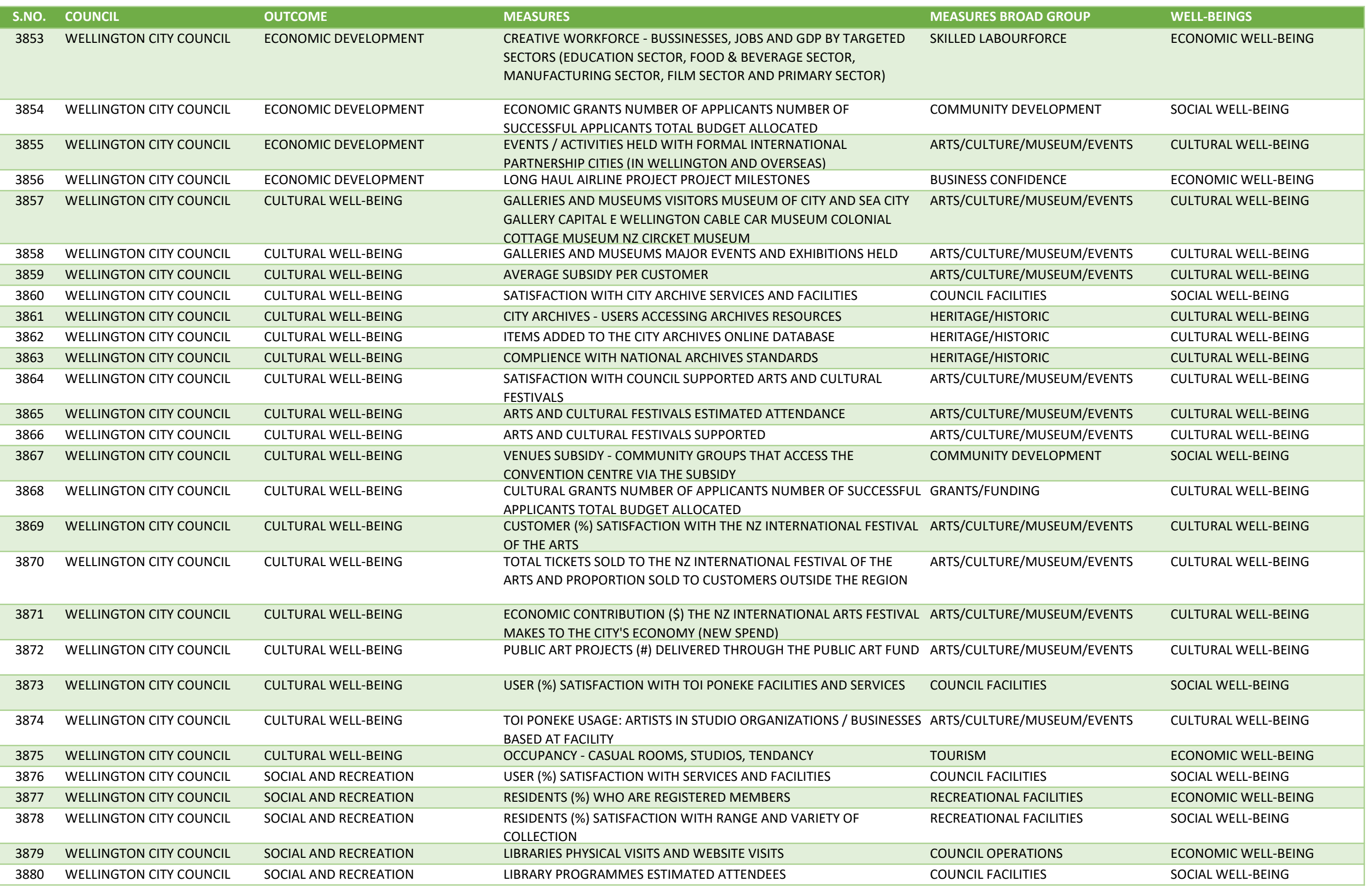


Appendix A: List of Community Well-beings Outcomes and Measures by Councils

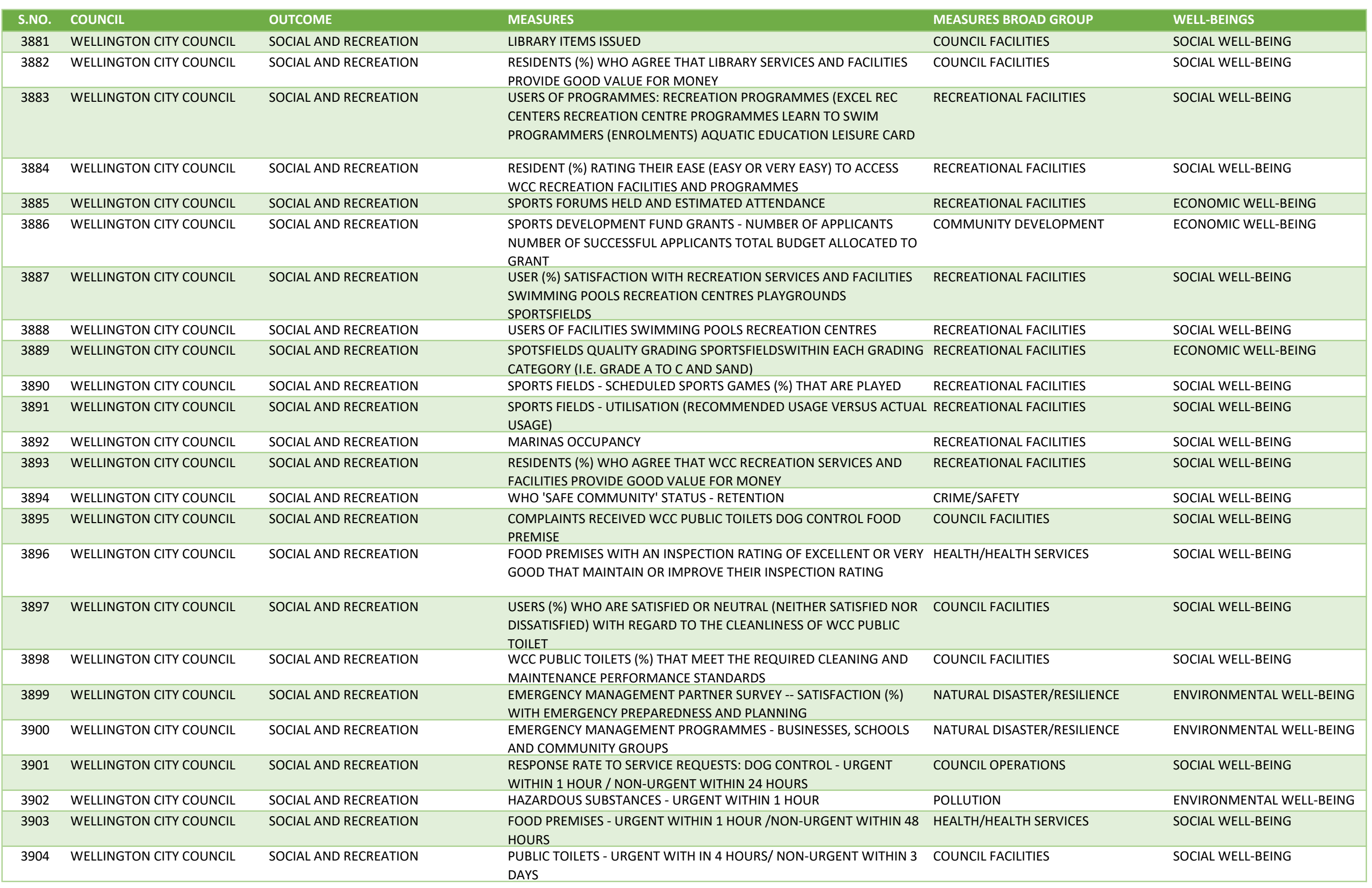


Appendix A: List of Community Well-beings Outcomes and Measures by Councils

\begin{tabular}{|c|c|c|c|c|c|}
\hline S.NO. & COUNCIL & OUTCOME & MEASURES & MEASURES BROAD GROUP & WELL-BEINGS \\
\hline 3905 & WELLINGTON CITY COUNCIL & SOCIAL AND RECREATION & $\begin{array}{l}\text { INSPECTION SCHEDULE COMPLETION INCLUDING LIQUOR LICENSED } \\
\text { PREMISES }\end{array}$ & COUNCIL OPERATIONS & SOCIAL WELL-BEING \\
\hline 3906 & WELLINGTON CITY COUNCIL & SOCIAL AND RECREATION & FOOD LICENSED PREMISES & HEALTH/HEALTH SERVICES & SOCIAL WELL-BEING \\
\hline 3907 & WELLINGTON CITY COUNCIL & SOCIAL AND RECREATION & TENANT SATISFACTION (\%) WITH SERVICES AND FACILITIES & COUNCIL FACILITIES & SOCIAL WELL-BEING \\
\hline 3908 & WELLINGTON CITY COUNCIL & SOCIAL AND RECREATION & $\begin{array}{l}\text { TENANT RATING (\%) OF THE OVERALL CONDITION OF THEIR } \\
\text { HOUSE/APARTMENT }\end{array}$ & HOUSE QUALITY & ECONOMIC WELL-BEING \\
\hline 3909 & WELLINGTON CITY COUNCIL & SOCIAL AND RECREATION & $\begin{array}{l}\text { CITY HOUSING SERVICES AND FACILITIES COMPLY WITH ALL } \\
\text { LEGISLATIVE REQUIREMENTS (E.G. RTA, BUILDING WOF) }\end{array}$ & HOUSE QUALITY & ECONOMIC WELL-BEING \\
\hline 3910 & WELLINGTON CITY COUNCIL & SOCIAL AND RECREATION & TENANT (\%) SENSE OF SAFETY IN THEIR COMPLEX AT NIGHT & CRIME/SAFETY & SOCIAL WELL-BEING \\
\hline 3912 & WELLINGTON CITY COUNCIL & SOCIAL AND RECREATION & AVERAGE WAITING TIME FOR APPLICANTS BY TARGET GROUPS & COUNCIL FACILITIES & SOCIAL WELL-BEING \\
\hline 3913 & WELLINGTON CITY COUNCIL & SOCIAL AND RECREATION & APPLICANTS (\%) HOUSED -- BY TARGET GROUPS & HOUSE AFFORDABILITY & ECONOMIC WELL-BEING \\
\hline 3914 & WELLINGTON CITY COUNCIL & SOCIAL AND RECREATION & $\begin{array}{l}\text { RESIDENTS (\%) WHO RATE SERVICES AND FACILITIES AS GOOD VALUE } \\
\text { FOR MONEY }\end{array}$ & COUNCIL FACILITIES & SOCIAL WELL-BEING \\
\hline 3915 & WELLINGTON CITY COUNCIL & SOCIAL AND RECREATION & $\begin{array}{l}\text { AGREED MILESTONES, DESIGN STANDARDS AND BUDGETS ARE MET IN } \\
\text { ACCORDANCE WITH THE AGREED WORKS PROGRAMME AND DEED OF } \\
\text { GRANT BETWEEN THE CROWN AND THE COUNCIL }\end{array}$ & GRANTS/FUNDING & CULTURAL WELL-BEING \\
\hline 3916 & WELLINGTON CITY COUNCIL & SOCIAL AND RECREATION & MAJOR UPGRADE PROJECTS CONSTRUCTION COMPLETION DATES & PLANNING/BUILDING CONSENTS & ECONOMIC WELL-BEING \\
\hline 3917 & WELLINGTON CITY COUNCIL & SOCIAL AND RECREATION & $\begin{array}{l}\text { COMMUNITY GROUPS (\%) WHO ARE SATISFIED WITH COUNCIL } \\
\text { RELATIONSHIPS }\end{array}$ & COUNCIL OPERATIONS & SOCIAL WELL-BEING \\
\hline 3918 & WELLINGTON CITY COUNCIL & SOCIAL AND RECREATION & $\begin{array}{l}\text { COMMUNITY FORUMS, COMMUNITY GROUPS MEETINGS, ADVISORY } \\
\text { GROUPS AND YOUTH EVENTS AND ESTIMATED ATTENDANCE }\end{array}$ & COMMUNITY DEVELOPMENT & SOCIAL WELL-BEING \\
\hline 3919 & WELLINGTON CITY COUNCIL & SOCIAL AND RECREATION & $\begin{array}{l}\text { SOCIAL AND RECREATION GRANTS NUMBER OF APPLICANTS NUMBER } \\
\text { OF SUCCESSFUL APPLICANTS TOTAL BUDGET ALLOCATED }\end{array}$ & GRANTS/FUNDING & SOCIAL WELL-BEING \\
\hline 3920 & WELLINGTON CITY COUNCIL & SOCIAL AND RECREATION & COMMUNITY CENTRES VISITORS AND OCCUPANCY & COUNCIL FACILITIES & SOCIAL WELL-BEING \\
\hline 3921 & WELLINGTON CITY COUNCIL & SOCIAL AND RECREATION & COMMUNITY HALLS VISITORS AND OCCUPANCY & COUNCIL FACILITIES & SOCIAL WELL-BEING \\
\hline 3922 & WELLINGTON CITY COUNCIL & SOCIAL AND RECREATION & $\begin{array}{l}\text { HOMELESSNESS STRATEGY - WE WILL REPORT ON THE WORK WE HAVE } \\
\text { DONE IN PARTNERSHIP WITH OTHERS }\end{array}$ & PLANNING/BUILDING CONSENTS & SOCIAL WELL-BEING \\
\hline 3923 & WELLINGTON CITY COUNCIL & URBAN DEVELOPMENT & $\begin{array}{l}\text { RESIDENTS (\%) WHO AGREE THE CITY IS DEVELOPING IN A WAY THAT } \\
\text { TAKES INTO ACCOUNT ITS UNIQUE URBAN CHARACTER AND NATURAL } \\
\text { ENVIRONMENT }\end{array}$ & $\begin{array}{l}\text { ENVIRONMENT/ENVIRONMENTAL } \\
\text { ATTITUDES }\end{array}$ & ENVIRONMENTAL WELL-BEING \\
\hline 3924 & WELLINGTON CITY COUNCIL & URBAN DEVELOPMENT & $\begin{array}{l}\text { URBAN HERITAGE AND CHARACTER PROTECTION -- WORK SCHEDULE } \\
\text { (NOTE: PLAN CHANGES, NOTIFIED AND WITH COUNCIL DECISION IN } \\
\text { PLACE BY THE END OF 2010) }\end{array}$ & HERITAGE/HISTORIC & CULTURAL WELL-BEING \\
\hline 3925 & WELLINGTON CITY COUNCIL & URBAN DEVELOPMENT & $\begin{array}{l}\text { DISTRICT PLAN PROPOSED CHANGES - WORK SCHEDULE (NOTE: PLAN } \\
\text { CHANGES, NOTIFIED AND WITH COUNCIL DECISION IN PLACE BY THE } \\
\text { END OF 2010) }\end{array}$ & PLANNING/BUILDING CONSENTS & ECONOMIC WELL-BEING \\
\hline 3926 & WELLINGTON CITY COUNCIL & URBAN DEVELOPMENT & $\begin{array}{l}\text { DISTRCT PLAN CHANGES - APPEALS THAT ARE MEDIATED (SETTLED) } \\
\text { BEFORE REACHING THE ENVIRONMENT COURT }\end{array}$ & PLANNING/BUILDING CONSENTS & ECONOMIC WELL-BEING \\
\hline 3930 & WELLINGTON CITY COUNCIL & URBAN DEVELOPMENT & $\begin{array}{l}\text { LAND INFORMATION MEMORANDUMS (LIMS) ISSUED WITHIN } 10 \\
\text { WORKING DAYS }\end{array}$ & PLANNING/BUILDING CONSENTS & ECONOMIC WELL-BEING \\
\hline
\end{tabular}


Appendix A: List of Community Well-beings Outcomes and Measures by Councils

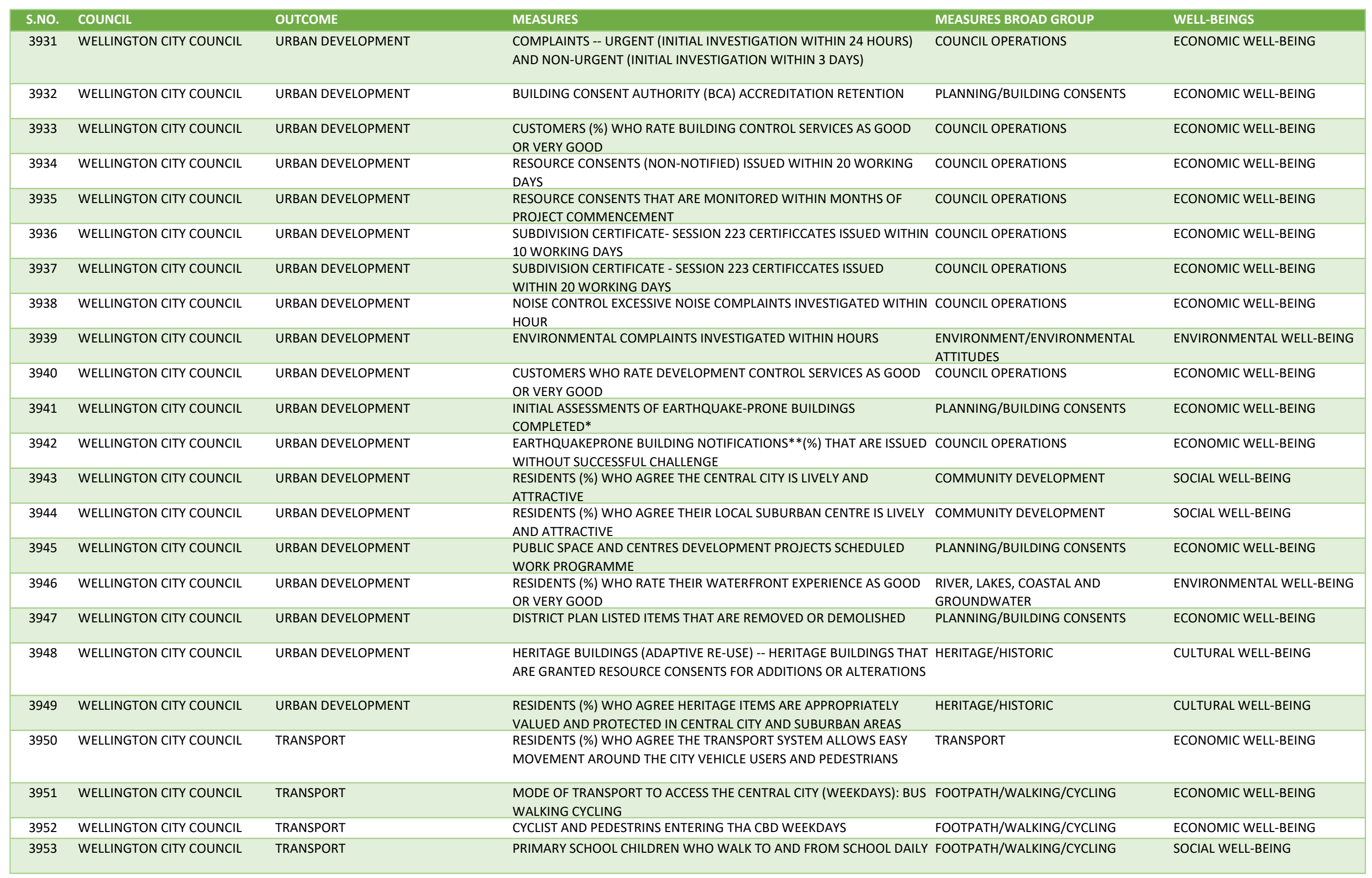


Appendix A: List of Community Well-beings Outcomes and Measures by Councils

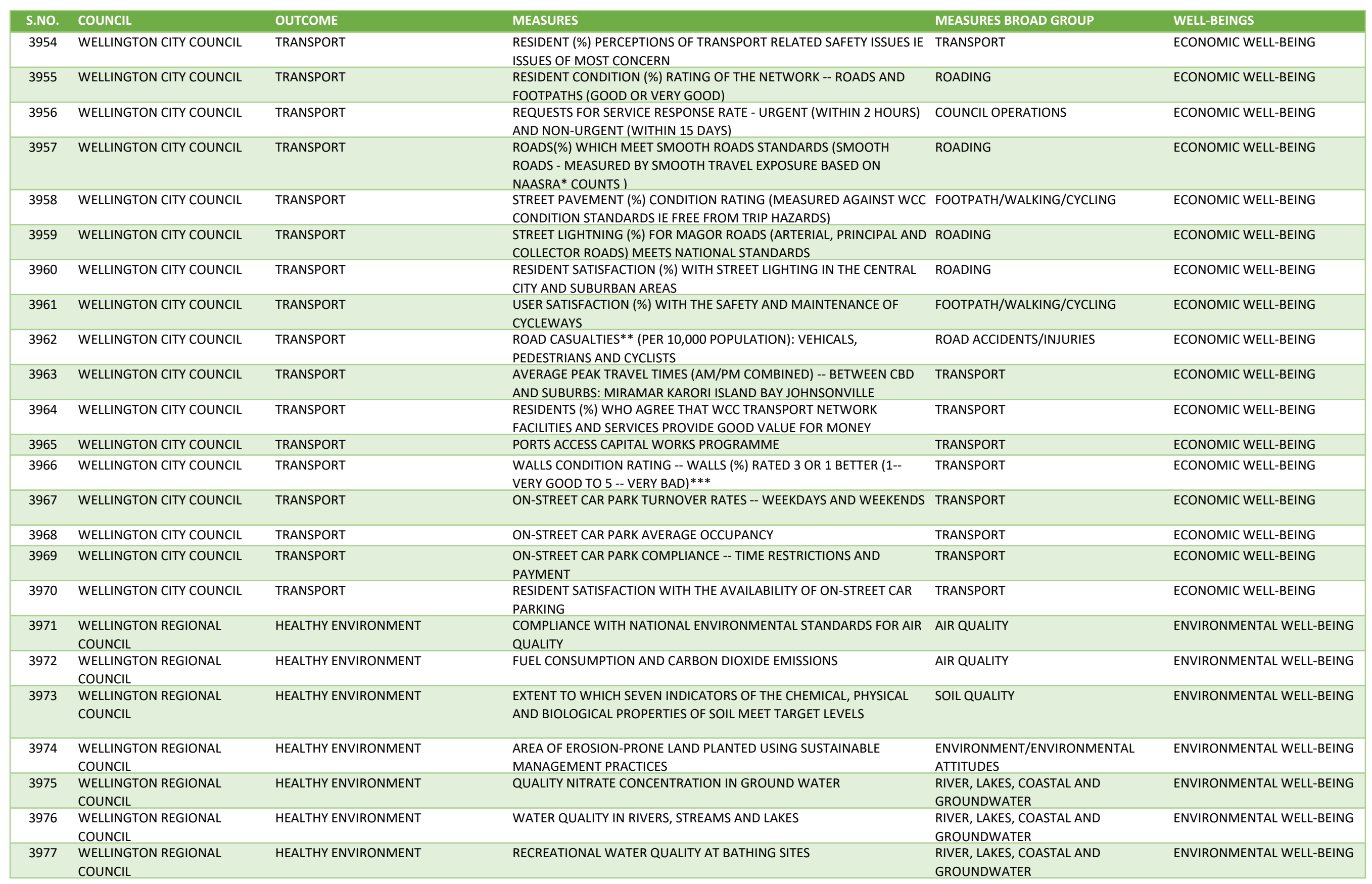


Appendix A: List of Community Well-beings Outcomes and Measures by Councils

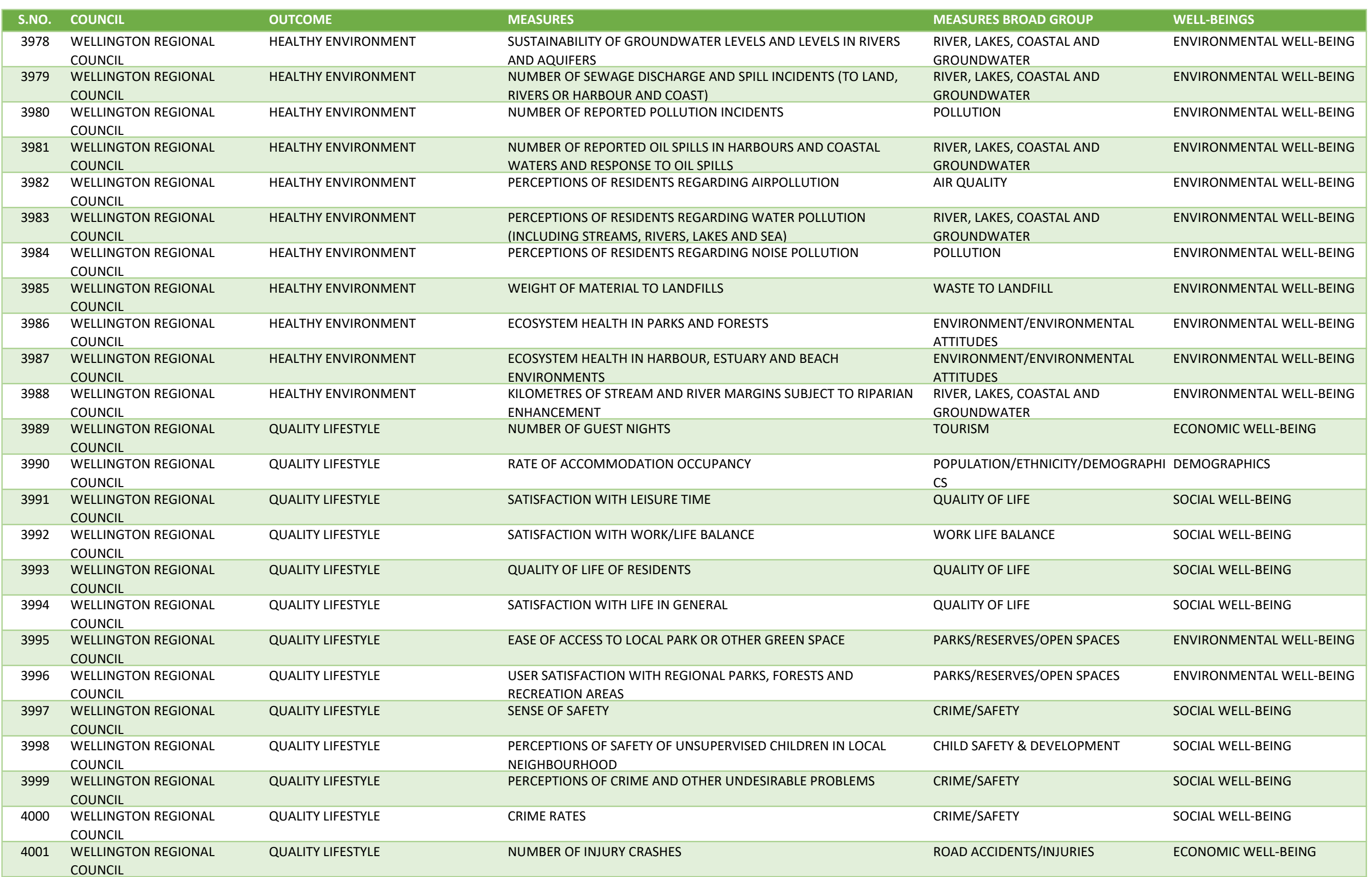


Appendix A: List of Community Well-beings Outcomes and Measures by Councils

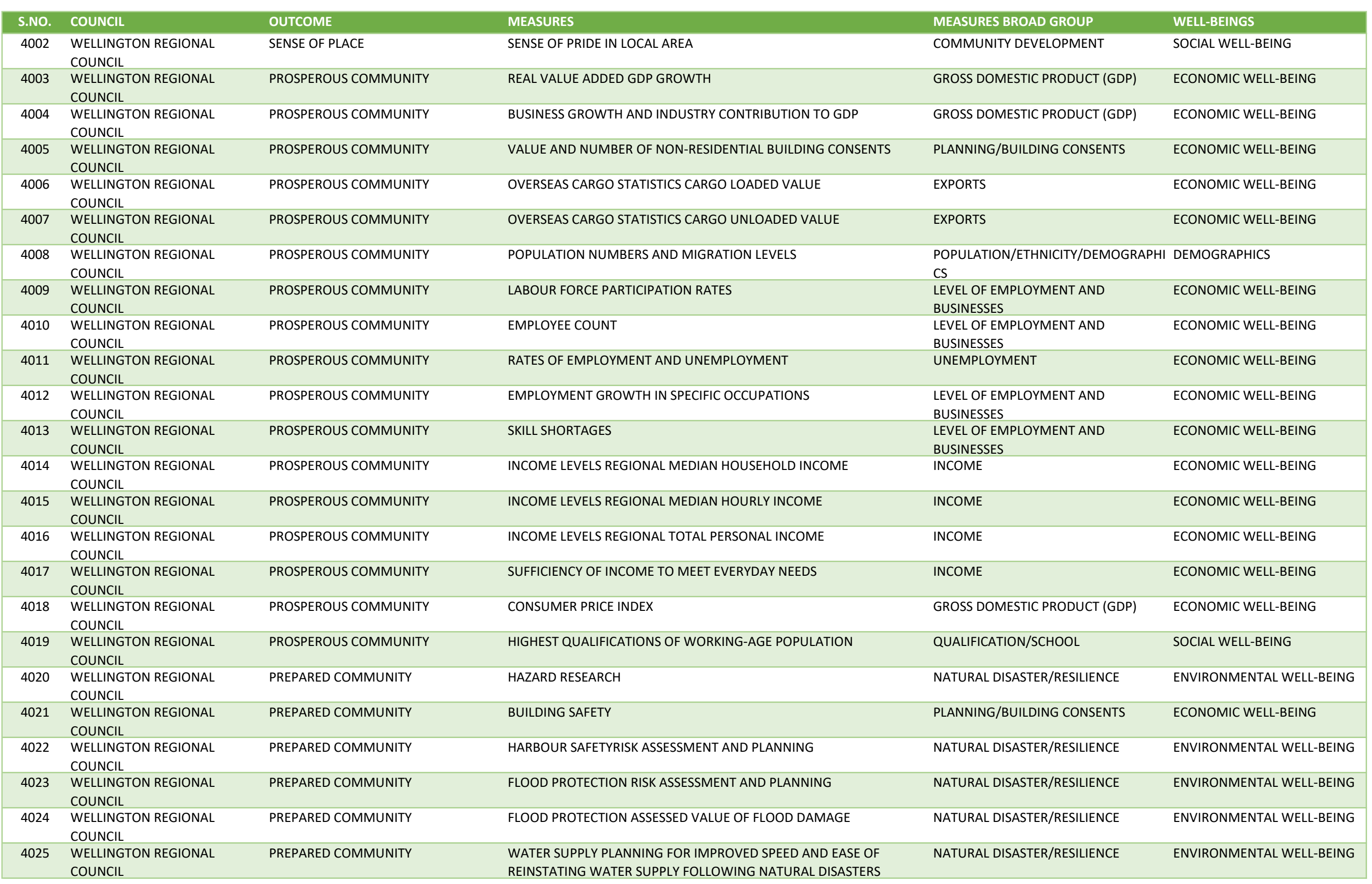


Appendix A: List of Community Well-beings Outcomes and Measures by Councils

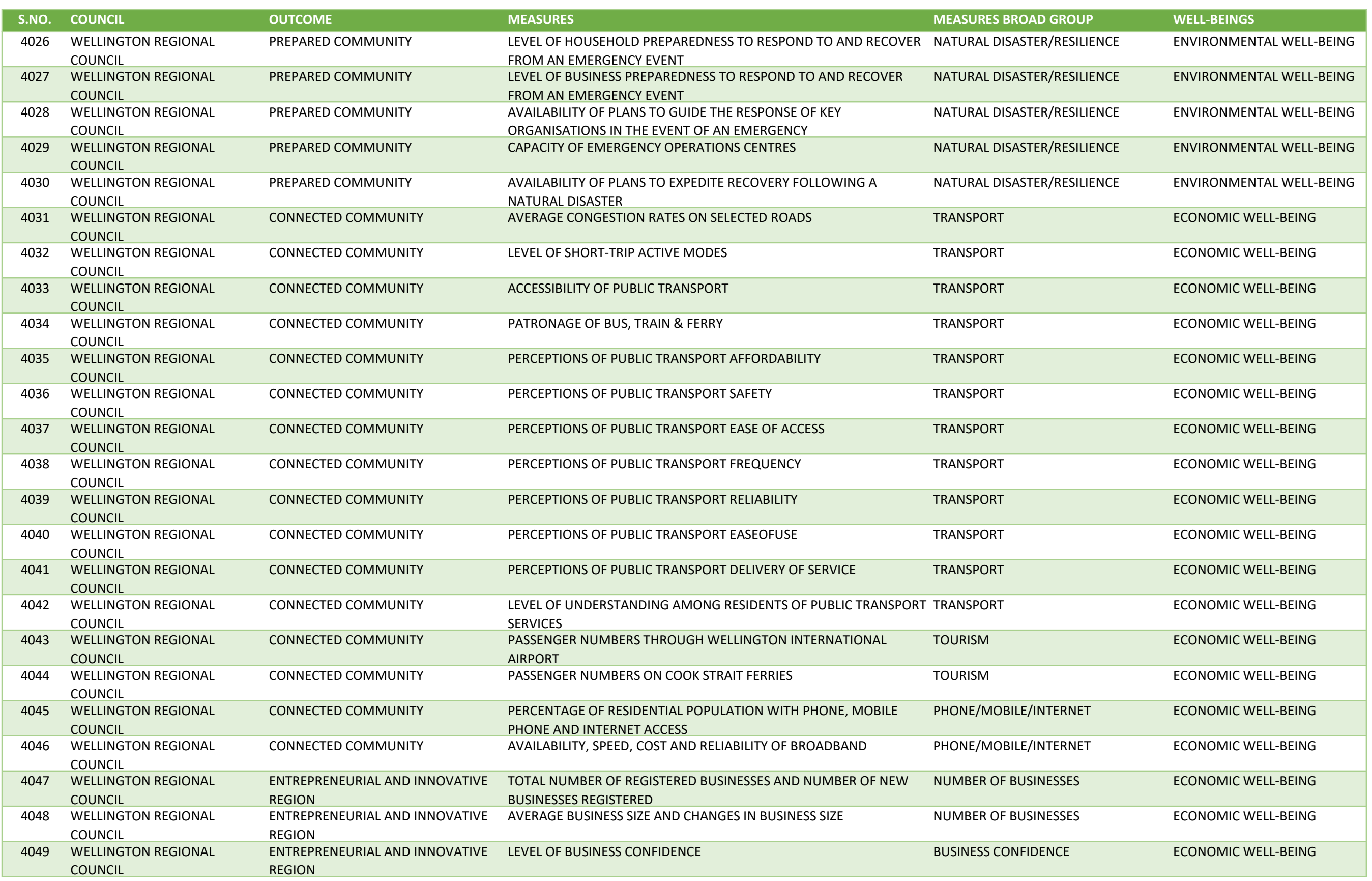


Appendix A: List of Community Well-beings Outcomes and Measures by Councils

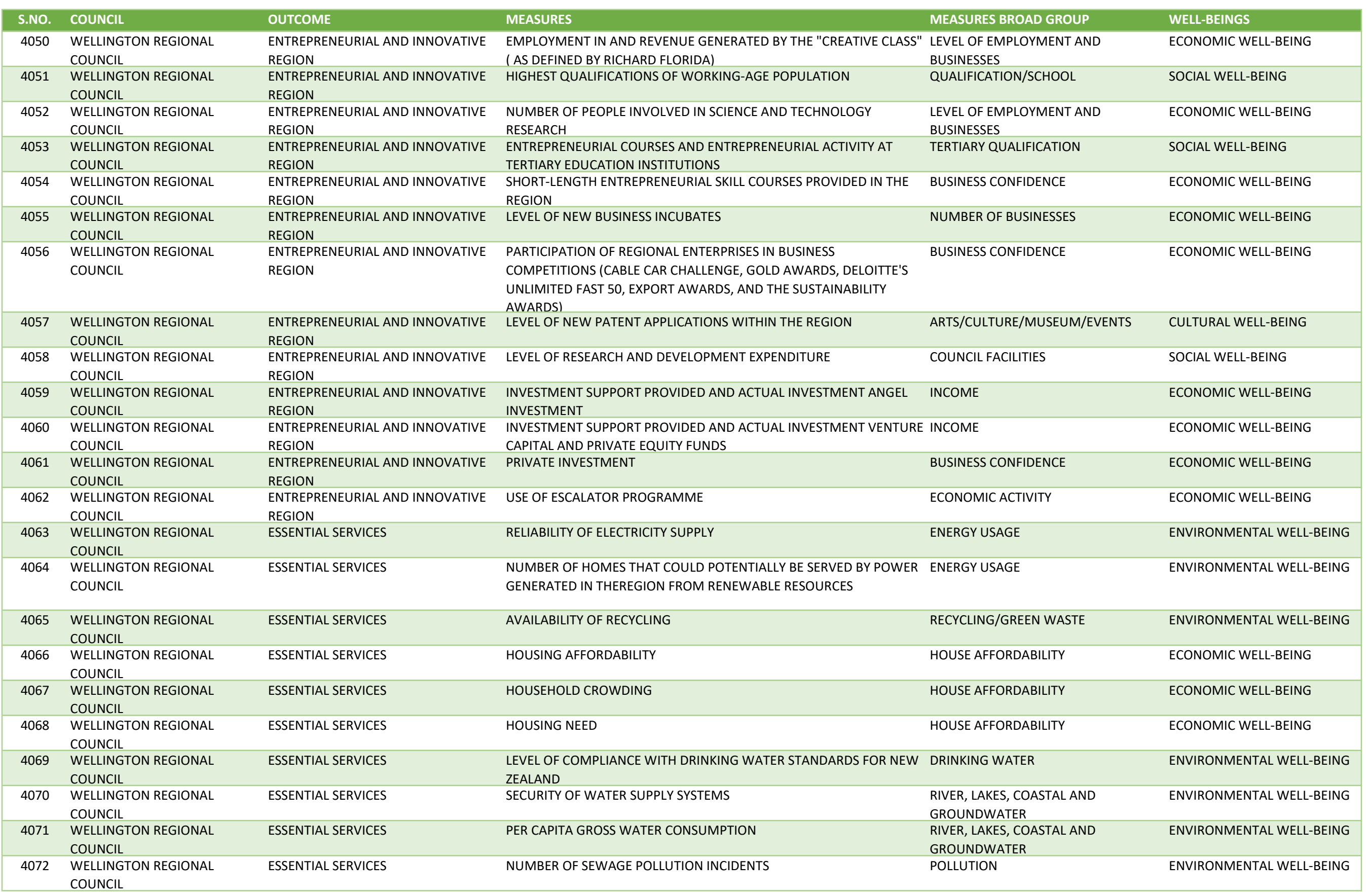


Appendix A: List of Community Well-beings Outcomes and Measures by Councils

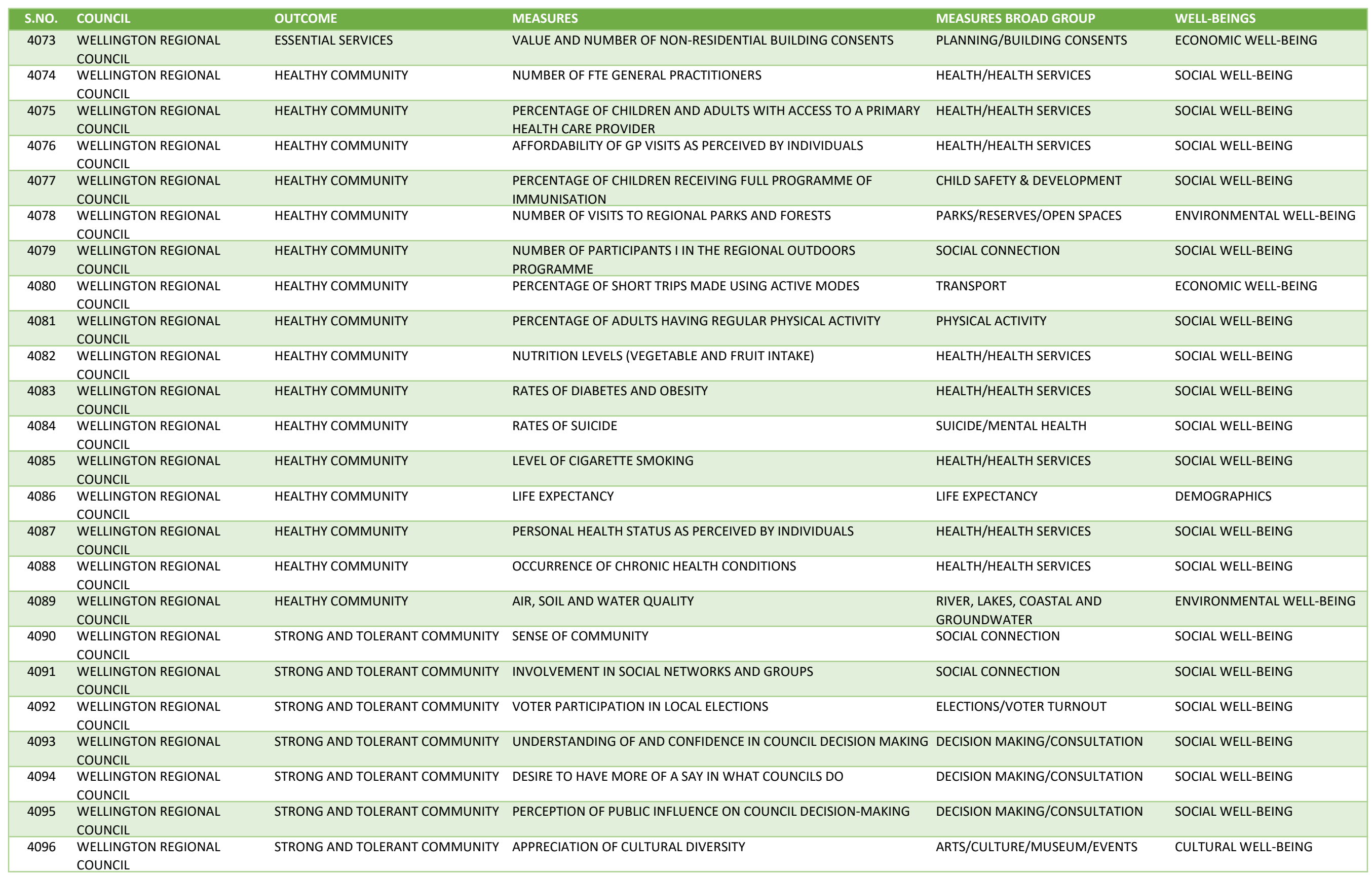


Appendix A: List of Community Well-beings Outcomes and Measures by Councils

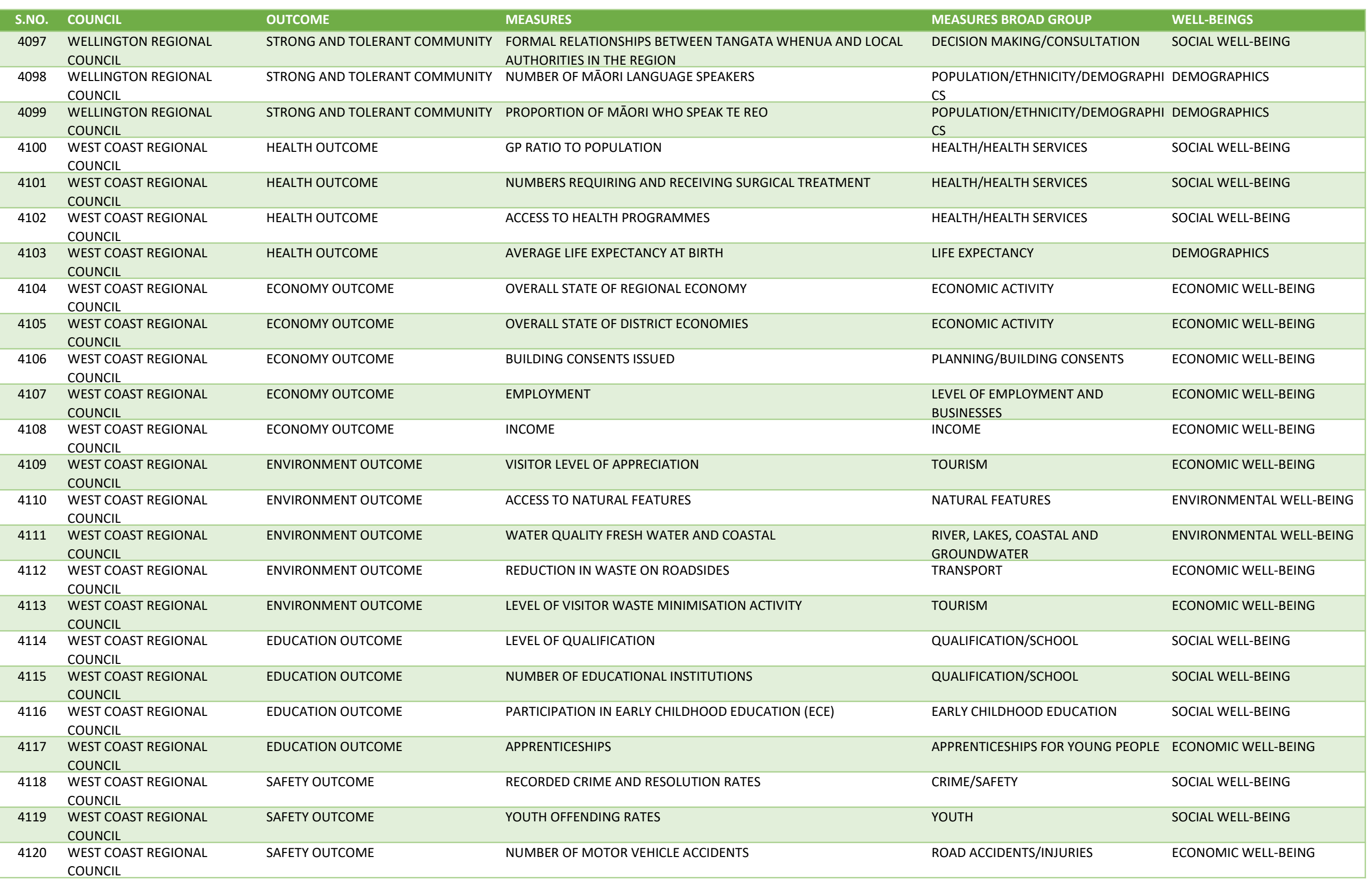


Appendix A: List of Community Well-beings Outcomes and Measures by Councils

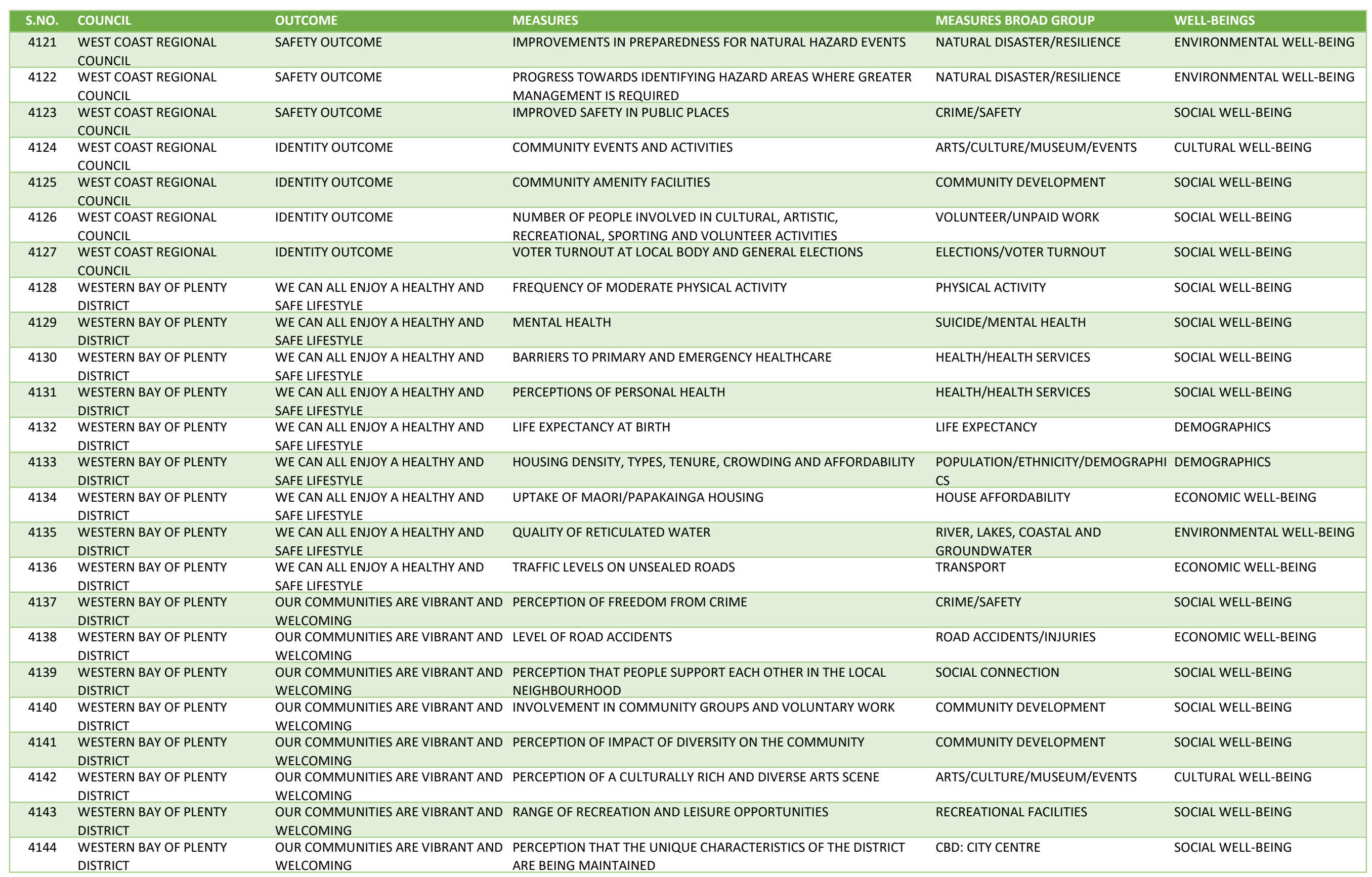


Appendix A: List of Community Well-beings Outcomes and Measures by Councils

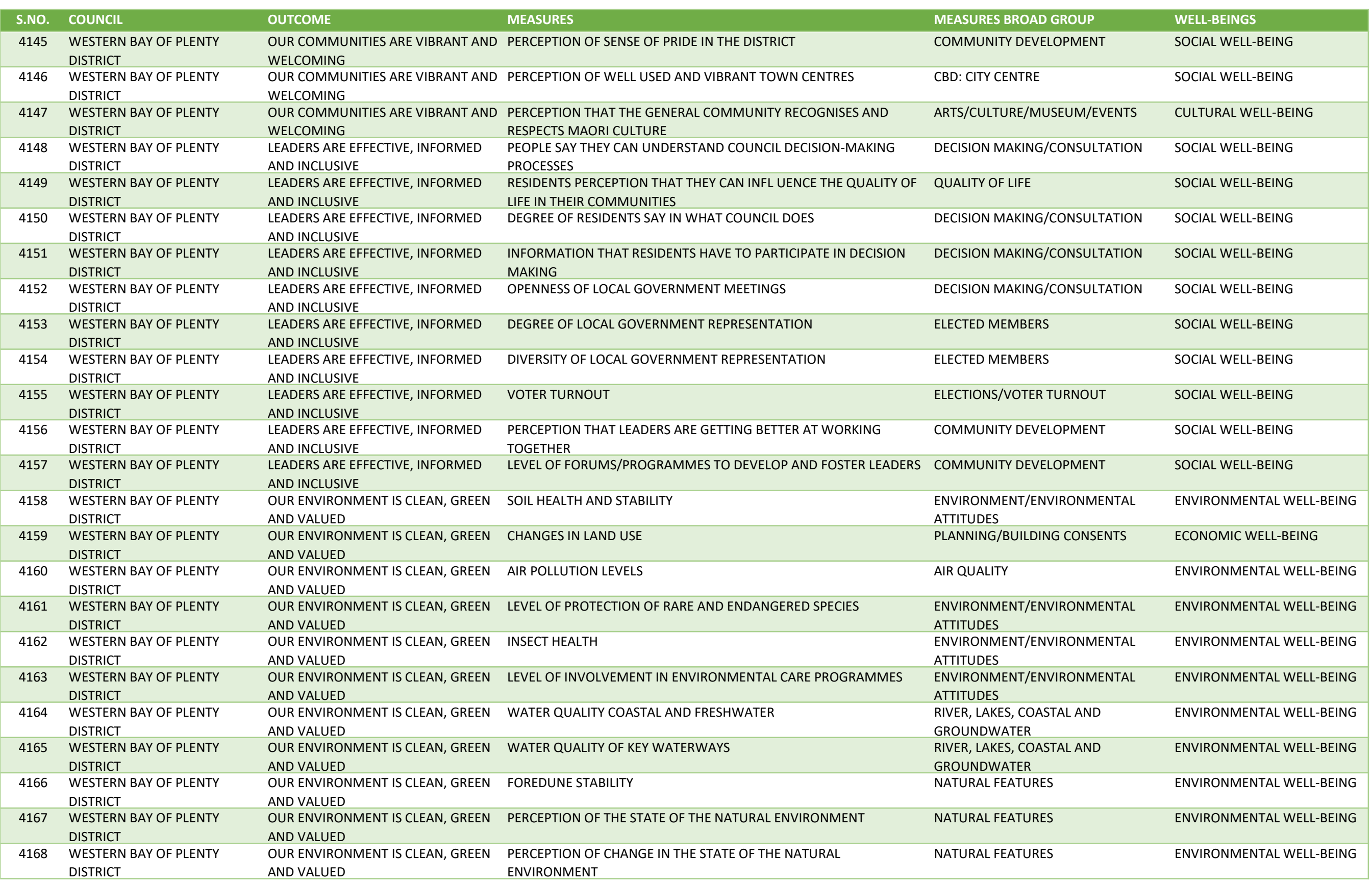


Appendix A: List of Community Well-beings Outcomes and Measures by Councils

\begin{tabular}{|c|c|c|c|c|c|}
\hline S.NO. & COUNCIL & OUTCOME & MEASURES & MEASURES BROAD GROUP & WELL-BEINGS \\
\hline 4169 & $\begin{array}{l}\text { WESTERN BAY OF PLENTY } \\
\text { DISTRICT }\end{array}$ & OUR ECONOMY IS THRIVING & VALUE OF EXPORTS BY SECTOR & EXPORTS & ECONOMIC WELL-BEING \\
\hline 4170 & $\begin{array}{l}\text { WESTERN BAY OF PLENTY } \\
\text { DISTRICT }\end{array}$ & OUR ECONOMY IS THRIVING & THE SKILLS GAP & $\begin{array}{l}\text { LEVEL OF EMPLOYMENT AND } \\
\text { BUSINESSES }\end{array}$ & ECONOMIC WELL-BEING \\
\hline 4171 & $\begin{array}{l}\text { WESTERN BAY OF PLENTY } \\
\text { DISTRICT }\end{array}$ & OUR ECONOMY IS THRIVING & PERCEPTION OF WORKS SKILLS MATCHED TO OCCUPATIONS & $\begin{array}{l}\text { LEVEL OF EMPLOYMENT AND } \\
\text { BUSINESSES }\end{array}$ & ECONOMIC WELL-BEING \\
\hline 4172 & $\begin{array}{l}\text { WESTERN BAY OF PLENTY } \\
\text { DISTRICT }\end{array}$ & OUR ECONOMY IS THRIVING & GROWTH OF THE ECONOMY & GROSS DOMESTIC PRODUCT (GDP) & ECONOMIC WELL-BEING \\
\hline 4173 & $\begin{array}{l}\text { WESTERN BAY OF PLENTY } \\
\text { DISTRICT }\end{array}$ & OUR ECONOMY IS THRIVING & APPRENTICESHIPS & APPRENTICESHIPS FOR YOUNG PEOPLE & ECONOMIC WELL-BEING \\
\hline 4174 & $\begin{array}{l}\text { WESTERN BAY OF PLENTY } \\
\text { DISTRICT }\end{array}$ & OUR ECONOMY IS THRIVING & YOUTH EMPLOYMENT & $\begin{array}{l}\text { NEET: NOT IN EMPLOYMENT, } \\
\text { EDUCATION OR TRAINING }\end{array}$ & ECONOMIC WELL-BEING \\
\hline 4175 & $\begin{array}{l}\text { WESTERN BAY OF PLENTY } \\
\text { DISTRICT }\end{array}$ & OUR ECONOMY IS THRIVING & COURSE DIVERSITY AT SECONDARY AND TERTIARY LEVELS & TERTIARY QUALIFICATION & SOCIAL WELL-BEING \\
\hline 4176 & $\begin{array}{l}\text { WESTERN BAY OF PLENTY } \\
\text { DISTRICT }\end{array}$ & OUR ECONOMY IS THRIVING & LEVEL OF SCHOOL QUALIFI CATIONS & QUALIFICATION/SCHOOL & SOCIAL WELL-BEING \\
\hline 4177 & $\begin{array}{l}\text { WESTERN BAY OF PLENTY } \\
\text { DISTRICT }\end{array}$ & OUR ECONOMY IS THRIVING & BUSINESS PARTICIPATION IN INITIATIVES AND PROGRAMMES & BUSINESS CONFIDENCE & ECONOMIC WELL-BEING \\
\hline 4178 & WESTLAND DISTRICT COUNCIL & HEALTH & RATIO OF PROVIDERS TO POPULATIONS & $\begin{array}{l}\text { POPULATION/ETHNICITY/DEMOGRAPHI } \\
\text { CS }\end{array}$ & DEMOGRAPHICS \\
\hline 4180 & WESTLAND DISTRICT COUNCIL & HEALTH & ACCESS TO HEALTH PROGRAMMES & HEALTH/HEALTH SERVICES & SOCIAL WELL-BEING \\
\hline 4181 & WESTLAND DISTRICT COUNCIL & HEALTH & DRINKING WATER QUALITY & DRINKING WATER & ENVIRONMENTAL WELL-BEING \\
\hline 4182 & WESTLAND DISTRICT COUNCIL & HEALTH & NUMBER OF PEOPLE TREATED LOCALLY VERSUS OUT OF THE REGION & HEALTH/HEALTH SERVICES & SOCIAL WELL-BEING \\
\hline 4183 & WESTLAND DISTRICT COUNCIL & EDUCATION & PARTICIPATION IN EARLY CHILDHOOD EDUCATION & EARLY CHILDHOOD EDUCATION & SOCIAL WELL-BEING \\
\hline 4184 & WESTLAND DISTRICT COUNCIL & EDUCATION & SCHOOL LEAVERS WITH HIGHER QUALIFICATIONS & QUALIFICATION/SCHOOL & SOCIAL WELL-BEING \\
\hline 4185 & WESTLAND DISTRICT COUNCIL & EDUCATION & NUMBER OF EDUCATIONAL INSTITUTIONS & QUALIFICATION/SCHOOL & SOCIAL WELL-BEING \\
\hline 4186 & WESTLAND DISTRICT COUNCIL & EDUCATION & NUMBER OF APPRENTICESHIPS & APPRENTICESHIPS FOR YOUNG PEOPLE & ECONOMIC WELL-BEING \\
\hline 4187 & WESTLAND DISTRICT COUNCIL & SAFETY & RECORDED CRIME AND RESOLUTION RATES & CRIME/SAFETY & SOCIAL WELL-BEING \\
\hline 4188 & WESTLAND DISTRICT COUNCIL & SAFETY & YOUTH OFFENDING RATES & YOUTH & SOCIAL WELL-BEING \\
\hline 4189 & WESTLAND DISTRICT COUNCIL & SAFETY & NUMBER OF YOUTH OFFENCES & YOUTH & SOCIAL WELL-BEING \\
\hline 4190 & WESTLAND DISTRICT COUNCIL & SAFETY & MOTOR VEHICLE CRASH RATES & ROAD ACCIDENTS/INJURIES & ECONOMIC WELL-BEING \\
\hline 4191 & WESTLAND DISTRICT COUNCIL & SAFETY & IMPROVEMENTS IN PREPAREDNESS FOR NATURAL HAZARD EVENTS & NATURAL DISASTER/RESILIENCE & ENVIRONMENTAL WELL-BEING \\
\hline 4192 & WESTLAND DISTRICT COUNCIL & SAFETY & $\begin{array}{l}\text { PROGRESS TOWARDS IDENTIFYING HAZARD AREAS WHERE GREATER } \\
\text { MANAGEMENT IS REQUIRED }\end{array}$ & NATURAL DISASTER/RESILIENCE & ENVIRONMENTAL WELL-BEING \\
\hline 4193 & WESTLAND DISTRICT COUNCIL & SAFETY & IMPROVED SAFETY IN PUBLIC AREAS & CRIME/SAFETY & SOCIAL WELL-BEING \\
\hline 4194 & WESTLAND DISTRICT COUNCIL & ENVIRONMENT & VISITOR LEVEL OF APPRECIATION & TOURISM & ECONOMIC WELL-BEING \\
\hline 4195 & WESTLAND DISTRICT COUNCIL & ENVIRONMENT & ACCESS TO NATURAL FEATURES & NATURAL FEATURES & ENVIRONMENTAL WELL-BEING \\
\hline 4197 & WESTLAND DISTRICT COUNCIL & ENVIRONMENT & REDUCTION OF WASTE ON ROADSIDES & TRANSPORT & ECONOMIC WELL-BEING \\
\hline 4198 & WESTLAND DISTRICT COUNCIL & ENVIRONMENT & LEVEL OF VISITOR WASTE MINIMISATION ACTIVITY & TOURISM & ECONOMIC WELL-BEING \\
\hline 4199 & WESTLAND DISTRICT COUNCIL & ECONOMY & OVERALL STATE OF DISTRICT ECONOMIES & ECONOMIC ACTIVITY & ECONOMIC WELL-BEING \\
\hline
\end{tabular}


Appendix A: List of Community Well-beings Outcomes and Measures by Councils

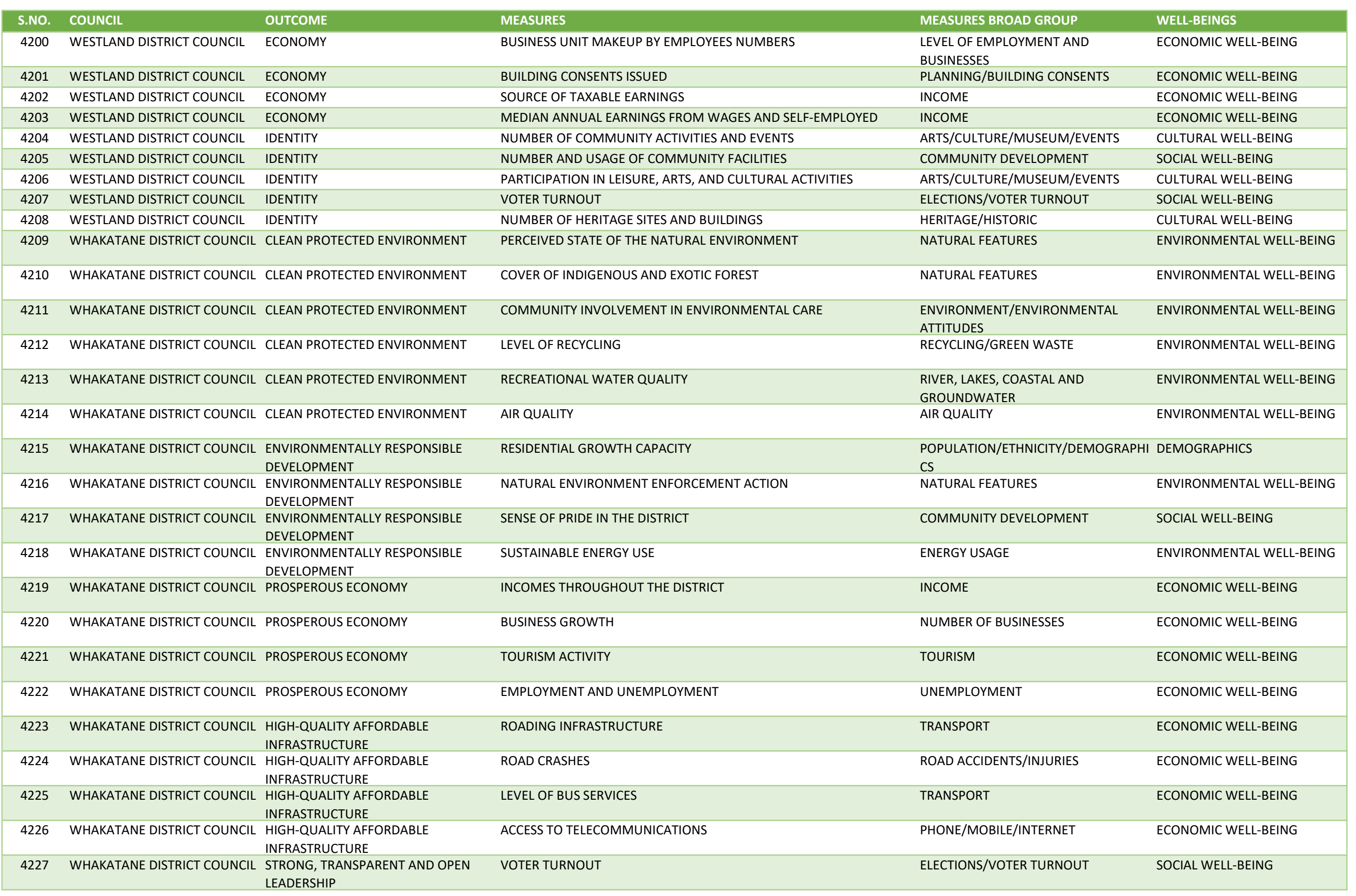


Appendix A: List of Community Well-beings Outcomes and Measures by Councils

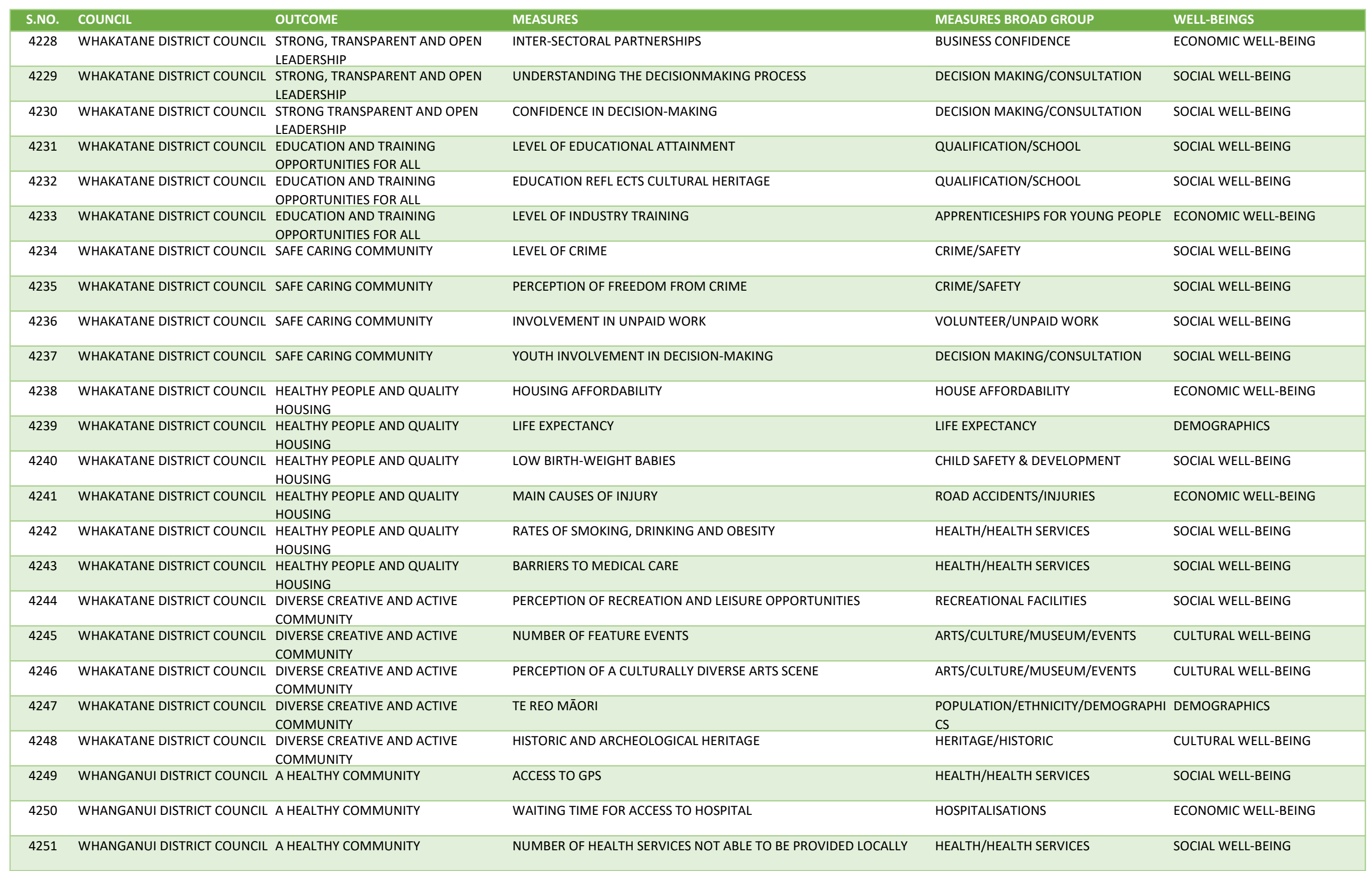


Appendix A: List of Community Well-beings Outcomes and Measures by Councils

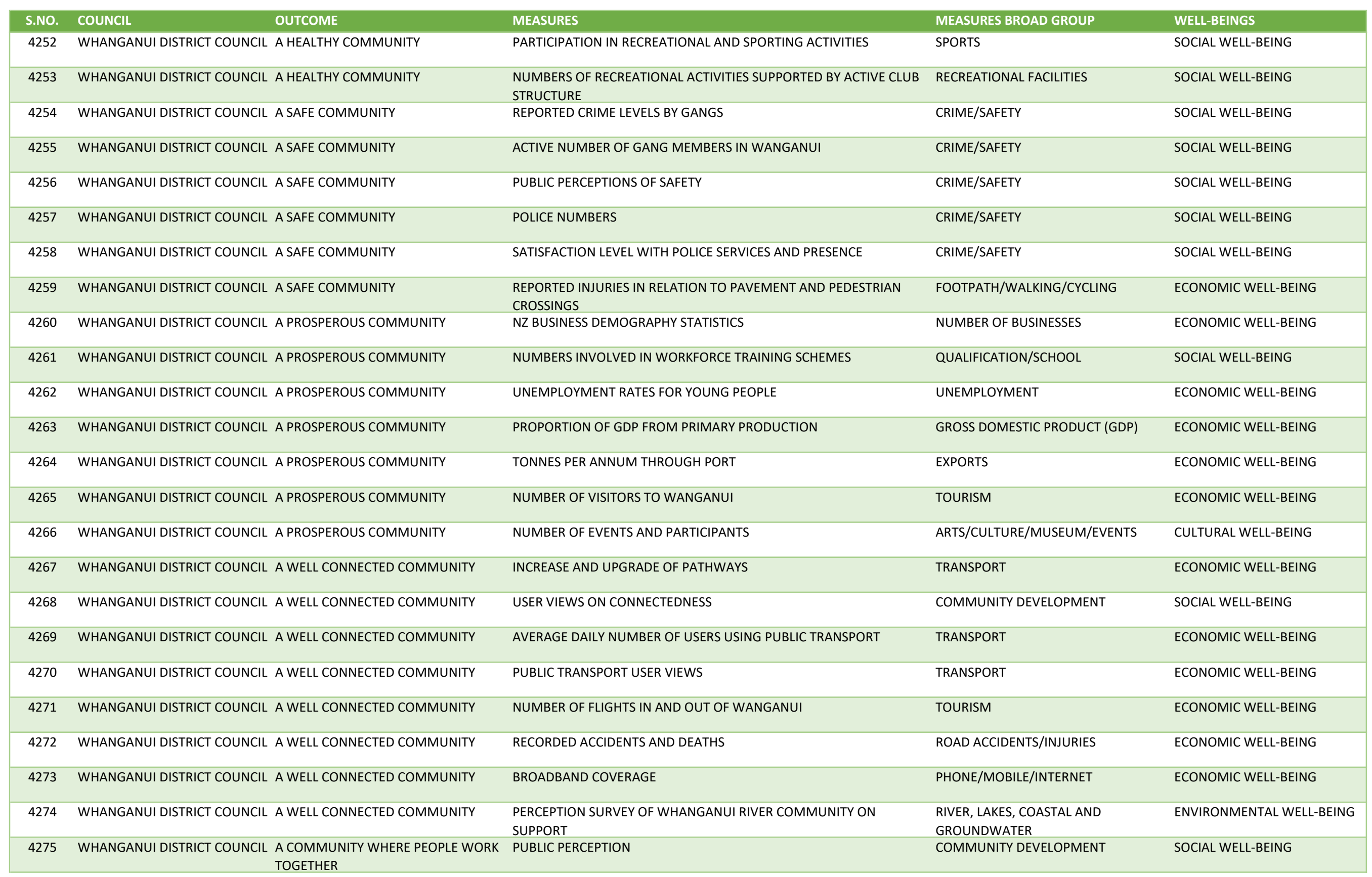


Appendix A: List of Community Well-beings Outcomes and Measures by Councils

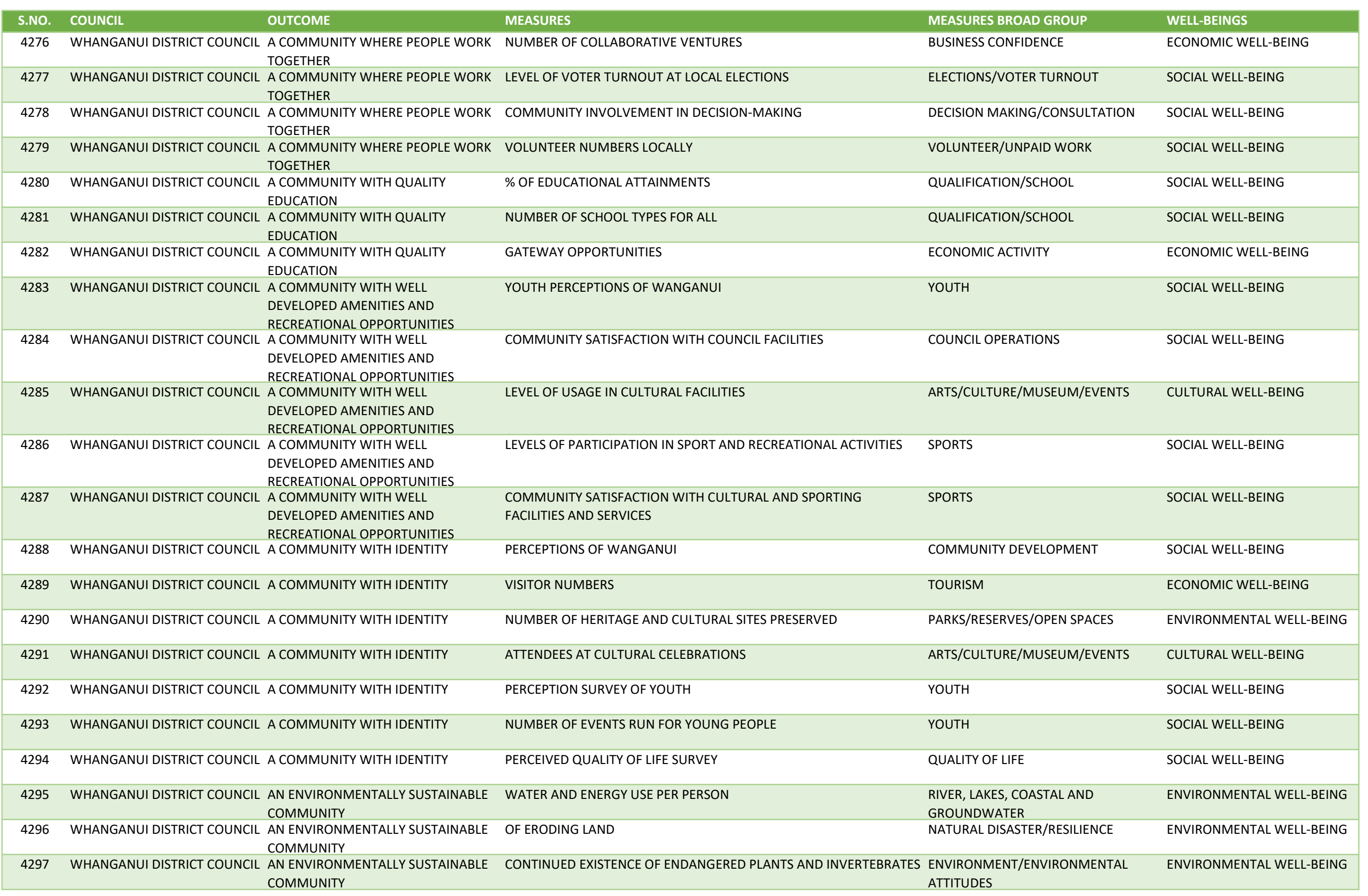


Appendix A: List of Community Well-beings Outcomes and Measures by Councils

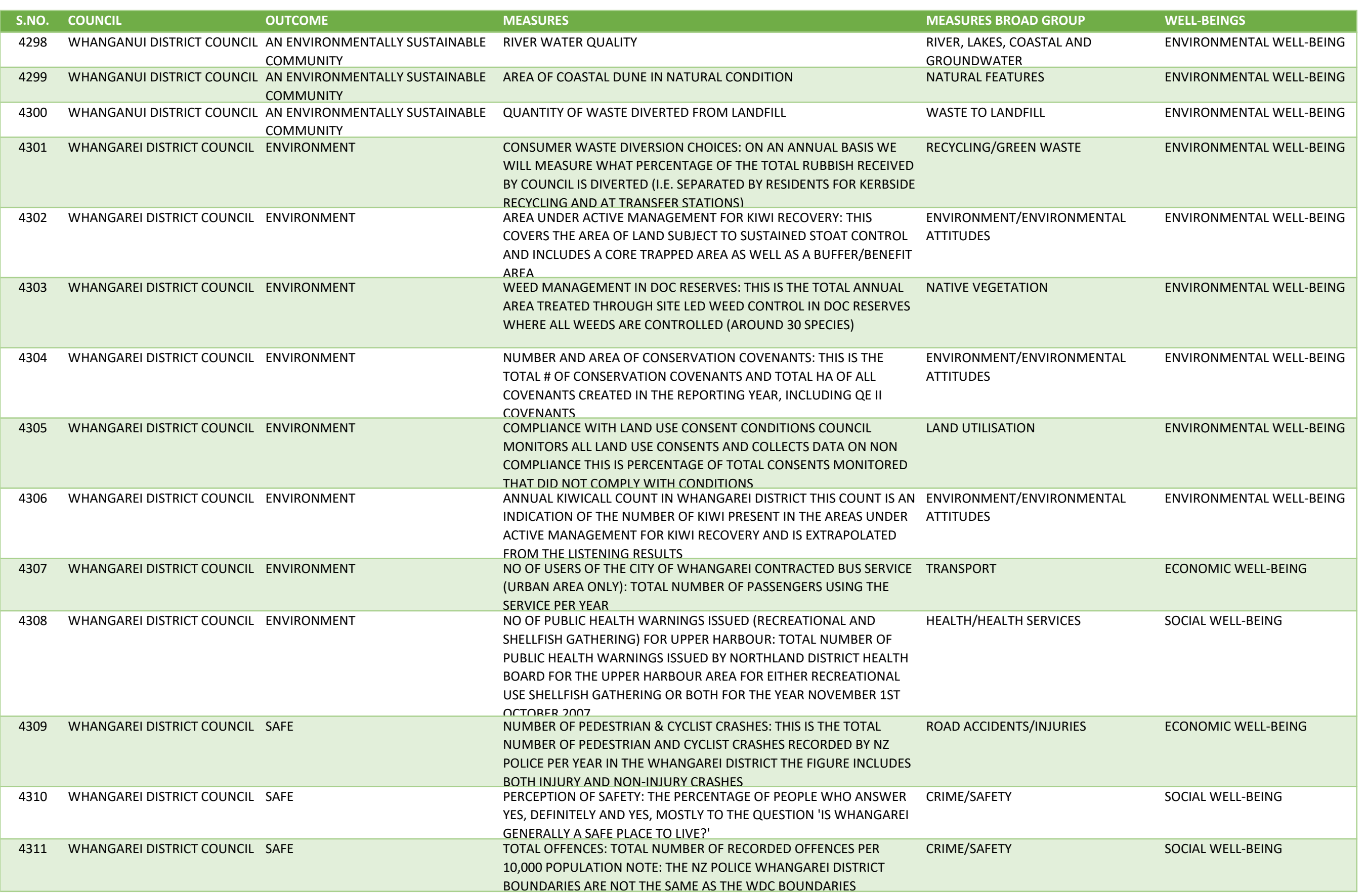




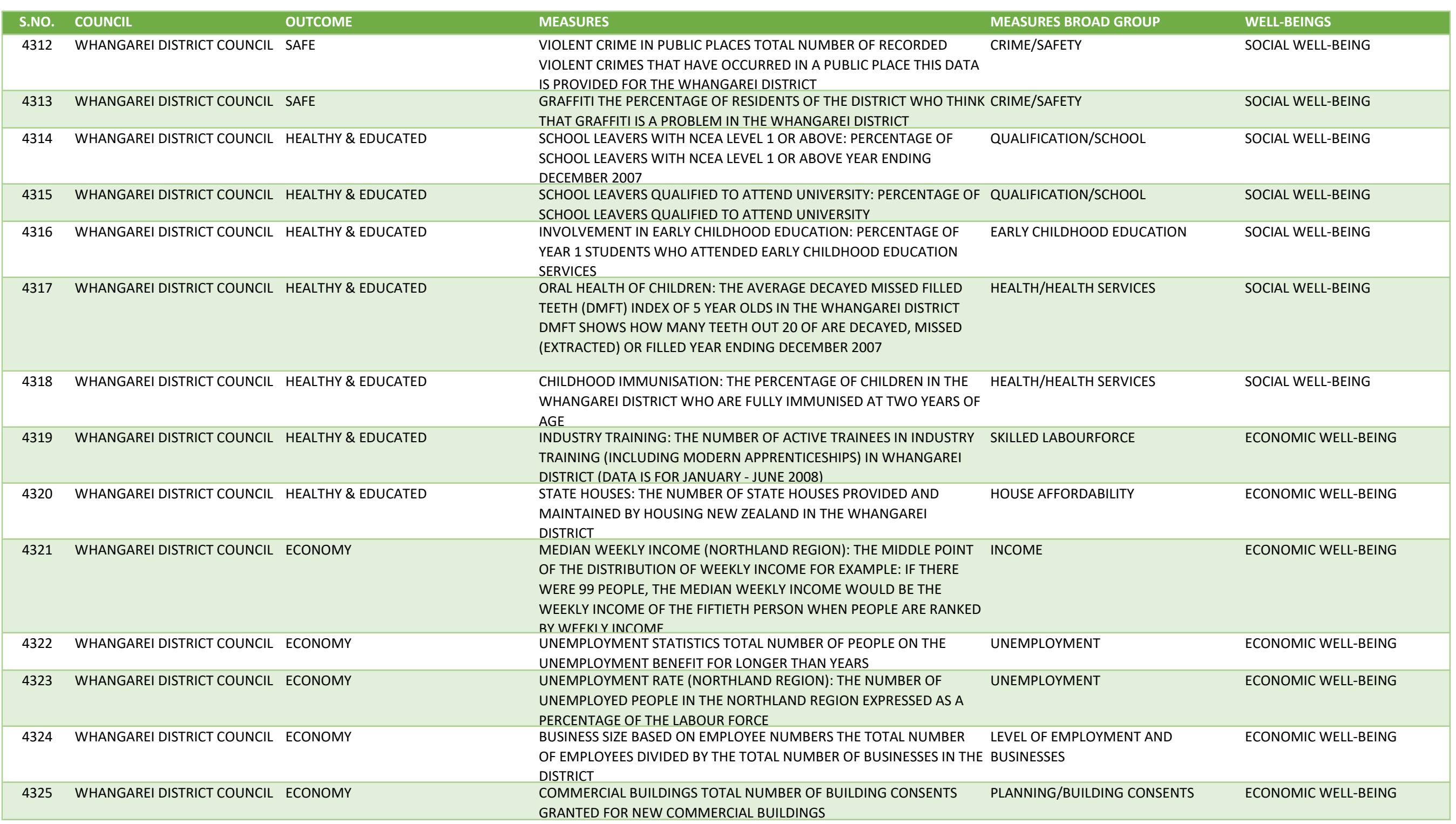


OUTCOME

4328 WHANGAREI DISTRICT COUNCIL COMMUNITY

4329 WHANGAREI DISTRICT COUNCIL COMMUNITY

4330 WHANGAREI DISTRICT COUNCIL COMMUNITY

4331 WHANGAREI DISTRICT COUNCIL COMMUNITY MEASURES

CRAFT TRUST (EXCLUDING SCHOOL HOLIDAY PROGRAMMES)

USAGE OF SPORT FIELDS THE AMOUNT OF USAGE OF SPORT

4334 WHANGAREI DISTRICT COUNCIL CULTURE \& HERITAGE

4335 WHANGAREI DISTRICT COUNCIL CULTURE \& HERITAGE

4336 WHANGAREI DISTRICT COUNCIL CULTURE \& HERITAGE

4337 WHANGAREI DISTRICT COUNCIL CULTURE \& HERITAGE
NET INTERNATIONAL MIGRATION (INCLUDING KIWIS LEAVING AND

RETURNING): PERMANENT AND LONG-TERM ARRIVALS INCLUDING

OVERSEAS MIGRANTS INTENDING TO STAY FOR 12 MONTHS OR MORE

AND NZ RESIDENTS RETURNING AFTER AN ABSENCE OF 12 MONTHS OR

MORE, LESS PERMANENT AND LONG-TERM DEPARTURES, INCLUDING

NZ RESIDENTS DEPARTING FOR MORE THAN 12 MONTHS AND

OVERSEAS VISITORS DEPARTING AFTER A STAY OF 12 MONTHS OR

MORE. THIS DOES NOT INCLUDE NATIONAL MIGRATION FIGURES

DISTRICT RESIDENTS PERCEPTION OF ECONOMIC GROWTH THE PERCENTAGE OF RESIDENTS OF THE DISTRICT WHO THINK THAT THE

LOCAL ECONOMY IS DOING WELL

促

PEOPLE ATTENDING CREATIVE WORKSHOPS RUN BY THE NORTHLAND

PROGRAMMES INVOLVING IN THE PERFORMING ARTS AND OTHER

EVENTS THE NUMBER OF EVENTS THAT ARE HELD AT FORUM NORTH

THAT ARE RUN BY COMMUNITY ORGANISATIONS

PLAY EQUIPMENT AVAIIABITY: PLAY EQUIPMENT WITHIN AVERAGE 500 METRES OR 15 MINUTES WALK OF URBAN POPULATION SPORTING CODES AS A PERCENTAGE OF TOTAL CAPACITY

USAGE OF WHANGAREI AQUATIC CENTRE THE TOTAL NUMBER OF

VISITS TO THE WHANGAREI AQUATIC CENTRE IN THE YEAR

COST OF VANDALISM: THE ANNUAL COST OF REPAIR WORK CARRIED OUT AS A RESULT OF VANDALISM TO COUNCIL'S PARKS, ASSETS AND FACILITIES

IWI MANAGEMENT PLANS: TOTAL \# OF IWI MANAGEMENT PLANS LODGED WITH COUNCIL WITHIN THE FINANCIAL YEAR

SITES OF SIGNIFICANCE TO MAORI: THE GOALS AND OBJECTIVES OF THE HERITAGE/HISTORIC

PROJECT TO IDENTIFY, MANAGE AND PROTECT SITES OF SIGNIFICANCE

TO MAORI ARE ACHIEVED IN ACCORDANCE WITH THE PROJECT PLAN

EPLATION/ETHNICITY/DEMOGRAPHI DEMOGRAPHICS

CS

SKILLED LABOURFORCE

ECONOMIC WELL-BEING

ARTS/CULTURE/MUSEUM/EVENTS CULTURAL WELL-BEING

PARKS/RESERVES/OPEN SPACES

ENVIRONMENTAL WELL-BEING

PAPAKAIANGA HOUSING: DEVELOPMENT AND IMPLEMENTATION OF PAPAKAINGA HOUSING POLICY

HERITAGE SITES REGISTERED WITH NEW ZEALAND HISTORIC PLACES

TRUST: TOTAL OF HISTORIC PLACES HISTORIC AREAS REGISTERED

PLANNING/BUILDING CONSENTS

CULTURAL WELL-BEING

HERITAGE/HISTORIC

CULTURAL WELL-BEING

ONLINE WITH RARANGA TAONGA IN THE WHANGAREI DISTRICT 
Appendix B: Community Well-being Statements by Councils

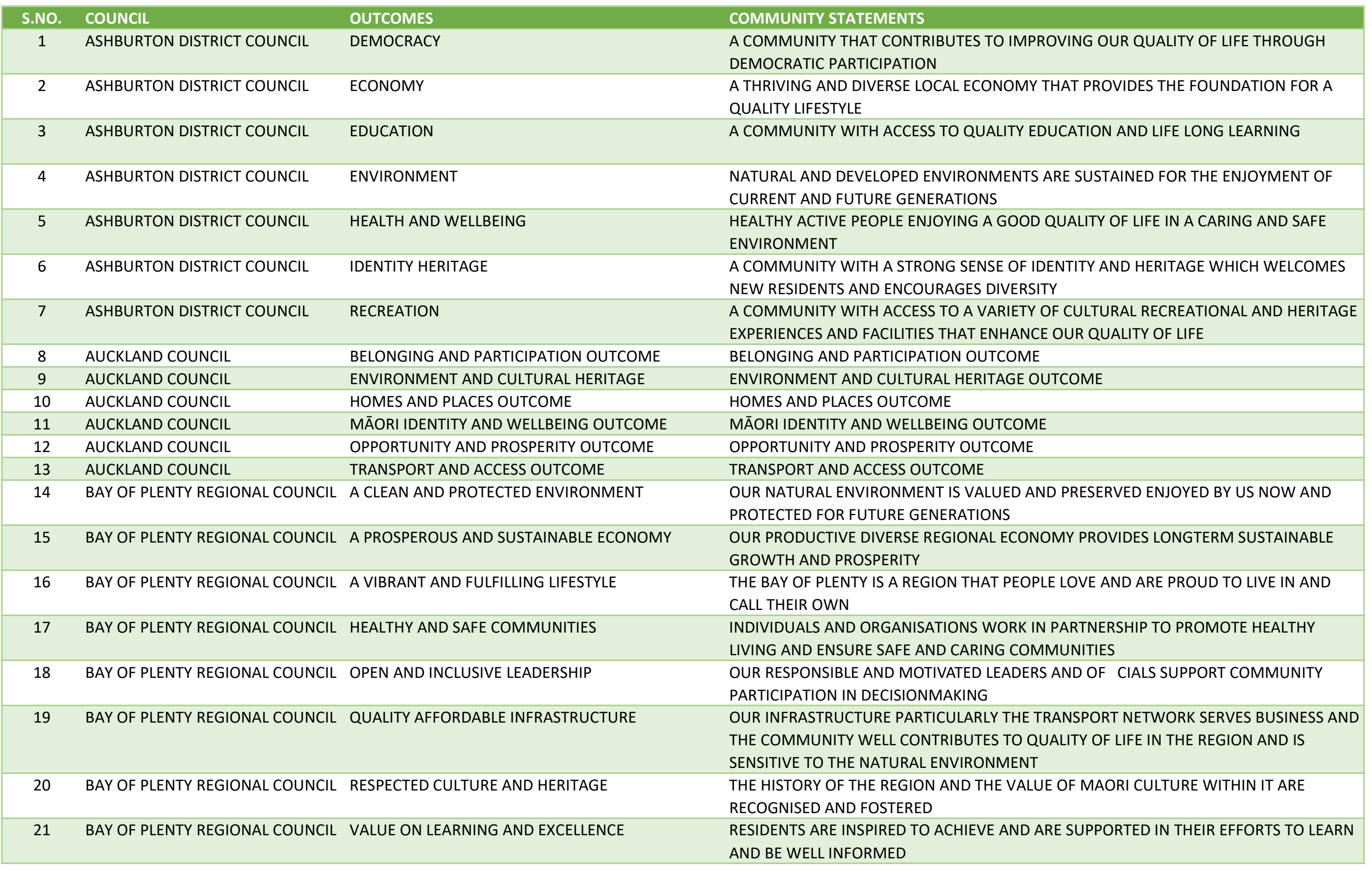


Appendix B: Community Well-being Statements by Councils

\begin{tabular}{|c|c|c|c|}
\hline 22 & BULLER DISTRICT COUNCIL & ECONOMY & ECONOMY \\
\hline 23 & BULLER DISTRICT COUNCIL & EDUCATION & EDUCATION \\
\hline 24 & BULLER DISTRICT COUNCIL & ENVIRONMENT & ENVIRONMENT \\
\hline 25 & BULLER DISTRICT COUNCIL & HEALTH & HEALTH \\
\hline 26 & BULLER DISTRICT COUNCIL & IDENTITY & IDENTITY \\
\hline 27 & BULLER DISTRICT COUNCIL & SAFETY & SAFETY \\
\hline 28 & CANTERBURY REGIONAL COUNCIL & CULTURAL WELLBEING & $\begin{array}{l}\text { THOSE FACTORS THAT ENCOMPASS THE SHARED BELIEFS VALUES CUSTOMS BEHAVIOURS } \\
\text { AND IDENTITIES REFLECTED THROUGH LANGUAGE STORIES EXPERIENCES VISUAL AND } \\
\text { PERFORMING ARTS CEREMONIES AND HERITAGE }\end{array}$ \\
\hline 29 & CANTERBURY REGIONAL COUNCIL & ECONOMIC WELLBEING & $\begin{array}{l}\text { THOSE FACTORS RELATING TO THE CAPACITY OF THE ECONOMY TO GENERATE THE } \\
\text { EMPLOYMENT AND WEALTH NECESSARY TO PROVIDE MANY OF THE PREREQUISITES FOR } \\
\text { SOCIAL WELLBEING SUCH AS HEALTH SERVICES }\end{array}$ \\
\hline 30 & CANTERBURY REGIONAL COUNCIL & ENVIRONMENTAL WELLBEING & $\begin{array}{l}\text { THOSE FACTORS THAT RELATE ULTIMATELY TO THE CAPACITY OF THE NATURAL } \\
\text { ENVIRONMENT TO SUPPORT IN A SUSTAINABLE WAY THE ACTIVITIES THAT CONSTITUTE } \\
\text { COMMUNITY LIFE }\end{array}$ \\
\hline 31 & CANTERBURY REGIONAL COUNCIL & SOCIAL WELLBEING & $\begin{array}{l}\text { THOSE FACTORS THAT ENABLE INDIVIDUALS THEIR FAMILIES HAPU AND COMMUNITIES } \\
\text { TO SET GOALS AND ACHIEVE THEM SUCH AS EDUCATION HEALTH THE STRENGTH OF } \\
\text { COMMUNITY NETWORKS FINANCIAL AND PERSONAL SECURITY RIGHTS AND FREEDOMS } \\
\text { AND LEVELS OF EQUITY }\end{array}$ \\
\hline 32 & CARTERTON DISTRICT COUNCIL & $\begin{array}{l}\text { A DISTRICT WHICH ENCOURAGES LIFELONG } \\
\text { LEARNING }\end{array}$ & A DISTRICT WHICH ENCOURAGES LIFELONG LEARNING \\
\hline 33 & CARTERTON DISTRICT COUNCIL & $\begin{array}{l}\text { A DISTRICT WHICH ENJOYS CREATIVITY AND } \\
\text { RECREATION }\end{array}$ & A DISTRICT WHICH ENJOYS CREATIVITY AND RECREATION \\
\hline 34 & CARTERTON DISTRICT COUNCIL & $\begin{array}{l}\text { A DISTRICT WHICH PROMOTES SUSTAINABLE } \\
\text { INFRASTRUCTURE AND SERVICES }\end{array}$ & A DISTRICT WHICH PROMOTES SUSTAINABLE INFRASTRUCTURE AND SERVICES \\
\hline 35 & CARTERTON DISTRICT COUNCIL & $\begin{array}{l}\text { A DISTRICT WHICH VALUES AND PROTECTS ITS } \\
\text { NATURAL ENVIRONMENT }\end{array}$ & A DISTRICT WHICH VALUES AND PROTECTS ITS NATURAL ENVIRONMENT \\
\hline 36 & CARTERTON DISTRICT COUNCIL & A HEALTHY DISTRICT & A HEALTHY DISTRICT \\
\hline 37 & CARTERTON DISTRICT COUNCIL & A SAFE DISTRICT & A SAFE DISTRICT \\
\hline 38 & CARTERTON DISTRICT COUNCIL & A VIBRANT AND PROSPEROUS ECONOMY & A VIBRANT AND PROSPEROUS ECONOMY \\
\hline 39 & CARTERTON DISTRICT COUNCIL & STRONG POSITIVE LEADERSHIP & STRONG POSITIVE LEADERSHIP \\
\hline 40 & $\begin{array}{l}\text { CENTRAL HAWKES BAY DISTRICT } \\
\text { COUNCIL }\end{array}$ & A LIFETIME OF GOOD HEALTH AND WELLBEING & $\begin{array}{l}\text { AN ENVIRONMENT IN WHICH ALL PEOPLE ARE ABLE TO ACHIEVE A LIFETIME OF GOOD } \\
\text { HEALTH AND WELLBEING THEY ARE PROTECTED FROM THE NEGATIVE EFFECTS OF } \\
\text { POLLUTION DRUG AND ALCOHOL ABUSE AND ILLNESS THEY HAVE CONFIDENCE IN AND } \\
\text { ACCESS TO HEALTH CARE PROVIDED IN OUR REGION }\end{array}$ \\
\hline 41 & $\begin{array}{l}\text { CENTRAL HAWKES BAY DISTRICT } \\
\text { COUNCIL }\end{array}$ & A STRONG PROSPEROUS AND THRIVING ECONOMY & $\begin{array}{l}\text { CENTRAL GOVERNMENT LOCAL GOVERNMENT AND INDIVIDUALS WORKING MORE } \\
\text { EFFECTIVELY TO CREATE CONDITIONS THAT PROMOTE ECONOMIC GROWTH }\end{array}$ \\
\hline
\end{tabular}




\begin{tabular}{|c|c|c|c|}
\hline 42 & $\begin{array}{l}\text { CENTRAL HAWKES BAY DISTRICT } \\
\text { COUNCIL }\end{array}$ & $\begin{array}{l}\text { AN ENVIRONMENT THAT IS SUSTAINED FOR FUTURE } \\
\text { GENERATIONS }\end{array}$ & $\begin{array}{l}\text { COMMUNITIES RESPECTING THE IMPORTANCE OF OUR NATURAL ENVIRONMENT AND } \\
\text { WORKING TOWARDS SUSTAINING THIS FOR THE WELLBEING OF FUTURE GENERATIONS }\end{array}$ \\
\hline 43 & $\begin{array}{l}\text { CENTRAL HAWKES BAY DISTRICT } \\
\text { COUNCIL }\end{array}$ & $\begin{array}{l}\text { COMMUNITIES THAT VALUE AND PROMOTE THEIR } \\
\text { UNIQUE CULTURE AND HERITAGE }\end{array}$ & $\begin{array}{l}\text { COMMUNITIES RECOGNISING ACHIEVING AND PROMOTING THEIR CULTURAL GOALS } \\
\text { ACKNOWLEDGING AND PROTECTING PLACES OF SIGNIFICANCE TO PASS TO FUTURE } \\
\text { GENERATIONS COMMUNITIES RESPECTING AND EMBRACING THE CULTURAL } \\
\text { ENVIRONMENT OF NGATI KAHUNGUNU }\end{array}$ \\
\hline 44 & $\begin{array}{l}\text { CENTRAL HAWKES BAY DISTRICT } \\
\text { COUNCIL }\end{array}$ & SAFE AND ACCESSIBLE RECREATIONAL FACILITIES & $\begin{array}{l}\text { A COORDINATED REGIONAL APPROACH WHERE ALL PEOPLE ARE ABLE TO ACCESS SAFE } \\
\text { RECREATIONAL FACILITIES IN OUR REGION }\end{array}$ \\
\hline 45 & $\begin{array}{l}\text { CENTRAL HAWKES BAY DISTRICT } \\
\text { COUNCIL }\end{array}$ & SAFE AND SECURE COMMUNITIES & $\begin{array}{l}\text { CENTRAL GOVERNMENT LOCAL GOVERNMENT AND THE COMMUNITY WORKING } \\
\text { TOGETHER TO CREATE AN ENVIRONMENT WHICH IS SAFE FOR ALL PEOPLE IN OUR } \\
\text { REGION }\end{array}$ \\
\hline 46 & $\begin{array}{l}\text { CENTRAL HAWKES BAY DISTRICT } \\
\text { COUNCIL }\end{array}$ & $\begin{array}{l}\text { STRONG REGIONAL LEADERSHIP AND A SENSE OF } \\
\text { BELONGING }\end{array}$ & $\begin{array}{l}\text { COORDINATED REGIONAL LEADERSHIP TO ACHIEVE ECONOMIC SOCIAL CULTURAL AND } \\
\text { ENVIRONMENTAL WELLBEING OF OUR COMMUNITIES A DEMOCRATIC ENVIRONMENT } \\
\text { WHERE ALL PEOPLE ARE ABLE TO PARTICIPATE IN THE LIFE OF THEIR COMMUNITIES AND } \\
\text { ACHIEVE A SENSE OF BELONGING }\end{array}$ \\
\hline 47 & $\begin{array}{l}\text { CENTRAL HAWKES BAY DISTRICT } \\
\text { COUNCIL }\end{array}$ & SUPPORTIVE CARING AND INCLUSIVE COMMUNITIES & $\begin{array}{l}\text { CARING COMMUNITIES WHERE PEOPLE FEEL RESPECTED AND VALUED AS IMPORTANT } \\
\text { MEMBERS OF OUR REGION THEY ARE ABLE TO ACCESS RESOURCES TO REACH THEIR FULL } \\
\text { POTENTIAL IN LIFE AND PARTICIPATE TOWARDS ACHIEVING STRONG COMMUNITIES }\end{array}$ \\
\hline 48 & $\begin{array}{l}\text { CENTRAL HAWKES BAY DISTRICT } \\
\text { COUNCIL }\end{array}$ & $\begin{array}{l}\text { TRANSPORT INFRASTRUCTURE AND SERVICES THAT } \\
\text { ARE SAFE EFFECTIVE AND INTEGRATED }\end{array}$ & $\begin{array}{l}\text { A SAFE AND AFFORDABLE TRANSPORT SYSTEM THAT MEETS THE NEEDS OF THE PEOPLE } \\
\text { IN OUR REGION }\end{array}$ \\
\hline 49 & CENTRAL OTAGO DISTRICT COUNCIL & A SAFE AND HEALTHY COMMUNITY & $\begin{array}{l}\text { THIS WOULD BE A VIBRANT COMMUNITY WITH A RANGE OF SERVICES AND FACILITIES } \\
\text { THIS WOULD ALSO BE A COMMUNITY THAT VALUED AND CELEBRATED ITS RICH HERITAGE }\end{array}$ \\
\hline 50 & CENTRAL OTAGO DISTRICT COUNCIL & A SUSTAINABLE ENVIRONMENT & $\begin{array}{l}\text { THIS WOULD BE AN ENVIRONMENT THAT PROVIDES A GOOD QUALITY OF LIFE THE } \\
\text { COMMUNITY WOULD ALSO HAVE A HEALTHY BALANCE BETWEEN ITS NATURAL AND } \\
\text { BUILT ENVIRONMENT }\end{array}$ \\
\hline 51 & CENTRAL OTAGO DISTRICT COUNCIL & A THRIVING ECONOMY & A THRIVING ECONOMY WOULD BE ATTRACTIVE TO BOTH BUSINESS AND RESIDENTS ALIKE \\
\hline 52 & CHATHAM ISLAND COUNCIL & CULTURAL WELLBEING & A CULTURALLY ENRICHED COMMUNITY \\
\hline 53 & CHATHAM ISLAND COUNCIL & ECONOMIC WELLBEING & AN ENTERPRISING INNOVATIVE COMMUNITY \\
\hline 54 & CHATHAM ISLAND COUNCIL & ENVIRONMENTAL WELLBEING & AN ENVIRONMENTALLY CONSCIOUS COMMUNITY \\
\hline 55 & CHATHAM ISLAND COUNCIL & SOCIAL WELLBEING & A SAFE HEALTHY COMMUNITY \\
\hline 58 & CHRISTCHURCH CITY COUNCIL & HEALTHY ENVIRONMENT & SUSTAINABLE USE OF RESOURCES \\
\hline 59 & CHRISTCHURCH CITY COUNCIL & HEALTHY ENVIRONMENT & UNIQUE LANDSCAPES AND INDIGENOUS BIODIVERSITY ARE VALUED \\
\hline
\end{tabular}


Appendix B: Community Well-being Statements by Councils

\begin{tabular}{|c|c|c|c|}
\hline 60 & CHRISTCHURCH CITY COUNCIL & LIVEABLE CITY & A WELL CONNECTED AND ACCESSIBLE CITY \\
\hline 61 & CHRISTCHURCH CITY COUNCIL & LIVEABLE CITY & ST CENTURY GARDEN CITY WE ARE PROUD TO LIVE IN \\
\hline 62 & CHRISTCHURCH CITY COUNCIL & LIVEABLE CITY & SUFFICIENT SUPPLY OF AND ACCESS TO A RANGE OF HOUSING \\
\hline 63 & CHRISTCHURCH CITY COUNCIL & LIVEABLE CITY & VIBRANT THRIVING CENTRAL CITY SUBURBAN AND RURAL CENTRES \\
\hline 64 & CHRISTCHURCH CITY COUNCIL & PROSPEROUS ECONOMY & A PRODUCTIVE ADAPTIVE AND RESILIENT ECONOMIC BASE \\
\hline 65 & CHRISTCHURCH CITY COUNCIL & PROSPEROUS ECONOMY & GREAT PLACE FOR PEOPLE BUSINESS AND INVESTMENT \\
\hline 66 & CHRISTCHURCH CITY COUNCIL & PROSPEROUS ECONOMY & INCLUSIVE EQUITABLE ECONOMY WITH BROADBASED PROSPERITY \\
\hline 67 & CHRISTCHURCH CITY COUNCIL & PROSPEROUS ECONOMY & MODERN AND ROBUST CITY INFRASTRUCTURE AND FACILITIES \\
\hline 68 & CHRISTCHURCH CITY COUNCIL & STRONG COMMUNITIES & ACTIVE PARTICIPATION IN CIVIC LIFE \\
\hline 69 & CHRISTCHURCH CITY COUNCIL & STRONG COMMUNITIES & OUR IDENTITY THROUGH ARTS CULTURE HERITAGE AND SPORT \\
\hline 70 & CHRISTCHURCH CITY COUNCIL & STRONG COMMUNITIES & SAFE AND HEALTHY COMMUNITIES \\
\hline 71 & CHRISTCHURCH CITY COUNCIL & STRONG COMMUNITIES & STRONG SENSE OF COMMUNITY \\
\hline 72 & CHRISTCHURCH CITY COUNCIL & STRONG COMMUNITIES & VALUING THE VOICES OF CHILDREN AND YOUNG PEOPLE \\
\hline 73 & CLUTHA DISTRICT COUNCI & $\begin{array}{l}\text { CREATING A SUSTAINABLE ENVIRONMENT THATS } \\
\text { LIVING UP TO OUR CLEAN GREEN IMAGE }\end{array}$ & $\begin{array}{l}\text { CREATING A SUSTAINABLE ENVIRONMENT THATS LIVING UP TO OUR CLEAN GREEN } \\
\text { IMAGE }\end{array}$ \\
\hline 74 & CLUTHA DISTRICT COUNCI & $\begin{array}{l}\text { DEVELOPING AND MAKING THE MOST OF } \\
\text { EDUCATION TRAINING AND LIFELONG LEARNING } \\
\text { OPPORTUNITIES }\end{array}$ & $\begin{array}{l}\text { DEVELOPING AND MAKING THE MOST OF EDUCATION TRAINING AND LIFELONG } \\
\text { LEARNING OPPORTUNITIES }\end{array}$ \\
\hline 75 & CLUTHA DISTRICT COUNCI & $\begin{array}{l}\text { ENCOURAGING STRONG POSITIVE AND } \\
\text { ENTERPRISING COMMUNITIES }\end{array}$ & ENCOURAGING STRONG POSITIVE AND ENTERPRISING COMMUNITIES \\
\hline 76 & CLUTHA DISTRICT COUNCI & ENSURING OUR ECONOMY GROWS AND DIVERSIFIES & ENSURING OUR ECONOMY GROWS AND DIVERSIFIES \\
\hline 77 & CLUTHA DISTRICT COUNCI & MAKING SURE THERES PLENTY TO SEE AND DO & MAKING SURE THERES PLENTY TO SEE AND DO \\
\hline 78 & CLUTHA DISTRICT COUNCI & $\begin{array}{l}\text { PROVIDING THE INFRASTRUCTURE WE NEED TO } \\
\text { MOVE FORWARD }\end{array}$ & PROVIDING THE INFRASTRUCTURE WE NEED TO MOVE FORWARD \\
\hline 79 & CLUTHA DISTRICT COUNCI & $\begin{array}{l}\text { WORKING TOWARDS A SAFER AND HEALTHIER } \\
\text { COMMUNITY }\end{array}$ & WORKING TOWARDS A SAFER AND HEALTHIER COMMUNITY \\
\hline 80 & DUNEDIN CITY COUNCIL & ACCESSIBLE CITY & $\begin{array}{l}\text { A CITY WITH A TRANSPORT SYSTEM THAT SUPPORTS ECONOMIC DEVELOPMENT AND } \\
\text { WHERE PEOPLE MOVE ABOUT EASILY AND SAFELY }\end{array}$ \\
\hline 81 & DUNEDIN CITY COUNCIL & ACTIVE CITY & $\begin{array}{l}\text { A CITY THAT PROVIDES AND ENCOURAGES PARTICIPATION IN A BROAD RANGE OF } \\
\text { SPORTING RECREATIONAL AND LEISURE ACTIVITIES }\end{array}$ \\
\hline 82 & DUNEDIN CITY COUNCIL & CULTURE AND LEARNING & $\begin{array}{l}\text { A CITY THAT CELEBRATES AND SUPPORTS CULTURE AND EXCELLENCE IN THE ARTS AND } \\
\text { EDUCATION }\end{array}$ \\
\hline 83 & DUNEDIN CITY COUNCIL & SAFE AND HEALTHY PEOPLE & A CITY WHERE RESIDENTS FEEL SAFE AND ENJOY A HEALTHY LIFESTYLE \\
\hline 84 & DUNEDIN CITY COUNCIL & SUPPORTIVE COMMUNITY & $\begin{array}{l}\text { A CITY WHERE RESIDENTS FEEL INCLUDED AND CONNECTED WITH THEIR WIDER } \\
\text { COMMUNITY }\end{array}$ \\
\hline
\end{tabular}


Appendix B: Community Well-being Statements by Councils

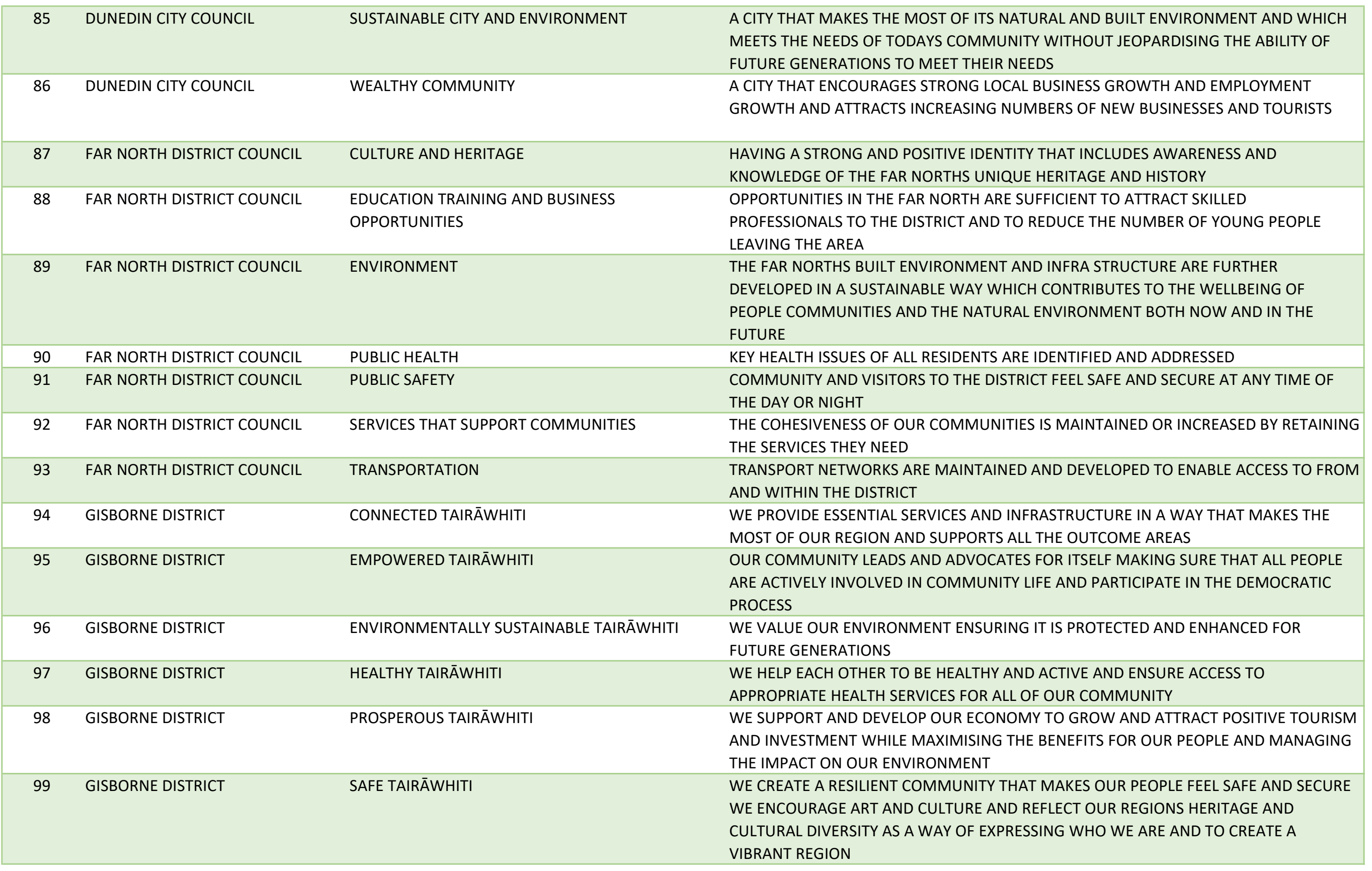


Appendix B: Community Well-being Statements by Councils

\begin{tabular}{|c|c|c|c|}
\hline 100 & GISBORNE DISTRICT & SKILLED AND EDUCATED TAIRĀWHITI & $\begin{array}{l}\text { WE ENSURE THERE ARE LOCAL EDUCATION AND TRAINING OPPORTUNITIES THAT MEET } \\
\text { THE NEEDS OF OUR PEOPLE AND SUPPORT OUR ECONOMY }\end{array}$ \\
\hline 101 & GISBORNE DISTRICT & VIBRANT TAIRĀWHITI & $\begin{array}{l}\text { WE TREASURE THE SPECIAL VALUES THAT MAKE TAIRĀWHITI UNIQUE AND SHAPE OUR } \\
\text { SENSE OF IDENTITY AND PRIDE }\end{array}$ \\
\hline 102 & GORE DISTRICT COUNCIL & $\begin{array}{l}\text { A DIVERSE ECONOMY BUILT FROM OUR STRENGTHS } \\
\text { FOR GROWTH AND PROSPERITY }\end{array}$ & A DIVERSE ECONOMY BUILT FROM OUR STRENGTHS FOR GROWTH AND PROSPERITY \\
\hline 103 & GORE DISTRICT COUNCIL & $\begin{array}{l}\text { A TREASURED ENVIRONMENT WHICH WE CARE FOR } \\
\text { AND WHICH SUPPORTS US NOW AND INTO THE } \\
\text { FUTURE }\end{array}$ & $\begin{array}{l}\text { A TREASURED ENVIRONMENT WHICH WE CARE FOR AND WHICH SUPPORTS US NOW } \\
\text { AND INTO THE FUTURE }\end{array}$ \\
\hline 104 & GORE DISTRICT COUNCIL & $\begin{array}{l}\text { A WELLEDUCATED AND SKILLED COMMUNITY } \\
\text { CONTINUALLY SEEKING FURTHER OPPORTUNITIES } \\
\text { TO LEARN }\end{array}$ & $\begin{array}{l}\text { A WELLEDUCATED AND SKILLED COMMUNITY CONTINUALLY SEEKING FURTHER } \\
\text { OPPORTUNITIES TO LEARN }\end{array}$ \\
\hline 105 & GORE DISTRICT COUNCIL & $\begin{array}{l}\text { SAFE PLACES IN A CARING SOCIETY THAT IS FREE } \\
\text { FROM CRIME }\end{array}$ & SAFE PLACES IN A CARING SOCIETY THAT IS FREE FROM CRIME \\
\hline 106 & GORE DISTRICT COUNCIL & SOUTHLAND IS A GREAT PLACE TO LIVE & SOUTHLAND IS A GREAT PLACE TO LIVE \\
\hline 107 & GORE DISTRICT COUNCIL & $\begin{array}{l}\text { STRONG EFFECTIVE LEADERSHIP TAKING US INTO } \\
\text { THE FUTURE }\end{array}$ & STRONG EFFECTIVE LEADERSHIP TAKING US INTO THE FUTURE \\
\hline 108 & GORE DISTRICT COUNCIL & WE ARE HEALTHY PEOPLE & WE ARE HEALTHY PEOPLE \\
\hline 109 & GREY DISTRICT COUNCIL & ECONOMY & $\begin{array}{l}\text { A THRIVING RESILIENT AND INNOVATIVE ECONOMY CREATING OPPORTUNITIES FOR } \\
\text { GROWTH AND EMPLOYMENT }\end{array}$ \\
\hline 110 & GREY DISTRICT COUNCIL & EDUCATION & $\begin{array}{l}\text { A DISTRICT THAT VALUES AND SUPPORTS LEARNING WITH ACCESSIBLE RELEVANT } \\
\text { EDUCATION AND TRAINING OPPORTUNITIES }\end{array}$ \\
\hline 111 & GREY DISTRICT COUNCIL & ENVIRONMENT & $\begin{array}{l}\text { THAT THE DISTINCTIVE CHARACTER OF THE ENVIRONMENT IS APPRECIATED AND } \\
\text { RETAINED }\end{array}$ \\
\hline 112 & GREY DISTRICT COUNCIL & HEALTH & HEALTHY COMMUNITIES WITH ACCESS TO QUALITY FACILITIES AND SERVICES \\
\hline 113 & GREY DISTRICT COUNCIL & IDENTITY & A HAPPENING DISTRICT WITH A STRONG COMMUNITY SPIRIT AND DISTINCTIVE LIFESTYLE \\
\hline 114 & GREY DISTRICT COUNCIL & SAFETY & A DISTRICT THAT IS A SAFE PLACE TO LIVE \\
\hline 115 & HAMILTON CITY COUNCIL & HEALTHY AND HAPPY & ACTIVE AND HEALTHY PEOPLE WITH ACCESS TO AFFORDABLE FACILITIES AND SERVICES \\
\hline 116 & HAMILTON CITY COUNCIL & INTELLIGENT AND PROGRESSIVE CITY & $\begin{array}{l}\text { BUSINESS GROWTH THAT IS IN HARMONY WITH THE CITYS IDENTITY AND COMMUNITY } \\
\text { SPIRIT }\end{array}$ \\
\hline 117 & HAMILTON CITY COUNCIL & SAFETY AND COMMUNITY SPIRIT & A SAFE FRIENDLY CITY WHERE ALL PEOPLE FEEL CONNECTED AND VALUED \\
\hline 118 & HAMILTON CITY COUNCIL & SUSTAINABLE AND WELLPLANNED & $\begin{array}{l}\text { AN ATTRACTIVE CITY THAT IS PLANNED FOR THE WELLBEING OF PEOPLE AND NATURE } \\
\text { NOW AND IN THE FUTURE }\end{array}$ \\
\hline 119 & HAMILTON CITY COUNCIL & UNIQUE IDENTITY & $\begin{array}{l}\text { A CITY WITH A STRONG IDENTITY THAT RECOGNISES THE SIGNIFICANCE OF ITS RIVER AND } \\
\text { HISTORY }\end{array}$ \\
\hline 120 & HAMILTON CITY COUNCIL & VIBRANT AND CREATIVE & A CITY THAT ENCOURAGES CREATIVITY FOR A VIBRANT LIFESTYLE \\
\hline
\end{tabular}


Appendix B: Community Well-being Statements by Councils

\begin{tabular}{|c|c|c|c|}
\hline 121 & HAMILTON CITY COUNCIL & WORKING TOGETHER & COLLABORATIVE DECISIONMAKING AND PLANNING ARE COMMON PRACTICE \\
\hline 122 & HASTINGS DISTRICT COUNCIL & MOVING AROUND & MOVING AROUND \\
\hline 123 & HASTINGS DISTRICT COUNCIL & OUR ECONOMY & OUR ECONOMY \\
\hline 124 & HASTINGS DISTRICT COUNCIL & OUR LIFESTYLE AND PEOPLE & OUR LIFESTYLE AND PEOPLE \\
\hline 125 & HASTINGS DISTRICT COUNCIL & RESOURCES WE NEED & RESOURCES WE NEED \\
\hline 126 & HASTINGS DISTRICT COUNCIL & WHERE WE LIVE & WHERE WE LIVE \\
\hline 127 & HASTINGS DISTRICT COUNCIL & ZERO WASTE & ZERO WASTE \\
\hline 128 & HAURAKI DISTRICT COUNCIL & $\begin{array}{l}\text { COMMUNITY OUTCOME VIBRANT AND SUSTAINABLE } \\
\text { BUSINESSES AND BUSINESS ECONOMIES IN OUR } \\
\text { DISTRICT }\end{array}$ & $\begin{array}{l}\text { COMMUNITY OUTCOME VIBRANT AND SUSTAINABLE BUSINESSES AND BUSINESS } \\
\text { ECONOMIES IN OUR DISTRICT }\end{array}$ \\
\hline 129 & HAURAKI DISTRICT COUNCIL & $\begin{array}{l}\text { CULTURAL VALUES OF TANGATA WHENUA } \\
\text { THROUGHOUT THE DISTRICT BE RESPECTED AND } \\
\text { SUPPORTED THROUGH FURTHER DEVELOPMENT OF } \\
\text { CONSULTATION PARTICIPATION AND PARTNERSHIPS }\end{array}$ & $\begin{array}{l}\text { CULTURAL VALUES OF TANGATA WHENUA THROUGHOUT THE DISTRICT BE RESPECTED } \\
\text { AND SUPPORTED THROUGH FURTHER DEVELOPMENT OF CONSULTATION PARTICIPATION } \\
\text { AND PARTNERSHIPS }\end{array}$ \\
\hline 130 & HAURAKI DISTRICT COUNCIL & $\begin{array}{l}\text { HAURAKI DISTRICT RESIDENTS BE GIVEN THE } \\
\text { OPPORTUNITY TO PARTICIPATE IN EDUCATION AND } \\
\text { TRAINING PROGRAMMES }\end{array}$ & $\begin{array}{l}\text { HAURAKI DISTRICT RESIDENTS BE GIVEN THE OPPORTUNITY TO PARTICIPATE IN } \\
\text { EDUCATION AND TRAINING PROGRAMMES }\end{array}$ \\
\hline 131 & HAURAKI DISTRICT COUNCIL & $\begin{array}{l}\text { INTEGRATED PROVISION OF QUALITY HEALTH AND } \\
\text { SOCIAL SERVICES THROUGHOUT OUR DISTRICT }\end{array}$ & $\begin{array}{l}\text { INTEGRATED PROVISION OF QUALITY HEALTH AND SOCIAL SERVICES THROUGHOUT OUR } \\
\text { DISTRICT }\end{array}$ \\
\hline 132 & HAURAKI DISTRICT COUNCIL & $\begin{array}{l}\text { MAINTAIN AND PROTECT THE VIBRANCY OF RURAL } \\
\text { COMMUNITIES WITHIN OUR DISTRICT }\end{array}$ & MAINTAIN AND PROTECT THE VIBRANCY OF RURAL COMMUNITIES WITHIN OUR DISTRICT \\
\hline 133 & HAURAKI DISTRICT COUNCIL & $\begin{array}{l}\text { OUR HAURAKI YOUTH BE PROVIDED WITH GREATER } \\
\text { OPPORTUNITIES TO PARTICIPATE IN THE } \\
\text { DECISIONMAKING PROCESSES PERTAINING TO THE } \\
\text { DEVELOPMENT OF OUR COMMUNITIES }\end{array}$ & $\begin{array}{l}\text { OUR HAURAKI YOUTH BE PROVIDED WITH GREATER OPPORTUNITIES TO PARTICIPATE IN } \\
\text { THE DECISIONMAKING PROCESSES PERTAINING TO THE DEVELOPMENT OF OUR } \\
\text { COMMUNITIES }\end{array}$ \\
\hline 134 & HAURAKI DISTRICT COUNCIL & $\begin{array}{l}\text { VIBRANT AND SUSTAINABLE BUSINESSES AND } \\
\text { BUSINESS ECONOMIES IN OUR DISTRICT }\end{array}$ & VIBRANT AND SUSTAINABLE BUSINESSES AND BUSINESS ECONOMIES IN OUR DISTRICT \\
\hline 135 & HAURAKI DISTRICT COUNCIL & $\begin{array}{l}\text { WE ENCOURAGE INCREASED OPPORTUNITIES TO } \\
\text { PARTICIPATE IN RECREATIONAL SPORTING AND } \\
\text { CULTURAL ACTIVITIES }\end{array}$ & $\begin{array}{l}\text { WE ENCOURAGE INCREASED OPPORTUNITIES TO PARTICIPATE IN RECREATIONAL } \\
\text { SPORTING AND CULTURAL ACTIVITIES }\end{array}$ \\
\hline 136 & HAWKES BAY REGIONAL COUNCIL & A LIFETIME OF GOOD HEALTH AND WELLBEING & $\begin{array}{l}\text { HAWKES BAY PEOPLE LIVE IN SAFE AND SECURE COMMUNITIES WHERE THEY ARE } \\
\text { SUPPORTED TO LIVE HEALTHY LIVES AND ACHIEVE THEIR FULL POTENTIAL }\end{array}$ \\
\hline 137 & HAWKES BAY REGIONAL COUNCIL & A LIFETIME OF GOOD HEALTH AND WELLBEING & $\begin{array}{l}\text { HAWKES BAY RESIDENTS WILL HAVE ACCESS TO HIGHQUALITY HEALTH AND DISABILITY } \\
\text { SERVICES }\end{array}$ \\
\hline 138 & HAWKES BAY REGIONAL COUNCIL & A LIFETIME OF GOOD HEALTH AND WELLBEING & HAWKES BAY WILL HAVE HIGH STANDARDS OF POPULATION HEALTH \\
\hline
\end{tabular}


Appendix B: Community Well-being Statements by Councils

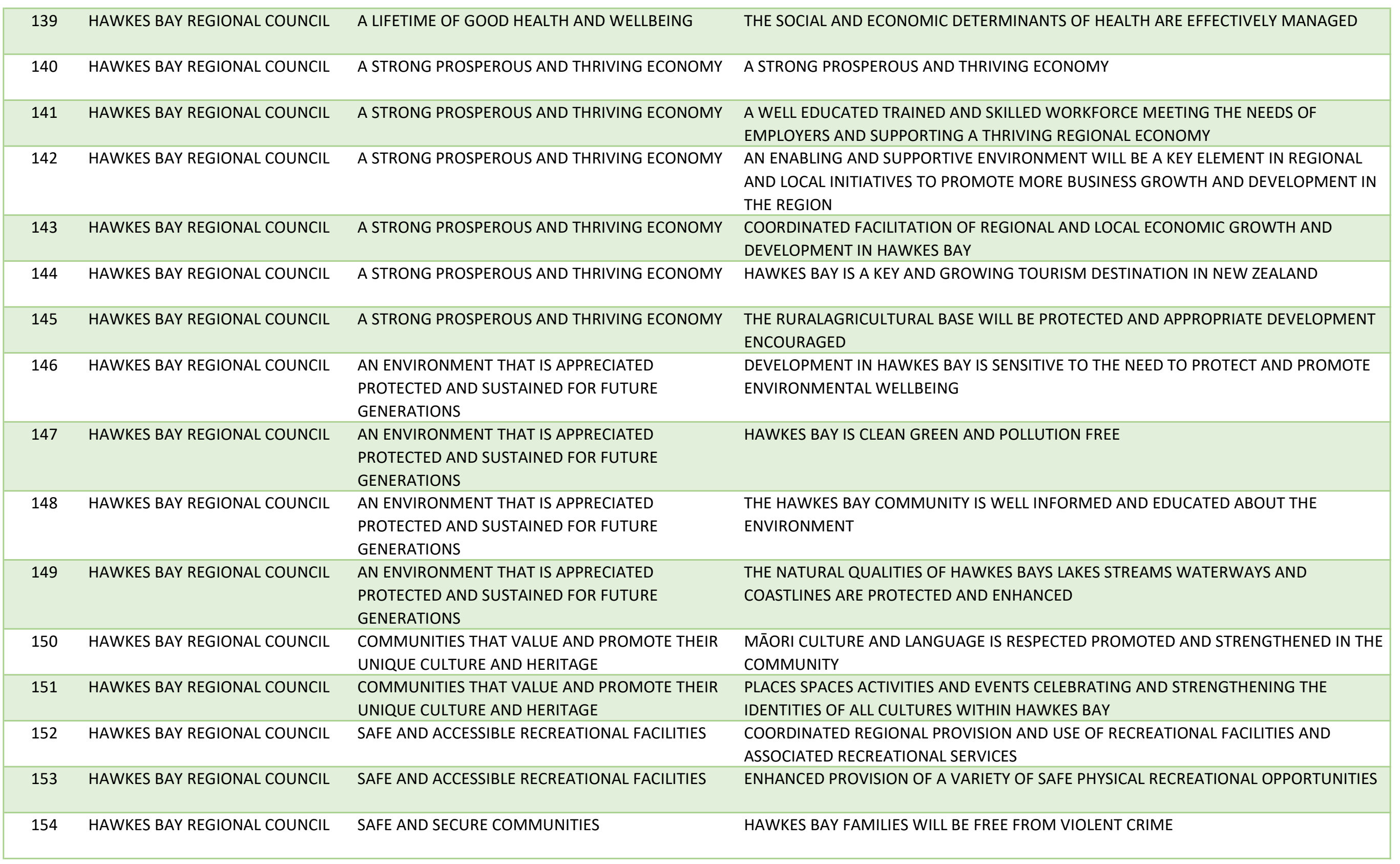


Appendix B: Community Well-being Statements by Councils

\begin{tabular}{|c|c|c|c|}
\hline 155 & HAWKES BAY REGIONAL COUNCIL & SAFE AND SECURE COMMUNITIES & HAWKES BAY PUBLIC SPACES WILL BE CRIME FREE \\
\hline 156 & HAWKES BAY REGIONAL COUNCIL & SAFE AND SECURE COMMUNITIES & PEOPLE IN HAWKES BAY WILL BE FREE FROM BURGLARY THEFT AND PROPERTY DAMAGE \\
\hline 158 & HAWKES BAY REGIONAL COUNCIL & SUPPORTIVE CARING AND INCLUSIVE COMMUNITIES & $\begin{array}{l}\text { HAWKES BAY PEOPLE ARE ENGAGED WITH THEIR COMMUNITY REGARDLESS OF AGE } \\
\text { ABILITY OR ETHNICITY }\end{array}$ \\
\hline 160 & HAWKES BAY REGIONAL COUNCIL & SUPPORTIVE CARING AND INCLUSIVE COMMUNITIES & $\begin{array}{l}\text { THE DELIVERY OF KEY SOCIAL SERVICES IN HAWKES BAY IS UNDERTAKEN IN A } \\
\text { COORDINATED AND ACCESSIBLE MANNER }\end{array}$ \\
\hline 161 & HAWKES BAY REGIONAL COUNCIL & $\begin{array}{l}\text { TRANSPORT INFRASTRUCTURE AND SERVICES THAT } \\
\text { ARE SAFE EFFECTIVE AND INTEGRATED }\end{array}$ & A SAFE AND EFFICIENT TRANSPORT NETWORK \\
\hline 162 & HAWKES BAY REGIONAL COUNCIL & $\begin{array}{l}\text { TRANSPORT INFRASTRUCTURE AND SERVICES THAT } \\
\text { ARE SAFE EFFECTIVE AND INTEGRATED }\end{array}$ & AN INCLUSIVE ACCESSIBLE AND AFFORDABLE TRANSPORT SYSTEM \\
\hline 163 & HAWKES BAY REGIONAL COUNCIL & $\begin{array}{l}\text { TRANSPORT INFRASTRUCTURE AND SERVICES THAT } \\
\text { ARE SAFE EFFECTIVE AND INTEGRATED }\end{array}$ & $\begin{array}{l}\text { AN INTEGRATED TRANSPORT SYSTEM WITH EFFICIENT LINKAGES SUPPORTING NATIONAL } \\
\text { AND REGIONAL ECONOMIC DEVELOPMENT }\end{array}$ \\
\hline 164 & HOROWHENUA DISTRICT COUNCIL & AN EXUBERANT ECONOMY & $\begin{array}{l}\text { WE ARE A WELCOMING ENABLING AND BUSINESS FRIENDLYDISTRICT THAT ENCOURAGES } \\
\text { LOCAL ECONOMIC DEVELOPMENT }\end{array}$ \\
\hline 165 & HOROWHENUA DISTRICT COUNCIL & AN EXUBERANT ECONOMY & $\begin{array}{l}\text { WE PROVIDE OPPORTUNITIES FOR PEOPLE OF ALL AGES AND AT ALLPHASES OF LIFE TO } \\
\text { ENJOY A QUALITY OF LIVING WITHIN OUR DISTRICTTHAT IS ECONOMICALLY SUSTAINABLE } \\
\text { AND AFFORDABLE }\end{array}$ \\
\hline 166 & HOROWHENUA DISTRICT COUNCIL & AN EXUBERANT ECONOMY & $\begin{array}{l}\text { WE RECOGNISE AND MANAGE THE EFFECTS OF POPULATIONGROWTH AND ACTIVELY } \\
\text { PROMOTE THE DISTRICT AS A DESTINATIONOF CHOICE }\end{array}$ \\
\hline 167 & HOROWHENUA DISTRICT COUNCIL & AN EXUBERANT ECONOMY & $\begin{array}{l}\text { WE VALUE THE ROLE OUR DISTRICTS NATURAL CULTURAL AND SOCIALASSETS PLAY IN } \\
\text { SUPPORTING ECONOMIC DEVELOPMENT }\end{array}$ \\
\hline 168 & HOROWHENUA DISTRICT COUNCIL & ENABLING INFRASTRUCTURE & $\begin{array}{l}\text { OUR COMMUNITY FACILITIES AND INFRASTRUCTURE ARE BUILT RESILIENTLY PREPARING } \\
\text { US TO COMBAT CLIMATE CHANGE AND NATURAL HAZARDS }\end{array}$ \\
\hline 169 & HOROWHENUA DISTRICT COUNCIL & ENABLING INFRASTRUCTURE & $\begin{array}{l}\text { OUR FACILITIES AND INFRASTRUCTURE SERVICES ARE PLANNED AND DEVELOPED FOR } \\
\text { EACH TOWN OR VILLAGE IN OUR DISTRICT TO MEET CURRENT AND FUTURE NEEDS }\end{array}$ \\
\hline 170 & HOROWHENUA DISTRICT COUNCIL & ENABLING INFRASTRUCTURE & $\begin{array}{l}\text { WASTE REDUCTION RECYCLING ENERGY CONSERVATION AND EFFICIENCY AND WATER } \\
\text { CONSERVATION ARE PROMOTED AS PART OF HOW WE ALL LIVE }\end{array}$ \\
\hline
\end{tabular}


Appendix B: Community Well-being Statements by Councils

\begin{tabular}{|c|c|c|c|}
\hline 172 & HOROWHENUA DISTRICT COUNCIL & PARTNERSHIP WITH TANGATA WHENUA & $\begin{array}{l}\text { WE ACKNOWLEDGE OUR PARTNERSHIP WITH THE TANGATA WHENUA OF OUR DISTRICT } \\
\text { THROUGH A PROACTIVE APPROACH TO THE TREATY OF WAITANGI AND ITS PRINCIPLES }\end{array}$ \\
\hline 173 & HOROWHENUA DISTRICT COUNCIL & PARTNERSHIP WITH TANGATA WHENUA & $\begin{array}{l}\text { WE SUPPORT MANA WHENUA TO MAINTAIN AND ENHANCE THEIR TRADITIONS WITH } \\
\text { THEIR ANCESTRAL LANDS AND WATERWAYS WAHI TAPU AND OTHER TAONGA }\end{array}$ \\
\hline 174 & HOROWHENUA DISTRICT COUNCIL & PARTNERSHIP WITH TANGATA WHENUA & WE VALUE WORKING TOGETHER TO ACHIEVE COMMON GOALS \\
\hline 175 & HOROWHENUA DISTRICT COUNCIL & PARTNERSHIP WITH TANGATA WHENUA & $\begin{array}{l}\text { WE WORK WITH LOCAL MARAE HAPU AND IWI TO SUPPORT THEIR DEVELOPMENT AND } \\
\text { CAPACITY BUILDING }\end{array}$ \\
\hline 176 & HOROWHENUA DISTRICT COUNCIL & STUNNING ENVIRONMENT & $\begin{array}{l}\text { WE ACTIVELY SUPPORT IMPROVING THE HEALTH OF OUR DISTRICTS RIVERS LAKES AND } \\
\text { WATERWAYS }\end{array}$ \\
\hline 177 & HOROWHENUA DISTRICT COUNCIL & STUNNING ENVIRONMENT & WE ARE PROUD OF OUR NATURAL AND BUILT ENVIRONMENTS \\
\hline 178 & HOROWHENUA DISTRICT COUNCIL & STUNNING ENVIRONMENT & $\begin{array}{l}\text { WE RECOGNISE THAT OUR NATURAL ENVIRONMENT PLAYS A VITAL ROLE IN SUSTAINING } \\
\text { THE DISTRICT }\end{array}$ \\
\hline 179 & HOROWHENUA DISTRICT COUNCIL & STUNNING ENVIRONMENT & $\begin{array}{l}\text { WE SUSTAINABLY MANAGE OUR ENVIRONMENT AND NATURAL RESOURCES TO ENSURE } \\
\text { THEY CAN BE ENJOYED NOW AND BY FUTURE GENERATIONS }\end{array}$ \\
\hline 180 & HOROWHENUA DISTRICT COUNCIL & THRIVING COMMUNITIES & $\begin{array}{l}\text { OUR COMMUNITIES ARE INCLUSIVE CONNECTED AND HAVE THEOPPORTUNITY TO } \\
\text { INFLUENCE LOCAL OUTCOMES AND DECISIONS }\end{array}$ \\
\hline 181 & HOROWHENUA DISTRICT COUNCIL & THRIVING COMMUNITIES & $\begin{array}{l}\text { OUR COMMUNITIES ARE RESILIENT AND PROVIDE FORINTERGENERATIONAL WELLBEING } \\
\text { THROUGH NETWORKS WHICH CAREFOR ALL AGES }\end{array}$ \\
\hline 182 & HOROWHENUA DISTRICT COUNCIL & THRIVING COMMUNITIES & $\begin{array}{l}\text { OUR COMMUNITIES HAVE A SENSE OF PLACE THAT MAKES PEOPLE FEEL PROUD TO LIVE } \\
\text { HERE }\end{array}$ \\
\hline 183 & HOROWHENUA DISTRICT COUNCIL & THRIVING COMMUNITIES & $\begin{array}{l}\text { OUR COMMUNITIES HAVE ACCESS TO HEALTH SOCIAL ANDRECREATION FACILITIES } \\
\text { WHICH ENABLE PEOPLE TO ENJOY POSITIVEHEALTHY LIFESTYLES }\end{array}$ \\
\hline 184 & HOROWHENUA DISTRICT COUNCIL & THRIVING COMMUNITIES & $\begin{array}{l}\text { OUR COMMUNITIES INDIVIDUALLY AND COLLECTIVELY PARTICIPATE INCOMMUNITY } \\
\text { DEVELOPMENT }\end{array}$ \\
\hline 185 & HOROWHENUA DISTRICT COUNCIL & THRIVING COMMUNITIES & $\begin{array}{l}\text { OUR COMMUNITIES LIVE IN A SAFE AND SUPPORTIVE ENVIRONMENTAND ARE } \\
\text { EMPOWERED TO MAKE POSITIVE AND HEALTHY LIFESTYLECHOICES }\end{array}$ \\
\hline 186 & HOROWHENUA DISTRICT COUNCIL & VIBRANT CULTURES & OUR COMMUNITYS CULTURAL DIVERSITY IS CELEBRATED \\
\hline 187 & HOROWHENUA DISTRICT COUNCIL & VIBRANT CULTURES & WE ARE PROUD OF THE HERITAGE AND DIVERSITY OF OUR DISTRICT AND OUR PEOPLE \\
\hline
\end{tabular}


Appendix B: Community Well-being Statements by Councils

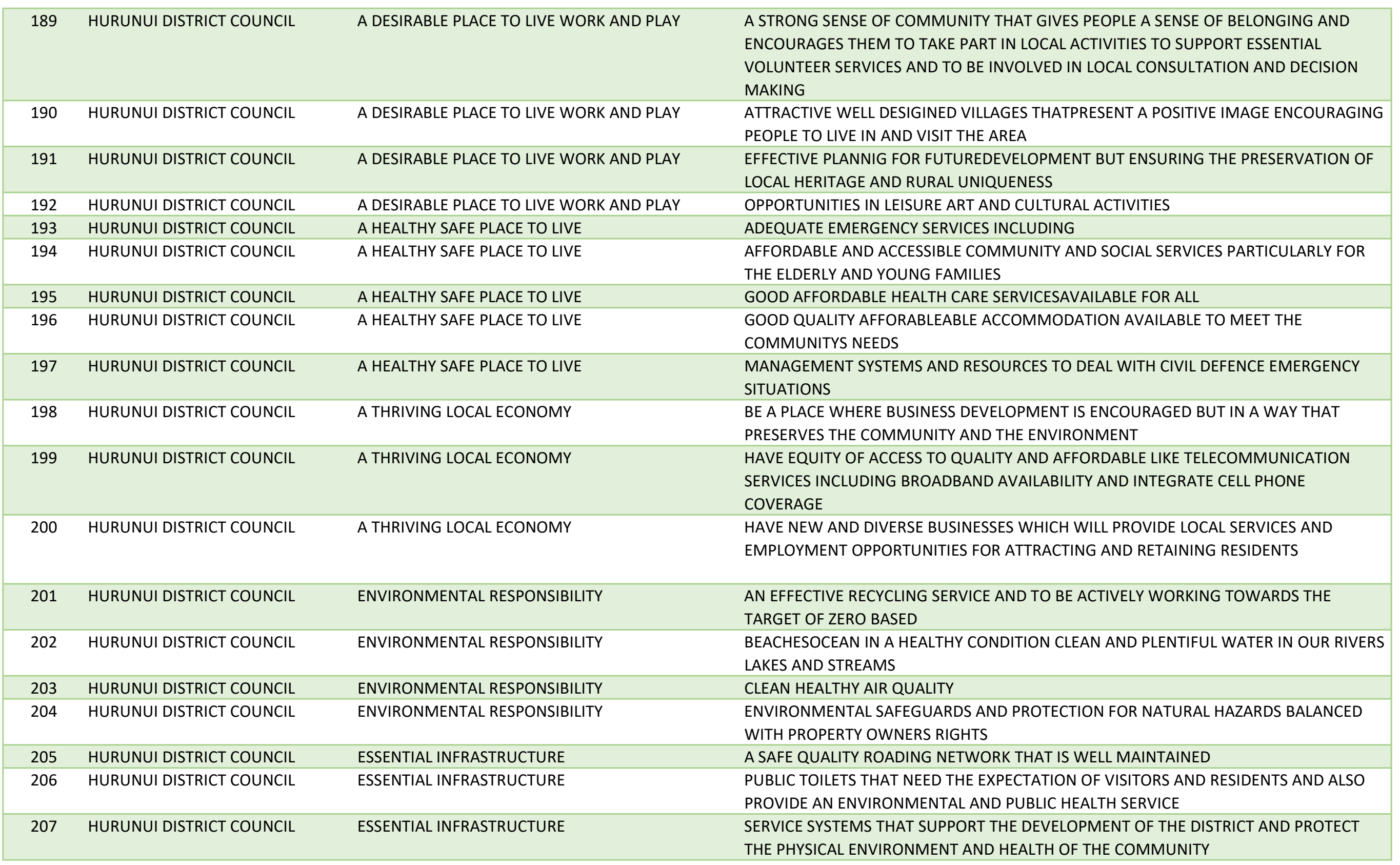


Appendix B: Community Well-being Statements by Councils

\begin{tabular}{|c|c|c|c|}
\hline 208 & HURUNUI DISTRICT COUNCIL & ESSENTIAL INFRASTRUCTURE & $\begin{array}{l}\text { WATER QUALITY AND QUANTITY MANAGEMENT SYSTEMS THAT PROVIDE GOOD CLEAN } \\
\text { HOUSEHOLD DRINKING WATER AND CONTINUE TO OF SUPPLY FOR AGRICULTURE HOW } \\
\text { TO CULTURE AND IN THE EVENT OF AN EMERGENCY }\end{array}$ \\
\hline 209 & HURUNUI DISTRICT COUNCIL & SKILLS AND EDUCATION FOR WORK AND LIFE & A VARIETY OF OPTIONS FOR LEARNING TO BE AVAILABLE TO ALL \\
\hline 210 & HURUNUI DISTRICT COUNCIL & SKILLS AND EDUCATION FOR WORK AND LIFE & PRACTICAL SKILLS AND WORKTRADEBASED TRAINING OPPORTUNITIES AVAILABLE \\
\hline 211 & HURUNUI DISTRICT COUNCIL & SKILLS AND EDUCATION FOR WORK AND LIFE & QUALITY EDUCATION AVAILABLE AT LOCAL SCHOOLS AND PRESCHOOLS \\
\hline 212 & INVERCARGILL DISTRICT COUNCIL & $\begin{array}{l}\text { A DIVERSE ECONOMY BUILT FROM OUR STRENGTHS } \\
\text { FOR GROWTH AND PROSPERITY }\end{array}$ & A DIVERSE ECONOMY BUILT FROM OUR STRENGTHS FOR GROWTH AND PROSPERITY \\
\hline 213 & INVERCARGILL DISTRICT COUNCIL & $\begin{array}{l}\text { A TREASURED ENVIRONMENT WHICH WE CARE FOR } \\
\text { AND WHICH SUPPORTS US NOW AND INTO THE } \\
\text { FUTURE }\end{array}$ & $\begin{array}{l}\text { A TREASURED ENVIRONMENT WHICH WE CARE FOR AND WHICH SUPPORTS US NOW } \\
\text { AND INTO THE FUTURE }\end{array}$ \\
\hline 214 & INVERCARGILL DISTRICT COUNCIL & $\begin{array}{l}\text { A WELLEDUCATED AND SKILLED COMMUNITY } \\
\text { CONTINUALLY SEEKING FURTHER OPPORTUNITIES } \\
\text { TO LEARN }\end{array}$ & $\begin{array}{l}\text { A WELLEDUCATED AND SKILLED COMMUNITY CONTINUALLY SEEKING FURTHER } \\
\text { OPPORTUNITIES TO LEARN }\end{array}$ \\
\hline 215 & INVERCARGILL DISTRICT COUNCIL & $\begin{array}{l}\text { SAFE PLACES IN A CARING SOCIETY THAT IS FREE } \\
\text { FROM CRIME }\end{array}$ & SAFE PLACES IN A CARING SOCIETY THAT IS FREE FROM CRIME \\
\hline 216 & INVERCARGILL DISTRICT COUNCIL & SOUTHLAND IS A GREAT PLACE TO LIVE & SOUTHLAND IS A GREAT PLACE TO LIVE \\
\hline 217 & INVERCARGILL DISTRICT COUNCIL & $\begin{array}{l}\text { STRONG EFFECTIVE LEADERSHIP TAKING US INTO } \\
\text { THE FUTURE }\end{array}$ & STRONG EFFECTIVE LEADERSHIP TAKING US INTO THE FUTURE \\
\hline 218 & INVERCARGILL DISTRICT COUNCIL & WE ARE HEALTHY PEOPLE & WE ARE HEALTHY PEOPLE \\
\hline 219 & KAIKOURA DISTRICT COUNCIL & A QUALITY STANDARD OF HOUSING & A QUALITY STANDARD OF HOUSING \\
\hline 220 & KAIKOURA DISTRICT COUNCIL & $\begin{array}{l}\text { AFFORDABLE ACCESS TO QUALITY COMMUNITY } \\
\text { FACILITIES }\end{array}$ & AFFORDABLE ACCESS TO QUALITY COMMUNITY FACILITIES \\
\hline 221 & KAIKOURA DISTRICT COUNCIL & $\begin{array}{l}\text { COMMUNITY INVOLVEMENT IN PLANNING THE } \\
\text { FUTURE AND MANAGING THE PRESENT }\end{array}$ & COMMUNITY INVOLVEMENT IN PLANNING THE FUTURE AND MANAGING THE PRESENT \\
\hline 222 & KAIKOURA DISTRICT COUNCIL & ENVIRONMENTAL PROTECTION AND ENHANCEMENT & ENVIRONMENTAL PROTECTION AND ENHANCEMENT \\
\hline 223 & KAIKOURA DISTRICT COUNCIL & $\begin{array}{l}\text { OPPORTUNITIES FOR QUALITY EDUCATION AND } \\
\text { EMPLOYMENT }\end{array}$ & OPPORTUNITIES FOR QUALITY EDUCATION AND EMPLOYMENT \\
\hline 224 & KAIKOURA DISTRICT COUNCIL & QUALITY WATER WASTEWATER SERVICES & QUALITY WATER WASTEWATER SERVICES \\
\hline 225 & KAIKOURA DISTRICT COUNCIL & SAFE EFFICIENT TRANSPORT NETWORK & SAFE EFFICIENT TRANSPORT NETWORK \\
\hline 226 & KAIKOURA DISTRICT COUNCIL & SUSTAINABLE DEVELOPMENT & SUSTAINABLE DEVELOPMENT \\
\hline 227 & KAIPARA DISTRICT COUNCIL & SAFETY AND A GOOD QUALITY OF LIFE & $\begin{array}{l}\text { KAIPARA DISTRICT IS A SAFE PLACE TO LIVE AND RAISE A FAMILY WHERE PEOPLE ENJOY A } \\
\text { GOOD QUALITY OF LIFE }\end{array}$ \\
\hline
\end{tabular}


Appendix B: Community Well-being Statements by Councils

\begin{tabular}{|c|c|c|c|}
\hline 228 & KAIPARA DISTRICT COUNCIL & SPECIAL CHARACTER AND HEALTHY ENVIRONMENT & $\begin{array}{l}\text { KAIPARA DISTRICT IS PROUD OF AND RENOWNED FOR ITS BEAUTIFUL ENVIRONMENT } \\
\text { AND SOUND MANAGEMENT OF NATURAL RESOURCES WHERE RESIDENTS ENJOY A CLEAN } \\
\text { HEALTHY ENVIRONMENT }\end{array}$ \\
\hline 229 & KAIPARA DISTRICT COUNCIL & STRONG COMMUNITIES & $\begin{array}{l}\text { KAIPARA DISTRICT IS BUILT ON STRONG COMMUNITIES WHERE PEOPLE HAVE A SENSE OF } \\
\text { BELONGING AND WORK TOGETHER TO SHAPE THEIR COLLECTIVE FUTURE }\end{array}$ \\
\hline 230 & KAIPARA DISTRICT COUNCIL & SUSTAINABLE ECONOMY & $\begin{array}{l}\text { KAIPARA DISTRICT HAS A DIVERSIFIED AND SUSTAINABLE ECONOMY THAT SUPPORTS THE } \\
\text { WELLBEING OF ITS COMMUNITIES AND RESIDENTS }\end{array}$ \\
\hline 232 & KAPITI COAST DISTRICT COUNCIL & $\begin{array}{l}\text { LOCAL CHARACTER IS RETAINED WITHIN A COHESIVE } \\
\text { DISTRICT }\end{array}$ & $\begin{array}{l}\text { COMMUNITY AND SECTOR NETWORKS THAT ACTIVELY CONTRIBUTE TO LOCAL AND } \\
\text { COMMUNITY COHESIVENESS ARE SUPPORTED }\end{array}$ \\
\hline 233 & KAPITI COAST DISTRICT COUNCIL & $\begin{array}{l}\text { LOCAL CHARACTER IS RETAINED WITHIN A COHESIVE } \\
\text { DISTRICT }\end{array}$ & $\begin{array}{l}\text { ECONOMIC INTERDEPENDENCIES OF ALL THE COMMUNITIES ARE RECOGNISED AND } \\
\text { CLUSTERING OF COMPLEMENTARY BUSINESSES IS ENCOURAGED FOR THE BENEFIT OF } \\
\text { LOCAL AREAS AND THE DISTRICT AS A WHOLE }\end{array}$ \\
\hline 234 & KAPITI COAST DISTRICT COUNCIL & $\begin{array}{l}\text { LOCAL CHARACTER IS RETAINED WITHIN A COHESIVE } \\
\text { DISTRICT }\end{array}$ & $\begin{array}{l}\text { LOCAL SCHOOLS ESPECIALLY THE SMALLER SCHOOLS IN THE SMALLER COMMUNITIES ARE } \\
\text { RECOGNISED AS KEY COMMUNITY FOCAL POINTS AND SOURCES OF COMMUNITY } \\
\text { COHESIVENESS AND THAT THEY ARE RETAINED INTO THE FUTURE }\end{array}$ \\
\hline 235 & KAPITI COAST DISTRICT COUNCIL & $\begin{array}{l}\text { LOCAL CHARACTER IS RETAINED WITHIN A COHESIVE } \\
\text { DISTRICT }\end{array}$ & $\begin{array}{l}\text { THE DESIGN OF BUILDINGS AND INFRASTRUCTURE IS MORE IN KEEPING WITH THE } \\
\text { CHARACTER OF THE KAPITI COAST }\end{array}$ \\
\hline 236 & KAPITI COAST DISTRICT COUNCIL & $\begin{array}{l}\text { LOCAL CHARACTER IS RETAINED WITHIN A COHESIVE } \\
\text { DISTRICT }\end{array}$ & $\begin{array}{l}\text { THE LEVEL AND QUALITY OF ACCESS WITHIN AND BETWEEN COMMUNITIES MEETS THE } \\
\text { NEEDS OF ALL USERS AND INCLUDES }\end{array}$ \\
\hline 237 & KAPITI COAST DISTRICT COUNCIL & $\begin{array}{l}\text { LOCAL CHARACTER IS RETAINED WITHIN A COHESIVE } \\
\text { DISTRICT }\end{array}$ & $\begin{array}{l}\text { THE ROLE NATURE AND CHARACTER OF EACH OF KAPITI COASTS TOWNS VILLAGES LOCAL } \\
\text { AND SPECIAL AREAS IS RESPECTED AND RETAINED AND SHAPES THE FUTURE FORM AND } \\
\text { QUALITY OF THE DISTRICT }\end{array}$ \\
\hline 238 & KAPITI COAST DISTRICT COUNCIL & $\begin{array}{l}\text { LOCAL CHARACTER IS RETAINED WITHIN A COHESIVE } \\
\text { DISTRICT }\end{array}$ & $\begin{array}{l}\text { THERE IS A HIGH LEVEL OF PARTICIPATION IN COMMUNITY DECISIONMAKING WITHIN } \\
\text { COMMUNITIES AND ACROSS THE DISTRICT PARTICULARLY IN RELATION TO DISTRICT } \\
\text { PLANNING }\end{array}$ \\
\hline 239 & KAPITI COAST DISTRICT COUNCIL & $\begin{array}{l}\text { THE COMMUNITY MAKES WISE USE OF LOCAL } \\
\text { RESOURCES AND PEOPLE HAVE THE ABILITY TO ACT } \\
\text { IN A SUSTAINABLE WAY ON A DAY TO DAY BASIS }\end{array}$ & $\begin{array}{l}\text { INFRASTRUCTURE IS DESIGNED AND MANAGED TO MEET THE COMMUNITYS BASIC } \\
\text { NEEDS AND TO INCREASE PEOPLES ABILITY TO ACT SUSTAINABLY AND TAKE } \\
\text { RESPONSIBILITY FOR THE EFFECTS OF THEIR ACTIONS INCLUDING }\end{array}$ \\
\hline
\end{tabular}




\begin{tabular}{|c|c|c|c|}
\hline 241 & KAPITI COAST DISTRICT COUNCIL & $\begin{array}{l}\text { THE COMMUNITY MAKES WISE USE OF LOCAL } \\
\text { RESOURCES AND PEOPLE HAVE THE ABILITY TO ACT } \\
\text { IN A SUSTAINABLE WAY ON A DAY TO DAY BASIS }\end{array}$ & PEOPLE ARE UNDERSTOOD TO BE A COMMUNITY RESOURCE AND THAT IN PARTICULAR \\
\hline 244 & KAPITI COAST DISTRICT COUNCIL & $\begin{array}{l}\text { THE COMMUNITY MAKES WISE USE OF LOCAL } \\
\text { RESOURCES AND PEOPLE HAVE THE ABILITY TO ACT } \\
\text { IN A SUSTAINABLE WAY ON A DAY TO DAY BASIS }\end{array}$ & THE KAPITI COAST DISTRICT CONTINUES TO HAVE A FUNCTIONING AIRPORT \\
\hline 245 & KAPITI COAST DISTRICT COUNCIL & $\begin{array}{l}\text { THE COMMUNITY MAKES WISE USE OF LOCAL } \\
\text { RESOURCES AND PEOPLE HAVE THE ABILITY TO ACT } \\
\text { IN A SUSTAINABLE WAY ON A DAY TO DAY BASIS }\end{array}$ & THE LOCAL RESOURCE AVAILABLE TO PEOPLE TO HARVEST IS ENHANCED INCLUDING \\
\hline 246 & KAPITI COAST DISTRICT COUNCIL & $\begin{array}{l}\text { THE COMMUNITY MAKES WISE USE OF LOCAL } \\
\text { RESOURCES AND PEOPLE HAVE THE ABILITY TO ACT } \\
\text { IN A SUSTAINABLE WAY ON A DAY TO DAY BASIS }\end{array}$ & $\begin{array}{l}\text { THE PRODUCTIVE POTENTIAL OF KAPITI COAST RURAL LANDS FOR FOOD FIBRE AND } \\
\text { OTHER AGRICULTURAL PRODUCTS IS PRESERVED OVER TIME PARTICULAR REGARD } \\
\text { SHOULD BE HAD FOR THE PROTECTION OF SOILS AND POTENTIAL OF THE HAUTERE TE } \\
\text { HORO AND OTAKI HINTERLANDS }\end{array}$ \\
\hline 247 & KAPITI COAST DISTRICT COUNCIL & $\begin{array}{l}\text { THE COMMUNITY MAKES WISE USE OF LOCAL } \\
\text { RESOURCES AND PEOPLE HAVE THE ABILITY TO ACT } \\
\text { IN A SUSTAINABLE WAY ON A DAY TO DAY BASIS }\end{array}$ & $\begin{array}{l}\text { THERE ARE MORE OPPORTUNITIES FOR INNOVATIVE DESIGN OF SUBDIVISIONS } \\
\text { BUILDINGS AND INFRASTRUCTURE TO REDUCE ENERGY AND RESOURCE USE }\end{array}$ \\
\hline 248 & KAPITI COAST DISTRICT COUNCIL & $\begin{array}{l}\text { THE COMMUNITY MAKES WISE USE OF LOCAL } \\
\text { RESOURCES AND PEOPLE HAVE THE ABILITY TO ACT } \\
\text { IN A SUSTAINABLE WAY ON A DAY TO DAY BASIS }\end{array}$ & $\begin{array}{l}\text { THERE ARE SYSTEMS THROUGHOUT THE DISTRICT FOR THE REDUCTION OF WASTE } \\
\text { ESPECIALLY RECYCLING INCLUDING GREENWASTE DOMESTIC AND COMMERCIAL } \\
\text { PACKAGING WASTE AND GREYWATER }\end{array}$ \\
\hline 249 & KAPITI COAST DISTRICT COUNCIL & $\begin{array}{l}\text { THE DISTRICT HAS A STRONG HEALTHY SAFE AND } \\
\text { INVOLVED COMMUNITY }\end{array}$ & $\begin{array}{l}\text { OLDER PEOPLE HAVE A HIGH LEVEL OF CONTROL AND INFLUENCE OVER THOSE THINGS } \\
\text { THAT ENSURE THEIR ACCESS TO SERVICES AND ENJOYMENT OF LIFE INCLUDING }\end{array}$ \\
\hline
\end{tabular}




\begin{tabular}{|c|c|c|c|}
\hline 250 & KAPITI COAST DISTRICT COUNCIL & $\begin{array}{l}\text { THE DISTRICT HAS A STRONG HEALTHY SAFE AND } \\
\text { INVOLVED COMMUNITY }\end{array}$ & $\begin{array}{l}\text { PEOPLE ARE ENCOURAGED AND ENABLED TO TAKE ACTION AND RESPONSIBILITY AND TO } \\
\text { LIVE IN A SUSTAINABLE WAY AND HAVE ACCESS TO COMMUNITY SPACES AND } \\
\text { OPPORTUNITIES TO SHARE RESOURCES AND KNOWLEDGE }\end{array}$ \\
\hline 252 & KAPITI COAST DISTRICT COUNCIL & $\begin{array}{l}\text { THE DISTRICT HAS A STRONG HEALTHY SAFE AND } \\
\text { INVOLVED COMMUNITY }\end{array}$ & $\begin{array}{l}\text { PEOPLE ARE VALUED AND RESPECTED FOR THE CONTRIBUTION THEY MAKE TO A STRONG } \\
\text { AND DIVERSE COMMUNITY ESPECIALLY WHERE THIS CONTRIBUTION IS THROUGH } \\
\text { VOLUNTEERING }\end{array}$ \\
\hline 253 & KAPITI COAST DISTRICT COUNCIL & $\begin{array}{l}\text { THE DISTRICT HAS A STRONG HEALTHY SAFE AND } \\
\text { INVOLVED COMMUNITY }\end{array}$ & $\begin{array}{l}\text { PEOPLE ARE WELCOMED INTO THE COMMUNITY AS NEW RESIDENTS AND HAVE THE } \\
\text { CHANCE PARTICIPATE IN AND CONTRIBUTE TO A RANGE OF ACTIVITIES EVENTS AND } \\
\text { OPPORTUNITIES }\end{array}$ \\
\hline 254 & KAPITI COAST DISTRICT COUNCIL & $\begin{array}{l}\text { THE DISTRICT HAS A STRONG HEALTHY SAFE AND } \\
\text { INVOLVED COMMUNITY }\end{array}$ & $\begin{array}{l}\text { PEOPLE HAVE A SENSE OF SAFETY FOR THEMSELVES THEIR FAMILY AND THEIR } \\
\text { COMMUNITY AND FEEL SECURE IN THEIR HOMES AND IN THE COMMUNITY }\end{array}$ \\
\hline 255 & KAPITI COAST DISTRICT COUNCIL & $\begin{array}{l}\text { THE DISTRICT HAS A STRONG HEALTHY SAFE AND } \\
\text { INVOLVED COMMUNITY }\end{array}$ & $\begin{array}{l}\text { PEOPLE HAVE READY ACCESS TO INFORMATION ABOUT THEIR LOCAL COMMUNITY } \\
\text { DISTRICT AND WIDER WORLD INCLUDING KNOWING AND VALUING LOCAL PEOPLE WHO } \\
\text { HAVE THAT KNOWLEDGE }\end{array}$ \\
\hline 257 & KAPITI COAST DISTRICT COUNCIL & $\begin{array}{l}\text { THE DISTRICT HAS A STRONG HEALTHY SAFE AND } \\
\text { INVOLVED COMMUNITY }\end{array}$ & $\begin{array}{l}\text { THE DISTRICT HAS HIGH QUALITY CIVIC AND LOCAL CENTRES WHERE PEOPLE CHOOSE TO } \\
\text { GO FOR GENERAL ENJOYMENT AS WELL AS SHOPPING }\end{array}$ \\
\hline 258 & KAPITI COAST DISTRICT COUNCIL & $\begin{array}{l}\text { THE DISTRICT HAS A STRONG HEALTHY SAFE AND } \\
\text { INVOLVED COMMUNITY }\end{array}$ & $\begin{array}{l}\text { THE DISTRICTS CENTRES ESPECIALLY PARAPARAUMU AND OTAKI ARE MORE ALIVE AT } \\
\text { NIGHT }\end{array}$ \\
\hline 259 & KAPITI COAST DISTRICT COUNCIL & $\begin{array}{l}\text { THE DISTRICT HAS A STRONG HEALTHY SAFE AND } \\
\text { INVOLVED COMMUNITY }\end{array}$ & $\begin{array}{l}\text { THE DISTRICTS MAIN PUBLIC PLACES INCLUDING BEACHES ARE SAFE ATTRACTIVE AND } \\
\text { ACCESSIBLE TO EVERYONE IN THE COMMUNITY INCLUDING THE ELDERLY FAMILIES AND } \\
\text { PEOPLE WITH DISABILITIES }\end{array}$ \\
\hline 260 & KAPITI COAST DISTRICT COUNCIL & $\begin{array}{l}\text { THE DISTRICT HAS A STRONG HEALTHY SAFE AND } \\
\text { INVOLVED COMMUNITY }\end{array}$ & $\begin{array}{l}\text { THERE ARE EXTENSIVE LINKAGES WITHIN THE DISTRICT IN ADDITION TO STATE HIGHWAY } \\
\text { INCLUDING }\end{array}$ \\
\hline 261 & KAPITI COAST DISTRICT COUNCIL & $\begin{array}{l}\text { THE DISTRICT HAS A STRONG HEALTHY SAFE AND } \\
\text { INVOLVED COMMUNITY }\end{array}$ & $\begin{array}{l}\text { THERE IS A COMPREHENSIVE PLANNED APPROACH TO RECREATION } \\
\text { FACILITIESPROGRAMMES WHICH PROVIDES FOR DISTRICTWIDE AS WELL AS LOCAL } \\
\text { SERVICES INCLUDING }\end{array}$ \\
\hline 262 & KAPITI COAST DISTRICT COUNCIL & $\begin{array}{l}\text { THE DISTRICT HAS A STRONG HEALTHY SAFE AND } \\
\text { INVOLVED COMMUNITY }\end{array}$ & $\begin{array}{l}\text { THERE IS A GREATER RANGE OF HOUSING OPTIONS AVAILABLE IN THE DISTRICT } \\
\text { CATERING FOR A VARIETY OF SOCIAL NEEDS INCLUDING }\end{array}$ \\
\hline 264 & KAPITI COAST DISTRICT COUNCIL & $\begin{array}{l}\text { THE DISTRICT HAS A STRONG HEALTHY SAFE AND } \\
\text { INVOLVED COMMUNITY }\end{array}$ & THERE IS A LOCAL HEALTH SERVICE WHERE \\
\hline
\end{tabular}




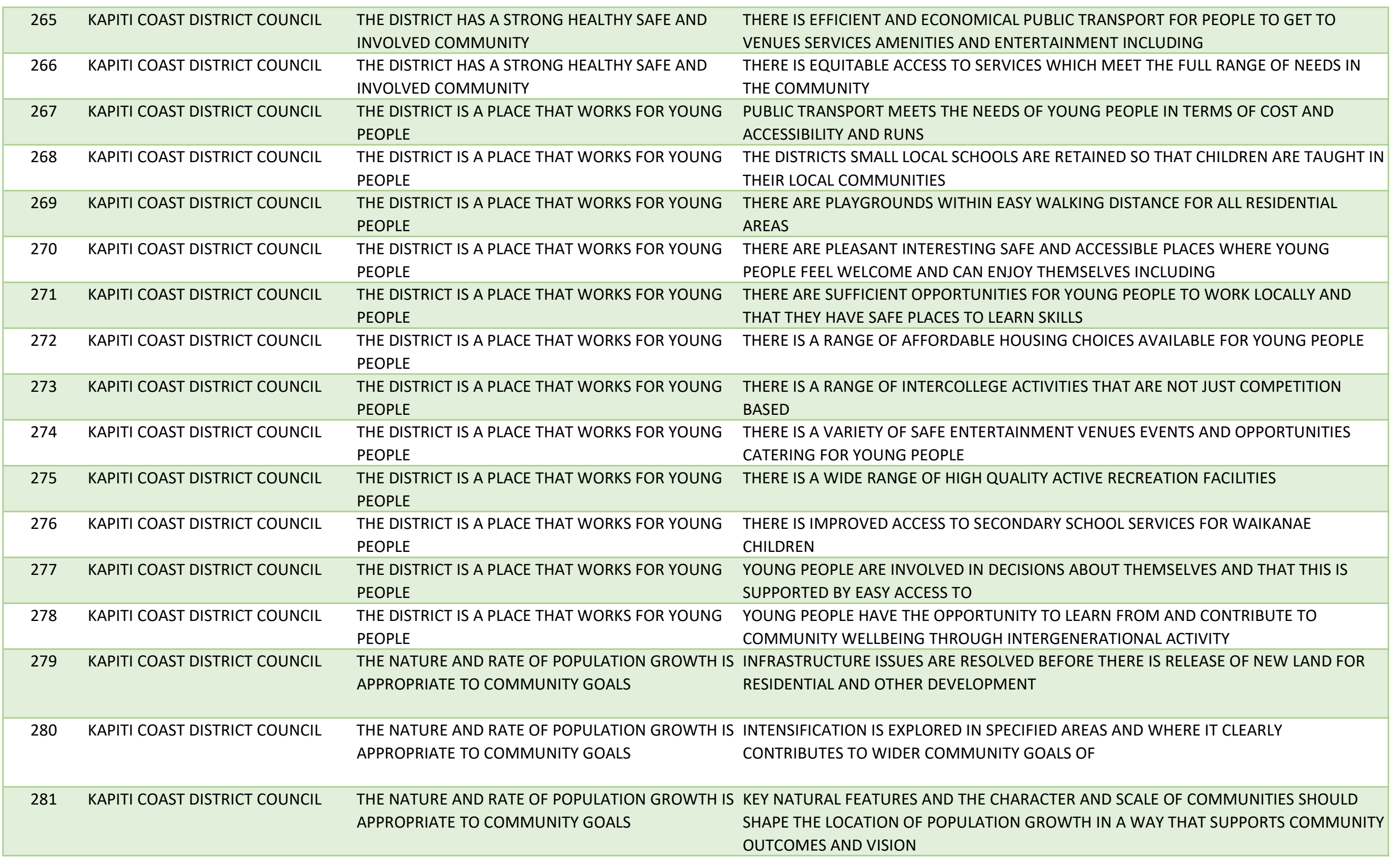




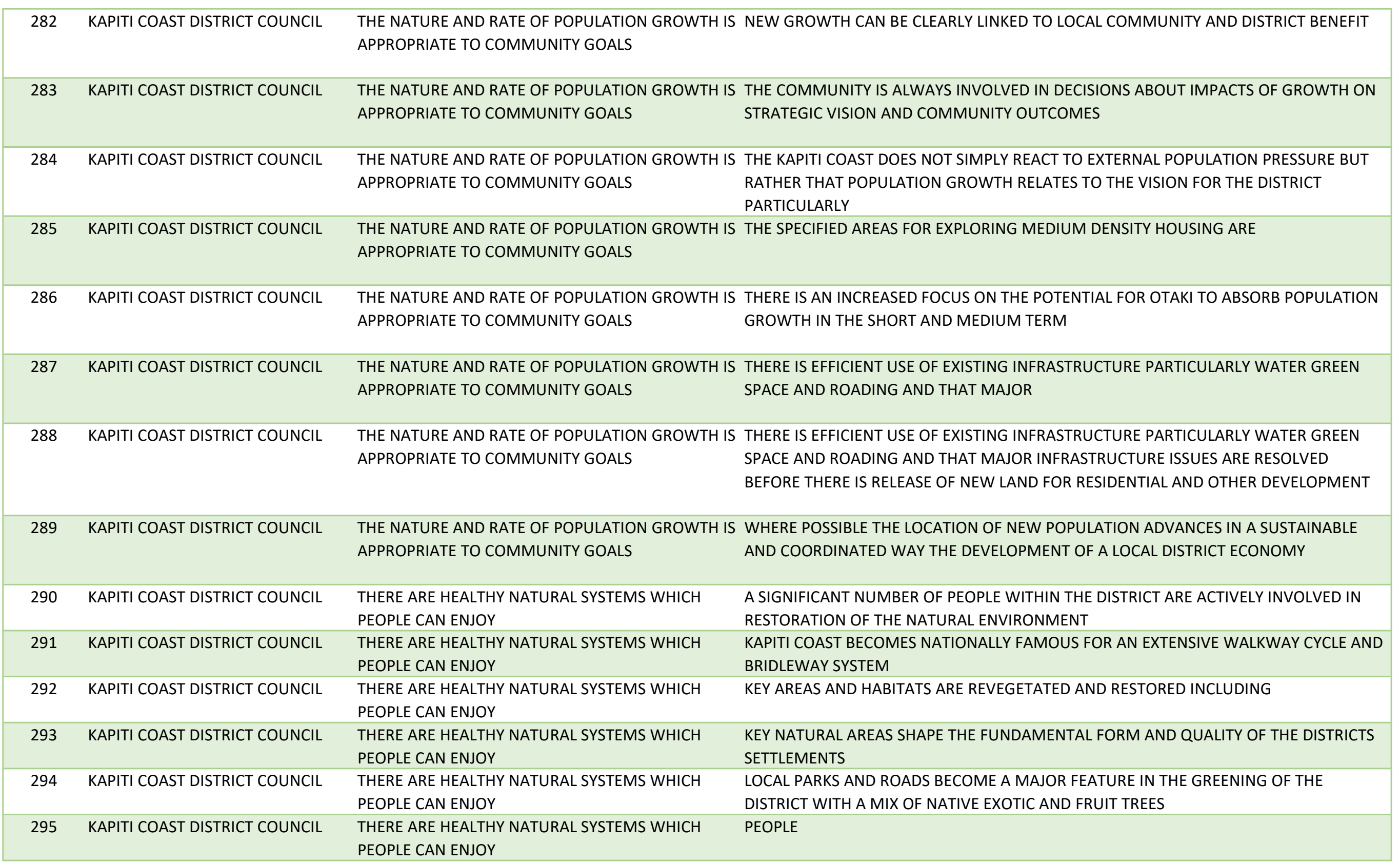


Appendix B: Community Well-being Statements by Councils

\begin{tabular}{|c|c|c|c|}
\hline 296 & KAPITI COAST DISTRICT COUNCIL & $\begin{array}{l}\text { THERE ARE HEALTHY NATURAL SYSTEMS WHICH } \\
\text { PEOPLE CAN ENJOY }\end{array}$ & THE COAST IS MANAGED \\
\hline 297 & KAPITI COAST DISTRICT COUNCIL & $\begin{array}{l}\text { THERE ARE HEALTHY NATURAL SYSTEMS WHICH } \\
\text { PEOPLE CAN ENJOY }\end{array}$ & $\begin{array}{l}\text { THE DISTRICT BECOMES KNOWN FOR BEST PRACTICE SUBDIVISION AND STREETSCAPE } \\
\text { DESIGN THAT INCLUDES TREES LANDFORMS WATERWAYS PARKS WALKWAYS CYCLEWAYS } \\
\text { AND BRIDLEWAYS AS FEATURES IN DEVELOPMENTS }\end{array}$ \\
\hline 298 & KAPITI COAST DISTRICT COUNCIL & $\begin{array}{l}\text { THERE ARE HEALTHY NATURAL SYSTEMS WHICH } \\
\text { PEOPLE CAN ENJOY }\end{array}$ & THE DISTRICT HAS BEST PRACTICE STORMWATER AND FLOOD MANAGEMENT INCLUDING \\
\hline 299 & KAPITI COAST DISTRICT COUNCIL & $\begin{array}{l}\text { THERE ARE HEALTHY NATURAL SYSTEMS WHICH } \\
\text { PEOPLE CAN ENJOY }\end{array}$ & $\begin{array}{l}\text { THE KEY FOCAL POINTS SUCH AS THE BEACHES KAPITI ISLAND THE TARARUA RANGES } \\
\text { OTAKI FORKS ARE MANAGED IN A WAY THAT WELCOMES VISITORS BUT PROTECTS THE } \\
\text { ESSENTIAL QUALITIES FOR WHICH THEY ARE VALUED }\end{array}$ \\
\hline 300 & KAPITI COAST DISTRICT COUNCIL & $\begin{array}{l}\text { THERE ARE HEALTHY NATURAL SYSTEMS WHICH } \\
\text { PEOPLE CAN ENJOY }\end{array}$ & THE QUALITY OF THE DISTRICTS STREAMS AND RIVERS ARE IMPROVED ESPECIALLY \\
\hline 301 & KAPITI COAST DISTRICT COUNCIL & $\begin{array}{l}\text { THERE ARE HEALTHY NATURAL SYSTEMS WHICH } \\
\text { PEOPLE CAN ENJOY }\end{array}$ & VULNERABLE AREAS OF NATIVE VEGETATION AND WILDLIFE ARE PROTECTED INCLUDING \\
\hline 302 & KAPITI COAST DISTRICT COUNCIL & THERE IS INCREASED CHOICE TO WORK LOCALLY & $\begin{array}{l}\text { ALL MAJOR EMPLOYMENT CENTRES HAVE ACCESS TO PUBLIC TRANSPORT PEDESTRIAN } \\
\text { AND CYCLING ACCESS }\end{array}$ \\
\hline 303 & KAPITI COAST DISTRICT COUNCIL & THERE IS INCREASED CHOICE TO WORK LOCALLY & $\begin{array}{l}\text { CHILDREN AND YOUNG PEOPLE CAN SEE THEIR CAREER PATH AND CAN AS MUCH AS } \\
\text { POSSIBLE GAIN THE NECESSARY SKILLS AND EMPLOYMENT WITHIN THE DISTRICT }\end{array}$ \\
\hline 304 & KAPITI COAST DISTRICT COUNCIL & THERE IS INCREASED CHOICE TO WORK LOCALLY & $\begin{array}{l}\text { EXISTING COMMERCIAL AND INDUSTRIAL AREAS ARE ATTRACTIVE AND INNOVATIVE IN } \\
\text { THEIR DESIGN AND USE }\end{array}$ \\
\hline 305 & KAPITI COAST DISTRICT COUNCIL & THERE IS INCREASED CHOICE TO WORK LOCALLY & $\begin{array}{l}\text { IT IS EASY TO ESTABLISH VALUE ADDED ECONOMIC ACTIVITY ESPECIALLY THAT LINKED } \\
\text { WITH FOOD PRODUCTION AND LOCAL MANUFACTURING IN THE DISTRICT }\end{array}$ \\
\hline 306 & KAPITI COAST DISTRICT COUNCIL & THERE IS INCREASED CHOICE TO WORK LOCALLY & $\begin{array}{l}\text { THE DISTRICT DEVELOPS A ROLE AS A TRANSPORT HUB INCLUDING THE DISTRIBUTION OF } \\
\text { FREIGHT }\end{array}$ \\
\hline 307 & KAPITI COAST DISTRICT COUNCIL & THERE IS INCREASED CHOICE TO WORK LOCALLY & $\begin{array}{l}\text { THE DISTRICTS RETAIL CENTRES INCLUDING LOCAL NEIGHBOURHOOD SHOPS AND } \\
\text { DAIRIES ARE SAFE AND ATTRACTIVE AND HAVE STABLE AND PROSPEROUS BUSINESSES }\end{array}$ \\
\hline 308 & KAPITI COAST DISTRICT COUNCIL & THERE IS INCREASED CHOICE TO WORK LOCALLY & $\begin{array}{l}\text { THE LOCAL LABOUR FORCE HAS EMPLOYMENT OPPORTUNITIES IN AN APPROPRIATE MIX } \\
\text { OF SKILLED AND UNSKILLED WORK AS MUCH AS POSSIBLE WITHIN THE DISTRICT }\end{array}$ \\
\hline 309 & KAPITI COAST DISTRICT COUNCIL & THERE IS INCREASED CHOICE TO WORK LOCALLY & $\begin{array}{l}\text { THERE IS CLEAR ACKNOWLEDGEMENT NURTURING AND USE OF THE ECONOMIC } \\
\text { BENEFITS THAT COME FROM BEING A DISTRICT KNOWN FOR GOOD QUALITY URBAN } \\
\text { ENVIRONMENTS HIGH QUALITY NATURAL LANDSCAPES AND DESIGN INNOVATION }\end{array}$ \\
\hline 310 & KAPITI COAST DISTRICT COUNCIL & THERE IS INCREASED CHOICE TO WORK LOCALLY & $\begin{array}{l}\text { THERE IS STRONG BUSINESS DEVELOPMENT FOCUS IN THE DISTRICT WHICH } \\
\text { ENCOMPASSES }\end{array}$ \\
\hline
\end{tabular}




\begin{tabular}{|c|c|c|c|}
\hline 311 & KAPITI COAST DISTRICT COUNCIL & THERE IS INCREASED CHOICE TO WORK LOCALLY & USABLE COMMERCIAL LAND IS READILY AVAILABLE IN THE DISTRICT \\
\hline 312 & KAWERAU DISTRICT COUNCIL & CLEAN ACCESSIBLE RESPECTED ENVIRONMENT & CLEAN ACCESSIBLE RESPECTED ENVIRONMENT \\
\hline 313 & KAWERAU DISTRICT COUNCIL & CONTINUED INDEPENDENCE AND VIABILITY & CONTINUED INDEPENDENCE AND VIABILITY \\
\hline 314 & KAWERAU DISTRICT COUNCIL & DIVERSE CREATIVE AND VITAL COMMUNITIES & DIVERSE CREATIVE AND VITAL COMMUNITIES \\
\hline 315 & KAWERAU DISTRICT COUNCIL & EDUCATION AND TRAINING OPPORTUNITIES FOR ALL & EDUCATION AND TRAINING OPPORTUNITIES FOR ALL \\
\hline 316 & KAWERAU DISTRICT COUNCIL & $\begin{array}{l}\text { FOCUSED COMMUNITY LEADERSHIP AND } \\
\text { GOVERNANCE }\end{array}$ & FOCUSED COMMUNITY LEADERSHIP AND GOVERNANCE \\
\hline 317 & KAWERAU DISTRICT COUNCIL & HEALTHY PEOPLE AND HOUSING & HEALTHY PEOPLE AND HOUSING \\
\hline 318 & KAWERAU DISTRICT COUNCIL & RELIABLE AND AFFORDABLE INFRASTRUCTURE & RELIABLE AND AFFORDABLE INFRASTRUCTURE \\
\hline 319 & KAWERAU DISTRICT COUNCIL & SAFE CARING COMMUNITIES & SAFE CARING COMMUNITIES \\
\hline 320 & KAWERAU DISTRICT COUNCIL & $\begin{array}{l}\text { STRONG AND PROSPEROUS ECONOMY ALIGNED TO } \\
\text { COMMUNITY VALUES }\end{array}$ & STRONG AND PROSPEROUS ECONOMY ALIGNED TO COMMUNITY VALUES \\
\hline 321 & LOWER HUTT CITY COUNCIL & COMMUNITY PROSPERITY & $\begin{array}{l}\text { TO ENJOY AND PROSPER FROM A STRONG AND GROWING ECONOMY THAT CONTINUES } \\
\text { TO ATTRACT AND RETAIN A HIGHLY SKILLED PRODUCTIVE POPULATION BASE AND } \\
\text { BUSINESS SECTOR }\end{array}$ \\
\hline 322 & LOWER HUTT CITY COUNCIL & CONNECTED & $\begin{array}{l}\text { THE REGION WILL BE CONNECTED LOCALLY AND GLOBALLY BY OFFERING WORLDCLASS } \\
\text { ACCESSIBILITY AND LINKAGES THAT INCLUDES HIGHCAPACITY COMMUNICATION } \\
\text { NETWORKS TOPQUALITY AIR AND SEA PORTS WELL FUNCTIONING HIGHWAYS AND LOCAL } \\
\text { LINKS AND EXCELLENT PUBLIC TRANSPORT SERVICES }\end{array}$ \\
\hline 323 & LOWER HUTT CITY COUNCIL & ENTREPRENEURIAL AND INNOVATIVE CULTURE & $\begin{array}{l}\text { WE WILL BE RECOGNISED AS A PLACE OF NEW IDEAS INNOVATION AND CREATIVITY } \\
\text { UNDERPIN THE DEVELOPMENT OF NEW FIELDS OF ENDEAVOUR AND BUSINESS AND } \\
\text { DRIVE STRONG SUSTAINABLE ECONOMIC GROWTH WE WILL CAPITALISE ON BEING THE } \\
\text { SEAT OF GOVERNMENT AND OUR EXCELLENT EDUCATIONAL AND RESEARCH } \\
\text { INSTITUTIONS WILL FOSTER CROSSFERTILISATION }\end{array}$ \\
\hline 324 & LOWER HUTT CITY COUNCIL & HEALTHY AND EDUCATED COMMUNITY & $\begin{array}{l}\text { OUR PHYSICAL AND MENTAL HEALTH IS PROTECTED LIVING AND WORKING } \\
\text { ENVIRONMENTS ARE SAFE AND EVERYONE HAS ACCESS TO HEALTH CARE EVERY } \\
\text { OPPORTUNITY IS TAKEN TO RECOGNISE AND PROVIDE FOR GOOD HEALTH LIFELONG } \\
\text { LEARNING AND EDUCATION OPPORTUNITIES ARE WORLD CLASS CONTRIBUTING TO AN } \\
\text { INTERNATIONALLY COMPETITIVE WORKFORCE AND REINFORCING THE REGION AS A } \\
\text { DESIRABLE PLACE TO LIVE }\end{array}$ \\
\hline 325 & LOWER HUTT CITY COUNCIL & HEALTHY ENVIRONMENT & $\begin{array}{l}\text { THE REGION WILL OFFER A BEAUTIFUL ECOLOGICALLY SUSTAINABLE NATURAL } \\
\text { ENVIRONMENT THAT ENVIRONMENT WILL OFFER NUMEROUS OPPORTUNITIES FOR } \\
\text { RECREATION AND HEALTHY LIVING }\end{array}$ \\
\hline
\end{tabular}


326 LOWER HUTT CITY COUNCIL LIFESTYLE

327 LOWER HUTT CITY COUNCIL

REGIONAL FOUNDATIONS

328 LOWER HUTT CITY COUNCIL

SENSE OF PLACE

329 LOWER HUTT CITY COUNCIL

STRONG AND TOLERANT COMMUNITIES
THE REGION WILL HAVE AN EXCEPTIONAL QUALITY OF LIFE WHERE LIVING IS EASY SAFE AND FUN AND THERE IS A WIDE RANGE OF AFFORDABLE HEALTHY LIFESTYLE OPTIONS UNDERPINNING A STRONG COMMUNITY SPIRIT WE WILL BE CELEBRATED FOR OUR ARTS SPORTS AND ENTERTAINMENT SCENE

PEOPLE AND BUSINESSES WILL HAVE HIGHQUALITY SECURE ESSENTIAL SERVICES FOR THEIR EVERYDAY LIVING THEY WILL BE RETAINED AND DEVELOPED IN A MANNER THAT IS CONSISTENT WITH THE SUSTAINABLE GROWTH OF THE REGION

WE WILL HAVE A RICH DIVERSE SENSE OF PLACE AS A SOPHISTICATED REGION ENCOMPASSING THE INTIMATE URBAN CORE CAPITAL CITY STATUS MAGNIFICENT HARBOURS RIVERS CENTRES OF LEARNING EXTENSIVE COASTLINES ITS ROLLING HINTERLAND AND DIVERSE CENTRES AND NEIGHBOURHOODS WHICH ARE ALL TREASURED BY ITS CITIZENS

WE WILL HAVE INCLUSIVE CARING FRIENDLY AND PARTICIPATIVE COMMUNITIES THAT EMBRACE NEWCOMERS ARE OPEN AND WELCOMING TO DIFFERENT LIFESTYLES AND CELEBRATE DIVERSITY THE RELATIONSHIP WITH TANGATA WHENUA AND ETHNIC COMMUNITIES WILL BE IMPORTANT TO THE VIBRANCY OF THE COMMUNITY TAPESTRY

\begin{tabular}{|c|c|c|c|}
\hline \multirow[t]{2}{*}{330} & \multirow[t]{2}{*}{ MACKENZIE DISTRICT COUNCIL } & \multicolumn{2}{|c|}{ A DEMOCRACY WHICH UPHOLDS THE RIGHTS OF THE THE OUTCOME DESIRED BY THE COMMUNITY IS TO HAVE A COUNCIL WHICH CHAMPIONS } \\
\hline & & INDIVIDUAL & THE RIGHTS OF THE INDIVIDUAL AND REPRESENTS ITS COMMUNITY \\
\hline \multirow[t]{2}{*}{331} & MACKENZIE DISTRICT COUNCIL & AN ATTRACTIVE AND HIGHLY VALUED NATURAL & THE OUTCOME DESIRED BY THE COMMUNITY IS TO HAVE THE OUTSTANDING NATURAL \\
\hline & & ENVIRONMENT & FEATURES OF THE DISTRICT PRESERVED ENHANCED ACCESSIBLE AND PROMOTED \\
\hline \multirow[t]{3}{*}{332} & MACKENZIE DISTRICT COUNCIL & FIT AND HEALTHY COMMUNITY & THE OUTCOME DESIRED BY THE COMMUNITY IS TO HAVE A VARIETY OF SPORTING \\
\hline & & & RECREATIONAL CULTURAL SPIRITUAL HEALTH WELFARE AND EDUCATIONAL RESOURCES \\
\hline & & & AVAILABLE TO SUSTAIN AND ENRICH THE LIVES OF ITS PEOPLE \\
\hline \multirow[t]{3}{*}{333} & MACKENZIE DISTRICT COUNCIL & SAFE EFFECTIVE AND SUSTAINABLE & THE OUTCOME DESIRED BY THE COMMUNITY IS TO HAVE SAFE EFFECTIVE AND \\
\hline & & INFRASTRUCTURE & SUSTAINABLE WATER WASTE COMMUNICATION ENERGY AND TRANSPORT SYSTEMS IN \\
\hline & & & PLACE WHEN REQUIRED THROUGH SOUND LONG TERM PLANNING AND FUNDING \\
\hline \multirow[t]{3}{*}{334} & MACKENZIE DISTRICT COUNCIL & SUPPORTIVE AND CONTRIBUTING COMMUNITY & THE OUTCOMES DESIRED BY THE COMMUNITY ARE TO FOSTER THE UNIQUE ATTRIBUTES \\
\hline & & & AND STRONG SENSE OF COMMUNITY THAT MAKES THE MACKENZIE SPECIAL AND TO \\
\hline & & & ENCOURAGE PEOPLE TO USE THEIR SKILLS FOR THE BENEFIT OF THE COMMUNITY \\
\hline \multirow[t]{3}{*}{335} & MACKENZIE DISTRICT COUNCIL & THRIVING ECONOMY & THE OUTCOME DESIRED BY THE COMMUNITY IS TO HAVE A WELLBALANCED ECONOMY \\
\hline & & & PROVIDING EMPLOYMENT AND INVESTMENT OPPORTUNITIES FOR ALL AGES AND \\
\hline & & & SUSTAINING A FULL RANGE OF SUPPORT SERVICES \\
\hline
\end{tabular}


Appendix B: Community Well-being Statements by Councils

\begin{tabular}{|c|c|c|c|}
\hline 336 & MANAWATU DISTRICT COUNCIL & $\begin{array}{l}\text { A COMMUNITY THAT HAS ACCESS TO EFFECTIVE } \\
\text { SERVICES }\end{array}$ & A COMMUNITY THAT HAS ACCESS TO EFFECTIVE SERVICES \\
\hline 337 & MANAWATU DISTRICT COUNCIL & $\begin{array}{l}\text { A SELFSUFFICIENT ENVIRONMENT THAT PRESERVES } \\
\text { VALUES AND DEVELOPS OUR NATURAL } \\
\text { ENVIRONMENT }\end{array}$ & $\begin{array}{l}\text { A SELFSUFFICIENT ENVIRONMENT THAT PRESERVES VALUES AND DEVELOPS OUR } \\
\text { NATURAL ENVIRONMENT }\end{array}$ \\
\hline 338 & MANAWATU DISTRICT COUNCIL & $\begin{array}{l}\text { A VITAL COMMUNITY THAT IS COHESIVE AND } \\
\text { CHARACTERISED BY COMMUNITY INVOLVEMENT }\end{array}$ & $\begin{array}{l}\text { A VITAL COMMUNITY THAT IS COHESIVE AND CHARACTERISED BY COMMUNITY } \\
\text { INVOLVEMENT }\end{array}$ \\
\hline 339 & MANAWATU DISTRICT COUNCIL & $\begin{array}{l}\text { PEOPLE ARE ABLE TO GO ABOUT THEIR BUSINESS } \\
\text { AND LEISURE ANY TIME OF THE DAY OR EVENING } \\
\text { WITHOUT FEAR FOR THEIR SAFETY }\end{array}$ & $\begin{array}{l}\text { PEOPLE ARE ABLE TO GO ABOUT THEIR BUSINESS AND LEISURE ANY TIME OF THE DAY OR } \\
\text { EVENING WITHOUT FEAR FOR THEIR SAFETY }\end{array}$ \\
\hline 340 & $\begin{array}{l}\text { MANAWATUWANGANUI REGIONAL } \\
\text { COUNCIL }\end{array}$ & A ROBUST ECONOMY & A ROBUST ECONOMY \\
\hline 341 & $\begin{array}{l}\text { MANAWATUWANGANUI REGIONAL } \\
\text { COUNCIL }\end{array}$ & CONFIDENCE IN DECISIONMAKING & CONFIDENCE IN DECISIONMAKING \\
\hline 342 & $\begin{array}{l}\text { MANAWATUWANGANUI REGIONAL } \\
\text { COUNCIL }\end{array}$ & CONNECTED COMMUNITIES & CONNECTED COMMUNITIES \\
\hline 343 & $\begin{array}{l}\text { MANAWATUWANGANUI REGIONAL } \\
\text { COUNCIL }\end{array}$ & HEALTHY ECOSYSTEMS & HEALTHY ECOSYSTEMS \\
\hline 344 & $\begin{array}{l}\text { MANAWATUWANGANUI REGIONAL } \\
\text { COUNCIL }\end{array}$ & HUMAN WELLBEING & HUMAN WELLBEING \\
\hline 345 & $\begin{array}{l}\text { MANAWATUWANGANUI REGIONAL } \\
\text { COUNCIL }\end{array}$ & NATURAL HAZARD RESILIENCE & NATURAL HAZARD RESILIENCE \\
\hline 346 & MARLBOROUGH DISTRICT COUNCIL & AFFORDABLE HOUSING & $\begin{array}{l}\text { A COMMUNITY WHERE PEOPLE HAVE ACCESS TO A RANGE OF AFFORDABLE AND QUALITY } \\
\text { HOUSING OPTIONS }\end{array}$ \\
\hline 347 & MARLBOROUGH DISTRICT COUNCIL & CREATIVITY & $\begin{array}{l}\text { AN ENLIVENED AND CREATIVE COMMUNITY IN WHICH DIFFERENT ARTS ARE WIDELY } \\
\text { PRACTISED AND ENJOYED }\end{array}$ \\
\hline 348 & MARLBOROUGH DISTRICT COUNCIL & ENERGY EFFICIENCY & $\begin{array}{l}\text { A COMMUNITY WHERE ENERGY USE IS EFFICIENT WITH A DECREASING DEPENDENCY ON } \\
\text { NONRENEWABLE SOURCES }\end{array}$ \\
\hline 349 & MARLBOROUGH DISTRICT COUNCIL & ENTERPRISE AND ENDEAVOUR & A COMMUNITY WHERE ENTERPRISE AND ENDEAVOUR IS SUPPORTED AND REWARDED \\
\hline 350 & MARLBOROUGH DISTRICT COUNCIL & ENVIRONMENTAL SUSTAINABILITY & $\begin{array}{l}\text { A COMMUNITY THAT SUSTAINS AND ENRICHES THE ENVIRONMENT FOR FUTURE } \\
\text { GENERATIONS }\end{array}$ \\
\hline 351 & MARLBOROUGH DISTRICT COUNCIL & ESSENTIAL SERVICES & $\begin{array}{l}\text { A COMMUNITY THAT IS SERVED BY A STRONG INFRASTRUCTURE OF ESSENTIAL SERVICES } \\
\text { WHERE DAILY LIFE AND BUSINESS IS ABLE TO BE CONDUCTED SAFELY AND EASILY }\end{array}$ \\
\hline
\end{tabular}


Appendix B: Community Well-being Statements by Councils

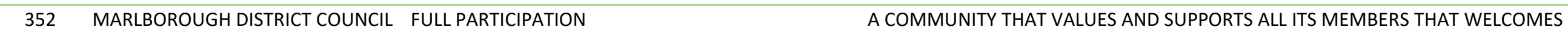
VISITORS AND NEW ARRIVALS AND CONTINUALLY ENHANCES FULL PARTICIPATION

354 MARLBOROUGH DISTRICT COUNCIL HEALTH CHOICES

355 MARLBOROUGH DISTRICT COUNCIL HERITAGE

356 MARLBOROUGH DISTRICT COUNCIL KNOWLEDGE AND LEARNING

357 MARLBOROUGH DISTRICT COUNCIL PHYSICAL ACTIVITY

358 MARLBOROUGH DISTRICT COUNCIL POSITIVE AGING

359 MARLBOROUGH DISTRICT COUNCIL POSITIVE YOUTH
353 MARLBOROUGH DISTRICT COUNCIL FUN AND RECREATION

A COMMUNITY THAT HAS FUN

A COMMUNITY WHERE PEOPLE ARE SERVED BY A HEALTH INFRASTRUCTURE THAT IS SUITED AND RESPONSIVE TO THEIR NEEDS AND WHERE THEY CAN MAKE HEALTHY CHOICES FOR THEIR OWN LIFESTYLES

A COMMUNITY THAT ACKNOWLEDGES VALUES AND ENJOYS ITS HERITAGE

A COMMUNITY WHERE KNOWLEDGE AND LEARNING IS PRIZED

A COMMUNITY WHERE PEOPLE OF ALL AGES ARE PHYSICALLY ACTIVE

A COMMUNITY WHERE PEOPLE CAN AGE POSITIVELY WHERE OLDER PEOPLE ARE HIGHLY VALUED FOR THEIR EXPERIENCE WISDOM AND CHARACTER AND WHERE THEY ARE RECOGNISED AS AN INTEGRAL PART OF FAMILIES AND COMMUNITIES

A COMMUNITY WHERE YOUNG PEOPLE ARE VIBRANT AND OPTIMISTIC ENCOURAGED TO TAKE UP CHALLENGES AND SUPPORTED IN THEIR LIFESTYLE CHOICES

A PROSPEROUS COMMUNITY WHERE ALL PEOPLE HAVE THE MEANS TO EARN ADEQUATE INCOMES AND ENJOY STANDARDS OF LIVING THAT ALLOW THEM TO PARTICIPATE FULLY IN SOCIETY AND TO HAVE CHOICES ABOUT HOW TO LIVE THEIR LIVES

361 MARLBOROUGH DISTRICT COUNCIL SAFETY AND SECURITY

A COMMUNITY WHERE PEOPLE ENJOY PERSONAL SAFETY AND SECURITY AND ARE FREE

FROM VICTIMISATION ABUSE VIOLENCE AND AVOIDABLE INJURY

MASTERTON DISTRICT COUNCIL

BUOYANT LOCAL ECONOMY

BUOYANT LOCAL ECONOMY

BUOYANT LOCAL ECONOMY

BUOYANT LOCAL ECONOMY

BUOYANT LOCAL ECONOMY

BUOYANT LOCAL ECONOMY

EDUCATED AND KNOWLEDGEABLE PEOPLE

EDUCATED AND KNOWLEDGEABLE PEOPLE

EDUCATED AND KNOWLEDGEABLE PEOPLE

EDUCATED AND KNOWLEDGEABLE PEOPLE

EDUCATED AND KNOWLEDGEABLE PEOPLE

EQUITABLE SOCIETY
BUILDING

ECONOMIC GROWTH

EMPLOYMENT OPPORTUNITIES

INCOME

TOURISM

UNEMPLOYMENT

EARLY CHILDHOOD EDUCATION

EDUCATIONAL ATTAINMENT

INTERNET ACCESS IN THE HOME

SCHOOL LEAVER QUALIFICATIONS

VOTING AT LOCAL BODY ELECTIONS
MĀORI LANGUAGE SPEAKERS 
Appendix B: Community Well-being Statements by Councils

\begin{tabular}{|c|c|c|c|}
\hline 374 & MASTERTON DISTRICT COUNCIL & EQUITABLE SOCIETY & NUMBER OF LANGUAGES SPOKEN \\
\hline 375 & MASTERTON DISTRICT COUNCIL & EQUITABLE SOCIETY & SOCIAL DEPRIVATION \\
\hline 376 & MASTERTON DISTRICT COUNCIL & GROW MASTERTON & DEMOGRAPHIC PROFILE \\
\hline 377 & MASTERTON DISTRICT COUNCIL & GROW MASTERTON & POPULATION GROWTH \\
\hline 378 & MASTERTON DISTRICT COUNCIL & SAFE AND EFFICIENT TRANSPORT & AIR QUALITY \\
\hline 379 & MASTERTON DISTRICT COUNCIL & SAFE AND EFFICIENT TRANSPORT & INJURY RATES \\
\hline 380 & MASTERTON DISTRICT COUNCIL & SAFE AND EFFICIENT TRANSPORT & PROVISION OF PUBLIC TRANSPORT \\
\hline 381 & MASTERTON DISTRICT COUNCIL & SAFE AND EFFICIENT TRANSPORT & ROAD CASUALTIES \\
\hline 382 & MASTERTON DISTRICT COUNCIL & SAFE AND EFFICIENT TRANSPORT & SATISFACTION WITH ROADS STREETS AND FOOTPATHS \\
\hline 383 & MASTERTON DISTRICT COUNCIL & SUSTAINABLE USE OF ENVIRONMENT & AIR QUALITY \\
\hline 384 & MASTERTON DISTRICT COUNCIL & SUSTAINABLE USE OF ENVIRONMENT & BEHAVIOUR CHANGE \\
\hline 385 & MASTERTON DISTRICT COUNCIL & SUSTAINABLE USE OF ENVIRONMENT & GREEN SPACE PARKS \\
\hline 386 & MASTERTON DISTRICT COUNCIL & SUSTAINABLE USE OF ENVIRONMENT & PEST MANAGEMENT \\
\hline 387 & MASTERTON DISTRICT COUNCIL & SUSTAINABLE USE OF ENVIRONMENT & SATISFACTION WITH PRESERVATION OF THE NATURAL ENVIRONMENT \\
\hline 388 & MASTERTON DISTRICT COUNCIL & SUSTAINABLE USE OF ENVIRONMENT & WATER QUALITY \\
\hline 389 & MASTERTON DISTRICT COUNCIL & VIBRANT STRONG AND HEALTHY COMMUNITIES & HOUSEHOLD ACCESS TO A MOTOR VEHICLE \\
\hline 390 & MASTERTON DISTRICT COUNCIL & VIBRANT STRONG AND HEALTHY COMMUNITIES & LIFE EXPECTANCY \\
\hline 391 & MASTERTON DISTRICT COUNCIL & VIBRANT STRONG AND HEALTHY COMMUNITIES & PERCEPTION OF SAFETY \\
\hline 392 & MASTERTON DISTRICT COUNCIL & VIBRANT STRONG AND HEALTHY COMMUNITIES & PHYSICAL ACTIVITY RATES \\
\hline 393 & MASTERTON DISTRICT COUNCIL & VIBRANT STRONG AND HEALTHY COMMUNITIES & QUALITY OF PLACE \\
\hline 394 & MASTERTON DISTRICT COUNCIL & VIBRANT STRONG AND HEALTHY COMMUNITIES & TELEPHONE AND INTERNET ACCESS IN THE HOME \\
\hline 395 & MASTERTON DISTRICT COUNCIL & VIBRANT STRONG AND HEALTHY COMMUNITIES & VOTING AT THE ELECTIONS \\
\hline 396 & $\begin{array}{l}\text { MATAMATAPIAKO DISTRICT } \\
\text { COUNCIL }\end{array}$ & BELONGING TO OUR COMMUNITY & DOING THINGS TOGETHER \\
\hline 397 & $\begin{array}{l}\text { MATAMATAPIAKO DISTRICT } \\
\text { COUNCIL }\end{array}$ & BELONGING TO OUR COMMUNITY & IMPROVING LIFE ON A DAY TO DAY BASIS \\
\hline 398 & $\begin{array}{l}\text { MATAMATAPIAKO DISTRICT } \\
\text { COUNCIL }\end{array}$ & BELONGING TO OUR COMMUNITY & PARTICIPATING IN DECISIONMAKING \\
\hline 399 & $\begin{array}{l}\text { MATAMATAPIAKO DISTRICT } \\
\text { COUNCIL }\end{array}$ & BELONGING TO OUR COMMUNITY & PROMOTION OF CITIZEN RESPONSIBILITY \\
\hline
\end{tabular}


Appendix B: Community Well-being Statements by Councils

\begin{tabular}{|c|c|c|c|}
\hline 400 & $\begin{array}{l}\text { MATAMATAPIAKO DISTRICT } \\
\text { COUNCIL }\end{array}$ & BELONGING TO OUR COMMUNITY & RECREATION AND ARTS \\
\hline 401 & $\begin{array}{l}\text { MATAMATAPIAKO DISTRICT } \\
\text { COUNCIL }\end{array}$ & BELONGING TO OUR COMMUNITY & VOLUNTEERS \\
\hline 402 & $\begin{array}{l}\text { MATAMATAPIAKO DISTRICT } \\
\text { COUNCIL }\end{array}$ & $\begin{array}{l}\text { COMMUNITY SAFETY AND SUPPORT LOOKING AFTER } \\
\text { PEOPLE }\end{array}$ & SAFETY \\
\hline 403 & $\begin{array}{l}\text { MATAMATAPIAKO DISTRICT } \\
\text { COUNCIL }\end{array}$ & $\begin{array}{l}\text { COMMUNITY SAFETY AND SUPPORT LOOKING AFTER } \\
\text { PEOPLE }\end{array}$ & SUPPORT FOR YOUNG PEOPLE \\
\hline 404 & $\begin{array}{l}\text { MATAMATAPIAKO DISTRICT } \\
\text { COUNCIL }\end{array}$ & ECONOMIC DEVELOPMENT PROSPERITY & BUSINESS FARMING AND INDUSTRY \\
\hline 405 & $\begin{array}{l}\text { MATAMATAPIAKO DISTRICT } \\
\text { COUNCIL }\end{array}$ & ECONOMIC DEVELOPMENT PROSPERITY & EMPLOYMENT \\
\hline 406 & $\begin{array}{l}\text { MATAMATAPIAKO DISTRICT } \\
\text { COUNCIL }\end{array}$ & ECONOMIC DEVELOPMENT PROSPERITY & TOURISM \\
\hline 407 & $\begin{array}{l}\text { MATAMATAPIAKO DISTRICT } \\
\text { COUNCIL }\end{array}$ & HEALTHY AIR WATER LAND HEALTHY PEOPLE & AIR POLLUTION \\
\hline 408 & $\begin{array}{l}\text { MATAMATAPIAKO DISTRICT } \\
\text { COUNCIL }\end{array}$ & HEALTHY AIR WATER LAND HEALTHY PEOPLE & HEALTHY PEOPLE \\
\hline 409 & $\begin{array}{l}\text { MATAMATAPIAKO DISTRICT } \\
\text { COUNCIL }\end{array}$ & HEALTHY AIR WATER LAND HEALTHY PEOPLE & PESTS AND NOXIOUS WEEDS \\
\hline 410 & $\begin{array}{l}\text { MATAMATAPIAKO DISTRICT } \\
\text { COUNCIL }\end{array}$ & HEALTHY AIR WATER LAND HEALTHY PEOPLE & POLLUTION AND WASTE \\
\hline 411 & $\begin{array}{l}\text { MATAMATAPIAKO DISTRICT } \\
\text { COUNCIL }\end{array}$ & HEALTHY AIR WATER LAND HEALTHY PEOPLE & PROTECTING THE LANDSCAPE \\
\hline 412 & $\begin{array}{l}\text { MATAMATAPIAKO DISTRICT } \\
\text { COUNCIL }\end{array}$ & HEALTHY AIR WATER LAND HEALTHY PEOPLE & WATER QUANTITY AND QUALITY \\
\hline 413 & $\begin{array}{l}\text { MATAMATAPIAKO DISTRICT } \\
\text { COUNCIL }\end{array}$ & HERITAGE OUR PAST & BUILT HERITAGE \\
\hline 414 & $\begin{array}{l}\text { MATAMATAPIAKO DISTRICT } \\
\text { COUNCIL }\end{array}$ & HERITAGE OUR PAST & KNOWLEDGE AND TREASURES \\
\hline 415 & $\begin{array}{l}\text { MATAMATAPIAKO DISTRICT } \\
\text { COUNCIL }\end{array}$ & HERITAGE OUR PAST & MOUNT TE AROHA \\
\hline 416 & $\begin{array}{l}\text { MATAMATAPIAKO DISTRICT } \\
\text { COUNCIL }\end{array}$ & HERITAGE OUR PAST & TE AROHA DOMAIN \\
\hline 417 & $\begin{array}{l}\text { MATAMATAPIAKO DISTRICT } \\
\text { COUNCIL }\end{array}$ & HERITAGE OUR PAST & WETLANDS SIGNIFICANT NATURAL FEATURES AND WAHI TAPU \\
\hline 418 & $\begin{array}{l}\text { MATAMATAPIAKO DISTRICT } \\
\text { COUNCIL }\end{array}$ & OUR SOCIAL INFRASTRUCTURE & OUTSIDE AGENCIES MEETING OUR COMMUNITIES NEEDS \\
\hline
\end{tabular}


Appendix B: Community Well-being Statements by Councils

\begin{tabular}{|c|c|c|c|}
\hline 419 & $\begin{array}{l}\text { MATAMATAPIAKO DISTRICT } \\
\text { COUNCIL }\end{array}$ & OUR SOCIAL INFRASTRUCTURE & SERVICES \\
\hline 420 & $\begin{array}{l}\text { MATAMATAPIAKO DISTRICT } \\
\text { COUNCIL }\end{array}$ & PLANNING AND DEVELOPMEN & INDUSTRIAL DEVELOPMENT \\
\hline 421 & $\begin{array}{l}\text { MATAMATAPIAKO DISTRICT } \\
\text { COUNCIL }\end{array}$ & PLANNING AND DEVELOPMEN & INTENSIVE FARMING \\
\hline 422 & $\begin{array}{l}\text { MATAMATAPIAKO DISTRICT } \\
\text { COUNCIL }\end{array}$ & PLANNING AND DEVELOPMEN & RURAL SUBDIVISION AND AMALGAMATION \\
\hline 423 & $\begin{array}{l}\text { MATAMATAPIAKO DISTRICT } \\
\text { COUNCIL }\end{array}$ & PLANNING AND DEVELOPMEN & SMALL TOWN CULTURE LARGE TOWN INFRASTRUCTURE \\
\hline 424 & $\begin{array}{l}\text { MATAMATAPIAKO DISTRICT } \\
\text { COUNCIL }\end{array}$ & PLANNING AND DEVELOPMEN & TOWN APPEARANCE \\
\hline 425 & $\begin{array}{l}\text { MATAMATAPIAKO DISTRICT } \\
\text { COUNCIL }\end{array}$ & PLANNING AND DEVELOPMEN & TOWN PLANNING \\
\hline 426 & $\begin{array}{l}\text { MATAMATAPIAKO DISTRICT } \\
\text { COUNCIL }\end{array}$ & PRIDE AND JUSTICE & PRIDE AND JUSTICE \\
\hline 427 & $\begin{array}{l}\text { MATAMATAPIAKO DISTRICT } \\
\text { COUNCIL }\end{array}$ & TRANSPORT PEOPLE GOING PLACES & PARKING \\
\hline 428 & $\begin{array}{l}\text { MATAMATAPIAKO DISTRICT } \\
\text { COUNCIL }\end{array}$ & TRANSPORT PEOPLE GOING PLACES & PEOPLE AND TRAFFIC FLOW \\
\hline 429 & NAPIER CITY COUNCIL & $\begin{array}{l}\text { A SAFE AND HEALTHY CITY THAT SUPPORTS } \\
\text { COMMUNITY WELLBEING }\end{array}$ & A SAFE AND HEALTHY CITY THAT SUPPORTS COMMUNITY WELLBEING \\
\hline 430 & NAPIER CITY COUNCIL & A SUSTAINABLE CITY & A SUSTAINABLE CITY \\
\hline 431 & NAPIER CITY COUNCIL & A VIBRANT INNOVATIVE CITY FOR EVERYONE & A VIBRANT INNOVATIVE CITY FOR EVERYONE \\
\hline 432 & NAPIER CITY COUNCIL & COUNCIL WORKS WITH AND FOR THE COMMUNITY & COUNCIL WORKS WITH AND FOR THE COMMUNITY \\
\hline 433 & NAPIER CITY COUNCIL & $\begin{array}{l}\text { EXCELLENCE IN INFRASTRUCTURE AND PUBLIC } \\
\text { SERVICES FOR NOW AND IN THE FUTURE }\end{array}$ & EXCELLENCE IN INFRASTRUCTURE AND PUBLIC SERVICES FOR NOW AND IN THE FUTURE \\
\hline 434 & NELSON CITY COUNCIL & A FUN CREATIVE CULTURE & WE ARE PROUD OF OUR CREATIVE LOCAL CULTURE AND REGIONAL IDENTITY \\
\hline 435 & NELSON CITY COUNCIL & A STRONG ECONOMY & WE ALL BENEFIT FROM A SUSTAINABLE INNOVATIVE AND DIVERSIFIED ECONOMY \\
\hline 436 & NELSON CITY COUNCIL & GOOD LEADERSHIP & OUR LEADERS ARE PROACTIVE INNOVATIVE AND INCLUSIVE \\
\hline 437 & NELSON CITY COUNCIL & HEALTHY LAND SEA AIR AND WATER & WE PROTECT THE NATURAL ENVIRONMENT \\
\hline 438 & NELSON CITY COUNCIL & KIND HEALTHY PEOPLE & WE ARE PART OF A WELCOMING SAFE INCLUSIVE AND HEALTHY COMMUNITY \\
\hline 439 & NELSON CITY COUNCIL & PEOPLEFRIENDLY PLACES & $\begin{array}{l}\text { WE BUILD HEALTHY ACCESSIBLE AND ATTRACTIVE PLACES AND LIVE IN A SUSTAINABLE } \\
\text { REGION }\end{array}$ \\
\hline
\end{tabular}


Appendix B: Community Well-being Statements by Councils

\begin{tabular}{|c|c|c|c|}
\hline 440 & NEW PLYMOUTH DISTRICT COUNCIL & CONNECTED RELATES TO OUR INFRASTRUCTURE & $\begin{array}{l}\text { A DISTRICT THAT DELIVERS ACCESSIBLE AND INTEGRATED INFRASTRUCTURE TRANSPORT } \\
\text { AND COMMUNICATION SYSTEMS WHICH MEET THE NEEDS OF RESIDENTS BUSINESSES } \\
\text { AND VISITORS }\end{array}$ \\
\hline 441 & NEW PLYMOUTH DISTRICT COUNCIL & PROSPEROUS OUR ECONOMY & $\begin{array}{l}\text { A DISTRICT THAT BOASTS A SUSTAINABLE RESILIENT AND INNOVATIVE ECONOMY THAT } \\
\text { PROSPERS WITHIN THE NATURAL AND SOCIAL ENVIRONMENT }\end{array}$ \\
\hline 442 & NEW PLYMOUTH DISTRICT COUNCIL & $\begin{array}{l}\text { SECURE AND HEALTHY OUR COMMUNITY } \\
\text { WELLBEING }\end{array}$ & $\begin{array}{l}\text { A DISTRICT THAT PROVIDES A SAFE HEALTHY AND FRIENDLY PLACE TO LIVE WORK OR } \\
\text { VISIT }\end{array}$ \\
\hline 443 & NEW PLYMOUTH DISTRICT COUNCIL & $\begin{array}{l}\text { SKILLED OUR LEARNING AND EDUCATION } \\
\text { OPPORTUNITIES }\end{array}$ & $\begin{array}{l}\text { A DISTRICT THAT VALUES AND SUPPORTS LEARNING SO ALL PEOPLE CAN PLAY A FULL } \\
\text { AND ACTIVE ROLE IN SOCIAL CULTURAL AND ECONOMIC LIFE }\end{array}$ \\
\hline 444 & NEW PLYMOUTH DISTRICT COUNCIL & SUSTAINABLE OUR ENVIRONMENT & $\begin{array}{l}\text { A DISTRICT THAT APPRECIATES ITS NATURAL ENVIRONMENT AND ITS PHYSICAL AND } \\
\text { HUMAN RESOURCES IN PLANNING DELIVERY AND PROTECTION }\end{array}$ \\
\hline 445 & NEW PLYMOUTH DISTRICT COUNCIL & TOGETHER OUR COMMUNITY COHESION & $\begin{array}{l}\text { A DISTRICT THAT IS CARING INCLUSIVE AND WORKS TOGETHER AND WHERE PEOPLE } \\
\text { HAVE A STRONG DISTINCTIVE SENSE OF IDENTITY }\end{array}$ \\
\hline 446 & NEW PLYMOUTH DISTRICT COUNCIL & $\begin{array}{l}\text { VIBRANT OUR CULTURE LEISURE AND RECREATION } \\
\text { OPPORTUNITIES }\end{array}$ & $\begin{array}{l}\text { A DISTRICT THAT PROVIDES HIGHQUALITY AND DIVERSE CULTURAL AND RECREATIONAL } \\
\text { EXPERIENCES AND WHERE INDEPENDENCE AND CREATIVITY ARE ENCOURAGED }\end{array}$ \\
\hline 447 & NORTHLAND REGIONAL COUNCIL & NORTHLAND HAS COHESIVE COMMUNITIES & NORTHLAND HAS COHESIVE COMMUNITIES \\
\hline 448 & NORTHLAND REGIONAL COUNCIL & NORTHLAND IS PROSPEROUS & NORTHLAND IS PROSPEROUS \\
\hline 449 & NORTHLAND REGIONAL COUNCIL & NORTHLAND RESIDENTS ARE EDUCATEDAND SKILLED & NORTHLAND RESIDENTS ARE EDUCATEDAND SKILLED \\
\hline 450 & NORTHLAND REGIONAL COUNCIL & NORTHLAND RESIDENTS ARE SAFE AND HEALTHY & NORTHLAND RESIDENTS ARE SAFE AND HEALTHY \\
\hline 451 & NORTHLAND REGIONAL COUNCIL & $\begin{array}{l}\text { NORTHLAND RESIDENTS HAVE ACCESS TO } \\
\text { RECREATIONAL AND LEISURE OPPORTUNITIES }\end{array}$ & NORTHLAND RESIDENTS HAVE ACCESS TO RECREATIONAL AND LEISURE OPPORTUNITIES \\
\hline 452 & NORTHLAND REGIONAL COUNCIL & $\begin{array}{l}\text { NORTHLAND RETAINS AND ENHANCES ITSREGIONAL } \\
\text { IDENTITY }\end{array}$ & NORTHLAND RETAINS AND ENHANCES ITSREGIONAL IDENTITY \\
\hline 453 & NORTHLAND REGIONAL COUNCIL & $\begin{array}{l}\text { NORTHLANDS INFRASTRUCTURE IS DEVELOPEDIN A } \\
\text { SUSTAINABLE WAY }\end{array}$ & NORTHLANDS INFRASTRUCTURE IS DEVELOPEDIN A SUSTAINABLE WAY \\
\hline 454 & NORTHLAND REGIONAL COUNCIL & $\begin{array}{l}\text { NORTHLANDS NATURAL ENVIRONMENT } \\
\text { ISSUSTAINABLY MANAGED }\end{array}$ & NORTHLANDS NATURAL ENVIRONMENT ISSUSTAINABLY MANAGED \\
\hline 455 & OPOTIKI DISTRICT COUNCIL & A STRONG AND DISTINCTIVE COMMUNITY SPIRIT & $\begin{array}{l}\text { OUR RURAL COMMUNITY IS FRIENDLY AND SUPPORTIVE AND WORKS TOGETHER TO } \\
\text { ACHIEVE COMMON GOALS WE WANT TO ENSURE THAT THE STRONG AND DISTINCTIVE } \\
\text { SPIRIT AND CHARACTER OF OUR COMMUNITY DOES NOT CHANGE OVER TIME THIS IS } \\
\text { WHO WE ARE AND WE ARE PROUD OF IT }\end{array}$ \\
\hline
\end{tabular}




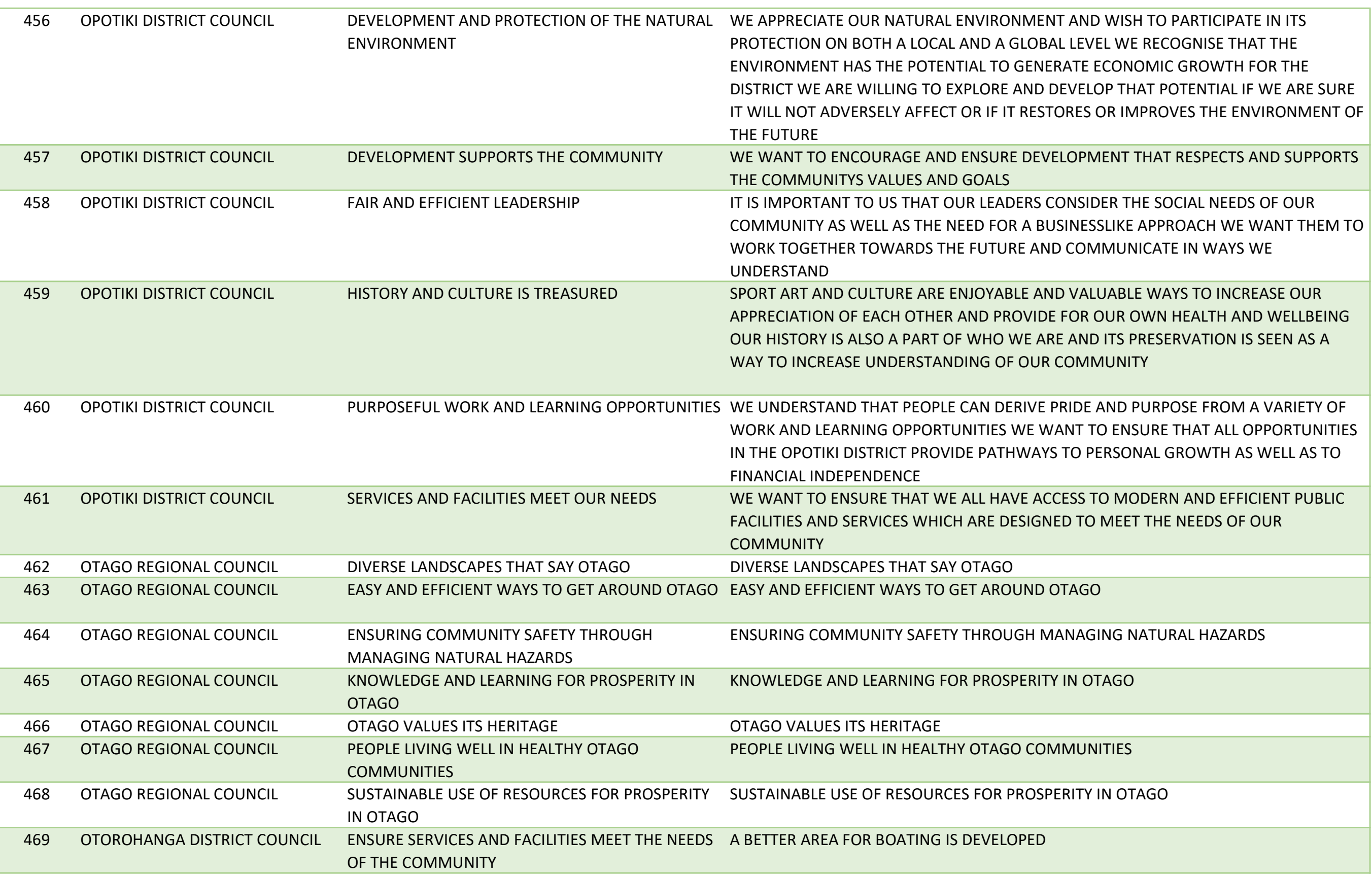


Appendix B: Community Well-being Statements by Councils

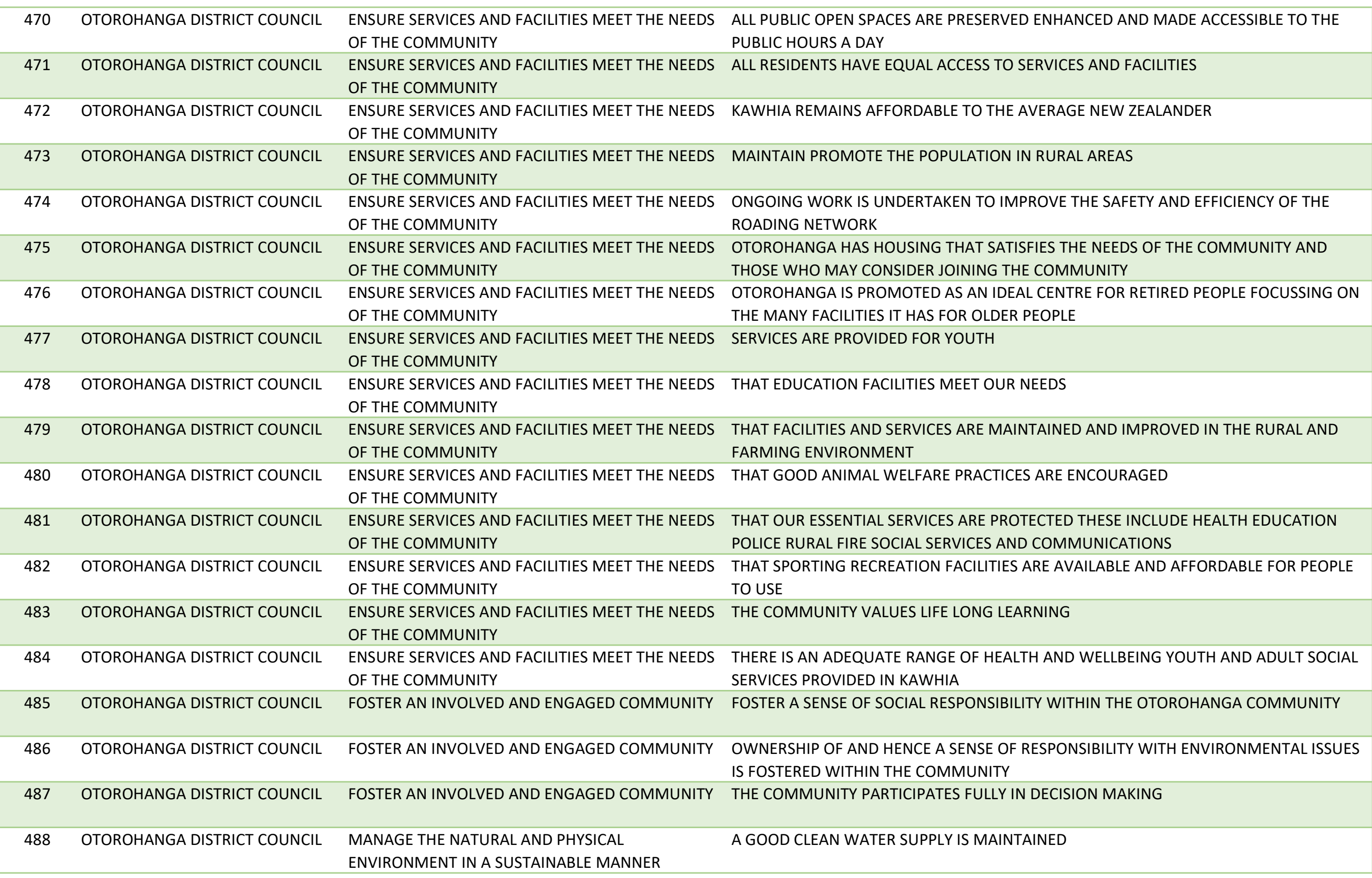




\begin{tabular}{|c|c|c|c|}
\hline 489 & OTOROHANGA DISTRICT COUNCIL & $\begin{array}{l}\text { MANAGE THE NATURAL AND PHYSICAL } \\
\text { ENVIRONMENT IN A SUSTAINABLE MANNER }\end{array}$ & $\begin{array}{l}\text { ALL BODIES OF WATER ARE RETURNED TO THEIR BEST POSSIBLE CONDITION THEY ARE } \\
\text { RECOGNISED AND ENHANCED AS LIVING ECOSYSTEMS AND AS HAVING CULTURAL } \\
\text { SIGNIFICANCE FOR TANGATA WHENUA LOCAL RESIDENTS PEOPLE OF THE LAND }\end{array}$ \\
\hline 491 & OTOROHANGA DISTRICT COUNCIL & $\begin{array}{l}\text { MANAGE THE NATURAL AND PHYSICAL } \\
\text { ENVIRONMENT IN A SUSTAINABLE MANNER }\end{array}$ & ENHANCE OUR CLEAN GREEN IMAGE \\
\hline 493 & OTOROHANGA DISTRICT COUNCIL & $\begin{array}{l}\text { MANAGE THE NATURAL AND PHYSICAL } \\
\text { ENVIRONMENT IN A SUSTAINABLE MANNER }\end{array}$ & THAT LANDUSE IS SUSTAINABLE \\
\hline 494 & OTOROHANGA DISTRICT COUNCIL & $\begin{array}{l}\text { MANAGE THE NATURAL AND PHYSICAL } \\
\text { ENVIRONMENT IN A SUSTAINABLE MANNER }\end{array}$ & THE COMMUNITY HAS READY ACCESS TO ITS NATURAL ENVIRONMENT \\
\hline 495 & OTOROHANGA DISTRICT COUNCIL & $\begin{array}{l}\text { MANAGE THE NATURAL AND PHYSICAL } \\
\text { ENVIRONMENT IN A SUSTAINABLE MANNER }\end{array}$ & THE COMMUNITY IS ENHANCED THROUGH APPROPRIATE PLANTINGS \\
\hline 496 & OTOROHANGA DISTRICT COUNCIL & $\begin{array}{l}\text { MANAGE THE NATURAL AND PHYSICAL } \\
\text { ENVIRONMENT IN A SUSTAINABLE MANNER }\end{array}$ & THE COMMUNITY IS WORKING TOWARDS PRODUCING ZERO WASTE TO LANDFILL BY \\
\hline 497 & OTOROHANGA DISTRICT COUNCIL & $\begin{array}{l}\text { MANAGE THE NATURAL AND PHYSICAL } \\
\text { ENVIRONMENT IN A SUSTAINABLE MANNER }\end{array}$ & THE CONCEPT OF SUSTAINABLE ENVIRONMENTAL MANAGEMENT IS PROMOTED \\
\hline 498 & OTOROHANGA DISTRICT COUNCIL & OTOROHANGA IS A SAFE PLACE TO LIVE & FOOTPATH AND OTHER WALKING AREAS ARE SAFE FOR PEDESTRIANS \\
\hline 499 & OTOROHANGA DISTRICT COUNCIL & OTOROHANGA IS A SAFE PLACE TO LIVE & OTOROHANGA IS A SAFE ENVIRONMENT TO LIVE AND PLAY \\
\hline 500 & OTOROHANGA DISTRICT COUNCIL & OTOROHANGA IS A SAFE PLACE TO LIVE & $\begin{array}{l}\text { PEDESTRIANS AND MOTORISTS SAFETY IN THE MAIN STREET OF OTOROHANGA IS } \\
\text { ADDRESSED }\end{array}$ \\
\hline 501 & OTOROHANGA DISTRICT COUNCIL & OTOROHANGA IS A SAFE PLACE TO LIVE & THAT RURAL COMMUNITIES ARE AWARE OF CIVIL DEFENCE PROCEDURES \\
\hline 502 & OTOROHANGA DISTRICT COUNCIL & OTOROHANGA IS A SAFE PLACE TO LIVE & ZERO TOLERANCE TO DRUGS IS PROMOTED IN OTOROHANGA \\
\hline 503 & OTOROHANGA DISTRICT COUNCIL & $\begin{array}{l}\text { PROMOTE THE ECONOMY AND OPPORTUNITIES FOR } \\
\text { SUSTAINABLE ECONOMIC DEVELOPMENT }\end{array}$ & $\begin{array}{l}\text { A BALANCE OF SUSTAINABLE ECONOMIC DEVELOPMENT APPROPRIATE TO THIS AREA IS } \\
\text { ACHIEVED WITHOUT OVER COMMERCIALISATION }\end{array}$ \\
\hline 504 & OTOROHANGA DISTRICT COUNCIL & $\begin{array}{l}\text { PROMOTE THE ECONOMY AND OPPORTUNITIES FOR } \\
\text { SUSTAINABLE ECONOMIC DEVELOPMENT }\end{array}$ & AN HISTORIC TOURIST ROUTE IS DEVELOPED AND PROMOTED \\
\hline
\end{tabular}


506 OTOROHANGA DISTRICT COUNCIL

SUSTAINABLE ECONOMIC DEVELOPMENT
EXISTING ECONOMIC STRENGTHS IN THE COMMUNITY ARE PROMOTED AND MAKE THE TOWN AN ATTRACTIVE DESTINATION FOR THE DEVELOPMENT OF SMALL TO MEDIUM SIZED BUSINESSES THAT THE BUSINESS COMMUNITY IS VIBRANT AND PROGRESSIVE AND A CORNERSTONE OF THE GREATER COMMUNITY
PROMOTE THE ECONOMY AND OPPORTUNITIES FOR SUSTAINABLE ECONOMIC DEVELOPMENT
OTOHANGA IS A MUSTSEE TOURIST DESTINATION ITS MANY ATTRIBUTES ARE ENHANCED INCLUDING ITS NATURAL FEATURES SUCH AS RIVERS GARDENS AND KAHIKITEA REMNANTS ITS POSITION AS A TRAVEL GATEWAY AND TOURISM NEW ZEALANDS CATEGORISATION OF OTOROHANGA AS AN IDEAL TOWN

\section{OTOROHANGA DISTRICT COUNCIL}

PROMOTE THE ECONOMY AND OPPORTUNITIES FOR SUSTAINABLE ECONOMIC DEVELOPMENT MAJOR SUSTAINABLE ECONOMIC BASE OF THE DISTRICT

509 OTOROHANGA DISTRICT COUNCIL

HOTE THE ECONOMY AND OPPORTUNI THAT OTHER ECONOMIC ACTIVITIES ARE RECOGNISED

510 OTOROHANGA DISTRICT COUNCIL CONTRIBUTIONS TO THE ECONOMY OF THE DISTRICT

STE THE ECONOMY AND OPPORTUNITIES FOR THERE ARE ADEQUATE FLOWON EFFECTS OF ANY ECONOMIC BENEFITS SUSTAINABLE ECONOMIC DEVELOPMENT

511 OTOROHANGA DISTRICT COUNCIL PROTECT THE SPECIAL CHARACTER OF OUR

512 OTOROHANGA DISTRICT COUNCIL HARBOURS AND THEIR CATCHMENTS

513 OTOROHANGA DISTRICT COUNCIL

514 OTOROHANGA DISTRICT COUNCIL

515 OTOROHANGA DISTRICT COUNCIL

516 OTOROHANGA DISTRICT COUNCIL

PROVIDE FOR THE UNIQUE HISTORY AND CULTURE

517 OTOROHANGA DISTRICT COUNCIL

518 OTOROHANGA DISTRICT COUNCIL

519 OTOROHANGA DISTRICT COUNCIL

520 OTOROHANGA DISTRICT COUNCIL

521 OTOROHANGA DISTRICT COUNCIL HARBOURS AND THEIR CATCHMENTS

PROTECT THE SPECIAL CHARACTER OF OUR

PROTECT THE SPECIAL CHARACTER OF OUR HARBOURS AND THEIR CATCHMENTS

PROTECT THE SPECIAL CHARACTER OF OUR HARBOURS AND THEIR CATCHMENTS FIISE FOR THE UNIQUE HISTORY AND CULTURE OF THE DISTRICT OF THE DISTRICT PROVIDE FOR THE UNIQUE HISTORY AND CULTURE OF THE DISTRICT PROVIDE FOR THE UNIQUE HISTORY AND CULTURE OF THE DISTRICT PROVIDE FOR THE UNIQUE HISTORY AND CULTURE OF THE DISTRICT PROVIDE FOR THE UNIQUE HISTORY AND CULTURE OF THE DISTRICT

FISH STOCKS IN THE HARBOUR ARE REPLACED

THAT KAWHIA AND AOTEA HARBOURS ARE ACKNOWLEDGED TO BE UNIQUE ENVIRONMENTS

THAT OUR ESTUARIES HARBOURS WATERWAYS AND WETLANDS ARE PROTECTED

THE HARBOUR AND SURROUNDS BE CLEANED UP PRESERVED AND PROTECTED SIMILAR TO THE RAGLAN HARBOUR CARE PROJECT

A TOWN THEME IS DEVELOPED BASED UPON KAWHIAS UNIQUE HISTORICAL ENVIRONMENTAL AND KIWI BACH CULTURE IN ORDER TO MAINTAIN ITS UNIQUE INDIVIDUALITY AND CULTURE

OTOROHANGA HAS A STRONG AND VIBRANT ARTS COMMUNITY

OUR CHILDREN ARE ABLE TO EXPERIENCE THE CULTURE OF RURAL AND COASTAL LIFE FULLY

OUR CULTURAL FESTIVALS ARE RECOGNISED

TANGATA WHENUA ARE RECOGNISED AND THE COMMUNITYS COMMITMENT TO TE TIRITI O WAITANGI THE TREATY OF WAITANGI IS ACKNOWLEDGED

PROVIDE FOR THE UNIQUE HISTORY AND CULTURE THE MAORI CHARACTER OF KAWHIA IS RECOGNISED AND ENHANCED OF THE DISTRICT 


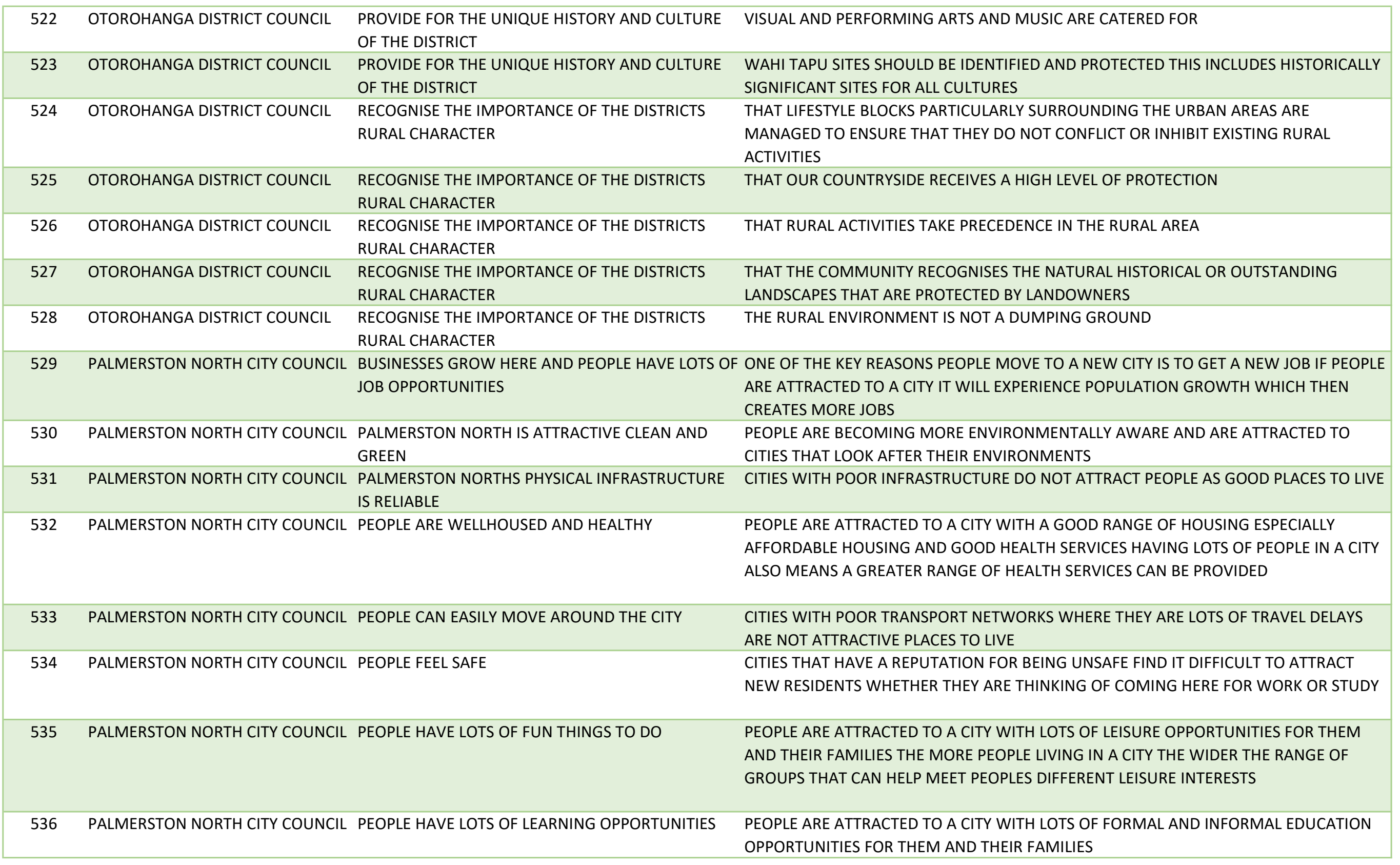




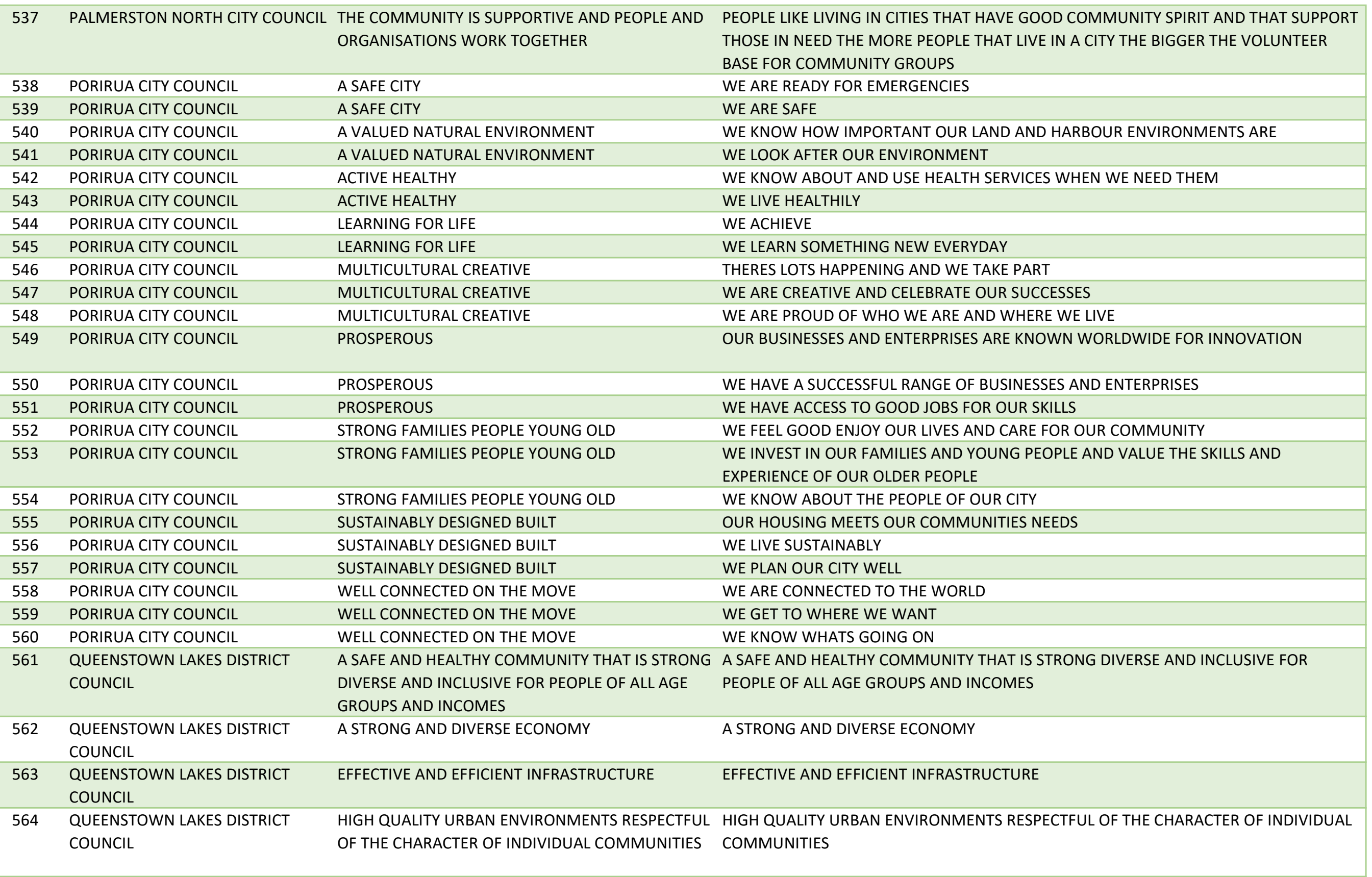




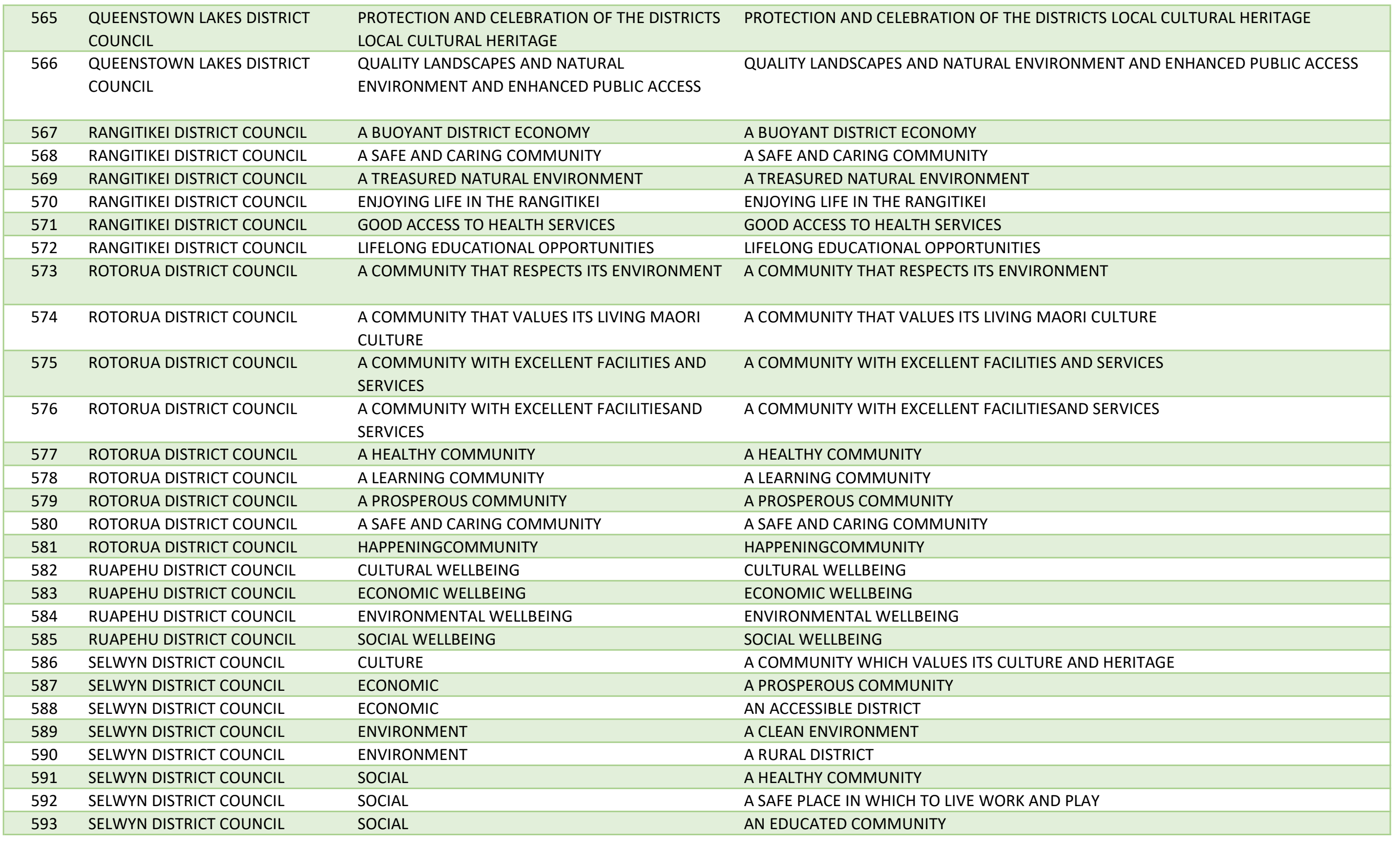


Appendix B: Community Well-being Statements by Councils

\begin{tabular}{|c|c|c|c|}
\hline 594 & $\begin{array}{l}\text { SOUTH TARANAKI DISTRICT } \\
\text { COUNCIL }\end{array}$ & PROSPEROUS & $\begin{array}{l}\text { A DISTRICT THAT BOASTS A SUSTAINABLE RESILIENT AND INNOVATIVE ECONOMY HAS } \\
\text { ACCESSIBLE AND INTEGRATED INFRASTRUCTURE COMMUNITY SERVICES AND OPEN } \\
\text { SPACE THAT PROSPER WITHIN THE NATURAL AND SOCIAL ENVIRONMENT }\end{array}$ \\
\hline 596 & $\begin{array}{l}\text { SOUTH TARANAKI DISTRICT } \\
\text { COUNCIL }\end{array}$ & TOGETHER & $\begin{array}{l}\text { A DISTRICT THAT LEADS WITH CARE WORKS TOGETHER ADVOCATES FOR ITS PEOPLE AND } \\
\text { ENABLES PEOPLE TO HAVE A STRONG AND DISTINCTIVE SENSE OF IDENTITY }\end{array}$ \\
\hline 597 & $\begin{array}{l}\text { SOUTH TARANAKI DISTRICT } \\
\text { COUNCIL }\end{array}$ & VIBRANT & $\begin{array}{l}\text { A DISTRICT THAT PROVIDES A HIGH QUALITY AND DIVERSE CULTURAL AND } \\
\text { RECREAOONAL EXPERIENCE AND ENCOURAGES INDEPENDENCE AND CREATIVITY }\end{array}$ \\
\hline 598 & SOUTH WAIKATO DISTRICT COUNCIL & A BETTER ENVIRONMENT FOR OUR CHILDREN & $\begin{array}{l}\text { COMMUNITY WASTE IS DISPOSED OF WITH MINIMAL IMPACT ON THE ENVIRONMENT } \\
\text { AND PEOPLE ARE ENCOURAGED TO RECYCLE AND REDUCE WASTE }\end{array}$ \\
\hline 599 & SOUTH WAIKATO DISTRICT COUNCIL & A BETTER ENVIRONMENT FOR OUR CHILDREN & $\begin{array}{l}\text { ECONOMIC GROWTH IS IN KEEPING WITH THE SPECIAL CHARACTER AND ENVIRONMENT } \\
\text { OF THE DISTRICT }\end{array}$ \\
\hline 601 & SOUTH WAIKATO DISTRICT COUNCIL & A BETTER ENVIRONMENT FOR OUR CHILDREN & THE DISTRICTS WATERWAYS AND SENSITIVE AREAS ARE MONITORED AND PROTECTED \\
\hline 602 & SOUTH WAIKATO DISTRICT COUNCIL & A BETTER ENVIRONMENT FOR OUR CHILDREN & $\begin{array}{l}\text { THE QUALITY OF THE DISTRICTS NATURAL ENVIRONMENT IS MONITORED PROTECTED } \\
\text { AND MAINTAINED AND PEOPLE ARE ENCOURAGED TO RESPECT AND UNDERSTAND THEIR } \\
\text { ENVIRONMENT }\end{array}$ \\
\hline 603 & SOUTH WAIKATO DISTRICT COUNCIL & A BETTER ENVIRONMENT FOR OUR CHILDREN & YOUNG PEOPLE ARE ACTIVELY INVOLVED IN CARING FOR THEIR ENVIRONMENT \\
\hline 604 & SOUTH WAIKATO DISTRICT COUNCIL & PROSPERITY FOR ALL & EXISTING BUSINESSES AND INDUSTRIES ARE RETAINED AND SUPPORTED \\
\hline 605 & SOUTH WAIKATO DISTRICT COUNCIL & PROSPERITY FOR ALL & NEW BUSINESS STARTUPS ARE ENCOURAGED \\
\hline 606 & SOUTH WAIKATO DISTRICT COUNCIL & PROSPERITY FOR ALL & $\begin{array}{l}\text { SOUTH WAIKATO HAS QUALITY INFRASTRUCTURE TO SUPPORT COMMUNITIES AND } \\
\text { BUSINESSES }\end{array}$ \\
\hline 607 & SOUTH WAIKATO DISTRICT COUNCIL & PROSPERITY FOR ALL & SOUTH WAIKATO YOUNG PEOPLE HAVE A FUTURE IN THE DISTRICTS WORKFORCE \\
\hline
\end{tabular}


Appendix B: Community Well-being Statements by Councils

\begin{tabular}{|c|c|c|}
\hline 609 & SOUTH WAIKATO DISTRICT COUNCIL PROSPERITY FOR ALL & $\begin{array}{l}\text { TOURISM IS DEVELOPED AS A KEY INDUSTRY AND THE POTENTIAL OF THE WAIKATO } \\
\text { RIVER IS REALISED }\end{array}$ \\
\hline 610 & SOUTH WAIKATO DISTRICT COUNCIL STANDING TALL TOGETHER & MĀORI AND PACIFIC ISLAND PEOPLES ARE CONSULTED IN WAYS APPROPRIATE TO THEM \\
\hline 612 & SOUTH WAIKATO DISTRICT COUNCIL STANDING TALL TOGETHER & $\begin{array}{l}\text { SOUTH WAIKATO HAS A POSITIVE IMAGE TO ANCHOR COMMUNITY PRIDE AND NEW } \\
\text { INVESTMENT }\end{array}$ \\
\hline 614 & SOUTH WAIKATO DISTRICT COUNCIL STANDING TALL TOGETHER & SOUTH WAIKATOS HISTORY IS PROTECTED AND PRESERVED \\
\hline 615 & SOUTH WAIKATO DISTRICT COUNCIL STANDING TALL TOGETHER & $\begin{array}{l}\text { YOUNG PEOPLE ARE INVOLVED IN ORGANISING EVENTS AND PARTICIPATING IN } \\
\text { DECISIONS THAT AFFECT THEM }\end{array}$ \\
\hline 616 & SOUTH WAIKATO DISTRICT COUNCIL TOWNS WE ARE PROUD OF & $\begin{array}{l}\text { SOUTH WAIKATO HAS ATTRACTIVE URBAN AREAS OPEN SPACES AND PRIVATE } \\
\text { PROPERTIES THAT PEOPLE TAKE PRIDE IN }\end{array}$ \\
\hline 617 & SOUTH WAIKATO DISTRICT COUNCIL TOWNS WE ARE PROUD OF & $\begin{array}{l}\text { THE DISTRICTS URBAN ENVIRONMENTS ARE KEPT CLEAN AND TIDY AND THERE IS A } \\
\text { WIDESPREAD CULTURE OF RESPECT FOR OUR COMMUNITIES AND IDENTITY }\end{array}$ \\
\hline 618 & SOUTH WAIKATO DISTRICT COUNCIL TOWNS WE ARE PROUD OF & URBAN DESIGN CATERS FOR THE ELDERLY AND MOBILITY IMPAIRED \\
\hline 619 & SOUTH WAIKATO DISTRICT COUNCIL VIBRANT GROWING COMMUNITIES & $\begin{array}{l}\text { AIR QUALITY AND HEALTH ARE IMPROVED THROUGH BETTER INSULATED HOMES AND } \\
\text { CLEAN BURNING }\end{array}$ \\
\hline 620 & SOUTH WAIKATO DISTRICT COUNCIL VIBRANT GROWING COMMUNITIES & $\begin{array}{l}\text { EVERYONE HAS THE OPPORTUNITY AND ENCOURAGEMENT TO LEAD A HEALTHY ACTIVE } \\
\text { LIFESTYLE }\end{array}$ \\
\hline 621 & SOUTH WAIKATO DISTRICT COUNCIL VIBRANT GROWING COMMUNITIES & FACILITIES SERVICES AND COMMUNITY EVENTS ARE AFFORDABLE AND ACCESSIBLE \\
\hline 622 & SOUTH WAIKATO DISTRICT COUNCIL VIBRANT GROWING COMMUNITIES & HEALTH CARE AND EDUCATION ARE WELL RESOURCED AND ACCESSIBLE FOR ALL PEOPLE \\
\hline 623 & SOUTH WAIKATO DISTRICT COUNCIL VIBRANT GROWING COMMUNITIES & PEOPLE ARE CONNECTED WITH EMPLOYMENT AND ACTIVITIES THEY WANT TO DO \\
\hline 624 & SOUTH WAIKATO DISTRICT COUNCIL VIBRANT GROWING COMMUNITIES & PEOPLE ARE SAFE AT HOME AND IN THE COMMUNITY \\
\hline 625 & SOUTH WAIKATO DISTRICT COUNCIL VIBRANT GROWING COMMUNITIES & $\begin{array}{l}\text { SOUTH WAIKATO COMMUNITIES ARE SUPPORTIVE OF EACH OTHER AND HAVE STRONG } \\
\text { COMMUNITY SPIRIT }\end{array}$ \\
\hline 627 & SOUTH WAIKATO DISTRICT COUNCIL VIBRANT GROWING COMMUNITIES & YOUNG PEOPLE ARE PROVIDED WITH CONSTRUCTIVE ACTIVITIES OUTSIDE OF SCHOOL \\
\hline
\end{tabular}


Appendix B: Community Well-being Statements by Councils

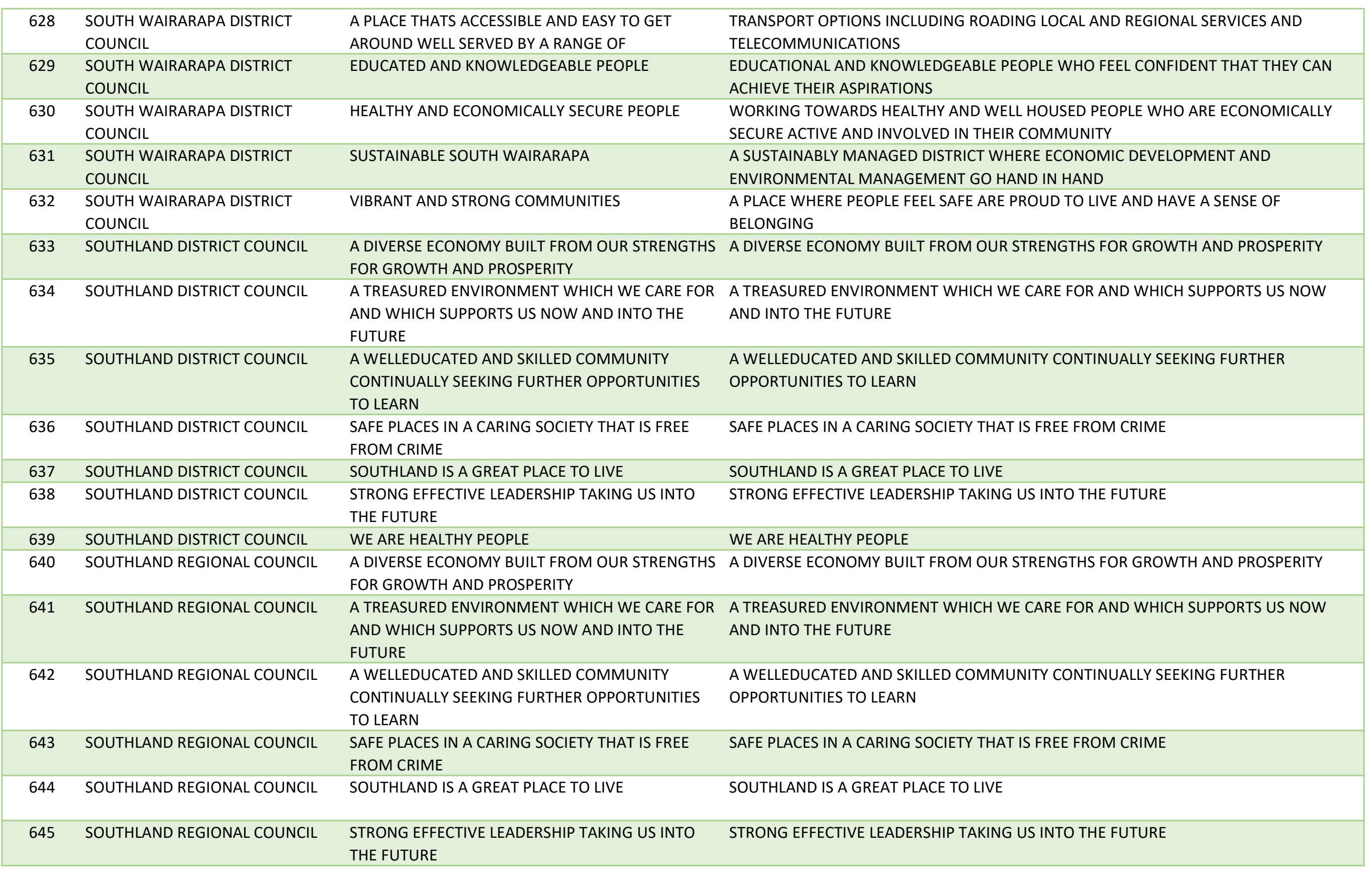


Appendix B: Community Well-being Statements by Councils

\begin{tabular}{|c|c|c|c|}
\hline 646 & SOUTHLAND REGIONAL COUNCIL & WE ARE HEALTHY PEOPLE & WE ARE HEALTHY PEOPLE \\
\hline 647 & STRATFORD DISTRICT COUNCIL & CONNECTED TARANAKI & $\begin{array}{l}\text { A REGION THAT DELIVERS ACCESSIBLE AND INTEGRATED INFRASTRUCTURE TRANSPORT } \\
\text { AND COMMUNICATIONS SYSTEMS WHICH MEET THE NEEDS OF RESIDENTS BUSINESS } \\
\text { AND VISITORS }\end{array}$ \\
\hline 648 & STRATFORD DISTRICT COUNCIL & PROSPEROUS TARANAKI & $\begin{array}{l}\text { A REGION THAT BOASTS A SUSTAINABLE RESILIENT AND INNOVATIVE ECONOMY THAT } \\
\text { PROSPERS WITHIN THE NATURAL AND SOCIAL ENVIRONMENT }\end{array}$ \\
\hline 649 & STRATFORD DISTRICT COUNCIL & SECURE HEALTHY TARANAKI & A REGION THAT PROVIDES A SAFE HEALTHY FRIENDLY PLACE TO LIVE WORK OR VISIT \\
\hline 650 & STRATFORD DISTRICT COUNCIL & SKILLED TARANAKI & $\begin{array}{l}\text { A REGION THAT VALUES AND SUPPORTS LEARNING SO THAT ALL PEOPLE CAN PLAY A FULL } \\
\text { AND ACTIVE ROLE IN ITS SOCIAL CULTURAL AND ECONOMIC LIFE }\end{array}$ \\
\hline 651 & STRATFORD DISTRICT COUNCIL & SUSTAINABLE TARANAKI & $\begin{array}{l}\text { A REGION THAT APPRECIATES ITS NATURAL ENVIRONMENT AND ITS PHYSICAL AND } \\
\text { HUMAN RESOURCES IN PLANNING DELIVERY AND PROTECTION }\end{array}$ \\
\hline 652 & STRATFORD DISTRICT COUNCIL & TOGETHER TARANAKI & $\begin{array}{l}\text { A REGION THAT IS CARING AND INCLUSIVE WORKS TOGETHER AND ENABLES PEOPLE TO } \\
\text { HAVE A STRONG AND DISTINCTIVE SENSE OF IDENTITY }\end{array}$ \\
\hline 653 & STRATFORD DISTRICT COUNCIL & VIBRANT TARANAKI & $\begin{array}{l}\text { A REGION THAT PROVIDES HIGH QUALITY AND DIVERSE CULTURAL AND RECREATIONAL } \\
\text { EXPERIENCES AND ENCOURAGES INDEPENDENCE AND CREATIVITY }\end{array}$ \\
\hline 654 & TARANAKI REGIONAL COUNCIL & CONNECTED TARANAKI & $\begin{array}{l}\text { A REGION THAT DELIVERS ACCESSIBLE AND INTEGRATED INFRASTRUCTURE TRANSPORT } \\
\text { AND COMMUNICATIONS SYSTEMS WHICH MEET THE NEEDS OF RESIDENTS BUSINESS } \\
\text { AND VISITORS }\end{array}$ \\
\hline 655 & TARANAKI REGIONAL COUNCIL & PROSPEROUS TARANAKI & $\begin{array}{l}\text { A REGION THAT BOASTS A SUSTAINABLE RESILIENT AND INNOVATIVE ECONOMY THAT } \\
\text { PROSPERS WITHIN THE NATURAL AND SOCIAL ENVIRONMENT }\end{array}$ \\
\hline 656 & TARANAKI REGIONAL COUNCIL & SECURE AND HEALTHY TARANAKI & A REGION THAT PROVIDES A SAFE HEALTHY AND FRIENDLY PLACE TO LIVE WORK OR VISIT \\
\hline 657 & TARANAKI REGIONAL COUNCIL & SKILLED TARANAKI & $\begin{array}{l}\text { A REGION THAT VALUES AND SUPPORTS LEARNING SO THAT ALL PEOPLE CAN PLAY A FULL } \\
\text { AND ACTIVE ROLE IN ITS SOCIAL CULTURAL AND ECONOMIC LIFE }\end{array}$ \\
\hline 658 & TARANAKI REGIONAL COUNCIL & SUSTAINABLE TARANAKI & $\begin{array}{l}\text { A REGION THAT APPRECIATES ITS NATURAL ENVIRONMENT AND ITS PHYSICAL AND } \\
\text { HUMAN RESOURCES IN PLANNING DELIVERY AND PROTECTION }\end{array}$ \\
\hline 659 & TARANAKI REGIONAL COUNCIL & TOGETHER TARANAKI & $\begin{array}{l}\text { A REGION THAT IS CARING AND INCLUSIVE WORKS TOGETHER AND ENABLES PEOPLE TO } \\
\text { HAVE A STRONG AND DISTINCTIVE SENSE OF IDENTITY }\end{array}$ \\
\hline 660 & TARANAKI REGIONAL COUNCIL & VIBRANT TARANAKI & $\begin{array}{l}\text { A REGION THAT PROVIDES HIGH QUALITY AND DIVERSE CULTURAL AND RECREATIONAL } \\
\text { EXPERIENCES AND ENCOURAGES INDEPENDENCE AND CREATIVITY }\end{array}$ \\
\hline 661 & TARARUA DISTRICT COUNCIL & A HIGH STANDARD OF INFRASTRUCTURE & $\begin{array}{l}\text { A TRANSPORT NETWORK THAT PROVIDES FOR THE SAFE AND CONVENIENT MOVEMENT } \\
\text { OF PEOPLE AND GOODS ATTRACTIVE WELL MAINTAINED TOWNS AND PUBLIC AMENITIES } \\
\text { THAT OUR COMMUNITY IS PROUD OF }\end{array}$ \\
\hline 662 & TARARUA DISTRICT COUNCIL & A SAFE COMMUNITY & A COMMUNITY FREE FROM CRIME VIOLENCE ABUSE OR HARM \\
\hline
\end{tabular}


Appendix B: Community Well-being Statements by Councils

\begin{tabular}{|c|c|c|c|}
\hline 663 & TARARUA DISTRICT COUNCIL & A STRONG PROSPEROUS ECONOMY & $\begin{array}{l}\text { A VIBRANT PROSPEROUS ECONOMY THAT CREATES EMPLOYMENT OPPORTUNITIES AND } \\
\text { SUPPORTS BUSINESS DEVELOPMENT PRODUCTIVE LAND USE TOURISM AND COMMUNITY } \\
\text { INITIATIVES A DISTRICT THAT ATTRACTS VISITORS AND ATTRACTS AND RETAINS } \\
\text { RESIDENTS }\end{array}$ \\
\hline 665 & TARARUA DISTRICT COUNCIL & ACCESS TO LOCAL SERVICES & $\begin{array}{l}\text { ACCESS TO LOCAL HOSPITAL AND HEALTH SERVICES EDUCATION AND TRAINING } \\
\text { PROGRAMMES TELECOMMUNICATION PUBLIC TRANSPORT CHILDCARE RETAIL AND } \\
\text { BANKING SERVICES THAT MEET THE NEEDS OF THE COMMUNITY }\end{array}$ \\
\hline 666 & TARARUA DISTRICT COUNCIL & CONNECTED COMMUNITY & $\begin{array}{l}\text { A COMMUNITY THAT HAS ACCESS TO AND PARTICIPATES IN SPORT RECREATION AND } \\
\text { SOCIAL ACTIVITIES OUR COMMUNITY HAS A GREATER SAY IN THE ISSUES THAT AFFECT } \\
\text { THEM AND THEIR WISHES AND NEEDS ARE LISTENED TO OUR COMMUNITYS CULTURE } \\
\text { AND HERITAGE IS CELEBRATED AND PRESERVED FOR FUTURE GENERATIONS ARTS AND } \\
\text { CULTURE ARE SUPPORTED BY THE COMMUNITY }\end{array}$ \\
\hline 667 & TASMAN DISTRICT COUNCIL & $\begin{array}{l}\text { OUR BUILT URBAN AND RURAL ENVIRONMENTS ARE } \\
\text { FUNCTIONAL PLEASANT SAFE AND SUSTAINABLY } \\
\text { MANAGED }\end{array}$ & $\begin{array}{l}\text { AS A COMMUNITY WE HAVE DEVELOPED METHODS AND STRATEGIES TO MANAGE } \\
\text { FUTURE DEVELOPMENT WHILE PROTECTING OUR GREEN SPACES AND OUR TREASURED } \\
\text { WAY OF LIFE }\end{array}$ \\
\hline 668 & TASMAN DISTRICT COUNCIL & $\begin{array}{l}\text { OUR BUILT URBAN AND RURAL ENVIRONMENTS ARE } \\
\text { FUNCTIONAL PLEASANT SAFE AND SUSTAINABLY } \\
\text { MANAGED }\end{array}$ & CREATIVE PLANNING PROCESSES CONTINUE TO VALUE RURAL LAND USE \\
\hline 669 & TASMAN DISTRICT COUNCIL & $\begin{array}{l}\text { OUR BUILT URBAN AND RURAL ENVIRONMENTS ARE } \\
\text { FUNCTIONAL PLEASANT SAFE AND SUSTAINABLY } \\
\text { MANAGED }\end{array}$ & $\begin{array}{l}\text { OUR BUILT ENVIRONMENT ENHANCES THE QUALITIES OF OUR UNIQUE AND SPECIAL } \\
\text { NATURAL ENVIRONMENT }\end{array}$ \\
\hline 670 & TASMAN DISTRICT COUNCIL & $\begin{array}{l}\text { OUR BUILT URBAN AND RURAL ENVIRONMENTS ARE } \\
\text { FUNCTIONAL PLEASANT SAFE AND SUSTAINABLY } \\
\text { MANAGED }\end{array}$ & OUR BUILT ENVIRONMENT IS ROBUST AND MEETS THE NEEDS OF ALL ITS USERS \\
\hline 671 & TASMAN DISTRICT COUNCIL & $\begin{array}{l}\text { OUR BUILT URBAN AND RURAL ENVIRONMENTS ARE } \\
\text { FUNCTIONAL PLEASANT SAFE AND SUSTAINABLY } \\
\text { MANAGED }\end{array}$ & OUR BUILT ENVIRONMENT IS WELL PLANNED AND WELL MAINTAINED \\
\hline 672 & TASMAN DISTRICT COUNCIL & $\begin{array}{l}\text { OUR BUILT URBAN AND RURAL ENVIRONMENTS ARE } \\
\text { FUNCTIONAL PLEASANT SAFE AND SUSTAINABLY } \\
\text { MANAGED }\end{array}$ & OUR FAMILYFOCUSED COMMUNITIES ARE ENVIRONMENTALLY SENSITIVE \\
\hline
\end{tabular}




\begin{tabular}{|c|c|c|c|}
\hline 674 & TASMAN DISTRICT COUNCIL & $\begin{array}{l}\text { OUR BUILT URBAN AND RURAL ENVIRONMENTS ARE } \\
\text { FUNCTIONAL PLEASANT SAFE AND SUSTAINABLY } \\
\text { MANAGED }\end{array}$ & TREES ARE A VALUED PART OF OUR LANDSCAPES \\
\hline 675 & TASMAN DISTRICT COUNCIL & $\begin{array}{l}\text { OUR BUILT URBAN AND RURAL ENVIRONMENTS ARE } \\
\text { FUNCTIONAL PLEASANT SAFE AND SUSTAINABLY } \\
\text { MANAGED }\end{array}$ & WE RETAIN OUR RURAL CHARACTER PEACEFULNESS AND SENSE OF BELONGING \\
\hline 676 & TASMAN DISTRICT COUNCIL & $\begin{array}{l}\text { OUR COMMUNITY UNDERSTANDS REGIONAL } \\
\text { HISTORY HERITAGE AND CULTURE }\end{array}$ & $\begin{array}{l}\text { SUPPORTING OUR DYNAMIC ARTS SECTOR PROMOTES CREATIVE THINKING IN ALL } \\
\text { ASPECTS OF COMMUNITY LIFE }\end{array}$ \\
\hline 677 & TASMAN DISTRICT COUNCIL & $\begin{array}{l}\text { OUR COMMUNITY UNDERSTANDS REGIONAL } \\
\text { HISTORY HERITAGE AND CULTURE }\end{array}$ & THE SPECIAL PLACE OF MĀORI IN OUR COMMUNITY IS RECOGNISED AND RESPECTED \\
\hline 678 & TASMAN DISTRICT COUNCIL & $\begin{array}{l}\text { OUR COMMUNITY UNDERSTANDS REGIONAL } \\
\text { HISTORY HERITAGE AND CULTURE }\end{array}$ & $\begin{array}{l}\text { WE ARE A FORWARDTHINKING AND TOLERANT SOCIETY WHERE CULTURAL DIVERSITY IS } \\
\text { EMBRACED }\end{array}$ \\
\hline 679 & TASMAN DISTRICT COUNCIL & $\begin{array}{l}\text { OUR COMMUNITY UNDERSTANDS REGIONAL } \\
\text { HISTORY HERITAGE AND CULTURE }\end{array}$ & WE CELEBRATE OUR HERITAGE \\
\hline 680 & TASMAN DISTRICT COUNCIL & $\begin{array}{l}\text { OUR COMMUNITY UNDERSTANDS REGIONAL } \\
\text { HISTORY HERITAGE AND CULTURE }\end{array}$ & $\begin{array}{l}\text { WE UNDERSTAND THAT CARING FOR OTHERS AND THE ENVIRONMENT CREATES A } \\
\text { STRONG SENSE OF COMMUNITY SPIRIT }\end{array}$ \\
\hline 681 & TASMAN DISTRICT COUNCIL & $\begin{array}{l}\text { OUR DIVERSE COMMUNITY ENJOYS ACCESS TO A } \\
\text { RANGE OF SPIRITUAL CULTURAL SOCIAL } \\
\text { EDUCATIONAL AND RECREATIONAL SERVICES }\end{array}$ & $\begin{array}{l}\text { MEMBERS OF OUR COMMUNITY EXPLORE THE POTENTIAL AND PLAN FOR NEW } \\
\text { FACILITIES AND SERVICES TOGETHER }\end{array}$ \\
\hline 682 & TASMAN DISTRICT COUNCIL & $\begin{array}{l}\text { OUR DIVERSE COMMUNITY ENJOYS ACCESS TO A } \\
\text { RANGE OF SPIRITUAL CULTURAL SOCIAL } \\
\text { EDUCATIONAL AND RECREATIONAL SERVICES }\end{array}$ & OUR COMMUNITY LIVES IN FAITH HOPE AND LOVE \\
\hline 683 & TASMAN DISTRICT COUNCIL & $\begin{array}{l}\text { OUR DIVERSE COMMUNITY ENJOYS ACCESS TO A } \\
\text { RANGE OF SPIRITUAL CULTURAL SOCIAL } \\
\text { EDUCATIONAL AND RECREATIONAL SERVICES }\end{array}$ & $\begin{array}{l}\text { OUR EASYTOACCESS BEACHES PARKS AND RESERVES CREATE AN ACTIVE AND VIBRANT } \\
\text { SOCIETY }\end{array}$ \\
\hline 684 & TASMAN DISTRICT COUNCIL & $\begin{array}{l}\text { OUR DIVERSE COMMUNITY ENJOYS ACCESS TO A } \\
\text { RANGE OF SPIRITUAL CULTURAL SOCIAL } \\
\text { EDUCATIONAL AND RECREATIONAL SERVICES }\end{array}$ & $\begin{array}{l}\text { OUR LEISURE AND RECREATION FACILITIES PROVIDE A RANGE OF OPTIONS FOR SOCIAL } \\
\text { INTERACTION AND ENCOURAGE PEOPLE TO BE ACTIVE AND INVOLVED }\end{array}$ \\
\hline 685 & TASMAN DISTRICT COUNCIL & $\begin{array}{l}\text { OUR DIVERSE COMMUNITY ENJOYS ACCESS TO A } \\
\text { RANGE OF SPIRITUAL CULTURAL SOCIAL } \\
\text { EDUCATIONAL AND RECREATIONAL SERVICES }\end{array}$ & OUR YOUTH ARE ENGAGED IN THINKING ABOUT AND CREATING OUR FUTURE \\
\hline 686 & TASMAN DISTRICT COUNCIL & $\begin{array}{l}\text { OUR DIVERSE COMMUNITY ENJOYS ACCESS TO A } \\
\text { RANGE OF SPIRITUAL CULTURAL SOCIAL } \\
\text { EDUCATIONAL AND RECREATIONAL SERVICES }\end{array}$ & THE PROVISION OF EDUCATION AND TRAINING OPPORTUNITIES ENHANCES OUR LIVES \\
\hline 687 & TASMAN DISTRICT COUNCIL & $\begin{array}{l}\text { OUR DIVERSE COMMUNITY ENJOYS ACCESS TO A } \\
\text { RANGE OF SPIRITUAL CULTURAL SOCIAL } \\
\text { EDUCATIONAL AND RECREATIONAL SERVICES }\end{array}$ & $\begin{array}{l}\text { WE ENCOURAGE THE CELEBRATION OF FESTIVALS AND EVENTS IMPORTANT IN FAMILY } \\
\text { LIFE }\end{array}$ \\
\hline
\end{tabular}


Appendix B: Community Well-being Statements by Councils

\begin{tabular}{|c|c|c|c|}
\hline 688 & TASMAN DISTRICT COUNCIL & $\begin{array}{l}\text { OUR GROWING AND SUSTAINABLE ECONOMY } \\
\text { PROVIDES OPPORTUNITIES FOR US ALL }\end{array}$ & OUR BUSINESSFRIENDLY PROCESSES ASSIST BUSINESSES TO SET UP IN TASMAN DISTRICT \\
\hline 689 & TASMAN DISTRICT COUNCIL & $\begin{array}{l}\text { OUR GROWING AND SUSTAINABLE ECONOMY } \\
\text { PROVIDES OPPORTUNITIES FOR US ALL }\end{array}$ & OUR CAN DO ATTITUDE IS THE FOUNDATION OF TASMAN DISTRICTS ECONOMIC SUCCESS \\
\hline 690 & TASMAN DISTRICT COUNCIL & $\begin{array}{l}\text { OUR GROWING AND SUSTAINABLE ECONOMY } \\
\text { PROVIDES OPPORTUNITIES FOR US ALL }\end{array}$ & $\begin{array}{l}\text { OUR DISTRICT SPECIALITY INDUSTRIES ARE MANAGED IN A RESPONSIBLE AND } \\
\text { SUSTAINABLE WAY }\end{array}$ \\
\hline 691 & TASMAN DISTRICT COUNCIL & $\begin{array}{l}\text { OUR GROWING AND SUSTAINABLE ECONOMY } \\
\text { PROVIDES OPPORTUNITIES FOR US ALL }\end{array}$ & $\begin{array}{l}\text { THE COMMUNITY CONTINUES TO VALUE THE CONTRIBUTION OF PRIMARY INDUSTRY TO } \\
\text { OUR DISTRICT }\end{array}$ \\
\hline 692 & TASMAN DISTRICT COUNCIL & $\begin{array}{l}\text { OUR GROWING AND SUSTAINABLE ECONOMY } \\
\text { PROVIDES OPPORTUNITIES FOR US ALL }\end{array}$ & THERE ARE STABLE JOBS ACROSS DIVERSE INDUSTRIES \\
\hline 693 & TASMAN DISTRICT COUNCIL & $\begin{array}{l}\text { OUR GROWING AND SUSTAINABLE ECONOMY } \\
\text { PROVIDES OPPORTUNITIES FOR US ALL }\end{array}$ & $\begin{array}{l}\text { WE ENCOURAGE BUSINESSES WHICH COMPLEMENT THE CLEAN GREEN CHARACTER OF } \\
\text { OUR AREA }\end{array}$ \\
\hline 694 & TASMAN DISTRICT COUNCIL & $\begin{array}{l}\text { OUR GROWING AND SUSTAINABLE ECONOMY } \\
\text { PROVIDES OPPORTUNITIES FOR US ALL }\end{array}$ & $\begin{array}{l}\text { WE WELCOME VISITORS AND NEWCOMERS AND SHARE OUR DISTINCTIVE LIFESTYLE } \\
\text { WITH THEM }\end{array}$ \\
\hline 695 & TASMAN DISTRICT COUNCIL & $\begin{array}{l}\text { OUR PARTICIPATORY COMMUNITY CONTRIBUTES TO } \\
\text { DISTRICT DECISIONMAKING AND DEVELOPMENT }\end{array}$ & $\begin{array}{l}\text { OUR COMMUNITY LEADERS EXERCISE WISDOM AND COMMON SENSE IN } \\
\text { DECISIONMAKING FOR THE FUTURE AND WORK TO BUILD STRONG HEALTHY } \\
\text { COMMUNITIES }\end{array}$ \\
\hline 696 & TASMAN DISTRICT COUNCIL & $\begin{array}{l}\text { OUR PARTICIPATORY COMMUNITY CONTRIBUTES TO } \\
\text { DISTRICT DECISIONMAKING AND DEVELOPMENT }\end{array}$ & $\begin{array}{l}\text { OUR GOVERNANCE MODEL ALLOWS ALL COMMUNITIES AND THEIR VIEWS TO BE } \\
\text { ADEQUATELY REPRESENTED }\end{array}$ \\
\hline 697 & TASMAN DISTRICT COUNCIL & $\begin{array}{l}\text { OUR PARTICIPATORY COMMUNITY CONTRIBUTES TO } \\
\text { DISTRICT DECISIONMAKING AND DEVELOPMENT }\end{array}$ & $\begin{array}{l}\text { OUR PLANNING IS PROACTIVE THOROUGH REALISTIC AND ANCHORED BY A SHARED } \\
\text { VISION A BIG PICTURE AGAINST WHICH WE REFERENCE OUR CHOICES }\end{array}$ \\
\hline 698 & TASMAN DISTRICT COUNCIL & $\begin{array}{l}\text { OUR PARTICIPATORY COMMUNITY CONTRIBUTES TO } \\
\text { DISTRICT DECISIONMAKING AND DEVELOPMENT }\end{array}$ & WE ACTIVELY WORK TOGETHER TO MAKE THE BEST LOCALLY SUPPORTED DECISIONS \\
\hline 699 & TASMAN DISTRICT COUNCIL & $\begin{array}{l}\text { OUR PARTICIPATORY COMMUNITY CONTRIBUTES TO } \\
\text { DISTRICT DECISIONMAKING AND DEVELOPMENT }\end{array}$ & WE HAVE TAKEN RESPONSIBILITY FOR OUR FUTURE \\
\hline 700 & TASMAN DISTRICT COUNCIL & $\begin{array}{l}\text { OUR PARTICIPATORY COMMUNITY CONTRIBUTES TO } \\
\text { DISTRICT DECISIONMAKING AND DEVELOPMENT }\end{array}$ & $\begin{array}{l}\text { WE THINK DISCUSS AND PLAN AHEAD TO ENSURE OUR POPULATION IS BALANCED AND } \\
\text { RESOURCED }\end{array}$ \\
\hline 701 & TASMAN DISTRICT COUNCIL & $\begin{array}{l}\text { OUR TRANSPORT AND ESSENTIAL SERVICES ARE } \\
\text { SUFFICIENT EFFICIENT AND SUSTAINABLY MANAGED }\end{array}$ & EFFECTIVE PUBLIC TRANSPORT EXISTS ALONG THE MAIN ROUTES \\
\hline 702 & TASMAN DISTRICT COUNCIL & $\begin{array}{l}\text { OUR TRANSPORT AND ESSENTIAL SERVICES ARE } \\
\text { SUFFICIENT EFFICIENT AND SUSTAINABLY MANAGED }\end{array}$ & $\begin{array}{l}\text { EVERYONE IN TASMAN DISTRICT HAS ACCESS TO CLEAN WATER AND OUR SEWERAGE } \\
\text { AND WASTE DISPOSAL SYSTEM ARE SUSTAINABLY AND EFFICIENTLY MANAGED }\end{array}$ \\
\hline
\end{tabular}




\begin{tabular}{|c|c|c|c|}
\hline 703 & TASMAN DISTRICT COUNCIL & $\begin{array}{l}\text { OUR TRANSPORT AND ESSENTIAL SERVICES ARE } \\
\text { SUFFICIENT EFFICIENT AND SUSTAINABLY MANAGED }\end{array}$ & $\begin{array}{l}\text { OUR COMMUNITIES ARE LINKED TOGETHER BY A NETWORK OF ROADS CYCLEWAYS AND } \\
\text { WALKWAYS }\end{array}$ \\
\hline 704 & TASMAN DISTRICT COUNCIL & $\begin{array}{l}\text { OUR TRANSPORT AND ESSENTIAL SERVICES ARE } \\
\text { SUFFICIENT EFFICIENT AND SUSTAINABLY MANAGED }\end{array}$ & $\begin{array}{l}\text { OUR EFFECTIVE ROADING SYSTEM IS WELL MAINTAINED AND SAFE FOR ALL USERS } \\
\text { INCLUDING NON MOTORISED USERS }\end{array}$ \\
\hline 705 & TASMAN DISTRICT COUNCIL & $\begin{array}{l}\text { OUR TRANSPORT AND ESSENTIAL SERVICES ARE } \\
\text { SUFFICIENT EFFICIENT AND SUSTAINABLY MANAGED }\end{array}$ & $\begin{array}{l}\text { OUR EXPANSIVE RECYCLING PROGRAMME IS SUPPORTED AND USED BY ALL OUR } \\
\text { COMMUNITIES }\end{array}$ \\
\hline 706 & TASMAN DISTRICT COUNCIL & $\begin{array}{l}\text { OUR TRANSPORT AND ESSENTIAL SERVICES ARE } \\
\text { SUFFICIENT EFFICIENT AND SUSTAINABLY MANAGED }\end{array}$ & $\begin{array}{l}\text { OUR FUTURE GROWTH AND DEVELOPMENT TRENDS ARE CAREFULLY RESEARCHED AND } \\
\text { MONITORED }\end{array}$ \\
\hline 707 & TASMAN DISTRICT COUNCIL & $\begin{array}{l}\text { OUR TRANSPORT AND ESSENTIAL SERVICES ARE } \\
\text { SUFFICIENT EFFICIENT AND SUSTAINABLY MANAGED }\end{array}$ & $\begin{array}{l}\text { OUR TRANSPORT AND ESSENTIAL SERVICE SYSTEMS ARE STEADILY AND REALISTICALLY } \\
\text { UPGRADED TO MEET OUR NEEDS }\end{array}$ \\
\hline 708 & TASMAN DISTRICT COUNCIL & $\begin{array}{l}\text { OUR UNIQUE AND SPECIAL NATURAL ENVIRONMENT } \\
\text { IS BOUNTIFUL HEALTHY CLEAN AND PROTECTED }\end{array}$ & OUR CHILDREN REAP THE REWARDS OF OUR STEWARDSHIP \\
\hline 709 & TASMAN DISTRICT COUNCIL & $\begin{array}{l}\text { OUR UNIQUE AND SPECIAL NATURAL ENVIRONMENT } \\
\text { IS BOUNTIFUL HEALTHY CLEAN AND PROTECTED }\end{array}$ & $\begin{array}{l}\text { OUR NATURAL ENVIRONMENT IS ENJOYED BY LOCAL PEOPLE AND VISITORS IN } \\
\text { SUSTAINABLE NUMBERS }\end{array}$ \\
\hline 710 & TASMAN DISTRICT COUNCIL & $\begin{array}{l}\text { OUR UNIQUE AND SPECIAL NATURAL ENVIRONMENT } \\
\text { IS BOUNTIFUL HEALTHY CLEAN AND PROTECTED }\end{array}$ & $\begin{array}{l}\text { OUR USE OF ENERGY RESOURCES IS ENVIRONMENTALLY FRIENDLY EFFICIENT AND } \\
\text { SUSTAINABLE }\end{array}$ \\
\hline 711 & TASMAN DISTRICT COUNCIL & $\begin{array}{l}\text { OUR UNIQUE AND SPECIAL NATURAL ENVIRONMENT } \\
\text { IS BOUNTIFUL HEALTHY CLEAN AND PROTECTED }\end{array}$ & OUR WATERWAYS ARE CLEAN AND TEEMING WITH LIFE \\
\hline 712 & TASMAN DISTRICT COUNCIL & $\begin{array}{l}\text { OUR UNIQUE AND SPECIAL NATURAL ENVIRONMENT } \\
\text { IS BOUNTIFUL HEALTHY CLEAN AND PROTECTED }\end{array}$ & PESTS ARE CONTROLLED WITH EFFICIENCY AND INGENUITY \\
\hline 713 & TASMAN DISTRICT COUNCIL & $\begin{array}{l}\text { OUR UNIQUE AND SPECIAL NATURAL ENVIRONMENT } \\
\text { IS BOUNTIFUL HEALTHY CLEAN AND PROTECTED }\end{array}$ & THE COAST IS PEACEFUL AND OPEN TO ALL \\
\hline 714 & TASMAN DISTRICT COUNCIL & $\begin{array}{l}\text { OUR UNIQUE AND SPECIAL NATURAL ENVIRONMENT } \\
\text { IS BOUNTIFUL HEALTHY CLEAN AND PROTECTED }\end{array}$ & THE ENVIRONMENT IS A TOP PRIORITY WHICH INFLUENCES OUR DECISIONMAKING \\
\hline
\end{tabular}




\begin{tabular}{|c|c|c|c|}
\hline 715 & TASMAN DISTRICT COUNCIL & $\begin{array}{l}\text { OUR UNIQUE AND SPECIAL NATURAL ENVIRONMENT } \\
\text { IS BOUNTIFUL HEALTHY CLEAN AND PROTECTED }\end{array}$ & WE RETAIN AND ENHANCE OUR NATURAL AREAS \\
\hline 716 & TASMAN DISTRICT COUNCIL & $\begin{array}{l}\text { OUR UNIQUE AND SPECIAL NATURAL ENVIRONMENT } \\
\text { IS BOUNTIFUL HEALTHY CLEAN AND PROTECTED }\end{array}$ & $\begin{array}{l}\text { WE SUSTAINABLY MANAGE THE INTERACTION BETWEEN THE COMMUNITY AND THE } \\
\text { ENVIRONMENT }\end{array}$ \\
\hline 717 & TASMAN DISTRICT COUNCIL & $\begin{array}{l}\text { OUR VIBRANT COMMUNITY IS SAFE WELL ENJOYS AN } \\
\text { EXCELLENT QUALITY OF LIFE AND SUPPORTS THOSE } \\
\text { WITH SPECIAL NEEDS }\end{array}$ & ITS STILL THE LIFESTYLE THAT COUNTS \\
\hline 718 & TASMAN DISTRICT COUNCIL & $\begin{array}{l}\text { OUR VIBRANT COMMUNITY IS SAFE WELL ENJOYS AN } \\
\text { EXCELLENT QUALITY OF LIFE AND SUPPORTS THOSE } \\
\text { WITH SPECIAL NEEDS }\end{array}$ & $\begin{array}{l}\text { OUR COMMUNITY HAS ACCESS TO SOCIAL AND SUPPORT SERVICES TO KEEP THEM } \\
\text { HEALTHY AND ACTIVE }\end{array}$ \\
\hline 719 & TASMAN DISTRICT COUNCIL & $\begin{array}{l}\text { OUR VIBRANT COMMUNITY IS SAFE WELL ENJOYS AN } \\
\text { EXCELLENT QUALITY OF LIFE AND SUPPORTS THOSE } \\
\text { WITH SPECIAL NEEDS }\end{array}$ & WE ENJOY A PERSONAL SENSE OF BELONGING TO LIFE IN THIS AREA \\
\hline 720 & TASMAN DISTRICT COUNCIL & $\begin{array}{l}\text { OUR VIBRANT COMMUNITY IS SAFE WELL ENJOYS AN } \\
\text { EXCELLENT QUALITY OF LIFE AND SUPPORTS THOSE } \\
\text { WITH SPECIAL NEEDS }\end{array}$ & WE ENJOY HEALTHY LIFESTYLES WORK AND LIVING SPACES \\
\hline 721 & TASMAN DISTRICT COUNCIL & $\begin{array}{l}\text { OUR VIBRANT COMMUNITY IS SAFE WELL ENJOYS AN } \\
\text { EXCELLENT QUALITY OF LIFE AND SUPPORTS THOSE } \\
\text { WITH SPECIAL NEEDS }\end{array}$ & WE HAVE ACCESS TO A RANGE OF ADEQUATE AND QUALITY HOUSING \\
\hline 722 & TASMAN DISTRICT COUNCIL & $\begin{array}{l}\text { OUR VIBRANT COMMUNITY IS SAFE WELL ENJOYS AN } \\
\text { EXCELLENT QUALITY OF LIFE AND SUPPORTS THOSE } \\
\text { WITH SPECIAL NEEDS }\end{array}$ & WE HAVE ACCESS TO THE HEALTH CARE FACILITIES THAT WE NEED \\
\hline 723 & TASMAN DISTRICT COUNCIL & $\begin{array}{l}\text { OUR VIBRANT COMMUNITY IS SAFE WELL ENJOYS AN } \\
\text { EXCELLENT QUALITY OF LIFE AND SUPPORTS THOSE } \\
\text { WITH SPECIAL NEEDS }\end{array}$ & WE RECOGNISE AND VALUE OUR VOLUNTEERS AND CAREGIVERS \\
\hline 724 & TASMAN DISTRICT COUNCIL & $\begin{array}{l}\text { OUR VIBRANT COMMUNITY IS SAFE WELL ENJOYS AN } \\
\text { EXCELLENT QUALITY OF LIFE AND SUPPORTS THOSE } \\
\text { WITH SPECIAL NEEDS }\end{array}$ & $\begin{array}{l}\text { WE VALUE AND INVOLVE OUR YOUTH AND PROVIDE THEM WITH QUALITY } \\
\text { OPPORTUNITIES }\end{array}$ \\
\hline 725 & TAUPO DISTRICT COUNCIL & HEALTHY PEOPLE HEALTHY COMMUNITIES & ACCESS TO FACILITIES AND SERVICES \\
\hline 726 & TAUPO DISTRICT COUNCIL & HEALTHY PEOPLE HEALTHY COMMUNITIES & ACKNOWLEDGING COMMUNITY DIVERSITY \\
\hline 727 & TAUPO DISTRICT COUNCIL & HEALTHY PEOPLE HEALTHY COMMUNITIES & COMMUNITY BELONGING \\
\hline 728 & TAUPO DISTRICT COUNCIL & HEALTHY PEOPLE HEALTHY COMMUNITIES & HEALTHY ACTIVE LIFESTYLES \\
\hline 729 & TAUPO DISTRICT COUNCIL & HEALTHY PEOPLE HEALTHY COMMUNITIES & QUALITY AFFORDABLE HOUSING \\
\hline 730 & TAUPO DISTRICT COUNCIL & HEALTHY PEOPLE HEALTHY COMMUNITIES & WELLBEING THROUGH TIKANGA MAORI \\
\hline 731 & TAUPO DISTRICT COUNCIL & KAITIAKITANGA & KAITIAKITANGA \\
\hline
\end{tabular}




\begin{tabular}{|c|c|c|c|}
\hline 732 & TAUPO DISTRICT COUNCIL & $\begin{array}{l}\text { LAKES RIVERS LANDSCAPES PLACES WE ARE PROUD } \\
\text { OF }\end{array}$ & APPRECIATING THE ENVIRONMENT \\
\hline 733 & TAUPO DISTRICT COUNCIL & $\begin{array}{l}\text { LAKES RIVERS LANDSCAPES PLACES WE ARE PROUD } \\
\text { OF }\end{array}$ & BALANCING GROWTH \\
\hline 734 & TAUPO DISTRICT COUNCIL & $\begin{array}{l}\text { LAKES RIVERS LANDSCAPES PLACES WE ARE PROUD } \\
\text { OF }\end{array}$ & LAKE AND RIVER WATER QUALITY \\
\hline 735 & TAUPO DISTRICT COUNCIL & $\begin{array}{l}\text { LAKES RIVERS LANDSCAPES PLACES WE ARE PROUD } \\
\text { OF }\end{array}$ & UNDERSTANDING AND MANAGING NATURAL RESOURCES \\
\hline 736 & TAUPO DISTRICT COUNCIL & SAFE AND SECURE & COMMUNITY SUPPORT \\
\hline 737 & TAUPO DISTRICT COUNCIL & SAFE AND SECURE & RESPECT FOR PEOPLE AND PROPERTY \\
\hline 738 & TAUPO DISTRICT COUNCIL & SAFE AND SECURE & SAFE PLACES AND ROADS \\
\hline 739 & TAUPO DISTRICT COUNCIL & SAFE AND SECURE & WELCOMING VISITORS \\
\hline 740 & TAUPO DISTRICT COUNCIL & THRIVING AND PROSPEROUS & BUSINESS AND ECONOMIC GROWTH \\
\hline 741 & TAUPO DISTRICT COUNCIL & THRIVING AND PROSPEROUS & EDUCATION AND TRAINING \\
\hline 742 & TAUPO DISTRICT COUNCIL & THRIVING AND PROSPEROUS & EMPLOYMENT OPPORTUNITIES \\
\hline 743 & TAUPO DISTRICT COUNCIL & THRIVING AND PROSPEROUS & INFRASTRUCTURE AND SERVICES \\
\hline 744 & TAUPO DISTRICT COUNCIL & THRIVING AND PROSPEROUS & QUALITY EDUCATION AND LIFELONG LEARNING \\
\hline 745 & TAUPO DISTRICT COUNCIL & VIBRANT AND DIVERSE & ARTS AND CULTURE \\
\hline 746 & TAUPO DISTRICT COUNCIL & VIBRANT AND DIVERSE & CELEBRATING MAORI CULTURE \\
\hline 747 & TAUPO DISTRICT COUNCIL & VIBRANT AND DIVERSE & CELEBRATING OUR CULTURE AND HERITAGE \\
\hline 748 & TAUPO DISTRICT COUNCIL & VIBRANT AND DIVERSE & SUPPORTING YOUNG PEOPLE \\
\hline 749 & TAURANGA CITY COUNCIL & ACTIVELY INVOLVED PEOPLE & $\begin{array}{l}\text { PEOPLE OF ALL AGES WITH A PASSION FOR TAURANGA HAVE SET A DIRECTION FOR THE } \\
\text { FUTURE OF THIS GREAT CITY MOTIVATED LEADERS FROM ALL WALKS OF LIKE ARE } \\
\text { WORKING TOGETHER TO GET THINGS DONE THIS SETS TAURANGA APART AS A CITY THAT } \\
\text { KNOWS WHERE IT IS HEADED AND IS COMMITTED TO GETTING THERE THE FUTURE } \\
\text { LOOKS BRIGHT }\end{array}$ \\
\hline 750 & TAURANGA CITY COUNCIL & BUILT TO FIT OUR HILLS HARBOUR AND COAST & $\begin{array}{l}\text { OUR CITY TAKES FULL ADVANTAGE OF AND FITS SENSITIVELY WITHIN ITS UNIQUE } \\
\text { LANDSCAPE AND COASTAL SETTING RADIATING FROM TAURANGAS VIBRANT CITY CENTRE } \\
\text { A NETWORK OF TRANSPORTATION AND OPEN SPACE CORRIDORS CONNECT AND DEFINE } \\
\text { A SERIES OF NEIGHBOURHOODS NESTLED INTO THE LANDSCAPE EACH NEIGHBOURHOOD } \\
\text { HAS DEFINED EDGES AND A VISIBLE CHARACTER HISTORY AND IDENTITY THAT IS } \\
\text { REFLECTED IN ITS ARCHITECTURE PLANTING AND STREETSCAPE THEY OFFER PEOPLE A } \\
\text { WIDE CHOICE OF HOUSING TYPES AND WAYS OF GETTING AROUND IT IS VERY EASY TO } \\
\text { WALK TO LOCAL SHOPS SCHOOLS AND AMENITIES AND PEDESTRIAN ACTIVITY MAKES } \\
\text { OUR NEIGHBOURHOODS FEEL SAFE EXCELLENT NEW BUILDINGS AND DEVELOPMENT } \\
\text { COMPLEMENT ESTABLISHED AREAS HERITAGE AND LANDSCAPE FEATURES }\end{array}$ \\
\hline
\end{tabular}


751 TAURANGA CITY COUNCIL CLEAN GREEN VALUED ENVIRONMENT

752 TAURANGA CITY COUNCIL

EASY AND SAFE TO MOVE AROUND

753 TAURANGA CITY COUNCIL

GREAT PLACE TO GROW UP

754 TAURANGA CITY COUNCIL

LIVING WELL WASTING LESS

755 TAURANGA CITY COUNCIL

STRONG SUSTAINABLE ECONOMY

756 TAURANGA CITY COUNCIL

VIBRANT HEALTHY AND DIVERSE COMMUNITIES

757 THAMES COROMANDEL DISTRICT COUNCIL

OUR COMMUNITIES ARE HEALTHY COHESIVE CARING AND SUPPORTIVE

OUR COMMUNITIES RECOGNISE AND VALUE THE NATURAL ENVIRONMENT
TAURANGA IS OUR PLACE A PLACE WITH STRONG COMMUNITY PRIDE AND VALUES IT IS A PLACE WHERE OUR BEAUTIFUL LANDSCAPE AND ENVIRONMENT IS RECOGNISED AND CONTRIBUTES TO OUR SENSE OF PLACE MAURI AND IDENTITY IT IS A PLACE WHERE THROUGH WORKING TOGETHER WE CAN ENCOURAGE OTHERS TO RESPECT AND INTERACT WITH NATURE AND ENJOY AND CHERISH A WIDE RANGE OF NATIVE AND EXOTIC ANIMALS AND PLANTS THAT FLOURISH IN OUR CITY IT IS A PLACE WHERE ITS NATURAL ATTRIBUTES AND BENEFICIAL ELEMENTS ARE PROTECTED AS THE CITY CONTINUES TO DEVELOP AND GROW TAURANGA IS A RICH NATURAL ENVIRONMENT A CITY THAT WE ARE PROUD TO LIVE IN AND GROW WITH

IT IS EASY AND SAFE TO MOVE AROUND TAURANGA ALL THE DIFFERENT WAYS TO TRAVEL ARE LINKED TOGETHER CYCLISTS WALKERS AND PEOPLE ON MOBILITY SCOOTERS ARE USING A NETWORK OF PATHWAYS FROM EVERY SUBURB TO THE CITY CENTRE LOCAL AREAS AND FACILITIES

WE THINK TAURANGA IS A GREAT PLACE TO GROW UP ITS THE LIFESTYLE EDUCATION AND CAREER OPPORTUNITIES ON OFFER THAT MAKE US WANT TO LIVE HERE WE FEEL LIKE WE BELONG WE ARE VALUED LISTENED TO RESPECTED AND ARE SUPPORTED TO ACHIEVE OUR GOALS THIS IS OUR COMMUNITY A SAFE AND HAPPENING PLACE A PLACE WHERE WE HAVE A SAY IN OUR FUTURE

THE WESTERN BAY OF PLENTY SUBREGION IS AN ECOFRIENDLY PARADISE A PLACE WE LIVE WORK LEARN AND PLAY IN A WAY THAT PROTECTS OUR ENVIRONMENT WE ARE VIEWED AS A NATIONAL LEADER IN CLEVER AND EFFICIENT RESOURCE AND ENERGY USE ALWAYS EXPLORING WAYS TO REDUCE THE IMPACT WE HAVE ON OUR ENVIRONMENT ITS A GREAT PLACE TO LIVE

THE WESTERN BAY OF PLENTY SUBREGION IS THE BUSINESS LOCATION OF CHOICE BASED ON SUN SEA SOIL AND PEOPLE SKILLS RESULTING IN A HIGHLY PRODUCTIVE COMPETITIVE EXPORTBASED ECONOMY

TAURANGA IS OUR PLACE A HAPPENING PLACE FULL OF OPPORTUNITY AND LIFE

THROUGH WORKING TOGETHER BUILDING ON OUR WONDERFUL ENVIRONMENT AND CELEBRATING OUR DIVERSITY AND THE BIG AND LITTLE SUCCESSES ALONG THE WAY WE HAVE CREATED A CITY WITH A REAL COMMUNITY HEART

A STRONG CARING AND HEALTHY COMMUNITY BENEFITS EVERYONE PEOPLE NEED TO FEEL THEY BELONG AND CAN CONTRIBUTE WE MUST CONTINUOUSLY RESPOND TO THE CHANGING NEEDS OF OUR COMMUNITIES

THE NATURAL ENVIRONMENT OF THE PENINSULA IS OF SUCH IMPORTANCE IT MUST BE PROTECTED FOR FUTURE GENERATIONS THE PRESSURES OF GROWTH AND DEVELOPMENT NEED TO BE CAREFULLY MANAGED SO THAT THE PENINSULAS UNIQUE VALUES AND QUALITIES ARE NOT LOST 


\begin{tabular}{|c|c|c|c|}
\hline 759 & $\begin{array}{l}\text { THAMES COROMANDEL DISTRICT } \\
\text { COUNCIL }\end{array}$ & $\begin{array}{l}\text { OUR LOCAL ECONOMIES REFLECT THE SPIRIT OF THE } \\
\text { PENINSULA }\end{array}$ & $\begin{array}{l}\text { OUR COMMUNITIES REFER TO THE SPIRIT OF THE PENINSULA TO MEAN MANY THINGS } \\
\text { ESPECIALLY THE STRONG LINKS TO THE NATURAL ENVIRONMENT AND THE LOCAL } \\
\text { COMMUNITY ATMOSPHERE THE MAJORITY OF BUSINESSES IN THE COMMUNITY ARE } \\
\text { SMALL TO MEDIUM IN SIZE AND ARE PREDOMINANTLY IN THE PRIMARY AND SERVICES } \\
\text { INDUSTRIES OPPORTUNITIES EXIST FOR VALUE ADDED ENTERPRISES THAT CREATE } \\
\text { SUSTAINABLE EMPLOYMENT }\end{array}$ \\
\hline 760 & $\begin{array}{l}\text { THAMES COROMANDEL DISTRICT } \\
\text { COUNCIL }\end{array}$ & $\begin{array}{l}\text { THE DIVERSITY AND CHARACTER OF OUR } \\
\text { COMMUNITIES AND THE UNIQUENESS OF THE } \\
\text { PENINSULA IS A VALUED PART OF OUR LIFESTYLE }\end{array}$ & $\begin{array}{l}\text { HERE ARE OVER COMMUNITIES AND SETTLEMENTS ON THE PENINSULA EACH HAS ITS } \\
\text { OWN DISTINCTIVE CHARACTER AND DIVERSITY AS A RESULT OF THE HISTORY THE } \\
\text { ENVIRONMENT THE LANDSCAPE AND THE PEOPLE THAT LIVE THERE BY EMBRACING THIS } \\
\text { DIVERSITY WE CAN BUILD ON THE STRENGTH AND PRIDE EACH COMMUNITY } \\
\text { CONTRIBUTES TO THE CHARACTER OF THE PENINSULA }\end{array}$ \\
\hline 761 & $\begin{array}{l}\text { THAMES COROMANDEL DISTRICT } \\
\text { COUNCIL }\end{array}$ & $\begin{array}{l}\text { THE NATURAL VALUES OF OUR COAST AND BEACHES } \\
\text { ARE RESPECTED AND ENHANCED }\end{array}$ & $\begin{array}{l}\text { BEING SURROUNDED BY SUCH DIVERSE COASTLINE AND THE RECREATIONAL } \\
\text { OPPORTUNITIES THAT COME WITH IT THERE ARE COMPELLING REASONS WHY THE } \\
\text { MAJORITY OF PEOPLE LIVE ON OR VISIT THE PENINSULA OUR COMMUNITIES WANT THE } \\
\text { NATURAL BEAUTY AND THE CLEAN BEACH ENVIRONMENT RETAINED AND WANTS TO SEE } \\
\text { THESE VALUES RESPECTED INCREASED USE OF OUR BEACHES AND COASTAL } \\
\text { ENVIRONMENT CREATES PRESSURES THAT CAN HAVE NEGATIVE IMPACTS UNLESS } \\
\text { CAREFULLY MANAGED EVERYBODY MUST TAKE INDIVIDUAL AND COLLECTIVE } \\
\text { RESPONSIBILITY }\end{array}$ \\
\hline 762 & $\begin{array}{l}\text { THAMES COROMANDEL DISTRICT } \\
\text { COUNCIL }\end{array}$ & $\begin{array}{l}\text { THE NEEDS OF BOTH LOCAL AND VISITOR } \\
\text { COMMUNITIES ARE MET THROUGH SOUND } \\
\text { PLANNING AHEAD OF GROWTH AND DEVELOPMENT }\end{array}$ & $\begin{array}{l}\text { THE PROXIMITY OF THE PENINSULA TO LARGE CITIES HAS RESULTED IN RAPID GROWTH } \\
\text { AND ASSOCIATED PRESSURES WHICH ARE EXPECTED TO CONTINUE IT IS RECOGNISED } \\
\text { THAT SOUND PLANNING ACROSS ALL SECTORS OF OUR COMMUNITIES IS ESSENTIAL TO } \\
\text { MEET THE NEEDS OF BOTH LOCAL AND VISITOR POPULATIONS }\end{array}$ \\
\hline 763 & $\begin{array}{l}\text { THAMES COROMANDEL DISTRICT } \\
\text { COUNCIL }\end{array}$ & $\begin{array}{l}\text { THE PENINSULAS LONG AND RICH HISTORY IS } \\
\text { VALUED AND PRESERVED }\end{array}$ & $\begin{array}{l}\text { OUR LONG AND RICH HISTORY IS AN IMPORTANT PART OF THE IDENTITY OF THE } \\
\text { PENINSULA WE MUST HAVE A COORDINATED APPROACH TO THE PRESERVATION OUR } \\
\text { HISTORY SO THAT IT REMAINS FOR FUTURE GENERATIONS }\end{array}$ \\
\hline 764 & TIMARU DISTRICT COUNCIL & ATTRACTIVE AND DESIRABLE DISTRICT & $\begin{array}{l}\text { THIS OUTCOME IS CONCERNED WITH ENSURING OUR DISTRICT REMAINS ATTRACTIVE TO } \\
\text { RESIDENTS VISITORS AND TOURISTS PROMOTING AND DEVELOPING ITS ATTRACTIONS } \\
\text { AND SMART PLANNING WILL HELP RAISE THE DISTRICTS PROFILE AND ENSURE IT MEETS } \\
\text { THE POTENTIAL DEMANDS OF GROWTH }\end{array}$ \\
\hline 765 & TIMARU DISTRICT COUNCIL & BALANCED AND SUSTAINABLE WATER USAGE & $\begin{array}{l}\text { WATER IS AN ESSENTIAL INGREDIENT FOR THE FUTURE OF THE TIMARU DISTRICT } \\
\text { COMPETING DEMANDS IN RECENT YEARS HAVE MADE THE FUTURE SUSTAINABLE } \\
\text { MANAGEMENT OF THE WATER RESOURCE A TOP PRIORITY }\end{array}$ \\
\hline
\end{tabular}




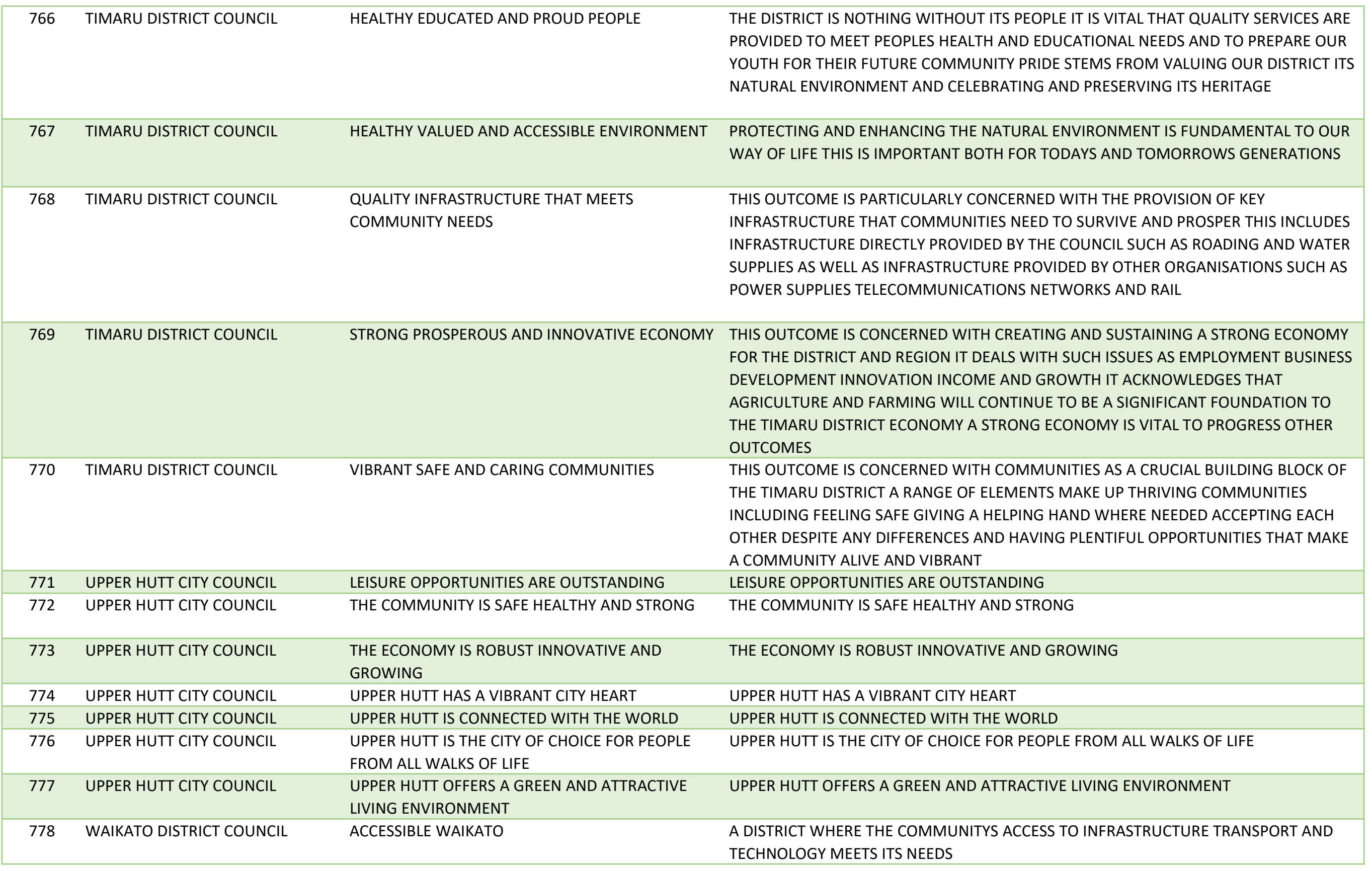


Appendix B: Community Well-being Statements by Councils

\begin{tabular}{|c|c|c|c|}
\hline 779 & WAIKATO DISTRICT COUNCIL & ACTIVE WAIKATO & $\begin{array}{l}\text { A DISTRICT THAT PROVIDES A VARIETY OF RECREATION AND LEISURE OPTIONS FOR THE } \\
\text { COMMUNITY }\end{array}$ \\
\hline 780 & WAIKATO DISTRICT COUNCIL & EDUCATED WAIKATO & $\begin{array}{l}\text { A DISTRICT WHERE EDUCATION OPTIONS ARE VARIED AND ALLOW OUR COMMUNITY TO } \\
\text { BE SKILLED FOR WORK AND LIFE }\end{array}$ \\
\hline 781 & WAIKATO DISTRICT COUNCIL & GREEN WAIKATO & $\begin{array}{l}\text { A DISTRICT WHERE OUR NATURAL RESOURCES ARE PROTECTED DEVELOPED AND } \\
\text { ENHANCED FOR FUTURE GENERATIONS }\end{array}$ \\
\hline 782 & WAIKATO DISTRICT COUNCIL & SAFE WAIKATO & $\begin{array}{l}\text { A DISTRICT WHERE PEOPLE FEEL SAFE AND SUPPORTED WITHIN THEIR COMMUNITIES } \\
\text { AND WHERE CRIME IS UNDER CONTROL }\end{array}$ \\
\hline 783 & WAIKATO DISTRICT COUNCIL & SUSTAINABLE WAIKATO & A DISTRICT WHERE GROWTH IS EFFECTIVELY MANAGED \\
\hline 784 & WAIKATO DISTRICT COUNCIL & THRIVING WAIKATO & $\begin{array}{l}\text { A DISTRICT WHERE BUSINESS AND INDUSTRY ARE ENCOURAGED AND SUPPORTED AND } \\
\text { EMPLOYMENT CONTRIBUTES TO A SUCCESSFUL LOCAL ECONOMY }\end{array}$ \\
\hline 785 & WAIKATO DISTRICT COUNCIL & VIBRANT WAIKATO & $\begin{array}{l}\text { A DISTRICT WHERE OUR HERITAGE AND CULTURE ARE RECOGNISED PROTECTED AND } \\
\text { CELEBRATED }\end{array}$ \\
\hline 786 & WAIKATO DISTRICT COUNCIL & WELL WAIKATO & $\begin{array}{l}\text { A DISTRICT WHERE PEOPLE CAN ACCESS QUALITY COMMUNITY HEALTH AND CARE } \\
\text { SERVICES }\end{array}$ \\
\hline 787 & WAIKATO REGIONAL COUNCIL & CULTURE AND IDENTITY & $\begin{array}{l}\text { THE WAIKATO REGION IDENTIFIES WITH AND VALUES ITS LAND AIR RIVERS AND } \\
\text { WATERWAYS MOUNTAINS FLORA AND FAUNA AND ITS PEOPLE }\end{array}$ \\
\hline 788 & WAIKATO REGIONAL COUNCIL & PARTICIPATION AND EQUITY & $\begin{array}{l}\text { THE WAIKATO REGION BUILDS STRONG INFORMED COMMUNITIES AND HAS A CULTURE } \\
\text { THAT ENCOURAGES PEOPLE AND COMMUNITIES TO PLAY THEIR PART }\end{array}$ \\
\hline 789 & WAIKATO REGIONAL COUNCIL & QUALITY OF LIFE & $\begin{array}{l}\text { THE WAIKATO REGION IS A GREAT PLACE TO LIVE PROVIDING THE SERVICES AND } \\
\text { OPPORTUNITIES WE NEED TO LIVE WELL }\end{array}$ \\
\hline 790 & WAIKATO REGIONAL COUNCIL & SUSTAINABLE ECONOMY & $\begin{array}{l}\text { THE WAIKATO REGION BALANCES A THRIVING ECONOMY WITH LOOKING AFTER ITS } \\
\text { PEOPLE PLACES AND ENVIRONMENT }\end{array}$ \\
\hline 791 & WAIKATO REGIONAL COUNCIL & SUSTAINABLE ENVIRONMENT & $\begin{array}{l}\text { THE WAIKATO REGION VALUES AND PROTECTS ITS DIVERSE INTERCONNECTED NATURAL } \\
\text { ENVIRONMENTS }\end{array}$ \\
\hline 792 & WAIMAKARIRI DISTRICT COUNCIL & $\begin{array}{l}\text { BUSINESSES IN THE DISTRICT ARE DIVERSE } \\
\text { ADAPTABLE AND GROWING }\end{array}$ & BUSINESSES IN THE DISTRICT ARE DIVERSE ADAPTABLE AND GROWING \\
\hline 793 & WAIMAKARIRI DISTRICT COUNCIL & $\begin{array}{l}\text { CORE UTILITY SERVICES ARE PROVIDED IN A TIMELY } \\
\text { SUSTAINABLE AND AFFORDABLE MANNER }\end{array}$ & $\begin{array}{l}\text { CORE UTILITY SERVICES ARE PROVIDED IN A TIMELY SUSTAINABLE AND AFFORDABLE } \\
\text { MANNER }\end{array}$ \\
\hline 794 & WAIMAKARIRI DISTRICT COUNCIL & $\begin{array}{l}\text { PEOPLE ARE FRIENDLY AND CARING CREATING A } \\
\text { STRONG SENSE OF COMMUNITY IN OUR DISTRICT }\end{array}$ & $\begin{array}{l}\text { PEOPLE ARE FRIENDLY AND CARING CREATING A STRONG SENSE OF COMMUNITY IN OUR } \\
\text { DISTRICT }\end{array}$ \\
\hline 795 & WAIMAKARIRI DISTRICT COUNCIL & $\begin{array}{l}\text { PEOPLE HAVE WIDE RANGING OPPORTUNITIES FOR } \\
\text { LEARNING AND BEING INFORMED }\end{array}$ & PEOPLE HAVE WIDE RANGING OPPORTUNITIES FOR LEARNING AND BEING INFORMED \\
\hline 796 & WAIMAKARIRI DISTRICT COUNCIL & $\begin{array}{l}\text { PUBLIC ORGANISATIONS GIVE EFFECT TO THE SPIRIT } \\
\text { OF THE TREATY OF WAITANGI }\end{array}$ & PUBLIC ORGANISATIONS GIVE EFFECT TO THE SPIRIT OF THE TREATY OF WAITANGI \\
\hline
\end{tabular}


Appendix B: Community Well-being Statements by Councils

\begin{tabular}{|c|c|c|c|}
\hline 797 & WAIMAKARIRI DISTRICT COUNCIL & $\begin{array}{l}\text { PUBLIC SPACES AND FACILITIES ARE PLENTIFUL } \\
\text { ACCESSIBLE AND HIGH QUALITY }\end{array}$ & PUBLIC SPACES AND FACILITIES ARE PLENTIFUL ACCESSIBLE AND HIGH QUALITY \\
\hline 798 & WAIMAKARIRI DISTRICT COUNCIL & THE AIR IS CLEAN & THE AIR IS CLEAN \\
\hline 799 & WAIMAKARIRI DISTRICT COUNCIL & $\begin{array}{l}\text { THE COMMUNITYS CULTURES ARTS AND HERITAGE } \\
\text { ARE CONSERVED AND CELEBRATED }\end{array}$ & THE COMMUNITYS CULTURES ARTS AND HERITAGE ARE CONSERVED AND CELEBRATED \\
\hline 800 & WAIMAKARIRI DISTRICT COUNCIL & $\begin{array}{l}\text { THE COMMUNITYS NEEDS FOR HEALTH AND SOCIAL } \\
\text { SERVICES ARE MET }\end{array}$ & THE COMMUNITYS NEEDS FOR HEALTH AND SOCIAL SERVICES ARE MET \\
\hline 801 & WAIMAKARIRI DISTRICT COUNCIL & $\begin{array}{l}\text { THE DISTINCTIVE CHARACTER OF OUR TOWNS } \\
\text { VILLAGES AND RURAL AREAS IS MAINTAINED }\end{array}$ & $\begin{array}{l}\text { THE DISTINCTIVE CHARACTER OF OUR TOWNS VILLAGES AND RURAL AREAS IS } \\
\text { MAINTAINED }\end{array}$ \\
\hline 802 & WAIMAKARIRI DISTRICT COUNCIL & THE LAND IS HEALTHY & THE LAND IS HEALTHY \\
\hline 803 & WAIMAKARIRI DISTRICT COUNCIL & $\begin{array}{l}\text { THERE ARE AREAS OF SIGNIFICANT INDIGENOUS } \\
\text { VEGETATION AND HABITATS FOR INDIGENOUS } \\
\text { FAUNA }\end{array}$ & $\begin{array}{l}\text { THERE ARE AREAS OF SIGNIFICANT INDIGENOUS VEGETATION AND HABITATS FOR } \\
\text { INDIGENOUS FAUNA }\end{array}$ \\
\hline 804 & WAIMAKARIRI DISTRICT COUNCIL & $\begin{array}{l}\text { THERE ARE WIDE RANGING OPPORTUNITIES FOR } \\
\text { PEOPLE TO CONTRIBUTE TO THE DECISIONMAKING } \\
\text { BY PUBLIC ORGANISATIONS THAT AFFECTS OUR } \\
\text { DISTRICT }\end{array}$ & $\begin{array}{l}\text { THERE ARE WIDE RANGING OPPORTUNITIES FOR PEOPLE TO CONTRIBUTE TO THE } \\
\text { DECISIONMAKING BY PUBLIC ORGANISATIONS THAT AFFECTS OUR DISTRICT }\end{array}$ \\
\hline 805 & WAIMAKARIRI DISTRICT COUNCIL & THERE IS A SAFE ENVIRONMENT FOR ALL & THERE IS A SAFE ENVIRONMENT FOR ALL \\
\hline 806 & WAIMAKARIRI DISTRICT COUNCIL & $\begin{array}{l}\text { THERE IS SUFFICIENT CLEAN WATER TO MEET THE } \\
\text { NEEDS OF COMMUNITIES AND ECOSYSTEMS }\end{array}$ & $\begin{array}{l}\text { THERE IS SUFFICIENT CLEAN WATER TO MEET THE NEEDS OF COMMUNITIES AND } \\
\text { ECOSYSTEMS }\end{array}$ \\
\hline 807 & WAIMAKARIRI DISTRICT COUNCIL & $\begin{array}{l}\text { TRANSPORT IS ACCESSIBLE CONVENIENT RELIABLE } \\
\text { AFFORDABLE AND SUSTAINABLE }\end{array}$ & TRANSPORT IS ACCESSIBLE CONVENIENT RELIABLE AFFORDABLE AND SUSTAINABLE \\
\hline 808 & WAIMATE DISTRICT COUNCIL & CULTURAL & WAIMATE DISTRICT PROMOTES RECREATION FUN AND CREATIVITY \\
\hline 809 & WAIMATE DISTRICT COUNCIL & ECONOMIC & $\begin{array}{l}\text { WAIMATE DISTRICT IS UNDERPINNED BY A STRONG SUCCESSFUL AND INNOVATIVE } \\
\text { ECONOMY }\end{array}$ \\
\hline 810 & WAIMATE DISTRICT COUNCIL & ENVIRONMENTAL & WAIMATE DISTRICT VALUES PROTECTS AND ENJOYS THE NATURAL ENVIRONMENT \\
\hline 811 & WAIMATE DISTRICT COUNCIL & GOVERNANCE & WAIMATE DISTRICT IS ABOUT COUNCIL AND THE COMMUNITY WORKING TOGETHER \\
\hline 812 & WAIMATE DISTRICT COUNCIL & SOCIAL & WAIMATE DISTRICT IS SAFE HEALTHY AND SUPPORTIVE OF LIFELONG LEARNING \\
\hline 813 & WAIPA DISTRICT COUNCIL & $\begin{array}{l}\text { ECONOMICALLY SECURE WAIPA ECONOMIC } \\
\text { WELLBEING }\end{array}$ & $\begin{array}{l}\text { A DIVERSITY OF EMPLOYMENT OPTIONS AND BUSINESS OPPORTUNITIES IS PROVIDED } \\
\text { WITHIN THE DISTRICT }\end{array}$ \\
\hline
\end{tabular}


Appendix B: Community Well-being Statements by Councils

\begin{tabular}{|c|c|c|c|}
\hline 814 & WAIPA DISTRICT COUNCIL & $\begin{array}{l}\text { ECONOMICALLY SECURE WAIPA ECONOMIC } \\
\text { WELLBEING }\end{array}$ & $\begin{array}{l}\text { OUR DISTRICT IS A TOURIST DESTINATION OF CHOICE AND OUR TOURISM INDUSTRY } \\
\text { PROMOTES OUR SPECIAL LOCAL FEATURES AND SUPPORTS ECONOMIC GROWTH }\end{array}$ \\
\hline 815 & WAIPA DISTRICT COUNCIL & $\begin{array}{l}\text { ECONOMICALLY SECURE WAIPA ECONOMIC } \\
\text { WELLBEING }\end{array}$ & $\begin{array}{l}\text { OUR STRONG DAIRY AND EQUINE INDUSTRIES HELP SUPPORT OUR PROSPEROUS } \\
\text { COMMUNITIES AND PLANNING AND PRACTICES ARE ENCOURAGED THAT PROTECT AND } \\
\text { SUSTAIN OUR PRODUCTIVE RESOURCESPUBLIC BUILDINGS AND PUBLIC TRANSPORT } \\
\text { OPTIONS ARE SAFE ACCESSIBLE TO PEOPLE OF ALL AGES AND ABILITIES AND SIGNAGE IS } \\
\text { CLEAR AND VISIBLE }\end{array}$ \\
\hline 817 & WAIPA DISTRICT COUNCIL & $\begin{array}{l}\text { ECONOMICALLY SECURE WAIPA ECONOMIC } \\
\text { WELLBEING }\end{array}$ & $\begin{array}{l}\text { PROVISION OF INDUSTRIAL AND RESIDENTIAL AREAS IS WELL BALANCED CAREFULLY } \\
\text { ZONED TO MAINTAIN OUR RURAL SEMIRURAL NATURE AND PLANNED WELL INTO THE } \\
\text { FUTURE }\end{array}$ \\
\hline 818 & WAIPA DISTRICT COUNCIL & $\begin{array}{l}\text { ECONOMICALLY SECURE WAIPA ECONOMIC } \\
\text { WELLBEING }\end{array}$ & $\begin{array}{l}\text { PUBLIC BUILDINGS AND PUBLIC TRANSPORT OPTIONS ARE SAFE ACCESSIBLE TO PEOPLE } \\
\text { OF ALL AGES AND ABILITIES AND SIGNAGE IS CLEAR AND VISIBLE }\end{array}$ \\
\hline 819 & WAIPA DISTRICT COUNCIL & $\begin{array}{l}\text { ECONOMICALLY SECURE WAIPA ECONOMIC } \\
\text { WELLBEING }\end{array}$ & $\begin{array}{l}\text { THE BUY LOCAL PHILOSOPHY IS ENCOURAGED AND LOCALLY SOURCED PRODUCTS AND } \\
\text { SERVICES ARE SUPPORTED }\end{array}$ \\
\hline 820 & WAIPA DISTRICT COUNCIL & $\begin{array}{l}\text { ECONOMICALLY SECURE WAIPA ECONOMIC } \\
\text { WELLBEING }\end{array}$ & $\begin{array}{l}\text { THE GROWTH WEALTH AND UNIQUENESS OF THE LOCAL MĀORI ECONOMY IS } \\
\text { ACKNOWLEDGED AND SUPPORTED }\end{array}$ \\
\hline 821 & WAIPA DISTRICT COUNCIL & $\begin{array}{l}\text { ECONOMICALLY SECURE WAIPA ECONOMIC } \\
\text { WELLBEING }\end{array}$ & $\begin{array}{l}\text { WE HAVE RELIABLE EFFICIENT AND WELL PLANNED TRANSPORT INFRASTRUCTURE } \\
\text { SYSTEMS THAT PROVIDE EASY ACCESS TO AND THROUGH OUR TOWNS }\end{array}$ \\
\hline 822 & WAIPA DISTRICT COUNCIL & $\begin{array}{l}\text { ECONOMICALLY SECURE WAIPA ECONOMIC } \\
\text { WELLBEING }\end{array}$ & $\begin{array}{l}\text { WE HAVE RELIABLE EFFICIENT AND WELL PLANNED WATER WASTEWATER AND } \\
\text { STORMWATER INFRASTRUCTURE THAT SUPPORTS DEVELOPMENT AND GROWTH WHILE } \\
\text { CONTINUING TO MEET THE NEEDS OF RESIDENTS }\end{array}$ \\
\hline 823 & WAIPA DISTRICT COUNCIL & $\begin{array}{l}\text { ENVIRONMENTALLY SUSTAINABLE WAIPA } \\
\text { ENVIRONMENTAL WELLBEING }\end{array}$ & $\begin{array}{l}\text { HERITAGE SITES AND LANDSCAPES OF SIGNIFICANCE TO MĀORI ARE PRESERVED AND } \\
\text { VALUED AND WHERE APPROPRIATE ARE IDENTIFIED AND CELEBRATED VISUALLY }\end{array}$ \\
\hline 824 & WAIPA DISTRICT COUNCIL & $\begin{array}{l}\text { ENVIRONMENTALLY SUSTAINABLE WAIPA } \\
\text { ENVIRONMENTAL WELLBEING }\end{array}$ & $\begin{array}{l}\text { MAUNGATAUTARI KAKEPUKU PIRONGIA AND OTHER MAUNGA IN THE DISTRICT ARE } \\
\text { PROTECTED AND PROMOTED AS KEY FEATURES OF THE WAIPA LANDSCAPE AND FOR } \\
\text { RECREATIONAL PURPOSES }\end{array}$ \\
\hline 825 & WAIPA DISTRICT COUNCIL & $\begin{array}{l}\text { ENVIRONMENTALLY SUSTAINABLE WAIPA } \\
\text { ENVIRONMENTAL WELLBEING }\end{array}$ & $\begin{array}{l}\text { OUR CLEAN GREEN ENVIRONMENT IS PRESERVED PROMOTED AND UTILISED FOR } \\
\text { RECREATION AND LEISURE AND CONTRIBUTES TO ENHANCING OUR IMAGE AS A TOURIST } \\
\text { DESTINATION }\end{array}$ \\
\hline
\end{tabular}




\begin{tabular}{|c|c|c|c|}
\hline 826 & WAIPA DISTRICT COUNCIL & $\begin{array}{l}\text { ENVIRONMENTALLY SUSTAINABLE WAIPA } \\
\text { ENVIRONMENTAL WELLBEING }\end{array}$ & $\begin{array}{l}\text { OUR SPECIAL NATURAL LANDSCAPES AND NATIVE BIODIVERSITY SUCH AS PEAT LAKES } \\
\text { RIPARIAN AND INDIGENOUS FOREST AREAS ARE PROTECTED MAINTAINED AND } \\
\text { ENHANCED ECOLOGICAL CORRIDORS ARE DEVELOPED AND ANIMAL AND PLANT PESTS } \\
\text { ARE CONTROLLED }\end{array}$ \\
\hline 827 & WAIPA DISTRICT COUNCIL & $\begin{array}{l}\text { ENVIRONMENTALLY SUSTAINABLE WAIPA } \\
\text { ENVIRONMENTAL WELLBEING }\end{array}$ & $\begin{array}{l}\text { WATER QUALITY IN OUR RIVERS AND LAKES IS MAINTAINED AND IMPROVED AND WATER } \\
\text { IS USED EFFICIENTLY }\end{array}$ \\
\hline 828 & WAIPA DISTRICT COUNCIL & $\begin{array}{l}\text { ENVIRONMENTALLY SUSTAINABLE WAIPA } \\
\text { ENVIRONMENTAL WELLBEING }\end{array}$ & $\begin{array}{l}\text { WE ACKNOWLEDGE THE NEED TO PURSUE A ZERO WASTE PHILOSOPHY INCLUDING } \\
\text { REDUCING OUR WASTE AND USING RESOURCES MORE EFFICIENTLY }\end{array}$ \\
\hline 829 & WAIPA DISTRICT COUNCIL & $\begin{array}{l}\text { ENVIRONMENTALLY SUSTAINABLE WAIPA } \\
\text { ENVIRONMENTAL WELLBEING }\end{array}$ & $\begin{array}{l}\text { WE ARE AWARE OF THE LINKS BETWEEN THE HEALTH OF OUR ENVIRONMENT AND OUR } \\
\text { OWN HEALTH LOCAL ORGANISATIONS AND INDIVIDUALS ARE REGULARLY INVOLVED IN } \\
\text { ENVIRONMENTAL CARE ACTIVITIES }\end{array}$ \\
\hline 830 & WAIPA DISTRICT COUNCIL & $\begin{array}{l}\text { ENVIRONMENTALLY SUSTAINABLE WAIPA } \\
\text { ENVIRONMENTAL WELLBEING }\end{array}$ & WE REDUCE OUR RELIANCE ON NONRENEWABLE ENERGY \\
\hline 831 & WAIPA DISTRICT COUNCIL & $\begin{array}{l}\text { ENVIRONMENTALLY SUSTAINABLE WAIPA } \\
\text { ENVIRONMENTAL WELLBEING }\end{array}$ & $\begin{array}{l}\text { WE USE LAND MANAGEMENT PRACTICES THAT PROTECT AND SUSTAIN OUR SOIL AND } \\
\text { LAND AND OUR DISTRICTS HIGH QUALITY SOILS ARE PROTECTED FROM INCREASING } \\
\text { DEVELOPMENT DEMANDS BY CONCENTRATING GROWTH AROUND EXISTING } \\
\text { SETTLEMENTS RATHER THAN INTRUDING ON RURAL AREAS }\end{array}$ \\
\hline 832 & WAIPA DISTRICT COUNCIL & HEALTHY AND ACTIVE WAIPA SOCIAL WELLBEING & $\begin{array}{l}\text { A GOOD RANGE OF FUN RECREATIONAL SPACES PLAYGROUNDS FACILITIES AND FAMILY } \\
\text { FRIENDLY AREAS ARE AVAILABLE WELL PLANNED AFFORDABLE ACCESSIBLE AND YOUTH } \\
\text { FOCUSSED }\end{array}$ \\
\hline 833 & WAIPA DISTRICT COUNCIL & HEALTHY AND ACTIVE WAIPA SOCIAL WELLBEING & $\begin{array}{l}\text { COMMUNITY SERVICES ARE COORDINATED AND DELIVERED EFFECTIVELY AND } \\
\text { EFFICIENTLY TO MAXIMISE RESOURCES WHILE MEETING THE NEEDS OF OUR RESIDENTS }\end{array}$ \\
\hline 834 & WAIPA DISTRICT COUNCIL & HEALTHY AND ACTIVE WAIPA SOCIAL WELLBEING & EVERYONE HAS ACCESS TO AFFORDABLE QUALITY HEALTH AND CHILDCARE SERVICES \\
\hline 835 & WAIPA DISTRICT COUNCIL & HEALTHY AND ACTIVE WAIPA SOCIAL WELLBEING & $\begin{array}{l}\text { FAMILIES AND CAREGIVERS ARE PROTECTED AND VALUED AND OUR COMMUNITIES } \\
\text { ACCEPT RESPONSIBILITY FOR OUR CHILDREN AND OTHER VULNERABLE MEMBERS }\end{array}$ \\
\hline 836 & WAIPA DISTRICT COUNCIL & HEALTHY AND ACTIVE WAIPA SOCIAL WELLBEING & GOOD QUALITY HOUSING IS AVAILABLE AND AFFORDABLE \\
\hline 837 & WAIPA DISTRICT COUNCIL & HEALTHY AND ACTIVE WAIPA SOCIAL WELLBEING & $\begin{array}{l}\text { INEQUITY IN INCOME AND ACCESS TO SERVICES AND EMPLOYMENT IS REDUCED AND } \\
\text { RESOURCES ARE SHARED APPROPRIATELY }\end{array}$ \\
\hline 838 & WAIPA DISTRICT COUNCIL & HEALTHY AND ACTIVE WAIPA SOCIAL WELLBEING & $\begin{array}{l}\text { IWI HAPŪ AND MĀORI WORK TOGETHER WITH CENTRAL GOVERNMENT LOCAL } \\
\text { GOVERNMENT AND COMMUNITY ORGANISATIONS IN MUTUALLY BENEFICIAL } \\
\text { PARTNERSHIPS }\end{array}$ \\
\hline 839 & WAIPA DISTRICT COUNCIL & HEALTHY AND ACTIVE WAIPA SOCIAL WELLBEING & $\begin{array}{l}\text { MĀORI ENJOY THE SAME QUALITY OF HEALTH EDUCATION HOUSING EMPLOYMENT AND } \\
\text { ECONOMIC OUTCOMES AS NONMĀORI }\end{array}$ \\
\hline
\end{tabular}


Appendix B: Community Well-being Statements by Councils

\begin{tabular}{|c|c|c|c|}
\hline 840 & WAIPA DISTRICT COUNCIL & HEALTHY AND ACTIVE WAIPA SOCIAL WELLBEING & $\begin{array}{l}\text { OUR COMMUNITIES AND AGENCIES WORK TOGETHER SO THAT WE ARE SAFE WE FEEL } \\
\text { SAFE CRIME IS REDUCED AND THERE IS A ZERO TOLERANCE APPROACH TO ANTISOCIAL } \\
\text { BEHAVIOUR }\end{array}$ \\
\hline 841 & WAIPA DISTRICT COUNCIL & HEALTHY AND ACTIVE WAIPA SOCIAL WELLBEING & $\begin{array}{l}\text { QUALITY EDUCATIONAL OPPORTUNITIES AT ALL LEVELS ARE LOCALLY ACCESSIBLE AND } \\
\text { PLANNED FOR FUTURE GROWTH }\end{array}$ \\
\hline 842 & WAIPA DISTRICT COUNCIL & HEALTHY AND ACTIVE WAIPA SOCIAL WELLBEING & $\begin{array}{l}\text { THE IMPORTANCE OF TE REO IS RECOGNISED AND PROMOTED THROUGH PROMOTION } \\
\text { AND SUPPORT OF APPROPRIATE EDUCATIONAL FACILITIES }\end{array}$ \\
\hline 843 & WAIPA DISTRICT COUNCIL & $\begin{array}{l}\text { VIBRANT AND CREATIVE WAIPA CULTURAL } \\
\text { WELLBEING }\end{array}$ & INDIVIDUAL AND GROUP ACHIEVEMENT IS RECOGNISED AND CELEBRATED DISTRICT WIDE \\
\hline 844 & WAIPA DISTRICT COUNCIL & $\begin{array}{l}\text { VIBRANT AND CREATIVE WAIPA CULTURAL } \\
\text { WELLBEING }\end{array}$ & $\begin{array}{l}\text { OUR COMMUNITIES RECOGNISE THAT WAIPA DISTRICT IS ENRICHED BY OUR MULTI } \\
\text { CULTURAL MAKEUP AND THE UNIQUE STATUS AND ROLE OF TANGATA WHENUA IS } \\
\text { RESPECTED AND REFLECTED IN COMMUNITY PROCESSES }\end{array}$ \\
\hline 845 & WAIPA DISTRICT COUNCIL & $\begin{array}{l}\text { VIBRANT AND CREATIVE WAIPA CULTURAL } \\
\text { WELLBEING }\end{array}$ & $\begin{array}{l}\text { THE SPECIAL CHARACTER AND HERITAGE OF OUR TOWNS AND VILLAGES IS RETAINED } \\
\text { AND PROMOTED }\end{array}$ \\
\hline 846 & WAIPA DISTRICT COUNCIL & $\begin{array}{l}\text { VIBRANT AND CREATIVE WAIPA CULTURAL } \\
\text { WELLBEING }\end{array}$ & $\begin{array}{l}\text { WE ALL HAVE MEANINGFUL OPPORTUNITIES TO PARTICIPATE IN MAKING DECISIONS } \\
\text { THAT HELP SHAPE THE WAIPA OF THE FUTURE }\end{array}$ \\
\hline 847 & WAIPA DISTRICT COUNCIL & $\begin{array}{l}\text { VIBRANT AND CREATIVE WAIPA CULTURAL } \\
\text { WELLBEING }\end{array}$ & $\begin{array}{l}\text { WE ARE PROUD OF OUR DISTRICTS UNIQUE IDENTITY AND ITS RICH AND DIVERSE } \\
\text { ARCHAEOLOGICAL NATURAL AND CULTURAL HERITAGE }\end{array}$ \\
\hline 848 & WAIPA DISTRICT COUNCIL & $\begin{array}{l}\text { VIBRANT AND CREATIVE WAIPA CULTURAL } \\
\text { WELLBEING }\end{array}$ & $\begin{array}{l}\text { WE HAVE A VIBRANT MĀORI COMMUNITY AND OUR MĀORI HERITAGE IS EMBRACED } \\
\text { AND CELEBRATED DISTRICT WIDE AND SHOWCASED AS AN INTEGRAL PART OF THE } \\
\text { VISUAL AMENITY OF OUR TOWNS }\end{array}$ \\
\hline 849 & WAIPA DISTRICT COUNCIL & $\begin{array}{l}\text { VIBRANT AND CREATIVE WAIPA CULTURAL } \\
\text { WELLBEING }\end{array}$ & $\begin{array}{l}\text { WE IDENTIFY WITH AND TAKE PART IN OUR COMMUNITIES AND ENJOY PARTICIPATING IN } \\
\text { CREATIVE RECREATIONAL AND CULTURAL ACTIVITIES THAT BUILD VIBRANT COMMUNITY } \\
\text { SPIRIT BOTH LOCALLY AND AT DISTRICT LEVEL }\end{array}$ \\
\hline 850 & WAIROA DISTRICT COUNCIL & $\begin{array}{l}\text { A COMMUNITY THAT VALUES AND PROMOTES ITS } \\
\text { CULTURE AND HERITAGE }\end{array}$ & $\begin{array}{l}\text { COMMUNITIES RECOGNISING ACHIEVING AND PROMOTING THEIR CULTURAL GOALS } \\
\text { ACKNOWLEDGING AND PROTECTING PLACES OF SIGNIFICANCE TO PASS TO FUTURE } \\
\text { GENERATIONS COMMUNITIES RESPECTING AND EMBRACING THE CULTURAL } \\
\text { ENVIRONMENT OF ALL CULTURES REPRESENTED IN OUR COMMUNITY }\end{array}$ \\
\hline 851 & WAIROA DISTRICT COUNCIL & A LIFE TIME OF GOOD HEALTH AND WELLBEING & $\begin{array}{l}\text { OUR COMMUNITY WANTS AN ENVIRONMENT IN WHICH ALL PEOPLE ARE ABLE TO } \\
\text { ACHIEVE A LIFETIME OF GOOD HEALTH AND WELLBEING THEY ARE PROTECTED FROM THE } \\
\text { NEGATIVE EFFECTS OF POLLUTION DRUG AND ALCOHOL ABUSE AND ILLNESS THEY HAVE } \\
\text { CONFIDENCE IN AND ACCESS TO HEALTH CARE PROVIDED IN OUR REGION }\end{array}$ \\
\hline 852 & WAIROA DISTRICT COUNCIL & A SAFE AND INTEGRATED TRANSPORT SYSTEM & $\begin{array}{l}\text { A SAFE AND AFFORDABLE TRANSPORT SYSTEM THAT MEETS THE NEEDS OF THE PEOPLE } \\
\text { IN OUR REGION }\end{array}$ \\
\hline 853 & WAIROA DISTRICT COUNCIL & A SAFE AND SECURE COMMUNITY & $\begin{array}{l}\text { CENTRAL GOVERNMENT LOCAL GOVERNMENT AND THE COMMUNITY WORKING } \\
\text { TOGETHER TO CREATE AN ENVIRONMENT WHICH IS SAFE FOR ALL PEOPLE IN OUR } \\
\text { REGION }\end{array}$ \\
\hline
\end{tabular}


Appendix B: Community Well-being Statements by Councils

\begin{tabular}{|c|c|c|c|}
\hline 854 & WAIROA DISTRICT COUNCIL & A STRONG PROSPEROUS AND THRIVING ECONOMY & $\begin{array}{l}\text { CENTRAL GOVERNMENT LOCAL GOVERNMENT AND INDIVIDUALS WORKING MORE } \\
\text { EFFECTIVELY TO CREATE CONDITIONS THAT PROMOTE ECONOMIC GROWTH }\end{array}$ \\
\hline 855 & WAIROA DISTRICT COUNCIL & $\begin{array}{l}\text { AN ENVIRONMENT THAT IS APPRECIATED } \\
\text { PROTECTED AND SUSTAINED FOR FUTURE } \\
\text { GENERATIONS }\end{array}$ & $\begin{array}{l}\text { COMMUNITIES RESPECTING THE IMPORTANCE OF OUR NATURAL ENVIRONMENT AND } \\
\text { WORKING TOWARDS SUSTAINING THIS FOR THE WELLBEING OF FUTURE GENERATIONS }\end{array}$ \\
\hline 856 & WAIROA DISTRICT COUNCIL & SAFE AND ACCESSIBLE RECREATIONAL FACILITIES & $\begin{array}{l}\text { A COORDINATED APPROACH WHERE ALL PEOPLE ARE ABLE TO ACCESS SAFE } \\
\text { RECREATIONAL FACILITIES }\end{array}$ \\
\hline 857 & WAIROA DISTRICT COUNCIL & $\begin{array}{l}\text { STRONG DISTRICT LEADERSHIP AND A SENSE OF } \\
\text { BELONGING }\end{array}$ & $\begin{array}{l}\text { COORDINATED LEADERSHIP TO ACHIEVE ECONOMIC SOCIAL CULTURAL AND } \\
\text { ENVIRONMENTAL WELLBEING OF OUR COMMUNITIES A DEMOCRATIC ENVIRONMENT } \\
\text { WHERE ALL PEOPLE ARE ABLE TO PARTICIPATE IN THE LIFE OF THEIR COMMUNITIES AND } \\
\text { ACHIEVE A SENSE OF BELONGING }\end{array}$ \\
\hline 858 & WAIROA DISTRICT COUNCIL & SUPPORTIVE CARING AND VALUED COMMUNITIES & $\begin{array}{l}\text { CARING COMMUNITIES WHERE PEOPLE FEEL RESPECTED AND VALUED AS IMPORTANT } \\
\text { MEMBERS OF OUR DISTRICT THEY ARE ABLE TO ACCESS RESOURCES TO REACH THEIR } \\
\text { FULL POTENTIAL IN LIFE AND PARTICIPATE TOWARDS ACHIEVING STRONG COMMUNITIES }\end{array}$ \\
\hline 859 & WAITAKI DISTRICT COUNCIL & $\begin{array}{l}\text { WAITAKI HAS A DIVERSE SUSTAINABLE AND } \\
\text { GROWING ECONOMY }\end{array}$ & $\begin{array}{l}\text { OUR CULTURAL AND NATURAL HERITAGE AND LANDSCAPES CONTRIBUTE SIGNIFICANTLY } \\
\text { TO ECONOMIC DIVERSITY AND SUCCESS }\end{array}$ \\
\hline 860 & WAITAKI DISTRICT COUNCIL & $\begin{array}{l}\text { WAITAKI HAS A DIVERSE SUSTAINABLE AND } \\
\text { GROWING ECONOMY }\end{array}$ & $\begin{array}{l}\text { OUR ECONOMIC DEVELOPMENT IS IN BALANCE WITH SOCIAL CULTURAL } \\
\text { ENVIRONMENTAL AND MANA WHENUA ASPIRATIONS }\end{array}$ \\
\hline 861 & WAITAKI DISTRICT COUNCIL & $\begin{array}{l}\text { WAITAKI HAS A DIVERSE SUSTAINABLE AND } \\
\text { GROWING ECONOMY }\end{array}$ & OUR INFRASTRUCTURE ENABLES AND RESPONDS TO ECONOMIC GROWTH \\
\hline 862 & WAITAKI DISTRICT COUNCIL & $\begin{array}{l}\text { WAITAKI HAS A DIVERSE SUSTAINABLE AND } \\
\text { GROWING ECONOMY }\end{array}$ & $\begin{array}{l}\text { SUSTAINABLE WELLPAID EMPLOYMENT PROVIDES WHANAU WITH A GREATER DEGREE OF } \\
\text { SELFDETERMINATION }\end{array}$ \\
\hline 863 & WAITAKI DISTRICT COUNCIL & $\begin{array}{l}\text { WAITAKI HAS A DIVERSE SUSTAINABLE AND } \\
\text { GROWING ECONOMY }\end{array}$ & WE HAVE LOW COMPLIANCE COSTS FOR BUSINESSES \\
\hline 864 & WAITAKI DISTRICT COUNCIL & $\begin{array}{l}\text { WAITAKI HAS A DIVERSE SUSTAINABLE AND } \\
\text { GROWING ECONOMY }\end{array}$ & $\begin{array}{l}\text { WE PROVIDE SUPPORT AND ADVICE FOR NEW AND EXISTING ENTERPRISES ESPECIALLY } \\
\text { SMALL AND MEDIUMSIZED BUSINESSES }\end{array}$ \\
\hline 865 & WAITAKI DISTRICT COUNCIL & $\begin{array}{l}\text { WAITAKI HAS ESSENTIAL TRANSPORT NETWORKS } \\
\text { AND SERVICES }\end{array}$ & REGULAR AIR SERVICES ARE AVAILABLE AT OAMARU AIRPORT \\
\hline 866 & WAITAKI DISTRICT COUNCIL & $\begin{array}{l}\text { WAITAKI HAS ESSENTIAL TRANSPORT NETWORKS } \\
\text { AND SERVICES }\end{array}$ & WE HAVE A SAFE AND AFFORDABLE ROADING NETWORK \\
\hline 867 & WAITAKI DISTRICT COUNCIL & $\begin{array}{l}\text { WAITAKI HAS ESSENTIAL TRANSPORT NETWORKS } \\
\text { AND SERVICES }\end{array}$ & $\begin{array}{l}\text { WE HAVE AFFORDABLE RELIABLE AND EFFECTIVE TRANSPORT SERVICES THAT MEET THE } \\
\text { NEEDS OF THE COMMUNITY }\end{array}$ \\
\hline 868 & WAITAKI DISTRICT COUNCIL & $\begin{array}{l}\text { WAITAKI HAS ESSENTIAL TRANSPORT NETWORKS } \\
\text { AND SERVICES }\end{array}$ & $\begin{array}{l}\text { WE RESPECT CULTURAL HERITAGE AND LANDSCAPE VALUES IN OUR CONSTRUCTION AND } \\
\text { MAINTENANCE OF TRANSPORT NETWORKS }\end{array}$ \\
\hline 869 & WAITAKI DISTRICT COUNCIL & WAITAKI HAS STRONG AND PROUD COMMUNITIES & NEW RESIDENTS ARE ATTRACTED AND WELCOMED TO THE DISTRICT \\
\hline
\end{tabular}


Appendix B: Community Well-being Statements by Councils

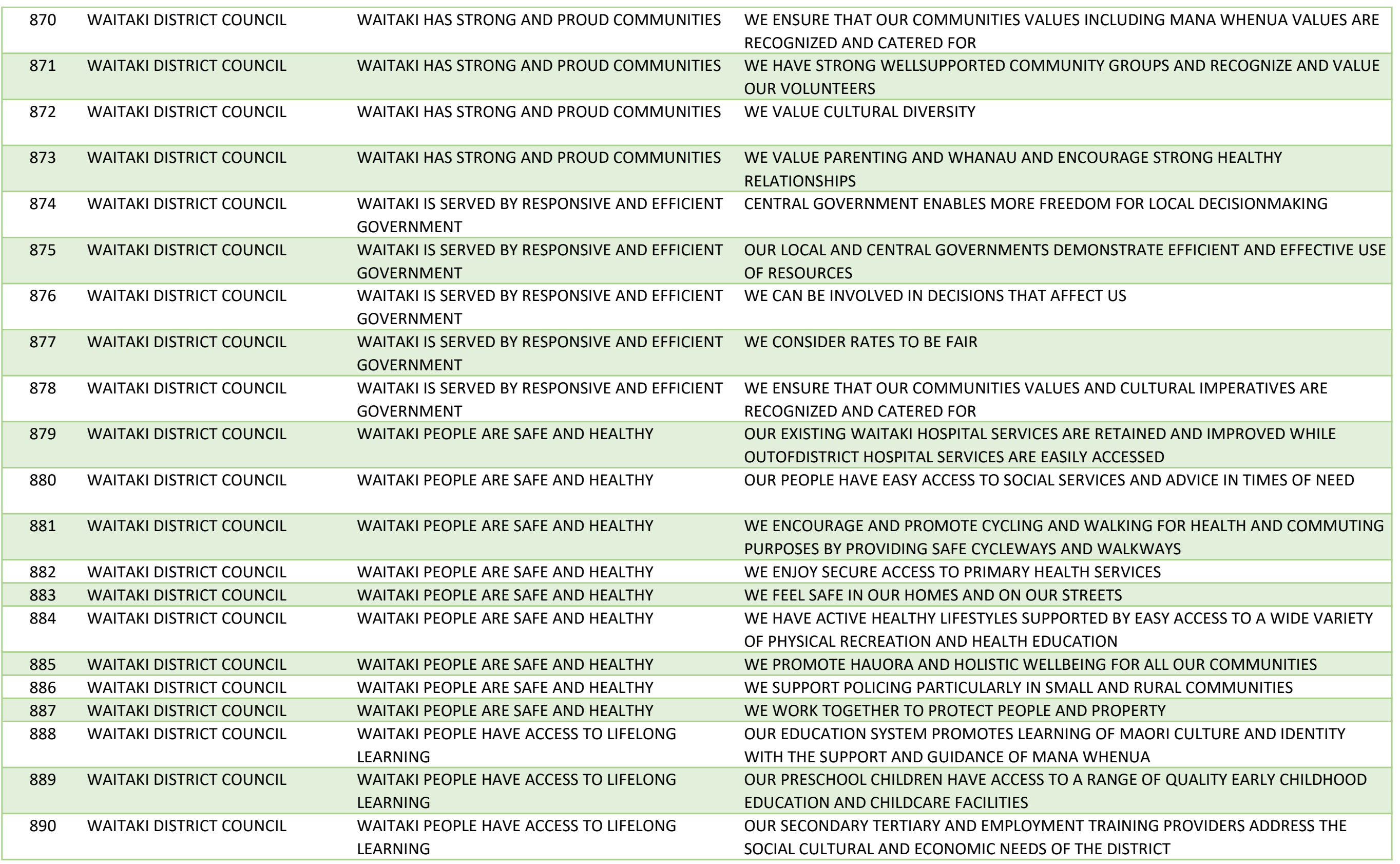


Appendix B: Community Well-being Statements by Councils

\begin{tabular}{|c|c|c|c|}
\hline 891 & WAITAKI DISTRICT COUNCIL & $\begin{array}{l}\text { WAITAKI PEOPLE HAVE ACCESS TO LIFELONG } \\
\text { LEARNING }\end{array}$ & OUR SMALL AND RURAL COMMUNITIES CAN ACCESS INTERACTIVE DISTANCE LEARNING \\
\hline 892 & WAITAKI DISTRICT COUNCIL & $\begin{array}{l}\text { WAITAKI PEOPLE HAVE ACCESS TO LIFELONG } \\
\text { LEARNING }\end{array}$ & SCHOOLS REMAIN AN INTEGRAL PART OF OUR COMMUNITIES \\
\hline 893 & WAITAKI DISTRICT COUNCIL & $\begin{array}{l}\text { WAITAKI PEOPLE HAVE ACCESS TO LIFELONG } \\
\text { LEARNING }\end{array}$ & $\begin{array}{l}\text { WE RETAIN A NETWORK OF QUALITY PRIMARY AND SECONDARY SCHOOLS ENABLING } \\
\text { ACCESS FOR CHILDREN WITHIN REASONABLE TRAVELLING DISTANCES }\end{array}$ \\
\hline 894 & WAITAKI DISTRICT COUNCIL & $\begin{array}{l}\text { WAITAKI RIVERS AND WATER ARE MANAGED FOR } \\
\text { THE FUTURE }\end{array}$ & WE HAVE QUALITY DRINKING WATER \\
\hline 895 & WAITAKI DISTRICT COUNCIL & $\begin{array}{l}\text { WAITAKI RIVERS AND WATER ARE MANAGED FOR } \\
\text { THE FUTURE }\end{array}$ & WE HAVE RELIABLE WATER SUPPLY FOR HOMES FARMS AND INDUSTRIES \\
\hline 896 & WAITAKI DISTRICT COUNCIL & $\begin{array}{l}\text { WAITAKI RIVERS AND WATER ARE MANAGED FOR } \\
\text { THE FUTURE }\end{array}$ & $\begin{array}{l}\text { WE PROTECT OUR WATER SUPPLIES AND WATER BODIES FROM POLLUTION AND } \\
\text { CONTAMINATION }\end{array}$ \\
\hline 897 & WAITAKI DISTRICT COUNCIL & $\begin{array}{l}\text { WAITAKI RIVERS AND WATER ARE MANAGED FOR } \\
\text { THE FUTURE }\end{array}$ & $\begin{array}{l}\text { WE RECOGNIZE MANA WHENUA VALUES ASSOCIATED WITH OUR WATERWAYS } \\
\text { INCLUDING RESPECTING THE MAURI OF WATERWAYS AND ENSURING ACCESS TO } \\
\text { MAHIKA KAI }\end{array}$ \\
\hline 898 & WAITAKI DISTRICT COUNCIL & $\begin{array}{l}\text { WAITAKI RIVERS AND WATER ARE MANAGED FOR } \\
\text { THE FUTURE }\end{array}$ & $\begin{array}{l}\text { WE RECOGNIZE THE SOCIAL AND RECREATIONAL VALUES ASSOCIATED WITH OUR } \\
\text { WATERWAYS }\end{array}$ \\
\hline 899 & WAITAKI DISTRICT COUNCIL & $\begin{array}{l}\text { WAITAKI RIVERS AND WATER ARE MANAGED FOR } \\
\text { THE FUTURE }\end{array}$ & WORKABLE PLANS FOR WAITAKI CATCHMENTS ARE IN PLACE \\
\hline 900 & WAITAKI DISTRICT COUNCIL & $\begin{array}{l}\text { WAITAKIS DISTINCTIVE NATURAL ENVIRONMENT IS } \\
\text { VALUED AND PROTECTED }\end{array}$ & $\begin{array}{l}\text { ALL LAND USE IS MANAGED SUSTAINABLY AND SENSITIVELY WITH PARTICULAR } \\
\text { ATTENTION TO COASTAL AND OTHER AREAS OF ENVIRONMENTAL AND CULTURAL } \\
\text { SIGNIFICANCE }\end{array}$ \\
\hline 901 & WAITAKI DISTRICT COUNCIL & $\begin{array}{l}\text { WAITAKIS DISTINCTIVE NATURAL ENVIRONMENT IS } \\
\text { VALUED AND PROTECTED }\end{array}$ & $\begin{array}{l}\text { WE ENJOY ACCESS TO OUR BEACHES LAKES AND RIVERS WHILE RESPECTING AND } \\
\text { UPHOLDING THE RIGHTS OF PROPERTY OWNERS }\end{array}$ \\
\hline 902 & WAITAKI DISTRICT COUNCIL & $\begin{array}{l}\text { WAITAKIS DISTINCTIVE NATURAL ENVIRONMENT IS } \\
\text { VALUED AND PROTECTED }\end{array}$ & $\begin{array}{l}\text { WE MAINTAIN THE INTEGRITY OF OUR NATURAL AND CULTURAL LANDSCAPES } \\
\text { INCLUDING WAAHI TAPU AND WAAHI TAOKA }\end{array}$ \\
\hline 903 & WAITAKI DISTRICT COUNCIL & $\begin{array}{l}\text { WAITAKIS DISTINCTIVE NATURAL ENVIRONMENT IS } \\
\text { VALUED AND PROTECTED }\end{array}$ & WE MANAGE OUR WASTE STREAM TO MINIMIZE RESIDUAL WASTE TO LANDFILL \\
\hline 904 & WAITAKI DISTRICT COUNCIL & $\begin{array}{l}\text { WAITAKIS UNIQUE CULTURE AND HERITAGE ARE } \\
\text { PRESERVED AND CELEBRATED }\end{array}$ & $\begin{array}{l}\text { A RANGE OF EASILY ACCESSED FACILITIES AND EVENTS ENABLE PARTICIPATION AND } \\
\text { ENJOYMENT OF SPORTING AND CULTURAL ACTIVITY BY ALL MEMBERS OF THE } \\
\text { COMMUNITY }\end{array}$ \\
\hline 905 & WAITAKI DISTRICT COUNCIL & $\begin{array}{l}\text { WAITAKIS UNIQUE CULTURE AND HERITAGE ARE } \\
\text { PRESERVED AND CELEBRATED }\end{array}$ & $\begin{array}{l}\text { ALL OUR COMMUNITIES SHARE THEIR CULTURE AND HERITAGE WITH GROWING } \\
\text { NUMBERS OF VISITORS }\end{array}$ \\
\hline 906 & WAITAKI DISTRICT COUNCIL & $\begin{array}{l}\text { WAITAKIS UNIQUE CULTURE AND HERITAGE ARE } \\
\text { PRESERVED AND CELEBRATED }\end{array}$ & $\begin{array}{l}\text { OUR DEVELOPMENT IN LOWER THAMES STREET AND THE OAMARU HARBOURSIDE AREA } \\
\text { PROVIDES AN ATTRACTIVE PEDESTRIANFRIENDLY DESTINATION THAT IS SYMPATHETIC TO } \\
\text { THE BUILT HERITAGE }\end{array}$ \\
\hline 907 & WAITAKI DISTRICT COUNCIL & $\begin{array}{l}\text { WAITAKIS UNIQUE CULTURE AND HERITAGE ARE } \\
\text { PRESERVED AND CELEBRATED }\end{array}$ & WE CELEBRATE THE UNIQUE HERITAGE AND CULTURE OF THE MANA WHENUA \\
\hline
\end{tabular}


Appendix B: Community Well-being Statements by Councils

\begin{tabular}{|c|c|c|c|}
\hline 908 & WAITOMO DISTRICT COUNCIL & COASTLINE PARKS AND RESERVES & $\begin{array}{l}\text { TO PRESERVE THE NATURAL CHARACTER OF THE DISTRICTS COASTLINE AND RESERVES } \\
\text { ENSURING PUBLIC ACCESS TO THE COASTLINE AND RESERVES }\end{array}$ \\
\hline 909 & WAITOMO DISTRICT COUNCIL & COMMUNICATIONS AND INFORMATION & $\begin{array}{l}\text { THE DISTRICT HAS A COMMUNICATION NETWORK EQUIVALENT TO THAT OF MAIN } \\
\text { URBAN CENTRES AND A COMMUNITY THAT IS CONVERSANT IN THE USE OF MODERN } \\
\text { COMMUNICATION TECHNOLOGY }\end{array}$ \\
\hline 910 & WAITOMO DISTRICT COUNCIL & CULTURAL HERITAGE AND SOCIAL HARMONY & $\begin{array}{l}\text { A DISTRICT THAT IS ENRICHED BY THE VALUES OF ALL ITS PEOPLE AND IN PARTICULAR } \\
\text { MAORI HERITAGE CULTURE BELIEFS AND WAY OF LIFE ARE AN INHERENT AND VALUED } \\
\text { PART OF COMMUNITY LIFE }\end{array}$ \\
\hline 911 & WAITOMO DISTRICT COUNCIL & EDUCATION & $\begin{array}{l}\text { TO BE A COMMUNITY WHERE THE LEVEL OF EDUCATION OF ALL RESIDENTS IS HIGH } \\
\text { ENOUGH TO TAKE ADVANTAGE OF OPPORTUNITIES THAT IMPROVE THE QUALITY OF LIFE } \\
\text { FOR THE COMMUNITY }\end{array}$ \\
\hline 912 & WAITOMO DISTRICT COUNCIL & EMPLOYMENT & $\begin{array}{l}\text { COLLABORATIVELY WORK TOWARDS CREATING A WIDE RANGE AND NUMBER OF } \\
\text { EMPLOYMENT OPPORTUNITIES IN THE REGION }\end{array}$ \\
\hline 913 & WAITOMO DISTRICT COUNCIL & ENERGY & $\begin{array}{l}\text { MINIMISE ENERGY CONSUMPTION THROUGH EFFICIENT USE AND INVESTMENT IN } \\
\text { ENERGY DEVELOPMENTCAPACITY IS CARRIED OUT IN A MANNER CONSISTENT WITH A } \\
\text { SUSTAINABLE ENVIRONMENT }\end{array}$ \\
\hline 914 & WAITOMO DISTRICT COUNCIL & GOVERNMENT SERVICES & $\begin{array}{l}\text { TO ENSURE THE COMMUNITY HAS APPROPRIATE AND TIMELY ACCESS TO SERVICES } \\
\text { PROVIDED BY CENTRAL GOVERNMENT }\end{array}$ \\
\hline 915 & WAITOMO DISTRICT COUNCIL & HEALTH & $\begin{array}{l}\text { TO PURSUE PERSONAL SOCIAL AND CULTURAL WELLBEING SUPPORTED BY LOCALLY } \\
\text { AVAILABLE HEALTH SERVICES }\end{array}$ \\
\hline 916 & WAITOMO DISTRICT COUNCIL & HOUSING AND ACCOMMODATION & $\begin{array}{l}\text { AVAILABILITY OF CHOICE IN HOUSING AND VISITOR ACCOMMODATION TO MEET THE } \\
\text { NEEDS OF ALL PEOPLE WHO WISH TO RESIDE OR VISIT THE DISTRICT IN A WAY THAT } \\
\text { CONTRIBUTES TO THE DISTRICT APPEAL AND IMAGE }\end{array}$ \\
\hline 917 & WAITOMO DISTRICT COUNCIL & IMAGE & $\begin{array}{l}\text { WORK TOWARDS CREATING A READILY PROSPEROUS IDENTIFIABLE AND ATTRACTIVE } \\
\text { IMAGE TO THE WORLD OUTSIDE WAITOMO BASED ON A STRONG INTERNAL SENSE OF } \\
\text { PRIDE SELFIMAGE AND SELFESTEEM }\end{array}$ \\
\hline 918 & WAITOMO DISTRICT COUNCIL & LAND USE FARMING FORESTRY AND MINING & $\begin{array}{l}\text { PLANNED INTENSIFICATION AND DIVERSITY OF USE TO ACHIEVE THE BEST ECONOMIC } \\
\text { BENEFIT OF LAND IN PRODUCTIVE USE }\end{array}$ \\
\hline 919 & WAITOMO DISTRICT COUNCIL & $\begin{array}{l}\text { MANUFACTURING PROCESS AND SERVICE } \\
\text { INDUSTRIES }\end{array}$ & $\begin{array}{l}\text { A DISTRICT THAT HAS PRIMARY SECTOR VALUEADDED BUSINESSES AND AN } \\
\text { INFRASTRUCTURE OF COMMERCE THAT COMPETES IN THE GLOBAL MARKET AND } \\
\text { PROVIDES LOCAL JOBS AND LOCAL WEALTH }\end{array}$ \\
\hline 920 & WAITOMO DISTRICT COUNCIL & PRESERVING THE ENVIRONMENT & $\begin{array}{l}\text { PRESERVE THE NATURAL ENVIRONMENT FOR FUTURE GENERATIONS ENSURING THAT } \\
\text { NATURAL RESOURCES ARE USED IN A SUSTAINABLE MANNER }\end{array}$ \\
\hline 921 & WAITOMO DISTRICT COUNCIL & RECREATION AND SOCIAL AMENITIES & $\begin{array}{l}\text { A DISTRICT WHERE THE COMMUNITY IS ABLE TO ENJOY SOCIAL CULTURAL AND SPIRITUAL } \\
\text { WELLBEING THROUGH COMMUNITY AMENITIES FACILITIES RECREATION SPORTS AND } \\
\text { ARTS ACTIVITIES THAT PROVIDE FOR A FULL QUALITY OF LIFE AND CHOICE OF } \\
\text { ENJOYMENT FOR ALL RESIDENTS }\end{array}$ \\
\hline
\end{tabular}


Appendix B: Community Well-being Statements by Councils

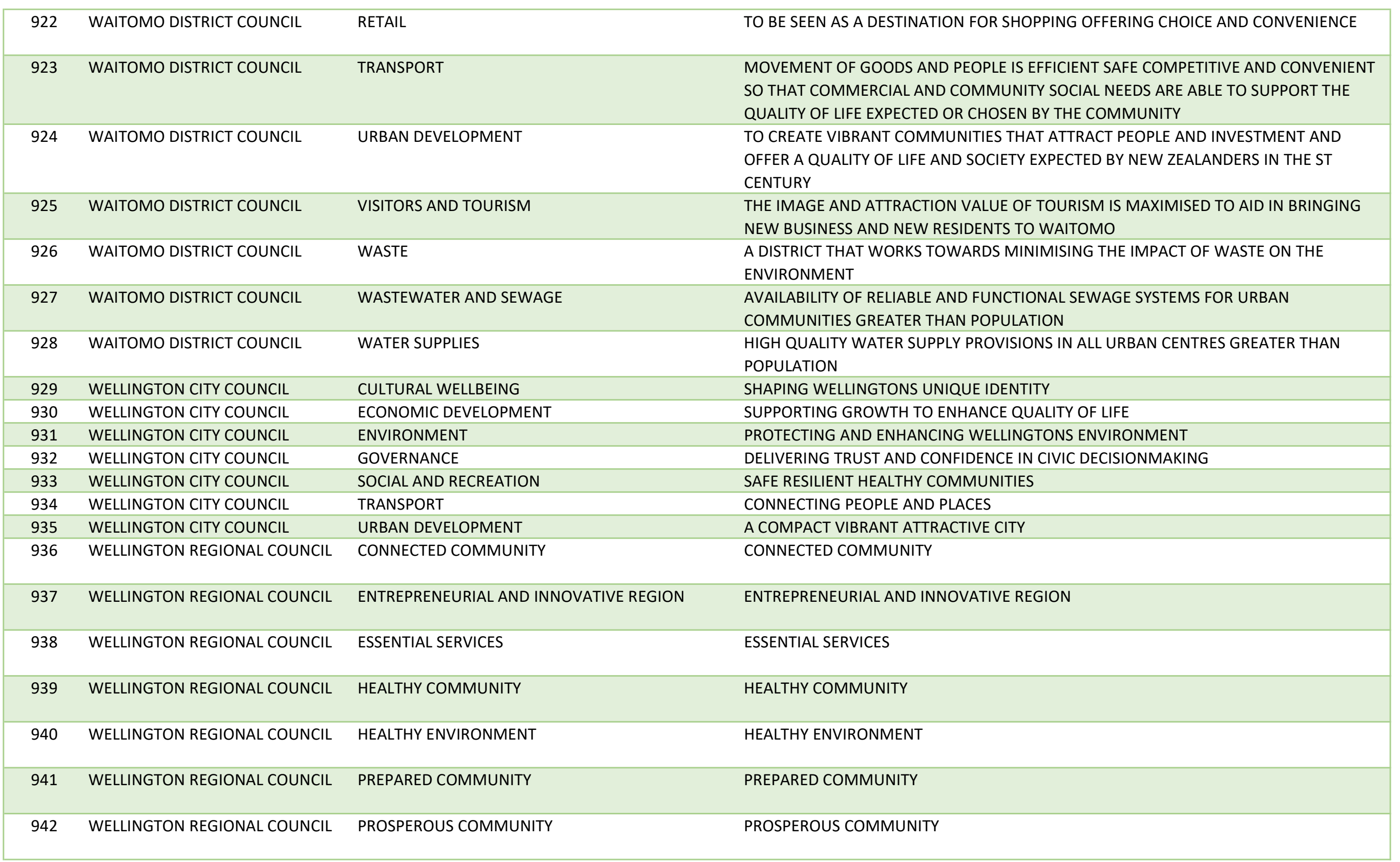


Appendix B: Community Well-being Statements by Councils

\begin{tabular}{|c|c|c|c|}
\hline 943 & WELLINGTON REGIONAL COUNCIL & QUALITY LIFESTYLE & QUALITY LIFESTYLE \\
\hline 944 & WELLINGTON REGIONAL COUNCIL & SENSE OF PLACE & SENSE OF PLACE \\
\hline 945 & WELLINGTON REGIONAL COUNCIL & STRONG AND TOLERANT COMMUNITY & STRONG AND TOLERANT COMMUNITY \\
\hline 946 & WEST COAST REGIONAL COUNCIL & ECONOMY OUTCOME & $\begin{array}{l}\text { A THRIVING RESILIENT AND INNOVATIVE ECONOMY CREATING OPPORTUNITIES FOR } \\
\text { GROWTH AND EMPLOYMENT }\end{array}$ \\
\hline 947 & WEST COAST REGIONAL COUNCIL & EDUCATION OUTCOME & $\begin{array}{l}\text { A REGION THAT VALUES AND SUPPORTS LEARNING WITH ACCESSIBLE RELEVANT } \\
\text { EDUCATION AND TRAINING OPPORTUNITIES }\end{array}$ \\
\hline 948 & WEST COAST REGIONAL COUNCIL & ENVIRONMENT OUTCOME & THE DISTINCTIVE CHARACTER OF THE ENVIRONMENT IS APPRECIATED AND RETAINED \\
\hline 949 & WEST COAST REGIONAL COUNCIL & HEALTH OUTCOME & HEALTHY COMMUNITY WITH ACCESS TO QUALITY FACILITIES AND SERVICES \\
\hline 950 & WEST COAST REGIONAL COUNCIL & IDENTITY OUTCOME & A HAPPENING REGION WITH A STRONG COMMUNITY SPIRIT AND DISTINCTIVE LIFESTYLE \\
\hline 951 & WEST COAST REGIONAL COUNCIL & SAFETY OUTCOME & A REGION THAT IS A SAFE PLACE TO LIVE \\
\hline 952 & WESTERN BAY OF PLENTY DISTRICT & LEADERS ARE EFFECTIVE INFORMED AND INCLUSIVE & $\begin{array}{l}\text { WE ARE REPRESENTED BY RESPONSIBLE AND MOTIVATED PEOPLE WHO SUPPORT OUR } \\
\text { PARTICIPATION IN DECISIONMAKING }\end{array}$ \\
\hline 953 & WESTERN BAY OF PLENTY DISTRICT & OUR COMMUNITIES ARE VIBRANT AND WELCOMING & $\begin{array}{l}\text { OUR COMMUNITIES CELEBRATE DIFFERENCES AND STRENGTHS WE HAVE PRIDE IN OUR } \\
\text { COMMUNITIES }\end{array}$ \\
\hline 954 & WESTERN BAY OF PLENTY DISTRICT & OUR ECONOMY IS THRIVING & $\begin{array}{l}\text { A DIVERSE PRODUCTIVE AND COMPETITIVE LOCAL ECONOMY SUPPORTS LONGTERM } \\
\text { SUSTAINABLE GROWTH }\end{array}$ \\
\hline 955 & WESTERN BAY OF PLENTY DISTRICT & OUR ENVIRONMENT IS CLEAN GREEN AND VALUED & $\begin{array}{l}\text { THE NATURAL ENVIRONMENT IS VALUED PROTECTED AND SUSTAINED FOR FUTURE } \\
\text { GENERATIONS }\end{array}$ \\
\hline 956 & WESTERN BAY OF PLENTY DISTRICT & WE CAN ALL ENJOY A HEALTHY AND SAFE LIFESTYLE & $\begin{array}{l}\text { WE ALL HAVE THE OPPORTUNITY TO BE HEALTHY AND SAFE AND HAVE ACCESS TO } \\
\text { SERVICES AND FACILITIES THAT SUPPORT THIS LIFESTYLE CHOICE }\end{array}$ \\
\hline 957 & WESTLAND DISTRICT COUNCIL & ECONOMY & $\begin{array}{l}\text { A THRIVING RESILIENT AND INNOVATIVE ECONOMY CREATING OPPORTUNITIES FOR } \\
\text { GROWTH AND EMPLOYMENT }\end{array}$ \\
\hline 958 & WESTLAND DISTRICT COUNCIL & EDUCATION & $\begin{array}{l}\text { A DISTRICT THAT VALUES AND SUPPORTS LEARNING WITH ACCESSIBLE RELEVANT } \\
\text { EDUCATION AND TRAINING OPPORTUNITIES }\end{array}$ \\
\hline 959 & WESTLAND DISTRICT COUNCIL & ENVIRONMENT & THE DISTINCTIVE CHARACTER OF THE ENVIRONMENT APPRECIATED AND RETAINED \\
\hline 960 & WESTLAND DISTRICT COUNCIL & HEALTH & HEALTHY COMMUNITIES WITH ACCESS TO QUALITY FACILITIES AND SERVICES \\
\hline 961 & WESTLAND DISTRICT COUNCIL & IDENTITY & A HAPPENING REGION WITH A STRONG COMMUNITY SPIRIT AND DISTINCTIVE LIFESTYLE \\
\hline 962 & WESTLAND DISTRICT COUNCIL & SAFETY & A DISTRICT THAT IS A SAFE PLACE TO LIVE \\
\hline
\end{tabular}


Appendix B: Community Well-being Statements by Councils

\begin{tabular}{|c|c|c|c|}
\hline 963 & WHAKATANE DISTRICT COUNCIL & CLEAN PROTECTED ENVIRONMENT & CLEAN PROTECTED ENVIRONMENT \\
\hline 964 & WHAKATANE DISTRICT COUNCIL & DIVERSE CREATIVE AND ACTIVE COMMUNITY & DIVERSE CREATIVE AND ACTIVE COMMUNITY \\
\hline 965 & WHAKATANE DISTRICT COUNCIL & EDUCATION AND TRAINING OPPORTUNITIES FOR ALL & EDUCATION AND TRAINING OPPORTUNITIES FOR ALL \\
\hline 966 & WHAKATANE DISTRICT COUNCIL & ENVIRONMENTALLY RESPONSIBLE DEVELOPMENT & ENVIRONMENTALLY RESPONSIBLE DEVELOPMENT \\
\hline 967 & WHAKATANE DISTRICT COUNCIL & HEALTHY PEOPLE AND QUALITY HOUSING & HEALTHY PEOPLE AND QUALITY HOUSING \\
\hline 968 & WHAKATANE DISTRICT COUNCIL & HIGHQUALITY AFFORDABLE INFRASTRUCTURE & HIGHQUALITY AFFORDABLE INFRASTRUCTURE \\
\hline 969 & WHAKATANE DISTRICT COUNCIL & PROSPEROUS ECONOMY & PROSPEROUS ECONOMY \\
\hline 970 & WHAKATANE DISTRICT COUNCIL & SAFE CARING COMMUNITY & SAFE CARING COMMUNITY \\
\hline 971 & WHAKATANE DISTRICT COUNCIL & STRONG TRANSPARENT AND OPEN LEADERSHIP & STRONG TRANSPARENT AND OPEN LEADERSHIP \\
\hline 972 & WHANGANUI DISTRICT COUNCIL & A COMMUNITY WHERE PEOPLE WORK TOGETHER & A COMMUNITY WHERE PEOPLE WORK TOGETHER \\
\hline 973 & WHANGANUI DISTRICT COUNCIL & A COMMUNITY WITH IDENTITY & A COMMUNITY WITH IDENTITY \\
\hline 974 & WHANGANUI DISTRICT COUNCIL & A COMMUNITY WITH QUALITY EDUCATION & A COMMUNITY WITH QUALITY EDUCATION \\
\hline 975 & WHANGANUI DISTRICT COUNCIL & $\begin{array}{l}\text { A COMMUNITY WITH WELL DEVELOPED AMENITIES } \\
\text { AND RECREATIONAL OPPORTUNITIES }\end{array}$ & A COMMUNITY WITH WELL DEVELOPED AMENITIES AND RECREATIONAL OPPORTUNITIES \\
\hline 976 & WHANGANUI DISTRICT COUNCIL & A HEALTHY COMMUNITY & A HEALTHY COMMUNITY \\
\hline 977 & WHANGANUI DISTRICT COUNCIL & A PROSPEROUS COMMUNITY & A PROSPEROUS COMMUNITY \\
\hline 978 & WHANGANUI DISTRICT COUNCIL & A SAFE COMMUNITY & A SAFE COMMUNITY \\
\hline 979 & WHANGANUI DISTRICT COUNCIL & A WELL CONNECTED COMMUNITY & A WELL CONNECTED COMMUNITY \\
\hline 980 & WHANGANUI DISTRICT COUNCIL & AN ENVIRONMENTALLY SUSTAINABLE COMMUNITY & AN ENVIRONMENTALLY SUSTAINABLE COMMUNITY \\
\hline 981 & WHANGAREI DISTRICT COUNCIL & COMMUNITY & A DISTRICT WITH COMMUNITY PROGRAMMES AND FACILITIES FOR ALL \\
\hline 982 & WHANGAREI DISTRICT COUNCIL & CULTURE HERITAGE & A COMMUNITY WHICH VALUES ITS CULTURE AND HERITAGE \\
\hline 983 & WHANGAREI DISTRICT COUNCIL & ECONOMY & A VIBRANT AND GROWING LOCAL ECONOMY \\
\hline 984 & WHANGAREI DISTRICT COUNCIL & ENVIRONMENT & $\begin{array}{l}\text { A SUSTAINABLE ENVIRONMENTALLY RESPONSIBLE DISTRICT WHICH VALUES ITS NATURAL } \\
\text { UNIQUENESS }\end{array}$ \\
\hline 985 & WHANGAREI DISTRICT COUNCIL & HEALTHY EDUCATED & A COMMUNITY WHICH IS HEALTHY AND EDUCATED \\
\hline 986 & WHANGAREI DISTRICT COUNCIL & SAFE & A DISTRICT WHICH IS SAFE AND CRIME FREE \\
\hline
\end{tabular}


Appendix C: Coverage of Community Well-being Measures in Living Standard Framework

\begin{tabular}{|c|c|c|c|c|c|c|c|}
\hline S.NO & HEADING & SUB HEADING & DOMAIN & INDICATOR & UNITS & AVAILABLE IN LTP & ALTERNATE DATA \\
\hline 1 & OUR COUNTRY & INDICATOR OVERVIEW & $\begin{array}{l}\text { CIVIC ENGAGEMENT AND } \\
\text { GOVERNANCE }\end{array}$ & PERCEIVED CORRUPTION & $\begin{array}{l}\text { CORRUPTION PERCEPTION INDEX SCORE ON A SCALE FROM } 0 \text { (HIGH CORRUPT) TO } \\
100 \text { (VERY CLEAN) }\end{array}$ & NO & \\
\hline 2 & OUR COUNTRY & INDICATOR OVERVIEW & $\begin{array}{l}\text { CIVIC ENGAGEMENT AND } \\
\text { GOVERNANCE }\end{array}$ & TRUST IN GOVERNMENT INSTITUTES & PERCENTAGE OF ADULTS WHO, OVERALL, TRUST THE PUBLIC SERVIE & NO & \\
\hline 3 & OUR COUNTRY & INDICATOR OVERVIEW & $\begin{array}{l}\text { CIVIC ENGAGEMENT AND } \\
\text { GOVERNANCE }\end{array}$ & VOTER TURNOUT & PERCENTAGE OF ENROLLED ELECTTORS WHO VOTED IN THE GENERAL ELECTION & YES & \\
\hline 4 & OUR COUNTRY & INDICATOR OVERVIEW & CULTURAL IDENTITY & ABILITY TO EXPRESS IDENTITY & $\begin{array}{l}\text { PERCENTAGE OF ADULTS WHO SAID OT WAS EASY OR VERY EASY TO EXPRESS } \\
\text { THEIR IDENITY IN NEW ZEALAND }\end{array}$ & YES & \\
\hline 5 & OUR COUNTRY & INDICATOR OVERVIEW & CULTURAL IDENTITY & TE REO MAORI SPEAKERS & $\begin{array}{l}\text { PERCENTAGE OF PEOPLE WHO CAN COVERS ABOUT A LOT OF EVERYDAY THINGS } \\
\text { IN TE REO MAORI }\end{array}$ & YES & \\
\hline 6 & OUR COUNTRY & INDICATOR OVERVIEW & ENVIROMENT & ACCESS TO THE NATURAL ENVIROMENT & $\begin{array}{l}\text { PERCENTAGE OF PEOPLE WH SAID IT WAS VER EASY TO GET TO THEIR NEAREST } \\
\text { PARK OR GREEN SPACE }\end{array}$ & YES & \\
\hline 7 & OUR COUNTRY & INDICATOR OVERVIEW & ENVIROMENT & AIR QUALITY & NATIONAL ANNUAL AVERAGE PM10 CONCENTRATION & YES & \\
\hline 8 & OUR COUNTRY & INDICATOR OVERVIEW & ENVIROMENT & PERCEIVED ENVIROMENT QUALITY & $\begin{array}{l}\text { PERCENTAGE OF PEOPLE WHO RATE THE "OVERALL STATE OF THE NATURAL } \\
\text { ENVIROMENT IN NEW ZEALAND" AS VERY GOOD OR GOOD }\end{array}$ & YES & \\
\hline 9 & OUR COUNTRY & INDICATOR OVERVIEW & ENVIROMENT & WATER QUALITY (SWIMMABILITY) & $\begin{array}{l}\text { PERCENTAGE OF TESTED RIVER SITES THAT ARE SAFE } \\
\text { TO SWIM IN UNDER NORMAL CONDITIONS }\end{array}$ & YES & \\
\hline 10 & OUR COUNTRY & INDICATOR OVERVIEW & HEALTH & HEALTH STATUS & PERCENTAGE OF ADULTS REPORTING GOOD OR VERY GOOD HEALTH & YES & \\
\hline 11 & OUR COUNTRY & INDICATOR OVERVIEW & HEALTH & HEALTHY LIFE EXPECTANCY & $\begin{array}{l}\text { NUMBER OF YEARS AN INFANT UNDER } 1 \text { YEAR OLD CAN EXPECT TO LIVE IN GOOD } \\
\text { HEALTH }\end{array}$ & YES & \\
\hline 12 & OUR COUNTRY & INDICATOR OVERVIEW & HEALTH & MENTAL HEALTH & PERCENTAGE OF ADULTS WITH HIGH LEVELS OF PSYCHOLOGICAL DISTRESS & YES & \\
\hline 13 & OUR COUNTRY & INDICATOR OVERVIEW & HEALTH & SUICIDE RATE & $\begin{array}{l}\text { DEATHS CAUSED BY INTENTIONAL SELF-HARM, AGE-STANDARDISED RATE PER } \\
100,000 \text { PEOPLE }\end{array}$ & YES & \\
\hline 14 & OUR COUNTRY & INDICATOR OVERVIEW & HOUSING & HOUSEHOLD CROWDING & PERCENTAGE OF PEOPLE LIVING IN A CROWDED HOUSE & YES & \\
\hline 15 & OUR COUNTRY & INDICATOR OVERVIEW & HOUSING & HOUSING COST & $\begin{array}{l}\text { PERCENTAGE OF HOUSEHOLDS WITH HOUSING COSTS GREATER THAN } 30 \% \text { OF } \\
\text { INCOME }\end{array}$ & YES & \\
\hline 16 & OUR COUNTRY & INDICATOR OVERVIEW & HOUSING & HOUSING QUALITY & PERCENTAGE OF PEOPLE REPORTING MAJOR REPAIRS NEEDED & YES & \\
\hline 17 & OUR COUNTRY & INDICATOR OVERVIEW & INCOME AND CONSUMPTION & CHILD POVERTY - MATERIAL HARDSHIP & $\begin{array}{l}\text { PERCENTAGE OF CHILDREN LIVING IN HOUSEHOLDS EXPERIENCING MATERIAL } \\
\text { HARDSHIP }\end{array}$ & MAYBE & FAMILY INCOME \\
\hline 18 & OUR COUNTRY & INDICATOR OVERVIEW & INCOME AND CONSUMPTION & CONSUMPTION & AVERAGE REAL WEEKLY HOUSEHOLD EXPENDITURE & YES & \\
\hline 19 & OUR COUNTRY & INDICATOR OVERVIEW & INCOME AND CONSUMPTION & DISPOSABLE INCOME & $\begin{array}{l}\text { MEDIAN REAL EQUIVALISED HOUSEHOLD INCOME AFTER TAXES AND TRANSFERS, } \\
\text { AND BEFORE HOUSING COSTS }\end{array}$ & YES & \\
\hline 20 & OUR COUNTRY & INDICATOR OVERVIEW & INCOME AND CONSUMPTION & FINANCIAL WELLBEING & $\begin{array}{l}\text { PERCENTAGE OF ADULTS WHO REPORT THEY DO NOT HAVE ENOUGH MONEY TO } \\
\text { MEET EVERYDAY NEEDS }\end{array}$ & YES & \\
\hline 21 & OUR COUNTRY & INDICATOR OVERVIEW & INCOME AND CONSUMPTION & HOUSEHOLD NET WORTH & AVERAGE HOUSEHOLD NET WORTH & MAYBE & FAMILY INCOME \\
\hline 22 & OUR COUNTRY & INDICATOR OVERVIEW & JOBS AND EARNINGS & EMPLOYMENT RATE & PERCENTAGE OF ADULTS WHO ARE EMPLOYED & YES & \\
\hline 23 & OUR COUNTRY & INDICATOR OVERVIEW & JOBS AND EARNINGS & HOURLY EARNINGS & MEDIAN HOURLY EARNINGS FOR WAGE AND SALARY EMPLOYEES (AGED 15+) & YES & \\
\hline 24 & OUR COUNTRY & INDICATOR OVERVIEW & JOBS AND EARNINGS & UNEMPLOYMENT RATE & PERCENTAGE OF LABOUR FORCE WHO ARE UNEMPLOYED & YES & \\
\hline 25 & OUR COUNTRY & INDICATOR OVERVIEW & JOBS AND EARNINGS & YOUTH NEET RATE & $\begin{array}{l}\text { PERCENTAGE OF YOUNG PEOPLE AGED } 15-24 \text { YEARS WHO ARE NOT IN } \\
\text { EMPLOYMENT, EDUCATION OR TRAINING (NEET) }\end{array}$ & YES & \\
\hline
\end{tabular}




\begin{tabular}{|c|c|c|c|c|c|c|c|}
\hline S.NO & HEADING & SUB HEADING & DOMAIN & INDICATOR & UNITS & AVAILABLE IN LTP & ALTERNATE DATA \\
\hline 26 & OUR COUNTRY & INDICATOR OVERVIEW & KNOWLEDGE AND SKILLS & COGNITIVE SKILLS AT AGE 15 & $\begin{array}{l}\text { PROGRAMME FOR INTERNATIONAL STUDENT ASSESSMENT (PISA) MEAN SCORE } \\
\text { FOR READING,MATHEMATICS AND SCIENCE }\end{array}$ & MAYBE & $\begin{array}{l}\text { NUMERACY AND } \\
\text { LITERACY CREDITS } \\
\text { AT NCEA LEVEL } 1\end{array}$ \\
\hline 27 & OUR COUNTRY & INDICATOR OVERVIEW & KNOWLEDGE AND SKILLS & $\begin{array}{l}\text { EDUCATIONAL ATTAINMENT OF THE ADULT } \\
\text { POPULATION (TERTIARY) }\end{array}$ & $\begin{array}{l}\text { PERCENTAGE OF ADULTS AGED } 25-64 \text { WITH A BACHELOR'S DEGREE OR HIGHER } \\
\text { QUALIFICATION }\end{array}$ & YES & \\
\hline 28 & OUR COUNTRY & INDICATOR OVERVIEW & KNOWLEDGE AND SKILLS & $\begin{array}{l}\text { EDUCATIONAL ATTAINMENT OF THE ADULT } \\
\text { POPULATION (UPPER SECONDARY) }\end{array}$ & $\begin{array}{l}\text { PERCENTAGE OF ADULTS AGED 25-64 WITH AT LEAST AN UPPER SECONDARY } \\
\text { EDUCATION(EQUIVALENT TO NCEA LEVEL 2) }\end{array}$ & YES & \\
\hline 29 & OUR COUNTRY & INDICATOR OVERVIEW & SAFETY & DOMESTIC VIOLENCE & $\begin{array}{l}\text { PERCENTAGE OF ADULTS WHO WERE VICTIMS OF FAMILY VIOLENCE IN THE PAST } \\
\text { YEAR }\end{array}$ & YES & \\
\hline 30 & OUR COUNTRY & INDICATOR OVERVIEW & SAFETY & FEELING SAFE & $\begin{array}{l}\text { PERCENTAGE OF ADULTS WHO FEEL SAFE WHEN WALKING ALONE IN THEIR } \\
\text { NEIGHBOURHOOD AFTER DARK }\end{array}$ & YES & \\
\hline 31 & OUR COUNTRY & INDICATOR OVERVIEW & SAFETY & INTENTIONAL HOMICIDE RATE & DEATHS CAUSED BY ASSAULT, AGE-STANDARDISED RATES PER 100,000 PEOPLE & MAYBE & CRIME RATE \\
\hline 32 & OUR COUNTRY & INDICATOR OVERVIEW & SAFETY & WORKPLACE ACCIDENT RATE & $\begin{array}{l}\text { NUMBER OF WORK-RELATED INJURY CLAIMS PER1,000 FULL-TIME EQUIVALENT } \\
\text { EMPLOYEES (FTES) }\end{array}$ & YES & \\
\hline 33 & OUR COUNTRY & INDICATOR OVERVIEW & SOCIAL CONNECTIONS & DISCRIMINATION & $\begin{array}{l}\text { PERCENTAGE OF ADULTS HOW EXPERIENCED DISCRIMINATION IN THE PAST } 12 \\
\text { MONTHS IN NEW ZEALAND }\end{array}$ & YES & \\
\hline 34 & OUR COUNTRY & INDICATOR OVERVIEW & SOCIAL CONNECTIONS & LONELINESS & $\begin{array}{l}\text { PERCENTAGE OF ADULTS WHO FELT LONELY AT LEAST SOME OF THE TIME IN THE } \\
\text { LAST FOUR WEEKS }\end{array}$ & MAYBE & MENTAL HEALTH \\
\hline 35 & OUR COUNTRY & INDICATOR OVERVIEW & SOCIAL CONNECTIONS & MĀORI CONNECTION TO MARAE & $\begin{array}{l}\text { PERCENTAGE OF MĀORI ADULTS WHO FEEL STRONGLY CONNECTED WITH THEIR } \\
\text { ANCESTRAL MARAE }\end{array}$ & MAYBE & $\begin{array}{l}\text { VOLUNTEER WORK } \\
\text { IN MARAE }\end{array}$ \\
\hline 36 & OUR COUNTRY & INDICATOR OVERVIEW & SOCIAL CONNECTIONS & SOCIAL NETWORK SUPPORT & $\begin{array}{l}\text { PERCENTAGE OF ADULTS WHO HAD FACE TO FACE CONTACT WITH FRIENDS WHO } \\
\text { DO NOT LIVE WITH THEM AT LEAST ONCE A WEEK }\end{array}$ & MAYBE & $\begin{array}{l}\text { SOCIAL } \\
\text { NETWORKING }\end{array}$ \\
\hline 37 & OUR COUNTRY & INDICATOR OVERVIEW & SUBJECTIVE WELLBEING & FAMILY WELLBEING & $\begin{array}{l}\text { PERCENTAGE OF PEOPLE WITH A SCORE OF } 7 / 10 \text { OR HIGHER FOR FAMILY } \\
\text { WELLBEING }\end{array}$ & MAYBE & $\begin{array}{l}\text { GREAT PLACE TO } \\
\text { LIVE }\end{array}$ \\
\hline 38 & OUR COUNTRY & INDICATOR OVERVIEW & SUBJECTIVE WELLBEING & GENERAL LIFE SATISFACTION & $\begin{array}{l}\text { PERCENTAGE OF PEOPLE WITH A SCORE OF } 7 / 10 \text { OR HIGHER FOR LIFE } \\
\text { SATISFACTION }\end{array}$ & MAYBE & $\begin{array}{l}\text { GREAT PLACE TO } \\
\text { LIVE }\end{array}$ \\
\hline 39 & OUR COUNTRY & INDICATOR OVERVIEW & SUBJECTIVE WELLBEING & SENSE OF PURPOSE IN ONE'S LIFE & $\begin{array}{l}\text { PERCENTAGE OF PEOPLE WITH A SCORE OF } 7 / 10 \text { OR HIGHER FOR FEELING THAT } \\
\text { LIFE IS WORTHWHILE }\end{array}$ & MAYBE & MENTAL HEALTH \\
\hline 40 & OUR COUNTRY & INDICATOR OVERVIEW & TIME USE & LEISURE AND PERSONAL CARE & $\begin{array}{l}\text { AVERAGE HOURS PER DAY DEVOTED TO FREE TIME AND PERSONAL CARE (E.G. } \\
\text { SLEEPING, EATING,PERSONAL HYGIENE) BY PEOPLE AGED } 12 \text { AND } \\
\text { OVER }\end{array}$ & YES & \\
\hline 41 & OUR COUNTRY & INDICATOR OVERVIEW & TIME USE & PAID WORK & AVERAGE ACTUAL WEEKLY HOURS WORKED BY EMPLOYED ADULTS & YES & \\
\hline 42 & OUR COUNTRY & INDICATOR OVERVIEW & TIME USE & SATISFACTION WITH WORK-LIFE BALANCE & $\begin{array}{l}\text { PERCENTAGE OF PEOPLE WHO ARE "VERYSATISFIED" OR "SATISFIED WITH THEIR } \\
\text { WORK-LIFE BALANCE }\end{array}$ & YES & \\
\hline 43 & OUR COUNTRY & INDICATOR OVERVIEW & TIME USE & UNPAID WORK & $\begin{array}{l}\text { AVERAGE HOURS PER DAY SPENT DOING UNPAID WORK (FOR OWN HOUSEHOLD, } \\
\text { OTHER HOUSEHOLD OR AN ORGANISATION) }\end{array}$ & YES & \\
\hline 44 & OUR FUTURE & NATURAL CAPITAL & NATURAL CAPITAL & BIODIVERSITY AND GENETIC RESUORCES & $\begin{array}{l}\text { PERCENTAGE OF ASSESSED INDIGENOUS SPECIES CLASSFIED AS THERATENED WITH } \\
\text { OR AT RISK OF EXTINCTION }\end{array}$ & MAYBE & PEST CONTROL \\
\hline 45 & OUR FUTURE & NATURAL CAPITAL & NATURAL CAPITAL & CLIMATE REGULATION & CARBON STORED IN FOREST BIOMASS & NO & \\
\hline 46 & OUR FUTURE & NATURAL CAPITAL & NATURAL CAPITAL & DRINKING WATER & $\begin{array}{l}\text { PERCERNTAGE OF PEOPLE SERVED WITH DRINKING WATER THAT MET ALL } \\
\text { STANDARDS }\end{array}$ & YES & \\
\hline 47 & OUR FUTURE & NATURAL CAPITAL & NATURAL CAPITAL & NET GREEN HOUSE GAS EMISSIONS & NET GREENHOUSE GAS EMISSIONS IN KILOTONNES OF CO2 EQUIVALENT & YES & \\
\hline 48 & OUR FUTURE & NATURAL CAPITAL & NATURAL CAPITAL & RENEWABLE ENERGY & RENEWABLE ENERGY AS A PERCENT OF TOTAL PRIMARY ENERGY SUPPLY & MAYBE & ENERGY USAGE \\
\hline 49 & OUR FUTURE & NATURAL CAPITAL & NATURAL CAPITAL & SUSTAINABLE FOOD PRODUCTION & $\begin{array}{l}\text { PERCENTAGE OF TESTED SITES WITHIN TARGETS FOR AT LEAST SIX OF THE SEVEN } \\
\text { TYPES OF SOIL TEST }\end{array}$ & MAYBE & SOIL QUALITY \\
\hline 50 & OUR FUTURE & NATURAL CAPITAL & NATURAL CAPITAL & WASTE MANAGEMENT & KILOGRAMS OF WASTE PER CAPITA & YES & \\
\hline
\end{tabular}




\begin{tabular}{|c|c|c|c|c|c|c|c|}
\hline S.NO & HEADING & SUB HEADING & DOMAIN & INDICATOR & UNITS & AVAILABLE IN LTP & ALTERNATE DATA \\
\hline 51 & OUR FUTURE & SOCIAL CAPITAL & SOCIAL CAPITAL & DISCRIMINATION & $\begin{array}{l}\text { PERCENTAGE OF ADULTS WHO EXPERIENCED DISCRIMINATION IN THE PAST } 12 \\
\text { MONTHS IN NEW ZEALAND }\end{array}$ & YES & \\
\hline 52 & OUR FUTURE & SOCIAL CAPITAL & SOCIAL CAPITAL & PERCEIVED CORRUPTION & $\begin{array}{l}\text { CORRUPTION PERCEPTION INDEX SCORE ON A SCALE FROM O (HIGH CORRUPT) TO } \\
100 \text { (VERY CLEAN) }\end{array}$ & NO & \\
\hline 53 & OUR FUTURE & SOCIAL CAPITAL & SOCIAL CAPITAL & SENSE OF BELONGING & $\begin{array}{l}\text { PERCENTAGE OF PEOPLE WITH A SCORE OF 7/10 OR HIGHER FOR SENSE OF } \\
\text { BELONGING TO NZ }\end{array}$ & YES & \\
\hline 54 & OUR FUTURE & SOCIAL CAPITAL & SOCIAL CAPITAL & TRUST HELD IN OTHERS & $\begin{array}{l}\text { PERCENTAGE OF PEOPLE WITH A SCORE OF } 7 / 10 \text { OR HIGHER FOR TRUST IN OTHER } \\
\text { PEOPLE IN NZ }\end{array}$ & MAYBE & $\begin{array}{l}\text { COMMUNITY } \\
\text { DEVELOPMENT }\end{array}$ \\
\hline 55 & OUR FUTURE & SOCIAL CAPITAL & SOCIAL CAPITAL & TRUST IN GOVERNMENT INSTITUTIONS & $\begin{array}{l}\text { PERCENTAGE OF ADULTS AGED } 18 \text { AND OVER WHO,OVERALL,TRUST THE PUBLIC } \\
\text { SERVICE }\end{array}$ & NO & \\
\hline 56 & OUR FUTURE & HUMAN CAPITAL & HUMAN CAPITAL & COGNITIVE SKILLS AT AGE 15 & $\begin{array}{l}\text { PROGRAMME FOR INTERNATIONAL STUDENT ASSESSMENT (PISA) MEAN SCORE } \\
\text { FOR READING,MATHEMATICS AND SCIENCE }\end{array}$ & MAYBE & $\begin{array}{l}\text { NUMERACY AND } \\
\text { LITERACY CREDITS } \\
\text { AT NCEA LEVEL } 1\end{array}$ \\
\hline 57 & OUR FUTURE & HUMAN CAPITAL & HUMAN CAPITAL & $\begin{array}{l}\text { EDUCATIONAL ATTAINMENT OF THE ADULT } \\
\text { POPLUTION (UPPER SECONDARY) }\end{array}$ & $\begin{array}{l}\text { PERCENTAGE OF ADULTS AGED } 25-24 \text { WITH AT LEAST AN UPPER SECONDARY } \\
\text { EDUCATION }\end{array}$ & YES & \\
\hline 58 & OUR FUTURE & HUMAN CAPITAL & HUMAN CAPITAL & LIFE EXPERCTANCY & LIFE EXPERCTANCY AT BIRTH & YES & \\
\hline 59 & OUR FUTURE & HUMAN CAPITAL & HUMAN CAPITAL & NON-COMMUNICABLE & $\begin{array}{l}\text { HEALTH LOSS CAUSED BY NON-COMMUNICABLE DISEASES, MEASURED IN } \\
\text { DISABLITIY-ADJUSTED LIFE YEARS (DALYS)PER 100,000 PEOPLE }\end{array}$ & NO & \\
\hline 60 & OUR FUTURE & $\begin{array}{l}\text { FINANCIAL AND } \\
\text { PHYSICAL CAPITAL }\end{array}$ & FINANCIAL AND PHYSICAL CAPITAL & INVESTMENT IN R\&D & INVESMENT IN RESEARCH AND DEVELOPMENT (R\&D) AS A PERCENTAGE OF GDP & YES & \\
\hline 61 & OUR FUTURE & $\begin{array}{l}\text { FINANCIAL AND } \\
\text { PHYSICAL CAPITAL }\end{array}$ & FINANCIAL AND PHYSICAL CAPITAL & MULTI-FACTOR PRODUCTIVTY (MFP) & ANNUAL PERCENTAGE GROWTH IN MULTIFACTOR PRODUCTIVITY (MFP) & NO & \\
\hline 62 & OUR FUTURE & $\begin{array}{l}\text { FINANCIAL AND } \\
\text { PHYSICAL CAPITAL }\end{array}$ & FINANCIAL AND PHYSICAL CAPITAL & NET INTANGIBLE FIXED ASSETS & NET INTANGIBLE FIXED ASSETS PER CAPITA & MAYBE & $\begin{array}{l}\text { ASSET } \\
\text { MANAGEMENT }\end{array}$ \\
\hline 63 & OUR FUTURE & $\begin{array}{l}\text { FINANCIAL AND } \\
\text { PHYSICAL CAPITAL }\end{array}$ & FINANCIAL AND PHYSICAL CAPITAL & $\begin{array}{l}\text { NET INTERNATIONAL INVESTMENT } \\
\text { POSITION }\end{array}$ & NET INTERNATIONAL INVESTMENT POSITION AS A PERCENTAGE OF GDP & NO & \\
\hline 64 & OUR FUTURE & $\begin{array}{l}\text { FINANCIAL AND } \\
\text { PHYSICAL CAPITAL }\end{array}$ & FINANCIAL AND PHYSICAL CAPITAL & TOTAL CROWN NET WORTH & TOTAL CROWN NET WORTH AS A PERCENTAGE OF GDP & NO & \\
\hline 65 & OUR FUTURE & $\begin{array}{l}\text { FINANCIAL AND } \\
\text { PHYSICAL CAPITAL }\end{array}$ & FINANCIAL AND PHYSICAL CAPITAL & TOTAL NET FIXED ASSETS & NET FIXED ASSETS PER CAPITA & MAYBE & $\begin{array}{l}\text { ASSET } \\
\text { MANAGEMENT }\end{array}$ \\
\hline
\end{tabular}




\begin{tabular}{|c|c|c|c|c|c|c|}
\hline S.NO. & TYPE & TOPIC & INDICATOR & DEFINITION & AVAILABLE IN LTP & ALTERNATE DATA \\
\hline 1 & WELLBEING INDICATOR & SUBJECTIVE WELLBEING & ABILITY TO BE YOURSELF & $\begin{array}{l}\text { THIS INDICATOR IS ABOUT THE EXTENT TO WHICH PEOPLE FEEL ABLE TO EXPRESS THEIR IDENTITY. IT SHOWS } \\
\text { THE PERCENTAGE OF PEOPLE AGED } 15 \text { YEARS AND OVER WHO REPORTED THAT IT WAS EASY OR VERY EASY }\end{array}$ & YES & \\
\hline 2 & WELLBEING INDICATOR & $\begin{array}{l}\text { CITIES AND } \\
\text { SETTLEMENTS }\end{array}$ & ACCESS TO NATURAL SPACES & $\begin{array}{l}\text { THIS INDICATOR WILL SHOW HOW SATISFIED NEW ZEALANDERS ARE WITH THEIR ABILITY TO ACCESS } \\
\text { NATURAL SPACES IN AOTEAROA NEW ZEALAND. THE TERM 'NATURAL SPACES' COVERS AREAS SUCH AS } \\
\text { WATERFRONTS, PUBLIC PARKS AND RESERVES, AND OTHER GREEN SPACES. }\end{array}$ & YES & \\
\hline 3 & WELLBEING INDICATOR & LAND & ACTIVE STEWARDSHIP OF LAND & THIS INDICATOR WILL SHOW HOW NEW ZEALAND LAND AND THE RESOURCES OF THAT LAND ARE MANAGED & YES & \\
\hline 4 & WELLBEING INDICATOR & HEALTH & AMENABLE MORTALITY & $\begin{array}{l}\text { AMENABLE MORTALITY IS DEFINED AS PREMATURE DEATHS (DEATHS UNDER AGE 75) THAT COULD } \\
\text { POTENTIALLY BE AVOIDED GIVEN EFFECTIVE AND TIMELY HEALTHCARE. THAT IS, EARLY DEATHS FROM } \\
\text { CAUSES (DISEASES OR INJURIES) FOR WHICH EFFECTIVE HEALTH INTERVENTIONS EXIST AND ARE ACCESSIBLE } \\
\text { TO EVERYONE IN NEED (IN NEW ZEALAND). THE LIST OF AMENABLE CAUSES OF DEATH COMPRISES } 35 \\
\text { CONDITIONS, GROUPED INTO SIX CATEGORIES: INFECTIONS, MATERNAL AND INFANT CONDITIONS, } \\
\text { INJURIES, CANCERS, CARDIOVASCULAR DISEASES AND DIABETES, OTHER CHRONIC DISEASES. THIS INDICATOR } \\
\text { SHOWS THE AGE-STANDARDISED RATE OF NEW ZEALANDERS, UNDER } 75 \text { YEARS OF AGE, WHO DIED }\end{array}$ & MAYBE & DEATH RATE \\
\hline 5 & WELLBEING INDICATOR & ECOSYSTEMS & BIODIVERSITY / NATIVE SPECIES & THIS INDICATOR WILL SHOW THE STATE OF OUR NATIVE SPECIES POPULATIONS. & MAYBE & BIODIVERSITY \\
\hline 6 & WELLBEING INDICATOR & $\begin{array}{l}\text { ECONOMIC STANDARD } \\
\text { OF LIVING }\end{array}$ & CHILD POVERTY: MATERIAL HARDSHIP & $\begin{array}{l}\text { PLEASE BE AWARE THE DATA CURRENTLY AVAILABLE FOR THIS INDICATOR HAS SOME LIMITATIONS. IT WILL } \\
\text { BE UPDATED IN 202O. FOR MORE INFORMATION, SEE CHILD POVERTY STATISTICS RELEASED. THIS INDICATOR } \\
\text { SHOWS THE PERCENTAGE OF NEW ZEALAND CHILDREN LIVING IN HOUSEHOLDS THAT EXPERIENCE MATERIAL } \\
\text { HARDSHIP. THE TERM 'MATERIAL HARDSHIP' MEANS THAT THE HOUSEHOLD IS MISSING OUT ON A HANDFUL } \\
\text { OF THINGS THAT COULD BE EXPECTED IN A TYPICAL HOUSEHOLD, FOR EXAMPLE: DOING WITHOUT OR } \\
\text { CUTTING BACK ON TRIPS TO THE SHOPS OR OTHER LOCAL PLACES, EATING FRESH FRUIT OR VEGETABLES. } \\
\text { ALSO SEE CHILD POVERTY: LOW INCOME. }\end{array}$ & MAYBE & HOUSEHOLD INCOME \\
\hline 7 & WELLBEING INDICATOR & $\begin{array}{l}\text { ECONOMIC STANDARD } \\
\text { OF LIVING }\end{array}$ & CHILD POVERTY: LOW INCOME & $\begin{array}{l}\text { PLEASE BE AWARE THE DATA CURRENTLY AVAILABLE FOR THIS INDICATOR HAS SOME LIMITATIONS. IT WILL } \\
\text { BE UPDATED IN 2O2O. FOR MORE INFORMATION, SEE CHILD POVERTY STATISTICS RELEASED. THIS INDICATOR } \\
\text { SHOWS THE PERCENTAGE OF NEW ZEALAND CHILDREN LIVING IN HOUSEHOLDS THAT EXPERIENCE LOW } \\
\text { INCOME. THE TERM 'LOW INCOME' MEANS THE HOUSEHOLD HAS AN EQUIVALISED DISPOSABLE INCOME, } \\
\text { AFTER HOUSING COSTS, BELOW HALF OF THE NEW ZEALAND MEDIAN INCOME. ALSO SEE CHILD POVERTY: } \\
\text { MATERIAL HARDSHIP. }\end{array}$ & MAYBE & HOUSEHOLD INCOME \\
\hline 8 & WELLBEING INDICATOR & $\begin{array}{l}\text { CITIES AND } \\
\text { SETTLEMENTS }\end{array}$ & COMMUTING TIME TO WORK & $\begin{array}{l}\text { THIS INDICATOR WILL SHOW THE AVERAGE TIME NEW ZEALANDERS TAKE TO COMMUTE TO WORK, BY } \\
\text { DIFFERENT FORMS OF TRANSPORT. }\end{array}$ & YES & \\
\hline 9 & WELLBEING INDICATOR & NATURAL CAPITAL & QUALITY OF WATER RESOURCES & $\begin{array}{l}\text { THIS INDICATOR WILL SHOW THE QUALITY OF NEW ZEALAND'S WATER RESOURCES BY TYPE OF RESOURCE. } \\
\text { THE TERM 'WATER RESOURCES' MEANS SOURCES OF FRESH OR BRACKISH WATER IN INLAND WATER BODIES } \\
\text { THAT ARE, OR COULD BE, USEFUL FOR HUMANS. THEY INCLUDE GROUND WATER AND SOIL WATER. THE } \\
\text { TERM 'BRACKISH WATER' MEANS WATER THAT HAS SOME SALT CONCENTRATIONS, MAKING IT HAVE A } \\
\text { STATE BETWEEN FRESH AND SEA WATER. SUCH WATER RESOURCES ARE USEFUL FOR: AGRICULTURE, } \\
\text { INDUSTRY, HOUSEHOLD ACTIVITIES }\end{array}$ & YES & \\
\hline 10 & WELLBEING INDICATOR & CLIMATE & $\begin{array}{l}\text { CONSUMPTION-BASED GREENHOUSE } \\
\text { GAS EMISSIONS }\end{array}$ & $\begin{array}{l}\text { THIS INDICATOR ATTEMPTS TO ESTIMATE THE EMISSIONS RESULTING FROM THE ECONOMIC ACTIVITY (BOTH } \\
\text { FOREIGN AND DOMESTIC) REQUIRED TO MEET A NATION'S DEMAND FOR GOODS AND SERVICES, } \\
\text { REFLECTING THE IMPLICATIONS OF A NATION'S CONSUMPIION AND LIFESTYLE CHOICES. CONSUMPTION- } \\
\text { BASED EMISSIONS ARE OFTEN REFERRED TO AS A NATION'S CARBON FOOTPRINT. CONSUMPTION-BASED } \\
\text { EMISSIONS ARE DESIGNED TO PROVIDE A COMPLEMENTARY PERSPECTIVE ON NEW ZEALAND'S EMISSIONS } \\
\text { PROFILE BY SHIFTING THE LENS FROM PRODUCER TO CONSUMER. CONSUMPTION-BASED GREENHOUSE GAS } \\
\text { EMISSIONS ARE MEASURED IN KILOTONNES OF CARBON DIOXIDE EQUIVALENT (KT CO }{ }_{2}-\text { E), WHILE THE } \\
\text { CARBON FOOTPRINT IS MEASURED IN TONNES CO }{ }_{2} \text {-E PER CAPITA. }\end{array}$ & YES & \\
\hline 11 & WELLBEING INDICATOR & SOCIAL CONNECTIONS & $\begin{array}{l}\text { CONTACT WITH FAMILY/WHĀNAU AND } \\
\text { FRIENDS }\end{array}$ & $\begin{array}{l}\text { THIS INDICATOR WILL SHOW THE EXTENT TO WHICH NEW ZEALANDERS HAVE CONTACT WITH FRIENDS AND } \\
\text { FAMILY/WHĀNAU AND THE QUALITY OF THAT CONTACT. }\end{array}$ & MAYBE & $\begin{array}{l}\text { COMMUNITY } \\
\text { DEVELOPMENT }\end{array}$ \\
\hline 12 & WELLBEING INDICATOR & $\begin{array}{l}\text { KNOWLEDGE AND } \\
\text { SKILLS }\end{array}$ & $\begin{array}{l}\text { CORE COMPETENCIES (NON-COGNITIVE } \\
\text { SKILLS) }\end{array}$ & $\begin{array}{l}\text { THIS INDICATOR WILL SHOW THE EXTENT TO WHICH NEW ZEALANDERS POSSESS BASIC ABILITIES / LIFE } \\
\text { SKILLS. EXAMPLES OF SUCH COMPETENCIES INCLUDE THE ABILITY TO: COMMUNICATE CLEARLY MAKE }\end{array}$ & NO & \\
\hline
\end{tabular}




\begin{tabular}{|c|c|c|c|c|c|c|}
\hline S.NO. & TYPE & TOPIC & INDICATOR & DEFINITION & AVAILABLE IN LTP & ALTERNATE DATA \\
\hline 13 & WELLBEING INDICATOR & GOVERNANCE & CORRUPTION & $\begin{array}{l}\text { THIS INDICATOR SHOWS THE PERCEIVED LEVEL OF CORRUPTION IN NEW ZEALAND'S PUBLIC SECTOR. IT } \\
\text { SHOWS THE CORRUPTION LEVEL ON A SCALE OF O TO 100, WHERE O IS HIGHLY CORRUPT AND } 100 \text { IS VERY } \\
\text { CLEAN. THE CORRUPTION PERCEPTIONS INDEX (CPI) RANKS COUNTRIES BY THEIR PERCEIVED LEVELS OF } \\
\text { CORRUPTION, AS DETERMINED BY EXPERT ASSESSMENTS AND OPINION SURVEYS. }\end{array}$ & NO & \\
\hline 14 & WELLBEING INDICATOR & CLIMATE & COSTS OF EXTREME WEATHER EVENTS & THIS INDICATOR WILL SHOW THE FINANCIAL COSTS OF EXTREME WEATHER EVENTS ON NEW ZEALANDERS. & NO & \\
\hline 15 & WELLBEING INDICATOR & NATURAL CAPITAL & CULTURAL ECOSYSTEM SERVICES & $\begin{array}{l}\text { THIS INDICATOR WILL SHOW HOW MUCH NON-MONETARY BENEFIT NEW ZEALANDERS GET FROM OUR } \\
\text { WELL-FUNCTIONING NATURAL ECOSYSTEMS. SUCH BENEFITS COULD BE PHYSICAL, EMOTIONAL, OR MENTAL. } \\
\text { EXAMPLES OF BENEFITS COULD INCLUDE: SPIRITUAL (INCLUDING UPHOLDING NATURE IN RELIGIOUS } \\
\text { VALUES) } \\
\text { COGNITIVE DEVELOPMENT (INCLUDING SCIENTIFIC DISCOVERIES OR SCHOOL TRIPS TO EXPLORE NATURAL } \\
\text { SYSTEMS) RECREATIONAL (INCLUDING ECOTOURISM AND OUTDOOR SPORTS AND RECREATION)CUSTOMARY } \\
\text { (FOR EXAMPLE, MĀORI HARVESTING OF TITTIT (MUTTONBIRD)). }\end{array}$ & NO & \\
\hline 16 & WELLBEING INDICATOR & GOVERNANCE & DEMOCRATIC PARTICIPATION & $\begin{array}{l}\text { THIS INDICATOR WILL SHOW PEOPLE'S CONFIDENCE IN DEMOCRATIC PROCESSES, THE IMPORTANCE THEY } \\
\text { ATTACH TO THEM, AND WHETHER THEY FEEL THEIR PARTICIPATION CAN MAKE A DIFFERENCE. THIS } \\
\text { MEASURE STILL NEEDS TO BE DEVELOPED. AS A PROXY, THIS MEASURE SHOWS THE PERCENTAGE OF PEOPLE }\end{array}$ & YES & \\
\hline 17 & WELLBEING INDICATOR & POPULATION & DISABILITY STATUS & $\begin{array}{l}\text { THIS INDICATOR SHOWS THE PERCENTAGE OF DISABLED NEW ZEALANDERS BY AGE GROUP. A DISABLED } \\
\text { PERSON IS SOMEONE WHO EXPERIENCES ACTIVITY LIMITATIONS BECAUSE OF A FUNCTIONAL DIFFICULTY. }\end{array}$ & YES & \\
\hline 18 & WELLBEING INDICATOR & SAFETY & DOMESTIC VIOLENCE & $\begin{array}{l}\text { THIS INDICATOR SHOWS THE PERCENTAGE OF NEW ZEALANDERS AGED } 15 \text { YEARS AND OLDER WHO SAID } \\
\text { THEY WERE VICTIMS OF FAMILY VIOLENCE IN THE LAST } 12 \text { MONTHS, WHETHER THIS WAS REPORTED TO } \\
\text { POLICE OR NOT. THE TERM 'FAMILY VIOLENCE' (OFTEN REFERRED TO AS DOMESTIC VIOLENCE) IS DEFINED } \\
\text { AS VIOLENCE OR ABUSE OF ANY TYPE BY ONE FAMILY MEMBER AGAINST ANOTHER FAMILY MEMBER. IT } \\
\text { COVERS A BROAD RANGE OF CONTROLLING BEHAVIOURS, COMMONLY OF A PHYSICAL, SEXUAL, AND/OR } \\
\text { PSYCHOLOGICAL NATURE. IT INCLUDES: CHILD ABUSE/NEGLECT, PARTNER ABUSE/NEGLECT, ELDER } \\
\text { ABUSE/NEGLECT, PARENTAL ABUSE, SIBLING ABUSE. THIS DATA RELATES TO PEOPLE AGED } 15 \text { YEARS AND } \\
\text { OVER, SO DOES NOT CONTAIN ANY RESPONSES FROM CHILDREN IN RELATION TO FAMILY VIOLENCE. }\end{array}$ & YES & \\
\hline 19 & WELLBEING INDICATOR & $\begin{array}{l}\text { WATER AND } \\
\text { SANITATION }\end{array}$ & DRINKING-WATER QUALITY & $\begin{array}{l}\text { THIS INDICATOR SHOWS THE PROPORTION OF NEW ZEALAND'S POPULATION THAT HAS DRINKING-WATER } \\
\text { THAT MEETS THE REQUIRED STANDARDS OF CLEANLINESS. THIS DATA IS BASED ON ALL REGISTERED-WATER } \\
\text { SUPPLIES THAT SERVED POPULATIONS OF MORE THAN } 100 \text { PEOPLE. }\end{array}$ & YES & \\
\hline 20 & WELLBEING INDICATOR & $\begin{array}{l}\text { KNOWLEDGE AND } \\
\text { SKILLS }\end{array}$ & $\begin{array}{l}\text { EARLY CHILDHOOD EDUCATION } \\
\text { PARTICIPATION }\end{array}$ & $\begin{array}{l}\text { THIS INDICATOR WILL AIM TO MEASURE THE EXTENT OF PARTICIPATION IN, AND QUALITY OF, EARLY } \\
\text { CHILDHOOD EDUCATION (ECE) IN NEW ZEALAND. THIS IS ASSOCIATED WITH POSITIVE OUTCOMES IN THE } \\
\text { SHORT AND LONG TERM. THIS MEASURE STILL NEEDS TO BE DEVELOPED. AS A PROXY, WE HAVE } \\
\text { INFORMATION ON THE PERCENTAGE OF CHILDREN IN THEIR FIRST YEAR OF SCHOOL WHO HAVE REGULARLY }\end{array}$ & YES & \\
\hline 21 & WELLBEING INDICATOR & NATURAL CAPITAL & ECOLOGICAL INTEGRITY & THIS INDICATOR WILL SHOW HOW WELL WE ARE MAINTAINING COMMUNITIES OF DIFFERENT TYPES OF & MAYBE & BIODIVERSITY \\
\hline 22 & WELLBEING INDICATOR & $\begin{array}{l}\text { KNOWLEDGE AND } \\
\text { SKILLS }\end{array}$ & EDUCATION EQUITY & $\begin{array}{l}\text { THIS INDICATOR WILL SHOW THE SPREAD OF EDUCATIONAL OUTCOMES ACROSS THE NEW ZEALAND } \\
\text { POPULATION. IT WILL SHOW THE GAP BETWEEN HIGH AND LOW EDUCATIONAL ACHIEVEMENTS. }\end{array}$ & YES & \\
\hline 23 & WELLBEING INDICATOR & $\begin{array}{l}\text { KNOWLEDGE AND } \\
\text { SKILLS }\end{array}$ & EDUCATIONAL ATTAINMENT & $\begin{array}{l}\text { THIS INDICATOR SHOWS THE PERCENTAGE OF THE POPULATION AGED } 25 \text { YEARS AND OLDER WITH AT LEAST } \\
\text { AN UPPER SECONDARY SCHOOL QUALIFICATION. THIS INCLUDES EVERYONE WITH AN EDUCATIONAL } \\
\text { ATTAINMENT OF AT LEAST NCEA LEVEL 2, OR AN EQUIVALENT LEVEL OF EDUCATION. NOTE: DUE TO A } \\
\text { SURVEY REDEVELOPMENT IN THE JUNE } 2016 \text { QUARTER, ONLY DATA FROM THIS QUARTER ONWARDS IS } \\
\text { DISPLAYED ON THIS PAGE. REFER TO THE DOWNLOADABLE DATA FILE AND METADATA FOR INFORMATION }\end{array}$ & YES & \\
\hline 24 & WELLBEING INDICATOR & NATURAL CAPITAL & EFFICIENCY OF LAND USE & $\begin{array}{l}\text { THIS INDICATOR WILL SHOW THE EXTENT TO WHICH LAND AROUND NEW ZEALAND IS BEING USED FOR ITS } \\
\text { OPTIMAL OR MAIN PURPOSE. }\end{array}$ & YES & \\
\hline 25 & WELLBEING INDICATOR & WORK & EMPLOYMENT RATE & $\begin{array}{l}\text { THIS INDICATOR SHOWS THE SEASONALLY ADJUSTED PERCENTAGE OF NON-INSTITUTIONALISED NEW } \\
\text { ZEALANDERS AGED } 15 \text { YEARS AND OLDER WHO WERE EMPLOYED. }\end{array}$ & YES & \\
\hline
\end{tabular}




\begin{tabular}{|c|c|c|c|c|c|c|}
\hline S.NO & TYPE & TOPIC & INDICATOR & DEFINITION & AVAILABLE IN LTP & ALTERNATE DATA \\
\hline 26 & WELLBEING INDICATOR & NATURAL CAPITAL & ENERGY CONSUMPTION & $\begin{array}{l}\text { THIS INDICATOR SHOWS HOW MUCH ENERGY IS BEING USED IN NEW ZEALAND. IT COVERS ENERGY (IN } \\
\text { GROSS TERAJOULES (TJ)) USED BY THREE SECTORS OF THE NEW ZEALAND ECONOMY: PRIMARY, INDUSTRY }\end{array}$ & YES & \\
\hline 27 & WELLBEING INDICATOR & NATURAL CAPITAL & ENERGY INTENSITY & $\begin{array}{l}\text { THIS INDICATOR SHOWS THE AMOUNT OF ENERGY THAT IS NEEDED TO PRODUCE A MONETARY UNIT OF } \\
\text { NEW ZEALAND'S GROSS DOMESTIC PRODUCT (GDP). ENERGY INTENSITY PROVIDES AN INDICATION OF THE } \\
\text { RELATIONSHIP BETWEEN ENERGY USE AND ECONOMIC GROWTH. IT IS CALCULATED AS ENERGY USE DIVIDED } \\
\text { BY GROSS DOMESTIC PRODUCT (GDP) AND TELLS US THE AMOUNT OF ENERGY REQUIRED TO PRODUCE } \\
\text { EACH DOLLAR OF GDP. A FALL IN THE INDICATOR, WHERE LESS ENERGY IS REQUIRED TO PRODUCE EACH } \\
\text { DOLLAR OF GDP, IS VIEWED AS AN IMPROVEMENT. }\end{array}$ & MAYBE & $\begin{array}{l}\text { SUSTAINABLE ENERGY } \\
\text { USE }\end{array}$ \\
\hline 28 & WELLBEING INDICATOR & NATURAL CAPITAL & ENERGY RESOURCES & $\begin{array}{l}\text { THIS INDICATOR WILL SHOW THE STOCK OF RESOURCES AVAILABLE IN NEW ZEALAND TO GENERATE ENERGY. } \\
\text { FOR EXAMPLE, THE STOCK INCLUDES HYDRO, WIND, GEOTHERMAL, OIL, GAS, AND COAL. }\end{array}$ & NO & \\
\hline 29 & WELLBEING INDICATOR & CULTURE & ENGAGEMENT IN CULTURAL ACTIVITIES & $\begin{array}{l}\text { THIS INDICATOR WILL SHOW HOW MUCH TIME PEOPLE AGED } 15 \text { YEARS AND OLDER SPEND PARTICIPATING } \\
\text { IN TRADITIONAL CULTURAL EXPERIENCES, SUCH AS CELEBRATIONS OR ACTIVITIES. THIS MEASURE STILL } \\
\text { NEEDS TO BE DEVELOPED. } \\
\text { AS A PROXY, WE HAVE INFORMATION ON THE PERCENTAGE OF PEOPLE AGED } 15 \text { YEARS AND OLDER WHO } \\
\text { DID AT LEAST ONE OF THE FOLLOWING IN THE PREVIOUS } 12 \text { MONTHS: USED TE REO MĀORI PHRASES OR } \\
\text { WORDS WATCHED A MĀORI TELEVISION PROGRAMME SUCH AS TE KARERE OR CODE, PARTICIPATED IN } \\
\text { KAPA HAKA, SANG A MĀORI SONG, PERFORMED A HAKA, GAVE A MIHI OR SPEECH, OR TOOK PART IN MĀORI } \\
\text { PERFORMING ARTS OR CRAFTS, ATTENDED A MARAE. }\end{array}$ & YES & \\
\hline 30 & WELLBEING INDICATOR & SAFETY & EXPERIENCE OF DISCRIMINATION & $\begin{array}{l}\text { THIS INDICATOR SHOWS THE PERCENTAGE OF PEOPLE AGED } 15 \text { YEARS AND OVER WHO REPORTED BEING } \\
\text { SUBJECTED TO SOME FORM OF DISCRIMINATION IN THE LAST } 12 \text { MONTHS. REASONS FOR DISCRIMINATION }\end{array}$ & YES & \\
\hline 31 & WELLBEING INDICATOR & SUBJECTIVE WELLBEING & EXPERIENCED WELLBEING & $\begin{array}{l}\text { THIS INDICATOR WILL SHOW NEW ZEALANDERS' EMOTIONAL STATE OR FEELINGS AT A GIVEN POINT IN } \\
\text { TIME. IT WILL INCLUDE PEOPLE'S FEELINGS OF JOY AND SADNESS, CONTENTMENT AND FEAR, AND } \\
\text { CONFIDENCE AND ANXIETY. }\end{array}$ & MAYBE & MENTAL HEALTH \\
\hline 32 & WELLBEING INDICATOR & WASTE & EXPORT OF WASTE (NET AND GROSS) & $\begin{array}{l}\text { THIS INDICATOR WILL SHOW HOW MUCH OF THE WASTE PRODUCED IN NEW ZEALAND IS EXPORTED FOR } \\
\text { DISPOSAL. AS A PROXY, WE HAVE INFORMATION ON NET EXPORTS OF WASTE BY TYPE. } 11 \text { DECEMBER 2019: } \\
\text { WE UPDATED THE NET WASTE VALUES FOR SLAG AND WOOL WASTE. PREVIOUSLY THESE WERE SHOWN IN }\end{array}$ & MAYBE & WASTE GENERATED \\
\hline 33 & WELLBEING INDICATOR & SUBJECTIVE WELLBEING & FAMILY WELLBEING & $\begin{array}{l}\text { THIS IS ONE OF TWO MEASURES USED FOR THE INDICATOR FAMILY/WHĀNAU WELLBEING. THIS MEASURE } \\
\text { SHOWS THE PERCENTAGE OF PEOPLE } 15 \text { YEARS AND OVER WHO RATED HOW WELL THEIR FAMILY WAS } \\
\text { DOING AS } 7 \text { OR HIGHER ON A SCALE OF O TO 10, WHERE O IS EXTREMELY BADLY AND } 10 \text { IS EXTREMELY WELL. } \\
\text { THE CONCEPT OF FAMILY IN THIS MEASURE IS NOT NECESSARILY THE SAME AS THE CONCEPT OF WHĀNAU. } \\
\text { THE WHĀNAU WELLBEING MEASURE IS STILL BEING DEVELOPED IN PARTNERSHIP WITH MĀORI. }\end{array}$ & MAYBE & GREAT PLACE TO LIVE \\
\hline 34 & WELLBEING INDICATOR & NATURAL CAPITAL & FISH STOCKS & $\begin{array}{l}\text { THIS INDICATOR WILL AIM TO SHOW THE TOTAL FISH STOCKS THAT EXIST WITHIN NEW ZEALAND'S } \\
\text { EXCLUSIVE ECONOMIC ZONE (EEZ). THIS HELPS US TO UNDERSTAND THE HEALTH OF OUR MARINE } \\
\text { ENVIRONMENT AND HOW WE CAN SUSTAINABLY USE THE OCEAN AS A RESOURCE. THIS MEASURE STILL } \\
\text { NEEDS TO BE DEVELOPED. AS A PROXY, WE HAVE DATA ON TOTAL ALLOWABLE CATCH, WHICH INCLUDES } \\
\text { TOTAL ALLOWABLE COMMERCIAL CATCH (TACC), CUSTOMARY FISHING, AND RECREATIONAL FISHING. THIS } \\
\text { DATA IS LIMITED AS A PROXY AS IT REPRESENTS THE TOTAL AMOUNT THAT CAN BE TAKEN FROM EACH FISH } \\
\text { STOCK MANAGED UNDER NEW ZEALAND'S QUOTA MANAGEMENT SYSTEM, RATHER THAN A MEASURE OF } \\
\text { THE FISH STOCK ITSELF. THE DATA ONLY REPRESENTS THE SUBSET OF FISH SPECIES THAT ARE AVAILABLE FOR } \\
\text { COMMERCIAL, CUSTOMARY, AND RECREATIONAL FISHING. }\end{array}$ & NO & \\
\hline 35 & WELLBEING INDICATOR & $\begin{array}{l}\text { FINANCIAL AND } \\
\text { PHYSICAL CAPITAL }\end{array}$ & FOREIGN DIRECT INVESTMENT & $\begin{array}{l}\text { THIS INDICATOR SHOWS THE VALUE OF NEW ZEALAND'S OVERSEAS DIRECT INVESTMENT (ODI) AND } \\
\text { FOREIGN DIRECT INVESTMENT IN NEW ZEALAND (FDI). }\end{array}$ & NO & \\
\hline 36 & WELLBEING INDICATOR & SOCIAL CAPITAL & GENERALISED TRUST & $\begin{array}{l}\text { THIS INDICATOR SHOWS THE PERCENTAGE OF PEOPLE AGED } 15 \text { YEARS AND OVER WHO RATED THEIR TRUST } \\
\text { IN MOST OTHER NEW ZEALANDERS AT } 7 \text { OR HIGHER ON A SCALE OF } 0 \text { TO 10, WHERE O IS NOT TRUSTED AT }\end{array}$ & MAYBE & $\begin{array}{l}\text { COMMUNITY } \\
\text { DEVELOPMENT }\end{array}$ \\
\hline 37 & WELLBEING INDICATOR & NATURAL CAPITAL & $\begin{array}{l}\text { GLOBAL CARBON DIOXIDE } \\
\text { CONCENTRATIONS }\end{array}$ & $\begin{array}{l}\text { THIS INDICATOR SHOWS THE GLOBAL CONCENTRATIONS OF CARBON DIOXIDE (CO2) IN THE ATMOSPHERE. } \\
\text { THESE ARE EXPRESSED IN PARTS PER MILLION (PPM), WHICH IS THE NUMBER OF CO2 MOLECULES IN EVERY }\end{array}$ & NO & \\
\hline
\end{tabular}




\begin{tabular}{|c|c|c|c|c|c|c|}
\hline S.NO. & TYPE & TOPIC & INDICATOR & DEFINITION & AVAILABLE IN LTP & ALTERNATE DATA \\
\hline 38 & WELLBEING INDICATOR & NATURAL CAPITAL & GROSS GREENHOUSE GAS EMISSIONS & $\begin{array}{l}\text { THIS INDICATOR SHOWS NEW ZEALAND'S TOTAL GROSS GREENHOUSE GAS (GHG) EMISSIONS, IN } \\
\text { KILOTONNES OF CARBON DIOXIDE EQUIVALENT (KT CO2-E). GROSS GHG EMISSIONS ARE THE TOTAL } \\
\text { EMISSIONS FROM AGRICULTURE, ENERGY, INDUSTRIAL PROCESSES AND PRODUCT USE, AND WASTE. GROSS } \\
\text { GHG EMISSIONS DO NOT INCLUDE THE LAND USE, LAND-USE CHANGE AND FORESTRY (LULUCF) SECTOR. } \\
\text { GROSS GHG EMISSIONS ARE REPORTED IN 'CO2 EQUIVALENTS' SO THAT DIFFERENT GHGS CAN BE } \\
\text { CONSISTENTLY COMPARED WITH EACH OTHER AND ADDED TOGETHER. }\end{array}$ & YES & \\
\hline 39 & WELLBEING INDICATOR & SAFETY & HARM AGAINST CHILDREN & $\begin{array}{l}\text { THIS INDICATOR WILL SHOW THE EXTENT TO WHICH CHILDREN ARE MALTREATED IN NEW ZEALAND. THE } \\
\text { TERM 'MALTREATED' INCLUDES PHYSICAL, SEXUAL, EMOTIONAL, AND PSYCHOLOGICAL ABUSE, AS WELL AS } \\
\text { NEGLECT. }\end{array}$ & YES & \\
\hline 40 & WELLBEING INDICATOR & HEALTH & HEALTH EQUITY & $\begin{array}{l}\text { THIS INDICATOR WILL SHOW THE SPREAD OF HEALTH OUTCOMES ACROSS THE NEW ZEALAND POPULATION. } \\
\text { IT WILL SHOW THE GAP BETWEEN HIGH AND LOW HEALTH OUTCOMES. }\end{array}$ & MAYBE & HEALTH \\
\hline 41 & WELLBEING INDICATOR & HEALTH & HEALTH EXPECTANCY & $\begin{array}{l}\text { THIS INDICATOR SHOWS THE AVERAGE NUMBER OF YEARS FROM BIRTH A PERSON CAN EXPECT TO LIVE } \\
\text { WITHOUT REQUIRING ASSISTANCE WITH EVERYDAY ACTIVITIES. THE GLOBAL BURDEN OF DISEASE STUDY } \\
\text { (GBD) IS A COMPREHENSIVE REGIONAL AND GLOBAL RESEARCH PROGRAM OF DISEASE BURDEN THAT } \\
\text { ASSESSES MORTALITY AND DISABILITY FROM MAJOR DISEASES, INJURIES, AND RISK FACTORS. THE BURDEN } \\
\text { OF DISEASE CAN BE VIEWED AS THE GAP BETWEEN CURRENT HEALTH STATUS AND AN IDEAL SITUATION IN }\end{array}$ & NO & \\
\hline 42 & WELLBEING INDICATOR & $\begin{array}{l}\text { FINANCIAL AND } \\
\text { PHYSICAL CAPITAL }\end{array}$ & HERITAGE ASSETS & $\begin{array}{l}\text { THIS INDICATOR WILL SHOW THE NUMBER OF HERITAGE ASSETS IN NEW ZEALAND. THE TERM 'HERITAGE } \\
\text { ASSET’ MEANS ANY CULTURAL RESOURCE THAT CONTRIBUTES TO NATIONAL IDENTITY AND WELLBEING. } \\
\text { THESE COULD BE RESOURCES RECOGNISED IN DISTRICT PLANS AS PLACES WITH HERITAGE VALUE, OR } \\
\text { ACTIVELY MANAGED HISTORIC SITES, OR THEY COULD HAVE A RECOGNISED CULTURAL OR SPIRITUAL } \\
\text { SIGNIFICANCE TO LOCAL COMMUNITIES. THIS MEASURE STILL NEEDS TO BE DEVELOPED. AS A PROXY, WE } \\
\text { HAVE INFORMATION ON REGISTERED HISTORIC PLACES. }\end{array}$ & YES & \\
\hline 43 & WELLBEING INDICATOR & $\begin{array}{l}\text { CITIES AND } \\
\text { SETTLEMENTS }\end{array}$ & HOMELESSNESS & $\begin{array}{l}\text { THIS INDICATOR WILL SHOW THE STABILITY AND SECURITY OF NEW ZEALANDERS' ACCOMMODATION. IT } \\
\text { WILL FOCUS ON THE NUMBER OF PEOPLE IN NEW ZEALAND WHO DO NOT HAVE SAFE AND SECURE } \\
\text { ACCOMMODATION. }\end{array}$ & YES & \\
\hline 44 & WELLBEING INDICATOR & SUBJECTIVE WELLBEING & HOPE FOR THE FUTURE & THIS INDICATOR WILL SHOW NEW ZEALANDERS' EXPECTATIONS FOR THEIR FUTURE WELLBEING. & MAYBE & $\begin{array}{l}\text { SUSTAINABLE } \\
\text { DEVELOPMENT }\end{array}$ \\
\hline 45 & WELLBEING INDICATOR & WORK & HOURLY EARNINGS & $\begin{array}{l}\text { THIS INDICATOR SHOWS THE MEDIAN HOURLY EARNINGS FOR WAGE AND SALARY EMPLOYEES AGED } 15 \\
\text { YEARS AND OVER IN NEW ZEALAND. }\end{array}$ & YES & \\
\hline 46 & WELLBEING INDICATOR & $\begin{array}{l}\text { CITIES AND } \\
\text { SETTLEMENTS }\end{array}$ & HOUSING AFFORDABILITY & $\begin{array}{l}\text { THIS INDICATOR SHOWS HOW AFFORDABLE NEW ZEALANDERS' ACCOMMODATION IS, BASED ON THE } \\
\text { PROPORTION OF HOUSEHOLDS SPENDING MORE THAN } 30 \text { PERCENT OF THEIR TOTAL HOUSEHOLD } \\
\text { DISPOSABLE (AFTER TAX) INCOME ON HOUSING COSTS. DISPOSABLE INCOME IS CONSIDERED A BETTER } \\
\text { INCOME MEASURE THAN GROSS (BEFORE TAX) INCOME TO REPRESENT THE ECONOMIC RESOURCES } \\
\text { AVAILABLE TO MEET THE NEEDS OF HOUSEHOLDS. }\end{array}$ & YES & \\
\hline 47 & WELLBEING INDICATOR & $\begin{array}{l}\text { CITIES AND } \\
\text { SETTLEMENTS }\end{array}$ & HOUSING QUALITY & $\begin{array}{l}\text { THIS INDICATOR WILL SHOW THE PHYSICAL CONDITION OF ACCOMMODATION IN NEW ZEALAND. IT WILL } \\
\text { INCLUDE HOW HABITABLE THE ACCOMMODATION IS. }\end{array}$ & YES & \\
\hline 48 & WELLBEING INDICATOR & NATURAL CAPITAL & ILLNESS ATTRIBUTABLE TO AIR QUALITY & $\begin{array}{l}\text { THIS INDICATOR SHOWS THE HEALTH IMPACTS ON NEW ZEALANDERS FROM EXPOSURE TO AIR POLLUTANTS. } \\
\text { THE INDICATOR FOCUSES ON HOSPITAL ADMISSIONS THAT ARE THE RESULT OF EXPOSURE TO FINE } \\
\text { PARTICLES (IE PARTICLES LESS THAN } 2.5 \text { MICROMETRES IN DIAMETER, PM2. 5) FROM HUMAN-GENERATED } \\
\text { SOURCES, FOR EXAMPLE, BURNING WOOD FOR HOME HEATING. THIS MEASURE STILL NEEDS TO BE } \\
\text { DEVELOPED. CURRENTLY WE ARE USING A PROXY MEASURE THAT MODELS THE HEALTH IMPACTS OF PM10. }\end{array}$ & MAYBE & AIR QUALITY \\
\hline 49 & WELLBEING INDICATOR & $\begin{array}{l}\text { ECONOMIC STANDARD } \\
\text { OF LIVING }\end{array}$ & INCOME & $\begin{array}{l}\text { THIS INDICATOR SHOWS NEW ZEALAND'S MEDIAN REAL EQUIVALISED HOUSEHOLD DISPOSABLE INCOME } \\
\text { BEFORE HOUSING COSTS. DISPOSABLE INCOME IS WHAT IS LEFT OF A HOUSEHOLD'S TOTAL INCOME, AFTER } \\
\text { TAX, THAT IS AVAILABLE FOR SPENDING OR SAVING. DISPOSABLE INCOME IS CONSIDERED A BETTER INCOME } \\
\text { MEASURE THAN GROSS (BEFORE TAX) INCOME TO REPRESENT THE ECONOMIC RESOURCES AVAILABLE TO } \\
\text { MEET THE NEEDS OF HOUSEHOLDS. }\end{array}$ & YES & \\
\hline
\end{tabular}




\begin{tabular}{|c|c|c|c|c|c|c|}
\hline S.NO. & TYPE & TOPIC & INDICATOR & DEFINITION & AVAILABLE IN LTP & ALTERNATE DATA \\
\hline 50 & WELLBEING INDICATOR & $\begin{array}{l}\text { ECONOMIC STANDARD } \\
\text { OF LIVING }\end{array}$ & INCOME ADEQUACY & $\begin{array}{l}\text { THIS INDICATOR SHOWS THE PERCENTAGE OF NEW ZEALAND HOUSEHOLDS THAT REPORTED THEIR } \\
\text { HOUSEHOLD INCOME IS ENOUGH OR MORE THAN ENOUGH TO MEET THEIR EVERYDAY NEEDS. EVERYDAY } \\
\text { NEEDS RELATE TO ACCOMMODATION, FOOD, CLOTHING AND OTHER NECESSITIES. }\end{array}$ & YES & \\
\hline 51 & WELLBEING INDICATOR & $\begin{array}{l}\text { ECONOMIC STANDARD } \\
\text { OF LIVING }\end{array}$ & INCOME INEQUALITY & $\begin{array}{l}\text { THIS INDICATOR SHOWS THE RATIO OF HIGH HOUSEHOLD INCOMES (IN THE 8OTH PERCENTILE) TO LOW } \\
\text { HOUSEHOLD INCOMES (IN THE 2OTH PERCENTILE) BASED ON THE HOUSEHOLD EQUIVALISED DISPOSABLE } \\
\text { INCOME OF NEW ZEALANDERS. THE HIGHER THIS RATIO, THE GREATER THE LEVEL OF INEQUALITY. THE } \\
\text { DEGREE OF INCOME INEQUALITY IS OFTEN REGARDED AS AN IMPORTANT ASPECT OF THE FAIRNESS OF THE }\end{array}$ & NO & \\
\hline 52 & WELLBEING INDICATOR & $\begin{array}{l}\text { FINANCIAL AND } \\
\text { PHYSICAL CAPITAL }\end{array}$ & INFRASTRUCTURE & $\begin{array}{l}\text { THIS INDICATOR SHOWS THE QUANTITY AND QUALITY OF NEW ZEALAND'S PHYSICAL INFRASTRUCTURE. } \\
\text { PHYSICAL INFRASTRUCTURE RELATES TO THE FUNDAMENTAL STRUCTURES THAT ARE NECESSARY FOR AN } \\
\text { ECONOMY TO FUNCTION. THIS INCLUDES: TRANSPORT NETWORKS (INCLUDING ROAD, RAIL, AND AIR } \\
\text { OPTIONS), THE NATION'S POWER GRID, SEWERAGE AND WASTE DISPOSAL SYSTEMS, NATIONAL } \\
\text { COMMUNICATION SYSTEMS. }\end{array}$ & YES & \\
\hline 53 & WELLBEING INDICATOR & SAFETY & INJURY PREVALENCE & $\begin{array}{l}\text { THIS INDICATOR SHOWS THE PREVALENCE OF SERIOUS INJURIES IN NEW ZEALAND PER 100, } 000 \text { PEOPLE. THE } \\
\text { TERM 'SERIOUS INJURIES' INCLUDES FATAL AND NON-FATAL INJURIES. THE TERM 'SERIOUS NON-FATAL’ } \\
\text { REFERS TO PEOPLE WHO ARE ADMITTED TO HOSPITAL WITH A 6. } 9 \% \text { OR GREATER PROBABILITY OF DEATH. }\end{array}$ & YES & \\
\hline 54 & WELLBEING INDICATOR & GOVERNANCE & INSTITUTIONAL TRUST: PARLIAMENT & $\begin{array}{l}\text { THIS IS ONE OF TWO MEASURES USED FOR THE INDICATOR INSTITUTIONAL TRUST. THIS MEASURE SHOWS } \\
\text { THE PERCENTAGE OF PEOPLE AGED } 15 \text { YEARS AND OVER WHO RATED THEIR TRUST IN NEW ZEALAND'S } \\
\text { PARLIAMENT AT } 7 \text { OR HIGHER ON A SCALE OF O TO 10, WHERE O IS NO TRUST AND } 10 \text { IS COMPLETE TRUST. } \\
\text { SEE THE OTHER MEASURE INSTITUTIONAL TRUST: POLICE. }\end{array}$ & NO & \\
\hline 55 & WELLBEING INDICATOR & GOVERNANCE & INSTITUTIONAL TRUST: POLICE & $\begin{array}{l}\text { THIS IS ONE OF TWO MEASURES USED FOR THE INDICATOR INSTITUTIONAL TRUST. THIS MEASURE SHOWS } \\
\text { THE PERCENTAGE OF PEOPLE AGED } 15 \text { YEARS AND OVER WHO RATED THEIR TRUST IN THE POLICE AT } 7 \text { OR } \\
\text { HIGHER ON A SCALE OF } 0 \text { TO } 10 \text {, WHERE O IS NO TRUST AND } 10 \text { IS COMPLETE TRUST. SEE THE OTHER }\end{array}$ & NO & \\
\hline 56 & WELLBEING INDICATOR & CULTURE & $\begin{array}{l}\text { INTERGENERATIONAL TRANSFER OF } \\
\text { KNOWLEDGE }\end{array}$ & $\begin{array}{l}\text { THIS INDICATOR WILL SHOW THE EXTENT TO WHICH CULTURAL KNOWLEDGE IS TRANSFERRED BETWEEN } \\
\text { GENERATIONS OF NEW ZEALANDERS. CULTURAL KNOWLEDGE RELATES TO EVERYTHING WE KNOW THAT } \\
\text { CHARACTERISES A PARTICULAR CULTURE. FOR EXAMPLE, IT CAN INCLUDE INFORMATION THAT EXPLAINS } \\
\text { WHY PEOPLE FROM A PARTICULAR CULTURE FOLLOW PARTICULAR CUSTOMS OR HABITS. IT CAN COVER }\end{array}$ & MAYBE & $\begin{array}{l}\text { ARTS, CULTURE AND } \\
\text { EVENTS }\end{array}$ \\
\hline 57 & WELLBEING INDICATOR & $\begin{array}{l}\text { FINANCIAL AND } \\
\text { PHYSICAL CAPITAL }\end{array}$ & $\begin{array}{l}\text { NET INTERNATIONAL INVESTMENT } \\
\text { POSITION }\end{array}$ & $\begin{array}{l}\text { THIS INDICATOR SHOWS THE LEVEL OF NEW ZEALAND'S FINANCIAL INTERACTION WITH THE REST OF THE } \\
\text { WORLD AT A PARTICULAR POINT IN TIME THROUGH FINANCIAL ASSETS AND LIABILITIE }\end{array}$ & NO & \\
\hline 58 & WELLBEING INDICATOR & WORK & JOB SATISFACTION & $\begin{array}{l}\text { THIS INDICATOR SHOWS THE PERCENTAGE OF EMPLOYED PEOPLE AGED } 15 \text { YEARS AND OVER WHO FELT } \\
\text { SATISFIED OR VERY SATISFIED WITH THEIR MAIN JOB IN THE LAST FOUR WEEKS. }\end{array}$ & YES & \\
\hline 59 & WELLBEING INDICATOR & WORK & JOB STRAIN & $\begin{array}{l}\text { THIS INDICATOR WILL SHOW THE PROPORTION OF WORKERS WHO FEEL THAT THEIR JOBS CREATE STRESSES } \\
\text { THAT CAN BE DETRIMENTAL TO THEIR PHYSICAL AND MENTAL HEALTH AND WELLBEING. THESE STRESSES } \\
\text { ARE THE RESULT OF THE WORKERS NOT HAVING ENOUGH RESOURCES TO MEET THE DEMANDS OF THEIR }\end{array}$ & YES & \\
\hline 60 & WELLBEING INDICATOR & GOVERNANCE & JUSTICE EQUITY & $\begin{array}{l}\text { THIS INDICATOR WILL SHOW THE EXTENT TO WHICH NEW ZEALANDERS FEEL THEY ARE ABLE TO ACCESS FAIR } \\
\text { AND IMPARTIAL JUSTICE IN THEIR COMMUNITY. }\end{array}$ & NO & \\
\hline 61 & WELLBEING INDICATOR & NATURAL CAPITAL & LAND ASSETS & THIS INDICATOR WILL SHOW THE AMOUNT OF LAND IN NEW ZEALAND THAT IS COVERED IN NATIVE & YES & \\
\hline 62 & WELLBEING INDICATOR & IDENTITY & $\begin{array}{l}\text { LANGUAGE DEVELOPMENT AND } \\
\text { RETENTION }\end{array}$ & $\begin{array}{l}\text { THIS INDICATOR WILL SHOW NEW ZEALANDERS' ABILITY TO SPEAK THE FIRST LANGUAGE OF THEIR ETHNIC } \\
\text { GROUP. }\end{array}$ & YES & \\
\hline 63 & WELLBEING INDICATOR & LEISURE & LEISURE AND PERSONAL TIME & $\begin{array}{l}\text { THIS INDICATOR WILL SHOW THE AVERAGE NUMBER OF HOURS NEW ZEALANDERS HAVE AVAILABLE EACH } \\
\text { DAY AS FREE TIME AND FOR PERSONAL CARE. }\end{array}$ & MAYBE & $\begin{array}{l}\text { RECREATIONAL } \\
\text { ACTIVITIES }\end{array}$ \\
\hline 64 & WELLBEING INDICATOR & NATURAL CAPITAL & LEVELS OF POLLUTANTS: $\mathrm{NO}_{2}$ & $\begin{array}{l}\text { THIS INDICATOR SHOWS THE OBSERVED CONCENTRATIONS OF NITROGEN DIOXIDE (NO2) AT SELECTED NEW } \\
\text { ZEALAND MONITORING SITES THAT EXCEED THE DAILY AIR QUALITY STANDARDS. NO2 IS A GAS THAT IS } \\
\text { EMITTED PRIMARILY FROM BURNING FOSSIL FUELS, MAINLY BY MOTOR VEHICLES BUT ALSO IN INDUSTRIAL } \\
\text { PROCESSES AND HOME HEATING. IT IS A POLLUTANT OF CONCERN BECAUSE IT CAN AFFECT HUMAN AND } \\
\text { ECOSYSTEM HEALTH. IT CAN CAUSE RESPIRATORY PROBLEMS, PARTICULARLY ASTHMA }\end{array}$ & MAYBE & AIR QUALITY \\
\hline
\end{tabular}




\begin{tabular}{|c|c|c|c|c|c|c|}
\hline \multirow{2}{*}{\begin{tabular}{|c|} 
S.NO. \\
65
\end{tabular}} & TYPE & TOPIC & INDICATOR & DEFINITION & AVAILABLE IN LTP & ALTERNATE DATA \\
\hline & WELLBEING INDICATOR & NATURAL CAPITAL & LEVELS OF POLLUTANTS: PM 10 & $\begin{array}{l}\text { THIS INDICATOR WILL SHOW THE CONCENTRATIONS OF FINE PARTICLES (IE PARTICLES LESS THAN } 2.5 \\
\text { MICROMETRES IN DIAMETER, PM2. 5) AT SELECTED NEW ZEALAND MONITORING SITES THAT EXCEED THE } \\
\text { DAILY AIR QUALITY STANDARDS. THIS MEASURE STILL NEEDS TO BE DEVELOPED. CURRENTLY, WE ARE USING } \\
\text { A PROXY MEASURE THAT SHOWS THE CONCENTRATIONS OF PM1O AT SELECTED NEW ZEALAND } \\
\text { MONITORING SITES THAT EXCEED THE DAILY AIR QUALITY STANDARDS. PM1O IS A POLLUTANT OF CONCERN } \\
\text { BECAUSE IT CAN CAUSE RESPIRATORY INFECTIONS, AND REDUCE LUNG DEVELOPMENT AND FUNCTIONING. } \\
\text { PM1O IS OF PARTICULAR CONCERN BECAUSE IT IS FOUND IN HIGH CONCENTRATIONS IN SOME AREAS. THE } \\
\text { MAIN SOURCES OF PM1O ARE BURNING WOOD OR COAL FOR HOME HEATING AND SEA SPRAY. }\end{array}$ & YES & \\
\hline 66 & WELLBEING INDICATOR & SUBJECTIVE WELLBEING & LIFE SATISFACTION & $\begin{array}{l}\text { THIS INDICATOR SHOWS THE PERCENTAGE OF PEOPLE AGED } 15 \text { YEARS AND OVER WHO RATED THEIR } \\
\text { SATISFACTION WITH THEIR LIVES AT } 7 \text { OR HIGHER ON A SCALE OF } 0 \text { TO 10, WHERE } 0 \text { IS NOT AT ALL SATISFIED }\end{array}$ & MAYBE & GREAT PLACE TO LIVE \\
\hline 67 & WELLBEING INDICATOR & $\begin{array}{l}\text { KNOWLEDGE AND } \\
\text { SKILLS }\end{array}$ & $\begin{array}{l}\text { LITERACY, NUMERACY, AND SCIENCE } \\
\text { SKILLS OF 15-YEAR-OLDS }\end{array}$ & $\begin{array}{l}\text { THIS INDICATOR SHOWS THE LEVEL OF } 15 \text {-YEAR-OLD NEW ZEALAND STUDENTS' KNOWLEDGE AND SKILLS IN } \\
\text { READING, MATHS, AND SCIENCE. IT USES RESULTS FROM THE PROGRAMME FOR INTERNATIONAL STUDENT } \\
\text { ASSESSMENT (PISA) THREE-YEAR TEST. }\end{array}$ & MAYBE & $\begin{array}{l}\text { NUMERACY AND } \\
\text { LITERACY CREDITS AT } \\
\text { NCEA LEVEL } 1\end{array}$ \\
\hline 68 & WELLBEING INDICATOR & SUBJECTIVE WELLBEING & LOCUS OF CONTROL & $\begin{array}{l}\text { THIS INDICATOR WILL BE ABOUT THE EXTENT TO WHICH PEOPLE FEEL ABLE TO DIRECT THEIR OWN LIVES. IT } \\
\text { WILL SHOW THE PERCENTAGE OF PEOPLE AGED } 15 \text { YEARS AND OLDER WHO RATED THEIR LEVEL OF } \\
\text { CONTROL OVER THEIR LIVES AS } 7 \text { OR HIGHER ON A SCALE OF } 0 \text { TO 10, WHERE O IS NO CONTROL AND } 10 \text { IS } \\
\text { COMPLETE CONTROL. PLEASE NOTE: FURTHER RESEARCH IS REQUIRED AROUND THIS INDICATOR. }\end{array}$ & NO & \\
\hline 69 & WELLBEING INDICATOR & SOCIAL CONNECTIONS & LONELINESS & $\begin{array}{l}\text { THIS INDICATOR SHOWS THE PERCENTAGE OF PEOPLE AGED } 15 \text { YEARS AND OVER WHO REPORTED FEELING } \\
\text { LONELY SOME, MOST, OR ALL OF THE TIME IN THE LAST FOUR WEEKS. }\end{array}$ & MAYBE & MENTAL HEALTH \\
\hline 70 & WELLBEING INDICATOR & $\begin{array}{l}\text { ECONOMIC STANDARD } \\
\text { OF LIVING }\end{array}$ & LOW INCOME & $\begin{array}{l}\text { THIS INDICATOR SHOWS THE PERCENTAGE OF PEOPLE LIVING IN HOUSEHOLDS THAT HAVE AN EQUIVALISED } \\
\text { DISPOSABLE INCOME (AFTER HOUSING COSTS) OF LESS THAN 50\% OF MEDIAN HOUSEHOLD EQUIVALISED } \\
\text { DISPOSABLE INCOME (AFTER HOUSING COSTS). DISPOSABLE INCOME IS WHAT IS LEFT OF A HOUSEHOLD'S } \\
\text { TOTAL INCOME, AFTER TAX, THAT IS AVAILABLE FOR SPENDING OR SAVING. }\end{array}$ & YES & \\
\hline 71 & WELLBEING INDICATOR & WASTE & $\begin{array}{l}\text { MATERIAL INTENSITY (INCLUDING } \\
\text { RECYCLING, LAND-FILL INFLOWS, } \\
\text { SECOND-HAND ECONOMY) }\end{array}$ & $\begin{array}{l}\text { THIS INDICATOR WILL AIM TO MEASURE WHAT MATERIALS WE USE TO PRODUCE THINGS, WHAT MATERIALS } \\
\text { ARE LEFT OVER FROM THE PRODUCTION, AND WHAT WE DO WITH THE LEFTOVER MATERIALS (SUCH AS } \\
\text { RECYCLING THEM, SENDING THEM TO LANDFILLS, OR REUSING THEM). THIS MEASURE STILL NEEDS TO BE } \\
\text { DEVELOPED. AS A PROXY, WE EXPECT TO HAVE INFORMATION ON THE QUANTITY OF SOLID WASTE SENT TO }\end{array}$ & YES & \\
\hline 72 & WELLBEING INDICATOR & $\begin{array}{l}\text { ECONOMIC STANDARD } \\
\text { OF LIVING }\end{array}$ & MATERIAL WELLBEING & $\begin{array}{l}\text { THIS INDICATOR SHOWS NEW ZEALANDERS' ECONOMIC STANDARD OF LIVING USING A NON-MONETARY } \\
\text { INDICATOR CALLED THE MATERIAL WELLBEING INDEX (MWI). THE MWI IS MADE UP OF } 24 \text { ITEMS THAT EACH } \\
\text { GIVE INFORMATION ON THE DAY TO DAY LIVING CONDITIONS THAT HOUSEHOLDS EXPERIENCE. THEY ARE } \\
\text { ABOUT THE BASICS SUCH AS FOOD, CLOTHES, ACCOMMODATION, ELECTRICITY, TRANSPORT, KEEPING } \\
\text { WARM, MAINTAINING HOUSEHOLD APPLIANCES IN WORKING ORDER, AND SO ON, AND ALSO ABOUT THE } \\
\text { FREEDOMS HOUSEHOLDS REPORT TO PURCHASE AND CONSUME NON-ESSENTIALS THAT ARE COMMONLY } \\
\text { ASPIRED TO. THE MWI SCORE RANGES FROM O-35 WITH HIGHER SCORES SHOWING HIGHER MATERIAL } \\
\text { WELLBEING. THIS INDICATOR SHOWS THE PERCENTAGE OF PEOPLE LIVING IN HOUSEHOLDS THAT HAVE A } \\
\text { MATERIAL WELLBEING INDEX SCORE OF } 15 \text { OR MORE. }\end{array}$ & NO & \\
\hline 73 & WELLBEING INDICATOR & HEALTH & $\begin{array}{l}\text { MENTAL HEALTH STATUS } \\
\text { (PSYCHOLOGICAL DISTRESS) }\end{array}$ & $\begin{array}{l}\text { THIS INDICATOR SHOWS THE PERCENTAGE OF PEOPLE } 15 \text { AND OVER WHO EXPERIENCED HIGH OR VERY } \\
\text { HIGH LEVELS OF PSYCHOLOGICAL DISTRESS IN THE PAST FOUR WEEKS USING THE KESSLER PSYCHOLOGICAL } \\
\text { DISTRESS SCALE (K10). THE K10 SCALE MEASURES HOW OFTEN A PERSON EXPERIENCES NON- } \\
\text { SPECIFICPSYCHOLOGICAL DISTRESS, SUCH AS FEELING NERVOUS, DEPRESSED OR HOPELESS IN THE PAST } \\
\text { FOUR WEEKS. PEOPLE WHO RECEIVE A SCORE OF } 12 \text { OR MORE HAVE A HIGH PROBABILITY OF HAVING AN }\end{array}$ & YES & \\
\hline 74 & WELLBEING INDICATOR & $\begin{array}{l}\text { KNOWLEDGE AND } \\
\text { SKILLS }\end{array}$ & MIGRATION BY SKILL TYPE & $\begin{array}{l}\text { THIS INDICATOR WILL SHOW THE NET FLOWS OF MIGRANTS INTO AND OUT OF NEW ZEALAND BY SKILL TYPE. } \\
\text { NET MIGRATION IS THE DIFFERENCE BETWEEN THE NUMBER OF PEOPLE WHO ARRIVE IN A COUNTRY TO LIVE } \\
\text { THERE AND THE NUMBER OF PEOPLE WHO LEAVE THAT COUNTRY TO LIVE IN ANOTHER COUNTRY. }\end{array}$ & YES & \\
\hline
\end{tabular}




\begin{tabular}{|c|c|c|c|c|c|c|}
\hline S.NO. & TYPE & TOPIC & INDICATOR & DEFINITION & AVAILABLE IN LTP & ALTERNATE DATA \\
\hline 75 & WELLBEING INDICATOR & NATURAL CAPITAL & MINERAL RESOURCES & $\begin{array}{l}\text { THIS INDICATOR WILL AIM TO MEASURE WHAT MINERAL RESOURCES ARE AVAILABLE FOR USE IN NEW } \\
\text { ZEALAND. THIS COVERS MINERAL (E. G. OIL, GAS, COAL), METALLIC (E. G. GOLD, SILVER), AND NON-METALLIC } \\
\text { (E. G. LIMESTONE, AGGREGATE, IRONSAND, CLAYS) ENERGY RESOURCES. }\end{array}$ & MAYBE & $\begin{array}{l}\text { RESOURCE } \\
\text { MANAGEMENT ACT }\end{array}$ \\
\hline 76 & WELLBEING INDICATOR & $\begin{array}{l}\text { FINANCIAL AND } \\
\text { PHYSICAL CAPITAL }\end{array}$ & $\begin{array}{l}\text { MODIFIED LAND: FROM A NATURAL } \\
\text { STATE }\end{array}$ & $\begin{array}{l}\text { THIS INDICATOR WILL SHOW THE AREA OF LAND AROUND NEW ZEALAND THAT HAS BEEN CHANGED FROM } \\
\text { ITS NATURAL STATE (FOR EXAMPLE, TO BE USED FOR PRODUCTION OR IN URBAN EXPANSION). }\end{array}$ & YES & \\
\hline 77 & WELLBEING INDICATOR & $\begin{array}{l}\text { FINANCIAL AND } \\
\text { PHYSICAL CAPITAL }\end{array}$ & $\begin{array}{l}\text { MODIFIED LAND: FROM A PRODUCTIVE } \\
\text { STATE }\end{array}$ & $\begin{array}{l}\text { THIS INDICATOR WILL SHOW THE AREA OF LAND AROUND NEW ZEALAND THAT HAS BEEN CHANGED FROM A } \\
\text { PRODUCTIVE STATE TO ANOTHER STATE (FOR EXAMPLE, TO BE USED IN URBAN EXPANSION). }\end{array}$ & YES & \\
\hline 78 & WELLBEING INDICATOR & $\begin{array}{l}\text { FINANCIAL AND } \\
\text { PHYSICAL CAPITAL }\end{array}$ & MULTIFACTOR PRODUCTIVITY & $\begin{array}{l}\text { THIS INDICATOR SHOWS HOW EFFICIENTLY NEW ZEALAND'S PRODUCTION INPUTS ARE USED AFTER } \\
\text { REMOVING THE EFFECTS OF LABOUR AND CAPITAL. GROWTH IN PRODUCTIVITY IS AN IMPORTANT FACTOR } \\
\text { IN A NATION'S LONG-TERM MATERIAL STANDARD OF LIVING. MULTIFACTOR PRODUCTIVITY CAPTURES THE } \\
\text { EFFECTS OF UNOBVIOUS INPUTS, SUCH AS TECHNOLOGICAL PROGRESS, UNEXPECTED WEATHER EVENTS, } \\
\text { EFFICIENCY GAINS, AND ECONOMIES OF SCALE. }\end{array}$ & NO & \\
\hline 79 & WELLBEING INDICATOR & $\begin{array}{l}\text { FINANCIAL AND } \\
\text { PHYSICAL CAPITAL }\end{array}$ & NET FIXED ASSETS & $\begin{array}{l}\text { THIS INDICATOR SHOWS THE VALUE OF NEW ZEALAND'S TANGIBLE AND INTANGIBLE FIXED ASSETS, TAKING } \\
\text { INTO ACCOUNT DEPRECIATION AND OBSOLETE ASSETS. TANGIBLE FIXED ASSETS ARE ASSETS THAT HAVE A } \\
\text { PHYSICAL FORM. THEY INCLUDE: BUILDINGS, VEHICLES, EQUIPMENT, MACHINERY, HYDRO-ELECTRIC } \\
\text { CONSTRUCTION, ROADING, LAND, STOCKS AND BONDS. INTANGIBLE FIXED ASSETS ARE THE OPPOSITE OF } \\
\text { TANGIBLE ASSETS. THEY HAVE NO PHYSICAL FORM BUT REPRESENT POTENTIAL REVENUE. THEY INCLUDE: } \\
\text { INTELLECTUAL PROPERTY, OIL AND GAS EXPLORATION, COMPUTER SOFTWARE, RESEARCH AND } \\
\text { DEVELOPMENT. }\end{array}$ & MAYBE & $\begin{array}{l}\text { ASSET MANAGEMENT } \\
\text { PLAN }\end{array}$ \\
\hline 80 & WELLBEING INDICATOR & NATURAL CAPITAL & NET GREENHOUSE GAS EMISSIONS & $\begin{array}{l}\text { THIS INDICATOR SHOWS NEW ZEALAND’S TOTAL NET GREENHOUSE GAS (GHG) EMISSIONS, IN KILOTONNES } \\
\text { OF CARBON DIOXIDE EQUIVALENT (KT CO2-E). NET GHG EMISSIONS INCLUDE GROSS GHG EMISSIONS } \\
\text { COMBINED WITH THE EMISSIONS AND REMOVALS FROM LAND USE, LAND-USE CHANGE AND THE FORESTRY } \\
\text { (LULUCF) SECTOR. THERE IS A DISTINCTION BETWEEN GROSS GHG EMISSIONS AND NET GHG EMISSIONS } \\
\text { BECAUSE SOME INDUSTRIES IN THE LULUCF SECTOR BOTH EMIT AND REMOVE GHGS. NET GHG EMISSIONS } \\
\text { ARE REPORTED IN ‘CO2 EQUIVALENTS’ SO THAT DIFFERENT GREENHOUSE GASES CAN BE COMPARED WITH } \\
\text { EACH OTHER AND ADDED TOGETHER. }\end{array}$ & NO & \\
\hline 81 & WELLBEING INDICATOR & $\begin{array}{l}\text { ECONOMIC STANDARD } \\
\text { OF LIVING }\end{array}$ & NET WORTH & $\begin{array}{l}\text { THIS INDICATOR SHOWS THE MEDIAN NET WORTH OF NEW ZEALAND HOUSEHOLDS. IT SHOWS HOW MUCH } \\
\text { HOUSEHOLDS ARE WORTH, TAKING INTO ACCOUNT THE RESIDENTS' ASSETS (SUCH AS PROPERTY AND } \\
\text { SUPERANNUATION SCHEMES) AND ANY DEBTS (SUCH AS MORTGAGES) THEY HAVE. }\end{array}$ & MAYBE & FAMILY INCOME \\
\hline 82 & WELLBEING INDICATOR & WORK & $\begin{array}{l}\text { NOT IN EMPLOYMENT, EDUCATION, OR } \\
\text { TRAINING (NEET) }\end{array}$ & $\begin{array}{l}\text { THIS INDICATOR SHOWS THE PERCENTAGE OF YOUNG PEOPLE AGED } 15 \text { TO } 24 \text { YEARS WHO ARE NOT IN } \\
\text { EMPLOYMENT, EDUCATION, OR TRAINING. }\end{array}$ & YES & \\
\hline 83 & WELLBEING INDICATOR & NATURAL CAPITAL & OCEAN ACIDIFICATION & $\begin{array}{l}\text { THIS INDICATOR SHOWS THE LEVEL OF ACIDIFICATION OF NEW ZEALAND SUBANTARCTIC WATERS WITHIN } \\
\text { ITS EXCLUSIVE ECONOMIC ZONE. THE INDICATOR MEASURES THE PH LEVELS OF THE SURFACE WATER. A } \\
\text { DECREASING PH REPRESENTS INCREASING ACIDIFICATION OF THE OCEANS. }\end{array}$ & MAYBE & WATER QUALITY \\
\hline 84 & WELLBEING INDICATOR & $\begin{array}{l}\text { ECONOMIC STANDARD } \\
\text { OF LIVING }\end{array}$ & OFFICIAL DEVELOPMENT ASSISTANCE & $\begin{array}{l}\text { THIS INDICATOR WILL SHOW THE IMPACT OF OFFICIAL DEVELOPMENT ASSISTANCE FUNDING THAT NEW } \\
\text { ZEALAND PROVIDES TO DEVELOPING COUNTRIES, BY PURPOSE OF AID AND COUNTRY BEING FUNDED. THIS } \\
\text { MEASURE STILL NEEDS TO BE DEVELOPED. AS A PROXY, WE HAVE INFORMATION ON THE LEVEL OF OFFICIAL } \\
\text { DEVELOPMENT ASSISTANCE IN MILLION US DOLLARS. OFFICIAL DEVELOPMENT ASSISTANCE (ODA) IS } \\
\text { DEFINED AS GOVERNMENT AID DESIGNED TO PROMOTE THE ECONOMIC DEVELOPMENT AND WELFARE OF }\end{array}$ & NO & \\
\hline 85 & WELLBEING INDICATOR & $\begin{array}{l}\text { CITIES AND } \\
\text { SETTLEMENTS }\end{array}$ & OVERCROWDING & $\begin{array}{l}\text { THIS INDICATOR SHOWS HOW MANY NEW ZEALANDERS LIVE IN A CROWDED HOUSEHOLD. TYPICALLY } \\
\text { MEASURES OF CROWDING INVOLVE A CALCULATION OF THE NUMBER OF ROOMS OR BEDROOMS AVAILABLE } \\
\text { FOR THE NUMBER OF PEOPLE IN THE HOUSE. WE USE THE CANADIAN NATIONAL OCCUPANCY STANDARD } \\
\text { (CNOS). USING THIS STANDARD, A PERSON IS LIVING IN A CROWDED HOUSE IF ONE OR MORE EXTRA }\end{array}$ & YES & \\
\hline 86 & WELLBEING INDICATOR & SAFETY & FEELINGS OF SAFETY & $\begin{array}{l}\text { THIS INDICATOR SHOWS THE PERCENTAGE OF NEW ZEALANDERS AGED } 15 \text { YEARS AND OVER WHO } \\
\text { REPORTED FEELING SAFE OR VERY SAFE WALKING ALONE IN THEIR NEIGHBOURHOOD AFTER DARK. }\end{array}$ & YES & \\
\hline 87 & WELLBEING INDICATOR & NATURAL CAPITAL & PRODUCTIVE LAND & $\begin{array}{l}\text { THIS INDICATOR WILL SHOW THE AMOUNT OF PRODUCTIVE LAND IN NEW ZEALAND. THE TERM } \\
\text { 'PRODUCTIVE LAND' CAN RELATE TO ANY LAND THAT IS USED AS PASTURE, CROP, OR FORESTRY. }\end{array}$ & YES & \\
\hline
\end{tabular}




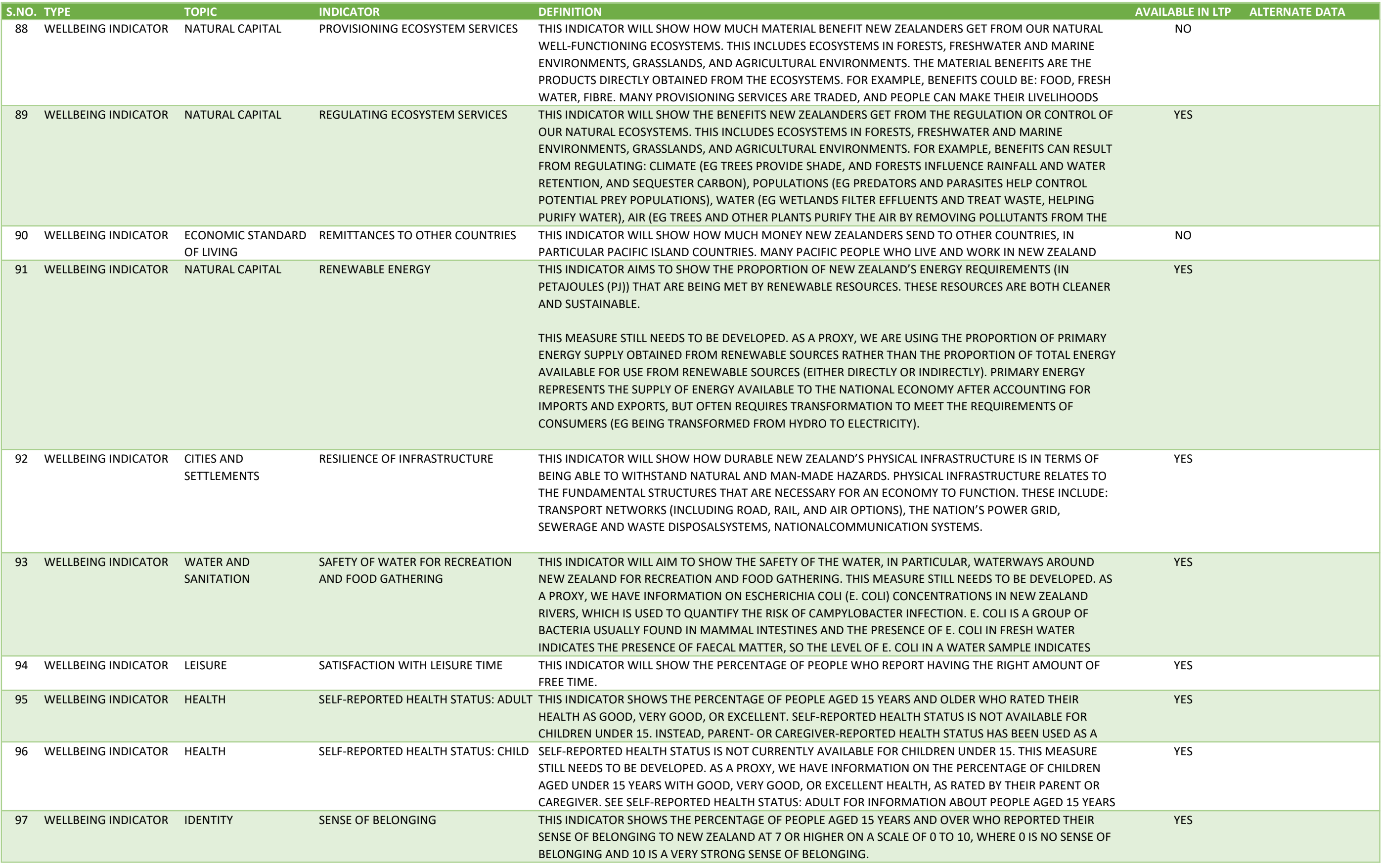




\begin{tabular}{|c|c|c|c|c|c|c|}
\hline S.NO. & TYPE & TOPIC & INDICATOR & DEFINITION & AVAILABLE IN LTP & ALTERNATE DATA \\
\hline 98 & WELLBEING INDICATOR & SUBJECTIVE WELLBEING & SENSE OF PURPOSE & $\begin{array}{l}\text { THIS INDICATOR IS ABOUT HOW WORTHWHILE PEOPLE THINK THE THINGS THEY DO IN LIFE ARE, REFLECTING } \\
\text { AN INDIVIDUAL'S SENSE OF PURPOSE. IT SHOWS THE PERCENTAGE OF PEOPLE AGED } 15 \text { YEARS AND OVER } \\
\text { WHO RATED HOW WORTHWHILE THEY FELT THE THINGS THEY DO ARE, AT } 7 \text { OR HIGHER ON A } 0 \text { TO } 10 \text { SCALE. } \\
\text { ON THIS SCALE O IS NOT AT ALL WORTHWHILE AND } 10 \text { IS COMPLETELY WORTHWHILE. }\end{array}$ & MAYBE & MENTAL HEALTH \\
\hline 99 & WELLBEING INDICATOR & SOCIAL CONNECTIONS & SOCIAL SUPPORT & $\begin{array}{l}\text { THIS INDICATOR SHOWS THE PERCENTAGE OF PEOPLE AGED } 15 \text { YEARS AND OVER WHO REPORTED IF THEY } \\
\text { URGENTLY NEEDED A PLACE TO STAY, IT WOULD BE EASY OR VERY EASY TO ASK SOMEONE THEY KNOW. }\end{array}$ & MAYBE & $\begin{array}{l}\text { CONNECTING WITH } \\
\text { CENTRAL }\end{array}$ \\
\hline 100 & WELLBEING INDICATOR & NATURAL CAPITAL & SOIL HEALTH & THIS INDICATOR WILL SHOW THE QUALITY OF DIFFERENT SOILS AROUND NEW ZEALAND. & YES & \\
\hline 101 & WELLBEING INDICATOR & HEALTH & SPIRITUAL HEALTH & $\begin{array}{l}\text { THIS INDICATOR WILL SHOW HOW CONTENT PEOPLE ARE WITH THEIR SPIRITUAL HEALTH. AMONG OTHER } \\
\text { THINGS, SPIRITUAL HEALTH ENCOMPASSES: UNSEEN AND UNSPOKEN ENERGIES, FAITH, SPIRITUAL }\end{array}$ & MAYBE & $\begin{array}{l}\text { COUNCIL SUPPORT TO } \\
\text { ETHINIC GROUPS }\end{array}$ \\
\hline 102 & WELLBEING INDICATOR & NATURAL CAPITAL & STOCK OF FRESHWATER RESOURCES & $\begin{array}{l}\text { THIS INDICATOR WILL SHOW THE AMOUNT OF CHANGE IN NEW ZEALAND'S STORES OF FRESHWATER } \\
\text { RESOURCES. THE TERM 'FRESHWATER RESOURCES' INCLUDES WATERWAYS SUCH AS STREAMS, RIVERS, } \\
\text { AQUIFERS, AND LAKES THAT PROVIDE OPTIONS FOR FOOD, AS WELL AS RECREATIONAL AND CUSTOMARY } \\
\text { USES. }\end{array}$ & YES & \\
\hline 103 & WELLBEING INDICATOR & HEALTH & SUICIDE & $\begin{array}{l}\text { THIS INDICATOR SHOWS THE PREVALENCE OF DEATHS CAUSED BY SELF-HARM IN NEW ZEALAND PER 100, } \\
000 \text { POPULATION. DATA FOR THE MOST RECENT YEAR ARE PROVISIONAL. }\end{array}$ & YES & \\
\hline 104 & WELLBEING INDICATOR & CULTURE & TE REO MĀORI SPEAKERS & $\begin{array}{l}\text { THIS INDICATOR SHOWS THE PERCENTAGE OF PEOPLE WHO REPORTED BEING ABLE TO TALK ABOUT } \\
\text { EVERYDAY THINGS IN TE REO MĀORI. TE REO MĀORI IS THE INDIGENOUS LANGUAGE OF AOTEAROA NEW } \\
\text { ZEALAND. THE LANGUAGE IS CENTRAL TO MĀORI CULTURE AND IDENTITY, AND FORMS PART OF THE } \\
\text { HERITAGE OF OUR COUNTRY. }\end{array}$ & YES & \\
\hline 105 & WELLBEING INDICATOR & WORK & UNDERUTILISATION & $\begin{array}{l}\text { THIS INDICATOR SHOWS THE NUMBER OF PEOPLE AGED } 15 \text { YEARS AND OLDER IN THE EXTENDED LABOUR } \\
\text { FORCE WHO ARE: UNEMPLOYED, UNDEREMPLOYED (PEOPLE WHO WORK PART-TIME AND WANT TO AND } \\
\text { CAN WORK MORE HOURS), POTENTIAL LABOUR FORCE (PEOPLE WHO WANT A JOB AND ARE AVAILABLE TO } \\
\text { WORK, BUT ARE NOT CURRENTLY LOOKING FOR A JOB AND PEOPLE WHO ARE UNAVAILABLE TO START }\end{array}$ & YES & \\
\hline 106 & WELLBEING INDICATOR & WORK & UNEMPLOYMENT & $\begin{array}{l}\text { THIS INDICATOR SHOWS THE PERCENTAGE OF PEOPLE IN THE NEW ZEALAND LABOUR FORCE OVER THE AGE } \\
\text { OF } 15 \text { YEARS WHO ARE UNEMPLOYED OVER A SINGLE REFERENCE WEEK. THIS INCLUDES PEOPLE WHO: } \\
\text { WERE NOT IN EMPLOYMENT, WERE AVAILABLE FOR WORK, HAD EITHER ACTIVELY SOUGHT WORK IN THE } \\
\text { PAST FOUR WEEKS ENDING WITH THE REFERENCE WEEK, OR WOULD BE STARTING A NEW JOB WITHIN THE }\end{array}$ & YES & \\
\hline 107 & WELLBEING INDICATOR & $\begin{array}{l}\text { ECONOMIC STANDARD } \\
\text { OF LIVING }\end{array}$ & VALUE OF UNPAID WORK & $\begin{array}{l}\text { THIS INDICATOR WILL SHOW THE MONETARY VALUE OF UNPAID WORK UNDERTAKEN IN NEW ZEALAND IN: } \\
\text { THE WORKER'S OWN HOUSEHOLD, ANOTHER HOUSEHOLD, A NOT-FOR-PROFIT ORGANISATION. }\end{array}$ & MAYBE & $\begin{array}{l}\text { UNPAID AND } \\
\text { VOLUNTARY WORK }\end{array}$ \\
\hline 108 & WELLBEING INDICATOR & SAFETY & VICTIMISATION & $\begin{array}{l}\text { THIS INDICATOR SHOWS THE PERCENTAGE OF NEW ZEALANDERS AGED } 15 \text { YEARS AND OLDER WHO SAID } \\
\text { THEY HAD A CRIME COMMITTED AGAINST THEM IN THE LAST } 12 \text { MONTHS. }\end{array}$ & YES & \\
\hline 109 & WELLBEING INDICATOR & SOCIAL CAPITAL & VOLUNTEERING & $\begin{array}{l}\text { THIS INDICATOR WILL SHOW HOW MUCH TIME PEOPLE SPEND DOING VOLUNTARY WORK FOR A GROUP OR } \\
\text { ORGANISATION. THIS MEASURE STILL NEEDS TO BE DEVELOPED. AS A PROXY, WE HAVE INFORMATION ON } \\
\text { THE PERCENTAGE OF PEOPLE AGED } 15 \text { YEARS AND OVER WHO REPORTED HAVING DONE VOLUNTARY WORK } \\
\text { FOR AT LEAST ONE ORGANISATION OR DIRECTLY FOR A PERSON FROM ANOTHER HOUSEHOLD IN THE }\end{array}$ & YES & \\
\hline 110 & WELLBEING INDICATOR & WASTE & $\begin{array}{l}\text { WASTE FLOWS IN WATERWAYS AND } \\
\text { COASTAL MARINE ENVIRONMENTS }\end{array}$ & $\begin{array}{l}\text { THIS INDICATOR WILL SHOW THE AMOUNT OF WASTE DISCHARGED INTO WATERWAYS AND COASTAL AREAS } \\
\text { AROUND NEW ZEALAND EACH YEAR. THE TERM 'WASTE' INCLUDES CHEMICALS, SEWAGE, AND SOLID } \\
\text { WASTE. }\end{array}$ & YES & \\
\hline 111 & WELLBEING INDICATOR & NATURAL CAPITAL & WASTE GENERATION & THIS INDICATOR WILL SHOW THE AMOUNT OF WASTE PRODUCED IN NEW ZEALAND. & YES & \\
\hline 112 & WELLBEING INDICATOR & NATURAL CAPITAL & WATER STRESS & THIS INDICATOR WILL SHOW THE AMOUNT OF FRESH WATER TAKEN FROM THE AVAILABLE FRESHWATER & YES & \\
\hline 113 & WELLBEING INDICATOR & WORK & WORK/LIFE BALANCE & $\begin{array}{l}\text { THIS INDICATOR SHOWS THE PERCENTAGE OF EMPLOYED NEW ZEALANDERS AGED } 15 \text { YEARS AND OLDER } \\
\text { WHO ARE SATISFIED OR VERY SATISFIED WITH THE BALANCE BETWEEN THEIR WORKING LIFE AND LIFE }\end{array}$ & YES & \\
\hline 114 & WELLBEING INDICATOR & WORK & WORKPLACE ACCIDENTS & $\begin{array}{l}\text { THIS INDICATOR WILL SHOW WORK-RELATED INJURIES PER 1, } 000 \text { FULL-TIME EQUIVALENT EMPLOYEES. THIS } \\
\text { MEASURE STILL NEEDS TO BE DEVELOPED. AS A PROXY WE HAVE INFORMATION ON THE RATE OF PEOPLE } \\
\text { PER 1, 000 IN THE EQUIVALENT OF FULL-TIME WORK (IE FULL-TIME EQUIVALENT EMPLOYEES, FTES) WHO } \\
\text { HAVE HAD A CLAIM ACCEPTED FOR A WORK-RELATED INJURY. IT IS BASED ON WORKPLACE CLAIMS TO THE } \\
\text { ACCIDENT COMPENSATION CORPORATION (ACC). CURRENTLY, THIS IS THE BEST MEASURE AVAILABLE FOR }\end{array}$ & YES & \\
\hline
\end{tabular}




\begin{tabular}{|c|c|c|c|c|c|c|}
\hline S.NO. & TYPE & TOPIC & INDICATOR & DEFINITION & AVAILABLE IN LTP & ALTERNATE DATA \\
\hline 115 & CONTEXT INDICATOR & POPULATION & AGE AND SEX STRUCTURE & $\begin{array}{l}\text { THIS INDICATOR SHOWS THE PERCENTAGE DISTRIBUTION OF THE ESTIMATED RESIDENT POPULATION OF } \\
\text { NEW ZEALAND, BY SEX AND FIVE-YEAR AGE GROUPS. }\end{array}$ & YES & \\
\hline 116 & CONTEXT INDICATOR & PRODUCTION & COMPONENTS OF FINAL USE & $\begin{array}{l}\text { THIS INDICATOR SHOWS THE LEVEL OF EXPENDITURE ON PRODUCTIVE ACTIVITY UNDERTAKEN IN NEW } \\
\text { ZEALAND, AS MEASURED BY THE EXPENDITURE APPROACH TO GROSS DOMESTIC PRODUCT (GDP). }\end{array}$ & NO & \\
\hline 117 & CONTEXT INDICATOR & POPULATION & DISABILITY STATUS & $\begin{array}{l}\text { THIS INDICATOR SHOWS THE PERCENTAGE OF DISABLED NEW ZEALANDERS BY AGE GROUP. A DISABLED } \\
\text { PERSON IS SOMEONE WHO EXPERIENCES ACTIVITY LIMITATIONS BECAUSE OF A FUNCTIONAL DIFFICULTY. }\end{array}$ & NO & \\
\hline 118 & CONTEXT INDICATOR & POPULATION & ETHNIC COMPOSITION & THIS INDICATOR SHOWS THE PERCENTAGE DISTRIBUTION OF THE TOTAL POPULATION IN NEW ZEALAND BY & YES & \\
\hline 119 & CONTEXT INDICATOR & POPULATION & FAMILY COMPOSITION & $\begin{array}{l}\text { THIS INDICATOR SHOWS THE PERCENTAGE DISTRIBUTION OF THE NEW ZEALAND POPULATION BY FAMILY } \\
\text { TYPE. THE TERM 'FAMILY TYPE' MEANS TWO OR MORE PEOPLE LIVING IN THE SAME HOUSEHOLD WHO ARE } \\
\text { ONE OF THE FOLLOWING: COUPLE WITHOUT CHILDREN, COUPLE WITH CHILD(REN), ONE PARENT WITH } \\
\text { CHILD(REN). }\end{array}$ & YES & \\
\hline 120 & CONTEXT INDICATOR & POPULATION & FERTILITY & THIS INDICATOR SHOWS THE NUMBER OF LIVE BIRTHS, AND BIRTH RATES, OF NEW ZEALAND WOMEN. & YES & \\
\hline 121 & CONTEXT INDICATOR & POPULATION & GEOGRAPHIC DISTRIBUTION & THIS INDICATOR SHOWS THE DISTRIBUTION OF THE ESTIMATED RESIDENT POPULATION BY REGIONAL & YES & \\
\hline 122 & CONTEXT INDICATOR & POPULATION & HOUSEHOLD COMPOSITION & $\begin{array}{l}\text { THIS INDICATOR SHOWS THE PERCENTAGE DISTRIBUTION OF THE NEW ZEALAND POPULATION BY } \\
\text { HOUSEHOLD COMPOSITION. HOUSEHOLD COMPOSITION CLASSIFIES HOUSEHOLDS ACCORDING TO THE } \\
\text { RELATIONSHIPS BETWEEN PEOPLE IN HOUSEHOLDS: ONE-FAMILY HOUSEHOLD (WITH OR WITHOUT OTHER } \\
\text { PEOPLE), TWO-FAMILY HOUSEHOLD (WITH OR WITHOUT OTHER PEOPLE), THREE OR MORE FAMILY }\end{array}$ & YES & \\
\hline 123 & CONTEXT INDICATOR & POPULATION & MIGRATION & $\begin{array}{l}\text { THIS INDICATOR SHOWS THE CONTRIBUTION OF INTERNATIONAL MIGRATION TO NEW ZEALAND'S RESIDENT } \\
\text { POPULATION. NET MIGRATION IS THE DIFFERENCE BETWEEN THE NUMBER OF PEOPLE WHO ARRIVE IN NEW } \\
\text { ZEALAND TO LIVE AND THE NUMBER OF PEOPLE WHO LEAVE TO LIVE IN ANOTHER COUNTRY. MIGRATION } \\
\text { ESTIMATES FOR THE MOST RECENT 16-MONTHS ARE PROVISIONAL. PROVISIONAL MIGRATION ESTIMATES } \\
\text { ARE REVISED EACH MONTH UNTIL FINALISED ABOUT } 17 \text { MONTHS AFTER THE REFERENCE PERIOD. } \\
\text { MIGRATION DATA TRANSFORMATION PROJECT HAS MORE INFORMATION ABOUT THE MIGRATION } \\
\text { ESTIMATES. }\end{array}$ & YES & \\
\hline 124 & CONTEXT INDICATOR & PRODUCTION & NATIONAL INCOME & $\begin{array}{l}\text { THIS INDICATOR SHOWS THE INCOME RECEIVED (LESS INCOME PAYABLE) BY NEW ZEALAND RESIDENTS } \\
\text { FROM BOTH DOMESTIC AND OVERSEAS SOURCES. THIS TAKES INTO ACCOUNT INCOME REDISTRIBUTED } \\
\text { THROUGH INTERNATIONAL TRANSFERS OR GROSS NATIONAL INCOME PLUS NET INTERNATIONAL }\end{array}$ & NO & \\
\hline 125 & CONTEXT INDICATOR & POPULATION & OVERSEAS-BORN POPULATION & $\begin{array}{l}\text { THIS INDICATOR SHOWS THE PERCENTAGE OF NEW ZEALAND'S POPULATION WHO WERE BORN OVERSEAS } \\
\text { AND THEIR DISTRIBUTION WITHIN AGE GROUPS AND REGIONS OF NEW ZEALAND. IT ALSO SHOWS THE } \\
\text { PERCENTAGE OF THE OVERSEAS-BORN POPULATION BY THEIR GLOBAL REGION OF BIRTH AND ETHNIC } \\
\text { GROUP. }\end{array}$ & MAYBE & ETHNIC POPULATION \\
\hline 126 & CONTEXT INDICATOR & POPULATION & POPULATION SIZE & THIS INDICATOR SHOWS THE TOTAL ESTIMATED SIZE OF NEW ZEALAND'S RESIDENT POPULATION. & YES & \\
\hline 127 & CONTEXT INDICATOR & PRODUCTION & PRODUCTION BY INDUSTRY & $\begin{array}{l}\text { THIS INDICATOR SHOWS THE LEVEL OF PRODUCTIVE ACTIVITY UNDERTAKEN IN NEW ZEALAND. IT IS TAKEN } \\
\text { FROM A VALUE-ADDED APPROACH TO GROSS DOMESTIC PRODUCT (GDP) BY INDUSTRY AND CONSTANT }\end{array}$ & MAYBE & $\begin{array}{l}\text { GROSS DOMESTIC } \\
\text { PRODUCT }\end{array}$ \\
\hline 128 & CONTEXT INDICATOR & PRODUCTION & REGIONAL PRODUCTION & $\begin{array}{l}\text { THIS INDICATOR SHOWS THE LEVEL OF PRODUCTIVE ACTIVITY UNDERTAKEN IN NEW ZEALAND BY INDUSTRY } \\
\text { FOR EACH GEOGRAPHIC REGION (AS MEASURED BY GROSS DOMESTIC PRODUCT (GDP)). }\end{array}$ & MAYBE & $\begin{array}{l}\text { GROSS DOMESTIC } \\
\text { PRODUCT }\end{array}$ \\
\hline 129 & CONTEXT INDICATOR & PRODUCTION & RETURNS TO FACTORS OF PRODUCTION & $\begin{array}{l}\text { THIS INDICATOR SHOWS THE LEVEL OF PRODUCTIVE ACTIVITY UNDERTAKEN IN NEW ZEALAND BY RETURNS } \\
\text { ON ALL THE FACTORS OF PRODUCTION IN THE ECONOMY (WAGES AND PROFITS). }\end{array}$ & NO & \\
\hline 130 & CONTEXT INDICATOR & POPULATION & SEXUAL IDENTITY & $\begin{array}{l}\text { THIS INDICATOR WILL SHOW THE DISTRIBUTION OF THE NEW ZEALAND POPULATION BY SEXUAL IDENTITY. } \\
\text { THE TERM 'SEXUAL IDENTITY' RELATES TO HOW A PERSON THINKS OF THEIR OWN SEXUALITY AND WHICH } \\
\text { TERMS THEY IDENTIFY WITH. AMONG OTHERS, SEXUAL IDENTITY TERMS INCLUDE: LESBIAN, GAY, STRAIGHT, } \\
\text { ASEXUAL, TAKATĀPUI, BISEXUAL, PANSEXUAL. SEE MORE INFORMATION ON THE STATISTICAL STANDARD } \\
\text { SEXUAL IDENTITY AND SEXUAL ORIENTATION (FRAMEWORK). }\end{array}$ & NO & \\
\hline 131 & CONTEXT INDICATOR & POPULATION & GENDER & $\begin{array}{l}\text { THIS INDICATOR WILL SHOW THE DISTRIBUTION OF THE NEW ZEALAND POPULATION BY GENDER. STATS NZ } \\
\text { IS CURRENTLY CONSULTING ON A PROPOSED UPDATE TO THE DEFINITION OF 'GENDER'. FOR MORE } \\
\text { INFORMATION, SEE SEX AND GENDER IDENTITY STATISTICAL STANDARDS: CONSULTATION. }\end{array}$ & YES & \\
\hline
\end{tabular}


Appendix D: Coverage of Community Well-being Measures in Indicators Aotearoa New Zealand

\begin{tabular}{lll} 
S.NO. TYPE & TOPIC & INDICATOR \\
\hline 132 CONTEXT INDICATOR & PRODUCTION & TOTAL NEW ZEALAND PRODUCTION \\
& &
\end{tabular}
DEFINITION

THIS INDICATOR SHOWS THE LEVEL OF PRODUCTIVE ACTIVITY UNDERTAKEN IN NEW ZEALAND (AS

MEASURED BY GROSS DOMESTIC PRODUCT (GDP)) FROM A VALUE-ADDED APPROACH BY CONSTANT PRICES.

AVAILABLE IN LTP ALTERNATE DATA

HIS INDICATOR SHOWS THE PERCENTAGE DISTRIBUTION OF THE TOTAL POPULATION BY URBAN/RURAL

MAYBE

GROSS DOMESTIC

URBAN/RURAL DISTRIBUTION

LOCATION. FOR A DEFINITION OF URBAN AND RURAL, SEE DEFINING URBAN AND RURAL NEW ZEALAND.

PRODUC

YES

PRODU

